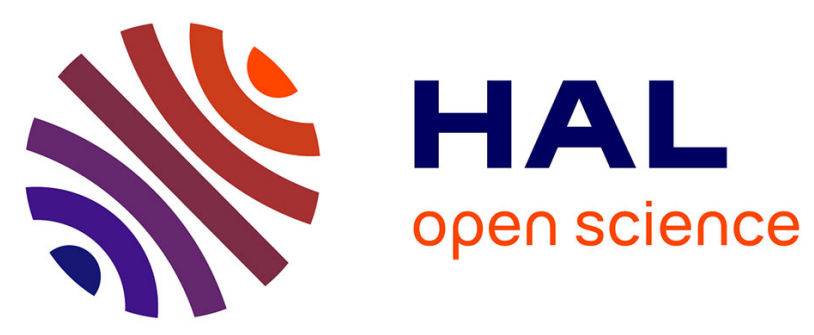

\title{
Systèmes alimentaires durables
}

Allison Loconto

\section{To cite this version:}

Allison Loconto. Systèmes alimentaires durables: Un manuel pour s'y retrouver. Institut national de recherche pour l'agriculture, l'alimentation et l'environnement. FAO, 260 p., 2020, Allison Loconto, 978-92-5-133270-2. 10.4060/ca9917fr . hal-03173213

\section{HAL Id: hal-03173213 \\ https://hal.inrae.fr/hal-03173213}

Submitted on 18 Mar 2021

HAL is a multi-disciplinary open access archive for the deposit and dissemination of scientific research documents, whether they are published or not. The documents may come from teaching and research institutions in France or abroad, or from public or private research centers.
L'archive ouverte pluridisciplinaire HAL, est destinée au dépôt et à la diffusion de documents scientifiques de niveau recherche, publiés ou non, émanant des établissements d'enseignement et de recherche français ou étrangers, des laboratoires publics ou privés.

\section{(2)(1) $\$$}

Distributed under a Creative Commons Attribution - NonCommercial - ShareAlikel 4.0 
A Organisation des Nations Unies pour l'alimentation et l'agriculture

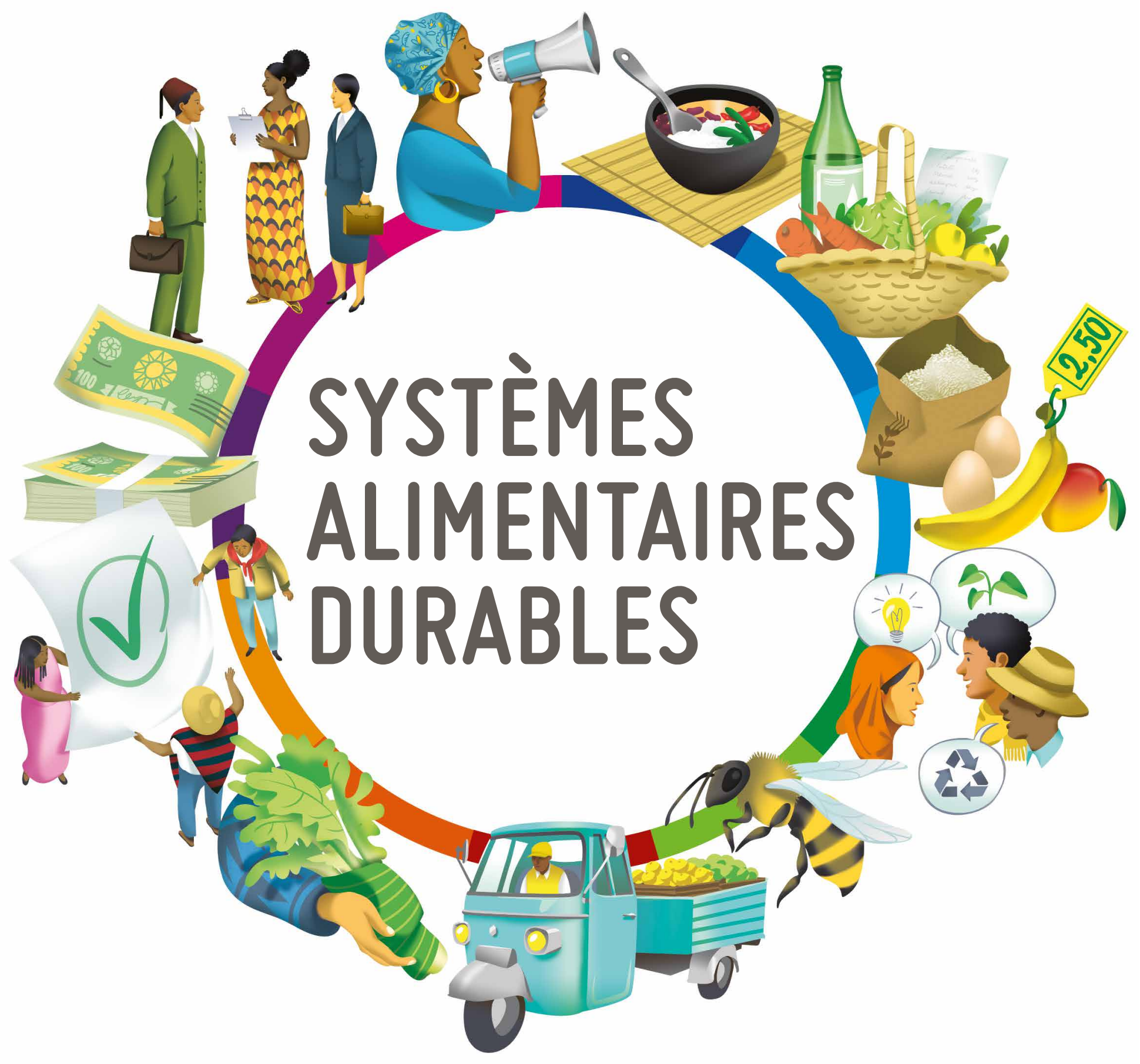

UN MANUEL POUR S'Y RETROUVER 


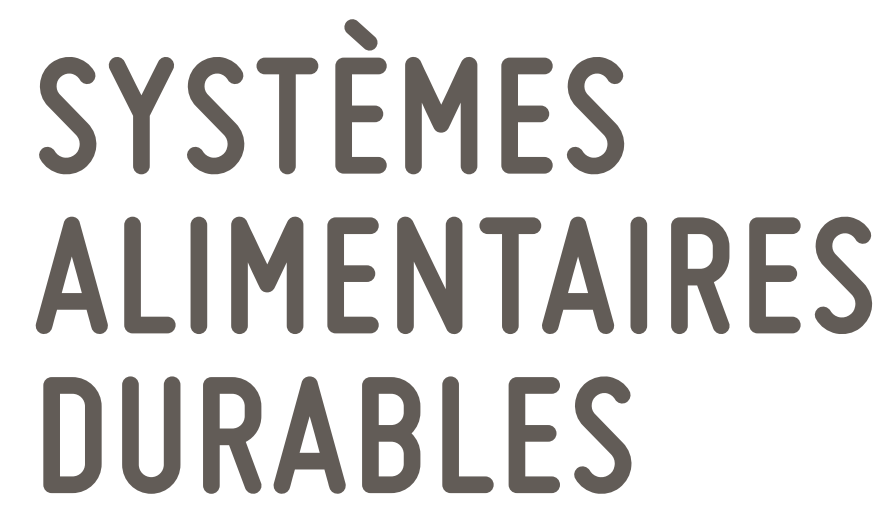

UN MANUEL POUR S'Y RETROUVER

Publié par:

l'Organisation des Nations Unies pour l'alimentation et l'agriculture (FAO) et Institut national de recherche pour l'agriculture, l'alimentation et l'environnement (INRAE)

Rome, 2020 
Citer comme suit:

FAO et INRAE. 2020. Systèmes alimentaires durables - Un manuel pour s'y retrouver. Rome.

https://doi.org/10.4060/ca9917fr

Les appellations employées dans ce produit d'information et la présentation des données qui y figurent n'impliquent de la part de l'Organisation des Nations Unies pour l'alimentation et l'agriculture (FAO) ni de l'Institut national de recherche pour l'agriculture, l'alimentation et l'environnement (INRAE), aucune prise de position quant au statut juridique ou au stade de développement des pays, territoires, villes ou zones ou de leurs autorités, ni quant au tracé de leurs frontières ou limites. Le fait qu'une société ou qu'un produit manufacturé, breveté ou non, soit mentionné ne signifie pas que la FAO ou INRAE approuvent ou recommandent ladite société ou ledit produit de préférence à d'autres sociétés ou produits analogues qui ne sont pas cités.

Les opinions exprimées dans ce produit d'information sont celles du/des auteur(s) et ne reflètent pas nécessairement les vues ou les politiques de la FAO ou INRAE.

ISBN 978-92-5-133270-2 [FAO]

(c) FAO, 2020

\section{(9) (1) (ब()}

Certains droits réservés. Cette œuvre est mise à la disposition du public selon les termes de la Licence Creative Commons Attribution-Pas d'Utilisation Commerciale-Partage dans les Mêmes Conditions 3.0 Organisations Intergouvernementales (CC BYNCSA3.0 IGO; https://creativecommons.org/licenses/by-nc-sa/3.0/igo/legalcode.fr).

Selon les termes de cette licence, cette œuvre peut être copiée, diffusée et adaptée à des fins non commerciales, sous réserve que la source soit mentionnée. Lorsque l'œuvre est utilisée, rien ne doit laisser entendre que la FAO cautionne tels ou tels organisation, produit ou service. L'utilisation du logo de la FAO n'est pas autorisée. Si l'œuvre est adaptée, le produit de cette adaptation doit être diffusé sous la même licence Creative Commons ou sous une licence équivalente. Si l'œuvre est traduite, la traduction doit obligatoirement être accompagnée de la mention de la source ainsi que de la clause de non-responsabilité suivante: "La traduction n'a pas été réalisée par l'Organisation des Nations Unies pour l'alimentation et l'agriculture (FAO). La FAO n'est pas responsable du contenu ni de l'exactitude de la traduction. L'édition originale [langue] est celle qui fait foi.»

Tout litige relatif à la présente licence ne pouvant être résolu à l'amiable sera réglé par voie de médiation et d'arbitrage tel que décrit à l'Article 8 de la licence, sauf indication contraire contenue dans le présent document. Les règles de médiation applicables seront celles de l'Organisation mondiale de la propriété intellectuelle (http://www.wipo.int/amc/fr/mediation/rules) et tout arbitrage sera mené conformément au Règlement d'arbitrage de la Commission des Nations Unies pour le droit commercial international (CNUDCI).

Matériel attribué à des tiers. Il incombe aux utilisateurs souhaitant réutiliser des informations ou autres éléments contenus dans cette œuvre qui y sont attribués à un tiers, tels que des tableaux, des figures ou des images, de déterminer si une autorisation est requise pour leur réutilisation et d'obtenir le cas échéant la permission de l'ayant-droit. Toute action qui serait engagée à la suite d'une utilisation non autorisée d'un élément de l'œuvre sur lequel une tierce partie détient des droits ne pourrait l'être qu'à l'encontre de l'utilisateur.

Ventes, droits et licences. Les produits d'information de la FAO sont disponibles sur le site web de la FAO (www.fao.org/publications) et peuvent être obtenus sur demande adressée par courriel à: publicationssales@fao.org. Les demandes visant un usage commercial doivent être soumises à: www.fao.org/contact-us/ licence-request. Les questions relatives aux droits et aux licences doivent être adressées à: copyright@fao.org. 


\section{RÉSUMÉ}

Les systèmes alimentaires durables sont essentiels pour assurer la sécurité alimentaire et une alimentation saine pour les générations futures. Pour faire la transition vers la durabilité, de nombreuses activités du système alimentaire doivent se transformer, et une myriade d'acteurs à travers le monde doivent agir localement. Certains changements sont plus faciles à mettre en place que d'autres, mais savoir comment naviguer à travers ces changements pour promouvoir des modes de consommation et de production durables exige un ensemble de compétences complexes. Ce manuel, adressé aux «innovateurs de systèmes alimentaires durables», est rédigé par un groupe d'innovateurs d'Asie, d'Afrique, des Amériques et d'Europe qui mènent des initiatives pour cultiver, partager, vendre et consommer des aliments plus durables dans leur contexte local. Le manuel inclut des expériences de changement dans l'organisation des systèmes alimentaires locaux pour les rendre plus durables. Il est rédigé sous la forme d'un «livre dont vous êtes le héros» où chaque lecteur ou groupe de lecteurs peut identifier ses priorités et développer son parcours d'apprentissage et d'action. Les sujets abordés dans ce manuel sont répartis en quatre groupes d'innovations organisationnelles, à savoir: mobiliser les consommateurs, produire durablement, mettre en marché les produits, et s'organiser équitablement. 

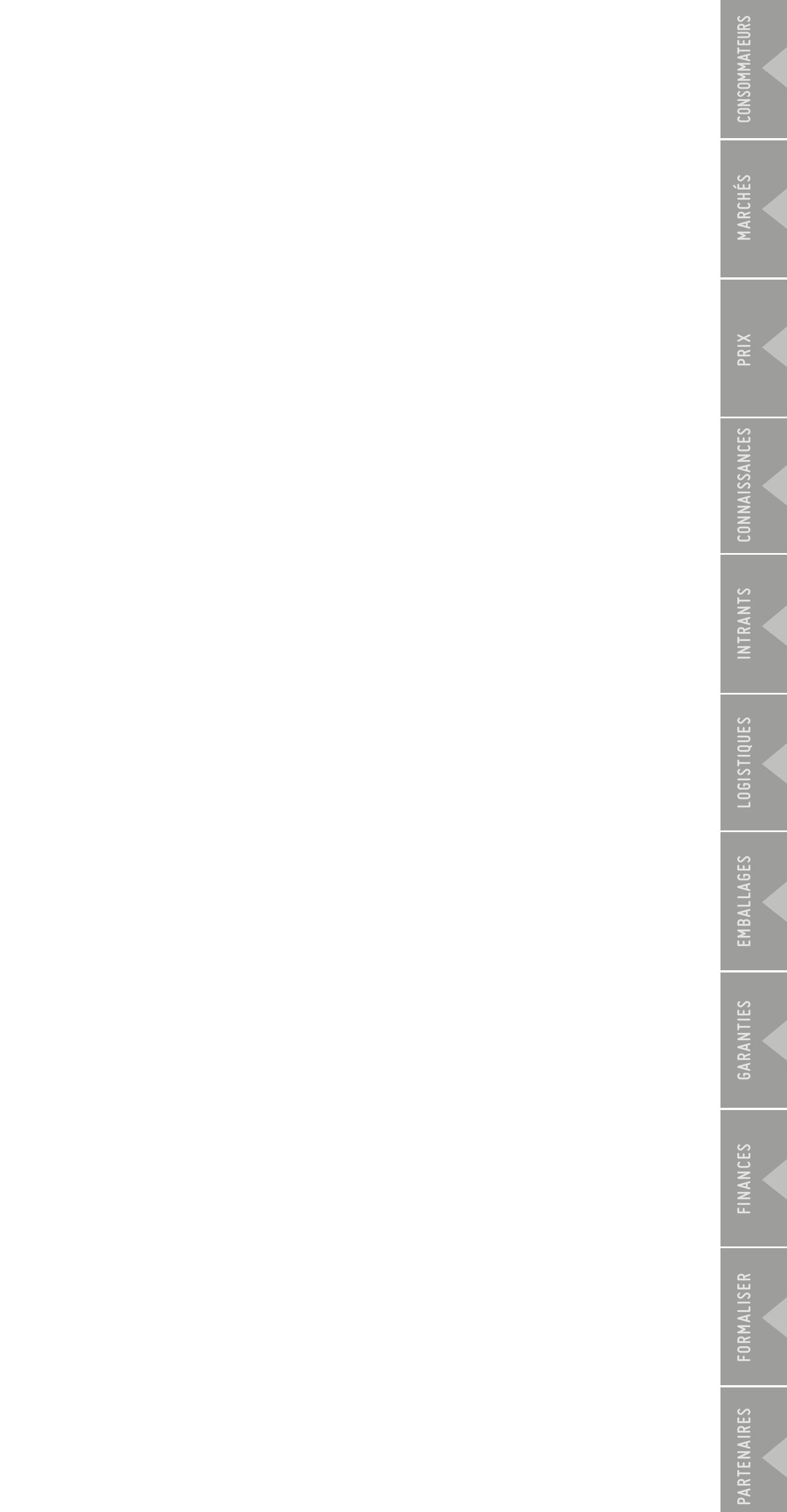


\section{TABLE DES MATIÈRES}

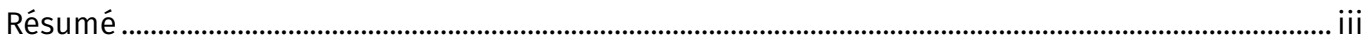

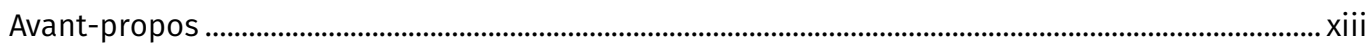

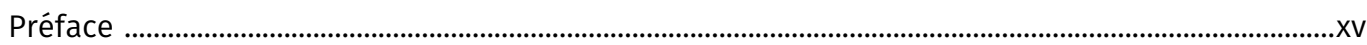

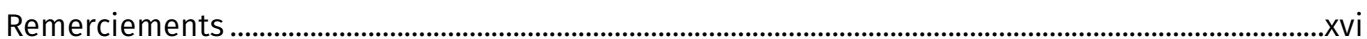

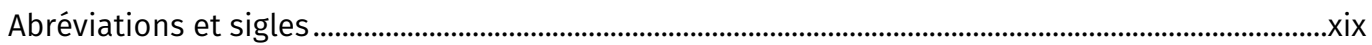

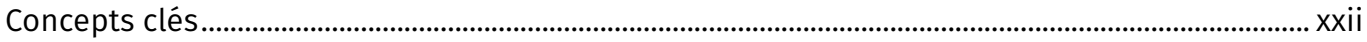

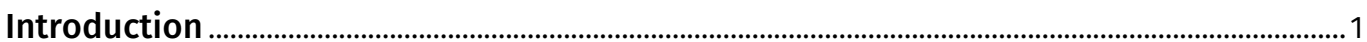

(Re)valoriser l'agriculture pour des systèmes alimentaires durables .........................................7

\section{MOBILISER LES CONSOMMATEURS}

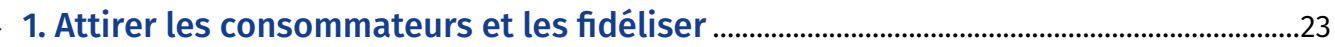

2. Connaître vos marchés .........................................................................................................................43

3. Trouver le «bon» prix ..........................................................................................................................61

\section{PRODUIRE DURABLEMENT}

4. Partager et co-créer des connaissances pour une production durable.......................87

5. Gérer et accéder aux intrants durables ......................................................................................103

\section{MISE EN MARCHÉ DES PRODUITS}

6. Rester connectés grâce à la logistique ..............................................................................125

7. Emballage de qualité et durable ..........................................................................................143

8. Garantir la durabilité .............................................................................................................................. 157

\section{COMMENT S'ORGANISER}

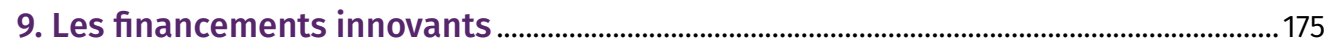

10. Formaliser votre travail collectif...........................................................................................195

11. La place des partenaires et des plaidoyers .......................................................................22 


\section{Tableaux}

Tableau 1: Exemple d'un barème des frais d'abonnement d'une AMAP ........................................................37

Tableau 2: Matrice de la capacité des producteurs et des besoins du marché ............................................4 46

Tableau 3: Questionnaire adressé au consommateur pour trouver un marché ...........................................52

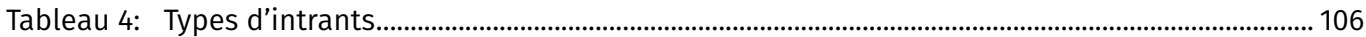

Tableau 5: Un modèle pour catégoriser les intrants..........................................................................................107

Tableau 6: Matrice pour identifier vos sources d'approvisionnement d'intrants ...................................... 121

Tableau 7: Typologie des différents systèmes de distribution .....................................................................135

Tableau 8: Exemples d'emballages différents selon les caractéristiques du produit ...............................146

Tableau 9: Exemples de différents formats d'emballage pour différents consommateurs.....................152

Tableau 10: Comment la confiance se construit-elle dans les différents systèmes de garantie ?..........165

Tableau 11: Comment les coûts sont-ils comparés d'un système de garantie à un autre ?.......................166

\section{Figures}

Figure 1: $\quad$ Chemins d'apprentissage possibles en commençant par n'importe quel chapitre.................6

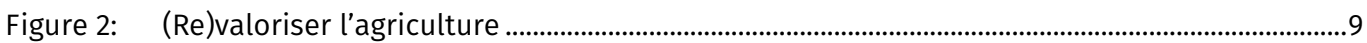

Figure 3: Exemple d'un modèle des résultats d'une étude de marché participative (EMP) sur les SPG

\section{Encadrés}

Encadré 1: L'inclusion sociale des consommateurs à faible revenu dans une AMAP ..............................28

Encadré 2: Les réseaux sociaux comme un outil de communication interne en Finlande .................... 30

Encadré 3: L'importance du message visuel sur le site de Lavka Lavka .......................................................31

Encadré 4: La démonstration culinaire pour renforcer les liens avec les consommateurs du SPG de Quezon .............................................................................. 36

Encadré 5: Des visites de consommateurs sur le terrain pour renforcer la relation

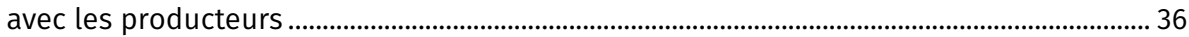

Encadré 6: La participation des consommateurs et les contraintes de temps dans les modèles

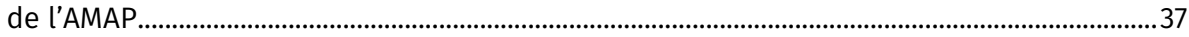

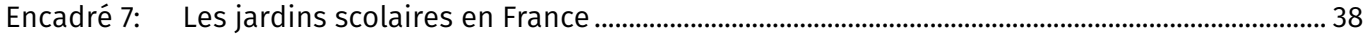


Encadré 8: Un programme scolaire pour éduquer les futurs consommateurs au Kenya ........................39

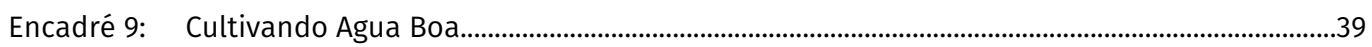

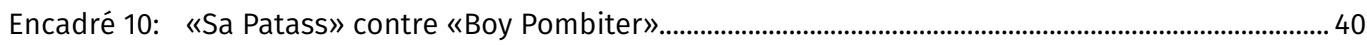

Encadré 11: Dégustations avec des chefs et des restaurants, Verde Oliva (Colombie) ............................ 48

Encadré 12: Des agriculteurs qui visitent une unité de transformation ...........................................................49

Encadré 13: Quand et comment organiser un groupe de discussion ?......................................................... 50

Encadré 14: La foire agroécologique de Carcelén (Équateur) ...........................................................................5 51

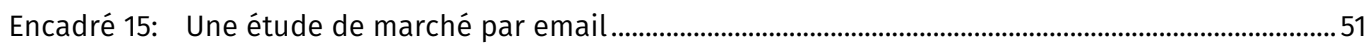

Encadré 16: Le marché mobile des agriculteurs de Pékin ................................................................................53

Encadré 17: Faire cavalier seul ou rejoindre un réseau ?.....................................................................................57

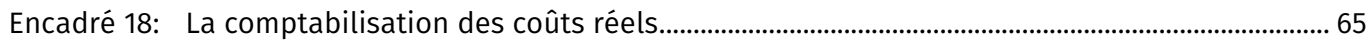

Encadré 19: Apprendre aux enfants la comptabilité (Ouganda) ..................................................................67

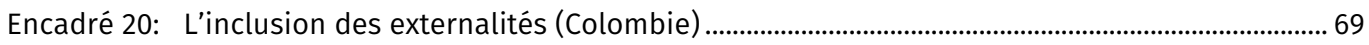

Encadré 21: Trois critères pour définir quand une transaction est acceptable pour les producteurs (Inde) .................................................................................................................70

Encadré 22: Enseigner la production alimentaire aux consommateurs (Ouganda).....................................73

Encadré 23: Les études de marché comme moyen de partager l'information (Ouganda).........................74

Encadré 24: Trois modèles différents d'intermédiaires transparents

(Corée du Sud et Inde) ...................................................................................................................75

Encadré 25: Les comités et les mécanismes de fixation des prix au sein de l'AMAP..................................77

Encadré 26: Une étude de marché participative (EMP) (Ouganda) …..............................................................78

Encadré 27: Le taux de partage des prix dans le secteur du thé (Tanzanie) .................................................79

Encadré 28: Le modèle de Shared Harvest Farm pour calculer le juste prix (Chine)..................................8 80

Encadré 29: Le gouvernement en tant qu'intermédiaire transparent (India) ............................................81

Encadré 30: Les programmes d'approvisionnement institutionnel (PAI)

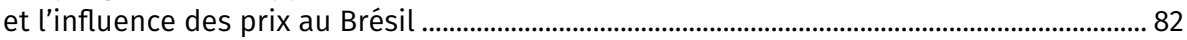

Encadré 31: Le programme TRIT pour les échanges d'agriculteurs (Tanzanie)..............................................99

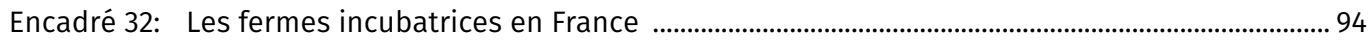

Encadré 33: La recherche-action pour promouvoir les pratiques agroécologiques (Équateur) ..............95

Encadré 34: Un partenariat société civile-secteur public pour promouvoir le concept d'éco-santé (Colombie) .............................................................................99

Encadré 35: Partenariats agriculteurs-chercheurs (Kenya)..........................................................................98

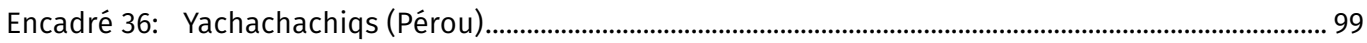

Encadré 37: Une école de formation de leaders en sécurité alimentaire et nutritionnelle et en souveraineté alimentaire (Colombie) .................................................... 100

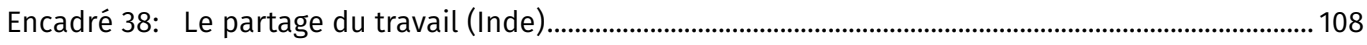

Encadré 39: Des engrais sains à base de déchets de restaurants (Colorado, États-Unis) ....................... 109

Encadré 40: Husk Power Systems (Inde) .....................................................................................................110

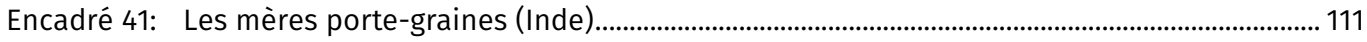




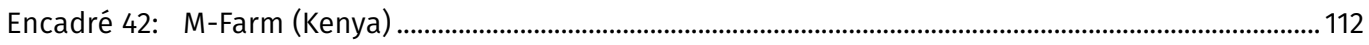

Encadré 43: Anicytor, un moteur d'énergie verte des outils agricoles (Éthiopie) .......................................114

Encadré 44: Agrovision - des outils manuels pour les agriculteurs (Inde) ..................................................115

Encadré 45: Wanakaset Network (Thaïlande) ......................................................................................................... 116

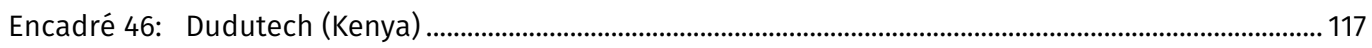

Encadré 47: Deux exemples de gestion d'eau (Zimbabwe, Inde, Tanzanie) ................................................118

Encadré 48: Contribuer au cycle naturel des pollinisateurs (Malaisie).............................................................119

Encadré 49: «Partager la poire en deux», transformer le désavantage en avantage (RDP Lao) ............130

Encadré 50: Le traitement du riz étuvé au Centre Songhaï (Bénin)...............................................................132

Encadré 51: EcoVida, un système de transport partagé (Brésil)......................................................................134

Encadré 52: Les déchets de la foire deviennent de l'engrais en ville (Brésil) ............................................ 137

Encadré 53: La traçabilité au service de la prévention des risques (Thaïlande).......................................138

Encadré 54: Un code couleur servant de système de traçabilité (Tanzanie)...............................................139

Encadré 55: Des tests participatifs de contamination (Inde) ......................................................................... 140

Encadré 56: La réglementation participative de la sécurité sanitaire des aliments (Inde)....................141

Encadré 57: Réduire, réutiliser, recycler (Chine) .................................................................................................145

Encadré 58: L'emballage dans un projet de pêche artisanale biologique (Thaïlande)..............................149

Encadré 59: Les emballages en fibres naturelles pour la livraison à domicile de FreshVeggie

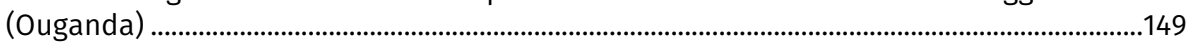

Encadré 60: Comment créer un design original et attrayant ? (Colombie) ................................................ 151

Encadré 61: L'externalisation de l'impression (Colombie) ...........................................................................153

Encadré 62: Les informations obligatoires sur l'étiquette (Colombie).........................................................154

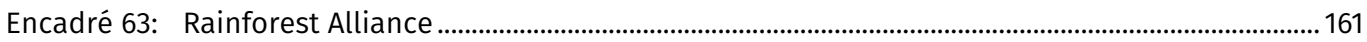

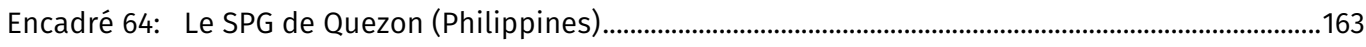

Encadré 65: Les supermarchés SPAR/SUPERSPAR (Namibie) ..........................................................................165

Encadré 66: La certification Fairtrade (FLOCERT) .............................................................................................. 167

Encadré 67: Le système participatif de garantie, l'enregistrement de la sécurité sanitaire des aliments et les marchés publics (Bolivie) ...........................................................................................170

Encadré 68: Des groupes d'épargne et de crédit communautaires (Kenya)..............................................179

Encadré 69: Crowdfunding ou financement participatif (Chine) .................................................................. 180

Encadré 70: Des contrôles verts menés par les consommateurs (Brésil) ................................................... 180

Encadré 71: Le projet Paramparagat Krishi Vikas Yojana (PKVY) du Ministère de l'agriculture (Inde)

Encadré 72: Utiliser des célébrités pour soutenir votre initiative (Pérou)....................................................183

Encadré 73: Le financement de projets d'innovation nationaux (Chili) ...................................................... 184

Encadré 74: Mobiliser des fonds publics de développement régional (Croatie) ...................................... 184

Encadré 75: Les fonds d'aide publique au développement (APD) en Inde et Allemagne.........................185

Encadré 76: Le Fonds national de développement agro-sylvo-pastoral (Sénégal).................................. 186 
Encadré 77: Le programme du Fonds communautaire pour l'écotourisme (Trinité-et-Tobago) ............187

Encadré 78: Un cas d'éco-services fournis par le gouvernement (Chine) ................................................... 188

Encadré 79: La certification du commerce équitable comme garantie de crédit (Amérique latine et Afrique) 189

Encadré 80: Les programmes RSE de l'industrie chocolatière (Côte d'Ivoire) .......................................... 190

Encadré 81: Cargonomia, un réseau de trois entreprises sociales et environnementales (Hongrie)......191

Encadré 82: Les monnaies locales, ce qui fonctionne et ce qui ne fonctionne pas (Colombie) ............192

Encadré 83: Le leadership organique: un concept de style de vie ................................................................200

Encadré 84: ChocoTogo - une entreprise de transformation détenue par ses employés (Togo).......... 202

Encadré 85: Hansalim, une structure coopérative agriculteurs-consommateurs (Corée du Sud).......204

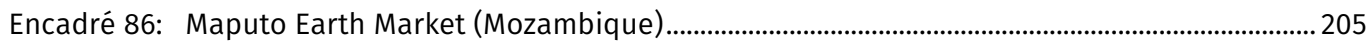

Encadré 87: Green Net Cooperative/Earth Net Foundation (Thaïlande) ....................................................206

Encadré 88: Des contrats de réseaux (Italie) .........................................................................................................207

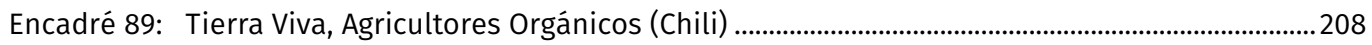

Encadré 90: La croissance d'un modèle de réseau (Colombie) .........................................................................210

Encadré 91: Impliquer les jeunes et les femmes dans les initiatives agroécologiques durables (Brésil) ....................................................................212

Encadré 92: SPG: Réseautage, partenariat et plaidoyer (Philippines) ...........................................................218

Encadré 93: La campagne de consommateurs ¡Que Rico Es ! (Équateur)....................................................220

Encadré 94: Programmes de panier: créer des liens sociaux (Équateur) ....................................................223

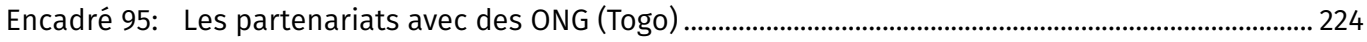

Encadré 96: Trouver et approcher les personnes pouvant influencer

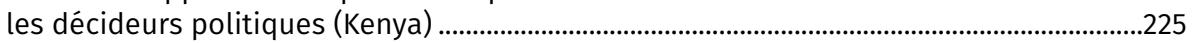




\section{Astuces}

Astuce 1: Comment un producteur peut Identifier des consommateurs potentiels .................................26

Astuce 2: $\quad$ Essayer de ne pas abuser de la générosité des autres ...................................................................32

Astuce 3: Maintenir l'engagement des consommateurs ......................................................................................33

Astuce 4: Créer une expérience d'achat agréable ................................................................................................34

Astuce 5: La surprise est un ingrédient important pour fidéliser les consommateurs ...........................35

Astuce 6: Le service personnalisé permet aux clients d'apprécier leur expérience,

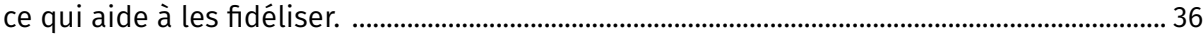

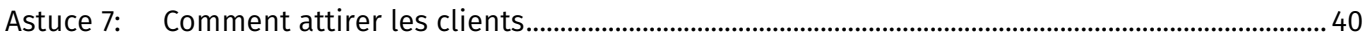

Astuce 8: Élaborer une matrice pour comparer votre capacité par rapport aux exigences du marché................................................................................................................ 45

Astuce 9: Faire en sorte que l'information recueillie soit facilement accessible et compréhensible pour les autres membres .........................................................................................47

Astuce 10: Vérifier la législation nationale concernant les programmes de restauration scolaire .......55

Astuce 11: Comment créer une AMAP .....................................................................................................................5

Astuce 12: Comment trouver des marchés durables ......................................................................................58

Astuce 13: Prendre note des tâches quotidiennes à la ferme ..........................................................................67

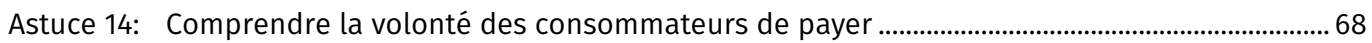

Astuce 15: La fixation des prix de produits transformés repose sur un calcul mixte.................................68

Astuce 16: Stabiliser les revenus des producteurs grâce à la diversification ...............................................71

Astuce 17: Aider les consommateurs à connaître le «bon» prix .......................................................................72

Astuce 18: Comment trouver un prix durable ................................................................................................... 83

Astuce 19: Savoir choisir entre une approche formelle et informelle ............................................................. 89

Astuce 20: Réfléchir à la durabilité de vos pratiques agricoles.....................................................................90

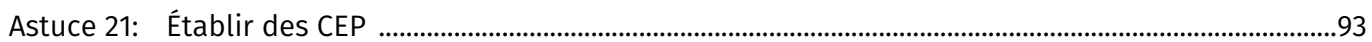

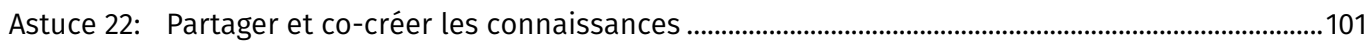

Astuce 23: Établir votre propre banque de semences ....................................................................................... 112

Astuce 24: Obtenir des solutions/des machines durables.............................................................................116

Astuce 25: Gérer et accéder aux intrants durables ......................................................................................... 121

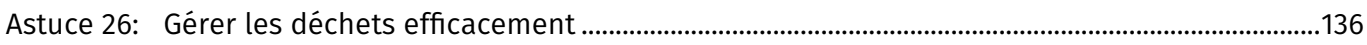

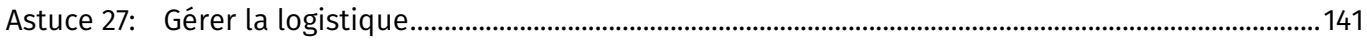

Astuce 28: Développer un design attrayant .........................................................................................................150

Astuce 29: Concevoir un emballage simple et attrayant....................................................................................152

Astuce 30: Emballer des produits durables qui se vendent .......................................................................155

Astuce 31: Recueillir des informations sur les systèmes de garantie opérant dans votre région.........164 
Astuce 32: Simplifier et standardiser le processus administratif ................................................................169

Astuce 33: Mettre en place un système de garantie lorsque personne ne le demande...........................170

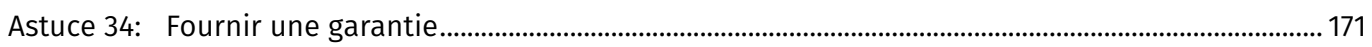

Astuce 35: Réinvestir le revenu issu de la vente de produits.......................................................................178

Astuce 36: Utiliser la budgétisation participative ........................................................................................181

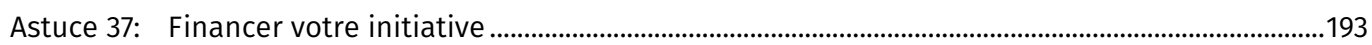

Astuce 38: Développer une initiative de système alimentaire durable .......................................................199

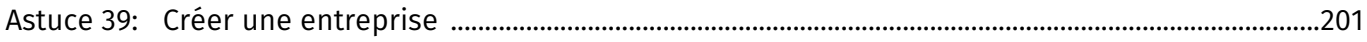

Astuce 40: Vérifier le procédé d'immatriculation des entreprises sociales de votre pays..................... 202

Astuce 41: Trouver et maintenir le bon équilibre..............................................................................................213

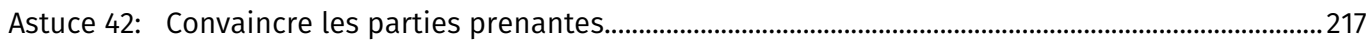

Astuce 43: Établir des partenariats pour le plaidoyer ....................................................................................221

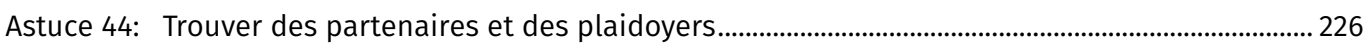



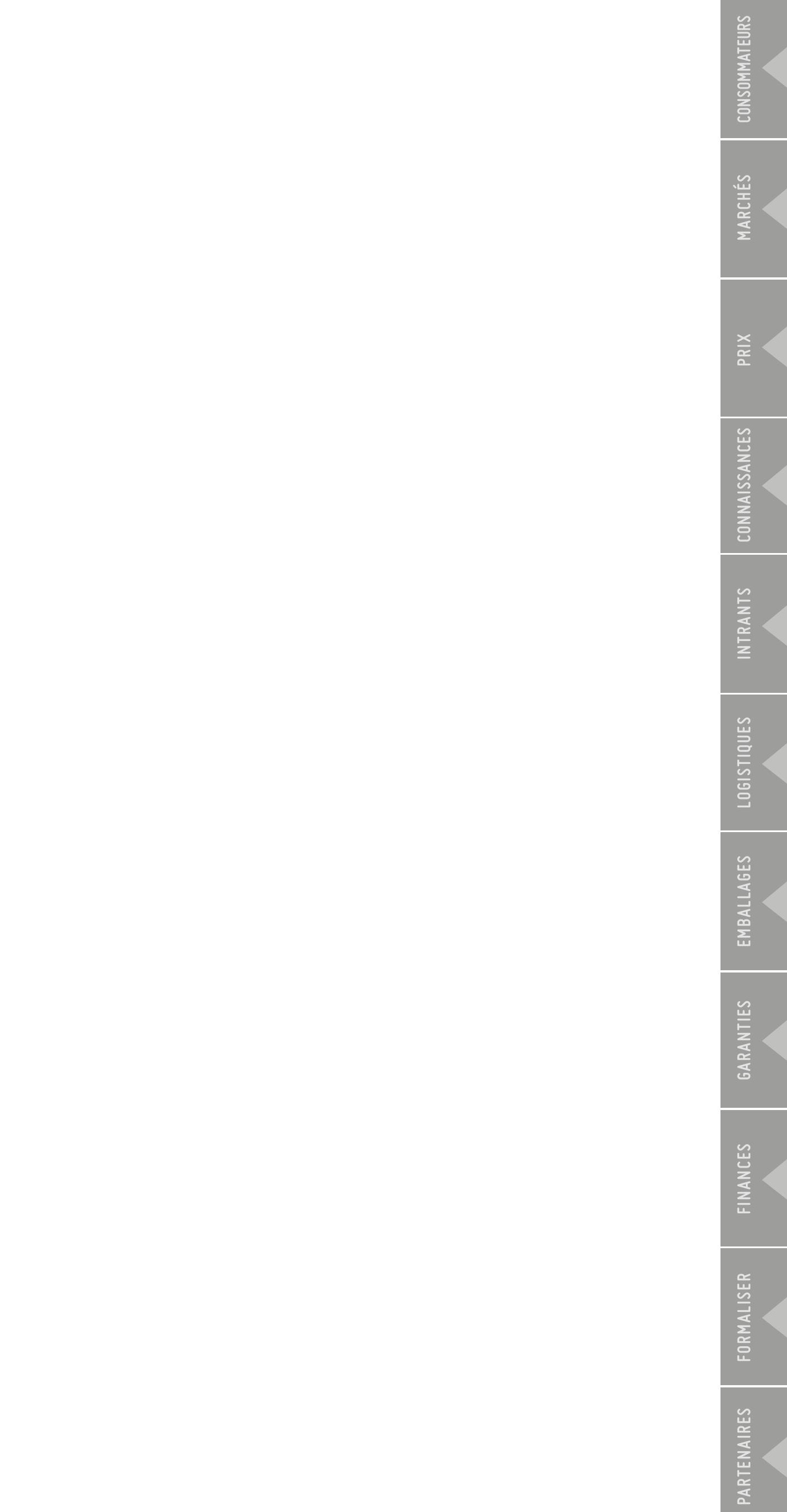


\section{AVANT-PROPOS}

En 2018, la FAO a organisé deux événements liés à l'innovation: une exposition et un débat sur l'innovation pour l'agroécologie lors du $2^{e}$ Symposium international sur l'agroécologie en avril 2018 et le Symposium sur l'innovation en novembre 2018. Ces deux événements ont établi l'innovation comme une solution aux défis auxquels les systèmes agricoles et alimentaires actuels sont confrontés dans leur quête de durabilité. Les deux évènements ont également placé l'innovation au sein de son programme d'accompagnement pour les États Membres en matière des objectifs du développement durable (ODD).

Le rapport 2019 du Groupe d'experts de haut niveau (HLPE) du Comité de la sécurité alimentaire mondiale sur l'agroécologie et d'autres approches novatrices de l'agriculture durable renforcent les enseignements tirés de la série Save and Grow de la FAO. La fermeture des cycles des nutriments, l'intégration des systèmes de production et l'atténuation intelligente du changement climatique offrent une myriade de possibilités d'innovation dans les systèmes de production agroécologiques. La nécessité de trouver de nouvelles façons de co-créer et de partager les connaissances sur l'agriculture durable est une priorité pour rendre les nouvelles connaissances, technologies et pratiques accessibles aux utilisateurs, afin d'assurer la généralisation des innovations. Une plus grande participation des agriculteurs, des consommateurs et des organisations locales à la recherche, à la commercialisation et à la garantie de la durabilité des produits alimentaires et agricoles ouvre de nouvelles voies pour des processus d'apprentissage dynamiques.

Les innovations du système alimentaire sont particulièrement nécessaires, car le système alimentaire mondial est responsable de l'état actuel de la malnutrition où la faim et l'obésité coexistent souvent au sein d'un même pays. Le Rapport sur l'état de l'insécurité alimentaire de 2019 met en lumière les causes de ces phénomènes, dont les conflits, la pauvreté et la marginalisation, mais aussi les inégalités existantes et croissantes dans l'accès et l'utilisation de l'alimentation. La crise de COVID-19, qui constitue l'arrière plan de la publication de ce livre, met en évidence la nécessité d'innover dans les systèmes alimentaires. Afin d'assurer leur résilience, ce sont notamment des innovations qui portent vers la rélocalisation et la diversification des systèmes dont nous avons besoin. Les changements apportés à la façon dont les systèmes alimentaires sont organisés peuvent prioriser l'accès aux aliments pour les communautés vulnérables, promouvoir l'agriculture durable, y compris la protection de la biodiversité, et encourager des régimes alimentaires sains et nutritifs. L'élément fondamental de ces changements est d'accroître les échanges entre les producteurs et les consommateurs et de modifier la façon dont ils influencent et interagissent au sein de leur environnement alimentaire. Plutôt que d'être des acteurs passifs, les consommateurs assument un rôle de plus en plus actif. En recherchant des aliments cultivés de manière durable, des systèmes commerciaux qui soutiennent les économies locales et qui incluent les petits exploitants agricoles, ils contribuent à la réorganisation des systèmes alimentaires tout en promouvant une alimentation plus saine. 
Ce manuel s'appuie des expériences novatrices dans plus de 20 pays et montre que les systèmes alimentaires sont déjà en transition vers la durabilité. Ces innovations remettent en question et modifient les normes, les institutions, les pratiques et les relations entre les acteurs du système alimentaire. Cette ressource est destinée aux acteurs qui veulent contribuer à ce mouvement. S'appuyant à la fois sur les connaissances scientifiques et expérientielles, les conseils et astuces fournis dans ce manuel nous inspirent à être positifs à la fois sur la capacité d'innovation des acteurs dans tous les coins du monde et sur les perspectives qui permettent l'émergence de systèmes alimentaires durables.

\section{Rémi Nono Womdim}

Directeur adjoint

Division de la production végétale et de la protection des plantes (AGP)

FAO

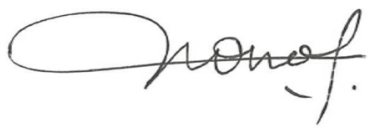

\section{Anna Lartey}

Directrice

Division de la nutrition et des systèmes alimentaires (ESN)

FAO

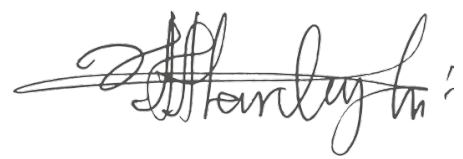




\section{PRÉFACE}

Ce manuel est le fruit d'un véritable projet collectif. Le processus a commencé en 2013 avec l'idée que nous devrions mieux comprendre pourquoi les acteurs locaux construisent des systèmes alimentaires locaux durables à travers la construction de marchés pour des produits alimentaires durables, même sans la certification formelle. Ce projet de recherche participative a permis de documenter des initiatives novatrices provenant de quatre continents et d'avancer les connaissances collectives sur les innovations institutionnelles nécessaires pour soutenir les transitions vers des systèmes alimentaires durables. Partant d'un petit groupe de trois collègues de la FAO et de l'INRAE, de nouveaux collaborateurs se sont joints à nous chaque année pour élargir et enrichir nos expériences géographiques et organisationnelles.

Le résultat de cette initiative est le présent manuel, qui est la troisième publication FAO/INRAE d'une série sur les marchés locaux pour une agriculture durable. Les idées et le texte de ce manuel ont été rédigés collectivement lors de deux ateliers d'écriture (à Chiang Mai en Thaïlande en 2016, à Delhi en Inde, en 2017) et des groupes de travail virtuels en 2017. En 2018, le manuel était testé par des initiatives novatrices à Delhi (Inde) et à Thiès (Sénégal). Cette approche a permis un ancrage fort de son contenu dans des expériences de terrain, qui assure un intérêt direct des utilisateurs.

Le message clé que nous voulons transmettre avec ce manuel est le suivant: pour permettre le changement d'un système alimentaire, il faut de la collaboration et des capacités de réflexion systémiques portant sur les divers connaissances, matériaux, technologies, relations, valeurs et processus naturels nécessaires afin de nous nourrir. Compte tenu de la riche diversité culturelle et biologique présente dans le monde, il n'est pas nécessaire que deux initiatives soient identiques pour qu'elles soient considérées durables. Plutôt que d'être une contrainte, cette réalité devrait être saisie comme une occasion d'innover, de créer de nouvelles règles sur la façon de cultiver, de commercialiser et de consommer les aliments. Par conséquent, l'élaboration d'une vision collective de l'avenir des systèmes alimentaires souhaités est une étape fondamentale pour prendre des mesures concrètes permettant la transformation des systèmes alimentaires. Nous espérons que ce manuel contribuera à cette démarche.

Allison Marie Loconto
Directrice adjointe
Laboratoire interdisciplinaire
sciences, innovations et
société (LISIS)
INRAE

\section{Anne-Sophie Poisot}

Coordinatrice des projets

Division de la production végétale et de la protection des plantes (AGP)

FAO

Pilar Santacoloma
Chargée des systèmes
agro-alimentaires
Division de la nutrition et des
systèmes alimentaires (ESN)
FAO
FAO

\author{
Marcello Vicovaro \\ Consultant des marchés \\ durables \\ Division de la nutrition et \\ des systèmes alimentaires \\ (ESN) \\ FAO
}




\section{REMERCIEMENTS}

Le présent manuel a été rédigé collectivement, dans le cadre d'une série d'ateliers et de groupes de travail et représente les contributions des personnes suivantes:

Allison Loconto, INRAE/FAO

Marcello Vicovaro, FAO

Pilar Santacoloma, FAO

Anne-Sophie Poisot, FAO

Ashish Gupta, Jaivik Haat/Gram Disha Trust

Michael Commons, Earth Net Foundation

Jocelyn Parot, URGENCI

Oscar Javier Nieto, Familia de la Tierra

Carmen Cabling, SPG de Quezon

Jaime Aguirre, Familia de la Tierra

Jelena Petrov, RERA SD

Julie Matovu, FreshVeggies Ltd

Judith Hitchman, URGENCI

Rosinah Mbenya, PELUM Kenya

Emmanuel Simbua, Institut de recherche sur le thé de Tanzanie (TRIT)

Ross Mary Borja, Fundación Ekorural

Patricia Flores, IFOAM Organics International

Andrea Moya, Claudia Helena Prieto Parque Temático en Salud Pública Chaquen, Subred Integrada De Prestación de Servicios de Salud Sur.

Mathew John, Fondation Keystone

José Antonio da Silva Marfil, Ecovida

Darli Bengh, Ecovida

Shi Yan, Récolte partagée

Chris May, IFOAM Organics International

René Piamonte, conseiller en biodynamie (Terrahabilis-Demeter)

Susan Boohaker, FAO/American University of Rome

Collier Lumpkin, FAO/American University of Rome

Zachary Reif, FAO/American University of Rome 
Nous avons réalisé ce travail dans le cadre de l'Initiative de base 3 (durabilité le long de toutes les chaînes de valeur) du Programme des systèmes alimentaires durables du réseau One Planet. Nous tenons à remercier la FAO (AGP, ESN et SLM), l'Institut national de recherche pour l'agriculture, l'alimentation et l'environnement (INRAE), l'Université Gustave Eiffel, l'Office fédéral de l'agriculture (OFAG), et le Programme Systèmes Alimentaires Résilients Integrated Approach Pilot (GEF IAP) du Fonds pour l'environnement mondial (FEM) pour leur soutien financier et institutionnel dans ce processus. Nous remercions également l'appui institutionnel que nous avons reçu de chaque organisation qui a collaboré avec nous pour la réalisation de cette publication.

Nous tenons à remercier tout particulièrement nos collègues de la FAO - Florence Tartanac, Renée van Dis, Jorge Fonseca, Guido Santini et Michael Clark - pour leurs commentaires sur des chapitres spécifiques et le texte général. Nous remercions deux réviseurs anonymes pour leurs commentaires qui ont contribué à l'amélioration du texte. Nous tenons également à remercier nos collègues de l'Inde et du Sénégal qui nous ont fait part de leurs commentaires sur le manuel lors de deux tests nationaux. Nous remercions Tara Dourian et Coumbaly Diaw pour la révision et Studio Bartoleschi pour la conception graphique. 

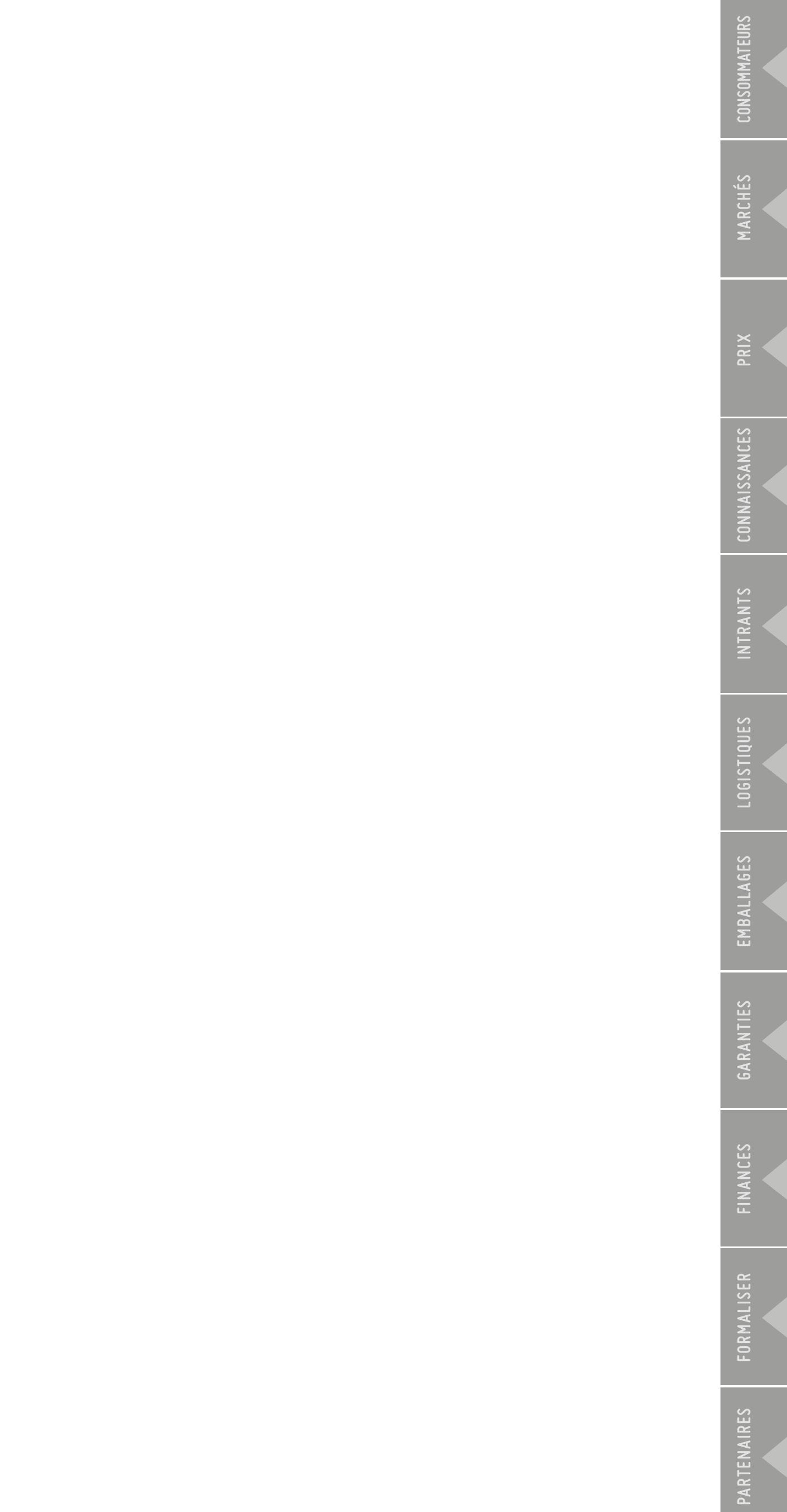


\section{ABRÉVIATIONS ET SIGLES}

\begin{tabular}{|c|c|}
\hline $\mathrm{ACl}$ & Alliance coopérative internationale \\
\hline AMAP & Association pour le maintien d'une agriculture paysanne \\
\hline APD & Aide publique au développement \\
\hline ANPE PERU & Association nationale des producteurs biologiques du Pérou \\
\hline APEGA & Association péruvienne de gastronomie \\
\hline ACOPA & Association des consommateurs de produits agroécologiques du Paraná - Brésil \\
\hline BAFS & Bureau des normes pour l'agriculture et la pêche - Philippines \\
\hline CACP & Commission des coûts et des prix agricoles - Inde \\
\hline $\mathrm{CDPH}$ & Centre pour le développement du potentiel humain \\
\hline CEDEAO & Communauté économique des États de l'Afrique de l'Ouest \\
\hline CEP & champs écoles des producteurs \\
\hline CONAB & Companhia Nacional de Abastecimento - Brésil \\
\hline COPACO & comités de participation communautaire en santé publique \\
\hline EAT & école d'agriculture de terrain \\
\hline EMP & étude de marché participative \\
\hline FAO & Organisation des Nations Unies pour l'alimentation et l'agriculture \\
\hline FDT & Familia de la Tierra - Colombie \\
\hline FFPM & force, faiblesses, possibilités et menaces \\
\hline FIDA & Fonds international de développement agricole \\
\hline FMF & formation des maitres formateurs \\
\hline FNDASP & Fonds national de développement agro-sylvo-pastoral - Sénégal \\
\hline FNRAA & Fonds national de recherche agricole et agro-alimentaire du Sénégal \\
\hline GEF & Fonds pour l'environnement mondial \\
\hline GPAA & Groupe de gestion du programme d'achats d'aliments - Brésil \\
\hline GTT & Groupe de travail technique - Philippines \\
\hline
\end{tabular}




\begin{tabular}{|c|c|}
\hline GVC & Gruppo di volontariato civile - Italie \\
\hline IFOAM & Fédération internationale des mouvements d'agriculture biologique \\
\hline IICA & Institut interaméricain de coopération pour l'agriculture \\
\hline INRAE & Institut national de recherche pour l'agriculture, l'alimentation et l'environnement \\
\hline ITC & Centre du commerce international \\
\hline JKUAT & Université d’agriculture Jomo Kenyatta - Kenya \\
\hline LANAC & Laboratoire national d'analyses et de contrôle \\
\hline LAl & lutte antiparasitaire intégrée \\
\hline MASIPAG & Magsasaka at Siyentista Para sa Pag-unlad ng Pagsasaka - Philippines \\
\hline MFGS & MASIPAG Farmers Guarantee System - Philippines \\
\hline NMSA & Mission nationale pour une agriculture durable - Inde \\
\hline NOA & Namibia Organic Association \\
\hline NRWRP & $\begin{array}{l}\text { Programme national de reboisement et de remise en état } \\
\text { des bassins hydrographiques }\end{array}$ \\
\hline 000 & objectifs de développement durable \\
\hline ONG & organisation non gouvernementale \\
\hline OSBL & organisme sans but lucratif \\
\hline OSC & organisations de société civile \\
\hline PAA & Programme d'approvisionnement alimentaire - Brésil \\
\hline PELUM & Participatory Ecological Land Use Management Association - Kenya \\
\hline PNAE & Programme national d'alimentation scolaire - Brésil \\
\hline PSM & prix de soutien minimal \\
\hline REDD & réduction des émissions liées à la déforestation et à la dégradation des forêts \\
\hline RERA-SD & $\begin{array}{l}\text { Institution publique de développement et de coordination de la région } \\
\text { Split-Dalmatie - Croatie }\end{array}$ \\
\hline SCl & système de contrôle interne \\
\hline SCOPE & Programme de permaculture dans les écoles et les collèges - Kenya \\
\hline SENA & Service national d'apprentissage - Colombie \\
\hline
\end{tabular}




\begin{tabular}{ll} 
SPG & système participatif de garantie \\
\hline SAD & système alimentaire durable \\
\hline TIDCO & Tourism and Industrial Development Company - Trinité-et-Tobago \\
\hline TRIT & Institut de recherche sur le thé de Tanzanie \\
\hline UE & Union européenne \\
\hline UEMOA & Union économique et monétaire Ouest Africaine \\
\hline VMOV & vision, mission, objectifs, valeurs \\
\hline WATCO & The Wakulima Tea Company - Tanzanie \\
\hline
\end{tabular}




\section{CONCEPTS CLÉS}

L'AGROÉCOLOGIE consiste à appliquer des concepts et principes écologiques de manière à optimiser les interactions entre les végétaux, les animaux, les humains et l'environnement, sans oublier les aspects sociaux dont il doit prendre en compte pour que le système alimentaire soit durable et équitable. En tant qu'outil d'analyse, les 10 éléments peuvent aider des pays à opérationnaliser l'agroécologie. Ils définissent les caractéristiques importantes des systèmes et des approches agroécologiques, ainsi que les principaux éléments à considérer pour créer un environnement favorable à l'agroécologie. Ils orientent ainsi les responsables politiques, les professionnels et les parties prenantes dans la planification, la gestion et l'évaluation de la transition agroécologique. Étant liés et interdépendants, les 10 éléments sont: la diversité; la co-création et le partage de connaissances; les synergies; l'efficacité; le recyclage; la résilience; les valeurs humaines et sociales; la culture et les traditions alimentaires; la gouvernance responsable; l'économie circulaire et solidaire (FAO, 2018a).

Les PANIERS, également connues sous le nom de box schemes ou canastas, sont un type particulier de relation directe avec le marché ou de chaîne d'approvisionnement courte où le producteur livre une caisse de produits directement aux consommateurs à un intervalle régulier. Ils sont souvent rattachés aux Associations pour le maintien d'une agriculture paysanne (AMAPs) bien que le niveau d'engagement puisse varier considérablement (voir la définition ci-dessous).

La CERTIFICATION est une procédure par laquelle une partie donne l'assurance écrite qu'un produit, un procédé ou un service soit conforme à certaines normes (ISO, 2012). La certification peut être considérée comme une forme de communication tout au long de la filière. Le certificat démontre à l'acheteur que le fournisseur respecte le cahier de charges, ce qui est plus convaincant que si le fournisseur seul donne l'assurance. Dans ce manuel, nous utilisons le mot «garantie» plutôt que «certification», car nous explorons comment une variété d'acteurs peuvent s'assurer que d'autres acteurs suivent des normes spécifiques de durabilité (FAO, 2003).

L'ÉCONOMIE CIRCULAIRE est basée sur le partage, la location, la réutilisation, la réparation, la restauration et le recyclage des produits et matériaux dans un circuit idéalement fermé. L'objectif est de réduire le gaspillage en maintenant les produits et les matériaux dans l'économie (Whitaker et al., 2017).

L' ASSOCIATION POUR LE MAINTIEN D'UNE AGRICULTURE PAYSANNE (AMAP) est un partenariat entre une ferme et des consommateurs où les risques et les avantages de l'agriculture sont partagés. Élaborée à l'origine sur le modèle teikei au Japon dans les années 1970, chaque AMAP est organisée différemment, mais elles partagent toutes quatre principes fondamentaux: 1) Partenariat: L'AMAP est fondée sur un partenariat, généralement officialisé sous la forme d'un contrat individuel entre chaque consommateur et le producteur, et 
caractérisé par un engagement réciproque à s'approvisionner mutuellement (en argent et en nourriture) sur une longue période, au-delà de tout acte d'échange unique. Les contrats, oraux ou écrits, durent plusieurs mois, une saison ou une année. 2) Local: Les AMAP s'inscrivent dans une démarche active de relocalisation de l'économie. Dans le mouvement de l'AMAP, le terme «local» n'est pas limité à une signification géographique. Plus précisément, les producteurs locaux doivent être bien intégrés dans leur environnement: leur travail doit bénéficier aux communautés qui les soutiennent. 3) Solidarité: Les AMAP reposent sur la solidarité entre les producteurs et les groupes de soutien. Elles impliquent de partager, à la fois, les risques et les bénéfices d'une production saine et adaptée au rythme naturel de l'agriculture respectueuse de la saisonnalité, de l'environnement, du patrimoine naturel et culturel et de la santé. Payer d'avance un prix équitable suffisant permet aux agriculteurs et à leur famille d'entretenir leur ferme et de vivre dignement. 4) Le tandem producteur-consommateur est basé sur le contact et la confiance directs, sans intermédiaire ni hiérarchie (Bashford et al., 2013; URGENCI, 2016).

Un SYSTÈME ALIMENTAIRE comprend tous les éléments (ressources naturelles, personnes, intrants, processus, infrastructures, institutions, produits, etc.) et activités liées à la production, à la transformation, à la distribution, à la préparation et à la consommation des aliments et aux extrants de ces activités, y compris les impacts socio-économiques et environnementaux (HLPE, 2014). Dans ce manuel, nous considérons également la gestion des déchets en fin de cycle comme un élément essentiel d'un système alimentaire.

L' INNOVATION est le processus par lequel des individus ou des organisations maîtrisent et mettent en œuvre la conception et la production de biens et de services qui sont nouveaux pour eux, pour leurs concurrents, pour leur pays ou pour le reste du monde (FAO, 2014). "L'innovation en agriculture touche toutes les dimensions du cycle de production tout au long de la chaîne de valeur - de la production végétale, forestière, halieutique ou animale à la gestion des intrants et des ressources, à l'organisation et à l'accès aux marchés. Il peut s'agir, par exemple, de planter de nouvelles variétés de cultures, de combiner des pratiques traditionnelles avec de nouvelles connaissances scientifiques, d'appliquer de nouvelles pratiques de lutte antiparasitaire et de post-récolte ou de s'engager sur les marchés par des moyens nouveaux et plus rémunérateurs. L'innovation n'est pas seulement une question de technologie, qui, à elle seule, peut tout simplement rester dans un tiroir. Il s'agit aussi, et c'est peut-être le plus important, de processus sociaux, économiques, institutionnels, organisationnels et politiques qui impactent la vie des agriculteurs familiaux» (FAO, 2018d).

Dans ce manuel, l' AGRICULTURE BIOLOGIQUE est utilisée pour désigner les fermes agroécologiques qui ont été certifiées selon une norme biologique publique ou privée. La certification peut être effectuée par une première partie (par exemple, en Inde), par un tiers ou par un système participatif de garantie (SPG). La fréquence d'utilisation de ce terme par les innovateurs locaux dépend de la réglementation nationale en matière d'agriculture biologique, de leur base de consommateurs, ainsi que des traditions et de l'histoire de l'agriculture biologique dans leur pays d'exploitation. Parfois, même lorsque certains producteurs sont certifiés biologiques, ils préfèrent utiliser le terme agroécologique pour désigner leurs pratiques. 
Les SYSTÈMES PARTICIPATIFS DE GARANTIE (SPG) sont des systèmes d'assurance de qualité axés sur les besoins locaux. Ils certifient les producteurs sur la base de la participation active des parties prenantes et reposent sur la confiance, les réseaux sociaux et l'échange de connaissances (IFOAM, 2019). Dans ce manuel, nous discutons les SPG aux côtés de la certification comme un moyen pouvant garantir des pratiques durables.

Le JUSTE PRIX est le prix qui est acceptable/raisonnable pour toutes les parties impliquées dans la transaction. Le juste prix est juste et durable. La comptabilisation des coûts réels peut aider les acteurs à obtenir le juste prix.

La CHAÎNE D’APPROVISIONNEMENT est une séquence de processus (de prise de décision et d'exécution) et de flux (matériels, d'information et d'argent) qui vise à répondre aux besoins des clients finaux à l'intérieur et entre différentes étapes d'un continuum, de la production à la consommation finale. La chaîne d'approvisionnement comprend non seulement le producteur et ses fournisseurs, mais aussi, en fonction des flux logistiques, les transporteurs, les entrepôts, les détaillants et les consommateurs eux-mêmes. Dans un sens plus large, les chaînes d'approvisionnement peuvent également inclure le développement de nouveaux produits, le marketing, l'exploitation, la distribution, les finances et le service à la clientèle (FAO, 2007).

Un SYSTÈME ALIMENTAIRE DURABLE (SAD) est un système alimentaire qui assure la sécurité alimentaire et la nutrition pour tous de manière à ne pas compromettre les bases économiques, sociales et environnementales de la sécurité alimentaire et de la nutrition des générations futures (HLPE, 2014).

L'ÉCONOMIE SOLIDAIRE est un mouvement qui vise à transformer le système économique et social actuel en un système basé sur des échanges coopératifs et solidaires. Ces derniers prennent typiquement la forme de circuits courts et directs qui relient les besoins individuels à ceux de la communauté. Ce type d'économie repose sur des pratiques de production, d'échange et d'utilisation de biens et services qui répondent aux besoins économiques et sociaux des communautés locales, territoriales et internationales. Elle promeut la démocratie économique, la justice sociale, la conscience environnementale, la souveraineté alimentaire, l'équité entre les sexes et une approche pluraliste et multiculturelle. L'économie solidaire est déjà en marche grâce à des milliers d'initiatives citoyennes, de pratiques solidaires et de réseaux de collaboration à travers le monde.

La TRAÇABILITÉ est définie comme la capacité de retracer l'historique, l'application ou l'emplacement d'un produit ou d'une activité par le biais d'une identification enregistrée. Elle comporte deux aspects principaux: d'une part, l'identification du produit par marquage et, d'autre part, l'enregistrement des données relatives au produit tout au long de la chaîne de production, transformation et distribution (FAO, 2016b). 
La TRANSPARENCE dans un système alimentaire se définit comme la disponibilité de l'information, en particulier celle du juste prix et d'une véritable comptabilité analytique, dans le domaine public ou à la demande de toute partie prenante dans une chaîne d'approvisionnement. Dans un système alimentaire durable, les informations non-biaisés sont librement accessibles, y compris ceux qui pourraient désavantager les producteurs et les consommateurs.

Un INTERMÉDIAIRE TRANSPARENT dans la chaîne de valeur alimentaire durable est une personne ou une entité qui s'efforce de fournir des échanges transparents, abordables et facilement accessibles entre le producteur et le consommateur final. Toutes les fonctions de ces acteurs, en particulier les fonctions monétaires, sont disponibles dans le domaine public. Idéalement, au minimum, ils s'engagent à fournir un accès équitable aux producteurs et, au mieux, au consommateur final aussi.

La COMPTABILITÉ AU COÛT RÉEL (également appelée comptabilité au coût complet, valeur totale ou impact total) «regroupe les biens non-marchand, tels que les actifs environnementaux et sociaux, dans l'équation du développement, afin de permettre l'analyse des coûts et avantages des décisions commerciales et/ou politiques. À cette fin, une valeur monétaire doit être attribuée à des aspects tels que les services écosystémiques ou la santé (entre autres). L'objectif ultime n'est pas de monétiser la nature ou les gens, mais plutôt de traduire les ressources invisibles (comme les actifs intellectuels, humains, sociaux et naturels qui ne sont pas saisis dans les comptes financiers historiques) en une devise commune pour la prise de décisions stratégiques les dépendances qui impactent la création de la valeur globale» (FAO, 2017). 

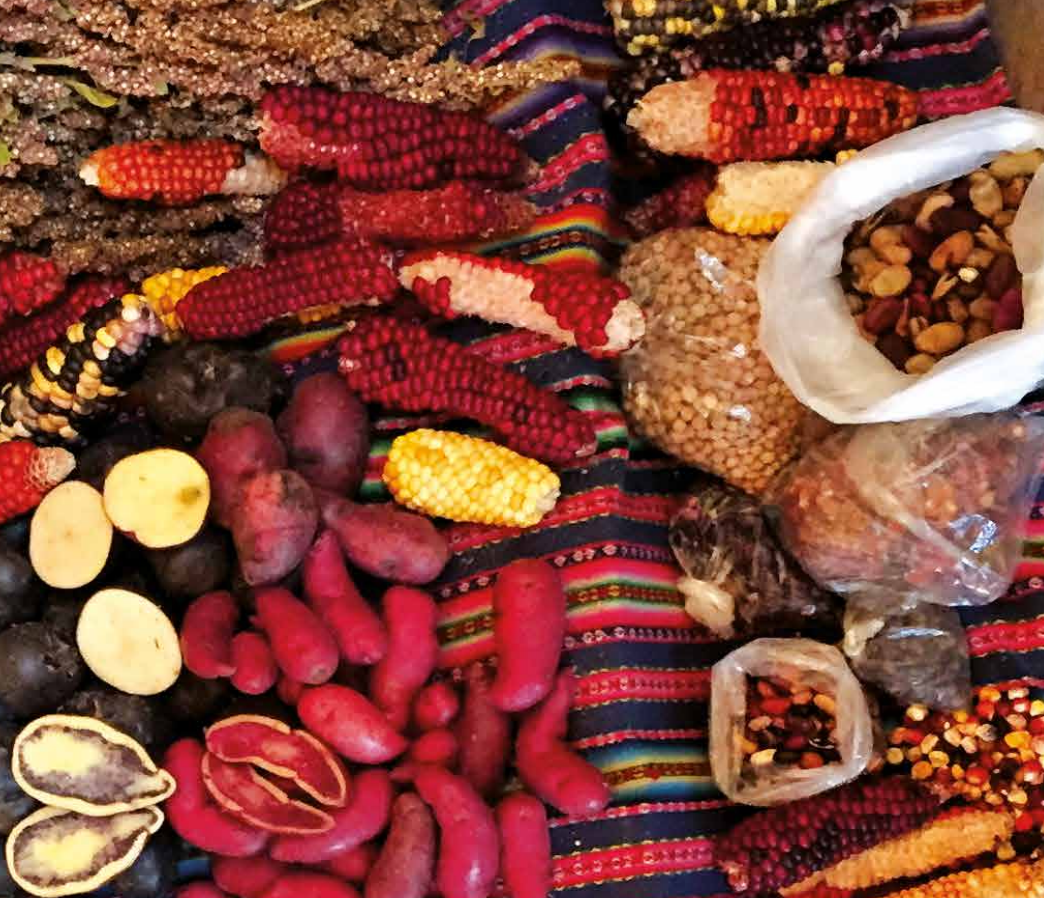

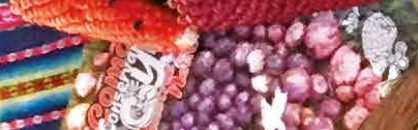

$\because-0$ s

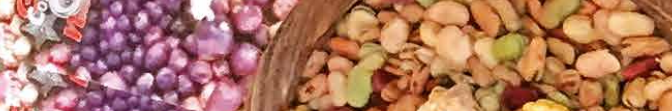
and $x+3=50$.

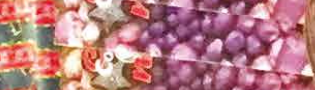

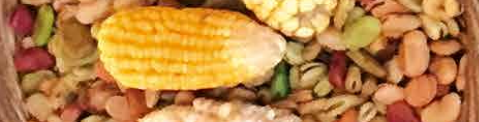

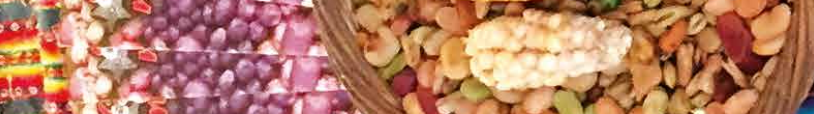

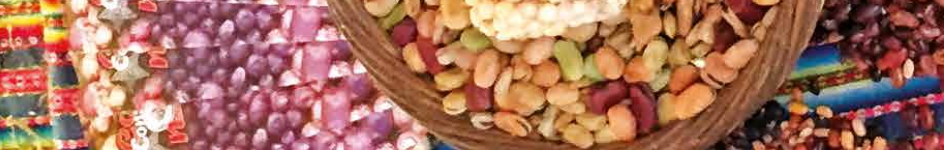

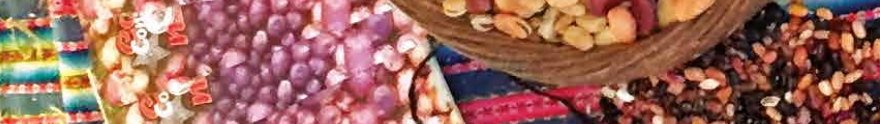

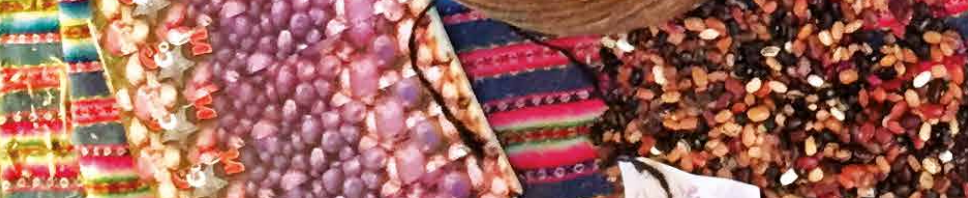

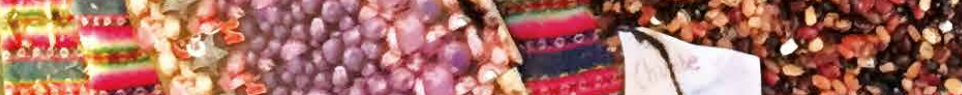

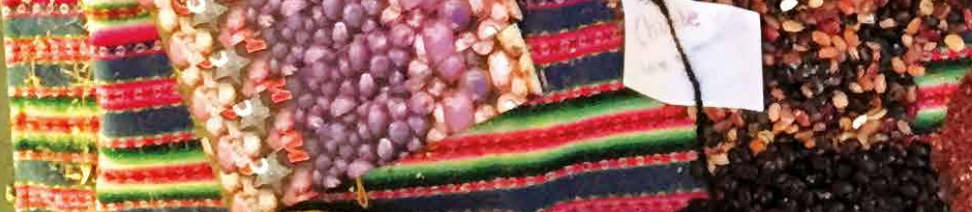
whis

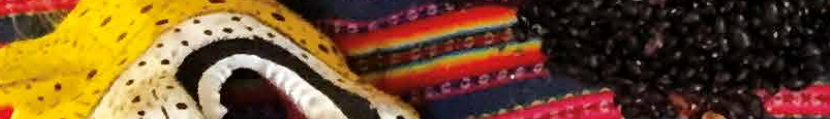

$:$
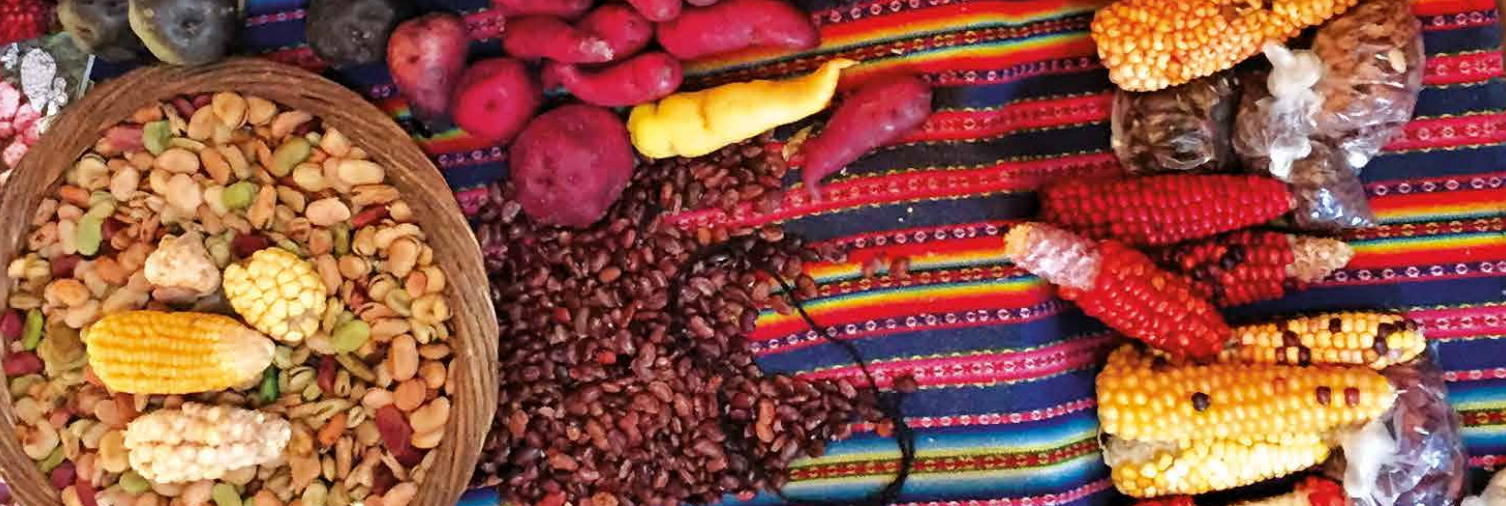

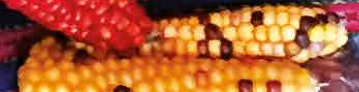

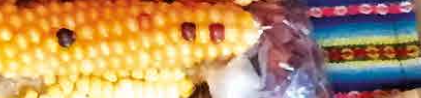
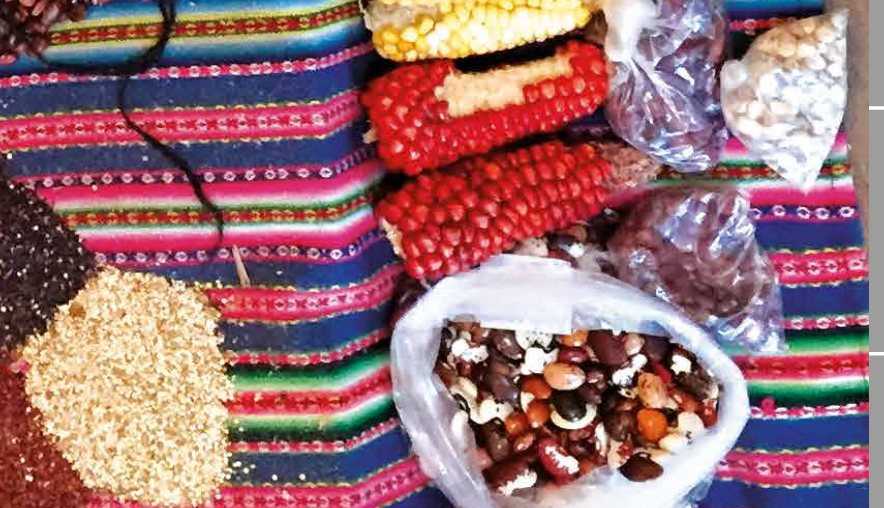


\section{INTRODUCTION}

«Nous devons promouvoir un changement transformateur dans la façon dont nous produisons et consommons les aliments. Nous devons proposer des systèmes alimentaires durables qui offrent des aliments sains et nutritifs, tout en préservant l'environnement.

L'agroécologie peut apporter plusieurs contributions à ce processus (...) l'agroécologie peut améliorer la résilience des agriculteurs, en particulier dans les pays en développement où la faim se concentre. Elle peut contribuer à stimuler l'économie locale. Elle peut sauvegarder les ressources naturelles et la biodiversité, ainsi que promouvoir l'adaptation au changement climatique et l'atténuation de ses effets. Elle peut aussi promouvoir la culture locale et les connaissances traditionnelles. Ces multiples avantages font de l'agroécologie une voie importante pour atteindre les objectifs de l'Agenda 2030 et relever les défis qui sont interdépendants».

José Graziano da Silva, Directeur général de la FAO Déclaration d'ouverture au $2^{e}$ Symposium international d'agroécologie. Rome, Italie 3 avril 2018

Nous vivons un moment critique où il est devenu évident que l'humanité doit prendre des mesures immédiates pour s'assurer que les systèmes alimentaires qui nous nourrissent aujourd'hui, demain et dans l'avenir soient durables (FAO, 2012). L'arrivée de l'anthropocène (Crutzen, 2006) et la vitesse sans précédent de la perte de biodiversité documentée par l'IPBES (Brondizio et al., 2019) ont suscité des inquiétudes importantes quant à la capacité de la Terre à soutenir les habitudes alimentaires et les modes de vie contemporains. Ceci est particulièrement vrai dans les débats portant sur la sécurité alimentaire dans les zones urbaines croissantes, tant dans les pays en développement que dans les pays développés (Fressoz and Bonneuil, 2016). En effet, l'étude de la Commission EAT-Lancet sur les régimes alimentaires sains soutient qu'étant donné «qu'une grande partie de la population mondiale est insuffisamment nourrie et que de nombreux systèmes et processus environnementaux sont poussés au-delà des limites prescrites pour la production alimentaire, une transformation globale du système alimentaire est nécessaire de toute urgence» (Willett et al., 2019). Cela signifie que nous ne pouvons pas nous concentrer uniquement sur la durabilité des pratiques de production qui fournissent des aliments (par exemple, les approches agroécologiques et la résistance au changement climatique), mais que nous devons également examiner la durabilité de la demande d'aliments sains et diversifiés. 
Pourtant, qui est responsable du rééquilibrage de l'offre et de la demande de denrées alimentaires aujourd'hui et dans l'avenir ? La création des objectifs de développement durable (ODD), comme un nouvel appui pour la communauté mondiale de gouverner les progrès réalisés pour surmonter les défis sociétaux interdépendants (Kanie and Biermann, 2017), suggère que ce rééquilibrage est complexe. Pour faire la transition vers des systèmes alimentaires durables, nous devons apporter des changements dans de nombreux éléments clés: comment nous cultivons et consommons les aliments; mais aussi comment nous utilisons et conservons les ressources naturelles; comment nous les transformons en aliments, en énergie et en matériaux qui sont transportés, distribués et parfois gaspillés; ce que nous en faisons lorsqu'ils ne sont plus utiles dans leur forme initiale; comment nous finançons nos activités; les façons dont nous organisons notre travail et nos communautés; et comment nous valorisons les différents types de connaissances que divers acteurs apportent aux activités des systèmes alimentaires. Les changements qui s'imposent touchent toutes les échelles d'action - du niveau local au niveau régional, national et international, en passant par le niveau territorial. Chacun joue un rôle - les changements ne peuvent être entraînés par les gouvernements ou le secteur privé seuls, les citoyens individuels et les groupes organisés de citoyens, les chercheurs et les praticiens de toutes sortes doivent aussi y contribuer.

Cette tâche semble alarmante, et si elle n'est déléguée qu'à un petit nombre d'acteurs qui travaillent à l'échelle mondiale, elle l'est certainement. Cependant, la diversité des systèmes alimentaires dans le monde offre des possibilités de changements progressifs et à petite échelle qui peuvent s'amalgamer et s'affilier pour effectuer des changements au niveau global. L'ODD 12 - consommation et production responsables - propose des objectifs pour assurer des modes de consommation et de production durables à l'avenir. Les travaux entrepris dans le cadre du Programme des systèmes alimentaires durables du réseau One Planet (UN Environment, 2020), qui contribuent à la réalisation de l'ODD 12, donnent un aperçu de la façon dont les initiatives d'acteurs dévoués à petite échelle peuvent surmonter les innombrables défis de la durabilité qui marquent les systèmes alimentaires conventionnels. De ces expériences, nous pouvons apprendre comment influer et inspirer un changement plus vaste dans le système alimentaire mondial.

C'est dans ce contexte que nous parlons d'innovation dans les systèmes alimentaires durables. Pour s'assurer que les systèmes alimentaires futures sont durables, il faut des idées, des pratiques et des modes d'organisation nouveaux (ou oubliés) qui garantissent la durabilité écologique, l'inclusion économique et l'équité sociale des activités qui amènent la nourriture provenant d'organismes vivants dans le sol ou dans l'eau jusqu'aux assiettes des consommateurs.

Des recherches antérieures et des années d'expérience pratique nous indiquent que les petits exploitants agricoles et les familles d'agriculteurs dans les pays en développement sont à la tête d'initiatives qui ont trouvé des solutions novatrices aux problèmes de durabilité de leurs systèmes alimentaires locaux (FAO, 2014, 2016a, 2018b, 2018c). Au fil des ans, ils ont utilisé de nombreux mots pour décrire ce qu'ils font: par exemple, les systèmes agroécologiques alimentaires, l'agriculture soutenue par la communauté, les systèmes alimentaires traditionnels, les marchés de producteurs, les systèmes de boîtes, les chaînes de valeur alimentaires courtes, les assemblées alimentaires, les systèmes agroalimentaires alternatifs, les systèmes alimentaires locaux, les marchés imbriqués, les réseaux alimentaires paysans. Trop souvent, ces activités passent inaperçues ou restent dans les communautés locales. Le partage horizontal des idées et des expériences est nécessaire pour 
élargir (augmenter la taille) ou étendre (reproduire et adapter ailleurs) l'impact de ces innovations afin de devenir plus inclusif, de sorte que davantage d'acteurs locaux puissent expérimenter par eux-mêmes pour trouver les idées et actions qui fonctionnent dans leurs propres communautés. Cette forme de réplication adaptée favorise l'innovation. Précisément le but de ce manuel, c'est defaciliter le partage d'idées et d'actions innovantes à travers le monde.

\section{AUDIENCE, OBJECTIFS ET PORTÉE}

Ce manuel a pour but d'aider les acteurs - ceux que nous appelons les innovateurs du système alimentaire - qui expérimentent activement la (re)valorisation de l'agriculture dans des systèmes alimentaires durables, c'est-à-dire qui modifient la façon de produire, transformer, transporter, stocker, vendre et consommer les produits alimentaires et agricoles. Selon les expériences des auteurs, ces acteurs peuvent être des agriculteurs, des chercheurs, des commerçants, des groupes de consommateurs, des consommateurs individuels engagés, des ONG, des fonctionnaires locaux et bien d'autres (FAO, 2016a).

Les changements dans les systèmes alimentaires sont complexes et il n'existe pas de feuilles de route déjà prêtes. Les innovateurs doivent plutôt être en mesure de naviguer à travers des possibilités et des défis divers qui se présentent au fur et à mesure que les changements se produisent. En tant que moteur du changement, les innovateurs doivent évaluer leur positionnement dans leur propre système alimentaire ainsi que leurs objectifs pour l'avenir du système afin de pouvoir créer la valeur selon leurs propres conditions. Si vous commencez à vous poser les questions suivantes, ce manuel devrait vous aider à trouver des réponses:

1. Est-ce que vos clients posent des questions sur vos pratiques durables et vous ne savez pas comment les communiquer?

2. Vous voulez accéder aux intrants nécessaires pour répondre aux besoins de votre marché ?

3. Vous avez une base de consommateurs et un groupe de producteurs dévoués, mais vous n'êtes pas en mesure de maintenir un approvisionnement régulier?

4. Vous avez entendu parler des systèmes participatifs de garantie et vous souhaitez en savoir plus à leur sujet ?

5. Vous voulez apprendre comment fixer le prix de vos produits pour différents marchés?

6. Vous voulez en savoir plus sur les intermédiaires transparents et comment ils pourraient soutenir votre initiative?

7. Vous voulez apprendre comment organiser une visite de ferme pour les consommateurs ?

8. Vous essayez de travailler avec d'autres initiatives mais vous n'êtes pas sûr des modalités de collaboration?

9. Vous voulez en savoir plus sur la production durable afin de mieux calculer vos prix ?

10. Vous avez épuisé vos moyens habituels pour financer votre initiative et vous êtes à la recherche de nouvelles idées? 
Ce guide offre des recommandations sous forme d'astuces, de listes de vérification et de matrices qui aideront les innovateurs du système alimentaire local à réfléchir de façon plus stratégique aux possibilités qui peuvent exister autour d'eux. Il s'appuie sur des exemples, rédigés par des innovateurs des systèmes alimentaires qui ont essayé chaque approche, visant à inspirer le lecteur à tester (ou à éviter, ou à adapter) une solution au problème en question. Il partage des astuces pour identifier les occasions d'innover, en mettant l'accent sur des solutions peu coûteuses et réalisables pour les pays en développement. Il est rédigé dans un langage facilement accessible et informel.

\section{COMMENT CE MANUEL A-T-IL ÉtÉ ÉLABORÉ?}

Ce manuel a été rédigé par des innovateurs de systèmes alimentaires durables qui ont partagés leurs propres expériences.

La collaboration a débuté en 2013 avec un appel lancé par la FAO et l'INRAE pour rassembler les innovations et les initiatives qui relient les petits producteurs durables et les consommateurs des marchés locaux. Un premier atelier chercheurs-praticiens a eu lieu à Bogotá, en Colombie, en 2015, au cours duquel des défis influant des systèmes alimentaires locaux durables ont été identifiés avec environ 50 participants dans 20 pays. Un deuxième atelier chercheurs-praticiens s'est tenu à Chiang Mai, en Thaïlande, en 2016. Durant, cet atelier, 5 participants de 15 pays ont élaboré la structure des chapitres du manuel et ont repéré les thématiques initiales pour lesquelles plus de connaissances étaient requises. En 2017, deux séries de trois groupes de travail ont été organisées pour rédiger et réviser le texte de chaque chapitre. En novembre 2017, le troisième atelier chercheurs-praticiens a eu lieu à Delhi, en Inde, où 25 personnes de 21 pays ont élaboré les parcours d'apprentissage utilisés dans ce manuel et ont finalisé le texte. En 2018, le manuel a été testé avec des partenaires: Jaivik Haat en Inde et le projet «Résilience climatique» au Sénégal financé par la FAO et le Fonds pour l'environnement mondial (GEF).

Les initiatives suivantes sur les systèmes alimentaires durables ont participé à la rédaction de ce manuel: Claudia Helena Prieto Parque Temático en Salud Pública Chaquen (Colombie), CICODEV (Sénégal), Earth Net Foundation (Thaïlande), Ecovida (Brésil), Familia de la Tierra (Colombie), FreshVeggies Ltd (Ouganda), Fonds national de développement agro-sylvo-pastoral (FNDASP) (Sénégal), Fundación Ekorural (Équateur), IFOAM Organics International (Pérou et NouvelleZélande), Jaivik Haat/Gram Disha Trust (Inde), Keystone Foundation (Inde), PELUM Kenya (Kenya), SPG de Quezon (Philippines), RERA SD (Croatie), Shared Harvest (Chine), Tea Research Institute of Tanzania (Tanzanie), Terrahabilis-Demeter (Pérou), le réseau international AMAP URGENCI (France et monde).

Le résultat est un guide pour les personnes qui travaillent dans les espaces urbains et ruraux afin de s'assurer que les aliments sont produits et consommés de façon durable. 


\section{COMMENT LIRE CE MANUEL?}

Le manuel est conçu comme «un livre dont vous êtes le héros». Il n'y a pas de façon «optimale» de le lire ou un point précis d'entrée.

- Il peut être lu à partir de n'importe quel chapitre en fonction d'intérêts spécifiques, et chaque chapitre peut être lu séparément. Par exemple, si vous vous intéressez principalement à quelques conseils sur la façon de soutenir la collaboration entre agriculteurs et chercheurs, vous pouvez vous concentrer sur le chapitre sur le partage et la co-création de connaissances (Chapitre 4).

- Il peut être utilisé dans le cadre d'un cours de formation ou d'une réunion de planification stratégique entre les intervenants qui collaborent à une initiative sur les systèmes alimentaires durables. Dans ce cas, il peut servir d'outil pour structurer une discussion et pour élaborer un plan d'action (Section 3).

Peu importe la façon dont vous choisirez de le lire, nous espérons que ce guide vous mènera sur un chemin d'apprentissage - un voyage d'innovation partant de votre idée jusqu'à la mise en œuvre d'un changement durable du système alimentaire.

Le chapitre suivant fournit un guide pour la conception de votre aventure d'apprentissage afin de vous aider à mettre en œuvre le changement que vous souhaitez voir.

Nous envisageons les chapitres du manuel comme un cercle d'interconnexions (Figure 1), car les possibilités de liaisons entre elles sont nombreuses.

La dernière section de chaque chapitre propose au lecteur des chapitres à consulter après lecture. Cela garantit que le manuel offre de multiples possibilités d'exploration et d'apprentissage. 
Figure 1: Chemins d'apprentissage possibles en commençant par n'importe quel chapitre

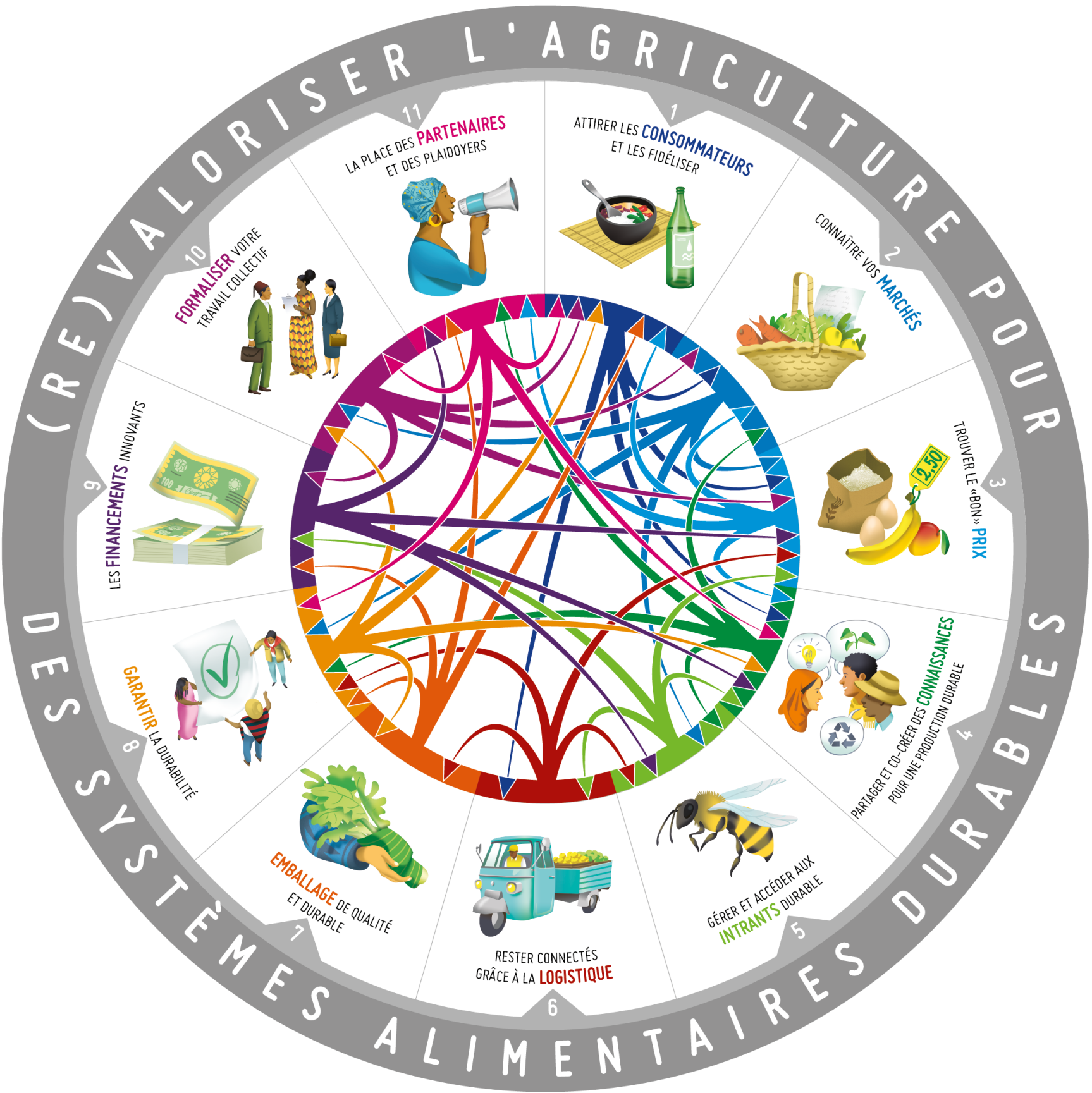




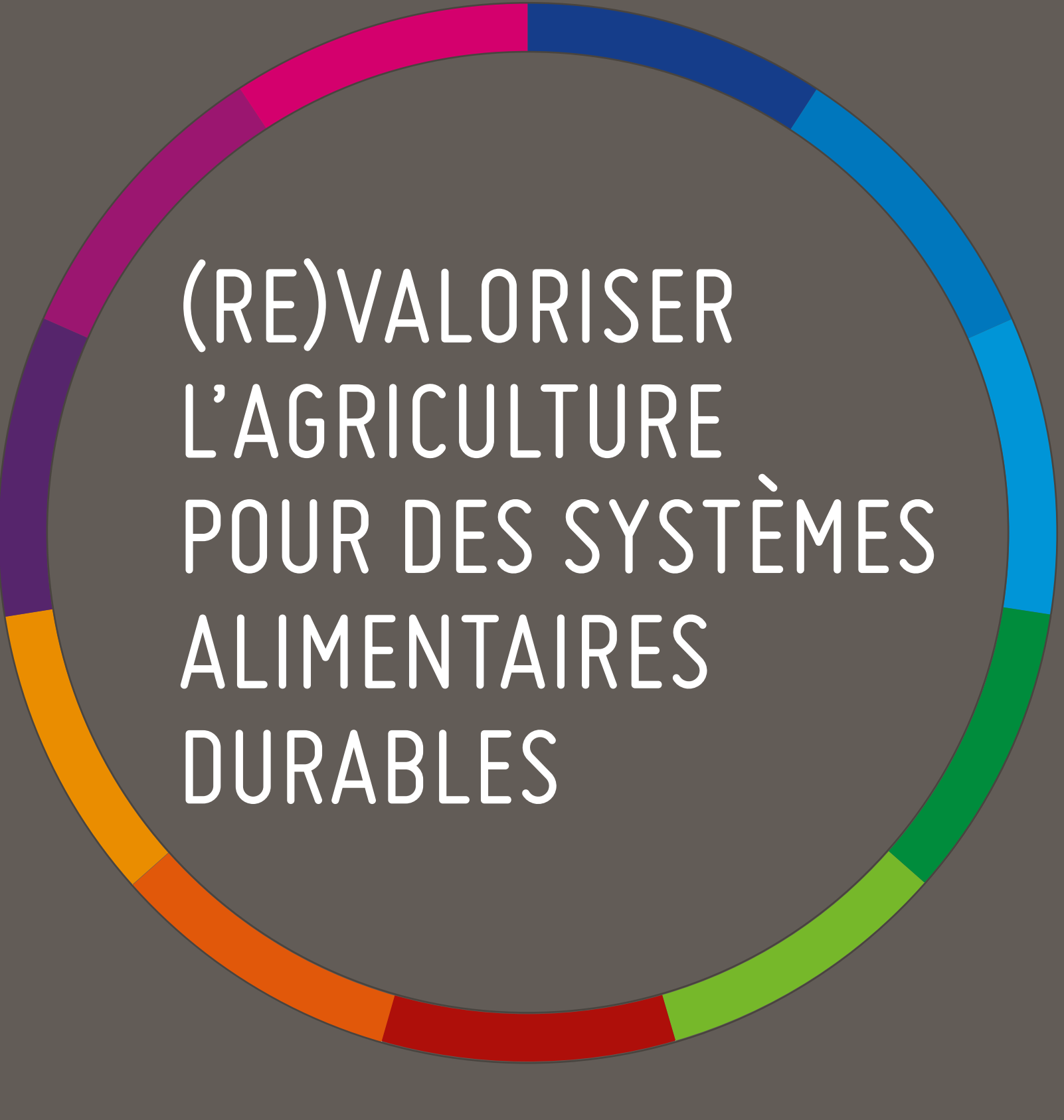


«Vous avez besoin d'un agriculteur au moins deux à trois fois par jour pour manger, Vous avez besoin d'un médecin ou d'un avocat qu'une fois par an (si vous n'avez vraiment pas de chance)»

Un agriculteur et activiste de l'Himachal Pradesh, Inde

\section{POURQUOI EST-CE IMPORTANT?}

- L'agriculture à la fois contribue au changement climatique et en subit les effets. Les changements positifs apportés aux pratiques agricoles, à l'élevage, à la pêche et à la foresterie sont également utiles pour réduire les émissions de gaz à effet de serre qui contribuent aux aggravations climatiques.

- En 2016, pour la première fois en près de 70 ans, le taux de malnutrition dans le monde a commencé à augmenter. Ceci est le résultat du double fardeau de la malnutrition et de l'obésité que l'on trouve dans un même pays. Sans de nouvelles méthodes de travail, la FAO a déclaré que l'objectif pour l'an 2030 d'un monde privé de faim et de malnutrition ne sera probablement pas atteint (FAO et al., 2017).

- L'agriculture est souvent «oubliée» ou considérée comme un «travail laborieux», ce qui ignore la beauté des paysages agricoles, la valeur que les agriculteurs apportent aux économies nationales ainsi que la santé et la diversité de tous les êtres vivants.

- L'expérience acquise dans le monde entier suggère que les approches agroécologiques peuvent changer la façon dont les producteurs, les consommateurs, les intermédiaires et les décideurs apprécient la durabilité et l'agriculture. Ceci, certainement, demande du travail et du temps.

- De plus en plus, les consommateurs du monde entier reconnaissent les liens étroits qui existent entre la façon dont les aliments sont produits et la qualité de ces produits (en termes de sécurité alimentaire, de nutrition et d'impact environnemental). Ils sont de plus en plus exigeants en termes de qualité et d'information sur les pratiques de production agricole et sur l'origine de leurs aliments.

- Avant de commencer à apporter des changements à votre système alimentaire, il est important d'envisager vos objectifs, les valeurs que vous voulez que votre système (re)produise et comment vous pensez y arriver.

Pour ces raisons et bien d'autres encore, la revalorisation de l'agriculture au sein de la société (et en particulier, par des changements dans nos systèmes alimentaires) est fondamentale pour assurer la durabilité de nos activités humaines. 


\section{QUE SIGNIFIE LA "VALEUR» DANS CE MANUEL?}

La valeur est le résultat d'un processus d'évaluation, de négociation et de la valorisation d'un bien ou d'un service (Vatin, 2013); c'est un compromis entre qualité et prix. Ce terme désigne également la façon dont un produit crée de la valeur pour les acteurs qui le fabriquent, l'utilisent et le commercialisent (FAO, 2018b). Enfin, la valeur des aliments cultivés et consommés est également liée aux valeurs sociales et culturelles de la communauté (par exemple, conservation de l'environnement, santé et bien-être, équité, solidarité, économie locale, tradition, dignité humaine) (Ostrom et al., 2017).

La façon dont un système alimentaire crée de la valeur pour les gens est liée à la façon dont le système est organisé et aux valeurs prioritaires. La littérature sur les économies diversifiées met l'accent sur les possibilités de créer de nouvelles économies (Gibson-Graham, 2008) en organisant l'entreprise, le travail, la propriété, les transactions et la finance par des moyens alternatifs qui peuvent redéfinir les priorités des valeurs sociétales et des types spécifiques d'activités productives. La Figure 2 est une représentation graphique simple d'une priorisation de la valeur de l'agriculture dans les systèmes alimentaires actuels: la valeur qu'elle produit pour le développement économique (c.-à.-d. contribuer à une économie de marché), la valeur qu'elle crée pour la santé humaine et la nutrition (c.-à.-d. nourrir l'économie alimentaire), et la valeur qu'elle crée pour les processus écologiques de la planète (c.-à.-d. participer à l'économie naturelle). En effet, nous ne cessons d'accroître les exigences humaines à l'égard de l'agriculture et de ses ressources naturelles de moins en moins abondantes afin de répondre aux besoins de l'expansion de l'alimentation et des économies de marché (FAO, 2012).

Figure 2: (Re)valoriser l'agriculture

LA VALEUR DE NOS ÉCONOMIES D'AUJOURD'HUI

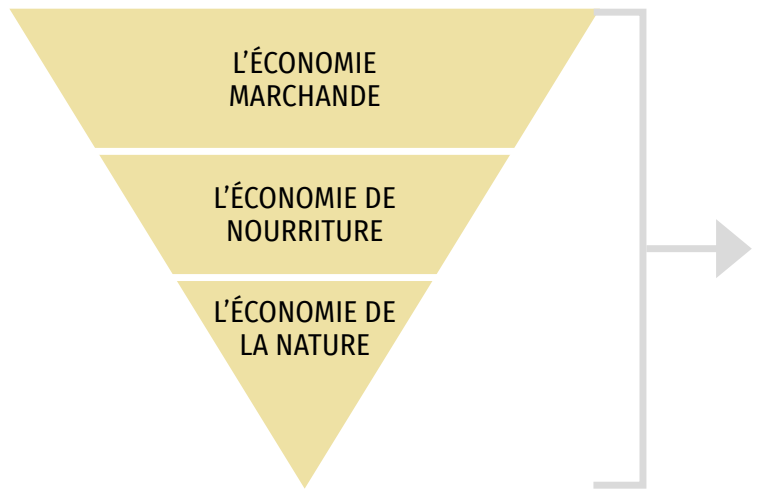

LA VALEUR QU'ON POURRAIT SOUHAITER POUR L'AVENIR

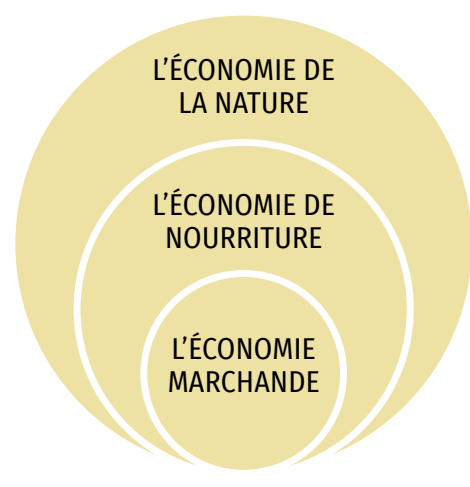

Source: Adapté d'un brainstorming des auteurs à Chiang Mai, 2016 et O. Nieto, Familia de la Tierra, Colombie. 
La valeur économique (ou monétaire) de l'agriculture est très importante dans les économies de marché car elle permet de mener une vie saine et productive. Cependant, pour que les générations futures puissent être productives et en bonne santé, la valeur économique de l'agriculture ne peut être la seule «valeur» attribuée à la façon dont nous produisons les aliments. Si nous ne considérons pas également la valeur sociale, nutritionnelle et écologique au sein du système alimentaire - en d'autres termes, si nous ne donnons pas plus d'importance à l'économie alimentaire et à l'économie de la nature - nous continuerons à perpétuer des modes de production et de consommation non-durables. Ceux-ci ne feront que reproduire les conséquences négatives environnementales, sanitaires et sociales dont nous avons déjà commencé à témoigner. En effet, si nous continuons sur cette voie, notre triangle hypothétique s'effondrera.

Les changements qui doivent être apportés au système sont ceux qui peuvent redéfinir l'ordre de priorité de la contribution de l'agriculture à ces trois économies. Nous pourrons alors travailler à partir d'une base solide et saine d'une économie de la nature, plutôt que d'une économie étroite axée sur l'extraction des ressources. Le renforcement des formes régénératives d'agriculture où les pratiques de production peuvent à la fois restaurer les écosystèmes et apporter la santé aux agriculteurs enrichira notre façon de nous nourrir et fera en sorte que cela soit économiquement viable et inclusif pour tous les acteurs. Nous devons donc réintégrer l'économie de marché, qui met l'accent sur la création de richesses dans l'économie de l'alimentation en apportant la santé à tous et en éliminant l'insécurité alimentaire et nutritionnelle. Ces valeurs doivent être atteintes à l'intérieur des limites des ressources planétaires (Rockstrom et al., 2009) si nous voulons atteindre les objectifs du développement durable.

\section{Cette redéfinition des valeurs est fondamentale pour "(re)valoriser" le rôle de l'agriculture dans le système alimentaire.}

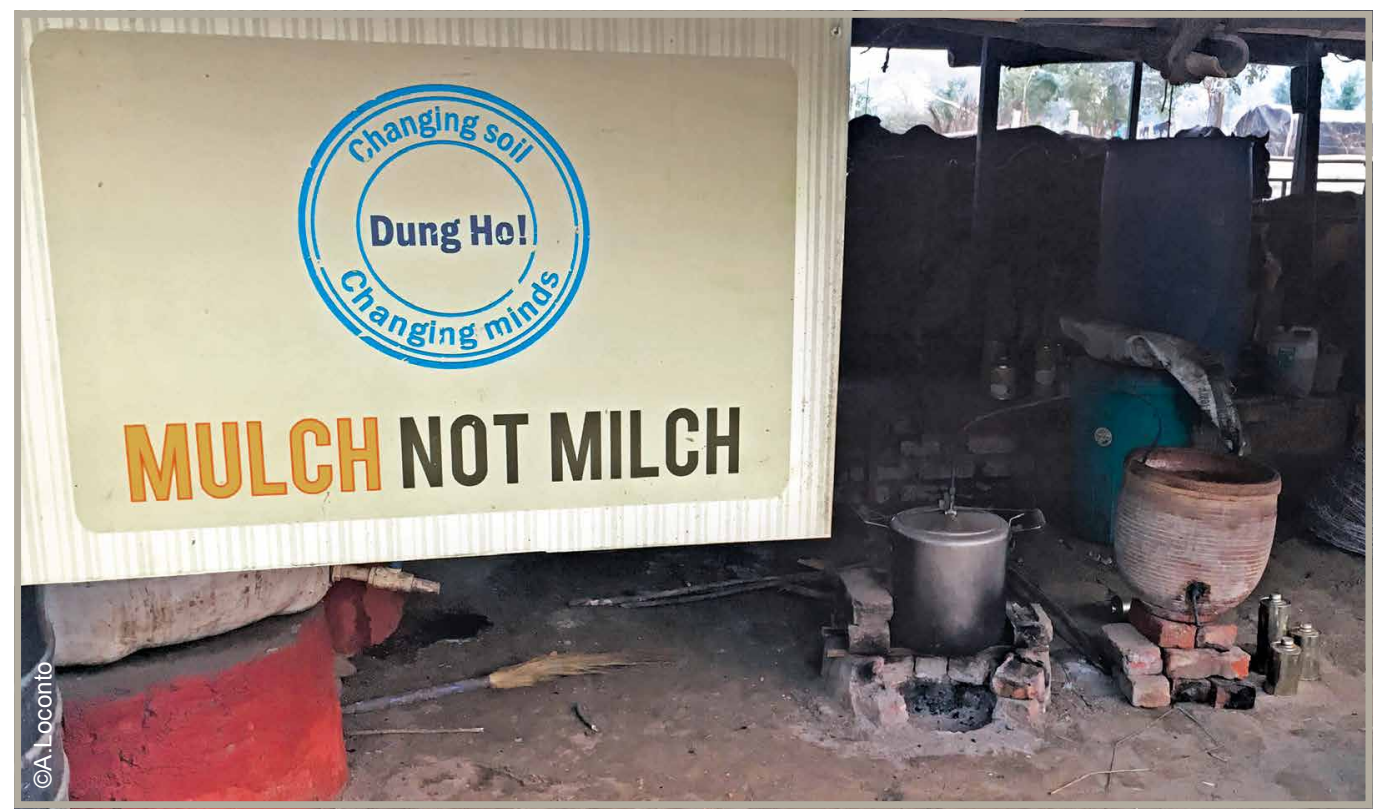


L'agriculture peut être réévaluée de diverses manières, en tenant compte des contributions économiques, culturelles, sociales et environnementales suivantes:

- Une augmentation au niveau de la diversification des systèmes d'exploitation agricole (par exemple, systèmes riz/poisson, riz/canards ou systèmes agroforestiers) peut fournir des produits et services diversifiés à haute valeur écologique, économique et culturelle.

- Il existe de nombreux exemples de tous les agroécosystèmes qui montrent que l'adoption de pratiques agroécologiques régénératives peut créer des valeurs écologiques, sanitaires et sociales grâce à une meilleure conservation de l'eau, une meilleure santé des sols, une biodiversité accrue, le retour de la faune, des environnements écologiques et de vies saines pour les agriculteurs et leur famille et des conditions de travail plus sûres.

- La résilience de la production et de la consommation agroécologiques apportera une valeur économique, sociale et écologique, tant pour les producteurs que pour les consommateurs. Par exemple, en période de stress environnemental comme les sécheresses ou les inondations, parmi d'autres, les producteurs peuvent se nourrir grâce à cette résilience.

- La «revalorisation» peut aussi être comprise d'une perspective de valeur ajoutée. En utilisant les technologies de transformation pour augmenter la durée de conservation, pour créer de nouveaux produits et sous-produits et pour rendre les produits traditionnels plus largement disponibles, les producteurs récupèrent ainsi leur valeur écologique, nutritionnelle, culturelle et économique. La fermentation est un exemple de transformation de matières premières en nouveaux produits à valeur ajoutée (par exemple, cornichons, kimchi, fromage, vin, charcuterie naturelle, pain au levain, sauce soja, tempeh, miso). D'autres transformations peuvent se produire dans les fermes ou dans les économies locales en utilisant des technologies biologiques naturelles pour ajouter de la valeur aux produits et aux écosystèmes, comme la transformation des résidus en champignons; la fabrication d'inoculants microbiens à partir de matières animales et végétales et de sols locaux; la préparation de pesticides botaniques ou de biopesticides fabriqués localement à l'aide de champignons ou de bactéries d'origine naturelle; le lombricompostage; ou le bio-charbon.

- La valeur médicinale et nutritionnelle peut être créée par la culture et la consommation de «variétés autochtones» et d'espèces négligées et sous-utilisées dont les propriétés médicinales, chimiques et nutritives ont été confirmées (et peuvent être vérifiées en laboratoire et par d'autres méthodes).

- Les innovations institutionnelles peuvent créer de la valeur grâce à la diversification des services que les exploitations agricoles peuvent fournir, par exemple:

- l'éducation: les agriculteurs durables peuvent être de merveilleux enseignants pour d'autres membres de communautés, proches et éloignés, en partageant de nouvelles façons de cultiver et de vivre;

- la solidarité: la confiance et l'amitié entre les consommateurs et les agriculteurs peuvent s'établir grâce à l'agriculture soutenue par la communauté et à d'autres formes de liens directs de production/consommation.

- la communauté: dans de nombreux pays en développement, les échanges de connaissances communautaires et la confiance interpersonnelle sont développés et revitalisés grâce aux systèmes participatifs de garantie pour l'agriculture biologique, où les producteurs dirigent le processus de certification. 
(Ré)valoriser l'agriculture dans des systèmes alimentaires durables signifie aussi de redonner la priorité aux valeurs économiques, sociales, culturelles, nutritionnelles et/ou écologiques dans les décisions qui sont prises sur comment, où et quand produire, commercialiser et consommer les aliments.

Elle exige des changements institutionnels pour la gestion des systèmes alimentaires et dans les infrastructures qui servent à rapprocher les producteurs et les consommateurs. Cela signifie que chaque acteur dans les systèmes alimentaires - qui possède les compétences nécessaires pour produire, transformer, commercialiser et consommer - doit trouver des moyens d'intégrer la durabilité.

Une «initiative sur les systèmes alimentaires locaux durables" désigne l'ensemble des activités liées aux pratiques de production, de transformation, d'échange, de recherche et d'éducation qui rassemblent des personnes et des organisations travaillant ensemble pour rendre la production et la consommation agroalimentaires locales plus durables.

Dans la plupart des cas, il s'agit du groupe d'acteurs qui est le principal facilitateur des pratiques durables au sein de leur système alimentaire local (par exemple, Familia de la Tierra, SPG Freshveggie, BhoomiKa).

Source: FAO, 2016; 2018.

Nous constatons qu'à travers le monde, un grand nombre de personnes se sont déjà engagées à réévaluer l'agriculture et la durabilité. Néanmoins, elles manquent parfois d'idées pratiques, d'outils et de compétences pour améliorer le fonctionnement des systèmes alimentaires. Cela signifie également que des nouveaux acteurs doivent apprendre à produire, commercialiser et consommer les aliments de manière durable et qu'ils auront besoin de nouveaux types de connaissances, de technologies et d'interactions.

Pour rééquilibrer l'offre et la demande d'aliments durables et pour faire en sorte que les anciens et les nouveaux acteurs assument la responsabilité des transitions vers des systèmes alimentaires durables, il faut que ces acteurs aient davantage de possibilités de réfléchir et de travailler ensemble. En s'appuyant sur leurs valeurs, les acteurs peuvent envisager de futurs systèmes alimentaires durables et agir localement pour en faire une réalité. Ce manuel peut être utilisé à cette fin.

Dans la section suivante, nous présentons un outil de réflexion qui peut être utilisé dans le cadre d'une initiative pour envisager des scénarios futurs, clarifier vos valeurs et utiliser le manuel pour planifier des actions futures. 


\section{UTILISER LE MANUEL POUR ENTREPRENDRE UN VOYAGE D’APPRENTISSAGE ET D’ACTION}

Ce manuel peut être utilisé comme support de formation ou de réflexion collective. Dans cette section, nous offrons des notes à l'intention des facilitateurs pour accompagner un groupe dans la lecture du guide. Nous utilisons un exemple développé lors de la mise à l'essai du manuel pour illustrer un scénario possible.

Le délai proposé est d'une heure 30 minutes pour préparer le scénario, puis d'au moins deux à trois heures pour examiner le manuel et élaborer un plan d'action. Si vous utilisez le manuel dans une session de formation, vous voudrez prendre plus de temps (au moins deux jours pour aborder tous les sujets), mais si vous l'utilisez dans une réunion d'une demi-journée ou d'une journée, vous pouvez faire une révision rapide.

Étapes simples pour démarrer le voyage:

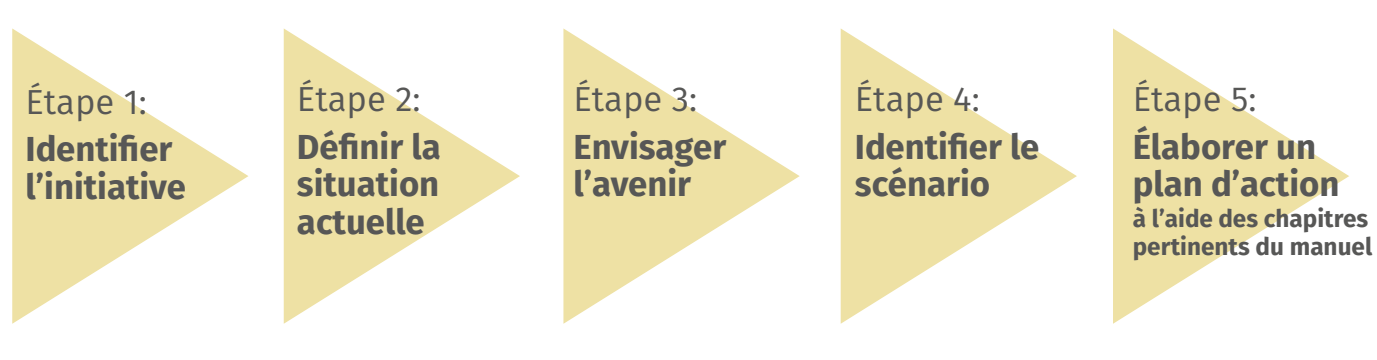

\section{Étape 1: Identifier l'initiative}

\section{Facilitateur:}

- Vous pouvez utiliser cet exercice avec un groupe de personnes qui travaillent déjà ensemble pour réfléchir collectivement aux défis, aux scénarios et aux solutions potentielles sur un projet ou une initiative de systèmes alimentaires spécifiques.

- Si vous utilisez cet exercice au cours d'une séance de formation ou d'ateliers de personnes qui travaillent sur différentes initiatives, animez une discussion [15 minutes] afin que le groupe identifie collectivement une initiative à laquelle il participera au cours de cet exercice.

Quelques questions importantes peuvent être posées: y a-t-il un problème spécifique que vous essayez de résoudre en ce moment ? Avez-vous récemment fait un exercice de planification stratégique où avez-vous des idées sur la direction que vous voulez prendre à l'avenir ? Vous pouvez choisir le projet d'un des participants comme étude de cas; le participant fournira les informations nécessaires sur son initiative, tandis que les autres membres du groupe l'aideront à réfléchir aux questions et aux solutions. 
Si vous faites partie d'un grand groupe, vous pouvez vous diviser en groupes de 8 à 10 participants maximum, qui travailleront sur différentes initiatives.

Identifier un rapporteur qui écrira les informations produites pendant l'exercice ainsi qu'un porte-parole qui présentera le scénario et le plan de travail à la fin de la séance.

\section{Étape 2: Définir la situation actuelle}

\section{Facilitateur:}

- Animez une discussion [30 minutes] qui soulignera les différents éléments de la situation actuelle du système ou de l'initiative alimentaire local que les participants veulent améliorer.

Le groupe doit fournir suffisamment d'informations pour rédiger une description du scénario actuel qui sera présentée.

\section{SCÉNARIO ACTUEL}

\begin{tabular}{|c|c|}
\hline \multicolumn{2}{|c|}{ Nom de l'initiative Campagne BhoomiKa (Situation en novembre 2017) } \\
\hline Pays/région Inde/Delhi & \\
\hline $\begin{array}{l}\text { Portée } \\
\text { À quel niveau (local, territorial, } \\
\text { national, global) d'intervention } \\
\text { travaillez-vous? }\end{array}$ & Niveau national \\
\hline $\begin{array}{l}\text { Valeur(s) } \\
\text { Quelle est la valeur (quelle } \\
\text { vision votre initiative tente- } \\
\text { t-elle de réaliser pour un } \\
\text { système alimentaire durable? }\end{array}$ & $\begin{array}{l}\text { De la nourriture propre, verte et équitable. } \\
\text { - Les valeurs gandhiennes. } \\
\text { - Connexions durables, interaction directe, transparence, } \\
\text { connaissances interpersonnelles } \\
\text { - Inclusif = tous les acteurs de la chaîne d'approvisionnement se } \\
\text { connaissent, y compris les petits producteurs. } \\
\text { - Agriculteurs/producteurs }\end{array}$ \\
\hline $\begin{array}{l}\text { Calendrier } \\
\text { Quel est le calendrier d'action? }\end{array}$ & $\begin{array}{l}\text { Fondée il y a un an, l'initiative prépare actuellement un plan de } \\
\text { projet sur trois ans. L'objectif est de mettre en place un organe } \\
\text { institutionnel soutenant une campagne nationale de sensibilisation } \\
\text { des citoyens et promouvant le soutien politique en faveur d'une } \\
\text { alimentation saine, verte et équitable. } \\
\text { - Échéancier = } 10 \text { ans }\end{array}$ \\
\hline
\end{tabular}




\section{Étape 3: Envisager l’avenir}

\section{Facilitateur :}

Animez une discussion [15 minutes] pour identifier la vision focale du scénario, en précisant quelle(s) valeur(s) définira(ont) l'état futur de votre initiative.

Définissez avec précision la question centrale, car cela aidera à saisir la valeur que vous essayez d'intégrer dans le futur système alimentaire durable. Orientez la discussion de façon à ce que les valeurs fondamentales que l'initiative vise à réaliser ressortent clairement. Aidez les participants à poser des questions au chef de scénario pour dévoiler les valeurs fondamentales. Consolidez ces valeurs dans une vision prospective qui rendra compte de l'état futur souhaité de l'initiative

Synthétisez votre vision comme une phrase «tweet»:

Bhoomika sera reconnue à l'échelle nationale comme une plateforme institutionnelle pour mettre en relation les petits producteurs et les consommateurs durables.

\section{Étape 4: Identifier le scénario}

En vous basant sur le scénario de la situation actuelle, discutez sur les incertitudes, les plus grands risques et les plus grandes opportunités qui pourraient survenir au cours des 5 à 10 prochaines années [30 minutes]. La discussion devrait faire ressortir plusieurs risques et opportunités. La prochaine étape consistera à choisir celle sur laquelle travailler dans le cadre de l'exercice. C'est le scénario.

Encourager les discussions sur ces éléments qui ont lieu aux niveaux national et international et dans le cadre de l'initiative elle-même. Ceux-ci doivent être à la fois positifs et négatifs pour chaque incertitude.

Exemple:

\section{Risque:}

Le nouveau cadre réglementaire du gouvernement exclut les systèmes participatifs de garantie pour la vérification/certification des aliments biologiques.

\section{Opportunité:}

Un nouveau scandale alimentaire déclenche une forte prise de conscience nationale des consommateurs et une demande croissante pour des aliments sûrs et durables.

Les participants ont choisi une question comme scénario qu'ils aborderont en définissant un plan d'action. 


\begin{tabular}{|c|c|c|}
\hline Incertitudes & Les plus grands risques & Les plus grandes opportunités \\
\hline $\begin{array}{l}\text { Changement social } \\
\text { Quels sont les changements } \\
\text { sociaux possibles qui } \\
\text { peuvent influencer votre } \\
\text { initiative? }\end{array}$ & $\begin{array}{l}\text { Les petits exploitants sont } \\
\text { marginalisés sans aucune aide. } \\
\text { Il n'y a «pas d'échappatoire à la } \\
\text { révolution verte.» } \\
\text { Due à la migration rurale- } \\
\text { urbaine, il y a un manque de } \\
\text { ressources humaines dans les } \\
\text { zones rurales. }\end{array}$ & $\begin{array}{l}\text { Il y a une forte demande des } \\
\text { consommateurs pour des «aliments } \\
\text { durables», une réalité qui est mis } \\
\text { en évidence par une croissance } \\
\text { annuelle à deux chiffres des } \\
\text { marchés biologiques nationaux. }\end{array}$ \\
\hline $\begin{array}{l}\text { Économie } \\
\text { Quels changements dans le } \\
\text { commerce, les marchés et } \\
\text { l'économie influenceront la } \\
\text { création de vos marchés? }\end{array}$ & $\begin{array}{l}\text { Dll y a un manque de transparence. } \\
\text { Le coût réel des aliments n'est } \\
\text { pas reconnu. } \\
\text { D Les moyens de subsistance pour } \\
\text { les petits exploitants agricoles } \\
\text { sont insuffisants. } \\
\text { Les marchés urbains sont } \\
\text { dominés par les supermarchés, } \\
\text { tandis que les marchés humides } \\
\text { disparaissent. } \\
\text { Les marchés ruraux dépendent } \\
\text { du système de rationnement } \\
\text { gouvernemental. }\end{array}$ & $\begin{array}{l}\text { Des marchés privés peuvent être } \\
\text { créés sur des terres privées. } \\
\text { L'accès à internet augmente. } \\
\text { Un nouveau mécanisme de } \\
\text { financement pourrait être utilisé } \\
\text { pour accélérer l'expansion de } \\
\text { l'initiative. }\end{array}$ \\
\hline $\begin{array}{l}\text { Connaissances et } \\
\text { technologie } \\
\text { Quels progrès scientifiques } \\
\text { ou nouvelles technologies } \\
\text { changeront la façon dont } \\
\text { vous cultivez, transformez } \\
\text { et commercialisez les } \\
\text { aliments dans le cadre de } \\
\text { votre initiative? }\end{array}$ & $\begin{array}{l}\text { Il y a une présence importante } \\
\text { d'OGM et d'une industrie } \\
\text { agrochimique locale }\end{array}$ & $\begin{array}{l}\text { Le commerce de détail moderne, } \\
\text { les réseaux sociaux, les marchés } \\
\text { de producteurs et les outils } \\
\text { à main appropriés offrent de } \\
\text { bonnes opportunités. } \\
\text { La production agroécologique } \\
\text { augmente (par exemple, } \\
\text { l'intensification durable du riz) } \\
\text { ll y a une transition de } \\
\text { l'agroécologie traditionnelle à } \\
\text { l'agroécologie moderne }\end{array}$ \\
\hline $\begin{array}{l}\text { Environnement } \\
\text { Dans quel type } \\
\text { d'environnement } \\
\text { agroécologique allez-vous } \\
\text { cultiver vos aliments? }\end{array}$ & $\begin{array}{l}\text { La biodiversité est faible. } \\
\text { L'eau est épuisée et la capacité de } \\
\text { sa gestion est faible. } \\
\text { Il y a des polluants organiques } \\
\text { persistants. }\end{array}$ & L'agriculture sur les terres arides \\
\hline $\begin{array}{l}\text { Politique } \\
\text { Quelles questions politiques } \\
\text { et de sécurité affecteront } \\
\text { votre initiative? }\end{array}$ & $\begin{array}{l}\text { La réglementation actuelle en } \\
\text { sécurité sanitaire des aliments } \\
\text { oblige l'obtention d'une } \\
\text { certification continue. } \\
\text { Des mécanismes de financement } \\
\text { cohérents pour les petits } \\
\text { producteurs biologiques (p. ex. } \\
\text { pour les intrants) sont absents. }\end{array}$ & $\begin{array}{l}\text { Les programmes } \\
\text { gouvernementaux appuient les } \\
\text { systèmes alimentaires durables } \\
\text { (depuis 2016) } \\
\text { Système traditionnel } \\
\text { d'amélioration de l'agriculture } \\
\text { Revenu monétaire basé sur le } \\
\text { temps pour les agriculteurs }\end{array}$ \\
\hline
\end{tabular}




\section{Étape 5: Élaborer un plan d’action à l'aide des chapitres pertinents du manuel}

\section{Facilitateur:}

Le temps prévu pour cette activité dépend de la façon dont l'atelier est organisé.

Demandez aux participants d'élaborer un plan d'action qui aidera soit à atténuer le plus grand risque, soit à tirer parti de la plus grande opportunité identifiée ci-dessus. Une option pourrait être de diviser le groupe en deux pour que chacun travaille sur le (les) risque(s), d'un côté et sur l'(les) opportunité(s), de l'autre.

Au cours des discussions, les participants déterminent d'abord le point d'entrée de leur scénario (c.-à-d. la question prioritaire ou le point de départ). Sur la base de cette question principale, identifiez le chapitre du livre qui pourrait fournir quelques conseils initiaux.

Utilisez les chapitres du manuel soit comme une ressource que les participants peuvent feuilleter pour obtenir des idées d'action, soit pour présenter le contenu du chapitre sélectionné au cours d'une formation (PowerPoint ou autre) pour fournir des idées et des exemples d'actions. Le plan d'action qui en résultera devrait fournir une voie pour répondre aux questions diverses abordées dans le guide (c.-à-d. commencer par le Chapitre 4, puis passer au Chapitre 8, etc.).

- Si vous êtes seul, le plan devient votre table des matières personnelle.

- Si vous participez à une réunion de stratégie ou de remue-méninges, cet exercice peut aider le groupe à définir l'ordre des questions et à générer des idées créatives pour résoudre les défis identifiés.

- Si vous participez à une formation, l'exercice peut vous aider à réorganiser l'ordre des séances dans le reste de votre atelier, de sorte qu'il soit fondé sur une approche interactive basée sur les problèmes, construite avec les participants, plutôt que sur un ordre prédéfini. 
C'est à vous de décider quel risque ou quelle opportunité guidera votre chemin d'apprentissage. Le plus grand risque est de nature social - les personnes compétentes ne sont pas disponibles. Ceux qui se consacrent à l'initiative ne sont pas disposés à y rester pendant 5 à 10 ans.

\begin{tabular}{|c|c|c|}
\hline Point d'entrée & Chapitre de référence & $\begin{array}{l}\text { Mesures proposées } \\
\text { (D'après les idées inclus dans les chapitres) }\end{array}$ \\
\hline $\begin{array}{l}\text { Entrer dans } \\
\text { l'organisation }\end{array}$ & $\begin{array}{l}\text { Chapitre 10: } \\
\text { Formaliser votre } \\
\text { travail collectif. }\end{array}$ & $\begin{array}{l}\text { Créer un diagramme qui montre aux membres } \\
\text { comment l'organisation BhoomiKa est/serait } \\
\text { structurée. } \\
\text { - Réviser la vision et s'assurer que tout le monde la } \\
\text { partage. } \\
\text { D Organiser les rôles et responsabilités de toutes les } \\
\text { personnes engagées dans le projet (y compris les } \\
\text { bénévoles). } \\
\text { - Gérer la croissance de l'organisation - assurez-vous de } \\
\text { répondre d'abord aux exigences légales. } \\
\text { - Recruter des personnes engagées au sein du réseau } \\
\text { BhoomiKa. }\end{array}$ \\
\hline $\begin{array}{l}\text { Être à court d'argent } \\
\text { pour la croissance }\end{array}$ & $\begin{array}{l}\text { Chapitre 9: } \\
\text { Les financements } \\
\text { innovants. }\end{array}$ & $\begin{array}{l}\text { - Déterminer les besoins financiers pour atteindre la } \\
\text { croissance (le plan d'affaires). } \\
\text { - Essayer la budgétisation participative. } \\
\text { - Développer un fonds d'investissement. } \\
\text { - Essayer d'obtenir l'investissement des consommateurs } \\
\text { (en savoir plus sur les modèles AMAP). } \\
\text { - Aider les agriculteurs à accéder aux fonds de groupe } \\
\text { disponibles. }\end{array}$ \\
\hline $\begin{array}{l}\text { Mobiliser les } \\
\text { consommateurs }\end{array}$ & $\begin{array}{l}\text { Chapitre 1: Attirer les } \\
\text { clients et les fidéliser. }\end{array}$ & $\begin{array}{l}\text { Pour maintenir l'engagement des consommateurs, } \\
\text { essayer quelques conseils proposés sur la p.26 auprès } \\
\text { des consommateurs potentiels. }\end{array}$ \\
\hline $\begin{array}{l}\text { Partenariats pour } \\
\text { le renforcement } \\
\text { des capacités des } \\
\text { agriculteurs et l'accès } \\
\text { aux marchés }\end{array}$ & $\begin{array}{l}\text { Chapitre 11: } \\
\text { La place des } \\
\text { partenaires et des } \\
\text { plaidoyers. }\end{array}$ & $\begin{array}{l}\text { Se mettre en relation avec le bon groupe de personnes } \\
\text { avec qui collaborer (par exemple, collaborer avec } \\
\text { Edible Route). }\end{array}$ \\
\hline $\begin{array}{l}\text { Le marketing comme } \\
\text { moyen de mobiliser } \\
\text { des capitaux }\end{array}$ & $\begin{array}{l}\text { Chapitre 2: } \\
\text { Connaître vos } \\
\text { marchés. }\end{array}$ & $\begin{array}{l}\text { - Fusionner avec un point de vente au détail; } \\
\text { - Assurez-vous que les ventes sur le marché continuent } \\
\text { quoi qu'il arrive. }\end{array}$ \\
\hline \multirow[t]{2}{*}{$\begin{array}{l}\text { Renforcement } \\
\text { des capacités des } \\
\text { agriculteurs }\end{array}$} & $\begin{array}{l}\text { Chapitre 4: } \\
\text { Partager et co-créer } \\
\text { des connaissances } \\
\text { pour une production } \\
\text { durable. }\end{array}$ & $\begin{array}{l}\text { Dartager les connaissances entre agriculteurs au sein } \\
\text { du réseau. } \\
\text { Développer l'apprentissage. }\end{array}$ \\
\hline & & $\begin{array}{l}\text { Aussi, ne pas oublier de laisser les choses au temps et au } \\
\text { hasard... }\end{array}$ \\
\hline
\end{tabular}

Une séance plénière de clôture pour présenter le travail de groupe encouragera ensuite le dialogue entre les participants. Cela permettra de comparer les plans divers et prendre des décisions collectives sur les actions qui peuvent être prises pour répondre aux différents scénarios. 


\section{LE CHEMIN D'APPRENTISSAGE: PAR OÙ COMMENCER, ET OÜ ALLER ENSUITE?}

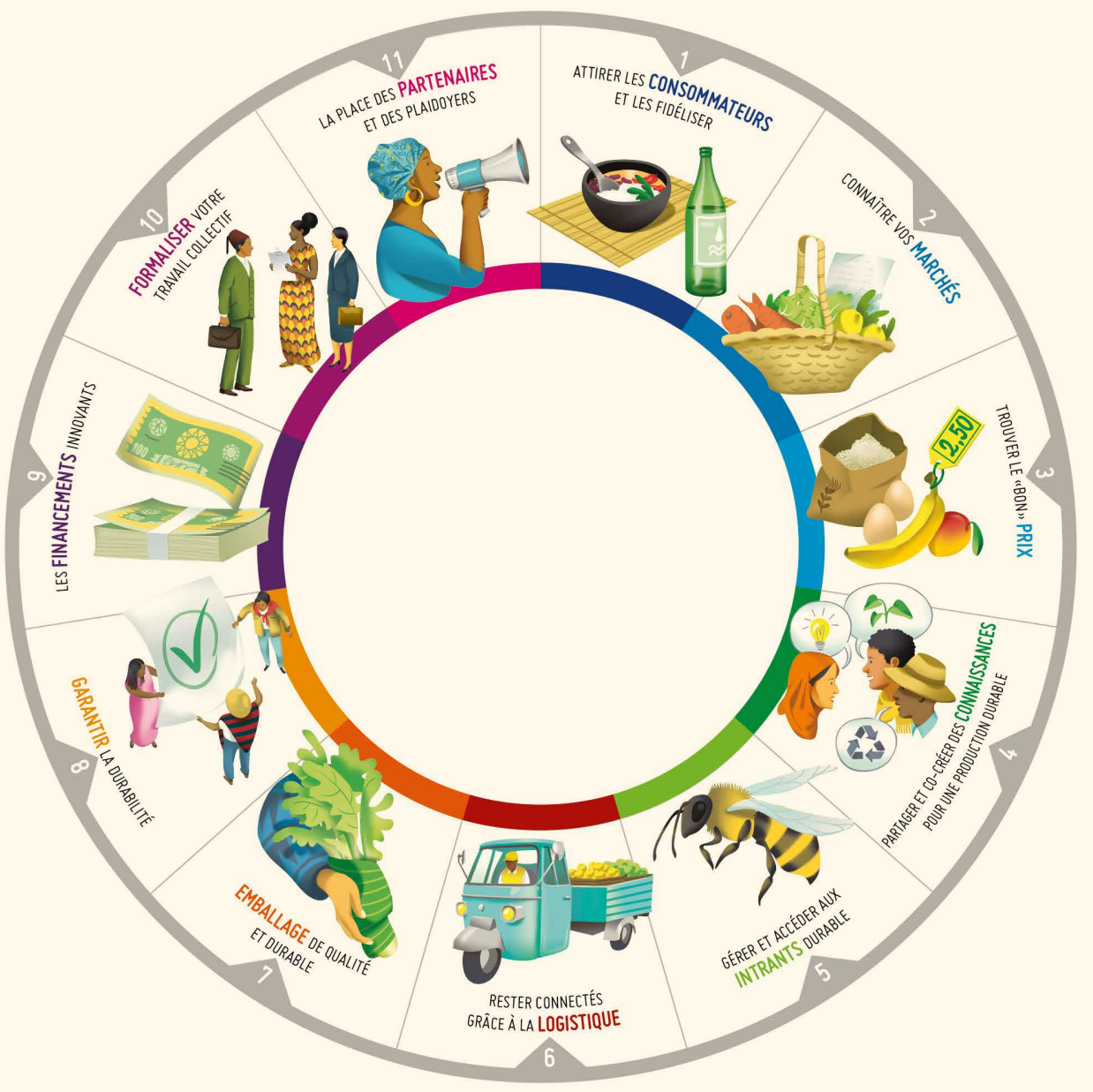

\section{Vous êtes maintenant prêt à commencer cette aventure d'apprentissage.}

Commencez par le point d'entrée qui vous intéresse ou qui intéresse la plupart des participants de votre groupe et commencez à lire et à utiliser le contenu du manuel.

Chaque chapitre vous indiquera:

- pourquoi le sujet est important;

- quelles sont les idées principales;

- des conseils pour faire face à des situations particulières;

- des exemples tirés de l'expérience des auteurs;

- des conseils à retenir sur les principales leçons du chapitre;

- des idées sur la marche à suivre pour continuer votre aventure.

Vous ne pouvez pas vous perdre, car chaque aventure sera différente ! 


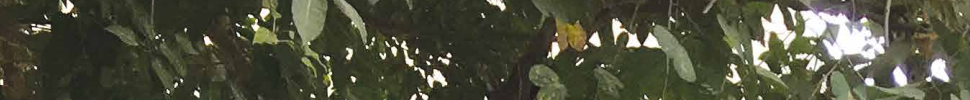

$\Rightarrow 1=1-2-3$

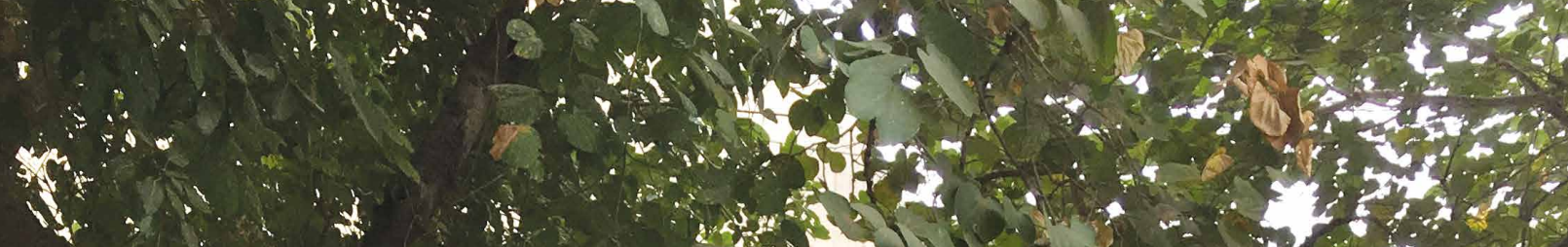

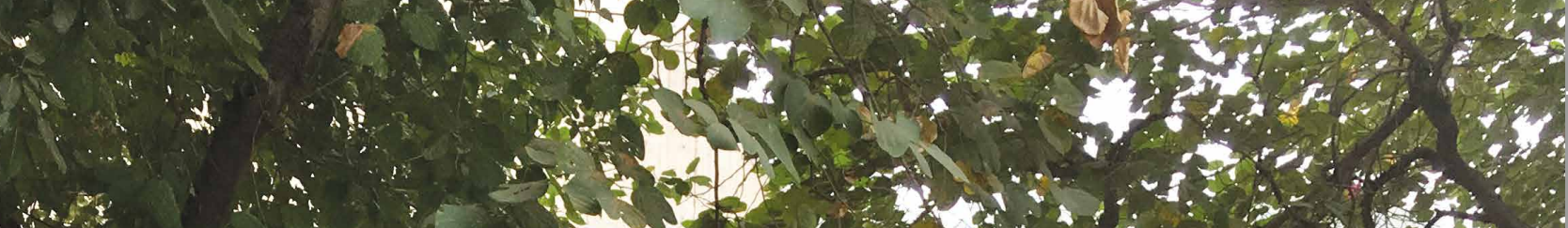
H.

(1) $)(\sqrt{2+x}$ NURSERY FARM

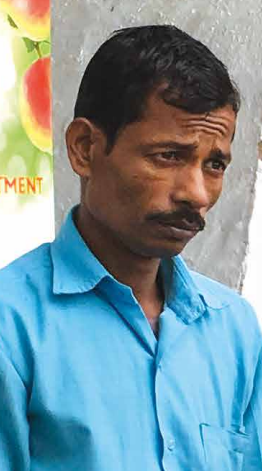

\section{1.}

-

int

2)

$00 \sqrt{2}$
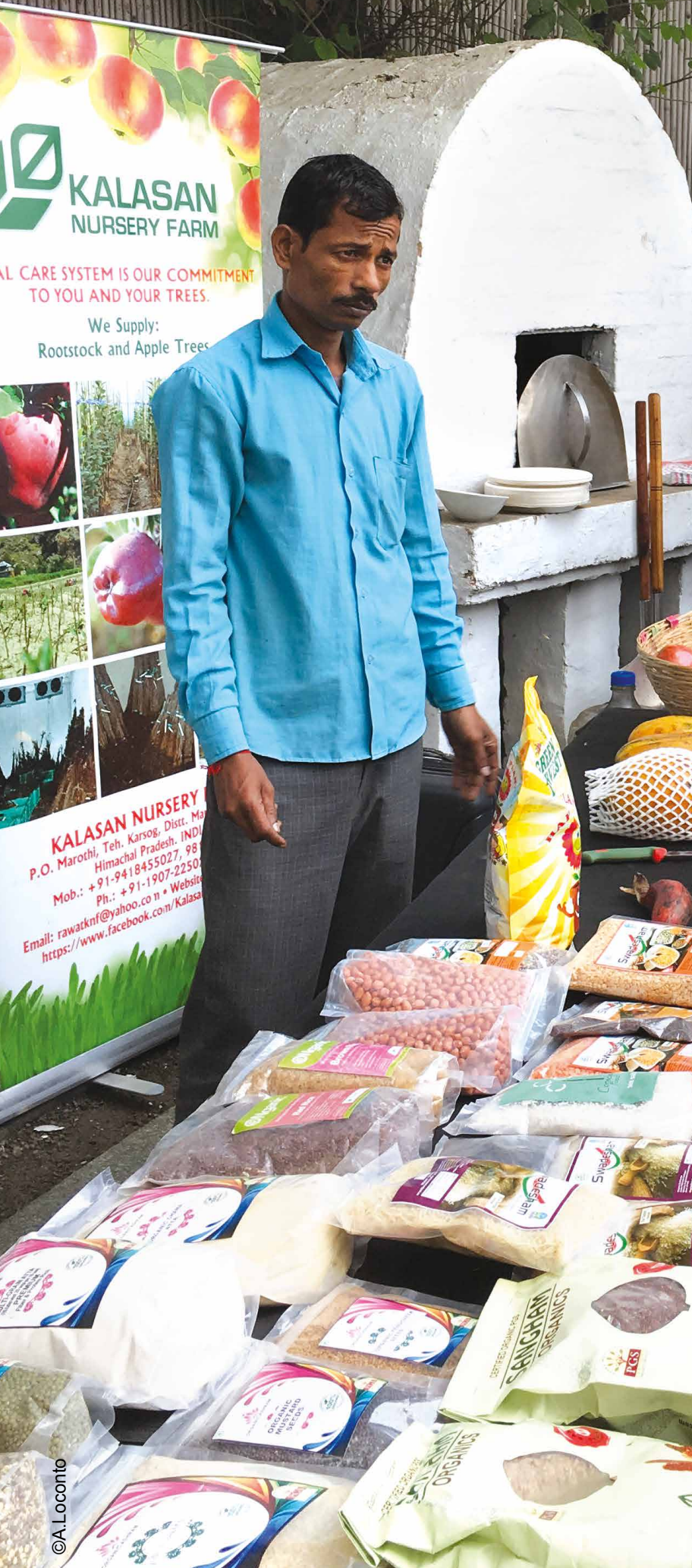


\section{ATTIRER LES CONSOMMATEURS ET LES FIDÉLISER}

CONNAITTRE VOS MARCHÉS

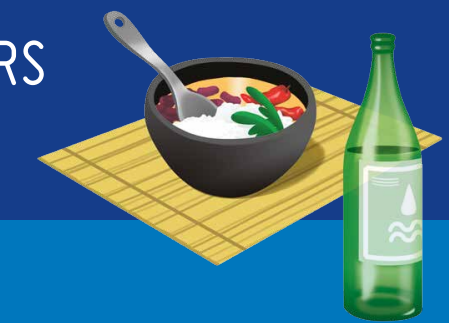

TROUVER LE «BON» PRIX
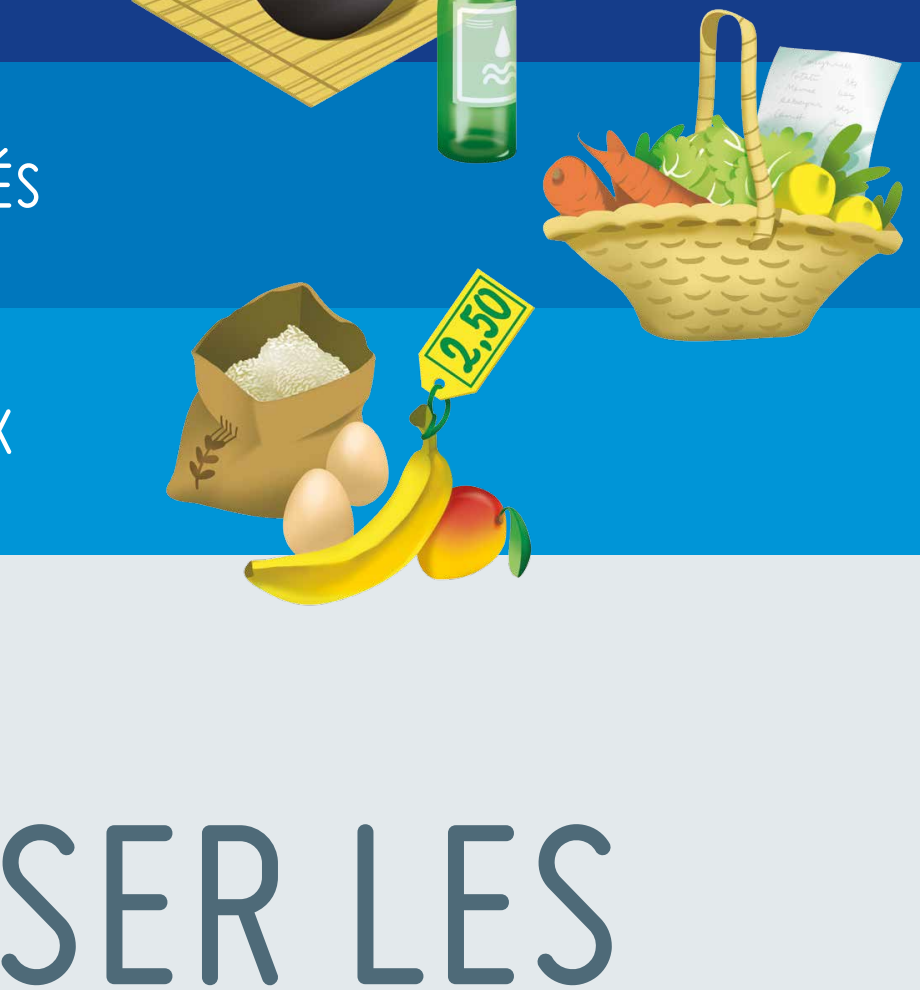

L'ensemble de ces chapitres traite de la consommation durable.

Les consommateurs jouent un rôle de plus en plus important pour garantir des systèmes alimentaires durables. Cela est dû à trois facteurs: dans les pays en développement, les producteurs sont les premiers consommateurs de leur propres produits; dans les dernières 20 années, les consommateurs urbains ont commencé à s'organiser et s'intéresser davantage à la durabilité des systèmes alimentaires; et parce que se nourrir est un acte quotidien et universel, chaque être humain at intérêt que cet acte continue dans l'avenir.

Donc, si vous cherchez des conseils sur la façon de trouver des consommateurs, d'établir des marchés et de négocier un prix équitable, commencez votre aventure ici.

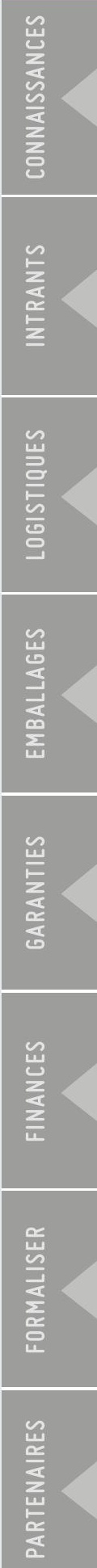



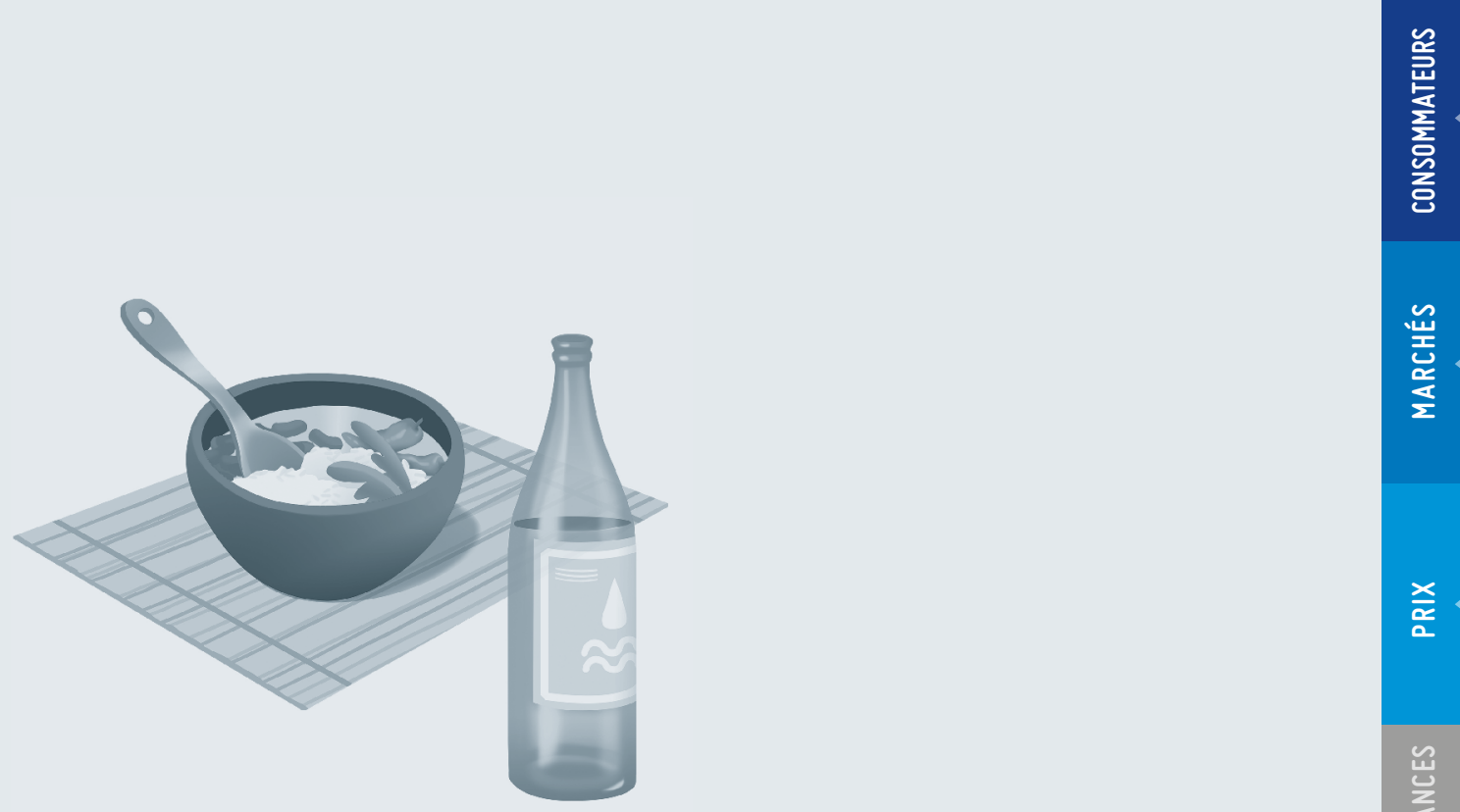

嵩

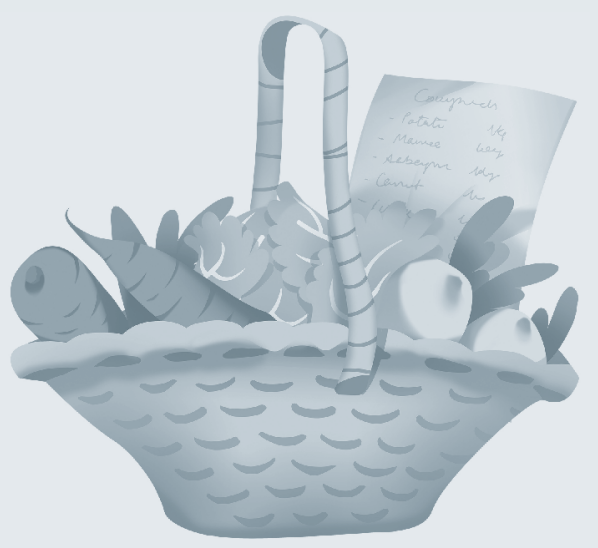

$\frac{x}{\frac{x}{\alpha}}$
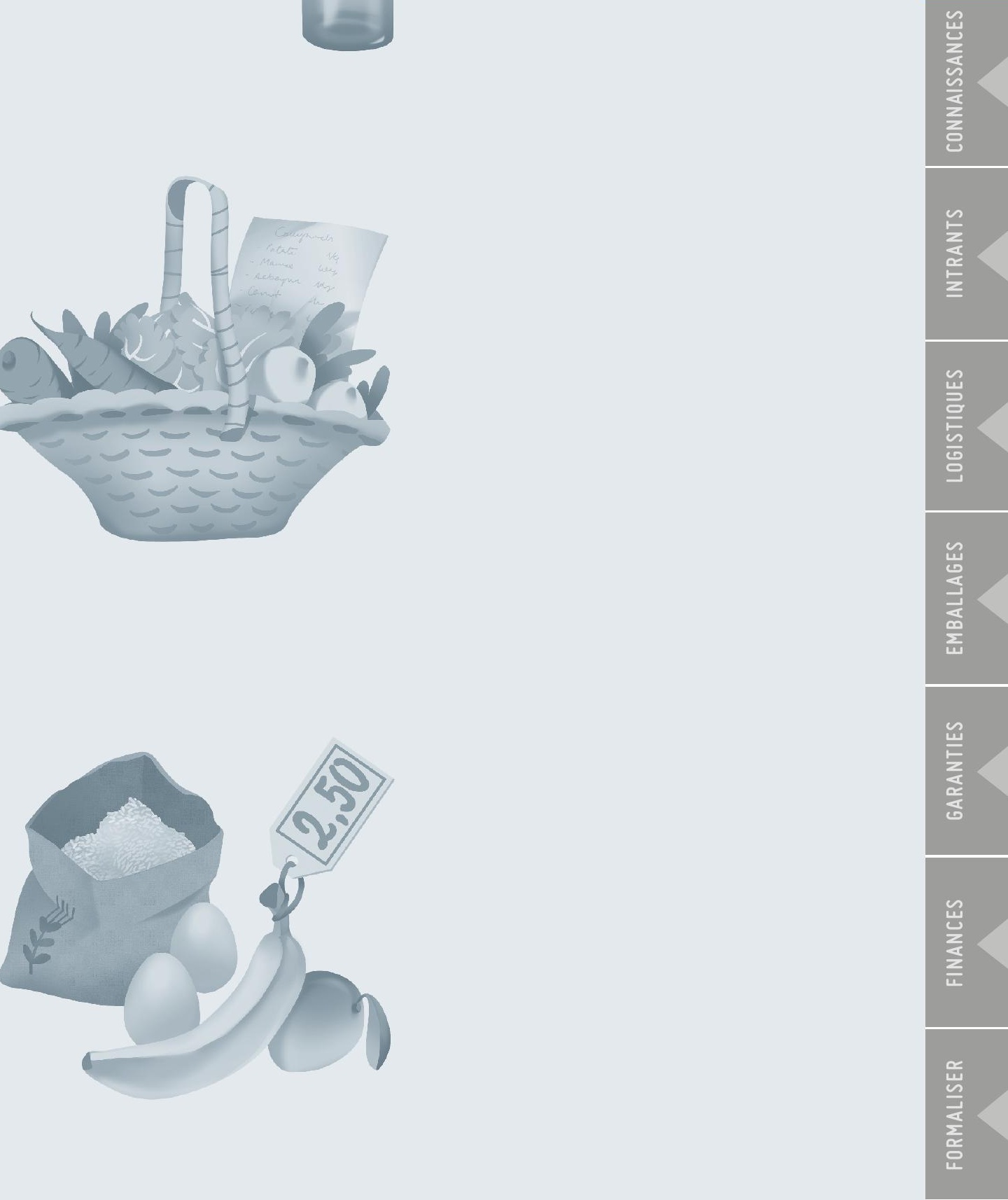


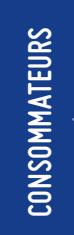

ATTIRER LES

CONSOMMATEURS

ET LES FIDÉLISER

$\frac{x}{a}$
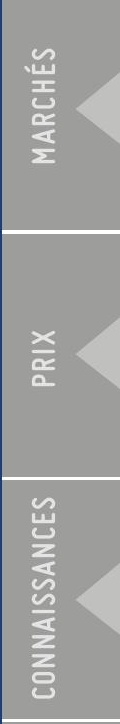

$\frac{n}{2}$
$\frac{a}{a}$
$\frac{a}{5}$
$\underline{z}$
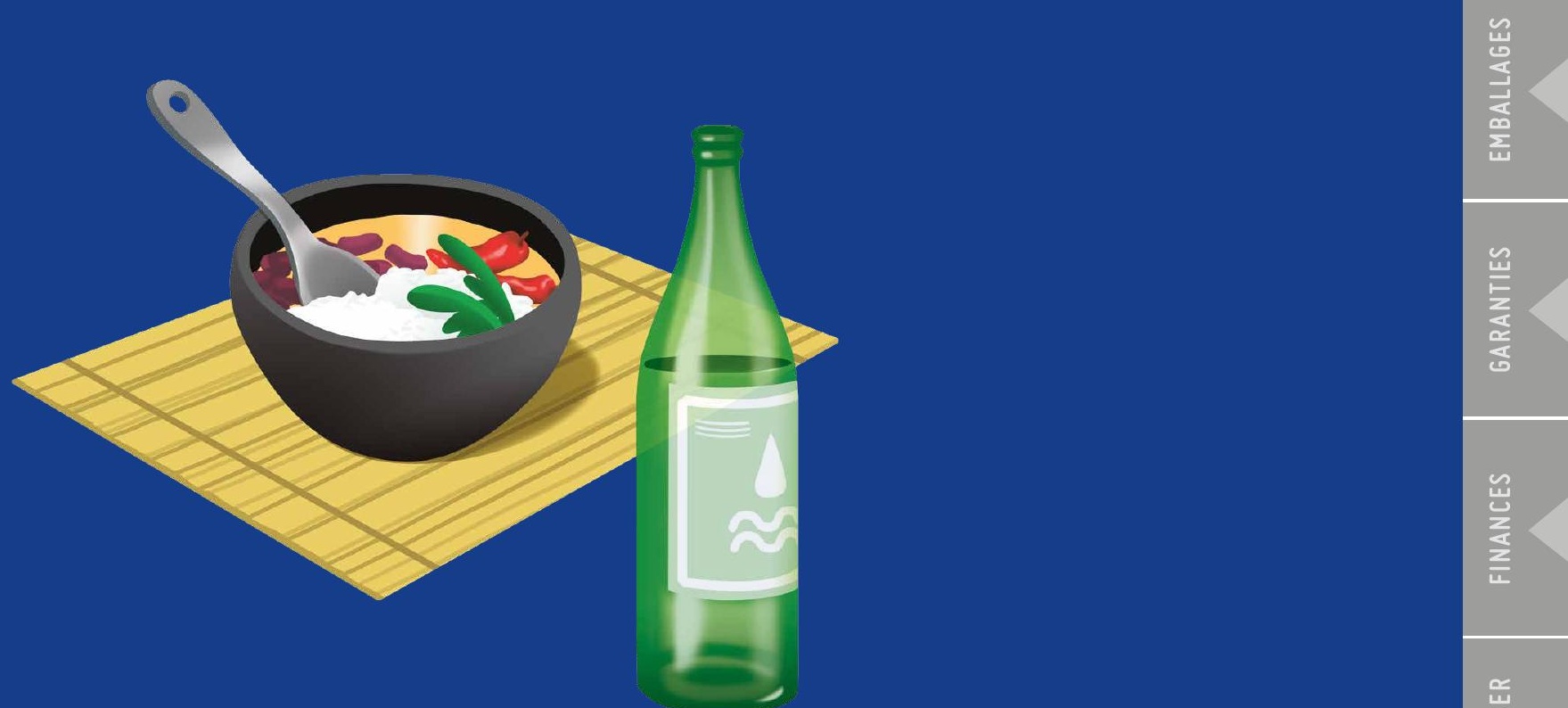

总

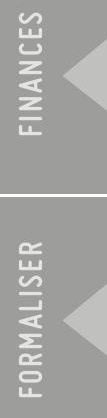




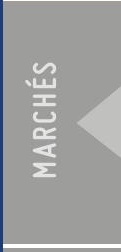

$\frac{x}{\alpha}$

留

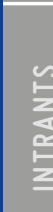

$\frac{\frac{n}{2}}{\frac{a}{2}}$

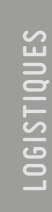

岃

世

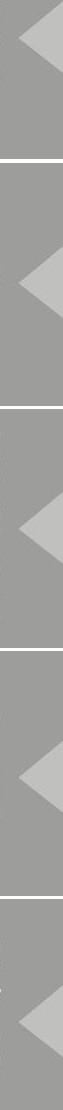




\section{POURQUOI EST-CE IMPORTANT?}

Dans les systèmes alimentaires durables, les producteurs et les consommateurs s'entendent sur la valeur des aliments produits de manière durable. Parfois, les consommateurs poussent les producteurs à imposer des pratiques plus durables, tandis qu'à d'autres moments, les producteurs doivent convaincre les consommateurs d'avoir des habitudes alimentaires plus durables. Dans ce contexte, les organisations publiques peuvent aussi assumer le rôle de consommateurs, en demandant que des aliments durables soient intégrés dans leurs modes d'approvisionnement.

Les producteurs et les consommateurs sont confrontés aux

Lorsque tous les acteurs (y compris les producteurs, les intermédiaires et les consommateurs) font des efforts pour promouvoir la consommation durable, l'ensemble du système devient plus durable. différents défis suivants:

- Les producteurs doivent trouver et développer progressivement des débouchés commerciaux viables. L'un de leurs principaux défis consiste à trouver des clients et à les fidéliser.

- Les consommateurs cherchent des aliments qu'ils apprécient et qu'ils peuvent se procurer facilement. Pour eux, le principal défi est de parvenir à une compréhension commune avec les producteurs ou les intermédiaires concernant les attentes de qualité, de quantité et de prix. Dans les deux cas, il faut recréer les liens entre les producteurs et les clients, sensibiliser les consommateurs, (réapprendre) la production alimentaire (y compris la saisonnalité, les différences de goût variétal), et (réapprendre) la consommation alimentaire en cuisinant à partir d'ingrédients frais et en partageant les recettes.

Présenter ces sujets aux enfants et aux jeunes peut être amusant et faire en sorte qu'il y ait des acteurs mieux informés dans les futurs systèmes alimentaires durables.

\section{QUI SONT LES CONSOMMATEURS DE PRODUITS DURABLES?}

Tout le monde peut devenir un consommateur de produits durables - il n'y a pas de critères fixes qui soient facilement reconnus. Cependant, l'intérêt pour l'alimentation durable est souvent déclenché par quatre préoccupations principales qui sont indépendantes du statut socioéconomique (FAO, 2018b):

- le souci de la santé;

- le souci de l'environnement;

- le souci de la protection des petits exploitants agricoles familiaux et des systèmes alimentaires locaux;

- l'intérêt de renouer avec les aliments cultivés et la façon dont ils le sont.

Les consommateurs peuvent également acheter des produits durables s'ils sont facilement accessibles, s'ils se situent dans leur marge budgétaire, s'ils ont le goût ou la qualité souhaitée, ou s'ils proviennent d'une marque à laquelle ils sont fidèles. 
Bien que la base de consommateurs potentiels soit importante, la création d'une véritable base de clients exige beaucoup de travail et de temps. Cibler des groupes spécifiques connus pour leur engagement envers la santé ou l'environnement peut s'avérer une bonne stratégie pour gagner du temps. Par exemple, cela peut inclure les étudiants intéressés par l'écologie, les végétariens ou les végétaliens, les personnes qui préparent fréquemment des repas à la maison, les mères allaitantes, les praticiens de yoga, les athlètes, les professeurs universitaires, les groupes religieux, les écoles, les hôpitaux, les garderies et restaurants biologiques ou ceux qui font la promotion des aliments sains et nutritifs.

Le niveau d'engagement que chaque consommateur prendra pour acheter des aliments et d'autres produits durables varie en fonction du temps disponible et du revenu tout au long de l'année (en plus des facteurs mentionnés ci-dessus).

Il y a aussi des différences entre les consommateurs qui partagent les valeurs de l'initiative et qui sont prêts à contribuer à son développement (une minorité) et les consommateurs alliés ou occasionnels. Ces derniers veulent acheter des produits durables, malgré le fait qu'ils ne partagent peut-être pas les valeurs de l'initiative. Pour mettre sur pied une initiative viable, vous devez identifier les consommateurs potentiels qui correspondent aux deux types d'engagement.

La disponibilité et la continuité de l'accès au marché constituent l'un des aspects clés de l'existence d'une base de consommateurs et de marchés fidèles. Prenons l'exemple d'un marché de fermier, dans lequel des fruits et légumes saisonniers sont accessibles: il y aura des moments durant l'année où certains produits ne seront pas disponibles. De plus, les consommateurs peuvent fluctuer en raison de voyages ou d'autres engagements personnels. Toutefois, le marché doit être régulièrement disponible, de manière à ce que le consommateur puisse compter dessus et en être informé. C'est l'un des aspects clés pour établir de la confiance avec les consommateurs déjà engagés ainsi qu'avec les nouveaux sur le long terme.

\section{ASTUCE 1}

\section{Comment un producteur peut Identifier des consommateurs potentiels}

- Tout d'abord, identifiez vos principaux consommateurs disponibles pour donner non seulement de l'argent, mais aussi du temps, des connaissances, des compétences et de l'influence politique. Ceux-ci sont les personnes disponibles pour devenir membres d'une initiative durable (souvent locale) et pour aider à recruter des membres, pour assister à la comptabilité, pour distribuer les produits, pour créer des sites web et des bulletins, etc. Commencez par élargir votre base de consommateurs avec des amis et des membres d'autres groupes auxquels vous appartenez (p. ex., religieux, culturel, professionnel, sportif).

- Deuxièmement, identifiez les consommateurs alliés, c'est à dire des groupes qui sont déjà organisés. Ils ont une connaissance approfondie des valeurs mais sont surtout intéressés par l'achat. Essayez de les approcher lors de foires commerciales, sur leur site web ou dans leurs magasins (p. ex. une chaîne de magasins d'aliments naturels).

- Enfin, identifiez les consommateurs occasionnels, c'est à dire les individus qui veulent seulement acheter et qui ne sont pas disponibles ou intéressés à consacrer leur temps ou à partager leurs valeurs. Rencontrez ces consommateurs en faisant de la publicité, en participant à des foires, à des marchés fermiers ou à des événements culturels. 


\subsection{Cherchez-vous des consommateurs qui achètent pour eux-mêmes et leur famille, ou qui achètent en vrac pour d'autres?}

Pour concevoir une bonne stratégie de marketing, divisez les consommateurs selon les quantités qu'ils achètent. Généralement, ils appartiennent à l'une des deux catégories suivantes:

- les consommateurs individuels qui achètent de la nourriture pour eux-mêmes et leur famille;

- les grands consommateurs (par exemple, les restaurants, les hôtels, les groupes d'achat) qui achètent pour des entreprises ou pour de grands groupes de consommateurs.

\subsubsection{Consommateurs individuels}

\section{Avantages}

+ Leur passion et leur enthousiasme pour les produits durables peuvent les faire devenir vos «meilleurs vendeurs» s'ils font la promotion des avantages de vos produits auprès de leurs amis et de leur famille. De plus, avec le cas d'un parent, l'attitude envers l'alimentation durable peut avoir une influence positive sur les enfants qui sont des futurs consommateurs potentiels.

+ En parlant avec vos clients, vous apprendrez beaucoup de choses sur eux, surtout en leur demandant quels sont leurs autres besoins. Cela vous donnera une bonne idée de ce qui est important pour eux et vous aidera à cibler les personnes clés.

+ Les échanges au cours des activités commerciales vous aideront à bâtir des relations plus étroites et à fidéliser votre clientèle. Vous pouvez même nouer des amitiés avec les consommateurs parce que l'alimentation réunie les gens.

\section{Inconvénients}

- Ils sont inconstants et peuvent avoir des priorités différentes quant à la continuité de leurs achats. Étant souvent à la recherche d'une large gamme de produits et achetant de petites quantités, ils vous obligeront à garder un stock important de produits. Ceci peut nuire à la qualité des produits et augmenter le gaspillage alimentaire.

\subsubsection{Grands consommateurs}

\section{Avantages}

+ Les institutions publiques, comme les écoles, les hôpitaux, etc. peuvent acheter des aliments produits de manière durable, vous donnant l'occasion d'atteindre une large gamme de personnes. Ceci peut favoriser le développement de modes alimentaires plus sains chez les élèves et les personnes ayant des problèmes de santé. Ces deux types de consommateurs peuvent être des alliés importants pour élargir la portée de votre initiative. Ils peuvent également présenter leur politique en matière de marchés publics durables.

+ Les clients grossistes comme les entreprises de restauration, les hôtels ou les restaurants, les détaillants et les groupes d'achat apprécient la «valeur ajoutée» qu'ils peuvent tirer de l'alimentation durable, par exemple une démonstration de responsabilité sociale et environnementale. Cela vous donne une chance de joindre les consommateurs qui ne se trouvent pas dans votre réseau immédiat (comme acheter directement de chez vous à votre ferme ou au marché fermier).

\section{Inconvénients}

- Il peut être difficile de satisfaire aux exigences légales des marchés publics.

- Ces consommateurs voudront commander de grandes quantités de produits, il est donc important de s'assurer que vous pouvez leur offrir la gamme et la quantité qu'ils recherchent. 
De nombreuses AMAP limitent le nombre

L'inclusion sociale des consommateurs à faible revenu

1 dans une AMAP

L'association pour le maintien d'une agriculture paysanne s'est construite historiquement sur deux piliers: la souveraineté alimentaire et l'économie solidaire, ce qui permet de mettre en place différentes formes de soutien pour les personnes dans le besoin. Il s'agit d'un bon mécanisme pour recruter des consommateurs qui veulent être des membres actifs d'une initiative.

L'une des principales façons dont le soutien et l'inclusion sociale sont utilisés dans une AMAP est par le biais d'un barème progressif de prix pour les abonnements, les retraités ou les étudiants peuvent payer moins que ceux qui travaillent, par exemple. C'est quelque chose qui varie d'un endroit à l'autre. L'une des caractéristiques fréquentes est cependant l'utilisation de la discrétion et de la confiance:

\section{Personne ne remettra en question la} déclaration de quelqu'un selon laquelle il a un problème financier. Tous s'efforceront de soutenir et de trouver des solutions. d'actions «subventionnées» dans le cadre de leur modèle économique. D'autres sont ouverts à la discussion. Un moyen novateur d'assurer à la fois l'inclusion sociale et la viabilité économique du modèle a été expérimenté dans de nombreuses AMAP allemandes: lors de la réunion annuelle entre producteurs et consommateurs, le producteur déclare le revenu total de l'AMAP dont il a besoin pour couvrir tous ses coûts (y compris les cotisations de santé et de retraite, les investissements en nouveaux équipements, en eau, etc). Chaque membre du groupe écrit le montant mensuel qu'il se sent capable de payer dans une enveloppe. Les montants sont ensuite additionnés. S'ils n'atteignent pas la somme spécifiée par le producteur, il y a un deuxième tour, où ceux qui sont en mesure de le faire augmentent le montant qu'ils sont prêts à payer... Dans plusieurs de ces groupes, il y a maintenant tellement d'expérience dans ce système, qu'il n'y a souvent pas de deuxième tour.

Il s'agit en effet d'un cas de «à chacun selon ses capacités, à chacun selon ses besoins».

Source: Judith Hitchman, URGENCI

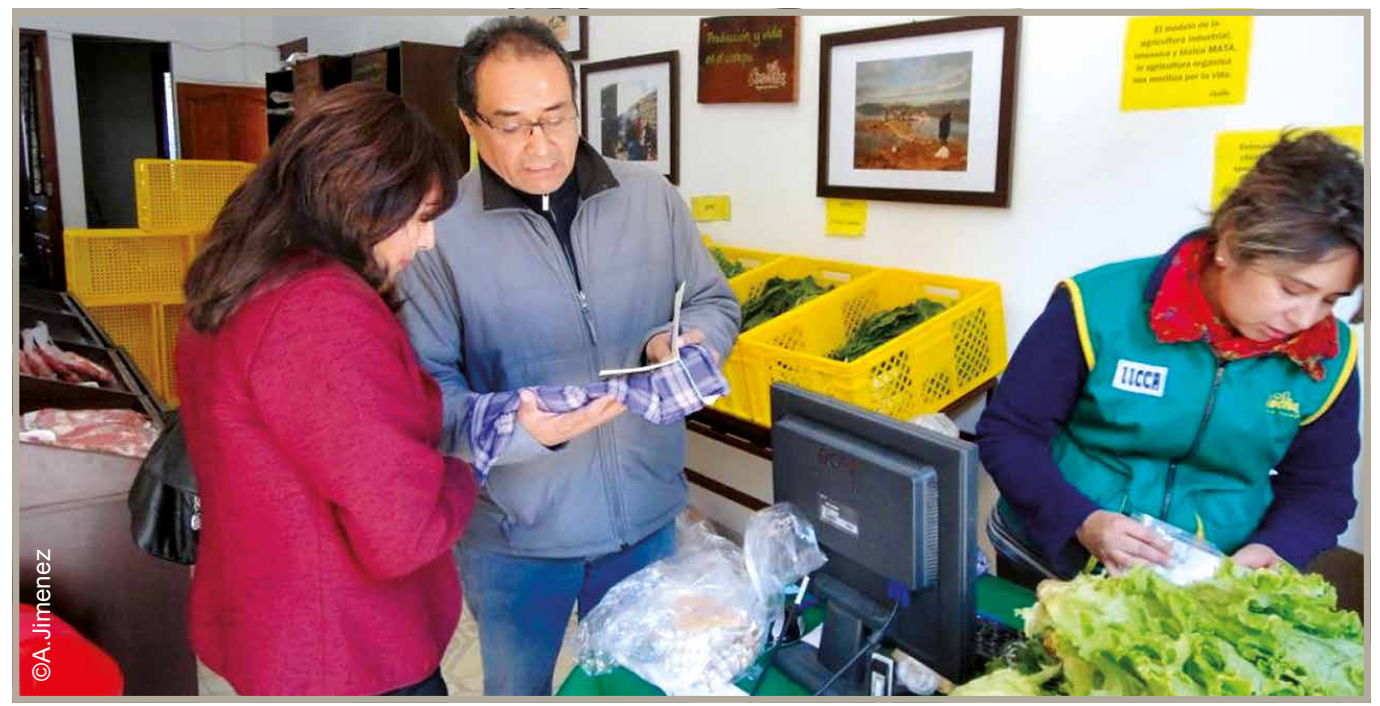




\section{COMMENT COMMUNIQUER AVEC VOS CONSOMMATEURS?}

Il n'existe pas de «solution miracle» pour la communication entre producteurs, intermédiaires et consommateurs. La meilleure approche consiste à identifier certains éléments communs qui attirent vos clients actuels et potentiels. Il est important d'adapter vos stratégies aux différents publics: la communication interne entre les membres de votre initiative ne nécessite pas le même rythme et le même langage que la communication externe.

\subsection{Communication interne}

\subsubsection{Simplicité, convivialité, résilience}

Que vous souhaitiez opter pour la haute ou la basse technologie, il y a toujours de nombreuses options.

Il y a des groupes très efficaces qui utilisent uniquement une liste de diffusion électronique.

Par exemple, dans le cas d'une AMAP ou d'un système de paniers alimentaires avec des livraisons hebdomadaires, les groupes qui

Il suffit de jeter un coup d'œil autour de vous...quelles sont les méthodes déjà utilisées? apprécient les réunions régulières en personne ont un besoin minimal de communication. Leurs livraisons sont uniformes, c'est-à-dire qu'elles ont le même contenu pour tous. L'envoi ou la collecte de commande n'est donc pas nécessaire, et la livraison devient donc le moment clé pour le partage d'information. La liste de diffusion est suffisante comme outil secondaire pour convoquer des réunions et des assemblées générales, pour partager des mises à jour, des recettes, l'agenda des tâches bénévoles, ainsi que des directives pour les journées d'aide à la ferme.

La transparence est un bon outil pour instaurer la confiance et établir des initiatives honnêtes qui fournissent toutes les informations nécessaires au consommateur. L'information sur la source des produits, les prix, la disponibilité saisonnière aident les consommateurs à faire des choix éclairés. L'opacité du marché a tendance à réduire la confiance des consommateurs au fil du temps.

\section{LISTE DE VÉRIFICATION}

Déterminer le meilleur mode de communication interne

1. La région dispose-t-elle d'une bonne connexion internet?

2. Vos membres ont-ils des téléphones cellulaires ou des téléphones intelligents?

3. Disposez-vous de suffisamment d'espace pour des réunions en personne?

4. Quelles sont les pratiques sociales de vos groupes cibles?

5. Les membres de votre groupe se réunissent-ils régulièrement ou se rencontrent-ils régulièrement pour d'autres raisons (par exemple pour aller chercher des enfants à l'école ou dans des lieux de culte)? 


\subsubsection{Les réseaux sociaux: efficaces pour la mobilisation, limités pour l'engagement}

D'autres initiatives optent pour les réseaux sociaux plutôt que pour les listes de diffusion électroniques. De nouvelles initiatives de marché «pop-up» se développent autour des groupes Facebook, WhatsApp ou WeChat. Les agriculteurs locaux affichent au groupe ce qu'ils peuvent fournir pour leur prochaine livraison, généralement organisée toutes les deux semaines. Les clients partagent leurs commandes en répondant simplement au post publié.

Les réseaux sociaux sont simples à installer et à utiliser; ils sont flexibles, et les consommateurs et les producteurs contrôlent le moment où ils accèdent à l'information. De plus, leur coût est faible (souvent gratuit !). Avant de passer trop de temps sur les réseaux sociaux, assurez-vous que vos consommateurs ont accès à internet et aux smartphones - s'ils n'utilisent pas déjà les réseaux sociaux, il est peu probable que vous les atteignez de cette façon!

Les réseaux sociaux comme un outil de communication interne

\section{2 en Finlande}

Au cours des trente dernières années, les ventes directes entre producteurs et consommateurs ont considérablement diminué en Finlande. Cependant, la demande de produits locaux et biologiques a récemment augmenté. Reko est une réponse à cette demande croissante. En suédois, Reko est une abréviation pour une consommation équitable. Les premiers cercles Reko ont vu le jour en 2013 à Jakobstad et Vasa, tous les deux situés dans la zone svécophone de la Finlande occidentale. Facebook est utilisé comme plateforme de communication, ce qui rend la manipulation très facile.

Un cercle Reko est basé sur un groupe Facebook privé. Les producteurs et les consommateurs intéressés à participer font une demande pour devenir membres du groupe. Lorsque les administrateurs, souvent un petit groupe de consommateurs, acceptent les demandes d'agriculteurs sur la base des principes Reko, l'entreprise peut démarrer.

Les livraisons ont lieu toutes les semaines ou toutes les deux semaines, selon la taille du groupe. Pour chaque livraison, les agriculteurs affichent une annonce sur Facebook et les consommateurs envoient des commandes sous forme de commentaires sur les annonces. Lorsqu'un cercle Reko a démarré, il nécessite très peu d'entretien. Les administrateurs doivent tout simplement accepter les demandes des nouveaux membres et confirmer les dates de livraison. La participation est gratuite, il n'y a pas de frais de logistique, et la vente directe est présente à 100 pour cent. Le fait qu'une telle initiative ait rassemblé plus de 400000 clients en quelques années, dans un pays de 5,5 millions d'habitants, en dit long sur le pouvoir des réseaux sociaux.

Source: adapté de https://urgenci.net/reko-awinning-concept-in-finland 


\subsection{Communication externe: attirer de nouveaux consommateurs}

\subsubsection{Faites attention à ce que vous dites !}

Le langage que vous utilisez doit être adapté aux consommateurs avec lesquels vous communiquez. Des concepts identiques peuvent être attirants ou offensants pour différentes personnes. Par exemple, des mots comme "durable» plairont généralement davantage aux consommateurs éduqués, tandis que drautres consommateurs sıidentifieront davantage à «biologique», «naturel» ou «local». Si vous présentez vos initiatives exactement dans les mêmes termes aux consommateurs de quartiers marginalisés ou appartenant à la classe moyenne, ils peuvent se sentir non-concernés. De nombreux témoignages soulignent une idée fondamentale: si l'on veut travailler avec des consommateurs de tous les segments de la société, les mots clés doivent être soigneusement choisis. La forme doit être différente, mais le contenu doit rester le même:

\section{Les consommateurs recherchent l'histoire des aliments qu'ils consomment.}

\subsubsection{Soyez conscient de votre image}

En moins d'une décennie, les divers réseaux sociaux sont devenus le mode préféré de communication virtuelle à travers le monde. Les réseaux sociaux ont remodelé les règles de communication sur internet. Utiliser une seule image ou une vidéo pour illustrer ou même pour composer le message, est presque devenu un impératif. Selon des recensements dans les réseaux sociaux, il est au moins deux fois moins probable que votre texte soit partagé s'il n'inclut pas de contenu visuel.

\section{L'importance du message visuel} sur le site de Lavka Lavka

Lavka Lavka est une coopérative agricole située à Moscou, en Russie. La coopérative gère une boutique en ligne, un marché fermier et un restaurant pour fournir à ses clients des aliments frais et de haute qualité provenant d'agriculteurs locaux, offrant ainsi un débouché commercial. Étant donné que Lavka Lavka vend une partie de ses produits en ligne, un site web (http://lavkalavka.com/page/chtotakoe-fermerskiy-kooperativ-lavkalavka) et une page Facebook (https://www.facebook. com/lavkalavkarestaurant) ont été créés

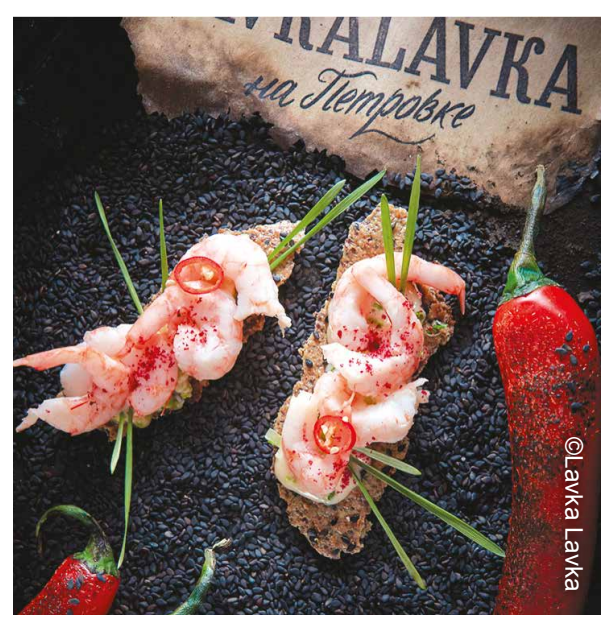


pour informer les consommateurs sur leur disponibilité d'aliments et pour raconter les histoires des agriculteurs. Le site web de Lavka Lavka est un portail d'information utilisé pour relier la ville à la campagne et les consommateurs aux producteurs.

La coopérative reconnaît que la nourriture n'est pas seulement une question d'alimentation, mais qu'il s'agit d'une expérience multi-sensorielle intégrant l'odorat, le toucher et la vue. Étant donné que la distance peut constituer un obstacle, les messages visuels sont utilisés comme une stratégie de communication pour partager les valeurs fondatrices de la coopérative, pour retenir et maintenir l'attention des clients, ainsi que pour établir le lien initial entre les consommateurs et les producteurs. Sur son site internet, Lavka Lavka présente de nombreuses photographies des différents agriculteurs, accompagnées de courtes biographies. En cliquant sur l'image de chacun, les consommateurs peuvent voir les produits disponibles auprès de cet agriculteur (voir la photo ci-dessous montrant M. Pavel

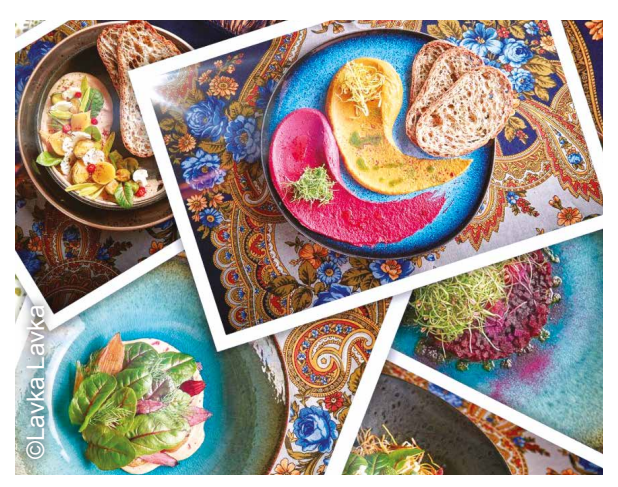

Abramos, producteur de céréales et de farine). De même, des images de spécialités culinaires traditionnelles et de produits alimentaires sont présentées sur la page web. Dans l'exemple ci-dessous, le plat ainsi que les ingrédients utilisés pour le préparer sont présentés, des éléments visuels qui attirent l'attention des consommateurs en les motivant à goûter le plat.

Source: adapté de http://restoran.lavkalavka.com/en et http://lavkalavka.com/page/chto-takoefermerskiy-kooperativ-lavkalavka

\subsubsection{Bâtir des alliances}

Les circuits de médias locaux sont des atouts, vu que la plupart des résidents de la région y ont généralement accès. L'aide des journalistes locaux est fondamentale au début du projet. Quelques articles (avec des photos) aideront à faire connaître votre initiative. Des centaines de personnes sauront qu'elle a été lancée et que la curiosité des gens est bienvenue.

Les réseaux locaux sont aussi des atouts. Dans la région où vous opérez, il existe probablement des réseaux associatifs, commerciaux, techniques, éducatifs et religieux qui rassemblent des gens qui partagent des valeurs similaires et qui recherchent le type d'initiative que vous mettez en place.

\section{ASTUCE 2}

Essayer de ne pas abuser de la générosité des autres

Soyez franc et transparent au sujet des intérêts et des valeurs communes, et de la façon dont vous prévoyez utiliser l'information partagée. Assurez-vous de connaître les exigences légales quant à la confidentialité et la protection des données dans votre pays. 


\section{COMMENT FIDÉLISER VOS CLIENTS ?}

Les consommateurs du monde entier ont tendance à constamment vouloir découvrir de nouveaux goûts et à vouloir trouver de bonnes affaires, de meilleurs prix, plus de qualité et des fournisseurs fiables. Il est donc difficile de maintenir votre base de clients actuelle s'il n'y a pas d'effort pour comprendre leurs besoins et y répondre. Habituellement, la vente est la meilleure occasion pour les producteurs de transformer leurs clients «explorateurs» en membres loyaux et engagés de l'initiative. La communication et la réciprocité jouent un rôle impératif dans cet enjeu.

Si les clients sont insatisfaits de votre style de vente, il est probable qu'ils ne feront plus jamais affaire avec vous. Ainsi, une expérience d'achat cohérente et agréable fait partie intégrante de la confiance et de la loyauté. La réciprocité, en tant que processus par lequel les clients sont, en quelque sorte, récompensés pour leur fidélité, est un autre facteur important pour assurer le retour de vos clients. Le développement d'une base de consommateurs loyaux exige une stratégie qui n'a pas besoin d'être coûteuse (comme par exemple, offrir trop de produits gratuitement), mais simplement intelligente!

\section{ASTUCE 3}

\section{Maintenir l'engagement des consommateurs}

1. Créez une relation étroite avec les consommateurs en organisant des événements à la ferme ou dans un espace commun, tels que des journées de travail communautaire, des événements sociaux, ou des activités pour les jeunes. ces événements créeront un sentiment d'appartenance où l'espace devient comme une «résidence secondaire» pour les consommateurs.

2. Essayez d'apprendre aux consommateurs à fabriquer leurs propres produits afin qu'ils puissent mieux comprendre les coûts de production. ceci est aussi important pour comprendre les prix fixés ou proposés par les producteurs.

3. Partagez l'histoire des produits. ces histoires bâtissent l'appréciation et la confiance des consommateurs envers la qualité des aliments achetés.

4. Informez les consommateurs sur le cycle de production et les saisons de production de vos principaux produits agricoles. pour ce faire, fournissez un calendrier aux consommateurs indiquant les moments auxquels ils recevront certains fruits et légumes, par exemple.

5. Partagez des recettes et offrez des ateliers sur la mise en conserve, l'entreposage, la cuisine et d'autres activités qui pourraient augmenter la valeur ajoutée.

6. Enregistrez le statut d'adhésion de vos consommateurs de manière efficace en choisissant le moment adéquat pour renouveler les abonnements. par exemple, vous pouvez le faire en basse saison, lorsqu'il y a moins de récolte à faire, ou pendant les périodes de pointe où le contact avec les consommateurs est plus régulier.

7. Maintenez votre communication avec les consommateurs actifs même pendant la basse saison.

8. Obtenez des retours de vos consommateurs en menant des enquêtes ou des sondages sur leurs préférences. lorsque vous rendez plus disponibles leurs produits préférés, ils sont susceptibles de revenir.

9. L'introduction de nouveaux produits (basés sur les retours des consommateurs) aidera à maintenir l'intérêt des consommateurs et à les fidéliser.

10. Enfin, effectuez des enquêtes de fin d'année et servez-vous en pour planifier la production agricole de l'an prochain. ces enquêtes vous informeront sur les préférences de vos consommateurs, sur les prix comparatifs des différents produits et sur les idées de nouveaux produits. 


\subsection{Créer une expérience d’achat agréable}

Créer une expérience d'achat agréable est l'étape fondamentale pour établir des relations durables avec vos clients et, par conséquent, pour maintenir leur engagement.

\section{ASTUCE 4}

\section{Créer une expérience d'achat agréable}

1. Servez très bien tous les clients.

2. Affichez les prix.

3. Démontrez la qualité.

4. Soulignez la diversité.

5. Créez et innovez.

6. Rendez le stand et le marché attractifs.

7. Faites la promotion du(des) produit(s).

8. Manipulez les aliments de façon saine et sécuritaire

9. Favorisez l'auto-apprentissage.
10. Ne faites jamais les graves erreurs suivantes:

a. vendre des produits de mauvaise qualité;

b. fixer des prix supérieurs aux prix du marché;

c. avoir un stand sale et mal-organisé;

d. mal servir les clients;

e. arrêter de venir au marché sans en parler aux clients et aux organisateurs;

f. manger et servir des clients en même temps;

g. vendre des produits sans le poids exact.

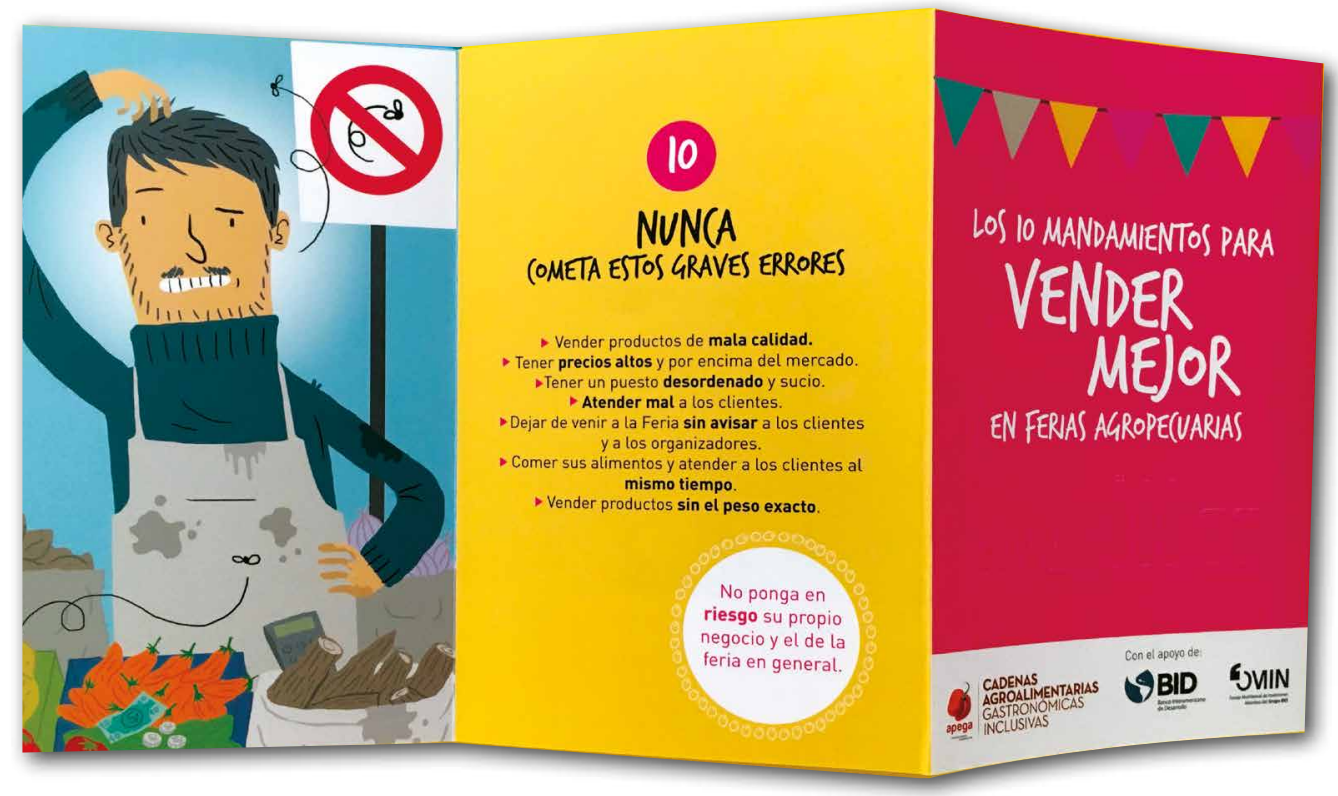

Source: Les 10 commandements pour mieux vendre dans les marchés de producteurs du Pérou, «Cadenas Agroalimentarias Gastronómicas Inclusivas» APEGA. 


\subsubsection{Les programmes de fidélisation}

Les programmes de fidélisation comprennent une variété d'initiatives visant à engager les consommateurs auprès des vendeurs. Ils reposent sur la réciprocité ou sur de petits cadeaux pour inciter les consommateurs à revenir régulièrement. Il peut s'agir de programmes officiels réservés aux membres ou simplement d'une d'offre de cadeau lorsque le consommateur effectue un achat d'un certain montant.

\section{ASTUCE 5}

La surprise est un ingrédient important pour fidéliser les consommateurs

En plus des approvisionnements habituels, les producteurs peuvent offrir certains de leurs produits en supplément. C'est un mode de fidélisation à faible coût !

\subsection{Suivi auprès de vos clients pour comprendre comment mieux les satisfaire}

La fréquence de l'échange d'informations est tout aussi importante que la formulation correcte. Il y a différentes façons de faire le suivi auprès des consommateurs: échanges de courriels, appels téléphoniques, bavardages lors des livraisons, dépliants et rencontres régulières. Les sondages de fin ou de début de saison ou les groupes de discussion sont des moyens efficaces d'évaluer à la fois la satisfaction des clients à l'égard des produits déjà consommés ainsi que leurs souhaits pour la prochaine saison. Les clients principaux/fidèles apprécient toute invitation pour influencer l'initiative: cela renforce leur sentiment d'appartenance. Lorsque vous créez des sondages, rappelez-vous que les logiciels libres offrant des outils efficaces sont nombreux.

En règle générale, si les consommateurs sont insatisfaits de leur expérience d'achat, ils préfèrent partir et ne plus revenir. Faites votre mieux pour éviter cette situation! Si elle se présente, même s'il est très difficile d'avoir des retours de ceux qui sont partis, essayez de noter les retraits et de comprendre leurs motivations. Qui est parti ? Quand ? Pourquoi ? Si vous répondez à ces questions, vous minimiserez les erreurs passées.

\subsection{Fournir des services de soutien pour encourager les clients fidèles}

Les services de soutien à la clientèle, comme les démonstrations culinaires et les visites à la ferme, sont très importants pour maintenir la fidélité et les incite à revenir. La qualité des services est généralement plus importante que la rapidité de la livraison. Une stratégie visant à passer plus de temps avec les clients donne des résultats plus positifs, car les clients ont tendance à se sentir importants et non pas pressés ou ignorés. Il est important que les services de soutien fournis à vos clients soient offerts par le moyen de communication qu'ils préfèrent (par exemple, le courrier électronique ou les réseaux sociaux). Dans la plupart des cas, les relations directes et en personne demeurent les plus courants dans les pays du monde entier. 


\section{ASTUCE 6}

Le service personnalisé permet aux clients d'apprécier leur expérience, ce qui aide à les fidéliser.

\section{La démonstration culinaire} pour renforcer les liens avec

\section{4} les consommateurs du SPG de Quezon

À deux reprises, le SPG de Quezon, aux Philippines, a organisé des démonstrations culinaires pour valoriser leur alimentation durable. Celles-ci ont eu lieu pendant la fête annuelle des récoltes du mois d'août, qui attire environ 300 à 500 visiteurs. Au cours de ces leçons, les agriculteurs préparent des plats pour les consommateurs en utilisant leurs propres produits vendus sur le marché: voici quelques exemples: salades, gâteaux de riz et poulet. En ciblant les consommateurs réguliers du marché, Quezon favorise un lien solide entre le producteur et le consommateur. Les démonstrations culinaires ont été annoncées lors des jours de marché réguliers et sur des affiches distribuées en partenariat avec le gouvernement municipal.

Source: Carmen Cabling, SPG de Quezon

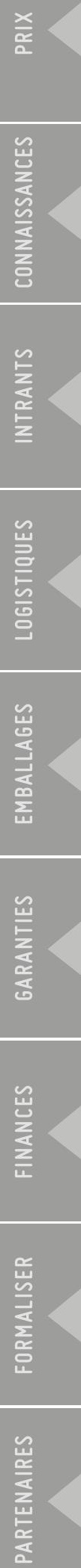

de chefs cuisiniers, sont invités à la ferme pour observer la diversité des cultures dans le champ. Accueillies par des producteurs locaux, ces visites durent environ deux heures et traitent de tous les sujets, des principaux processus de production à l'utilisation des cultures en cuisine. Ces visites sont avantageuses tant pour les producteurs que pour les consommateurs, car elles favorisent des relations plus solides et plus significatives.

Source: Oscar Nieto, Familia de la Tierra

\subsection{Partage et rotation des responsabilités}

Pour maintenir l'engagement des consommateurs, il est utile, si possible, de partager les responsabilités entre eux. Le bénévolat n'est pas durable à long terme si tout ne repose que sur une poignée de consommateurs engagés. Les responsabilités devraient être redistribuées régulièrement. Par exemple, des tâches simples mais longues, comme planifier la logistique ou 
vérifier que tout le monde signe une liste de distribution, devraient faire l'objet d'une rotation et être la tâche d'un autre bénévole. L'organisation des réunions et des conférences doit aussi être une responsabilité partagée.

\section{Vous voulez éviter la "fatigue des membres" à tout prix !}

\section{La participation des} consommateurs et les contraintes

6 de temps dans les modèles de l'AMAP

Du point de vue du consommateur, faire partie d'une AMAP varie en fonction du modèle et du pays en question. Il n'existe pas de formule toute faite. Cependant, le partage des risques et des avantages demeure constant. Si la récolte est abondante, le consommateur reçoit une quantité généreuse de légumes (ou d'autre produit pour lequel il est engagé). D'autre part, si des conditions climatiques défavorables ont complètement anéanti une récolte, le consommateur continue néanmoins de payer le producteur. Dans le cas d'une tempête ou d'une inondation, les consommateurs interviendront souvent pour aider le producteur à nettoyer les dégâts.

De nombreuses initiatives d'AMAP connaissent des taux de roulement de consommateurs atteignant jusqu'à 30 pour cent, surtout au cours de la première année d'exécution. Le coût est rarement la raison du décrochage, car la plupart des AMAP ont des prix très concurrentiels comparés aux fruits et légumes biologiques (ou même parfois conventionnels) trouvés dans les supermarchés. Au contraire, le temps d'engagement requis semble être la principale raison motivant l'abandon des consommateurs. Ce sont des gens qui n'avaient généralement pas anticipé les contraintes de la préparation et de la cuisson de tant de légumes frais différents.

Afin d'inciter les consommateurs à s'engager, notamment en termes de temps consacré à l'AMAP, des membres peuvent échanger quelques heures de travail mensuel à la ferme contre une partie ou la totalité de leur part. De cette manière, le montant qu'ils paient pour leur nourriture est démonétisée et demeure valorisée (voir tableau ci-dessous montrant un exemple de ce type de monétisation adopté par un groupe d'AMAP au Royaume-Uni).

L'adoption de tels mécanismes (et il y en a beaucoup d'autres), incite les membres à consacrer du temps à l'AMAP et augmente considérablement l'inclusion sociale des personnes à faible pouvoir d'achat ayant beaucoup de temps libre pour devenir membres.

Source: Judith Hitchman, URGENCI

Tableau 1: Exemple d'un barème des frais d'abonnement d'une AMAP

\begin{tabular}{l|c|c} 
Type d'abonnement & Heures de travail minimum & Frais annuel \\
Niveau 1 & 14 heures par mois & 0 GBP \\
\hline Niveau 2 & 7 heures par mois & $75 \mathrm{GBP}$ \\
\hline Niveau 3 & 4 heures par mois & $150 \mathrm{GBP}$ \\
\hline Niveau 4 & 7 heures par an & $\begin{array}{c}250 \mathrm{GBP} \text { (pas disponible aux } \\
\text { nouveaux membres) }\end{array}$ \\
\hline
\end{tabular}




\section{5. ÉDUQUER LES FUTURS CONSOMMATEURS}

Un système alimentaire n'est pas durable s'il ne satisfait pas les besoins des générations futures. Éduquer les enfants à manger et à profiter d'une alimentation saine et diversifiée est d'une importance cruciale pour leur santé, ainsi que pour garantir la survie et la croissance de votre initiative durable.

\section{塄 \\ Les jardins scolaires en France}

\section{7}

Les jardins scolaires sont peut-être le meilleur moyen d'amener les enfants et leurs familles à comprendre et à s'engager pour une alimentation durable. Dans l'école primaire Jean Guéhenno, à Caen, en Normandie, comme dans plusieurs écoles à travers le monde, les jardins scolaires sont utilisés comme un outil pédagogique polyvalent. Même si l'espace est limité en milieu urbain, il est toujours possible de cultiver des plantes comestibles. De plus, cultiver ses propres aliments est une activité pédagogique passionnante et une façon de mieux comprendre, dès un jeune âge, la nature, les plantes, le soleil, l'eau, le vent et les graines.

Il y a plusieurs possibilités quant aux activités jardinières. Par exemple, cuisiner des produits du jardin peut se faire en chantant des chansons sur les légumes ou sur le jardinage. Les élèves peuvent apprendre les différences entre les plantes et la végétation dans différentes parties du monde. L'économie de la production et de la consommation peut être calculée. Les principes de la durabilité peuvent être enseignées de manière concrète.
Un jardin scolaire est plus qu'un simple projet de classe. Il s'agit d'un projet d'école: différents enseignants peuvent choisir différentes façons de s'associer au même jardin. C'est aussi un projet communautaire. Les parents et les militants de l'alimentation locale peuvent s'impliquer dans les activités. Dans l'école Jean Guéhenno, les parents participent à la visite de la ferme pédagogique voisine, dont les producteurs ont participé à l'aménagement du jardin scolaire. Ces parents bénévoles sont formés sur place comme animateurs d'atelier pour la journée: les parents sont chargés d'organiser un jeu sur la classification des plantes en «familles»; un autre facilite un test à l'aveugle sur les légumes. L'essentiel est de tirer pleinement parti de la visite de la ferme en la préparant à l'avance et en rappelant ce qui y a été appris pendant les leçons en classe. Un jardin scolaire est un moyen très efficace de maintenir ces connaissances vivantes, car il exige un engagement régulier.

Source: Jocelyn Parot, URGENCI

Pour en savoir plus à ce sujet, visitez: https://eathink2015.org/en/download/SchoolGarden-Guide-WEB.PDF

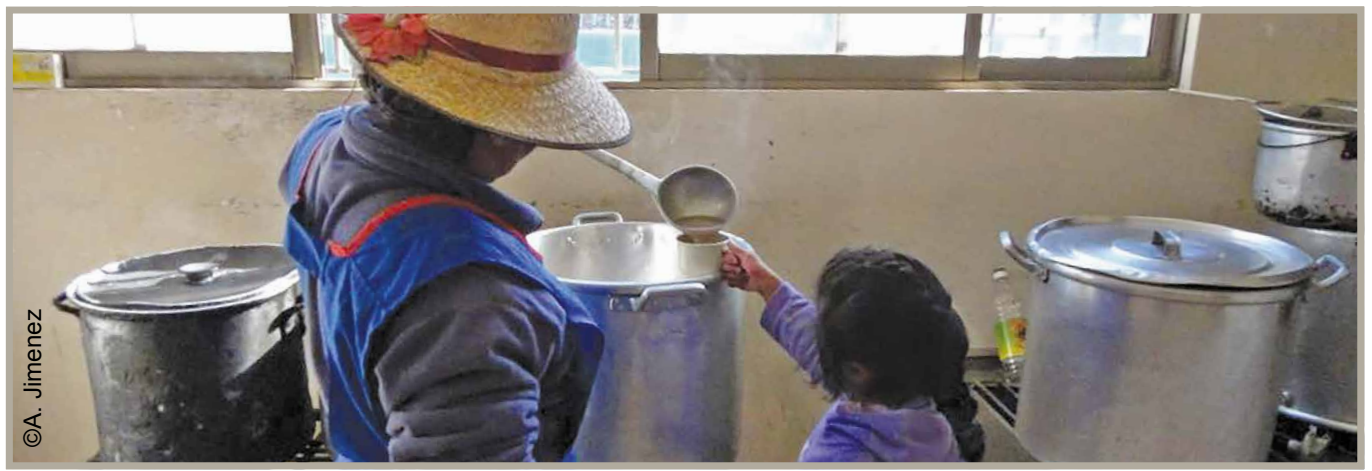




\section{Un programme scolaire} pour éduquer les futurs

\section{8 consommateurs au Kenya}

Le Programme de permaculture dans les écoles et les collèges - SCOPE Kenya - est une organisation en réseau qui compte actuellement 18 membres ONG, travaillant avec des écoles et des communautés dans 12 départements Kényans. L'objectif principal de SCOPE Kenya est d'éduquer et de préparer les jeunes écoliers et non-écoliers à comprendre la valeur nutritionnelle et la consommation pour une santé améliorée et à participer à la production agricole.

Pour ce faire, le programme SCOPE adopte l'approche de la gestion intégrée des terres, un processus holistique en étapes qui implique de travailler avec tous les acteurs de l'école: élèves, enseignants, parents et dirigeants locaux. Toute la cour de récréation est utilisée pour concevoir et établir un jardin scolaire qui adopte des principes et des pratiques en permaculture.

L'approche est basée sur les étapes suivantes:

1. Une analyse de situation: les acteurs observent la situation réelle pour développer une compréhension commune des problèmes actuels et des ressources potentielles dont ils disposent pour débuter;

2. Une formation d'objectifs holistiques: les acteurs définissent leur vision de l'environnement scolaire;

3. Une conception intégrée: les acteurs redessinent leur territoire en créant des liens entre les différents éléments de leur environnement;

4. Un plan d'action: les acteurs élaborent un plan de mise en œuvre et de suivi de leur projet.

Ce processus donne aux jeunes la possibilité de grandir dans des environnements où ils apprennent et pratiquent la production, la préparation et la consommation d'aliments sains.

Il est important que le jardin devienne une partie vivante du programme scolaire et que les élèves participent activement aux différentes activités - de la planification à l'alimentation en passant par la culture et la récolte.

Source: Rosinah Mbenya, PELUM

Pour en savoir plus, visitez:

http://www.fao.org/docrep/009/a0218e/ a0218e00.htm
塄

9

Inclure les enfants dans des actions

éducatives, transformatrices et durables

sont d'une importance capitale. Ceci est une manière efficace pour qu'ils deviennent les consommateurs actuels et futurs d'aliment produits durablement. Le Programme Cultivando agua boa de la Fondation Itaipu Binacional développe des actions de préservation de l'eau et de l'environnement, en soutenant l'agriculture agroécologique. De plus, des interventions éducatives sont mises en place dans les écoles primaires publiques de l'ouest du Paraná, Brésil, permettant aux enfants de comprendre les régimes alimentaires sains - une thématique qui est inclue dans le programme pédagogique. En parallèle, les chefs des cantines scolaires sont eux aussi formés sur le sujet. Un concours de recettes saines a été organisé, rassemblant les différents chefs. Toutes ces recettes ont été écrites et regroupées dans un livre. Ces actions impliquent l'ensemble de la communauté scolaire, les familles des enfants, les agriculteurs agroécologiques, les médias locaux et régionaux et d'autres organismes sociaux.

Source: Darli Benghi, Ecovida, Brésil 


\section{«Sa Patass» contre «Boy Pombiter»}

10

Dans le cadre du Programme SADMAD (Système alimentaire durable pour la lutte contre la malnutrition dans la région de Dakar), une étude sur les habitudes alimentaires des ménages et des étudiants dans plusieurs quartiers de Dakar a été réalisée par CICODEV (une organisation de défense des consommateurs). l'étude a montré qu'il y avait une forte consommation de patates douces avec près de 95 pour cent sous forme de frites ou de tubercules non transformés. Cependant, ils ont également constaté une forte présence de produits industriels transformés vendus aux enfants par des marchands à l'extérieur des écoles. l'équipe s'est rendu compte que la conception d'un outil de communication autour de la patate douce pourrait stimuler la sensibilisation des enfants à augmenter leur consommation de produits locaux nutritifs.

Il est bien connu que la lutte est le sport national préféré des hommes, des femmes et des enfants de toutes les classes sociales au Sénégal. De plus, la majorité des lutteurs sont des modèles et vivent dans la banlieue de Dakar, à proximité des zones ciblées par le Programme SADMAD. C'était le moyen idéal pour communiquer un message de service public portant sur les qualités nutritionnelles de la patate douce par rapport à celles de la patate blanche. Avec des lutteurs livrant ce message sous forme de caricature en wolof (une langue locale largement parlée), CICODEV a pu atteindre un public local et national. La vidéo a été conçue en collaboration avec le Laboratoire national d'analyses et de contrôle (LANAC) de la Direction du commerce intérieur, un graphiste, M. Ousmane Diallo, M. Becaye Mbaye, journaliste et figure emblématique de la lutte au Sénégal et Mme Khar Mbaye, grande chanteuse et icône nationale qui lui prête sa voix.

Source: Khady Ndoye, CICODEV, Sénégal

Pour visionner la vidéo en wolof, visitez:

https://www.youtube.com/ watch?v=BlHnHzOAoPU\&t=3s

\section{ASTUCE 7}

\section{Comment attirer les clients}

- Pour éviter l'échec, soyez conscient des besoins et des attentes de vos clients. Communiquez clairement ce que vous pouvez leur offrir et comment.

- Tous les agriculteurs ne sont pas des commerçants efficaces. Il est important de se rendre au marché pour montrer et raconter l'histoire derrière un produit.

- Pour développer une bonne communication, des compétences spécifiques sont nécessaires. Par exemple, le type de langage que vous utilisez est un élément clé pour parler avec des consommateurs particuliers. Soyez conscient que c'est impossible de tout faire seul(e)...une personne apte à la communication peut être utile !

- La sensibilisation des consommateurs - par la communication, l'éducation et l'engagement - est fondamentale pour élargir votre clientèle. 


\section{LE CHEMIN D’APPRENTISSAGE, OÙ ALLER ENSUITE?}

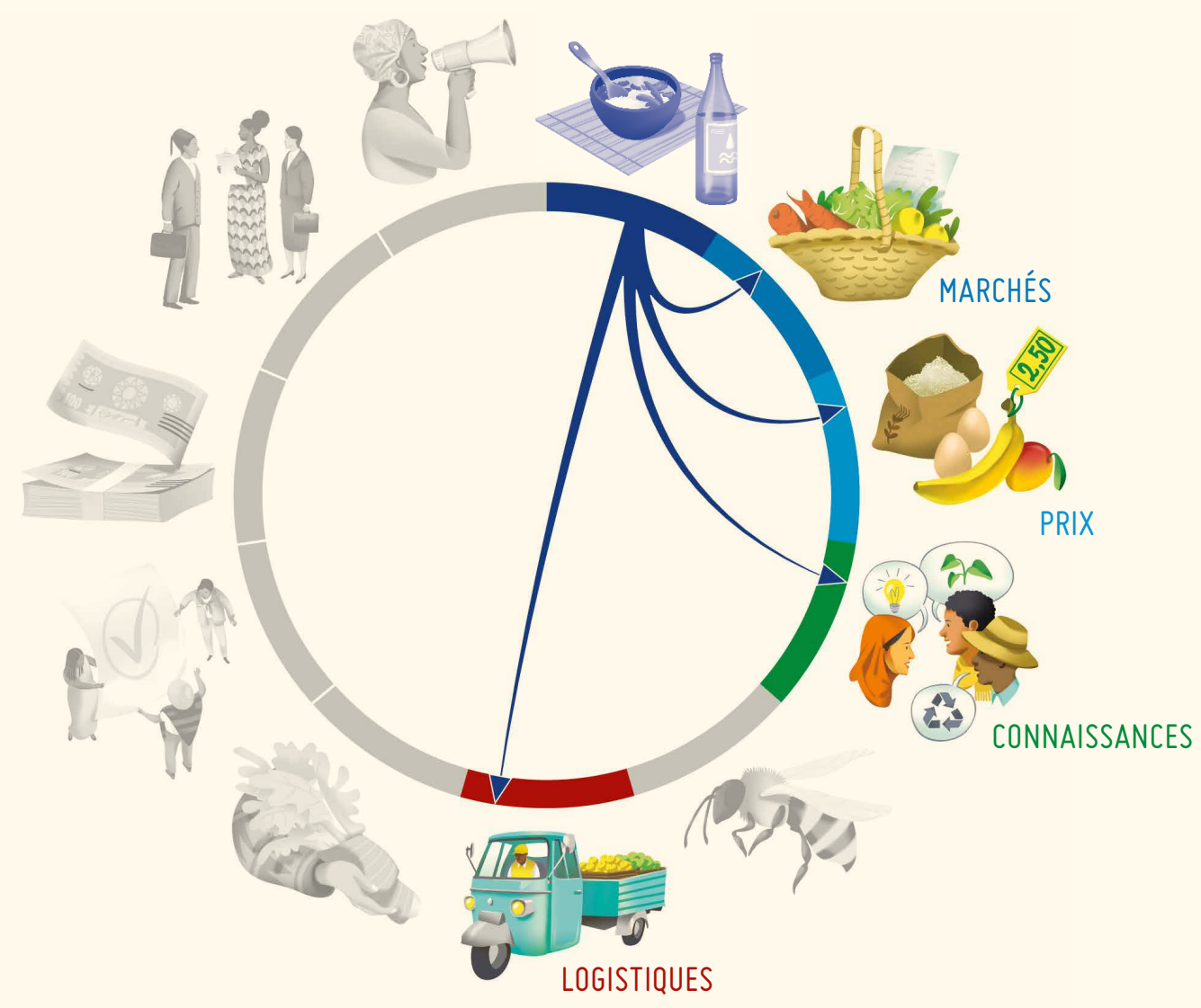

- Vous avez une base de consommateurs et un groupe de producteurs fidèles, mais vous n'arrivez pas à maintenir un approvisionnement stable ? Consultez le Chapitre 6: Rester connectés grâce à la logistique.

- Vous souhaitez en savoir plus sur les mécanismes d'établissement des prix novateurs comme celui de l'AMAP dans ce chapitre ? Consultez le Chapitre 3: Trouver le «bon» prix.

- Vous êtes intéressé par des approches éducatives et d'apprentissage qui vont au-delà des jardins scolaires ? Sautez au Chapitre 4: Partager et co-créer des connaissances pour une production durable pour en savoir plus.

- Vous voulez en savoir plus sur les exigences du marché que vos consommateurs demandent ? Allez au Chapitre 2: Connaître vos marchés.

Ou peut-être un autre défi vous est venu à l'esprit pendant votre lecture, quel chapitre peut vous aider à le résoudre? 

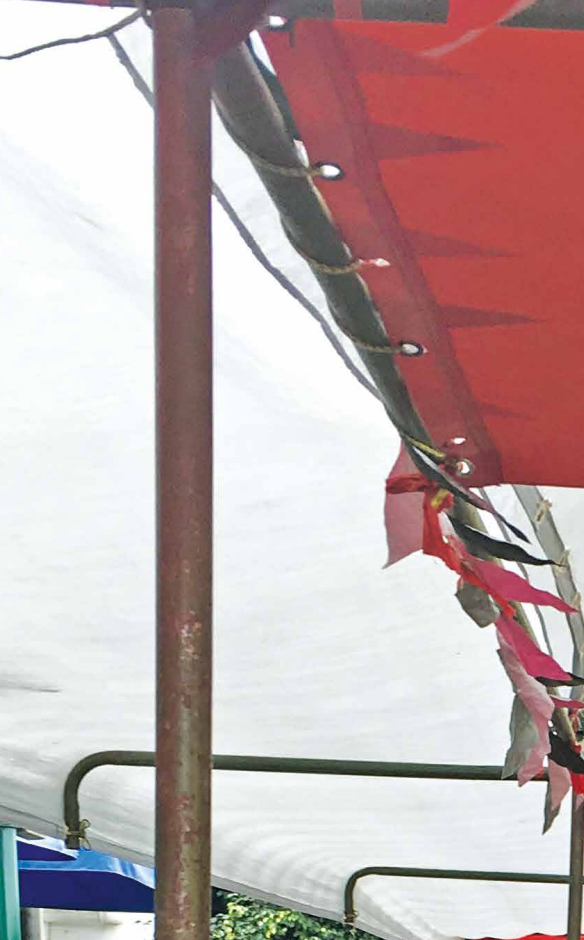

铲

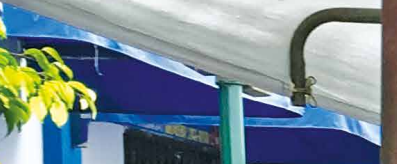


$\frac{. ّ}{\text { 岌 }}$

O N

VOS

MARCHÉS
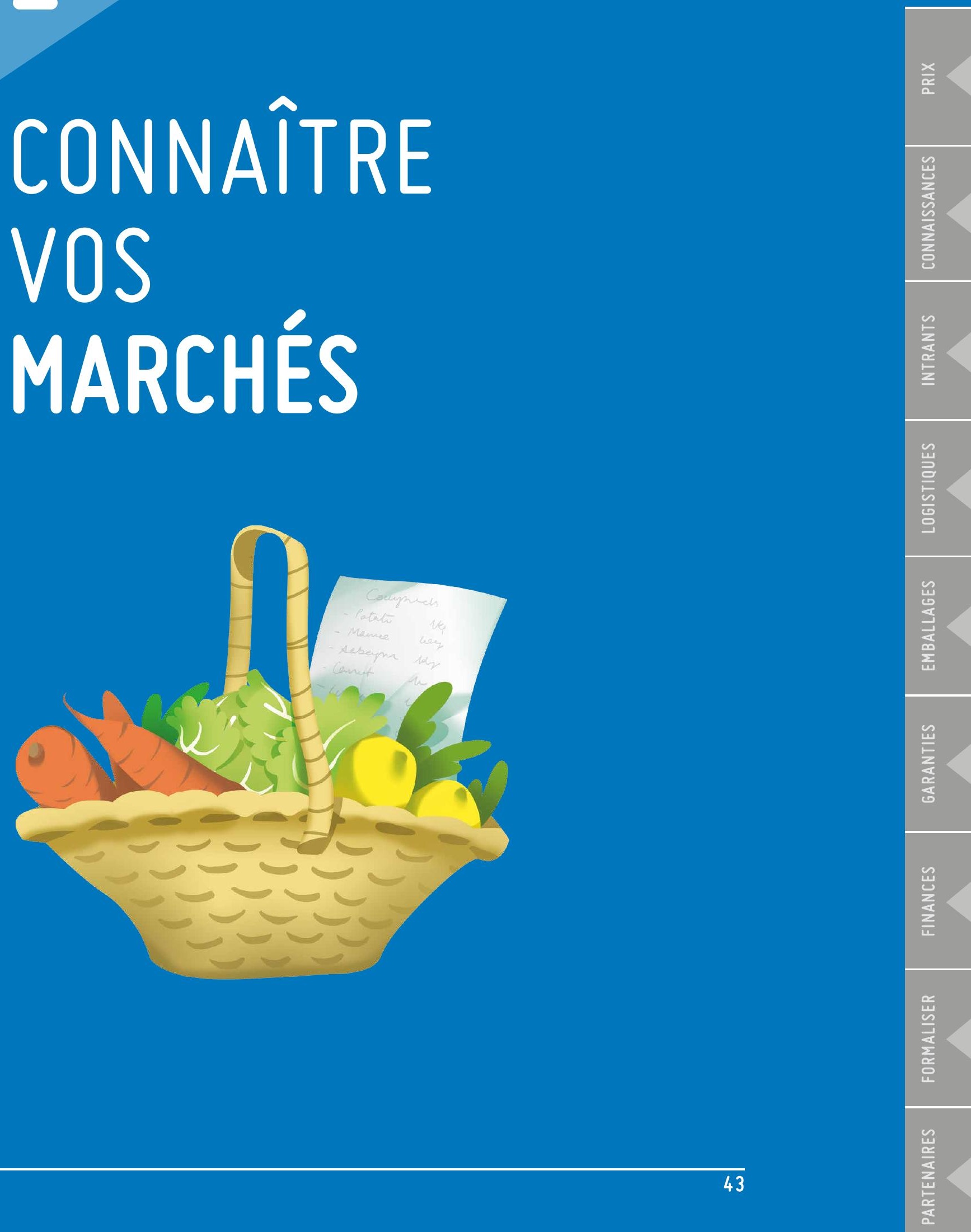


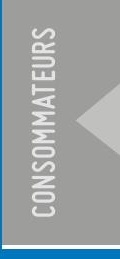

抏

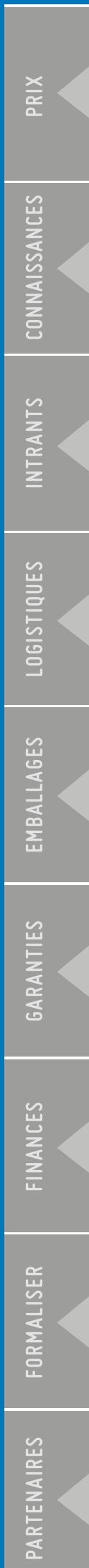




\section{POURQUOI EST-CE IMPORTANT?}

Les marchés sont des espaces d'échanges de produits qui sont à la fois physiques et virtuels. Ils peuvent être situés dans des lieux précis ou être de simples accords ou contrats verbaux ou numériques concernant la valeur des produits échangés. Faisant partie de ces deux types de situation, les marchés durables sont construits par des acteurs pour l'échange équitable de produits élaborés de manière durable.

Dans ce chapitre, nous discutons d'où se trouvent ces marchés durables, en offrant des conseils sur:

- comment identifier les marchés durables auxquels vous avez accès;

- comment déterminer quels sont les meilleurs marchés pour vos produits;

comment créer des nouveaux marchés durables dans vos communautés.

Ce type d'information est souvent appelé «étude de marché» ou «analyse de marché». Il s'agit d'une étape importante dans la commercialisation de vos produits, car elle vous permet d'obtenir des informations sur ce que vous prévoyez produire chaque saison, sur la façon de développer votre entreprise et sur la façon de diversifier les produits que vous offrez. Ce type d'information vous aidera à prendre de meilleures décisions dans vos activités de production, de transformation, de conditionnement et de distribution.

Les activités que nous abordons dans ce chapitre peuvent également être utiles pour tester de nouveaux produits, élargir vos marchés ou inclure de nouveaux acteurs dans votre initiative.

\section{QUE DOIS-JE PRODUIRE, POUR QUEL MARCHÉ?}

La demande ne conduit pas automatiquement à un marché. D'abord, vous devez repérer les marchés qui existent déjà pour vos produits, ce qu'ils offrent et ce qu'ils exigent.

\section{ASTUCE 8}

\section{Élaborer une matrice pour comparer votre capacité par rapport aux exigences du marché}

En suivant le modèle présenté ci-dessous, utilisez les colonnes pour énumérer tous les marchés que vous connaissez. Puis, utilisez les lignes pour énumérer certains des principaux besoins en fonction de votre capacité d'approvisionnement de ces marchés. Ce que vous énumérez dans les lignes et les colonnes dépendra de la façon dont votre système de production et d'approvisionnement est configuré.

1. Faites une liste de votre capacité à fournir des produits, réorganisez la liste selon vos priorités (par exemple, vous avez vos propres camions pour l'expédition, vous avez de grandes quantités de niébés emballés en vrac) afin d'organiser les informations que vous avez recueillis et mieux les utiliser.

2. Utilisez un système de classement * pour comparer l'importance de chaque capacité pour chaque marché. Vous devrez peut-être modifier la signification de chaque ligne. Définissez ce que le signe * signifie dans une légende.

3. Une fois que certains marchés clés ont été identifié et classifié, comme ceux où votre capacité répond le mieux à leur demande, posez-vous les questions suivantes:

a. Cette demande est-elle déjà bien établie ou est-elle en émergence ?

b. La demande est-elle constante tout au long de l'année ou seulement pendant certaines saisons ?

c. Vous pouvez mettre ces différences en évidence avec des couleurs distinctes ou identifier les colonnes avec des symboles uniques. 
Tableau 2: Matrice de la capacité des producteurs et des besoins du marché

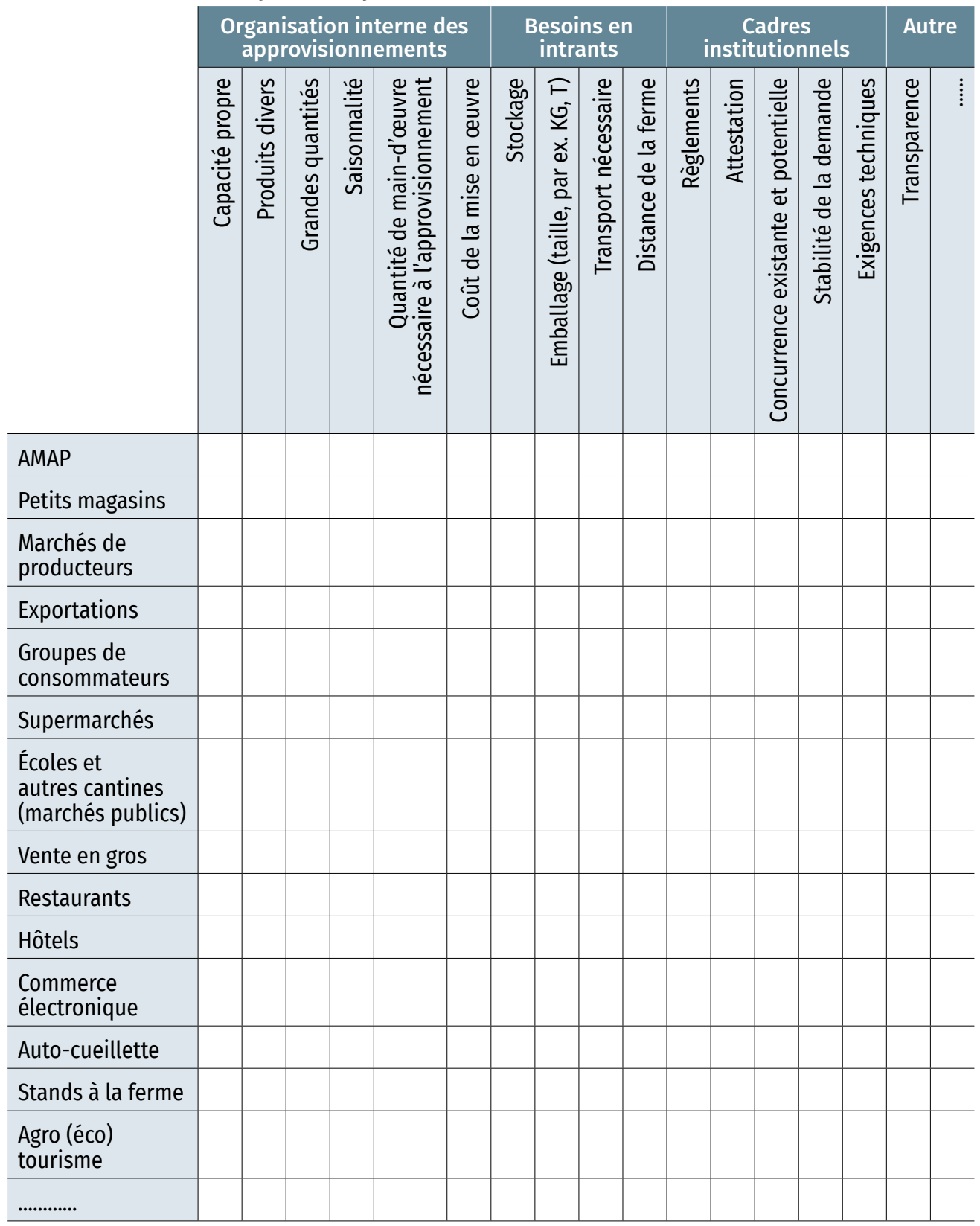

Une fois terminé, vous devriez analyser comment répondre à tous ces besoins en fonction des marchés choisis. Obtenir des devis et des budgets pour les services et les intrants nécessaires, y compris les coûts d'investissement pour l'infrastructure de production et la logistique des transactions.

Légende: ${ }^{*}=$ moins de demande, ${ }^{* * * * * * *}=$ plus de demande

- Pour mettre en œuvre l'information extraite de votre matrice, un plan d'affaires sera un outil très utile.

- Organisez un atelier avec vos membres pour remplir la matrice ensemble. Certains de vos membres ont peut-être déjà de l'expérience avec certains marchés et il est important de s'appuyer sur leurs réseaux. 


\section{COMMENT TROUVER L'INFORMATION NÉCESSAIRE POUR REMPLIR LA MATRICE ?}

Pour obtenir l'information, effectuez une recherche sur internet, téléphonez aux personnes que vous connaissez qui dirigent les marchés ou qui y vendent déjà leurs produits. Visitez les marchés et interrogez les responsables quant aux exigences de leurs fournisseurs.

\section{ASTUCE 9}

Faire en sorte que l'information recueillie soit facilement accessible et compréhensible pour les autres membres

1. Créez un modèle pour un rapport de marché que vous pouvez mettre à jour régulièrement avec de nouvelles informations au fur et à mesure que vous les recevez. Les principaux sujets qui devraient figurer dans le rapport sont ceux qui permettent à l'initiative de prendre des mesures et de prendre de meilleures décisions concernant la production et la commercialisation des produits. Ces sujets peuvent inclure les partenariats stratégiques, les marchés stratégiques et l'ordre de préférence pour l'inclusion de nouveaux marchés. Par exemple, la première étape consiste à lancer l'initiative avec les membres, puis ensuite avec la municipalité, puis avec les foires locales, puis l'exportation (il peut s'agir d'actions parallèles et/ou séquentielles).

2. Toutes les décisions à prendre en matière de commercialisation doivent être basés sur l'analyse du marché et inclure des termes tirés du classement dans le tableau des capacités.

3. En fonction de vos moyens de communication habituels, ces informations peuvent être mises à disposition sur un site internet ou dans vos bureaux. Ces rapports peuvent être autant formels qu'informels - ne vous inquiétez pas si votre rapport de marché est une seule liste sur une feuille de papier. Commencez ici et laissez-le s'approfondir avec le temps !

Vous pouvez obtenir des informations plus spécifiques en vous adressant directement à différents acteurs. Voici quelques approches ayant eu du succès.

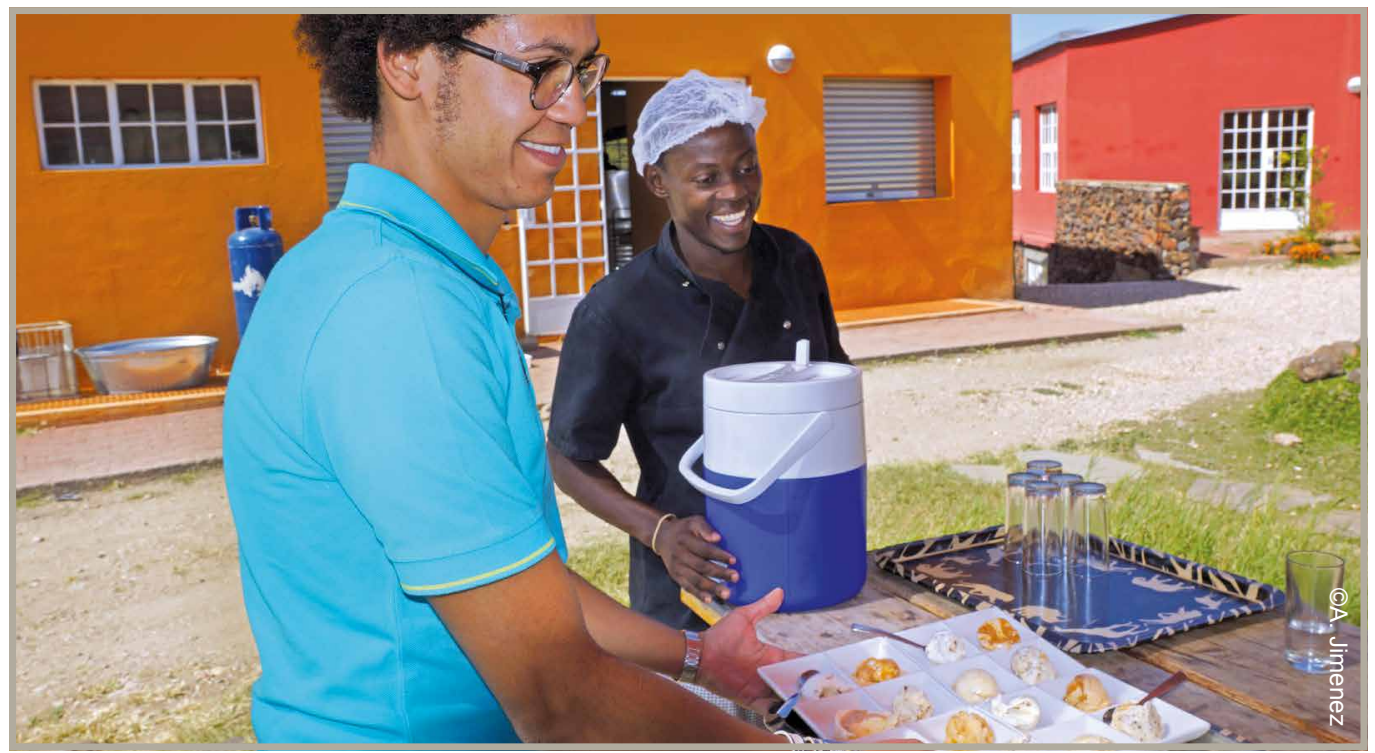




\subsection{Dégustations}

Les dégustations sont parmi les meilleurs moyens d'obtenir de l'information sur la commercialisation de vos produits. Essayez d'en organiser avec différents groupes pour voir si différents marchés réclament différentes versions de votre produit.

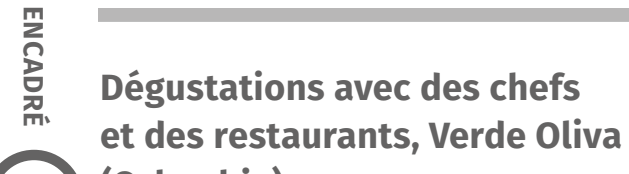

\section{1 (Colombie)}

Une façon d'assurer l'engagement des cuisiniers et des professeurs de gastronomie est de leur faire goûter les variétés de pommes de terre. Pour cela, nous avons réalisé différentes activités de dégustation avec des jeunes chefs d'écoles de gastronomie, avec des consommateurs et avec des chefs renommés de Bogotá. Lors d'un de ces évènements, organisé par Familia de la Tierra et cinq restaurants, des différentes variétés de pommes de terre autochtones ont été présentées sous la forme de cinq créations par cinq chefs. Plus de 20 personnes ont contribué à l'élaboration du menu de dégustation, qui s'est déroulée dans un des restaurants. Développée collectivement avec l'équipe de conception du restaurant, l'affiche a été utilisée pour promouvoir l'événement.

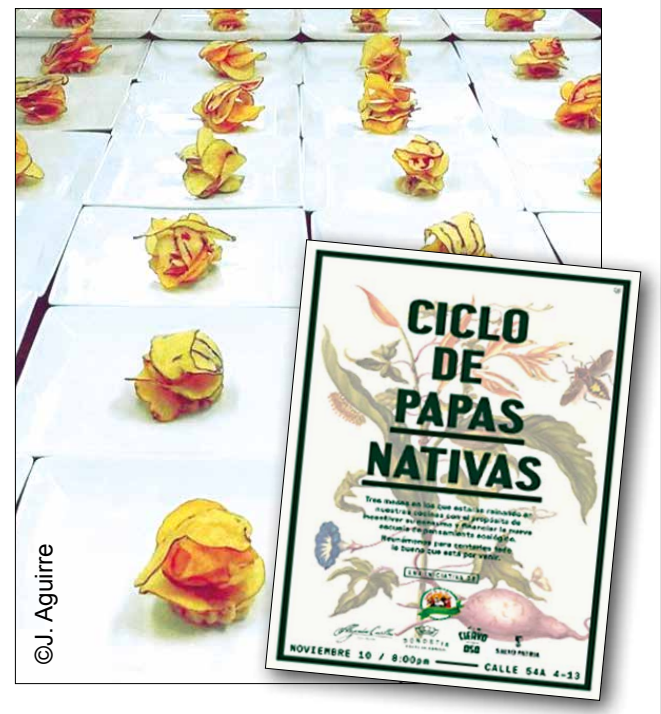

Ce type d'événement permet d'avoir un retour du marché sur la production, la préparation et les goûts des consommateurs pour ce produit.

Source: Oscar Nieto, FdT

\subsection{Visites de fermes et d'installations de transformation}

Lorsque vous organisez une visite pour les consommateurs, changez sa portée par rapport à celle que vous organisez pour les producteurs. Ce type de visite devrait, tout d'abord, présenter certaines pratiques agricoles et, en contrepartie, devrait vous permettre d'obtenir des informations importantes qui vous aideront à développer des produits ou des services nouveaux. Alors, concentrez-vous sur le partage de certaines nouvelles idées et encouragez les consommateurs à participer dans certaines activités. 


\section{Des agriculteurs qui visitent une unité de transformation}

12

Dans le cas des chips à base de variétés de pommes de terre autochtones produites par Familia de la Tierra, en Colombie, l'organisation des visites dans les unités de transformation est essentielle pour les producteurs-membres de l'association. Lorsque les producteurs sont informés du processus de transformation pour obtenir une valeur ajoutée, ils voient comment leurs pommes de terre sont frites et emballées et comprennent pourquoi ils doivent sélectionner les pommes de terre pour faire des chips. Le but principal de ces visites est le partage d'information pour améliorer l'ensemble du processus et renforcer la confiance entre les acteurs du système.

Source: Oscar Nieto, FdT

\subsection{Groupes de discussion}

Les groupes de discussion sont un moyen efficace de recueillir de l'information auprès d'un échantillon de consommateurs potentiels. Ils peuvent être organisés à différentes fins (à la fois pour des recherches approfondies et pour des essais de produits) et à différents moments au cours de la saison. Par exemple, si vous organisez plusieurs cours de formation communautaires, profitez-en pour organiser des groupes de discussion. Pensez aux informations/connaissances que vous vouliez acquérir et aux personnes clés qui tiennent ces informations et organisez le groupe de discussion en conséquence. Lorsque vous organisez des groupes de discussion pour la recherche, les participants ne sont généralement pas rémunérés. Par contre, lorsqu'ils sont posés sur des questions précises sur vos produits ou sur une campagne publicitaire que vous voulez lancer, leurs opinions devraient recevoir une forme de rémunération, car elles sont précieuses !

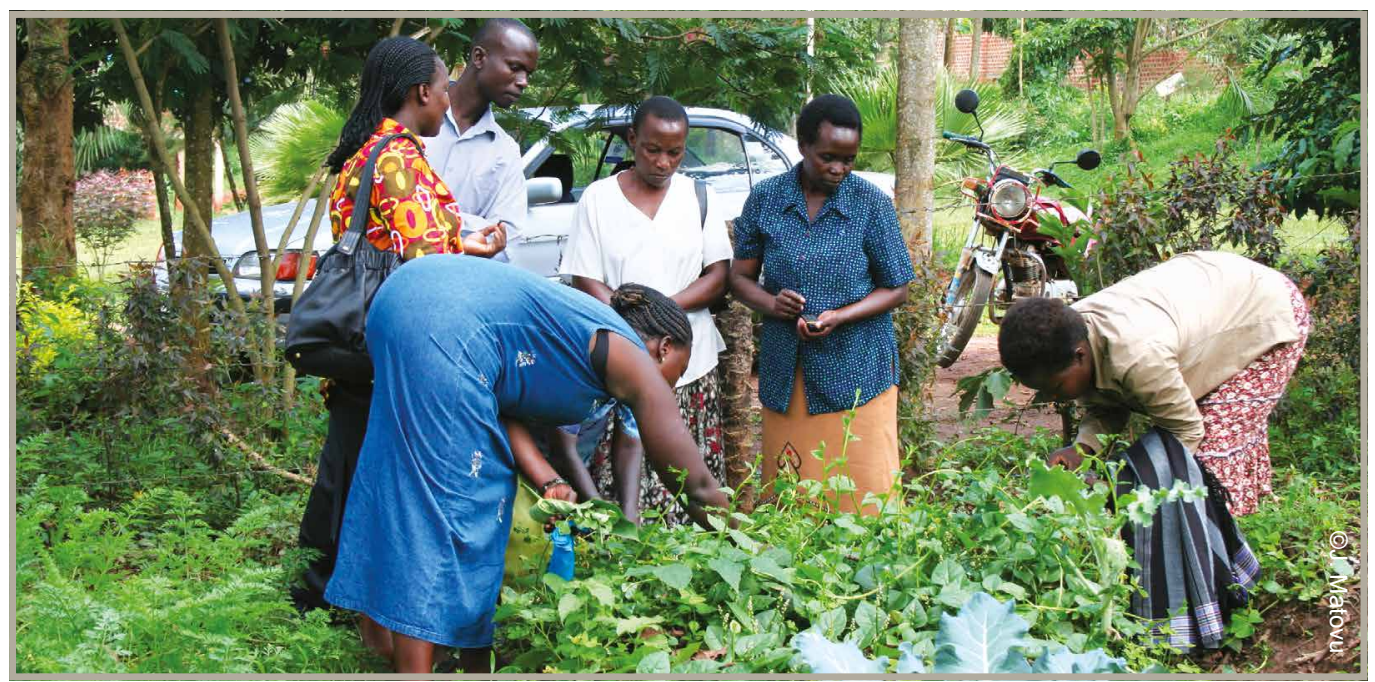




\section{Quand et comment organiser un groupe de discussion?}

Pour contribuer à la compréhension des réseaux alimentaires alternatifs, et plus particulièrement des circuits de marchés agroécologiques, la Fundación EkoRural a réalisé une étude de cas sur les relations producteurs-consommateurs. Ces relations se sont développées autour d'un programme communautaire de paniers alimentaires en Équateur, organisé par la fondation Utopia. Cette étude visait à mieux comprendre les relations économiques et sociales entre les consommateurs d'Utopia et Nueva Generación de Tzimbuto (NG), l'association paysanne qui fournit Utopia. Plusieurs entretiens ont été menés pour éclairer les différentes conceptions de l'agroécologie en tant que système de production, les attentes et la communication de la qualité des produits entre producteurs et consommateurs, les facteurs régissant les choix d'achat, les moteurs de la participation à l'initiative, les difficultés rencontrées et la création de liens sociaux entre consommateurs, entre producteurs et entre consommateurs et producteurs.

Des groupes de discussion et des séances d'information de suivi ont été organisés pour recouper l'information obtenue au cours des entrevues, pour fournir d'autres occasions de discussion et pour obtenir des précisions sur des sujets d'intérêt particulier.

\section{Dynamique de groupe pendant la discussion}

Pour mieux comprendre et contextualiser l'information discutée au cours d'un groupe de discussion, il est important de prêter attention à la dynamique de groupe (Morgan, 1996).

«Au cours de la discussion, tous les participants n'ont pas eu leur mot à dire. Certaines personnes parlaient plus que d'autres et parfois de petits désaccords se présentaient sur ce qui serait la meilleure approche. Les participants avaient également différents styles de contribution. Une dame parlait beaucoup, mais se répétait aussi parfois, ou prenait le temps de développer ses idées tout en parlant. Une autre participante parlait moins mais semblait réfléchir plus attentivement à ses paroles. L'animateur a tenté d'inclure l'opinion de toutes les personnes présentes en demandant aux plus silencieux de s'exprimer et en passant à la question suivante lorsque certains répétaient des points déjà mentionnés».

Source: Ross Mary Borja, Fundación Ekorural

\subsection{Activités pendant les foires et expositions}

Les foires agroécologiques offrent un espace pour la vente et l'achat de produits agroécologiques, ainsi que pour des événements culturels, des ateliers, etc. Et toutes ces activités contribuent à rapprocher le producteur du consommateur et à l'échange d'informations et d'expériences. Ces événements constituent donc une très bonne occasion de recueillir de l'information sur le marché. 
qui servent d'axes fondamentaux pour le développement d'un instrument d'enquête qui leur permettra de recueillir des données plus détaillées sur le comportement et les préférences de leurs consommateurs:

Tableau 3: Questionnaire adressé au consommateur pour trouver un marché

\begin{tabular}{|c|c|c|c|}
\hline \multirow{10}{*}{$\begin{array}{l}\text { Identifier les } \\
\text { consommateurs }\end{array}$} & \multirow{10}{*}{$\begin{array}{l}\text { Préférences et } \\
\text { habitudes de } \\
\text { consommation }\end{array}$} & $\begin{array}{l}\text { Connaissez-vous la différence entre } \\
\text { un produit alimentaire biologique et } \\
\text { traditionnel? }\end{array}$ & Fermée \\
\hline & & Mangez-vous des aliments biologiques? & Fermée \\
\hline & & $\begin{array}{l}\text { Croyez-vous que la consommation } \\
\text { d'aliments biologiques est bénéfique pour } \\
\text { votre santé ? }\end{array}$ & Fermée \\
\hline & & $\begin{array}{l}\text { Quel type de nourriture biologique mangez- } \\
\text { vous? }\end{array}$ & $\begin{array}{l}\text { À choix } \\
\text { multiple }\end{array}$ \\
\hline & & $\begin{array}{l}\text { À quelle fréquence consommez-vous des } \\
\text { aliments biologiques? }\end{array}$ & Fermée \\
\hline & & $\begin{array}{l}\text { Savez-vous ce qu'est yacón et quels sont } \\
\text { ses effets bénéfiques pour votre corps? }\end{array}$ & Fermée \\
\hline & & $\begin{array}{l}\text { Aimeriez-vous trouver où consommer des } \\
\text { produits dérivés du yacón? }\end{array}$ & Fermée \\
\hline & & $\begin{array}{l}\text { Combien d'argent dépensez vous chaque } \\
\text { mois pour des produits biologiques? }\end{array}$ & Fermée \\
\hline & & Où achetez-vous vos produits biologiques? & $\begin{array}{l}\text { À choix } \\
\text { multiple }\end{array}$ \\
\hline & & $\begin{array}{l}\text { Par rapport aux produits traditionnels, } \\
\text { combien seriez-vous prêt à payer pour des } \\
\text { aliments biologiques? }\end{array}$ & Fermée \\
\hline \multirow{5}{*}{$\begin{array}{l}\text { Identifiez les } \\
\text { points de vente } \\
\text { possibles }\end{array}$} & \multirow{9}{*}{$\begin{array}{l}\text { Facteurs clés } \\
\text { de succès }\end{array}$} & $\begin{array}{l}\text { Quelle distance êtes-vous prêt à parcourir } \\
\text { pour faire vos courses dans un magasin } \\
\text { d'aliments biologiques? }\end{array}$ & Fermée \\
\hline & & $\begin{array}{l}\text { Quels endroits de la ville seriez-vous prêt } \\
\text { à atteindre pour faire vos achats dans un } \\
\text { magasin d'aliments biologiques? }\end{array}$ & Fermée \\
\hline & & $\begin{array}{l}\text { Dans quels quartiers de Bogota iriez-vous } \\
\text { pour acheter des produits biologiques? }\end{array}$ & Fermée \\
\hline & & $\begin{array}{l}\text { Sur une échelle de } 1 \text { à } 5,1 \text { étant très } \\
\text { mauvais et } 5 \text { très bon, comment évalueriez- } \\
\text { vous la facilité de déplacement (transports } \\
\text { en commun) dans ces quartiers? }\end{array}$ & $\begin{array}{l}\text { Échelle } \\
\text { numérique }\end{array}$ \\
\hline & & $\begin{array}{l}\text { Sur une échelle de } 1 \text { à } 5,1 \text { étant très } \\
\text { mauvais et } 5 \text { très bon, comment évalueriez- } \\
\text { vous la sécurité dans ces quartiers? }\end{array}$ & $\begin{array}{l}\text { Échelle } \\
\text { numérique }\end{array}$ \\
\hline \multirow{4}{*}{$\begin{array}{l}\text { Identifier les } \\
\text { caractéristiques } \\
\text { idéales du point } \\
\text { de vente }\end{array}$} & & $\begin{array}{l}\text { Pensez-vous que les produits doivent } \\
\text { porter la certification du Ministère de la } \\
\text { santé et des aliments? }\end{array}$ & Fermée \\
\hline & & $\begin{array}{l}\text { Pensez-vous qu'il est important que les } \\
\text { produits portent un label de certification } \\
\text { biologique? }\end{array}$ & Fermée \\
\hline & & $\begin{array}{l}\text { Marquez les produits que vous aimeriez } \\
\text { voir en vente dans un magasin d'aliments } \\
\text { biologiques. }\end{array}$ & $\begin{array}{l}\text { À choix } \\
\text { multiple }\end{array}$ \\
\hline & & $\begin{array}{l}\text { Sélectionnez les critères qui, selon vous, } \\
\text { sont les plus importants pour un bon } \\
\text { magasin d'aliments biologiques. Vous } \\
\text { pouvez en sélectionner plusieurs. }\end{array}$ & $\begin{array}{l}\text { À choix } \\
\text { multiple }\end{array}$ \\
\hline
\end{tabular}

Source: Andrea Moya, Agroindustrias Nutriandina SAS, Colombie 


\section{COMMENT METTRE EN PLACE DES MARCHÉS QUI N'EXISTENT PAS ENCORE?}

Une fois que vous connaissez les marchés et les opportunités qui existent, commencez à planifier et à établir de nouveaux marchés.

\subsection{Mise en place d'un marché de producteurs}

\subsubsection{Comment obtenir de l'espace physique?}

Obtenir l'espace pour votre marché peut être un défi. À moins que votre initiative n'ait son propre bâtiment, vous devrez emprunter, louer ou acheter un espace. Les entreprises privées, les églises et les mairies peuvent vous prêter leurs aires de stationnements, leurs jardins, leurs parcs ou leurs places, mais vous devrez négocier avec elles et respecter leurs règles pour l'utilisation équitable de leur propriété.

\section{Le marché mobile des agriculteurs de Pékin}

Lorsque le premier marché a ouvert ses portes en septembre 2010, il n'était fréquenté que par ceux qui connaissaient les organisateurs. Il s'est également tenu à différents endroits de la ville, connue sous le nom de «marché mobile». Depuis, le nombre de vendeurs est passé de moins de 10 à plus de 40, et le nombre de fans de Weibo (un outil de réseau social) est passé à plus de 80 000. Plus de 200 fournisseurs ont également postulé pour rejoindre le marché en 2013. Il n'a pas encore établi de règles écrites pour la sélection de nouveaux fournisseurs, mais il s'efforce de conserver une certaine diversité et un équilibre entre «les valeurs mondiales et locales, commerciales et socialement justes, modernes et traditionnelles».

Le marché demeure mobile et presque toutes les sources d'information concernant la localisation proviennent directement des suggestions des consommateurs. Ces emplacements comprennent, sans s'y limiter, le
BMW 4 S Event Centre, Canadian International School of Beijing, Iron Chef Kitchen, Vintage Arts Square, Beijing New World Women's Shopping Mall, Children's Event Centre at Chongguang Mall, Nali Patio at Sanlitun Bar Street, Red Wall Garden Hotel, Shijia Hutong. En plus de trouver des endroits pour héberger le marché sans encourir de coûts monétaires, les consommateurs offrent aussi des conseils en matière d'organisation et d'information. La nature mobile du marché lui permet de se tenir simultanément à différents endroits de la ville et encourage les consommateurs à rester engagés afin de ne pas rater le prochain lieu ou le marché va se dérouler.

Source: Li et Loconto, 2018

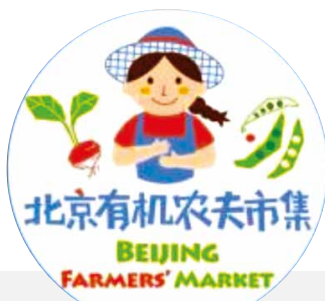

Pour en savoir plus, visitez:

https://www.facebook.com/farmersmarketbj 


\subsubsection{Déterminer qui et quoi peut être vendu sur le marché}

Avant d'installer un marché de producteurs, il est important que les règles d'échange soient déterminées. Parfois, il peut y avoir des restrictions sur le type de vendeur. Par exemple, estce qu'il inclura seulement les agriculteurs ou les commerçants aussi ? Dans d'autres cas, les produits seront restreints - est-ce qu'il offrira exclusivement des produits certifiés biologiques, seulement des produits de saison ou frais, ou des produits transformés aussi ? Il est important de se rassembler avec les autres acteurs qui veulent vous aider à gérer le marché de producteurs et de formuler une «charte» qui guidera la gestion du marché.

\section{LISTE DE VÉRIFICATION}

Rédiger une charte de marché paysan

1. Qui peut vendre sur le marché ?

2. Qui peut acheter?

3. Y a-t-il des restrictions quant aux types de produits vendus?

4. Les prix seront-ils fixes (dans une fourchette par produit ou concurrentiels en fonction des taux du marché)?

5. Quelles formes de paiement seront permises (argent comptant, crédit, bons d'État)?

6. Comment les paiements seront-ils traités ? Dans un lieu centralisé géré par le marché ou individuellement avec chaque vendeur?

7. À quelle fréquence le marché aurait-il lieu?

8. Où aurait-il lieu ?

9. Comment seront payés les frais opérationnels du marché (espace, électricité, eau, publicité, etc.) ?

10. Y aurait-il de la place pour des activités de loisir et d'éducation?

11. Qui le gérera et quelle sera la récompense pour le travail?

\subsection{Mise en place d’un programme d’alimentation scolaire}

Les programmes d'alimentation scolaire sont nombreux et souvent complexes. Cependant, les parents demandent de plus en plus aux écoles d'inclure des aliments plus sains et plus durables dans les repas de cantine. Vérifiez ce que les écoles de votre région font pour leurs programmes de repas scolaires et voyez s'il y a des associations parentales intéressées à établir un programme d'approvisionnement de produits durables. 


\section{ASTUCE 10}

Vérifier la législation nationale concernant les programmes de restauration scolaire

Ceci est une étape importante car les écoles ne sont pas toujours autorisées à déterminer leur propre stratégies d'approvisionnement alimentaire.

\subsection{Mise en place d'une AMAP}

L'association pour le maintien de l'agriculture paysanne (AMAP) est un terme utilisé pour décrire un certain nombre de modèles de ventes directes des producteurs aux consommateurs. Ils émergent dans le monde entier et sont relativement faciles à installer et ont juste besoin d'un groupe de producteurs et de consommateurs dévoués dans une communauté dévouée!

\section{ASTUCE 11}

Comment créer une AMAP

Que l'AMAP soit une initiative communautaire ou agricole, les étapes suivantes sont recommandées.

\section{Comprendre le concept}

Il est important de comprendre quel type de production est attendu d'une AMAP (diversité des produits, pratiques biologiques ou agroécologiques, continuité de la production, etc.) et quel type de relation doit être développé au sein de sa communauté. Il vaut la peine de communiquer avec d'autres initiatives de style AMAP dans votre région ou votre pays.

En tant que consommateur, préparez-vous à satisfaire ces engagements et demandez-vous si vous êtes capable de les respecter. L'un des principaux engagements est le paiement initial. Un autre est la participation aux distributions, l'aide à la gestion de groupe et, parfois, au travail agricole.

Le partage des risques et des avantages entre les agriculteurs et les consommateurs est un élément clé d'une AMAP. Cela signifie que s'il y a une récolte exceptionnelle, le consommateur aura une part beaucoup plus importante et aura besoin de congeler ou de conserver ses aliments à la maison pendant la basse saison. D'autre part, dans le cas d'un événement climatique, le contenu du panier hebdomadaire peut être très minime.

\section{Planifier}

En tant que producteur, il est important d'évaluer votre capacité et vos possibilités. Avez-vous une idée du nombre de ménages qui pourraient être approvisionnés avec ces produits ? Pourriez-vous estimer combien de temps de travail est nécessaire ? Avez-vous la capacité de l'accomplir seul ou devriez-vous embaucher des travailleurs?

\section{Trouver des membres}

Pour mettre sur pied une AMAP, il faut trouver un noyau de consommateurs qui pourraient devenir des partenaires.

- Si vous vendiez déjà vos produits par d'autres canaux de commercialisation, demandez à vos clients s'ils seraient intéressés à joindre votre AMAP.

- Demandez à vos amis ou à vos voisins avec qui vous avez déjà un rapport de confiance. 
- Recherchez des groupes existants: garderies, organismes environnementaux, groupes civiques, milieux de travail... De plus, des lieux comme les écoles alternatives ou les centres de yoga pourraient être des bons espaces pour trouver des gens qui s'intéressent à l'alimentation saine et aux relations communautaires. N'oubliez pas de communiquer avec les AMAP régionales.

- Communiquez avec votre réseau local de l'AMAP ou avec des ONG travaillant déjà sur ce thème.

\section{Organiser des réunions publiques}

Avec les premiers alliés, préparez des réunions publiques pour trouver de nouveaux consommateurs. N'ayez pas peur si vous n'avez pas beaucoup d'expérience. L'AMAP est un partenariat, et les consommateurs doivent participer à la recherche de solutions. Voici un ordre du jour possible:

> Qu'est-ce que l'AMAP?

- Pourquoi manger des aliments cultivés localement ? Quels sont les risques de l'agriculture industrielle?

- Pourquoi les petits agriculteurs ont-ils besoin d'aide?

- Quels sont les avantages à devenir membre de l'AMAP ?

- Évaluez le niveau d'engagement des participants.

- Répartissez les tâches/rôles et créez un groupe central.

Il est très utile d'inviter un agriculteur expérimenté sur l'AMAP à votre réunion.

La transparence est fondamentale. Toutes vos questions ou préoccupations devraient faire l'objet d'une discussion.

\section{Recueillir les engagements et établir le calendrier de la première saison}

Combien de temps cela prend-il ? Le calendrier pour l'établissement d'une AMAP varie selon votre situation initiale et la communauté qui vous entoure. Cependant, de façon générale, le plus de temps que vous consacrez à la planification initiale, à la réflexion et à la structure du projet et aux discussions avec les participants, le mieux vous serez préparé au fonctionnement de l'AMAP.

Source: Faites partie de la CSA ! Livret d'appui à la formation sur l'agriculture soutenue par la communauté, 2016.
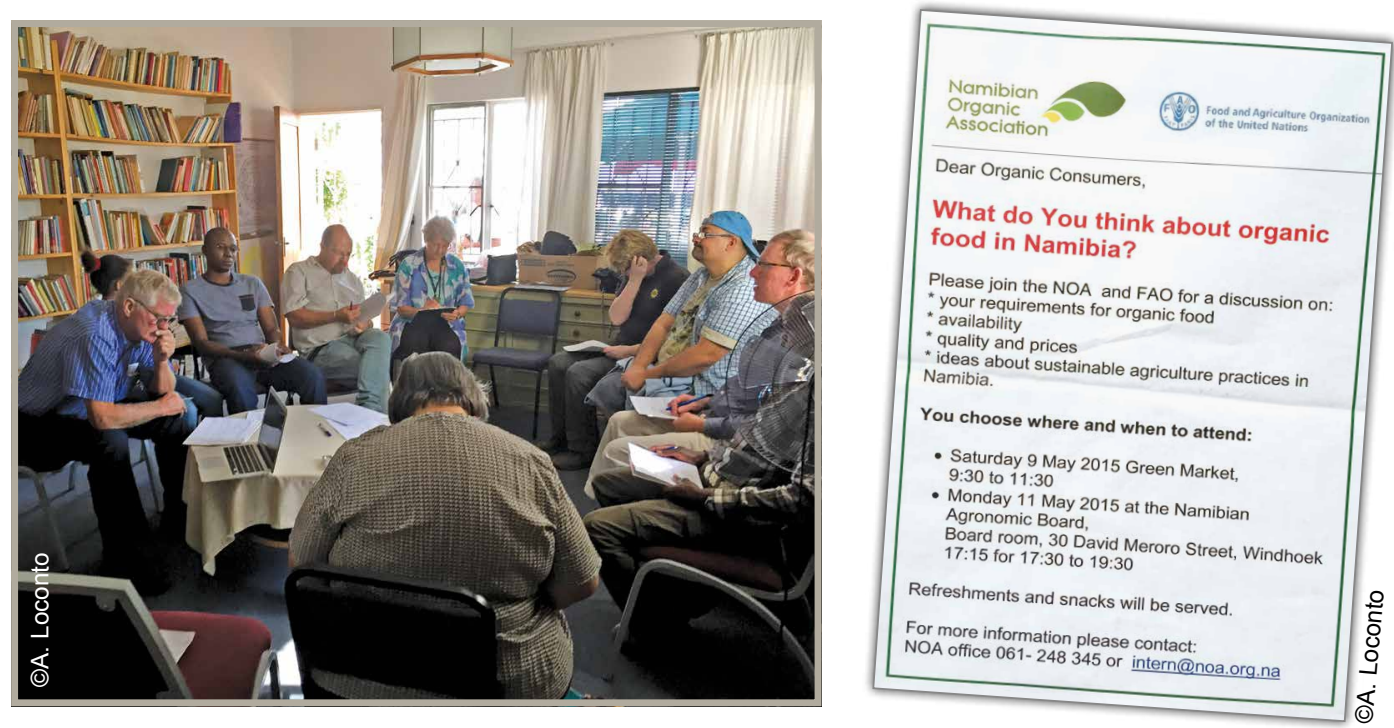


\subsection{Mise en place d'une plateforme internet}

De nombreuses initiatives utilisent l'internet pour mettre en place une vitrine en ligne où les producteurs, individuels ou en groupes, peuvent inscrire leurs produits. Les consommateurs peuvent les visualiser soit pour les commander en livraison, soit pour l'auto-cueillette. Ces plateformes en ligne peuvent faire partie d'une initiative, d'une nouvelle start-up ou d'un réseau mondial de plateformes électroniques.

\section{Faire cavalier seul ou rejoindre un réseau?}

17

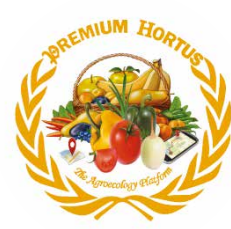
pour l'agroécologie spécialisée dans la
PREMIUM HORTUS est une plateforme technologique production biologique, le commerce électronique de produits agroécologiques

et le soutien aux producteurs en République du Bénin. Disponible sous forme d'applications web et mobiles et comme solution de paiement en ligne, le site PREMIUM HORTUS permet au consommateur de s'abonner, de choisir le contenu du panier, de faire des commandes, de payer en ligne et de faire livrer des fruits, des légumes, des cocktails et des produits biologiques à domicile en toute sécurité. Il y a deux programmes qui contribuent à rendre ce service plus inclusif des ménages les plus pauvres: CALIM + est un crédit alimentaire qui aide les membres à effectuer des paiements lorsqu'ils manquent d'argent; et CONSOM'Acteur est une application qui aide les consommateurs à documenter leurs habitudes et leurs besoins alimentaires. PREMIUM HORTUS ramasse les surplus de nourriture et les livre à ceux qui en ont besoin.

PREMIUM HORTUS garantit aux producteurs agroécologiques un accès permanent aux semences naturelles, aux bio-fertilisants et aux biopesticides spécifiques en dehors de ses sites agroécologiques au Bénin. Il renforce le professionnalisme et la résilience des petits producteurs et des exploitations agricoles familiales par le biais d'échanges, de renforcement des capacités, d'un soutien technique personnalisé en gestion d'entreprise verte et de solutions technologiques adaptées.

Source: Johannes Goudjanou, Premium Hortus

Pour en savoir plus, visitez: http://premiumhortus.com

\section{OPEN FOOD} NETWORK

Open Food Network (OFN) est un réseau mondial collaboratif d'organisations locales à but non lucratif qui ont conjointement développé des logiciels Open Source et les ont mis à la disposition des centres alimentaires du monde entier par le biais de plateformes en ligne localisées (comme OFN UK, OFN Australie, Open Food France, Katuma, OFN Canada, etc. La mission de cette communauté est de construire une infrastructure numérique partagée (et des connaissances partagées) pour permettre aux opérateurs de circuits courts alimentaires de gérer leurs activités, de coopérer plus efficacement et de renforcer la souveraineté alimentaire. Tout comme les consommateurs et les producteurs se réunissent pour créer des centres alimentaires, les centres alimentaires de 
l'OFN se réunissent pour créer une infrastructure numérique et partager son utilisation.

En pratique, la plateforme permet aux producteurs de gérer leurs catalogues de produits et de les partager avec de multiples distributeurs (centres alimentaires). Ces centres peuvent se connecter avec un ou plusieurs producteurs pour afficher leurs produits dans un magasin et organiser les ventes collectives et obtenir des données agrégées pour organiser la logistique et satisfaire les commandes en vrac ou individuelles.

Dans le cas de l'OFN, une plateforme peut être composée de plusieurs éléments, notamment:

$\checkmark$ un producteur vendant directement aux consommateurs par le biais d'une boutique en ligne;

$\checkmark$ une AMAP ou un groupement d'achat qui organise des achats collectifs réguliers basés sur des abonnements et qui gère plusieurs sous-groupes d'achat;

> un entrepreneur qui s'approvisionne en aliments auprès de producteurs locaux ou biologiques et qui organise des ventes aux particuliers, aux groupes, aux restaurants et aux détaillants.

La plateforme facilite également la mise en réseau et la commercialisation, comme la possibilité de créer un groupe avec les producteurs et d'identifier les centres d'un écosystème particulier. Ceux-ci peuvent être affichés sur une carte, comme celle de la Biodynamic Association au Royaume-Uni.

Source: Myriam Bouré, Open Food Network

Pour en savoir plus, visitez:

https://openfoodnetwork.org

\section{ASTUCE 12}

\section{Comment trouver des marchés durables}

- Assurez-vous de connaître les exigences du marché et vos capacités à y répondre avant de décider où vendre vos produits.

- Dialoguez autant que possible avec les membres de votre initiative, les consommateurs, les producteurs, les intermédiaires et les autres acteurs intéressés. Ils sont votre meilleure source d'information sur les marchés durables.

- S'il n'y a pas de marchés durables là où vous êtes, construisez-les !

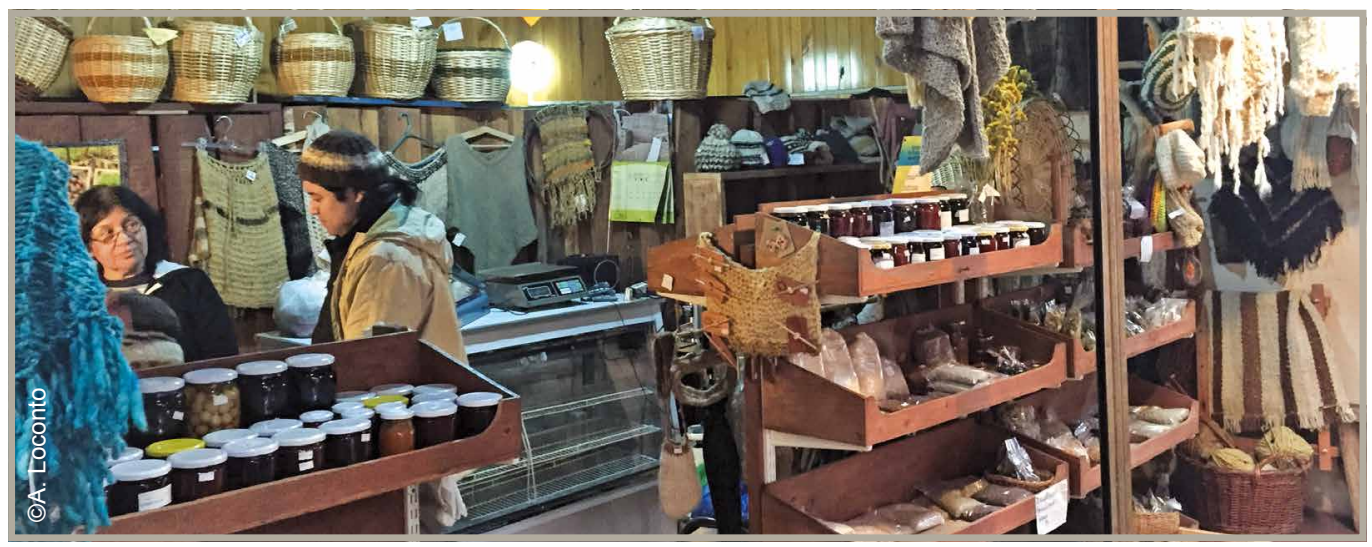




\section{LE CHEMIN D’APPRENTISSAGE, OÙ ALLER ENSUITE?}

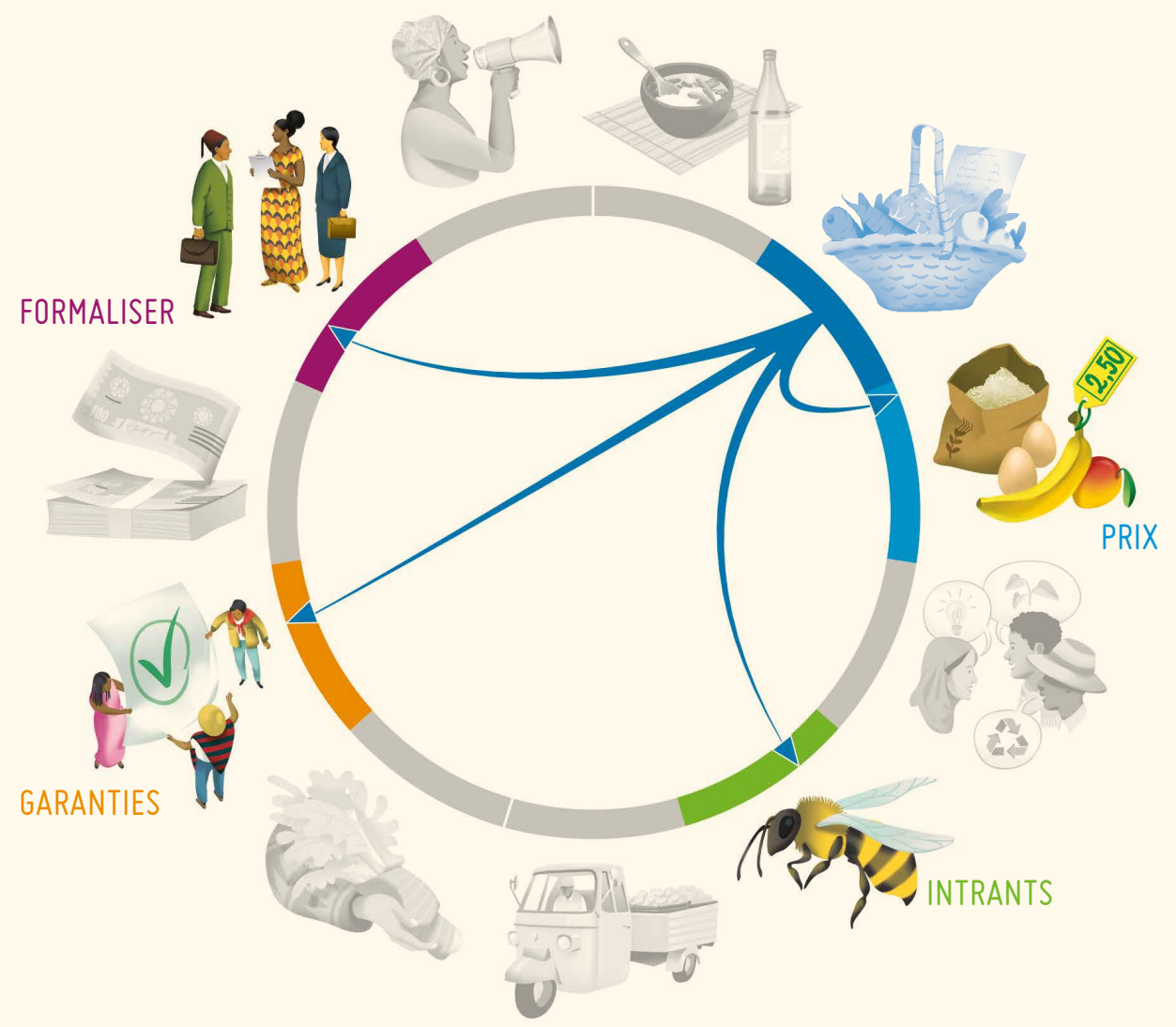

- Vous voulez savoir si le modèle AMAP est une bonne option pour commercialiser vos produits ? Allez au Chapitre 10: Formaliser votre travail collectif.

- Vous voulez accéder aux intrants nécessaires pour répondre aux exigences de votre marché ? Consultez le Chapitre 5: Gérer et accéder aux intrants durables pour en savoir plus.

- Vous voulez apprendre comment fixer le prix de vos produits pour vos différents marchés? Consultez le Chapitre 3: Trouver le «bon» prix pour étudier cette question.

- Est-ce que vos clients vous interrogent sur vos pratiques durables? Vous voulez leur expliquer votre système de garantie ? Allez au Chapitre 8: Garantirr la durabilité.

Ou peut-être vous avez une meilleure idée ? Laissez celle-ci vous guidez vers un autre chapitre. 

3

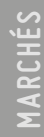

D?

LE "BON" PRIX

$\tilde{\Sigma}$

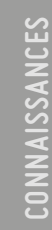

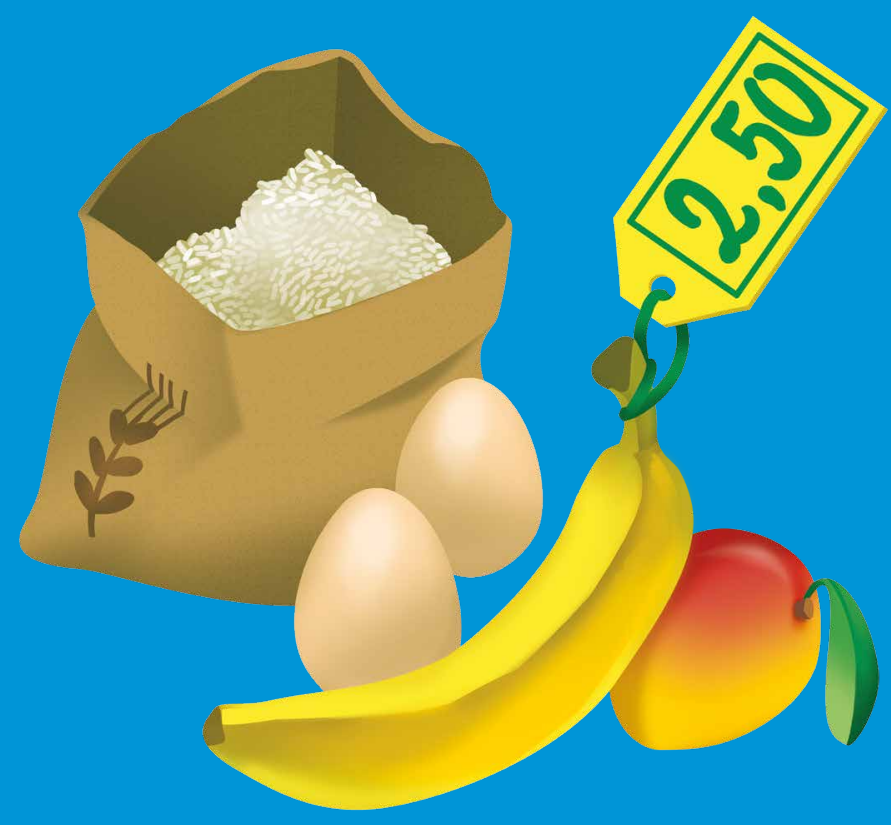

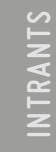

뜰

出
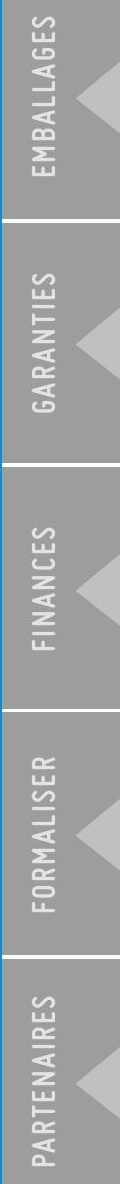


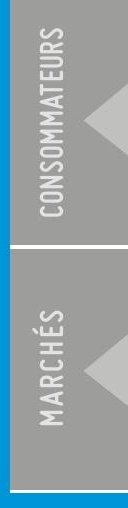

$\frac{x}{\alpha}$

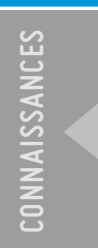

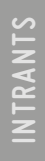

岸
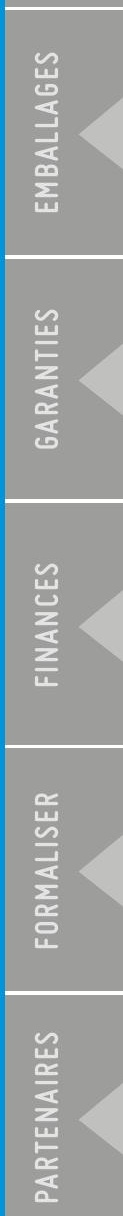


\section{POURQUOI EST-CE IMPORTANT?}

Comment comptabiliser l'ensemble des coûts de la production durable et comment négocier les prix avec les différents types d'acheteurs et de consommateurs ? C'est un problème principal de l'installation et de l'organisation du marché. De plus, l'incorporation de critères de durabilité à tous les aspects du système alimentaire rend cet équilibre encore plus difficile à établir.

La véritable comptabilisation des coûts, ou autrement dit, la connaissance qu'ont les agriculteurs des coûts réels de production, a pour but de rééquilibrer les calculs de prix. Toutefois, les consommateurs ont l'habitude d'acheter des aliments peu coûteux, ce qui rend difficile la renégociation de prix.

Par conséquent, l'établissement d'un prix «juste» pour les aliments - un prix équitable et durable pour toutes les parties de la transaction - est parmi les défis économiques plus pertinents de notre époque. C'est une étape fondamentale pour s'assurer que l'agriculture est valorisée.

Dans de nombreux pays, la façon dont les prix des produits alimentaires sont établis peut considérablement affecter la subsistance des petits producteurs. Cela est dû au fait que les petits producteurs participent peu, voire pas du tout, à la fixation des prix. De plus, l'évolution des marchés mondiaux des produits, des intrants et des marchés financiers impacte les marchés nationaux et locaux, où ces producteurs sont des preneurs de prix plutôt que des négociateurs. Néanmoins, dans les systèmes alimentaires durables, des innovations locales sont développées pour contrer le commerce conventionnel et le pouvoir de marché, promouvant ainsi un accord participatif sur les prix.

Dans ce chapitre, nous discuterons de ces innovations et tenterons de partager des exemples de modèles innovateurs de petite échelle qui contestent le mécanisme des «prix du marché» en faveur des petits producteurs.

\subsection{Coût par rapport au prix}

Il y a une distinction entre le coût du produit et son prix. Le coût des produits s'entend comme le coût des intrants nécessaires pour fabriquer une unité de production tels que le temps, la main-d'œuvre ou l'argent. Par exemple, en 2015, le coût de production de $1 \mathrm{~kg}$ de riz dans l'État du Tamil Nadu, dans le sud de l'Inde, équivalait en moyenne à 15 INR (TNAU, 2015). D'autre part, le prix signifie ce que le producteur obtient du marché. Par exemple, les marchés publics de riz au Tamil Nadu en 2015 étaient de 14,7 INR par kg (Kamaraj, 2015).

Pour les petits exploitants à travers le monde, il est toujours difficile de trouver le «bon» prix pour leurs produits, qui couvre leurs coûts, qui offre une marge raisonnable pour leurs frais de subsistance et pour investir dans la prochaine campagne agricole, et qui est durable. Toutefois, il s'agit surtout d'un déséquilibre, puisque les prix fixés sont généralement déterminés par des intermédiaires qui participent très peu ou pas du tout à la négociation avec les producteurs.

Étant donné que les producteurs ne calculent pas au-delà des coûts des intrants achetés, une partie du coût de production est souvent subventionnée par eux. Les coûts de main-d`œuvre (même ssil sıagit de leur propre main-d`œuvre) et les coûts de transaction sur le marché (p. ex. les coûts de transport vers le marché) sont rarement inclus. Les externalités environnementales 
et sociales ne sont pratiquement jamais prises en compte. Aussi, les économies issues d'une collaboration avec d'autres agriculteurs ou de la réalisation d'une économie d'échelle sont aussi souvent exclues. La négociation des prix représente un défi important pour les petits exploitants.

Dans les systèmes alimentaires durables, trouver des prix qui prennent en compte les coûts des producteurs font partie intégrante de la durabilité sociale et économique du système. Certains intermédiaires et consommateurs cherchent spécifiquement à assurer une indemnisation adéquate, alors que d'autres ne le font pas.

\subsection{Pourquoi le prix doit-il être "recalculé»?}

L'importance de recalculer le prix des produits agricoles est due à la nécessité d'inclure les coûts et avantages cachés tels que les dommages environnementaux, la récupération des actifs naturels, les services (ou externalités) écosystémiques et les attributs de qualité qui valorisent les produits agricoles. Celles-ci peuvent inclure: les particularités de semences autochtones, les couleurs particulières, le bon goût, la santé, la production biologique, la production locale ou artisanale, etc.

Comparé à la fixation conventionnelle des prix, cette approche a pour but d'inclure les coûts cachés dans le prix. De plus, elle veille à ce que les agriculteurs et les consommateurs profitent également de l'échange. Déterminer des prix équitables qui peuvent être maintenus par des échanges continus est fondamental pour que les acteurs puissent réévaluer le rôle de l'agriculture dans la société et créer des systèmes alimentaires durables.

Bien qu'il existe quelques formules pour calculer ces prix, en pratique, déterminer le «bon» prix dépend surtout d'une négociation empathique et de l'utilisation de nouveaux modèles commerciaux. Idéalement, ces derniers permettent de mieux calculer les coûts réels de production et de distribution et de partager les avantages entre les acteurs du système alimentaire.

EXEMPLE DE FORMULE POUR CALCULER LES PRIX DANS LES SYSTÈMES ALIMENTAIRES DURABLES

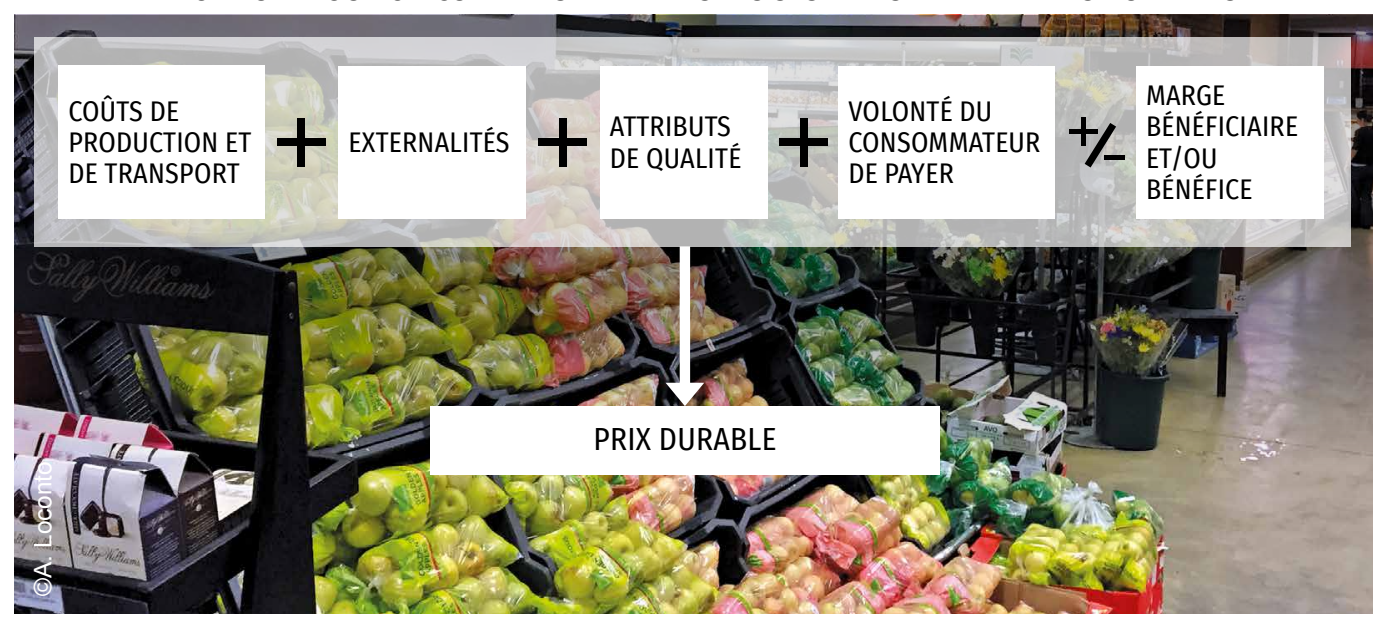


La comptabilisation des coûts réels

18

La comptabilisation du coût réel est

également connue sous les noms de coût complet, valeur totale ou impact total. II s'agit d'une approche qui permet de compter les biens non-marchand tels que les actifs environnementaux et sociaux, afin d'analyser les coûts et les avantages des décisions commerciales et/ou politiques. L'inclusion des externalités négatives et positives dans le calcul des coûts aide les producteurs à mieux comprendre comment leurs efforts devraient être compensés. Ce nouveau calcul devrait tenir compte des frais liés à la détermination participative des prix et/ou des avantages des paiements pour les services écosystémiques. Les connaissances accrues des producteurs leur permettent de mieux négocier les prix. Le Protocole sur le capital naturel a créé une boîte à outils pour recalculer le coût total de production:

https://naturalcapitalcoalition.org/wp-content/ uploads/2017/11/NCP-SEEA-Toolkit-Sep-2017IDEEA-Group-1.pdf

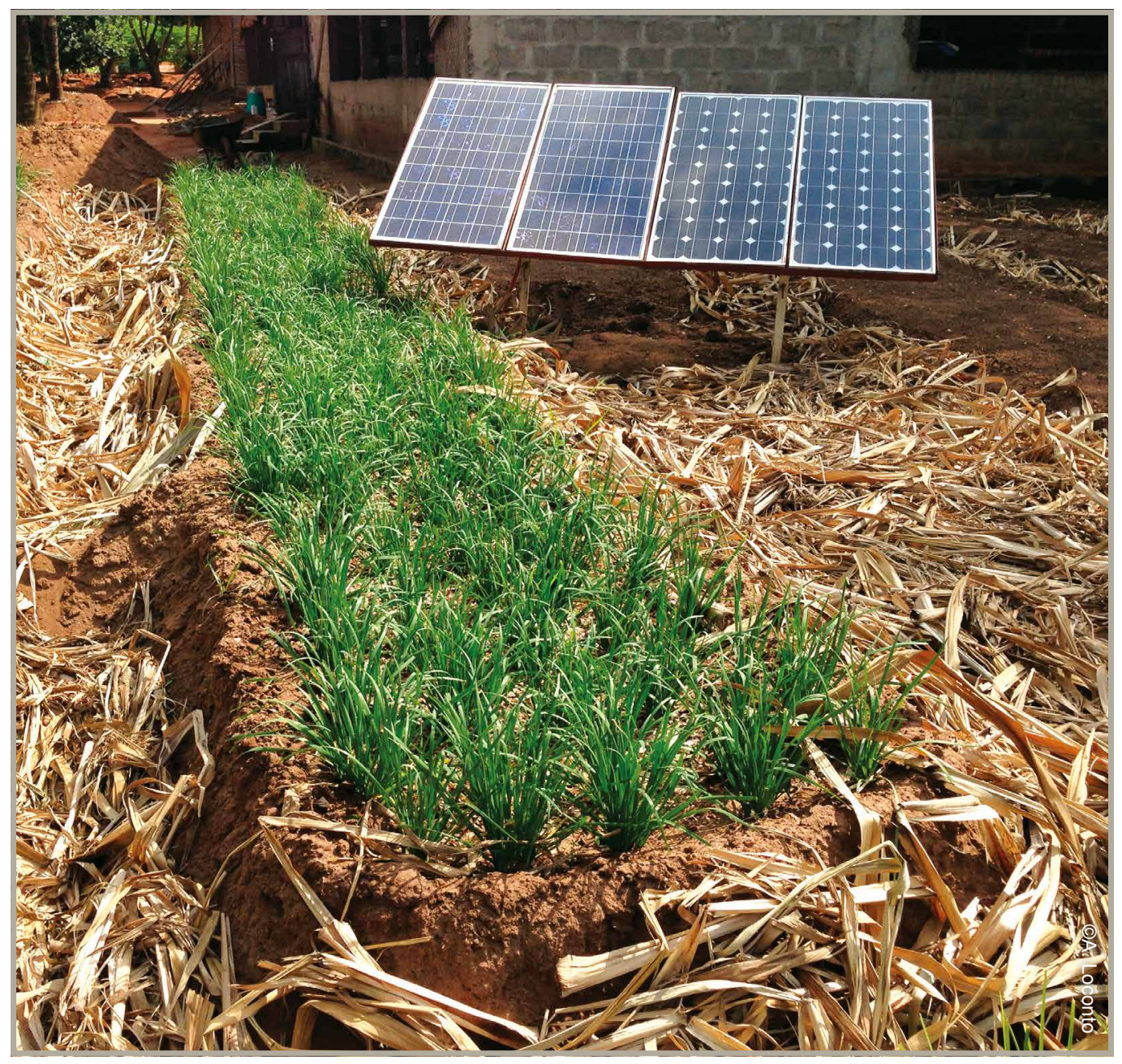




\section{COMMENT GÉRER DES MÉCANISMES DE TARIFICATION PARTICIPATIFS ET TRANSPARENTS?}

La plupart des méthodes visant à créer des mécanismes de tarification participatifs et transparents sont expérimentales. Elles se créent dans des situations où les consommateurs sont conscients des coûts réels de production et sont prêts à les payer. Dans ce cas, ils sont soit conscients du prix réel de leurs aliments, soit intéressés à soutenir ce prix, soit influencés par l'effort de négociation des producteurs.

Les mécanismes de tarification sont plus efficaces lorsque les acteurs comprennent les situations et les contraintes des autres acteurs

\subsection{Producteurs}

\subsubsection{Le défi}

Dans les systèmes alimentaires durables, seuls quelques producteurs parviennent à participer à la fixation des prix, ce qui pose un défi et réduit les marges sur le produit final vendu sur le marché (en raison des déséquilibres de pouvoir mentionnés ci-dessus). La distance qui sépare les agriculteurs des marchés et des négociations de prix jouent un rôle important dans leur capacité de contrôler les prix.

La vente de produits sur les marchés locaux est généralement facile car il y a une discussion verbale du prix avec les consommateurs, favorisant la communication et l'équité. Les marchés éloignés sont plus difficiles, tant en termes d'accès que de transparence, étant donné que des intermédiaires sont souvent nécessaires. Les prix peuvent également être influencés par la saisonnalité puisque celle-ci impacte directement l'offre du marché. Le surplus caractéristique d'une haute saison signifie que les prix du marché ainsi que ceux payés par les consommateurs sont plus bas durant cette période.

Les agriculteurs sont souvent confrontés à un défi important: trouver un compromis entre le volume vendu et le prix reçu. Selon la durée de conservation des produits, de nombreux agriculteurs doivent choisir entre deux options: soit ils attendent que les prix du marché s'améliorent et ils conservent leurs stocks (c.-à.-d. une parution lente des produits), soit ils vendent leur production (souvent la totalité) lorsque les prix sont bas et la demande est élevée. Ce choix est souvent fait à cause d'un manque d'entreposage disponible ou à cause du besoin d'argent comptant pour les dépenses quotidiennes.

\subsubsection{Comment les producteurs peuvent-ils calculer les différents composants d'un prix durable?}

Dans les systèmes alimentaires durables, la fixation des prix doit tenir compte de la transparence et de l'équité, ainsi que des coûts réels qui s'ajoutent aux coûts de production et aux coûts sousjacents tels que les services écosystémiques. Elle doit inclure un bénéfice raisonnable pour égaler 
les prix actuels des systèmes alimentaires conventionnels. Calculer la production et les coûts invisibles n'est pas une tâche simple, car elle exige plusieurs connaissances et compétences. Il existe néanmoins des moyens simples de calculer les prix pour des produits nouveaux ou déjà commercialisés. Les mesures à prendre sont les suivantes:

1. Pendant la phase de production, prenez note des tâches quotidiennes pour documenter le temps consacré, les coûts et les intrants.

\section{ASTUCE 13}

\section{Prendre note des tâches quotidiennes à la ferme}

Tenir des registres peut être un travail long et fastidieux, exigeant une prise de notes détaillées, une énumération précise et des décisions comptables exhaustives. Mais cela n'a pas ainsi ! Il n'y a pas de problème à commencer par de petits pas - il suffit d'écrire exactement ce que vous faites à la ferme. Par exemple, notez:

- Combien de graines avez-vous achetées?

- Combien avez-vous dépensé ?

- Quand avez-vous semé ces graines ?

- Combien d'eau avez-vous utilisé pour irriguer ces plantes?

À la fin de ce processus, vous connaîtrez les montants dépensés pour l'achat de produits agricoles et d'intrants, ainsi que le temps réellement consacré à votre exploitation agricole. Ces notes vous permettront donc de calculer, avec précision, les coûts compris dans la production de vos cultures.

\section{Apprendre aux enfants la comptabilité (Ouganda)}

19 FreshVeggie, un programme Ougandais, pratique également la prise de note avec des jeunes élèves dans leur cours de développement de projet de petite entreprise. Des enfants d'école primaire reçoivent quelques canards et sont chargés de les élever dans le but de les vendre pour financer leur scolarisation. Pour ces enfants, la prise de note est fondamentale dans le processus d'apprentissage et de fixation de prix. En commençant jeune, ils sont préparés aux réalités agricoles futures.

Source: Julie Matovu, FreshVeggie

Déterminez la position de votre produit par rapport à son prix et à sa qualité afin de mieux cibler les marchés et les consommateurs les plus avantageux.

Rappelez-vous la règle d’or: le modèle "prix élevé-qualité médiocre" n’est pas durable pour les consommateurs, tandis que l'approche "prix bas-qualité élevée" n'est pas durable pour les producteurs... c'est à vous de trouver le juste milieu ! 
Evaluez le prix de votre produit en le comparant avec celui d'autres producteurs dans les marchés auxquels vous souhaitez participer. Cela vous permettra de mieux comprendre le prix attendu.

Si vous introduisez un nouveau produit, incluez ses attributs d'originalité et de qualité dans son prix. Pour ce faire, sachez d'abord si les consommateurs sont prêts à l'acheter.

\section{ASTUCE 14}

Comprendre la volonté des consommateurs de payer

a. Pour obtenir des retours honnêtes sur la qualité et pour identifier les prix que les consommateurs sont prêts à payer, présentez une gamme de produits concurrentiels y compris les vôtres, à un petit groupe de consommateurs potentiels. En incluant aussi des renseignements sur les prix indiqués, ils peuvent alors déterminer et justifier leur premier choix. Si nécessaire, vous pouvez ensuite ajuster votre positionnement, vos prix, ou votre emballage.

b. N'hésitez pas à utiliser les marchés directs - permettez aux clients de négocier le prix à condition que vous ayez une marge de manœuvre suffisante. Vous verrez une tendance tant qu'aux prix que les consommateurs proposent et sur lesquels ils s'entendent. Une bonne affaire est atteinte lorsque le consommateur veut acheter une plus grande quantité du produit à un prix unitaire plus bas, aidant ainsi le producteur à liquider ses stocks.

\section{Si le produit est transformé, les coûts de transformation doivent être inclus.}

\section{ASTUCE 15}

\section{La fixation des prix de produits transformés repose sur un calcul mixte}

Lorsque vous vendez un produit transformé, tenez compte des éléments suivants:

- Combinez plusieurs méthodes de tarification pour déterminer les prix en fonction des coûts, des avantages de l'innovation, de la saturation du marché et de la volonté du consommateur de payer.

- Cette combinaison devrait être définie localement selon les conditions de chaque marché, et de l'expérience de l'entrepreneur ou du groupe d'entrepreneurs qui dirige le processus.

- Évaluez vos possibilités de transformation et les coûts associés. Essayez de trouver des entreprises qui peuvent à la fois transformer et expliquer tous les coûts associés. Cela vous aidera à déterminer un prix équitable, car vous n'assumerez pas tous les coûts liés à l'achat et à l'entretien de l'équipement.

- Testez le prix de votre produit sur le marché pour déterminer celui qui convient.

- N'oubliez pas que le prix est un accord entre acheteurs et vendeurs qui peut être modifié à tout moment lorsque de nouvelles informations sont disponibles. Par conséquent, la révision des prix devrait être effectuée périodiquement ou lorsqu'un événement particulier se produit. 


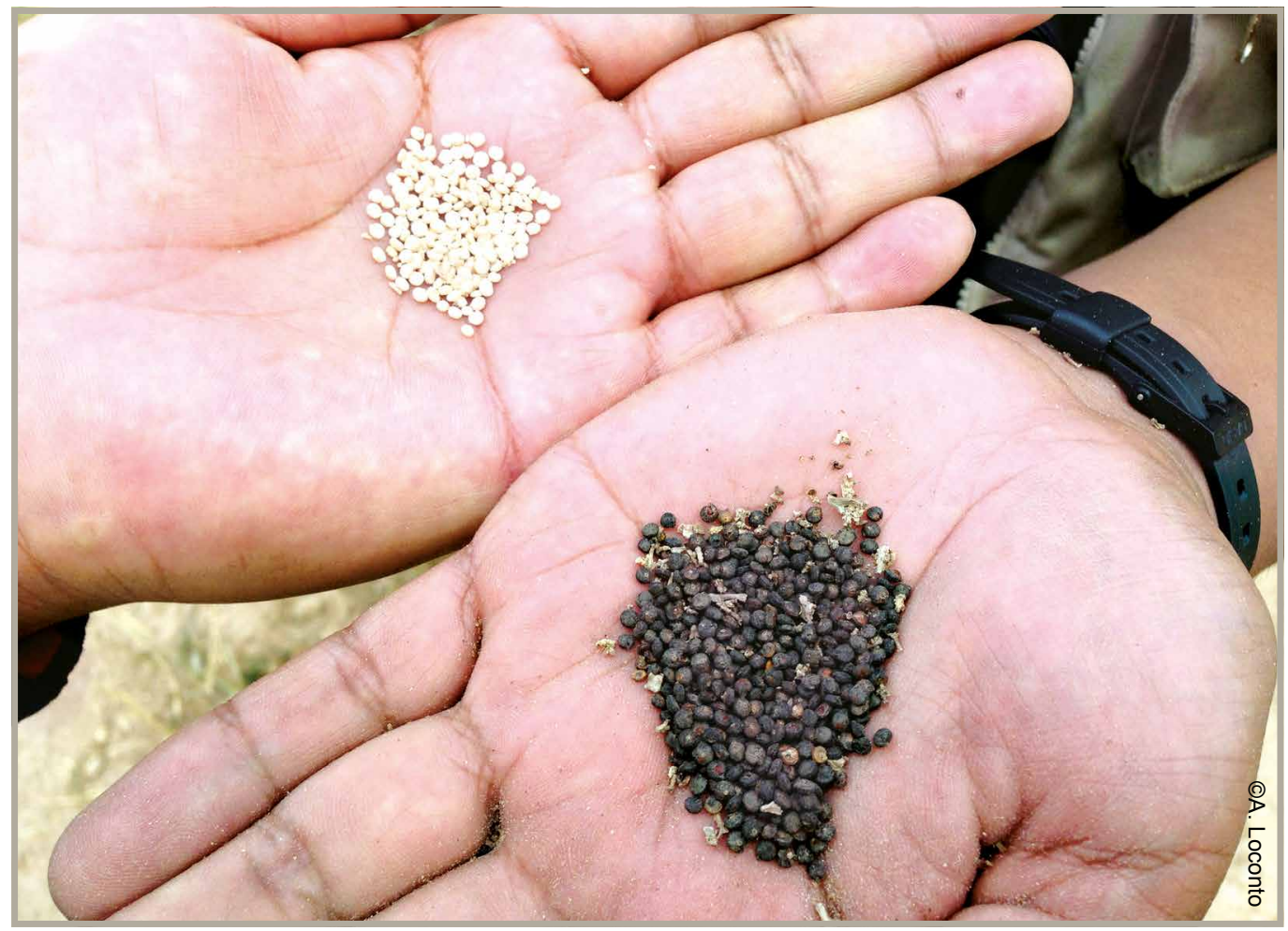

Nouez des partenariats avec des chercheurs et des fournisseurs de services qui vous aideront à comprendre les coûts cachés de vos produits, pour ensuite les inclure dans votre calcul de prix.

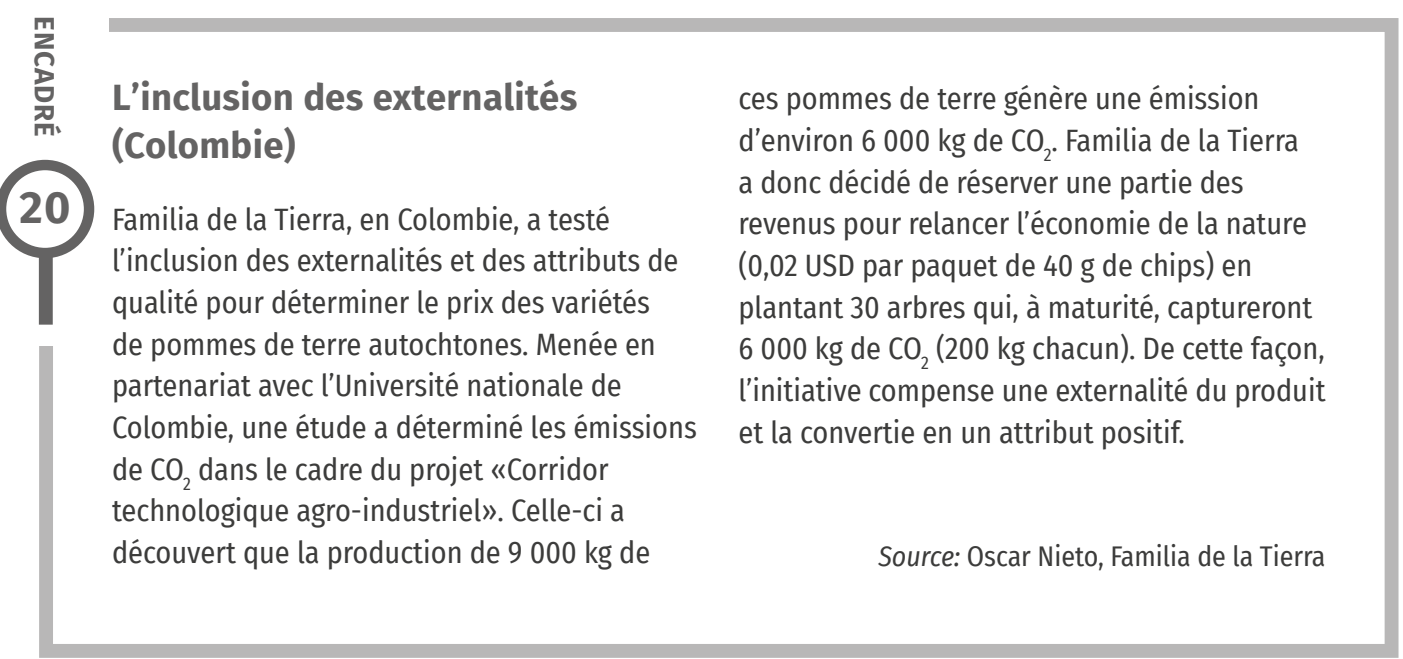


6 Une fois que les composantes du prix ont été identifiées et comprises, les producteurs doivent disposer de l'information nécessaire pour négocier un prix équitablement durable avec les autres acteurs. N'oubliez pas qu'un producteur seul n'est souvent pas en mesure de quantifier la volonté des consommateurs de payer les coûts de transformation ou les externalités. Des intermédiaires transparents ou des organisations de producteurs peuvent aider les producteurs à mener ces activités, et ce travail doit être considéré dans le prix.

\section{Trois critères pour définir quand} une transaction est acceptable

\section{1} pour les producteurs (Inde)

Des critères minimaux et communs facilitent les transactions consommateur-producteur, en particulier pour les petits exploitants. La prise en compte de ces critères doit se dérouler au moment de délimiter les paramètres de transaction dans le système alimentaire durable. Pour les petits exploitants en Inde, et peut-être dans le reste du monde, trois critères principaux s'appliquent:

a. Le producteur parvient à vendre tous les produits cultivés au cours d'une saison;

b. Le producteur reçoit le prix exact de la production d'une manière qui satisfait son coût d'intrant et son profit;

\section{c. Le producteur reçoit l'argent de la transaction en temps voulu.}

Ces critères sont de nature transactionnelle et ont pour but de satisfaire la parité d'échange de produits contre de l'argent ou un autre type de troc. Un scénario idéal exige que les trois critères ci-dessus soient satisfaits pour que le producteur puisse considérer le prix comme étant équitable. Toutefois, compte tenu des conditions du marché, deux de ces trois critères sont souvent acceptables pour la plupart des producteurs. Dans le cas contraire, les petits exploitants deviennent plus vulnérables, car ils sont peu protégés face aux chocs du marché et des prix. Tout espace de négociation doit tenir compte de ces critères simples tout en communiquant avec les petits exploitants.

Source: Ashish Gupta, Jaivik Haat

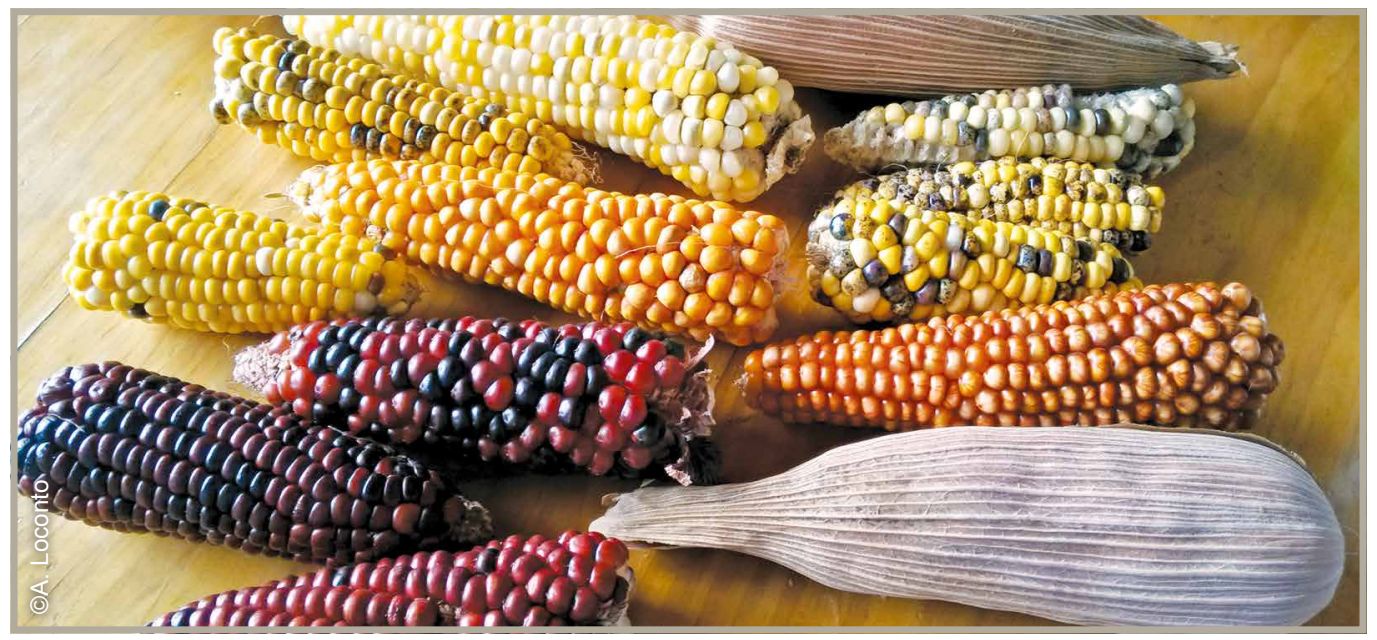


Enfin et surtout, une fois qu'un prix durable a été négocié avec les autres acteurs du système, les producteurs doivent essayer de stabiliser leurs revenus autant que possible afin de cultiver la confiance des consommateurs.

\section{ASTUCE 16}

\section{Stabiliser les revenus des producteurs grâce à la diversification}

a. Innovation permanente: L'innovation pour le développement de produits permet au producteur de maintenir et d'améliorer ses revenus à travers les marchés locaux, régionaux et, dans certains cas, mondiaux. Cette innovation répond à des questions telles que: Qui est mon consommateur cible ? Quelles sont les caractéristiques de mon produit ? Que pourrait-il inclure en plus ? Quelles alternatives peuvent être offertes?

b. Diversification des cultures: La diversification des aliments cultivés permet à l'agriculteur et au réseau d'agriculteurs de maintenir leur niveau de revenu par le biais d'un profit moyen. Lorsqu'un produit baisse de prix sur le marché, un autre produit peut avoir une meilleure valeur de revenu et «équilibrer» le profit du producteur.

c. Diversification des produits agricoles selon leur présentation: Un emballage innovant et durable permet aux fermes individuelles ou aux réseaux de fermes de développer un symbole commun ou une marque stratégique dans le marché. Cet emballage unique peut souvent signifier un meilleur prix, soit un revenu et un profit plus élevés pour le producteur.

d. Diversification des produits en fonction de leur état de maturation dans le cycle végétatif: Les possibilités de commercialisation des produits frais ne se limitent pas seulement à leur dimension spatiale, c'est-àdire au nombre de plantes dont les variétés sont plantées. Elles peuvent également être réparties dans le temps et dans la maturation de leur cycle de croissance: germination, croissance, floraison, fructification et production des semences. Selon le calendrier du cycle végétatif, il est possible de récolter une version «bébé», une version normale (standard commercial), des fleurs comestibles pour la cuisine gastronomique, ainsi que des semences de la même plante pour distribuer localement et régionalement.

e. Segmentation des prix et du marché: La fixation des prix différents pour différents groupes de consommateurs, se délimite en fonction de la volonté (et de la capacité) des consommateurs à payer. En suivant cette stratégie, le meilleur prix possible de chaque niche ou marché sera obtenu. Si vos consommateurs et vos listes de prix sont bien définis, votre revenu sera plus stable à moyen terme.

\subsection{Consommateurs}

\subsubsection{Le défi}

Les consommateurs, tout comme les producteurs, sont un élément clé d'un système alimentaire durable. Malgré les défis posés par la mise en place de nouveaux systèmes d'approvisionnement et les prix initiaux élevés, les consommateurs sont souvent favorables à ces initiatives. Ce soutien s'appuie fondamentalement sur le désir d'avoir une famille en bonne santé, ainsi que de préserver le bien-être général de l'environnement et des agriculteurs. Aujourd'hui, la disponibilité de produits de qualité et de prix équitables pose un défi principal pour les consommateurs. Comme les coûts des produits fabriqués de façon éthique et durable sont parfois plus élevés, ceux-ci peuvent retomber sur les épaules des consommateurs. 
Les consommateurs parfois prennent part dans l'établissement des prix, comme dans les comités de fixation des prix. Cependant, mise à part les AMAP, les consommateurs sont souvent exclus des discussions. Le défi consiste à créer un système d'approvisionnement transparent dans lequel les producteurs et les consommateurs comprennent la valeur des produits et ont le droit de la questionner. N'oubliez pas: une relation amicale entre les producteurs et les consommateurs fait partie intégrante des systèmes alimentaires durables.

\section{ASTUCE 17}

Aider les consommateurs à connaître le «bon» prix

a. La transparence et la confiance sont essentielles. Informez vos consommateurs et partagez votre histoire avec eux. Chaque fois que l'occasion se présente, y compris pendant les livraisons et les paiements, partagez davantage ce que vous faites en tant que groupe de producteurs, expliquez pourquoi vous êtes uniques et comment vous vous distinguez des autres.

b. Créez des activités qui favorisent l'interaction entre les consommateurs et les producteurs, comme des visites de fin de semaine où les consommateurs peuvent passer du temps à la ferme. Encouragez-les à amener leurs enfants pour les faire participer aux activités de remplissage des paniers, à rencontrer les producteurs qui fabriquent leurs produits préférés et à comprendre les attributs qui les différencient des produits traditionnels. C'est l'histoire complète des prix fixés.

\subsubsection{Comment les consommateurs peuvent-ils connaître le "bon" prix?}

Connaître le juste prix et la saisonnalité des produits agricoles est essentiel pour maintenir et construire une base de marché dans un système alimentaire durable. Les consommateurs, tout comme les producteurs, doivent participer à la fixation des prix.

Pour permettre aux consommateurs de comprendre et de participer à l'établissement d'un prix équitable, différentes stratégies peuvent être adoptées:

a. Invitez les consommateurs à la ferme (ou à l'usine de transformation) pour qu'ils puissent observer directement la cultivation et la qualité de vos produits;

b. Créez des comités d'établissement des prix pour négocier un prix équitable avec les consommateurs. Cette étape est spécialement utile si vous disposez d'un intermédiaire transparent entre les producteurs et les consommateurs;

c. Communiquez l'information sur les qualités du produit dans un langage compréhensible. Certains consommateurs préfèrent lire les étiquettes et reconnaître les marques, tandis que d'autres aiment en discuter avec le producteur, les commerçants ou leurs amis;

d. Enseignez aux consommateurs comment produire leurs propres aliments afin qu'ils comprennent les coûts de production;

e. Encouragez les consommateurs à questionner les prix à la source du produit et avec les intermédiaires. Incitez ces derniers à être transparents quant aux coûts et risques inhérents à la mise en disposition de produits au nom des producteurs.

f. Informez les consommateurs des prix et des changements de prix. 
艿

\section{2}

Afin de répondre à la demande croissante des consommateurs pour des produits agricoles de haute qualité et le défi de leur faire comprendre les des coûts de production, les membres du SPG FreshVeggie, avec l'appui d'un consultant privé, ont conçu une trousse de jardinage à domicile pour les consommateurs locaux. Pour environ 57 USD, le forfait de base comprend une variété de semis de légumes (assez pour produire environ $100 \mathrm{~kg}$ de légumes assortis en 90 jours) et des conseils techniques de l'équipe FreshVeggie sur place. Les consommateurs peuvent donc acheter ce forfait et faire venir un expert pour établir un jardin dans leur propre cour. De cette façon, avec des succès et des échecs dans la croissance de leurs légumes, les consommateurs apprennent et apprécient les coûts réels des systèmes alimentaires durables. Source: Julie Matovu, FreshVeggie

\section{Juls consults}

l'Agroentrepreneurait et le developpement durable communautaire

Nous sommes là pour répondre aux besoins de nos clients en leur proposant des solutions pratiques à leurs problèmes de planification et d'établissement des petites exploitations agricoles. Nous renforçons les capacités des organisations communautaires en matière de développement durable et nous offrons une service de conseil agricole.

Nous encourageons le développement durable des communautés par le biais de services de l'agroentrepreneurait biologique. Nos services comprennent une formation pratique à la production végétale et animale (volaille et petits ruminants). Notre approche se base sur la promotion de la nourriture saine pour la consommation familiale et commerciale: des cultures saines dans le potager à la nourriture saine dans l'assiette.

Notre paquet technique potager basique:

- Un assortiment de 160 - 220 plants et de semences à semis direct des legumes divers avec les besoins nutritionnels de votre famille à cœur ${ }^{1}$;

- 30 plants d'épinards

- 30 plants de brocoli

- 30 plants de choux vert/blanc/rouge

- 10 plants de tomate

- 30 plants d'ognion [printemps/poireaux/créole rouge]

- 30 plants de laitue (pour les salades)

- 30 carottes

-30 betterave

- $5 g$ des herbes aromatiques mélangées

- $10 \mathrm{~g}$ des semences ddodo/jjobyo/Nakati

- 2 visites pratiques à domicile: une consultation de

planification et une activité d'aménagement des parcelles

- Un plateau de semences

- Un guide de la culture biologique potager

- Accompagnement gratuit (à distance) pendant la saison pour les cultures établies dans l'exploitation.

1 La récolte moyenne attendue est de 90 à $100 \mathrm{~kg}$ de légumes frais par saison.

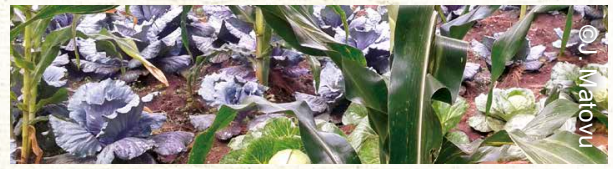

Coût du paquet technique: UGX200,000 = (seulement deux cent mille shillings) qui inclut les frais

d'expertise et de transport pour les deux visites à la ferme. Un acompte initial de $50 \%$ est demandé lors de la réseervation, qui doit être confirmé au moins trois semaines avant l'établissement de l'exploitation.

Juls Consults est associé à la société Freshveggies PGS².

\section{DES SUPPLÉMENTS PERSONNALISÉS:}

Nous pouvons élaborer des suppléments sur mesure pour répondre à vos besoins spécifiques. Par exemple: - Sacs d'empotage

- Engrais organiques équilibrés certifiés

- Livraison de sacs en pots prêts à l'emploi contenant de la terre stérilisée et/ou traitée et des engrais organiques [intrants, main-d'œuvre, transport].

- Intrants organiques supplémentaires

- Kit d'irrigation goutte à goutte

Nos coordonnées:

Ggombe B, Bukasa Parish, Wakiso District.

P.O.BOX 11133, Kampala.

Email: matovujuls@gmail.com

Appellez: Julie $0701636688 / 0772636688$

or George 07560473377

2 Freshveggies PGS est un réseau informel de petits producteurs biologiques - composé principalement par des femmes - dans les zones périurbaines de Kampala, Ouganda. L'initiative vise à assurer une production agricole durable chez les petits producteurs afin d'augmenter les revenus des ménages et de proposer des aliments sains aux consommateurs locaux. Il s'agit d'une innovation marchande qui rassemble les agriculteurs avec des consommateurs locaux afin d'échanger des connaissances et d'améliorer leurs compétences en matière d'agriculture biologique. Les systèmes participatifs de garantie (SPG) sont des systèmes de contrôle de la qualité adaptés au contexte local. Le FreshVeggies SPG s'appui sur la participation active de ses membres pour certifier les producteurs. Ce système est fondé sur la confiance, les réseaux sociaux et l'échange de connaissances. 


\section{Les études de marché comme} moyen de partager l'information

\section{3 (Ouganda)}

Les membres du SPG FreshVeggie réalisent des mini études de marché auprès des clients. Il s'agit de formulaires simples et faciles à remplir qui sont envoyés par courriel ou dans le panier de livraison au début de chaque année. Sur ce formulaire, les prix ou tout changement de prix sont annoncés, des nouveaux produits sont introduits, et les clients ont la possibilité de suggérer de nouveaux produits. Cela favorise un renseignement personnel des prix dans un délai convenable.

Source: Julie, Matovu, FreshVeggie

\subsection{Intermédiaires transparents}

\subsubsection{Le défi}

Alors que de nombreux systèmes alimentaires durables tentent d'éliminer les intermédiaires par la vente directe et les chaînes d'approvisionnement courtes, ils reviennent souvent en raison d'un manque de compétences. Ces intermédiaires, en plus de faciliter les échanges, ils investissent dans l'entreposage et le transport - des activités que les producteurs et les consommateurs ne peuvent pas gérer ou financer.

Le plus grand défi pour les intermédiaires consiste à répondre aux besoins des marchés, tant pour le producteur que pour le consommateur, même si ces deux besoins ne correspondent pas. Les intermédiaires transparents sont généralement conscients des problèmes de l'empreinte carbone, de la perte de biodiversité autochtone en raison du soutien des variétés de cultures hybrides et d'autres thématiques liées à la durabilité. Ainsi, un système alimentaire durable exige des intermédiaires transparents qui aident les agriculteurs à mettre en place leurs produits sur les marchés tout en répondant aux préoccupations des consommateurs (par exemple, la durabilité et la mise en place de chaînes d'approvisionnement courtes). Il existe différents modèles d'intermédiaires transparents selon le type de services offerts aux producteurs et aux consommateurs. En plus des fonctions de logistique et d'agrégation, les intermédiaires fournissent un large éventail de services pour encourager l'échange de connaissances et l'action collective. 
塄

\section{Trois modèles différents d'intermédiaires transparents (Corée du Sud et Inde)}

Nous pouvons mettre en évidence trois modèles d'intermédiaire transparent en fonction de la taille de leur base de consommateurs et des services offerts.

1. Modèle de grande taille: Coopérative Hansalim, Corée du Sud

La Coopérative Hansalim crée un accès transparent entre producteurs et consommateurs en leur offrant une équité organisationnelle afin qu'ils puissent participer activement aux diverses activités de la coopérative, y compris la fixation des prix. Le slogan de Hansalim est «Hansalim va dans le sens des producteurs», l'objectif étant que le producteur puisse maintenir sa production en toute sécurité et en tout confort. Les employés de la coopérative, les consommateurs et les producteurs tiennent des assemblées annuelles pour déterminer les prix avant la prochaine saison. En cas de forte volatilité des prix sur les marchés, ce qui est spécialement le cas pour les

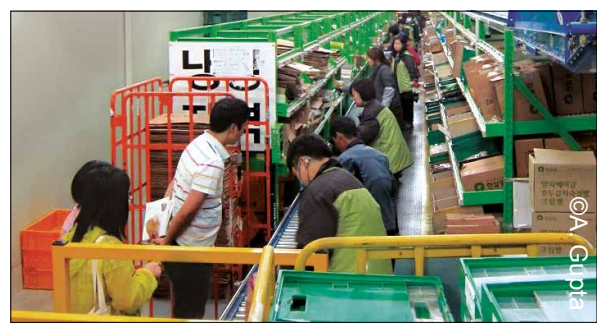

marchandises importées conventionnelles, la coopérative a accès à des fonds renouvelables de sécurité pour la production et la stabilisation des prix. Toutefois, le prix des produits de Hansalim reste relativement stable. Près de 75 pour cent du prix final du marché est payé aux producteurs dans ce modèle. En 2016, le chiffre d'affaires global de Hansalim était de 362 millions d'USD.

Source: Hansalim Rapport annuel 2016

\section{Modèle de taille moyenne: Coopérative Dharani FaM, Inde}

La coopérative aide les agriculteurs bio du SPG à regrouper et commercialiser leurs produits sous la marque «Timbaktu Organic». Ses membres reçoivent des directives de pesage pour leurs produits et une prime de prix de 25 à 30 pour cent relatif aux prix actuels du marché. De plus, les producteurs bénéficient d'une rémunération forfaitaire annuelle de 3 à 7 pour cent. Ces prix sont généralement fixés en accord avec les agriculteurs avant le début de la saison. Malgré ces marges, le prix global des produits alimentaires durables est inférieur d'environ 20 pour cent à celui des produits de grandes marques sur le marché. Les agriculteurs ont un accès transparent aux

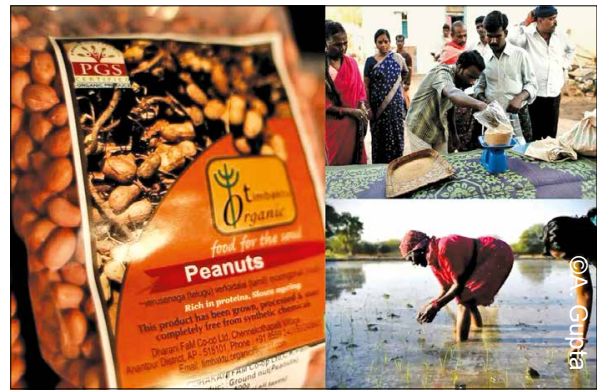

mécanismes d'approvisionnement, de fixation des prix et de commercialisation en tout temps. En 2016, le chiffre d'affaires global de Timbaktu Collective était de 31000 USD.

Source: Timbaktu Rapport annuel collectif 2016 
3. Modèle de petite taille: Jaivik Haat, Inde

Jaivik Haat est une entreprise sociale privée qui approvisionne des aliments biologiques et naturels au détail à Delhi, en Inde. Cette entreprise offre des opportunités de vente au détail aux petits agriculteurs et aux agriculteurs marginaux qui pratiquent l'agroécologie et en particulier aux agriculteurs certifiés SPG. Les producteurs ont un accès transparent au marché et ont un retour de 60 à 70 pour cent du prix final à la consommation. Les marges opérationnelles représentent environ 35 pour cent du prix final du marché. Pour fixer les prix, Jaivik Haat a deux méthodes. Dans la première, les producteurs (ou leurs groupes) fixent le prix en tenant compte du coût de leurs intrants, et Jaivik Haat rajoute ensuite ses marges pour gérer la chaîne d'approvisionnement. Dans la seconde, si les agriculteurs ne peuvent pas fixer les prix en raison d'une faible confiance des consommateurs, Jaivik Haat s'empare de leurs produits et tente de les vendre au meilleur prix possible dans les marchés où l'échange de l'argent pour le produit est immédiat. Après la vente, 60 à 70 pour cent des prix moyens finaux sont retournés aux producteurs. Dans ces

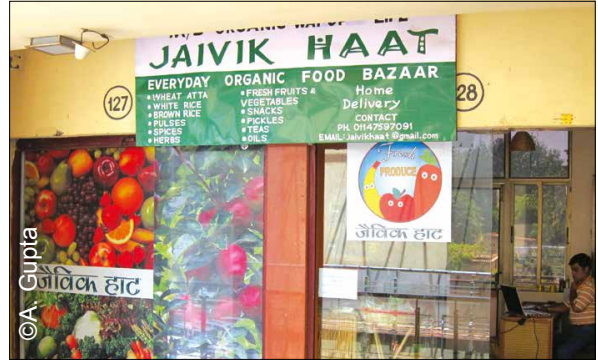

deux modèles, les retours d'information des consommateurs jouent un rôle indirect dans la détermination des prix. Dans le premier cas, si le producteur fixe des prix trop élevés, le volume de vente n'est pas atteint. Ce retour d'information permet donc de baisser le prix de production pour satisfaire un volume de vente minimum. Ainsi, l'apprentissage par la pratique du marché aide à déterminer les prix. Dans le second cas, Jaivik Haat négocie le meilleur prix possible sur le marché en fonction des coûts d'opportunité. Tant le marché que les consommateurs aident à négocier un coût réel basé sur des transactions de personne à personne et non sur des estimations fixées par des tiers.

Source: Ashish Gupta, Jaivik Haat

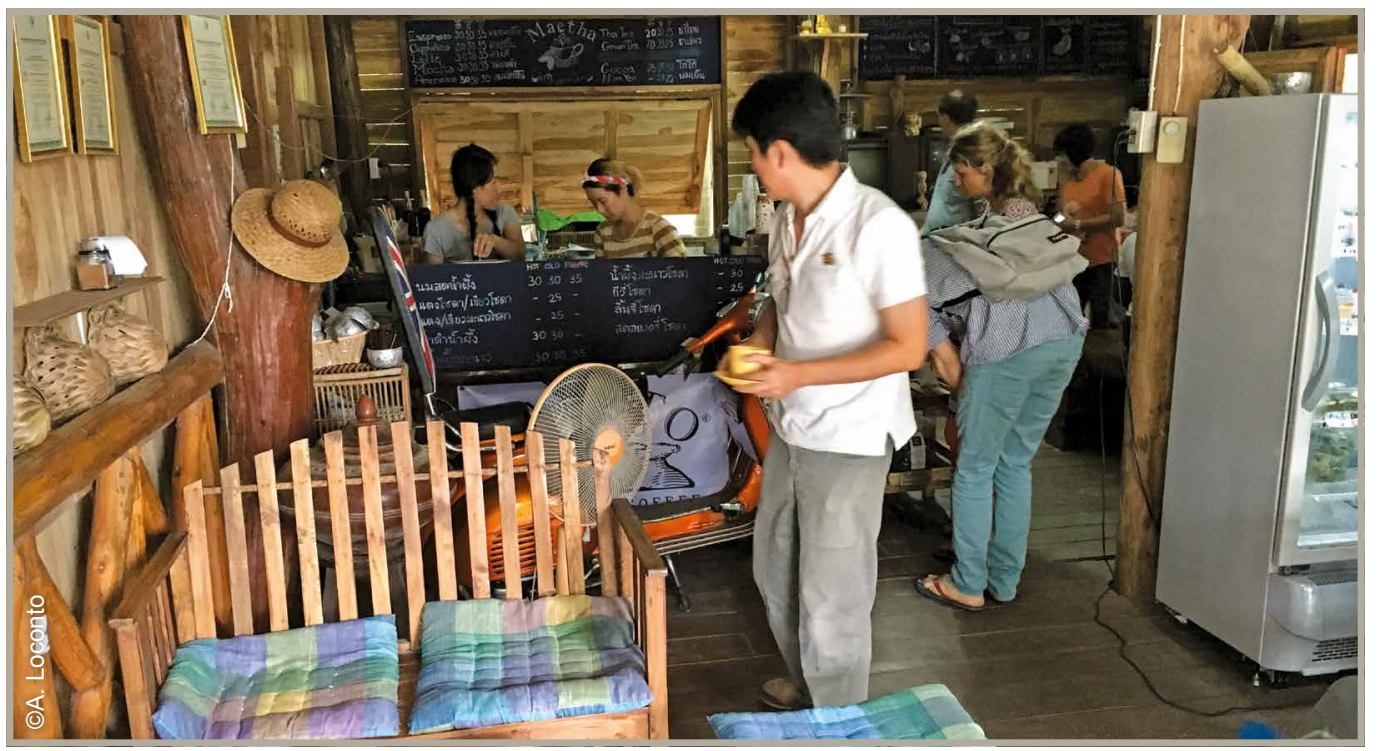




\subsubsection{Comment des intermédiaires transparents peuvent-ils faciliter la négociation entre les autres acteurs du système?}

Étant donné que des intermédiaires transparents sont souvent nécessaires pour relier les producteurs et les consommateurs, ils doivent fixer des prix satisfaisants pour tous les acteurs dans la chaîne de valeur.

D'abord, ils doivent bien connaitre la structure de coûts des producteurs ainsi que les prix fixés par les concurrents sur leur marché (ou même les prix projetés des marchés futurs).

Ensuite, afin de fixer des prix durables, ils peuvent faciliter la négociation entre producteurs et consommateurs (ou autres acteurs du système alimentaire) au moyen de diverses méthodes:

1. Comités de fixation des prix

\section{Les comités et les mécanismes de fixation des prix au sein de l'AMAP}

25 L'établissement de prix justes et équitables est au cœur d'une initiative d'AMAP. Bien que la tarification puisse varier en fonction de l'adhésion à un groupe, du lieu géographique ou des fondements philosophiques, trois exemples stratégiques de divers membres de I'URGENCI démontrent quelques possibilités:

a. Coûts réels de la fixation des prix agricoles: Évaluez le budget agricole de l'AMAP pour la saison de production. Incluez tous les coûts de production (c.-à-d. semences, outils et intrants), les coûts de main-d'œuvre, les coûts d'amortissement des machines, etc. Divisez ce coût total par le nombre de membres de l'AMAP (par exemple, si le coût total pour la saison de production est de 1000 USD et que vous avez 20 membres, chaque membre payera 50 USD par action). De ce fait, le prix de chaque action spécifique est fixé.

b. Contributions volontaires: Dans ce modèle de tarification, les prix sont déterminés directement par les membres du groupe. L'agriculteur évalue le budget de l'AMAP et le présente aux membres. Ensuite, les membres notent secrètement le montant que chacun peut payer pour la saison et l'agriculteur additionne tous ces montants. Si la somme respecte le budget, les prix sont fixés. Si elle ne le respecte pas, les membres proposent des prix à nouveau, jusqu'à ce que le budget soit satisfait.

c. Le prix en fonction de la valeur marchande: La troisième méthode - peut-être la plus courante - d'établir un prix se base sur les prix du marché agricole. Informezvous du prix que les consommateurs sont prêts à payer pour des programmes de paniers à légumes et ciblez ce prix. Ensuite, délimitez la valeur du panier en fonction du prix des articles individuels, sur les marchés de producteurs locaux ou dans des chaînes de vente directe similaires ou d'approvisionnement courtes, jusqu'à ce que le prix cible soit atteint. Par exemple, vous avez déterminé que les consommateurs sont prêts à payer jusqu'à 15 USD pour une livraison hebdomadaire; vous pouvez calculer ensuite le prix du panier en fonction des prix du marché.

Source: 2016 Faites partie du livret de l'AMAP 
2 Étude de marché participative

\section{Une étude de marché participative (EMP) (Ouganda)}

Dans le cadre de son programme MAIL (Market access improved livelihoods), le SPG FreshVeggie, basé en Ouganda, relie directement les producteurs et les consommateurs dans leur programme d'études de marché participatives (EMP) ciblées, ce qui conduit à des résultats concrets dans la modélisation des prix. Les membres du SPG FreshVeggie interrogent les consommateurs potentiels dans les marchés locaux, à leur domicile et sur leur lieu de travail. Ils recueillent aussi des informations sur les produits «préférés» et sollicitent des futurs acheteurs de ces produits. Selon le nombre de participants à SPG FreshVeggie et les distances à parcourir pour évaluer les consommateurs potentiels, l'EMP dure entre deux et cinq jours.

- Premier jour: Présenter aux participants le processus d'EMP, développer des questions pour l'EMP, répondre aux questions sur le processus.
- Jour 2 et 3: Exécuter l'EMP sur place dans les marchés, les résidences et les lieux de travail locaux; recueillir et noter l'information.

- Jour 4 et 5: Collecter et synthétiser l'information recueillie, organiser un exercice d'évaluation et de partage d'opinions avec les clients éventuels, discuter les prix et conclure les ententes d'achat.

De cette façon, les producteurs peuvent établir des relations directes avec les consommateurs potentiels et, ce faisant, discuter concrètement les coûts associés aux produits agricoles de haute qualité. De leur côté, les consommateurs se sont familiarisés avec l'intensité de main d'œuvre requise dans un projet agricole. Des lors, ils ont souvent plus tendance à soutenir les besoins financiers de ces producteurs.

Source: Julie Matovu, SPG FreshVeggie, Ouganda

Figure 3: Exemple d'un modèle des résultats d'une étude de marché participative (EMP) sur les SPG

\begin{tabular}{|c|c|c|c|c|c|c|c|}
\hline \multicolumn{8}{|c|}{$\begin{array}{l}\text { Résumé de l'information générée } \\
\text { l'activité PMR. }\end{array}$} \\
\hline \multicolumn{8}{|c|}{ Produit: p. ex. tomates biologiques } \\
\hline $\begin{array}{l}\text { Emplacement } \\
\text { de la } \\
\text { propriété/ } \\
\text { lieu de travail } \\
\text { /point de } \\
\text { vente du } \\
\text { marché }\end{array}$ & $\begin{array}{l}\text { Qualité/ } \\
\text { Type/ } \\
\text { Taille } \\
\text { souhaitée }\end{array}$ & $\begin{array}{l}\text { Quantité } \\
\text { requise }\end{array}$ & $\begin{array}{l}\text { Fréquence } \\
\text { de } \\
\text { livraison } \\
\text { et } \\
\text { emballage } \\
\text { spécial } \\
\text { requis }\end{array}$ & $\begin{array}{l}\text { Autres } \\
\text { sources } \\
\text { connues } \\
\text { de } \\
\text { produit } \\
\text { requis }\end{array}$ & $\begin{array}{l}\text { Prix/ } \\
\text { Fourchette } \\
\text { de prix }\end{array}$ & $\begin{array}{l}\text { Conditions } \\
\text { de } \\
\text { paiement }\end{array}$ & $\begin{array}{l}\text { Le } \\
\text { répondant } \\
\text { accepte les } \\
\text { ventes de } \\
\text { SPG } \\
\text { (Oui/Non) }\end{array}$ \\
\hline & & & & & & & \\
\hline & & & & & & & \\
\hline & & & & & & & \\
\hline
\end{tabular}




\section{Le taux de partage des prix dans le secteur du thé (Tanzanie)}

En Tanzanie, le thé est produit à la fois par les grands domaines et par les petits paysans. Le secteur immobilier possède la majorité des usines de transformation, tandis que les petits exploitants sont traités comme des agriculteurs sous-traitant (sous contrat) qui ne possèdent que leurs petits jardins familiaux. Après un long débat sur la structure de prix pour les agriculteurs sous-traitants, il a été décidé d'utiliser le coût de production tout au long de la chaîne d'approvisionnement pour partager les portions (sur la base de la théorie économique de la productivité marginale). Elle analyse le profit en maximisant la quantité d'intrants (c'est-à-dire les services en fonction de la production) achetés par une entreprise dans la production des extrants.

Le modèle du coût de production (CDP) est une approche équitable et viable à utiliser dans le secteur du thé tanzanien. Fondamentalement, tous les coûts de production pertinents sont utilisés pour déterminer le taux de partage du prix du marché du thé vendu. La méthode est résumée dans la figure ci-dessous: il s'agit d'une formule simple qui combine le coût de production des petits producteurs et des usines pour obtenir le coût total de la production, de la transformation et de la vente du thé. Ce modèle est envisageable du côté de l'entrée (les feuilles vertes) ou celui de la sortie (le thé noir). Toutefois, pour avoir un calcul commun, les mesures des feuilles vertes sont utilisées.

Basé sur les résultats de cette formule, un accord est conclu sur le partage entre les cultivateurs sous-traitants et les transformateurs à partir des prix finaux réalisés aux enchères. Ces prix incluent tout bénéfice réalisé de sorte qu'il soit également partagé entre les producteurs et les transformateurs. Comme les prix finaux du thé sont fortement déterminés par les attributs de qualité, cette formule et cette approche obligent tous les acteurs de la chaîne de valeur à travailler fort pour s'assurer que le produit final envoyé aux enchères atteigne le plus haut niveau de qualité possible et donc le meilleur prix.

Source: Institut tanzanien de recherche sur le thé (TRIT), 2017

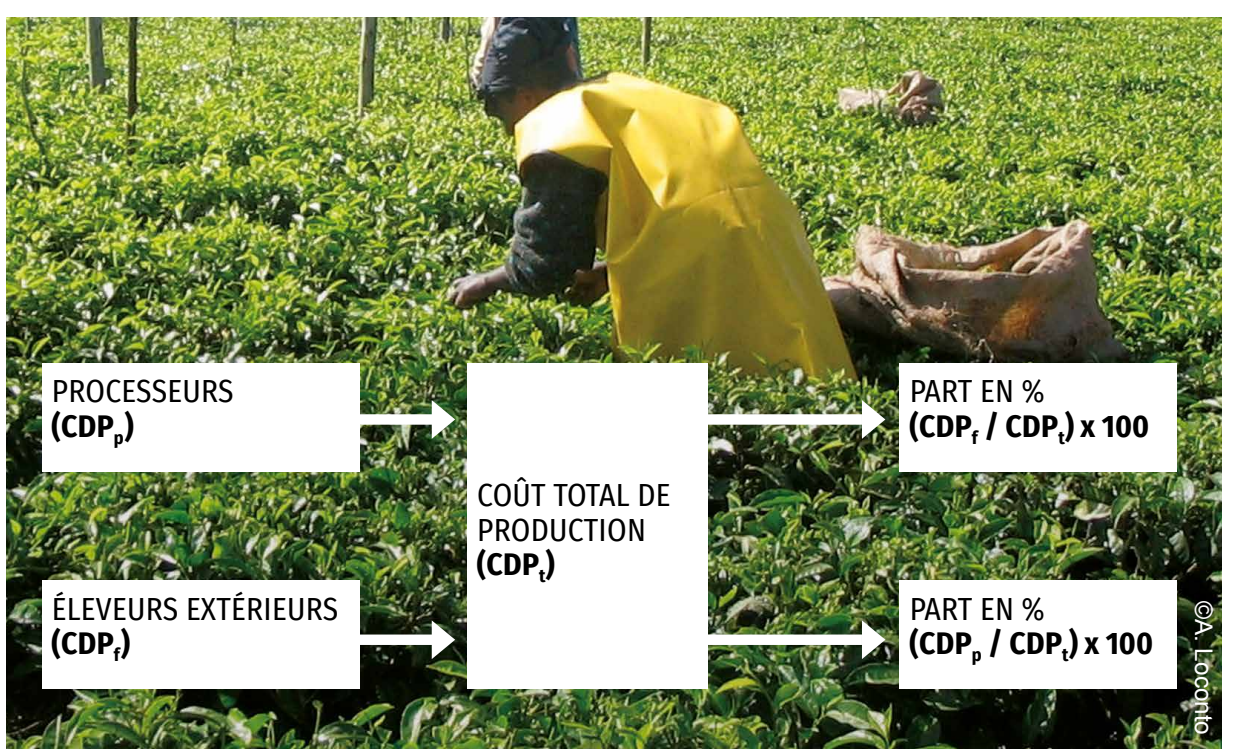




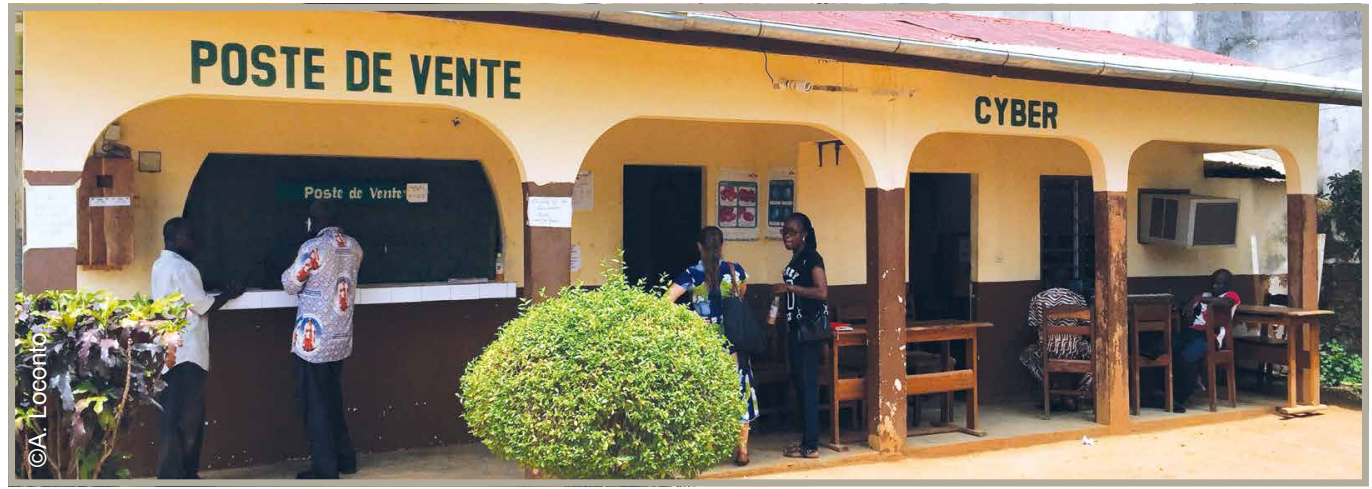

\subsubsection{Comment les intermédiaires transparents peuvent-ils fixer une marge équitable pour leurs activités?}

Les intermédiaires transparents doivent établir des prix de marché qui tiennent compte à la fois des besoins des producteurs et des consommateurs. Dans l'ensemble, la rémunération raisonnable d'un intermédiaire transparent est d'environ 30 pour cent du prix de vente, ce qui lui permet de vivre décemment et d'assurer les fonds nécessaires pour ses fonctions de base, notamment la logistique et l'agrégation. En même temps, le producteur retient 70 pour cent du prix de vente. Ainsi, les agriculteurs bénéficient d'une part équitable des prix du marché et les consommateurs reçoivent des informations transparentes sur les coûts immanents de la chaîne d'approvisionnement.

\section{Le modèle de Shared Harvest Farm pour calculer le juste prix (Chine)}

1. CALCULEZ LE COÛT (INTRANTS, TERRAIN, ETC.) + MAIN D'GUVRE + SALAIRES.
2.

COMPAREZ AVEC LES PRIX DU MARCHÉ BIOLOGIQUE POUR CALCULER LE PRIX DE VOS PRODUITS.
3.

CONSIDÉREZ QUE 15 À 30 POUR CENT DES COÛTS DE PRODUCTION SONT CONSACRÉS À L'EXPLOITATION ET AUX FRAIS GÉNÉRAUX.
4.

L'AGRICULTEUR OBTIENT DONC 70-85 POUR CENT DU PRIX FINAL DU MARCHÉ.

\subsection{Décideurs politiques}

Il est difficile de déterminer qui devrait absorber le coût réel de la production alimentaire durable. Plutôt que les consommateurs aient à payer pour les produits finaux, certains proposent que les gouvernements et les décideurs politiques paient pour les services écosystémiques. Au-delà de ces types de transactions qui sont hors-marché, les décideurs peuvent influencer la fixation des prix en adoptant deux stratégies distinctes: 
1 Les décideurs peuvent jouer le rôle d'intermédiaires transparents et faciliter le flux d'informations sur les prix, tant pour les producteurs que pour les consommateurs. Dans certains cas, lorsque les négociations entre les acteurs du système alimentaire sont particulièrement difficiles ou lorsque les producteurs sont vulnérables, les décideurs peuvent également fixer des prix minimaux.

\section{Le gouvernement en tant} qu'intermédiaire transparent (India)

En Inde, l'approvisionnement en produits conventionnels dans les marchés à plein-vent est un exemple de fonction «d'intermédiaire transparent». Les prix d'un certain nombre de produits de base sont déterminés à l'avance en tant que «prix de soutien minimal» (PSM) par un organisme gouvernemental, la Commission des coûts et des prix agricoles (CACP) (http:// cacp.dacnet.nic.in/). La CACP dispose d'un modèle pour calculer les dépenses des agriculteurs. Ceux-ci prennent en compte les coûts d'environ 27 cultures dont le paddy, le blé, les légumineuses et les cultures commerciales comme le jute, la canne à sucre, etc. Le gouvernement, fonctionnant comme un acheteur direct des produits d'agriculteurs, a pour objectif de les soutenir en contrôlant les prix. En cas de volatilité des prix sur le marché, le gouvernement accorde aussi des primes périodiques sur les prix en sus des prix calculés.

Le calcul de base du PSM repose sur divers facteurs, notamment le coût des intrants pour les agriculteurs, la parité des prix, l'historique des prix, l'offre et la demande, etc. Ainsi, la méthodologie de calcul de la CACP est:

Coût A1 = Coût de la culture, y compris les intrants, la main-d'œuvre, l'amortissement, les taxes, les intérêts, les frais divers, etc.

Coût A2 = A1 + loyer payé pour le bail foncier

Coût B1 = A2 + intérêts sur la valeur des immobilisations (hors terrains)

Coût B2 = B1 + valeur locative du terrain en propriété et loyer payé pour le terrain loué.

Coût C1 = B1 + valeur imputée de la main-d'œuvre familiale

Coût C2 = B2 + valeur imputée de la main-d'œuvre familiale

Coût C2* = Coût C2+ Valeur ajoutée de la main-d'œuvre basée sur les taux du marché

Coût final C3 = Coût C2* +10 \% de C2* (pour les coûts de gestion par agriculteur)

Bien que le concept de ce soutien soit largement apprécié en Inde, sa mise en œuvre dépend de facteurs divers tels que le marché, la situation politique et les déficits publics. Selon ces facteurs, les décideurs choisissent la formule la plus convenable parmi celles ci-dessus.
Le prix calculé comme tel a des répercussions importantes sur la détermination des prix du marché. Cependant, il ignore les externalités environnementales qui font partie intégrante de l'agriculture durable. Il y aurait probablement besoin de les inclure à l'avenir.

Source: Ashish Gupta, Jaivik Haat, Inde 
2. Les décideurs politiques peuvent créer un marché spécifique pour les produits durables en s'appuyant sur les marchés publics, offrant ainsi des prix plus élevés aux producteurs et un débouché commercial sûr.

\section{恖 Les programmes d'approvisionnement institutionnel (PAI) et l'influence des prix au Brésil}

Programa de Aquisição de Alimentos/ Le Programme d'approvisionnement alimentaire (PAA) et Programa Nacional de Alimentação Escolar/ Le Programme national d'alimentation scolaire (PNAE)

La portée des programmes d'approvisionnement des établissements publics pourrait être très large puisqu'il s'agit de fournir des aliments aux écoles publiques, aux organismes de secours alimentaire, aux prisons et aux hôpitaux. En 2003, le Brésil a mis en œuvre l'un des premiers programmes institutionnels des achats publiques de l'alimentation (PIPA) grâce à une relation directe entre la demande structurée de petits producteurs et une stratégie nationale pour la sécurité alimentaire. Alors que la plupart des systèmes gouvernementaux exigent qu'au moins trois propositions de prix soient soumises avant d'être réviser, le gouvernement brésilien a décidé de renoncer à ce processus en exigeant que «les aliments doivent être produits par les agriculteurs familiaux; les prix ne doivent pas être supérieurs à ceux affichés sur les marchés régionaux; et une limite financière annuelle pour chaque unité agricole familiale doit être respectée» (Swensson, 2015, 16).
Le Groupe de gestion du programme d'achats d'aliments (GPAA) établi les critères de définition du prix de référence de la PAA. Les critères actuels de fixation des prix de référence sont:

a. Le prix de référence pour tous les produits définis à partir de la moyenne déduite des trois enquêtes sur les prix réalisés dans les marchés de gros locaux/régionaux au cours des douze derniers mois;

b. D'autres agences peuvent utiliser les prix de référence définis par la Companhia Nacional de Abastecimento

c. (CONAB), la Société nationale d'approvisionnement, pour rationaliser le processus;

d. Le prix de référence des produits biologiques peut être augmenté jusqu'à 30 pour cent par rapport au prix de référence des produits conventionnels;

e. Le prix de référence reste valable pendant 12 mois.

De cette façon, le gouvernement est responsable de l'établissement d'un prix de référence équitable pour tous les produits, et les agriculteurs sont donc en mesure de fixer leurs prix en fonction de cette mesure externe afin d'être éligibles pour les achats institutionnels.

Source: Swensson, 2015. 
Les décideurs politiques sont confrontés à d'autres défis. S'il y a un consensus général sur la nécessité de promouvoir la durabilité des systèmes alimentaires, plusieurs soutiennent cependant les pratiques agricoles conventionnelles, par défaut d'une approche traditionnelle de modernisation, à savoir les politiques de subventions aux intrants. Ces types de subventions ont des effets pervers sur les prix du marché, ce qui donne la fausse impression que les aliments durables sont beaucoup plus chers. Les décideurs politiques peuvent faire beaucoup plus d'effort pour remédier à cette situation, en commençant par une meilleure compréhension des coûts réels des aliments.

http://www.fao.org/nr/sustainability/full-cost-accounting/en

\section{ASTUCE 18}

Comment trouver un prix durable

- Aucun acteur ne dispose à lui seul de toutes les informations nécessaires pour fixer un prix équitable et durable - la transparence aide chacun à élargir ses connaissances.

- Essayez d'être empathique dans vos négociations. Mettez-vous à la place de l'autre personne et essayez de considérer sa situation lorsque vous déterminez votre prix.

- Les comités des prix et la négociation collective, avec ou sans l'aide d'un intermédiaire transparent, peuvent aboutir à des prix plus équitables.

- Les prix durables couvrent la totalité des coûts de production, les volumes produits et les qualités souhaitées. De plus, ils peuvent être payés ponctuellement et maintenus dans le temps.

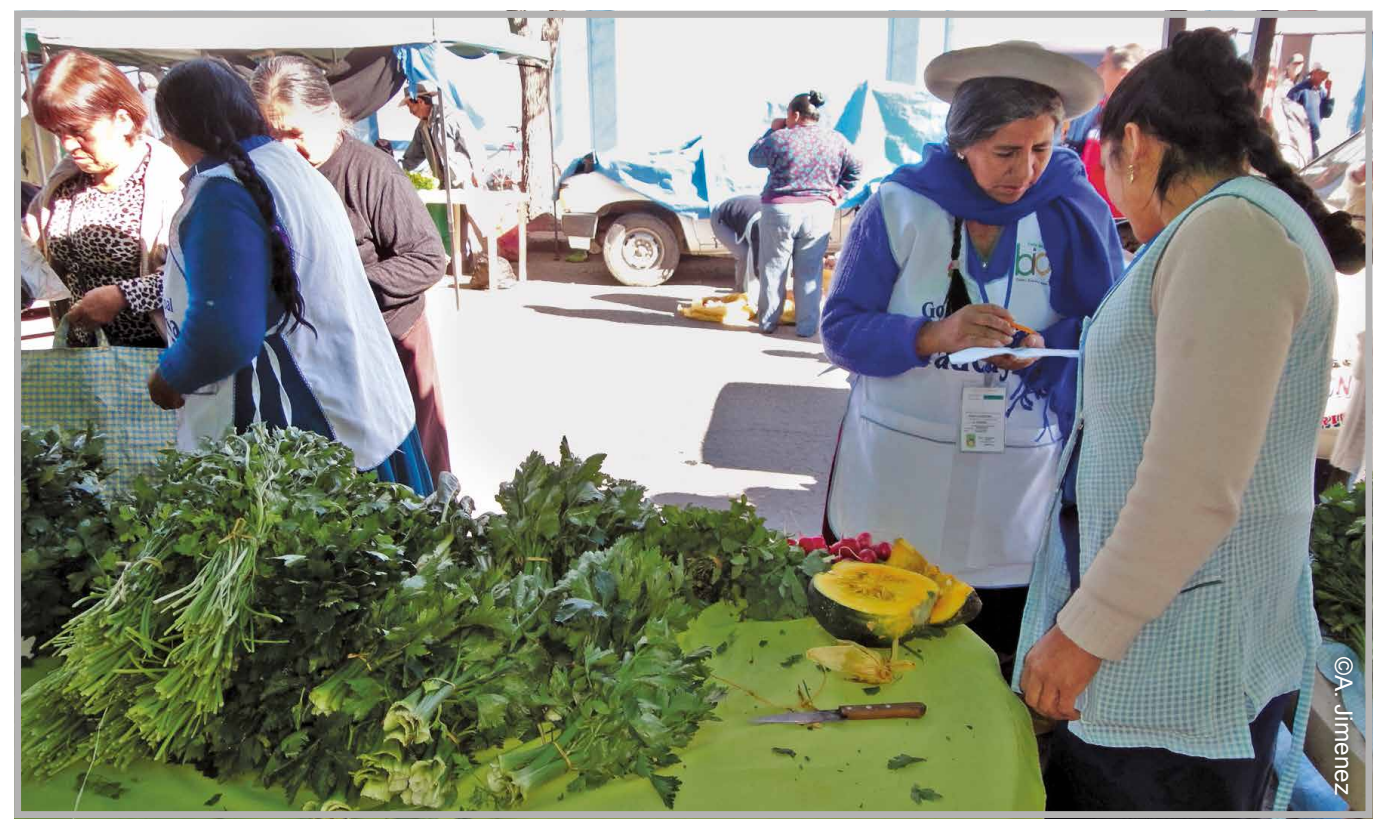




\section{LE CHEMIN D’APPRENTISSAGE, OÙ ALLER ENSUITE?}

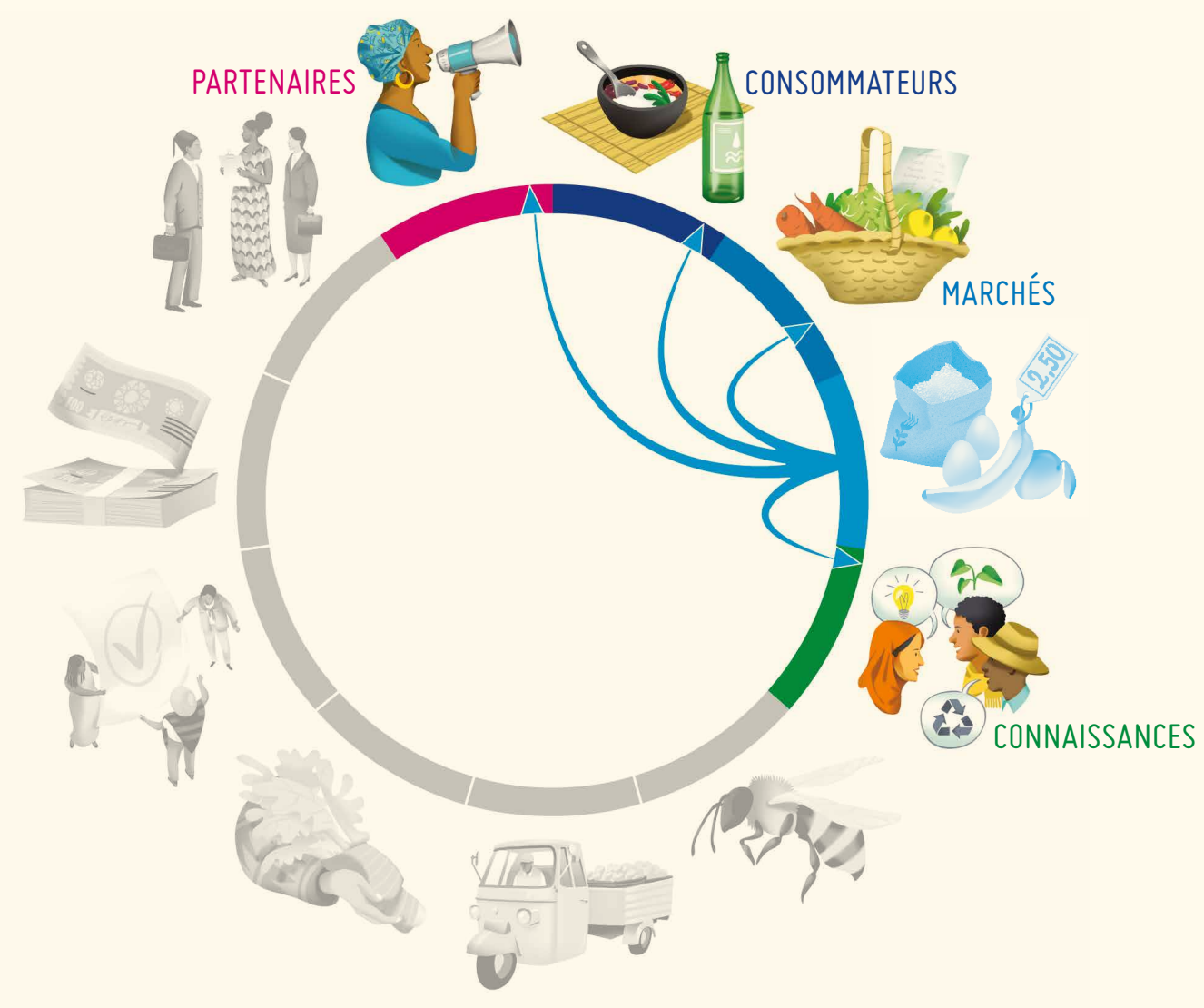

- Vous souhaitez connaître certains partenariats novateurs créés pour déterminer les prix ? Pour en savoir plus, consultez le Chapitre 11: La place des partenaires et des plaidoyers.

- Si vous pensez organiser une visite de ferme pour les consommateurs, consultez le Chapitre 2: Connaître vos marchés.

- Vous ne savez pas où trouver des consommateurs pour mettre sur pied un comité d'établissement de prix ? Allez au Chapitre 1: Attirer les clients et les fidéliser.

- Vous voulez en savoir plus sur la production durable afin de mieux calculer vos prix ? Continuer au Chapitre 4: Partager et co-créer des connaissances pour une production durable.

Ou dirigez-vous vers un autre chapitre qui vous intéresse... 


\section{PRODUIRE \\ DURABLEMENT}

\section{PARTAGER ET CO-CRÉER DES CONNAISSANCES POUR UNE PRODUCTION DURABLE}

GÉRER ET ACCÉDER AUX INTRANTS DURABLES
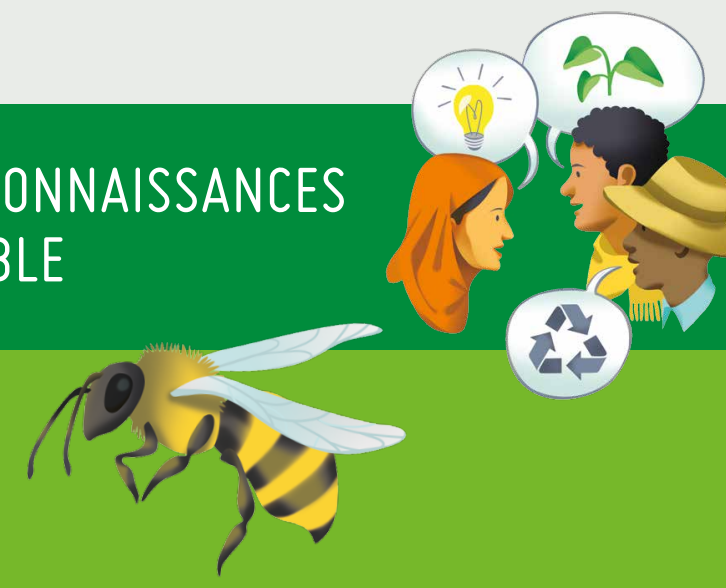

Dans ce groupe de chapitres, nous explorons la dimension productive des systèmes alimentaires durables.

Nous n'entrerons cependant PAS dans le détail des pratiques de production durable, puisque ce n'est pas l'objectif de ce manuel. Ces informations sont beaucoup trop vastes et dépendent fortement du contexte; elles peuvent être retrouvées ailleurs. Par exemple, ces outils suivants peuvent vous être utiles:

- http://www.fao.org/sustainability/frameworks-approaches/en

- http://www.fao.org/agroecology

- https://www.ifoam.bio/en/organic-30/organic-food-system-program

- https://www.ifoam.bio/sites/default/files/organic_food_system_in_ practice_feb2017web.pdf

Nous nous concentrons plutôt sur deux aspects de l'organisation des systèmes de production durable: comment partager et co-créer des connaissances sur la production durable et comment gérer et accéder aux intrants durables.

Ces deux aspects sont pleins d'innovations, et nous partageons quelques idées tirées des expériences des auteurs qui pourraient vous aider à construire votre propre chemin. 

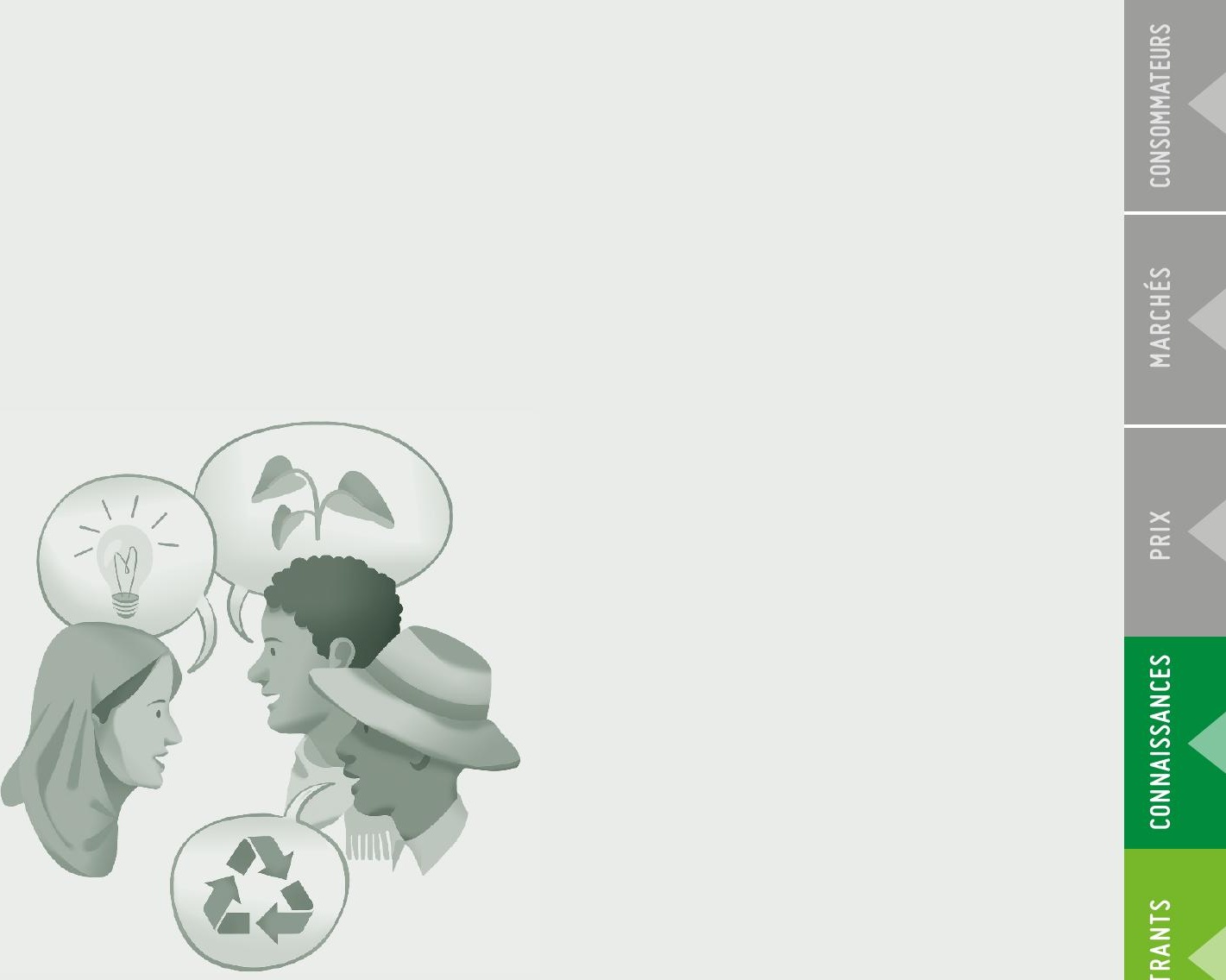

$\frac{x}{a}$

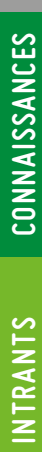

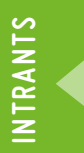

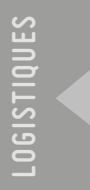

出
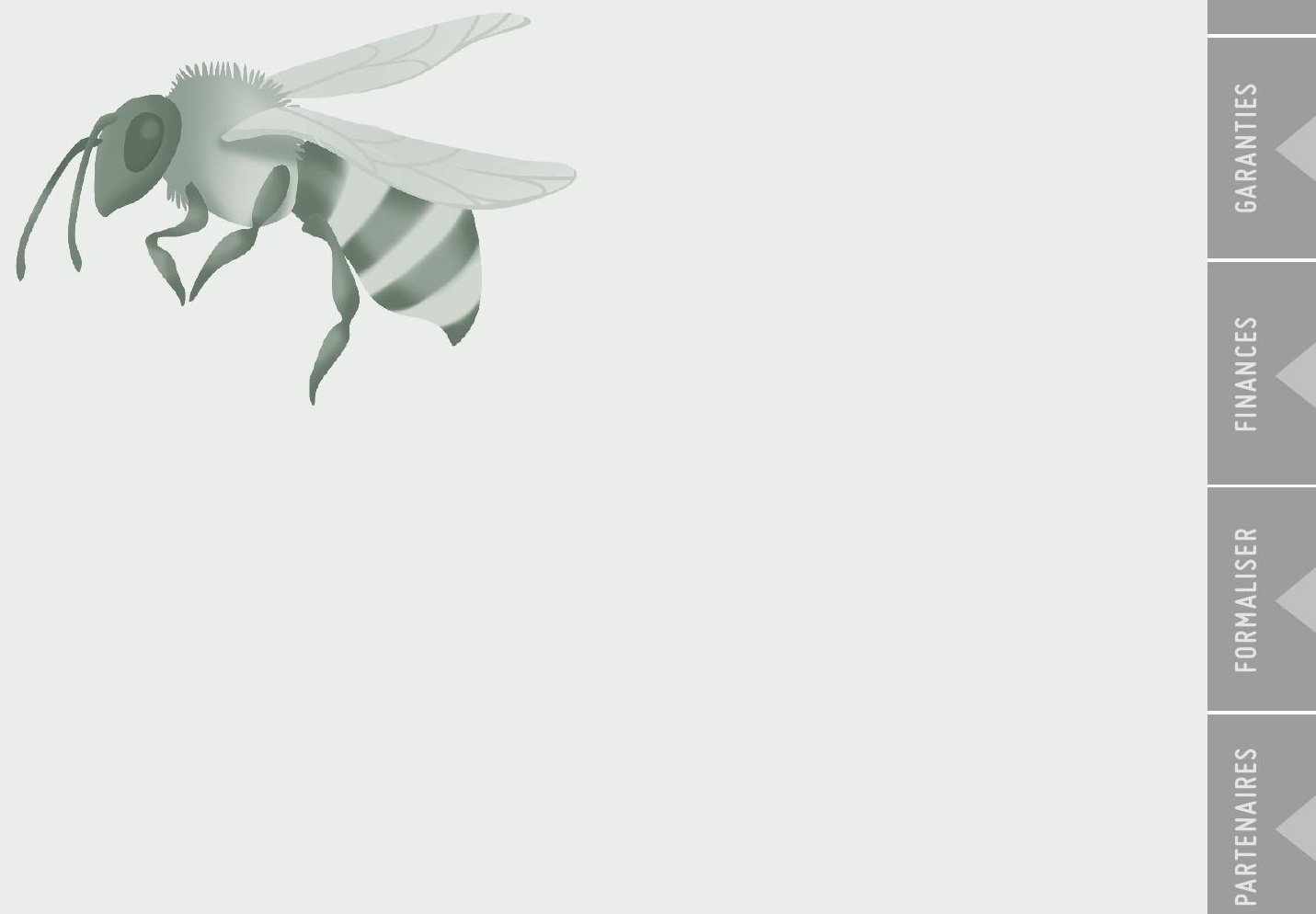



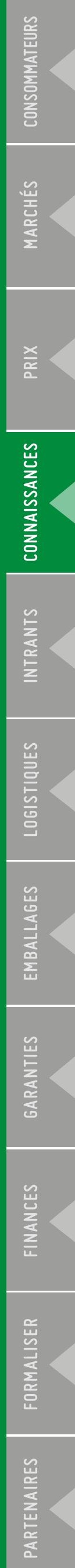




\section{POURQUOI EST-CE IMPORTANT?}

Pour soutenir leur production durable, les producteurs doivent accéder à une grande variété d'informations et de connaissances sur les cycles naturels, les interventions humaines et les interactions entre les systèmes.

Un aspect clé de la durabilité est l'égalité d'opportunités pour innover et pour partager des connaissances avec les générations actuelles et futures. Tous les acteurs doivent savoir ce que signifie «produire durablement»: cela sensibilise davantage l'ensemble du système alimentaire. Pourtant, la mise en place de plateformes de co-création et de partage de connaissances exige de la créativité, de la collaboration et des compétences.

Ce chapitre traite du «comment» fonctionnent les approches innovantes de partage et de co-création du savoir, et du «comment» les différents types d'acteurs s'organisent (à la fois formellement et informellement) pour y parvenir.

\section{ASTUCE 19}

Savoir choisir entre une approche formelle et informelle

La création et le partage formels des connaissances (partenariats légalement encadrés, connaissances institutionnalisées, programmes récurrents) sont plus appropriés lorsqu'il est nécessaire de s'assurer d'une amélioration continue des capacités et les connaissances. Les approches informelles sont plus simples et ponctuelles.

\section{COMMENT ABORDER LA CONNAISSANCE POUR UNE PRODUCTION DURABLE?}

Il y a deux approches particulièrement intéressantes qui sont les mieux adaptées au partage et à la co-création de connaissances pour une production durable:

1. L'apprentissage par la pratique priorise la pratique comme moyen d'acquérir des connaissances et du savoir-faire. Il comprend une variété de méthodes, comme les visites d'échange entre agriculteurs, la recherche-action et la recherche participative. Cette approche éducative est utilisée dans divers contextes et repose surtout sur l'idée que la connaissance est une activité appliquée. Ceci étant dit, apprendre l'agriculture durable survient de ses propres expériences et pratiques.

2. L'expérimentation menée par les agriculteurs se concentre davantage sur leur rôle comme vecteurs clés de l'innovation. Ils dirigent plusieurs types de plateformes d'innovation: platesformes d'agriculteurs-acteurs publics, plates-formes agriculteurs-sociétés civiles, plateformes agriculteurs-secteur privé, plateformes d'innovation multi-acteurs et écoles pratiques agricoles. L'expérimentation conduite par les agriculteurs est un type de recherche initiée et menée par eux dans leurs propres champs; elle peut aussi être initiée au sein des groupes d'agriculteurs existants. Elle leur permet d'identifier les options technologiques adaptées aux conditions agroécologiques et socio-économiques locales. 


\section{ASTUCE 20}

Réfléchir à la durabilité de vos pratiques agricoles

Être conscient de vos pratiques ainsi que de leur caractère durable est une étape clé pour déterminer vos manques de connaissance et comment les acquérir.

L'auto-évaluation peut être aussi simple que de comparer vos pratiques quotidiennes aux normes de pratiques durables souhaitées. Ceci vous aidera à mieux comprendre et à vous approprier le langage de la durabilité, ainsi que de communiquer vos activités avec plus d'aise à d'autres personnes sans «se perdre» ou «se cacher» dans des mots compliqués. Documenter vos évaluations encourage une prise de décision plus éclairée et deviendra éventuellement nécessaire si vous demandez une certification.

Commencez à étudier le contenu de votre formation ou de toute norme que vous essayez de respecter. Des outils reconnus à l'échelle internationale peuvent aussi vous guider:

- Directives SAFA (FAO): Les indicateurs de durabilité de la FAO ont été développés par des praticiens et des experts pour l'évaluation critique de la production durable.

Pour en savoir plus, visitez:

http://www.fao.org/nr/sustainability/sustainability-assessments-safa/en

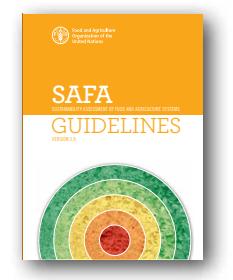

- SHARP (FAO): Le Schéma holistique pour l'auto-évaluation paysanne de la résilience climatique (SHARP) a été développé pour répondre aux besoins des petits exploitants agricoles et des bergers afin d'évaluer leur résilience climatique au niveau des ménages et des communautés individuelles.

Pour en savoir plus:

http://intranet.fao.org/departments/cio/products_and_services/ detail/c/25635

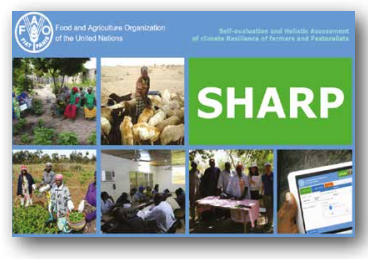

- Guide des meilleures pratiques pour l'agriculture et les chaînes de valeur (IFOAM): Ce guide vise à encourager, guider et inspirer le travail en coopération à travers l'agriculture durable. Il aspire à donner aux individus et aux organisations les moyens d'améliorer leurs propres performances et pratiques, leur qualité de vie et le bien-être de leurs communautés.

Pour en savoir plus:

https://www.ifoam.bio/sites/default/files/best_practice_guideline_v1.0.pdf
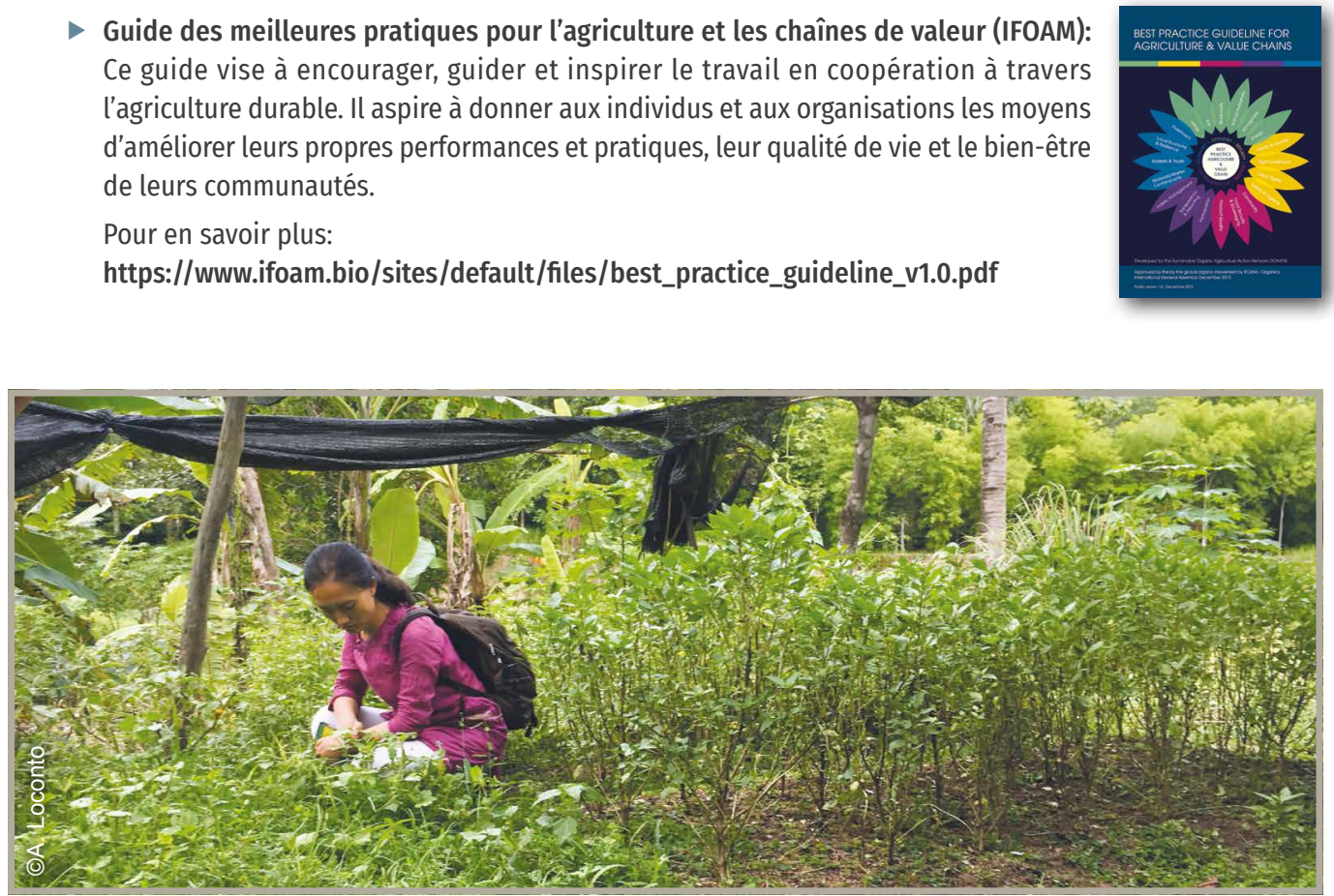


\section{DES MÉCANISMES DIFFÉRENTS POUR DES OBJECTIFS DIFFÉRENTS}

\subsection{Visites d'échange entre agriculteurs}

Les visites d'échange ont pour objectif de partager les connaissances entre les producteurs pratiquant une agriculture durable. Elles peuvent être formelles et programmées comme une activité récurrente dans le cadre de votre initiative afin d'assurer un flux continu d'informations entre les membres. Vous pouvez également les organiser de façon ad hoc, c'est à dire autour d'une question d'actualité pour l'initiative ou lorsqu'un élément d'information spécifique est nécessaire.

Rappelez-vous que ces visites sont efficaces pour le partage de connaissances lorsque des relations de confiance entre les agriculteurs sont établis.

Dans ce cas, ils ne se soucieront pas de l'appropriation des connaissances et ils chercheront les informations pertinentes avec plus d'aise.

\section{Le programme TRIT pour les échanges d'agriculteurs (Tanzanie)}

Des visites d'échange d'agriculteurs sont mises en œuvre par le Tea Research Institute of Tanzania (TRIT) dans le cadre d'un programme de transfert de technologie pour soutenir le partage des connaissances entre les petits producteurs de thé. L'activité s'organise de façon à ce que les agriculteurs plus compétents en matière d'adoption technologique accueillent des agriculteurs qui manquent des connaissances dans ce sujet. Cette approche repose sur l'hypothèse clé que les agriculteurs ont plus tendance à adopter une technologie déjà pratiquée avec succès par d'autres agriculteurs que si elle est promue par un agent extérieur (par exemple, un agent de vulgarisation).

Il y a différentes façons d'organiser ces visites, dont deux principales:

Visites externes inter-districts: Au-delà des nouvelles connaissances acquises lors d'une visite, la découverte d'autres lieux

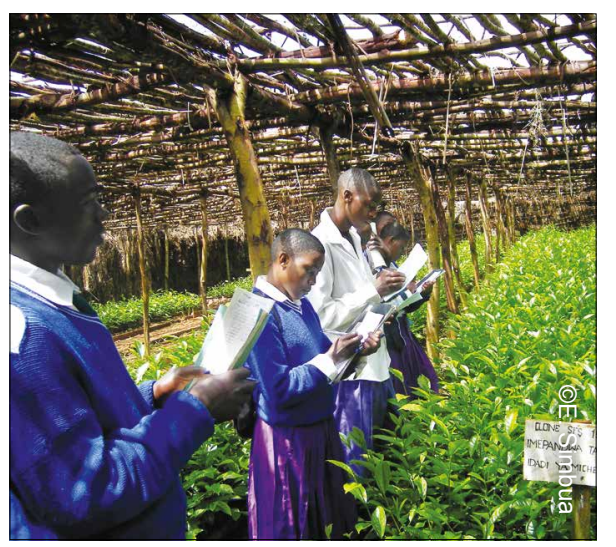

ou districts géographiques permet aux agriculteurs d'en apprendre davantage sur certains sujets, notamment: les environnements nouveaux, la rencontre de nouvelles personnes qui peuvent avoir des cultures différentes, l'essai de nouveaux aliments. Dans l'ensemble, tous ces éléments favorisent le rapprochement entre les agriculteurs et créent un espace d'apprentissage positif. 
- Visites internes inter-villages: Elles sont plus simples et pertinentes pour des thèmes très spécifiques, souvent liés à la production ou à la valeur ajoutée. Elles peuvent être de courte durée et permettre aux agriculteurs d'échanger plus librement leurs expériences, étant donné qu'ils partagent le même environnement agroécologique. Bien que ces visites ne recréent pas entièrement l'expérience touristique, elles offrent des occasions de réseautage et de formation de groupe.

Il y a d'importants éléments logistiques à considérer lors de ces visites d'échange:

1. Nombre de participants: Un nombre de participants gérable permet une participation harmonieuse et non-chaotique de tous. L'expérience au TRIT consistait d'un groupe de 20 à 30 participants - ils ont voyagé ensemble dans un minibus et ont socialisé tout au long du trajet.

2. Durée de la visite: Les visites d'échange sont courtes et efficaces. Généralement, les visites d'échange internes durent plusieurs heures en une journée tandis que les visites interrégionales durent de 1 à 3 heures. Les visites prolongées au-delà de 3 jours ont tendance à ennuyer les participants.
3. Soutien logistique: Le TRIT aide les participants à payer les frais de déplacement pour accommoder leur logement pour un séjour d'une nuit. Pour les visites courtes d'une journée, des rafraîchissements sont offerts afin de favoriser un espace de discussion et d'échange. TRIT possède deux minibus de 30 places qui sont habituellement utilisés pour transporter les participants à divers endroits.

Notre expérience générale avec de telles visites d'échange institutionnalisées montre que l'approche est efficace pour offrir des plateformes de partage d'expériences. Lors de ces visites, les paysans hôtes se sentent fiers et honorés d'accueillir les visiteurs et veulent les impressionner. Tout aussi vrai est le sentiment réciproque de gratitude qu'expriment les visiteurs envers leurs hôtes. À leur tour, les visiteurs seront contents de rendre la faveur et de partager leurs pratiques en tant qu'hôtes. Cependant, ces programmes sont malheureusement souvent dépendants d'une institution facilitatrice telle que le TRIT, ce qui impacte leur durabilité.

Source: Emmanuel Simbua, TRIT

\subsection{Champs écoles des producteurs}

Les champs écoles des producteurs (CEP) sont considérés un outil efficace pour partager les connaissances avec les agriculteurs en favorisant leur réflexion par l'expérimentation pratique et directe (approche dirigée par les agriculteurs). L'accent est mis sur l'adaptation locale des pratiques et des technologies existantes. Cet outil est particulièrement pertinent pour promouvoir la transition vers une agriculture durable. Les CEP peuvent créer des communautés de pratiques, qui peuvent devenir des initiatives novatrices si les participants s'engagent dans d'autres activités, telles que la transformation ou la commercialisation de produits durables. 


\section{ASTUCE 21}

\section{Établir des CEP}

1. Une formation des maitres formateurs (FMF) sur la méthode d'apprentissage des CEP doit avoir lieu.

- Chaque facilitateur doit préparer un rapport de formation qu'il remet à la direction de son organisation et à ses collègues.

2. L'animateur sensibilise le public aux CEP pour obtenir leur appui et pour les informer de l'établissement de l'école.

- Normalement, cette activité se déroule lors d'une réunion ou d'une assemblée villageoise convoquée et elle est ouverte à tous les producteurs intéressés d'y participer.

- L'animateur fournit des informations sur l'historique, les objectifs, les résultats techniques et les conditions de mise en place des CEP. L'animateur recueille les opinions des participants sur les principaux défis auxquels ils sont confrontés et sur l'installation de l'école.

3. Des parcelles pour les CEP sont sélectionnées.

- Cette sélection a lieu au moins un mois avant la formation et en consultation avec les organisations d'agriculteurs ou les communautés.

- Les parcelles sélectionnées doivent être: représentatives des contraintes de la zone, sécurisées autant que possible (conflits fonciers éventuels, dégâts animaux, inondations, etc.) et facilement accessibles.

4. Un diagnostic communautaire participatif des contraintes et des possibilités se déroule.

- Il s'agit d'une enquête sur les pratiques et les problèmes des producteurs pour définir le contenu de la formation. Ayant lieu au moins un mois avant le début du calendrier agricole, ce diagnostic doit être réalisé par l'animateur de manière participative par le biais d'entretiens avec les producteurs et acteurs locaux.

5. Le succès et l'impact de la formation sont largement influencés par la sélection de participants.

- Les critères généraux suivants peuvent servir de guide de sélection: être producteur de la culture étudiée; être volontaire; être ouvert à l'innovation; pouvoir participer régulièrement à des sessions de formation; accepter la diffusion de connaissances acquises d'autres producteurs et accepter des visites sur leur parcelle.

6. Des séances de formation sont préparées et animées.

- Un programme de formation est défini selon les résultats des enquêtes de base. Des sujets agronomiques et d'autres sujets (comme la dynamique de groupe, la connaissance des insectes, la production de compost, etc.) peuvent être inclus dans le programme. Le programme devrait encourager les producteurs à répondre aux inquiétudes des autres.

- Le matériel de formation, ainsi que les supports visuels, sont préparés à l'avance.

- L'autoréflexion, l'interaction entre agriculteurs et l'expérimentation directe sont stimulées.

- Un bon environnement d'apprentissage est créé en gérant efficacement les discussions et en veillant à ce que les hommes et les femmes, jeunes et moins jeunes, participent également.

- Les résultats sont évalués en saison et en fin de saison.

7. Une cérémonie de clôture est organisée ou les certificats sont remis. Il s'agit de sensibiliser et de partager les résultats des CEP avec la communauté (en termes du niveau de connaissance des participants et d'amélioration de la production). 


\section{Les fermes incubatrices en France}

En France, on observe une tendance croissante

de fermes incubatrices pour les petits exploitants agricoles. Récemment mis en place, le Réseau national d'espace test agricoles (Reneta) est la preuve de cette évolution.

Parmi les exemples les plus réussis, on peut citer l'incubatrice Les Champs des possibles, qui regroupent 10 sites de test d'activité en région parisienne. Elle a été créée par le réseau AMAP local pour répondre au besoin d'augmenter le nombre de nouveaux arrivants dans l'agriculture contribuant aux systèmes alimentaires locaux. Comme mentionné dans Peter Volz et al. (2017, p. 34), «Les fermes incubatrices permettes aux nouveaux producteurs d'accéder à la terre et a l'équipement, au mentorat et au statut juridique pendant une période d'essai. L'objectif est de les aider à acquérir de nouvelles compétences et de la confiance en soi, à tester les pratiques agricoles, à se constituer une base de consommateurs et à développer leur réseau professionnel avant de se lancer dans leur propre exploitation. Il s'agit d'une dimension essentielle pour combler le fossé entre la formation et l'entrée dans l'agriculture pour les agriculteurs potentiels dont la formation manque souvent d'expérience pratique». Depuis sa création en 2009, Les «Champs des possibles» a permis l'incubation de 40 futurs agriculteurs et l'installation de 14 nouveaux agriculteurs. Elle s'est également étendue sur de nouveaux sites d'essais.

Les Champs des possibles a également entamé une coopération avec l'association locale d'agriculture biologique, une société foncière nationale appelée Terre de Liens et le réseau AMAP. L'objectif était de mettre en place une plateforme informelle pour promouvoir l'accès à l'agriculture dans la région. Ils ont tous estimé qu'il y avait un besoin de coopération entre les différentes organisations: une première fournissant une formation et un mentorat sur les compétences agronomiques; une deuxième sur les systèmes de distribution et les relations communautaires; une troisième sur les essais agricoles; et une dernière sur la recherche et l'obtention de terres. La coopération entre toutes ces différentes structures était donc nécessaire pour fournir un large ensemble d'activités cohérentes. L'objectif est d'aider les futurs agriculteurs à «passer de leurs plans initiaux à l'agriculture réelle, en sécurisant chaque étape de leur entrée» (Volz et al., 2017, p. 36).

Source: Jocelyn Parot, URGENCI

Pour en savoir plus: http://www.accesstoland.eu/IMG/pdf/reneta_ overview_farmincubators_france_france_en.pdf http://www.terredeliens-iledefrance.org/lepole-abiosol

http://www.leschampsdespossibles.fr

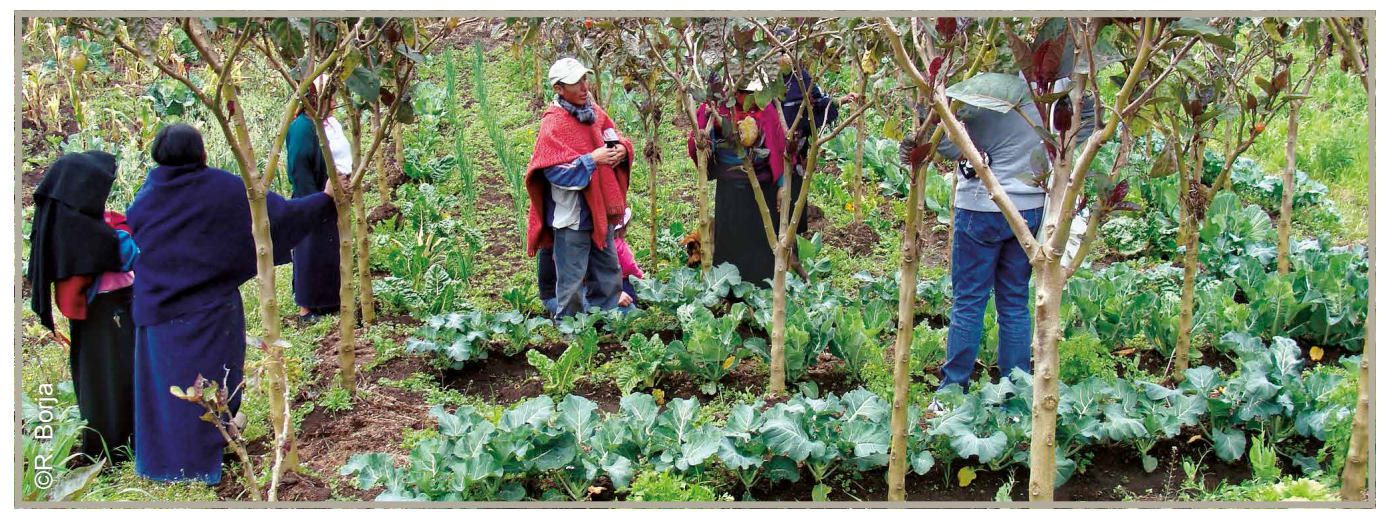




\subsection{Recherche participative et recherche-action}

La recherche participative et la recherche-action sont des outils efficaces pour combler les lacunes des connaissances d'agriculteurs et pour favoriser l'apprentissage collectif entre différents acteurs d'un système alimentaire.

- La recherche participative exige que des questions de recherche se co-construisent avec les participants et que la collecte et l'analyse de données se fasse en participant aux activités du projet.

- La recherche-action n'inclut pas toujours des questions co-construites. Cependant, vu son engagement avec les partenaires, son but est d'apporter des changements au cours du projet.

La recherche-action pour promouvoir les pratiques agroécologiques (Équateur)

EkoRural est une ONG équatorienne qui promeut les innovations sociotechniques dans les zones rurales en s'appuyant sur une approche endogène et centrée sur la population. Elle tient pour but principal de faciliter les relations entre producteurs et consommateurs. En 2010, un processus d'intensification agroécologique a été établi en mettant en relation une organisation paysanne de Tzimbuto, Asociación Nueva Generación, avec des consommateurs urbains de l'organisation Canasta Comunitaria Utopia, située à Riobamba. Ceci a été réalisé en adoptant une approche de recherche-action où les produits, les pratiques, les relations, les flux et les règles ont été enregistrés et analysés de façon critique par les participants afin de trouver des solutions collectives. Le processus s'est déroulé selon les étapes suivantes:

\section{Documenter et analyser les attentes:}

Ekorural, ayant beaucoup d'expérience avec la recherche-action axé sur les personnes, a documenté l'offre de l'organisation paysanne Asociación Nueva Generación.
Par la suite, elle a analysé les attentes des membres de Canasta Comunitaria Utopia et de Associación Nueva Generación.

2. Partager les attentes: Des réunions ont été organisées où les représentants d'EkoRural ont facilité le partage d'attentes entre les membres des deux organisations, créant ainsi un espace de compréhension et d'accord mutuel. En outre, les agriculteurs ont participé à des événements d'échange et d'apprentissage pour se familiariser avec la Canasta Comunitaria Utopia. Ses consommateurs-membres ont visité les agriculteurs de Asociación Nueva Generación se sont davantage sensibilisés à la réalité rurale et à l'importance de la consommation locale.

3. Régler le schéma de livraison: bien que le programme de livraison a débuté en 2010, l'alliance était fragile puisque les consommateurs ont noté des problèmes dans la qualité des produits livrés. Les producteurs manquaient d'expérience en production agroécologiques et en vente directe. Ils éprouvaient également des difficultés à coordonner et à organiser la collecte, à contrôler la qualité et à livrer les produits. 
4. Surmonter les lacunes: pour combler les lacunes par le dialogue, pendant les deux premières années du projet, EkoRural a subventionné le transport des agriculteurs de Tzimbuto pour qu'ils puissent visiter la Canasta Comunitaria Utopia. Asociación Nueva Generación a mis l'accent sur les produits de qualité, ainsi que sur le respect des quantités et de la diversification des produits, comme demandés par les consommateurs.

5. S'aligner à nouveau: pour respecter l'engagement avec Canasta Comunitaria Utopia, EkoRural a soutenu les membres de Asociación Nueva Generación dans la modification de leurs pratiques agricoles, afin de mieux refléter et renforcer l'agriculture agroécologiques andine. Grâce aux visites d'échange entre agriculteurs, de nouvelles espèces et variétés autochtones, des plantations échelonnées, la rotation des cultures et la planification agricole ont été introduites, permettant d'élargir l'offre de produits agroécologiques. Les systèmes de livraison et de contrôle de qualité se sont également améliorés.

6. Partager des retours en continu: EkoRural a facilité l'organisation de réunions régulières pour permettre aux membres des deux organisations d'échanger sur leurs expériences et leurs préoccupations. Les consommateurs évaluent chaque

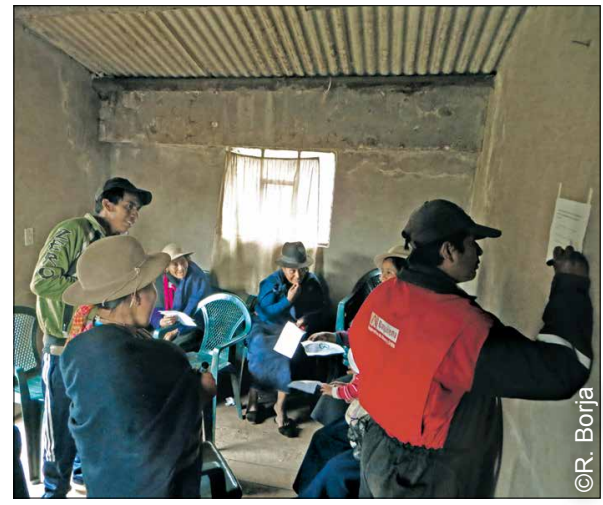

réunion à laquelle participent aussi les représentants des producteurs. En outre, ces derniers ont été systématiquement invités aux assemblées générales de Canasta Comunitaria Utopia, permettant aux producteurs d'exprimer leurs impressions quant à la relation avec les consommateurs et de discuter les prix et les accords de collaboration.

Grâce à l'approche adoptée, une innovation interactive s'est créée où les producteurs et les consommateurs partagent des valeurs et des objectifs communs, décident des prix considérés justes et passent d'une relation orientée vers la maximisation des profits à une relation basée sur des valeurs de réciprocité et de confiance.

Source: Ross Mary Borja, Fundación Ekorural

\subsection{Innovation interactive}

Une innovation interactive est une configuration multi-acteurs mise en place pour faciliter et entreprendre diverses activités autour de défis et d'opportunités identifiés dans les systèmes alimentaires durables. Il n'y a pas de configuration standardisée et les acteurs peuvent être différents. En plus de la société civile et des agriculteurs, elles impliquent souvent des acteurs publics et/ou privés. 


\section{Un partenariat société civile-} secteur public pour promouvoir le concept d'éco-santé (Colombie)

En 1994, le Gouvernement colombien a créé un cadre juridique pour assurer que les citoyens et les communautés participent à la planification, à la gestion et à l'évaluation des plans de santé publique. Dans ce cadre juridique, des comités de participation communautaire en santé publique (COPACO) ont été instaurés au niveau municipal, offrant un espace de discussion et de consultation. Dans la municipalité de Sumapaz (un district de Bogotá), en concertation avec la COPACO locale, l'hôpital Nazareth a obtenu des ressources financières du Secrétaire de la santé publique pour créer le Centre pour le développement du potentiel humain (CDPH). Étant donné que l'hôpital de Nazareth est le seul situé en zone rural du district, le centre répond aux besoins de santé de la population rurale au moyen d'une approche écosystémique de la santé, un concept nommé EcoHealth. Le CDPH est constitué de deux branches principales: le Parque Temático Chaquen et le programme d'éco-thérapie.

1. Le Parque Temático Chaquen est une stratégie innovante pour affronter les causes des problèmes de santé dans les zones rurales: conditions de travail dangereuses, pratiques agricoles inadéquates, régimes alimentaires inadéquats, populations dispersées, faibles revenus et relations hostiles avec l'environnement. Les activités du parc sont organisées autour de différentes dimensions:

- Accès à la nourriture: Les familles qui visitent le parc bénéficient d'un accès amélioré à la nourriture. Ce résultat dérive en grande partie de la diversification, de la restauration et de la conservation des variétés autochtones qui sont cultivées et répandues dans le parc. Celles-ci sont ensuite distribuées aux familles, favorisant ainsi une alimentation diversifiée.

- Consommation et utilisation biologique: Les connaissances des consommateurs en matière d'alimentation saine et d'aliments sains sont renforcées afin d'atteindre un mode de nutrition adéquat.

- Sécurité et qualité des aliments: Les agriculteurs ont été incités à éliminer l'utilisation des pesticides et à produire des aliments biologiques avec de meilleures conditions de travail.

- Habitat sain: Les agriculteurs ont été formés et encadrés pour adopter des systèmes de production propres, ainsi que de bonnes pratiques agricoles qui ont été démontrées aux visiteurs du parc.

- Sécurité et environnement de travail sain: Un environnement de travail sain est promu par des actions et des formations tant au niveau communautaire et individuel que dans les secteurs formels et informels.

2. Le programme de réadaptation d'écothérapie est innovateur pour les populations vulnérables. Plus précisément, les techniques et les stratégies d'intervention recréent des rôles sociaux significatifs et valorisés pour les personnes vulnérables. L'objectif et de faire croitre leur niveau d'emploi et leur inclusion sociale. À Bogota, une centaine de personnes sans-abris souffrant de diverses maladies mentales sont incluses dans le programme d'éco-thérapie et tentent une autre façon d'améliorer leur santé. Ils reçoivent des soins horticoles, écologiques, médicaux, psychologiques et psychiatriques pour vérifier, identifier, décrire et agir contre leur maladie.

Source: Andrea Moya, Claudia Helena Prieto, Parque Temático en Salud Pública Chaquen, Subred Integrada 


\section{更

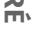

35

Les agriculteurs constituent une riche diversité de savoir-faire et de pratiques traditionnelles. Toutefois, leurs expertises ont toujours été sous-évaluées et il y a eu peu de convergence entre l'innovation informelle et la recherche et le développement formel. Le modèle dominant de soutien aux agriculteurs demeure une approche descendante, ou le «transfert de technologie». Il repose sur les scientifiques qui déterminent les priorités de recherche, génèrent la technologie et la transmettent, par l'intermédiaire de spécialistes, en une matière pour que les vulgarisateurs la transfèrent ensuite aux agriculteurs. Cette approche exclut les agriculteurs du développement et de la diffusion de nouvelles technologies.

Elle a conduit à une faible utilisation de ces technologies, que les agriculteurs considèrent souvent comme non-pertinentes à leur situation sociale, économique et environnementale. Les agriculteurs sont de plus en plus reconnus pour leurs savoirfaire et expériences qui peuvent appuyer la recherche et le développement agricoles. En tant qu'utilisateurs finaux de la technologie, ils devraient participer activement à toutes les étapes de ce processus.
PELUM Kenya et l'Université d'agriculture Jomo Kenyatta (JKUAT) collaborent pour s'engager dans un processus interactif impliquant la communauté et des acteurs divers. Ces derniers partagent des points de vue similaires et des compétences différentes afin de valider, adopter et développer les innovations des agriculteurs. Le soutien scientifique des innovations renforce le savoir-faire des agriculteurs tandis que la collaboration entre chercheurs et agriculteurs renforce la confiance des agriculteurs dans leur capacité d'adaptation des technologies. Le partenariat a amélioré les approches dirigées par les agriculteurs, d'où ils ont également participé à des processus de recherche formels.

Le partenariat vise à combler le fossé entre les agriculteurs et les chercheurs, à faciliter les approches de recherche dirigées par les agriculteurs et à permettre aux agriculteurs d'accéder aux résultats de recherche et aux innovations techniques des autres agriculteurs du JKUAT. PELUM a construit son programme en s'inspirant du modèle MASIPAG aux Philippines, qui connait beaucoup de succès depuis de nombreuses années.

Source: Carmen Cabling, SPG de Quezon

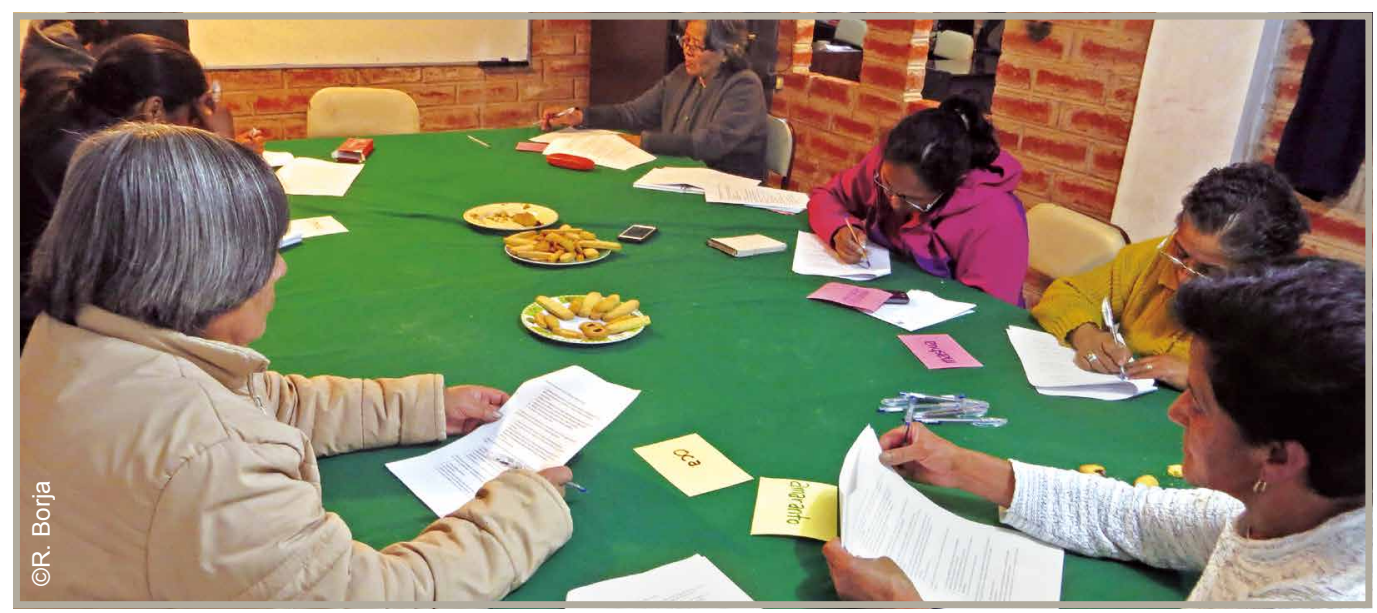




\subsection{Des conseils dirigés par les citoyens}

Dans plusieurs pays, les pouvoirs publics commencent à se rendre compte que la méthode descendante n'a pas été très efficace et n'a pas toujours proposé des solutions durables aux producteurs. D'autres actions novatrices sont mises en place à l'échelle locale pour améliorer l'engagement des citoyens. Les représentants locaux jouent le rôle d'intermédiaire dans les relations communautés-institutions publiques et sont souvent des atouts clés pour relever les défis de la durabilité.

\section{Yachachachiqs (Pérou)}

Selon le Recensement national de l'agriculture

36 2012) au Pérou, 10 pour cent des agriculteurs n'ont pas accès à des services de formation, de conseil ou d'assistance technique. Au vu de cet écart, les ONG développent les compétences et les capacités des leaders communautaires. Aujourd'hui, ces agriculteurs et responsables communautaires sont intégrés dans les services de vulgarisation pour le développement rural. Ils sont appelés Yachachachiq, ce qui se traduit en «celui qui enseigne» ou «l'enseignant». PRODERM (1986-1991) a été parmi les premiers projets de développement rural à inclure des paysans comme agents de vulgarisation. D'autres se sont appuyés sur la même approche dans les années 1990 et 2000. Les vulgarisateurs se sont concentrés sur l'inclusion des leaders paysans dans le partage des connaissances. Cet échange est devenu un vecteur d'autonomisation locale, comme en témoigne les communautés participant à ces projets qui ont pu co-créer le nouveau système de vulgarisation. Actuellement, le
MIDIS (Ministère du développement et de l'inclusion sociale) met en œuvre le programme Haku Wiñay («Ma Ferme Entrepreneuriale») avec des Yachachachiqs dans 40000 foyers répartis dans 19 régions du Pérou. En 2017, ils disposaient d'un budget annuel de 200 millions de PEN (environ 60 millions d'USD), soit le plus petit programme du MIDIS (équivalent à 4 à 5 pour cent du budget annuel total). Il traite des sujets suivants:

le renforcement des systèmes de production familiale;

> l'amélioration d'habitat sain;

- la promotion d'entreprises rurales inclusives;

la progression des compétences financières.

Source: Patricia Flores, IFOAM Organics International

Pour en savoir plus, visitez: http://intranet.foncodes.gob.pe/haku2016/ index.php/blog/yachachiqs 


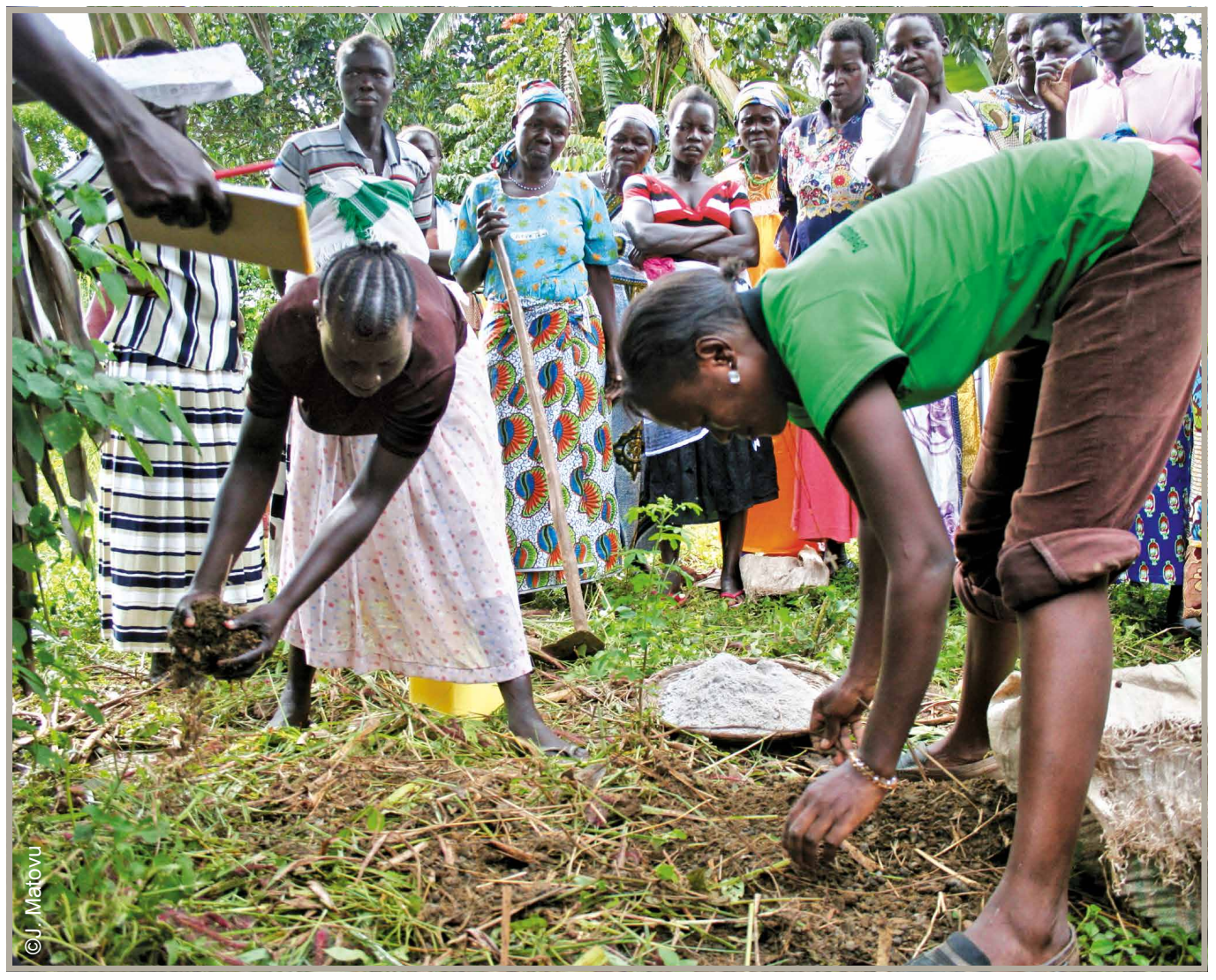

$$
\text { 塄 }
$$
nutritionnelle et en souveraineté alimentaire (Colombie)

L'École de gestion de la souveraineté alimentaire et de la sécurité alimentaire et nutritionnelle en Colombie promeut l'autonomie des communautés locales quant à la souveraineté alimentaire et à la sécurité alimentaire et nutritionnelle. L'école encourage le partage des connaissances et soutient la société civile, permettant ainsi à la communauté de participer à la formulation, à la mise en œuvre et au suivi des politiques alimentaires publiques. Le processus a commencé dans certaines zones urbaines autour de Bogota où les dirigeants locaux ont été identifiés et formés sur la manière d'identifier et de répondre aux besoins territoriaux et de s'appuyer sur les ressources locales. La formation d'animateurs permet également aux collectivités locales de s'impliquer dans la planification de développement territorial. Chaque session de formation suit une approche participative axée sur l'apprentissage par la pratique, permettant aux participants d'élaborer leurs propres propositions politiques.

Source: Andrea Moya, Claudia Helena Prieto Parque Temático en Salud Pública Chaquen, Subred Integrada de Prestación de Servicios de Salud 


\section{QUELLE STRATÉGIE VOUS CONVIENT LE MIEUX?}

Le partage de connaissances et d'expériences devrait être une activité essentielle des systèmes alimentaires durables, car ils évoluent, avec leurs défis et opportunités, constamment. La communication entre les acteurs du système alimentaire au sujet des pratiques de production durable peut également créer des occasions de résoudre certains défis rencontrés.

D

Identifiez vos principaux besoins en termes de connaissances et expérimentez avec différentes stratégies.

\begin{tabular}{ll}
$\begin{array}{l}\text { Vous voulez lancer une initiative durable et vous n'avez pas } \\
\text { beaucoup d'informations sur les pratiques agricoles durables }\end{array}$ & - Visites d'échange entre agriculteurs \\
\hline $\begin{array}{l}\text { Vous souhaitez favoriser le sentiment de communauté chez les } \\
\text { agriculteurs }\end{array}$ & $\begin{array}{l}\text { - Visites d'échange entre agriculteurs } \\
\text { - Des conseils dirigés par les citoyens }\end{array}$ \\
$\begin{array}{l}\text { Vous voulez améliorer la compréhension mutuelle entre les } \\
\text { différents acteurs du système }\end{array}$ & $\begin{array}{l}\text { - Recherche participative et } \\
\text { recherche-action }\end{array}$ \\
\hline $\begin{array}{l}\text { Vous voulez combler un manque de connaissances par une } \\
\text { approche ascendante qui favorise l'apprentissage collectif }\end{array}$ & $\begin{array}{l}\text { - Recherche participative et } \\
\text { recherche-action }\end{array}$ \\
$\begin{array}{l}\text { Vous souhaitez diffuser les connaissances sur les pratiques } \\
\text { agricoles durables auprès des acteurs de votre système }\end{array}$ & - Innovation interactive \\
\hline $\begin{array}{l}\text { Vous souhaitez profiter des opportunités existantes pour lier } \\
\text { l'agriculture durable aux biens publics }\end{array}$ & - Innovation interactive \\
\hline $\begin{array}{l}\text { Vous voulez intégrer la communauté locale dans les } \\
\text { processus de prise de décision sur le développement du } \\
\text { système alimentaire }\end{array}$ & - Des conseils dirigés par les citoyens \\
\hline
\end{tabular}

La collaboration multi-acteurs est un vecteur de succès pour ces approches. Identifiez les acteurs qui complémentent vos connaissances ou qui sont prêts à prendre un risque avec vous. Pensez non seulement à ceux déjà impliqués dans votre initiative, mais aussi à d'autres partenaires potentiels dans votre communauté locale.

N'oubliez pas:

si vous ne demandez pas, vous ne le saurez jamais !

\section{ASTUCE 22}

\section{Partager et co-créer les connaissances}

- Assurez-vous d'autoévaluer vos pratiques - cela vous aidera à mieux communiquer vos activités aux autres.

- Sachez comment accéder aux connaissances sur la production durable quand vous en avez besoin - c'est un étape clé !

- Il y a un large éventail de méthodes d'apprentissage et de co-création de connaissances. Sachez pourquoi vous recherchez des connaissances et choisissez ensuite la stratégie qui vous convient.

- La recherche et/ou l'expérimentation collective n'est pas seulement un moyen de co-créer des connaissances, c'est aussi un moyen de s'assurer que le contenu appris est aussi retenu. 


\section{LE CHEMIN D’APPRENTISSAGE, OÙ ALLER ENSUITE?}
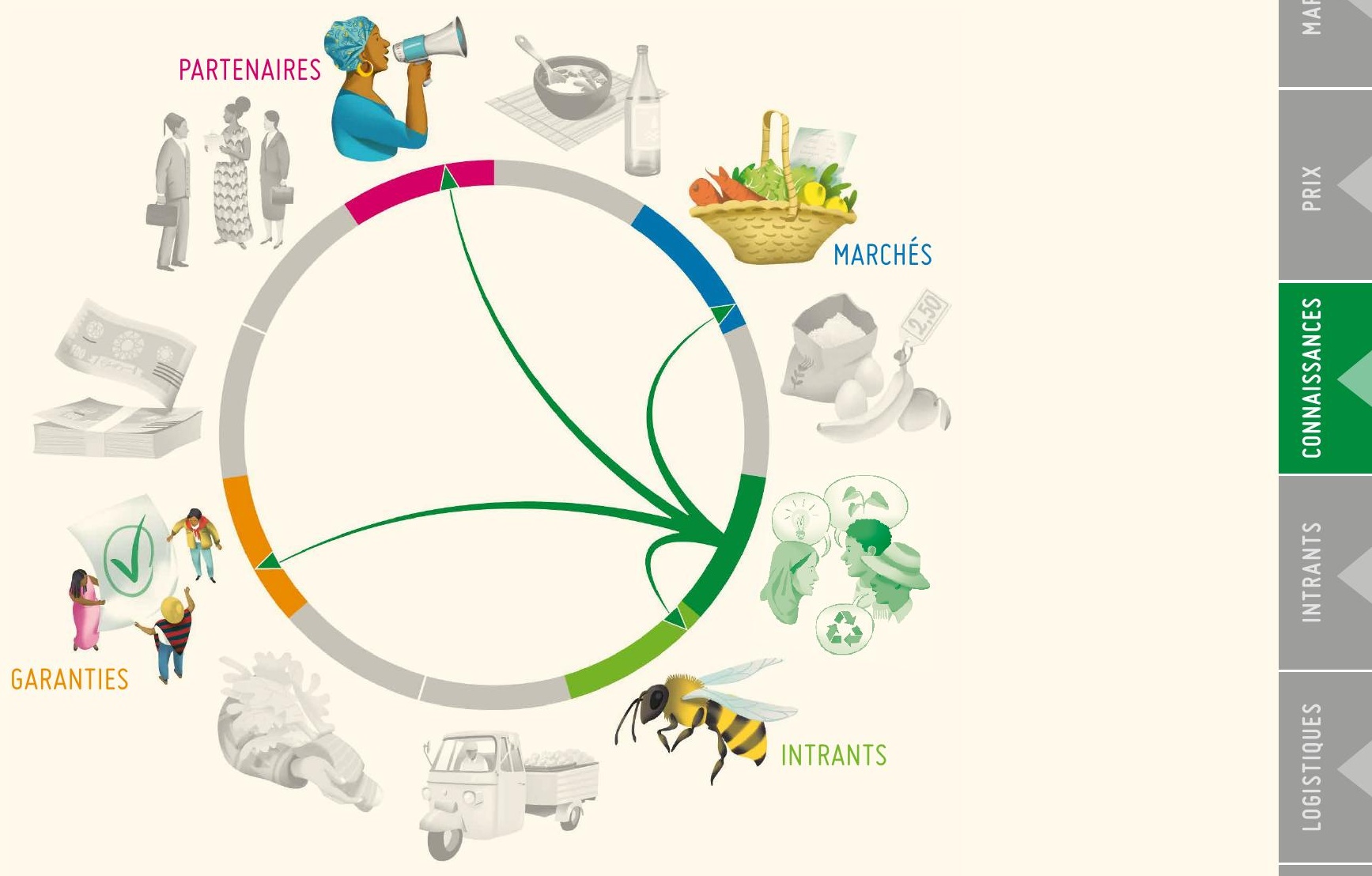

- Vous avez identifié un partenaire avec qui vous travaillez bien et avec qui vous souhaitez trouver des solutions à long terme ? Lisez le Chapitre 11: La place des partenaires et des plaidoyers.

- Vous avez autoévalué vos pratiques et vous voulez obtenir une certification ? Allez au Chapitre 8: Garantir des systèmes alimentaires durables.

- Vous avez introduit une nouvelle culture sur votre champ et voulez-vous savoir comment la vendre ? Jetez un coup d'œil au Chapitre 2: Connaître vos marchés.

- Vous voulez en savoir plus sur les intrants pour une production durable ? Continuez au Chapitre 5: Gérer et accéder aux intrants durables.

Ou peut-être vous avez besoin d'autres informations ? Choisissez un autre chapitre... 


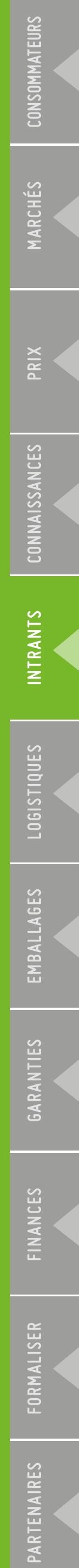


\section{POURQUOI EST-CE IMPORTANT?}

Les systèmes alimentaires durables doivent respecter la durabilité dans la façon dont les aliments sont produits et consommés, ce qui garantit aussi la durabilité des éléments utilisés pour les produire. En effet, lorsqu'ils essaient de s'assurer que les intrants dans leur système (matériaux et services) sont durables, ils font face à de nombreux défis tels que:

- l'indisponibilité d'intrants non-synthétiques dans de nombreuses zones rurales;

- la présence de produits contrefaits ou inefficaces, sans aucun moyen de tenir les entreprises responsables;

- des prix plus élevés, erratiques et non réglementés;

- le risque d'une éventuelle contamination par des OGM ou des substances actives et nonactives toxiques;

- la disparition d'une technologie autochtone efficace, en particulier pour conserver les semences;

- l'espace limité pour exploiter d'autres réseaux pour échanger des semences et des intrants non-conventionnels;

- le manque de recherche sur de nombreux intrants durables et solutions écologiques pour lutter contre les parasites et les maladies;

- le manque d'intégrité, de transparence et d'honnêteté de certains vulgarisateurs et intermédiaires chargés d'offrir des conseils sur les intrants et leur utilisation durable.

Accéder à des intrants durables est souvent un défi, principalement pour deux raisons:

a. Ils doivent être produits à la ferme, ce qui exige du temps et des ressources dont vous ne disposez peut-être pas en en quantité suffisante.

b. Ils doivent être achetés ou échangés avec d'autres, mais les fournisseurs locaux d'intrants ne proposent pas toujours des options durables. De plus, les réseaux d'échange ne sont peut-être pas encore établis dans votre localité.

En planifiant la production durable, réfléchissez clairement à la façon d'accéder puis de gérer l'utilisation des intrants durables. Considérez-les en relation avec les autres activités de votre système agricole et alimentaire. 


\section{DE QUELS INTRANTS AVEZ-VOUS BESOIN POUR CULTIVER DES ALIMENTS DURABLES?}

Les intrants sont les matériaux et les services (y compris écosystémiques et sociaux) nécessaires à la production alimentaire (et autres produits agricoles). Ils sont considérés comme les ressources et les services nécessaires à l'agriculture écologique et qui contribuent à la durabilité de l'agroécosystème et du système alimentaire.

Elles sont réparties en six catégories principales:

\section{Tableau 4: Types d'intrants}

\begin{tabular}{l|l}
\hline Types d'intrants & Exemples \\
\hline $\begin{array}{l}\text { Qui doit consacrer son temps } \\
\text { et ses connaissances à la } \\
\text { production? }\end{array}$ & $\begin{array}{l}\text { Connaissances et compétences des agriculteurs, main d'œuvre supplémentaire } \\
\text { (familiale, embauchée, partagée, etc.), services vétérinaires, services de } \\
\text { maçonnerie, services de vulgarisation. }\end{array}$ \\
\hline $\begin{array}{l}\text { Que faut-il cultiver, collecter } \\
\text { ou recycler? }\end{array}$ & $\begin{array}{l}\text { Aliments pour animaux, fumier animal, fourrage collecté ou récolté, plantes } \\
\text { médicinales, races animales, semences, matériel végétal, plantes pour cultures } \\
\text { intercalaires, plantes comme insectifuges. }\end{array}$ \\
\hline $\begin{array}{l}\text { Quels sont les outils } \\
\text { nécessaires pour produire } \\
\text { durablement? }\end{array}$ & $\begin{array}{l}\text { Outils de plantation (p. ex. talles, tracteurs, traction animale, engrais et fumier), } \\
\text { outils de récolte (p. ex. paniers, filets, machines à couper), outils de protection } \\
\text { des plantes (p. ex. vêtements de protection, bio-insecticides), matériaux de } \\
\text { construction pour structures agricoles (p. ex. ciment, toiture, bois). }\end{array}$ \\
\hline $\begin{array}{l}\text { À quel type de terrain avez- } \\
\text { vous accès? }\end{array}$ & $\begin{array}{l}\text { Parcelles communautaires, terres louées, terres individuelles, terres } \\
\text { communales pastorales, toits, terres contractées, étangs naturels ou artificiels, } \\
\text { littoral. }\end{array}$ \\
\hline $\begin{array}{l}\text { Quels services et conditions } \\
\text { écologiques et biologiques } \\
\text { sont nécessaires? }\end{array}$ & $\begin{array}{l}\text { Facteurs environnementaux appropriés (humidité, température), micro- } \\
\text { organismes autochtones, flore et faune du sol, pollinisateurs, eau, } \\
\text { micronutriments et macronutriments issus des cycles naturels, énergie/ } \\
\text { électricité, animaux intégrés dans le système de production pour fertiliser } \\
\text { naturellement, aérer les sols, etc. }\end{array}$ \\
\hline $\begin{array}{l}\text { Que faut-il pour } \\
\text { communiquer sur ce qui se } \\
\text { fait à la ferme? }\end{array}$ & $\begin{array}{l}\text { Services de certification, services d'enregistrement des exploitations agricoles, } \\
\text { services de communication, services de commercialisation. }\end{array}$ \\
\hline
\end{tabular}

\section{Production à la ferme ou hors ferme?}

Les intrants nécessaires pour produire de façon durable peuvent provenir de la ferme elle-même (à la ferme) ou de l'extérieur de la ferme (hors ferme). La production de vos propres intrants est souvent le moyen le plus rentable et le plus durable d'accéder aux services écologiques et biologiques. En recyclant les nutriments au sein de l'exploitation agricole ou de l'agroécosystème, vous pouvez réduire la perte de nutriments qui se produit parfois lorsque vous utilisez des intrants externes.

Cependant, votre ferme ne dispose toujours pas de tous les éléments nutritifs, de la technologie ou de la main-d'œuvre nécessaires pour atteindre la productivité souhaitée. À travers le monde entier, d'autres modèles d'entreprises émergent, qui offrent des moyens intéressants d'accéder à ces intrants. Parmi des exemples, il y a des start-ups qui fournissent directement des nouvelles technologies ou des services aux agriculteurs, ou des systèmes d'échange communautaires pour partager les intrants, les technologies et le travail. 


\section{DANS QUELLE MESURE VOS INTRANTS SONT-ILS DURABLES ET COMMENT LES RENDRE PLUS DURABLES?}

Tout comme il est important d'autoévaluer la durabilité de votre système de production, vous pouvez faire de même avec vos intrants. Pour commencer, notez, pour chacune des catégories ci-dessus, les intrants dont vous aurez besoin pour lancer votre projet agricole, pour réhabiliter des terres abandonnées ou pour convertir un système conventionnel en un système durable.

Après avoir dressé la liste de tous les intrants possibles, évaluez chacun que vous prévoyez avoir, que vous avez actuellement ou que vous pourriez manquer. Un bon exercice consiste à réfléchir au caractère durable ou non de chaque intrant et à l'évaluer par rapport à ce qu'il devrait être - l'état souhaitable. Enfin, notez les actions pratiques qui vous permettront de progresser vers une solution plus durable pour accéder aux intrants.

Le tableau suivant peut servir de modèle:

Tableau 5: Un modèle pour catégoriser les intrants

\begin{tabular}{|c|c|c|c|}
\hline \multirow{2}{*}{$\begin{array}{l}\text { Catégories d'intrant } \\
\text { Intrant }\end{array}$} & \multicolumn{2}{|c|}{ Caractéristiques de l'intrant } & \multirow{2}{*}{\begin{tabular}{|l} 
Recommandations \\
Mesures à prendre pour \\
progressivement atteindre la \\
durabilité
\end{tabular}} \\
\hline & Durable (souhaitable) & $\begin{array}{l}\text { Insoutenable } \\
\text { (indésirable) }\end{array}$ & \\
\hline Semences & $\begin{array}{l}\text { Des taux de germination } \\
\text { élevés, peuvent } \\
\text { être sauvegardés et } \\
\text { réensemencés, peuvent } \\
\text { satisfaire un profil de } \\
\text { qualité souhaité (goût, } \\
\text { couleur, taille), accès } \\
\text { facile. }\end{array}$ & $\begin{array}{l}\text { Faible germination, non } \\
\text { reproductible, ne peut } \\
\text { être sauvegardée et } \\
\text { réensemencée, profil } \\
\text { de qualité souhaité, } \\
\text { coût élevé, accès } \\
\text { difficile. }\end{array}$ & $\begin{array}{l}\text { Collaborer avec d'autres } \\
\text { agriculteurs pour organiser un } \\
\text { échange de semences, négocier } \\
\text { la disponibilité de semences } \\
\text { de meilleure qualité avec le } \\
\text { fournisseur local, établir des } \\
\text { liens avec les distributeurs de } \\
\text { semences certifiées. }\end{array}$ \\
\hline Engrais & $\begin{array}{l}\text { Animaux et compost } \\
\text { intégrés dans un système } \\
\text { de production mixte } \\
\text { capable de suffisamment } \\
\text { maintenir un microbiome } \\
\text { de sol sain. }\end{array}$ & $\begin{array}{l}\text { Épandage excessif } \\
\text { d'engrais synthétiques } \\
\text { achetés et } \\
\text { inaccessibilité au } \\
\text { fumier ou au compost } \\
\text { d'origine animale. }\end{array}$ & $\begin{array}{l}\text { Introduire les animaux dans le } \\
\text { système de production, composter } \\
\text { les déchets issus de la cuisine } \\
\text { et de la ferme, collaborer avec } \\
\text { d'autres agriculteurs pour accéder } \\
\text { à des engrais qui sont appliqués } \\
\text { pour équilibrer le microbiome. }\end{array}$ \\
\hline Terrain & $\begin{array}{l}\text { Obtenir le droit de } \\
\text { posséder ou d'utiliser } \\
\text { et d'apporter des } \\
\text { modifications pour le } \\
\text { présent et l'avenir. }\end{array}$ & $\begin{array}{l}\text { Pas d'accès garanti, } \\
\text { menaces pour la } \\
\text { sécurité, pas facilement } \\
\text { accessible, pas la } \\
\text { bonne superficie; } \\
\text { qualité de sol faible. }\end{array}$ & $\begin{array}{l}\text { Si vous squattez sur un terrain, } \\
\text { essayez de l'enregistrer. Si } \\
\text { possible, achetez un terrain } \\
\text { loué ou assurez-vous que le } \\
\text { contrat de location inclut au } \\
\text { moins trois saisons. Parlez avec } \\
\text { la communauté afin de mettre } \\
\text { en place un système sûr pour la } \\
\text { culture ou l'utilisation durable des } \\
\text { terres communautaires. }\end{array}$ \\
\hline $\begin{array}{l}\text { Maintenant, à vous } \\
\text { d'essayer... }\end{array}$ & & & \\
\hline
\end{tabular}




\section{OPTIONS POSSIBLES: EXAMINER DIFFÉRENTES FAÇONS DE SE PROCURER DES INTRANTS}

Une fois que votre liste d'intrants souhaités est établie, déterminez comment les accéder de façon durable sur votre exploitation. Dans cette section, nous présentons des exemples de stratégies novatrices de se procurer une gamme d'intrants.

Rappelez-vous qu'une même approche n'est pas convenable pour toutes les situations. D'après notre expérience, de nombreuses solutions pour améliorer l'approvisionnement et la gestion des intrants prennent racine dans de bonnes intentions. Cependant, une fois mises en œuvre, ces solutions présentent de nombreux défis pour les petits agriculteurs. L'une des principales préoccupations consiste à déterminer si le coût de l'intrant l'emportera sur les avantages potentiels de production ou si la façon d'accéder à l'intrant ou de le gérer s'inscrit bien dans votre agenda quotidien. Lorsque vous élaborez votre stratégie d'approvisionnement, réfléchissez bien aux avantages et aux inconvénients de chaque innovation.

\subsection{Temps ou main d'œuvre}

\subsubsection{Partager le travail plutôt que de le payer: les banques de temps}

Il n'y a aucun doute que le maintien d'une ferme durable est un effort d'équipe, qui repose fortement sur le partage du travail. Il a été prouvé que le fait d'être impliqué dans un système qui fournit de l'aide aux agriculteurs et aux étudiants hors-ferme s'est avéré utile pour soutenir une petite exploitation.

\section{Le partage du travail (Inde)}

Dans l'Himachal Pradesh, en Inde, dans un grand nombre de villages, il existe un système informel de partage du travail appelé Jawari. Il s'agit d'un système autonome qui fonctionne de manière autosuffisante dans une communauté close basée sur des réseaux interpersonnels et sur des relations de confiance. Dans ce système, un petit propriétaire peut faire appel à un Jawari pour terminer tout travail à la ferme ou à l'extérieur (comme la construction d'une nouvelle étable). En retour, l'agriculteur est obligé de répondre aux demandes de partage du travail d'autres petits agriculteurs. Il n'y a pas d'échange monétaire dans ce système reposant sur la confiance et la réciprocité.
Le petit paysan accueille la main-d'œuvre en tant qu'invité sur sa ferme et, si nécessaire, s'occupe de sa nourriture et de son logement durant la période de travail.

\section{Avantage}

* Lorsque le flux de trésorerie est faible, vous pouvez toujours avoir accès à de la maind'œuvre supplémentaire quand vous en avez besoin.

Inconvénient

- Accueillir la main-d'œuvre chez soi peut être un défi personnel/social.

Source: Ashish Gupta, Jaivik Haat 


\subsection{Matières premières pour la production}

\subsubsection{Engrais non agricoles}

Lorsque les besoins en engrais dépassent la capacité de les produire à la ferme, de nouveaux modèles d'affaires doivent se construire pour y répondre.

䌟

(39)
Des engrais sains à base de déchets de restaurants (Colorado, États-Unis)

Wastefarmers est une entreprise certifiée B Corp à Arvada, Colorado, qui se spécialise dans la fabrication d'engrais sains pour le sol à partir du gaspillage récupéré des restaurants, des entreprises et des écoles. Ces engrais organiques sont rassemblés auprès de ces organismes et transportés dans une «brasserie microbe» pour être compostés puis vendus aux agriculteurs. La brasserie microbe et la ferme où elle est située servent de lieux d'activités éducatives et de visites gratuites pour des milliers de personnes chaque année. Les employés ont droit à du temps rémunéré à la ferme et à des possibilités de formation. Les $B$ Corps sont des entreprises à but lucratif certifiées par l'organisation sans but lucratif (OSBL), B Lab, pour répondre à des normes rigoureuses de performance sociale et environnementale, de responsabilité et de transparence.

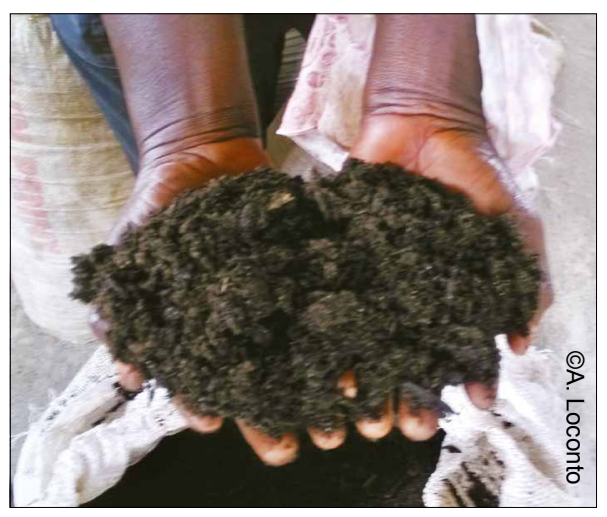

\section{Avantages}

- Les petits agriculteurs ont du mal à créer et à gérer de grandes quantités de compost, même si la biomasse est disponible, car la main-d'œuvre est exigeante. Il existe très peu d'outils abordables et utilisables par les agriculteurs. La création d'entreprises capables de produire de grandes quantités d'engrais organiques est un bon moyen de réduire les déchets urbains et industriels.

+ Il crée des emplois supplémentaires dans les zones rurales et urbaines afin de fournir un service qui réduit le stress sur les décharges et augmente l'utilisation du compost organique.

+ Le modèle d'entreprise responsable et les conditions de travail offrent un modèle d'entreprise équitable pour la durabilité.

\section{Inconvénients}

- Des investissements importants dans l'infrastructure (p. ex. routes, grandes usines de transformation) sont nécessaires pour assurer le transport de déchets et d'engrais.

- La collaboration avec les acteurs publics est nécessaire pour autoriser et coordonner l'achat ou le don de déchets qui peuvent être collectés dans les bâtiments publics et éventuellement dans les décharges.

\section{Source: https://www.wastefarmers.com}

Pour en savoir plus, visitez: https://www.wastefarmers.com http://www.bcorporation.net/community/ waste-farmers 


\subsubsection{Production d’énergie à la ferme}

Un certain nombre de nouvelles technologies à petite échelle apparaissent dans le monde entier pour transformer les déchets agricoles en énergie.

\section{Husk Power Systems (Inde)}

La mission de «Husk Power Systems» est de créer un écosystème autosuffisant dans les villages ruraux et les fermes en Inde qui n'ont pas un accès régulier à l'électricité. Cette petite entreprise a développé un générateur électrique équipé de instruments à gaz de la biomasse qui roule à base d'écorces de riz inutilisées. En produisant de l'électricité, un générateur seul peut alimenter jusqu'à 600 familles. Les usines pilotes de Husk Power Systems ont été rentables pendant six mois, de sorte que son modèle a attiré des investissements orientés vers la croissance.

\section{Avantage}

+ Un excellent moyen de recycler les déchets organiques et de fournir l'électricité en milieu rurale.

\section{Inconvénient}

- Le coût de la machine est élevé et sa mise en œuvre dans les fermes rurales peut être compliquée.

Source: www.huskpowersystems.com/innerPage. php?pageT=Community\%20Impact\&page_id=81

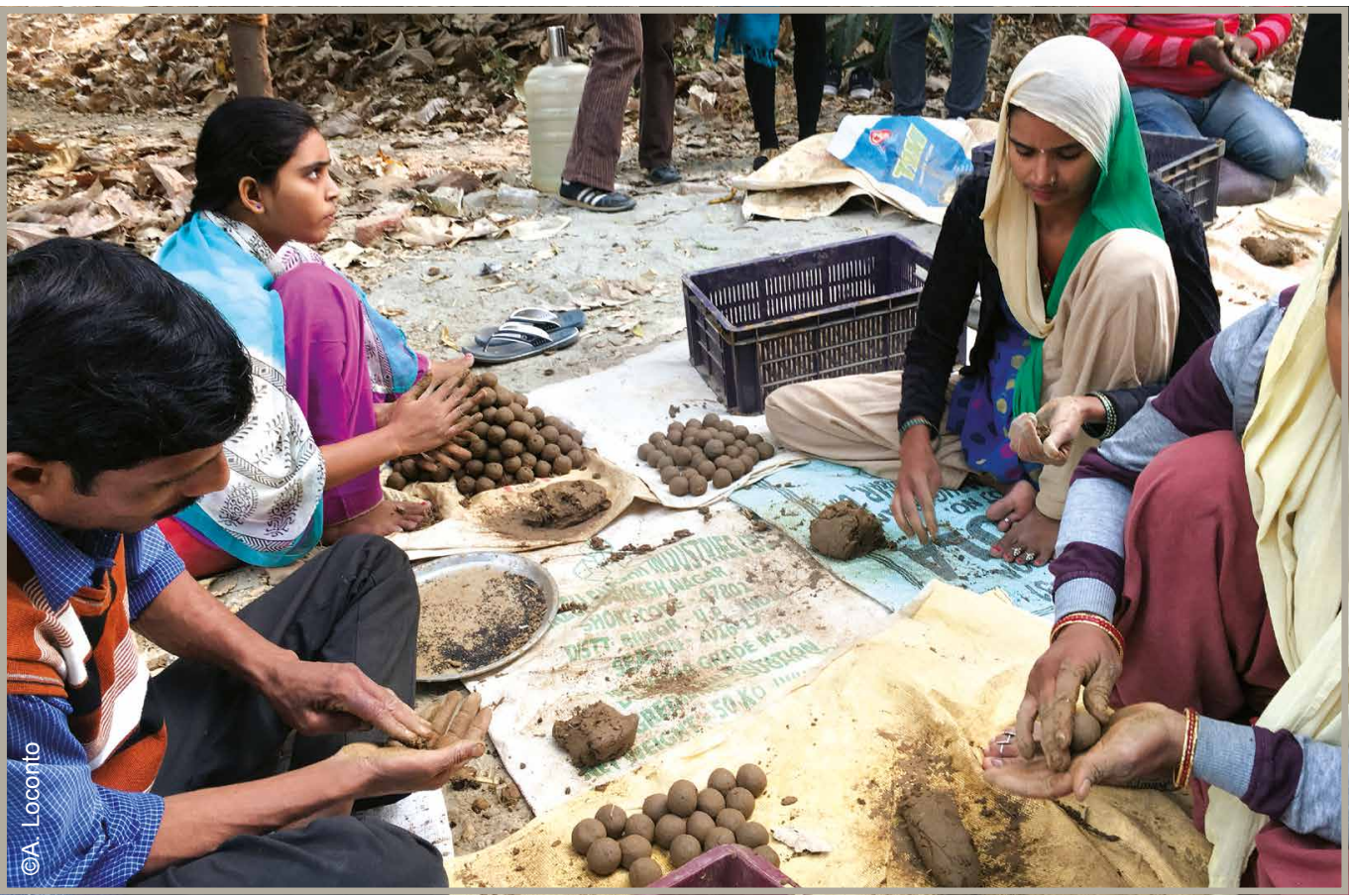




\subsection{3. Échanges de semences}

Dans de nombreux pays, les systèmes officiels de distribution de semences certifiées sont dominants. Ils fournissent systématiquement des informations sur la qualité et l'utilisation des semences. Cependant, de nombreuses variétés correspondant aux régimes alimentaires traditionnels (et innovateurs) ou qui résistent à certains parasites ou à la sécheresse ne font pas partie de ces systèmes. Celles-ci ont typiquement été conservées à la ferme. Les échanges traditionnels de semences ainsi que les techniques innovantes utilisées pour les conserver sont présentes à travers le monde entier.

\section{学 \\ Les mères porte-graines (Inde)}

Pour une communauté de petits agriculteurs dans l'État d'Odisha en Inde, la trousse agricole conventionnelle constituée de semences certifiées, d'engrais chimiques et de pesticides, de petites pompes d'irrigation, de machines post-récolte et d'outils agricoles ne s'est pas avéré durable. De ce fait, un groupe de femmes tribales que l'on nomme les «mères porte-graines», se spécialise dans l'identification, la collecte et la multiplication des semences pour l'ensemble de la communauté. Leur système cartographie la biodiversité des petites exploitations agricoles du village et identifie les semences adaptées à chacune d'elles, multiplie les semences et les partage avec les autres agriculteurs, et suit des procédures de sélection des semences de lignée pure. Le modèle multiplicatif exige que les familles cultivent entre 13 et 467 variétés de semences de millets, légumineuses, légumes secs, légumes verts, riz paddy et tubercules. Des foires d'échange et de distribution de semences ont régulièrement lieu avec les mères porte-graines qui offrent des conseils aux agriculteurs quant à la sélection de semences, les méthodes appropriées de stockage et la gestion des cultures. Les mères porte-graines traitent les semences comme si celles-ci étaient leurs propres enfants. En conséquence, au total, 5321 ménages ont enrichi leurs jardins de basse-cour, produisant de la nourriture disponible entre 6 et 11 mois par an. Le projet remet en question les notions considérées "acquises» de l'agriculture conventionnelle et encourage de nouvelles techniques de sélection biologique et participative.

\section{Avantages}

+ La diversité des variétés augmente la biodiversité et la résilience de l'agroécosystème ;

* La capacité d'échanger des semences plutôt que de les acheter réduit le coût des intrants pour les agriculteurs ;

+ La variété des cultures et des semences cultivées augmente la disponibilité de nourriture tout au long de l'année, qui entraine un effet positif sur la sécurité alimentaire.

\section{Inconvénients}

- Les banques de semences et les systèmes d'échange communautaires doivent être très vigilants quant à la qualité des semences qu'ils échangent. Il est important que chaque communauté ait une personne capable de vérifier la qualité des semences.

Source: Organisation pour la reconstruction rurale et les activités intégrées de services sociaux (ORRISSA).

Pour en savoir plus, visitez:

http://www.fao.org/3/a-bl924e.pdf 


\section{ASTUCE 23}

Établir votre propre banque de semences

Consultez ce manuel d'animateur:

Les Banques de Semences Communautaires

http://www.fao.org/3/a-i3987f.pdf
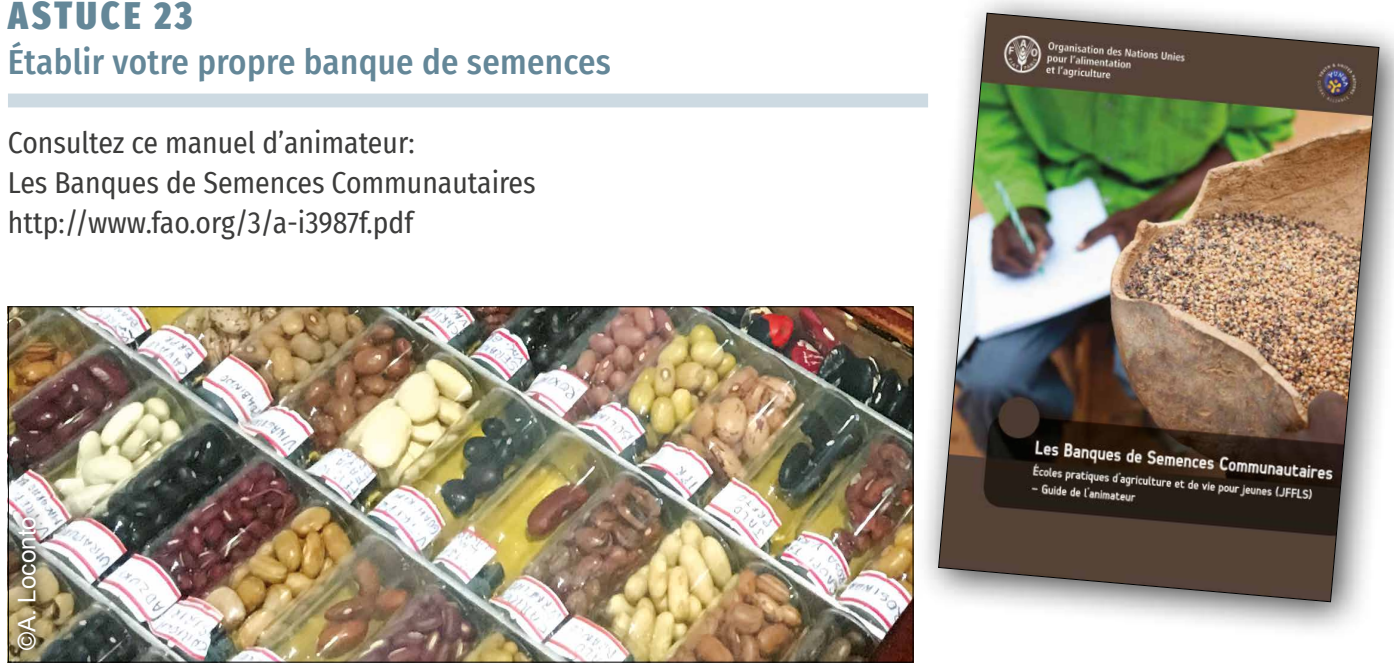

\section{M-Farm (Kenya)}

L'accès aux marchés environnants pour les

stocks, les semences et les idées inspirantes pour les pratiques agricoles est un élément clé de l'agriculture durable. M-farm est une start-up qui vise à relier les petits agriculteurs aux marchés environnants en leur fournissant des informations sur les prix en temps réel. Cette application pour smartphone partage des informations sur les prix des cultures dans quatre zones de marché au Kenya: Nairobi, Kisumu, Eldoret et Kitale. L'application permet également aux agriculteurs d'acheter des semences en utilisant M-Pesa (le système monétaire mobile du Kenya).

\section{Avantages}

+ M-farm aide les agriculteurs à acheter et à vendre leurs récoltes plus efficacement, ce qui rend leur agriculture plus durable à long terme. De plus, l'application pour smartphone offre un mode de communication innovant.

\section{Inconvénients}

- Puisque cette start-up est relativement récente, la preuve de sa durabilité est limitée. De plus, il existe de nombreuses applications de ce type, mais il est difficile de mesurer leurs impacts sur la vie quotidienne des petits agriculteurs sans augmenter le coût de leurs intrants. Il est donc utile d'avoir accès à ces initiatives et bien d'autres, mais il faut trier celles qui fonctionnent en conservant les coûts d'intrants stables.

- Vous devez disposer d'un smartphone et d'un accès à l'internet. M-Farm est également lié à M-Pesa, donc vous devez déjà être inscrit à ce service.

Source: Site web de M-Farm: https://www.mfarm.co.ke

Pour en savoir plus, visitez: https://www.mfarm.co.ke 


\subsection{Outils et machines}

Il est difficile de respecter la durabilité si tous les aspects de la production et de la transformation sont effectués manuellement. Plusieurs outils et machines ont grandement contribué à mécaniser certains processus de production durable. Souvent, les problèmes auxquels sont confrontés les petits exploitants agricoles sont liés aux difficultés d'accès à la taille d'outil appropriée à leur exploitation. Pour faciliter l'accès, voici quelques suggestions:

- réduire les coûts de production, créez des outils et des machines gérés par la communauté et partagés par tous les membres;

- formez les artisans locaux à la réparation ou à la modification des équipements en fonction de l'environnement;

- créez des machines ou des pièces de rechange essentielles pour les véhicules motorisés qui peuvent être la propriété collective de la coopérative;

- créez un magasin local de location d'équipement pour louer des petites machines ou d'autres outils;

- créez des petites entreprises de services agricoles qui peuvent être embauchées pour effectuer des activités de labourage, de désherbage, d'arpentage ou de récolte.

\subsubsection{Traction animale}

Investir dans les méthodes/ intrants de traction animale est avantageux si le champ se trouve principalement dans des zones semi-humides/semi-arides et dans les hauts-plateaux. Cependant, avec la mécanisation, cette forme de traction devient de moins en moins intéressante. Cela peut être dû à de nombreuses complications associées à la santé de l'animal, à l'entraînement et à l'acquisition des outils appropriés pour la mécanisation à l'aide d'animaux. Toutefois, vous trouverez ci-dessous certains avantages associés à ce type d'intrant:

> utilisée correctement, elle peut augmenter la productivité;

- la traction animale s'accompagne d'une technologie peu coûteuse et non-dangereuse pour l'environnement;

- c'est un type de technologie qui peut être acquis localement;

- elle n'exige pas de changements radicaux aux systèmes agricoles;

- elle est très efficace pour les petits agriculteurs;

- le fumier produit par l'animal en marchant dans le champ est un excellent engrais.

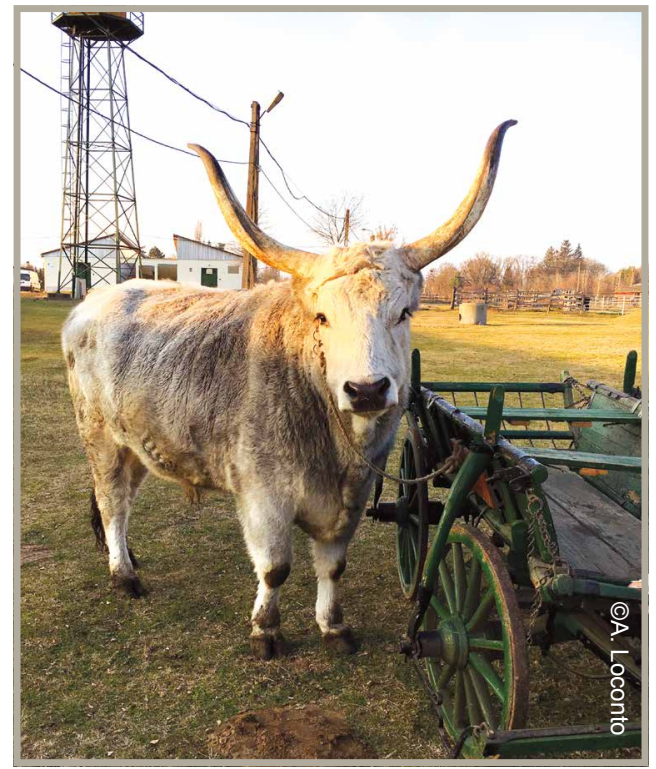




\section{Anicytor, un moteur d'énergie
营
verte des outils agricoles \\ (43) (Éthiopie)}

Les outils traditionnels ne comblent pas l'écart technique/d'ingénierie nécessaire pour répondre aux besoins technologiques des agriculteurs. Le changement climatique a des répercussions mondiales et des paysages entiers sont détruits à cause de l'utilisation inappropriée des outils agricoles traditionnels et à l'expansion des terres agricoles. Pour résoudre ces problèmes, la traction animale se transforme en «pédalage» animal, donnant lieu à une technologie efficace à base d'énergie verte et à forte intensité de main-d'œuvre pour les pays en développement. Les animaux ont des pattes puissantes: les pattes avant sont entrainées par l'inertie et le rapport de masse corporelle, tandis que les pattes arrière sont plus hautes et musclées, générant la force requise. Lorsque l'animal marche, cette énergie potentielle est transformée en énergie cinétique. Ainsi, en attachant des leviers et des engrenages aux pattes de l'animal, le mouvement avant-arrière et l'énergie cinétique sont transformés en une rotation complète (360 degrés). Il s'agit d'un moteur mécanique qui peut entraîner des roues et d'autres outils agricoles montés - d'où le nom scientifique d'«anicytor»: Tracteur à vélo/à pédales pour animaux. L'innovation est compatible avec tout animal à quatre pattes et avec l'homme. Sa taille et sa hauteur sont ajustables en fonction de la taille de l'animal et de l'usage prévu.

\section{Avantages}

+ Puisqu'elle n'utilise pas de combustible et elle est fabriquée de matériaux disponibles localement, elle réduit le taux de gaspillage et de pollution. elle peut être utilisée pour le nettoyage des rues et la collecte des déchets;

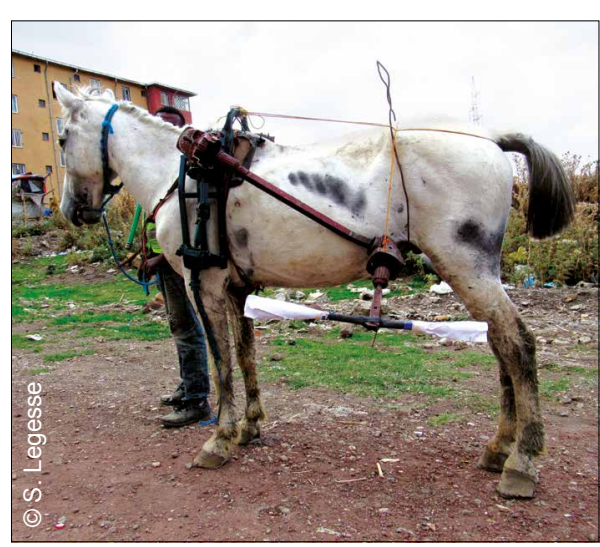

+ elle peut être fabriquée à partir de matériaux disponibles localement pour remplacer les tracteurs importés;

+ vu que la plupart des agriculteurs dans les pays en développement ont des besoins technologiques, ils sont le groupe cible de la traction animale;

+ des possibilités d'emploi doivent être créées en rendant l'agriculture intéressante et plus accessible pour les jeunes ruraux,la traction animale peut créer des nouveaux emplois pour la construction de ses pièces détachées, l'assemblage, la vente en gros, la vente au détail, et la prestation de services en utilisant cet outil.

\section{Inconvénients}

- Lors de son usage, il est nécessaire de s'assurer que les animaux sont traités éthiquement et sans cruauté.

Source: Sitotaw Legesse, Sitotaw et Yeshi Engineering Share Cop, Éthiopie

Pour en savoir plus, visitez: https://agriprofocus.com/profile/sitotaw. legesse.22606 


\subsubsection{Outils portatifs pour les petits exploitants}

Les petits exploitants travaillent principalement manuellement. Au fil du temps, des outils et des instruments agricoles ont été développés pour diluer l'aspect laborieux, en particulier pour les agricultrices. Par exemple, dans les rizières, le repiquage et le désherbage sont des activités essentielles de la culture, pour lesquelles les agricultrices éprouvent souvent des difficultés. Dans les montagnes, où la terre est aménagée en terrasses et la superficie des terres cultivables est non-uniforme, cette tâche est encore plus difficile à accomplir. Il est donc important que les petits exploitants disposent d'outils abordables et appropriés, conçus de manière ergonomique, efficaces en termes de rendement et légers à transporter.

\section{Agrovision - des outils manuels pour les agriculteurs (Inde)}

44 Dans l'est de l'Inde, une entreprise fabrique des outils manuels spécialisés pour les agriculteurs familiaux. Dans le pays, il y a un certain nombre de forgerons et de fabricants d'outils qui travaillent à différents niveaux locaux. Mais peu d'entre eux travaillent avec des outils manuels innovants. L'entreprise met à disposition à titre gratuit ses outils et ses designs. Elle encourage également les artisans locaux à adapter les outils aux conditions locales, ce qui rend les outils complètement open source. Souvent, il arrive que les outils créés pour les sols argileux lourds ne fonctionnent pas effectivement pour les sols sableux, d'où la nécessité de prendre en compte l'environnement agricole local dans la conception d'outils. Parmi les outils les plus populaires fabriqués par Agrovision sont ceux liés à la culture du riz paddy tels que Cono et Paddy Weeder, généralement utilisé par les agriculteurs qui innovent dans leur système de production en appliquant le Système d'intensification du riz. De plus, des outils tels que les semoirs multiples, les désherbeurs à terre sèche, les houes rotatives avec accessoires sont également utilisés. En général, ces outils sont efficaces, abordables et adaptables aux besoins locaux.

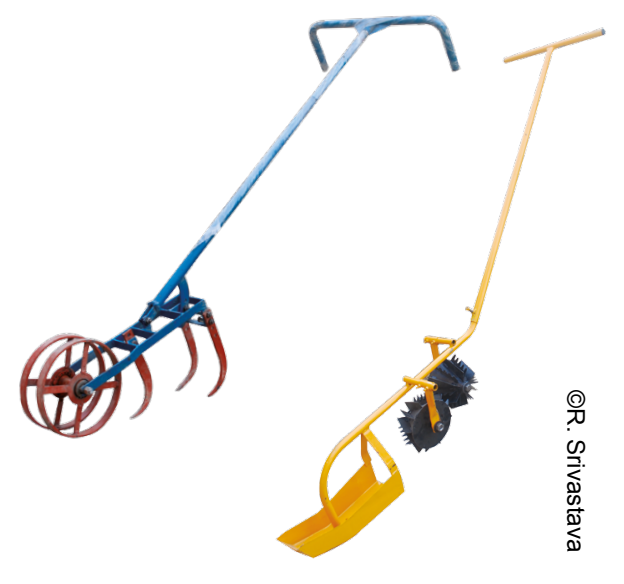

Avantages

+ Les outils sont open source et facilement adaptables;

+ leur poids léger les rend plus faciles à utiliser pour les femmes;

+ ils permettent de mécaniser la production à un coût relativement faible.

\section{Inconvénients}

- Ce sont encore des outils à main, ce qui signifie qu'un minimum de travail laborieux est nécessaire.

Source: Ashish Gupta, Jaivik Haat 


\section{ASTUCE 24}

Obtenir des solutions/des machines durables

- essayez la technologie vous-même et proposez une option réalisable.

- lancez un processus participatif qui permet aux membres de la communauté de proposer des solutions.

- achetez des outils manuels ou mécanisés sur le marché, bien que cela puisse augmenter les risques associés à la qualité, à la disponibilité et au coût.

- cherchez des technologies qui s'adaptent à vos conditions climatiques changeantes et aux répercussions des intrants après la récolte.

\subsection{Améliorer les services écosystémiques et biologiques}

\subsubsection{Le bio-charbon pour augmenter la matière organique du sol}

Bien qu'il s'agisse d'un processus lent, ajouter du bio-charbon au sol peut augmenter sa part de matière organique, le rendant plus fertile et plus résistant aux changements climatiques.

\section{尊 Wanakaset Network (Thaillande)}

P. Yai, membre du réseau Wanakaset, distille des huiles essentielles issues d'herbes et de fruits. Étant donné que les membres de Wanakaset pratiquent l'agroforesterie, ils ont une grande quantité de branches et de bâtons. P. Yai utilise un poêle à biomasse qui convertit efficacement cette biomasse en chaleur. En produisant très peu de fumée, la chaleur fait bouillir les cuves de distillation pendant les nombreuses heures nécessaires pour récupérer toutes les huiles essentielles.

Green Net traite les noix de cajou biologiques qui doivent être étuvées (pour ouvrir les coquilles) et séchées. Ensuite, les coquilles de noix de cajou sont utilisées comme combustible pour un four à biomasse qui produit la chaleur et la vapeur pour ce processus. Cela réduit considérablement le besoin et l'utilisation de gaz naturel ou d'électricité. Un certain nombre de rizeries plus modernes produisent de l'énergie à partir d'écorces de riz moulues pour alimenter leurs moulins. Ces écorces se transforment en charbon/cendre qui est ensuite utilisé comme un bon amendement au sol (comme le biocharbon) ou vendu aux fabricants de puces informatiques (puisque on y trouve du silicium de haute qualité).

\section{Avantages}

+ Une utilisation fiable des déchets;

+ un moyen efficace de recycler les nutriments dans les écosystèmes;

* facile à utiliser pour les agriculteurs et c'est un équipement avantageux à avoir dans un système agricole.

\section{Inconvénients}

- Les poêles à bio-charbon plus grands et plus efficaces sont souvent inaccessibles pour les petits agriculteurs, quant à leurs coûts et exigences d'entretien. Cependant, de nombreux petits agriculteurs produisent et utilisent différentes formes de biocharbon avec succès. En Asie du Sud-Est, les unités courantes de fabrication artisanale produisent du charbon de bois (utilisé comme combustible), du vinaigre de bois (utilisé comme bio-pesticide) et du biocharbon (petits morceaux) utilisés dans le compost et les terreaux.

Source: Michael Commons, GreenNet 


\subsubsection{Services de lutte antiparasitaire intégrée (LAI)}

Cependant, pour éviter l'utilisation de pesticides et d'herbicides, un système intégré qui «combat la nature avec la nature» présente une solution viable.

Dudutech est une entreprise qui fournit des insectes et des champignons bénéfiques aux agriculteurs afin de réduire leur utilisation d'insecticides et de pesticides qui stressent les cultures et qui sont non-durables. Ces intrants biologiques (champignons, insectes, bactéries) combattent les organismes nuisibles de manière mutuellement bénéfique pour les deux parties. Par exemple, le mycorhize, un champignon qui lutte contre la sècheresse, forme une relation symbiotique avec les plantes en échangeant de l'eau et de l'engrais contre de la sève végétale et du sucre. Le mycorhize augmente considérablement le volume efficace des racines, ce qui réduit le stress des plantes et leurs permet de continuer à pousser en période de sécheresse. Dudutech approvisionne des paquets d'intrants biologiques aux agriculteurs du Kenya et d'Afrique du Sud, en offrant une gamme de 17 intrants différents.

\section{Avantages}

+ Cette entreprise a plus de 100 employés qualifiés dans une région rurale et agricole du Kenya.

+ La capacité d'embaucher un expert en lutte antiparasitaire intégrée (LAI) pour desservir la ferme permet aux agriculteurs d'avoir accès à des connaissances sur la LAI qu'ils n'ont peut-être pas encore acquises.
Le site web de cette entreprise est très utile et il offre des suggestions sur le paquet à utiliser pour des problèmes spécifiques. Par exemple, pour un cas d'oïdium, le site web conseillera à l'agriculteur d'utiliser «TRICHOTECH», un emballage distribué par Dudutech pour combattre ce type d'oïdium. Ce type de programme de gestion intégrée des cultures a une approche holistique qui peut s'avérer fructueuse à long terme.

\section{Inconvénients}

- Ces forfaits sont parfois inabordables pour les petits agriculteurs, car la majorité des clients sont des grands exploitants.

- La vente de micro-organismes incubés dans des laboratoires ou des usines signifie qu'il y a peu de contrôle sur la qualité du produit final. Les concentrations microbiennes ont tendance à avoir une durée de conservation plus courte et une efficacité réduite avec le temps. De plus, la concentration promise par les fabricants ne tient pas compte des conditions locales du sol, de l'humidité et des méthodes d'application par les agriculteurs. Dans la plupart des cas, étant donné que les produits sont appliqués à base d'estimations moyennes, leurs effets réels sont difficiles à mesurer.

Source: Allison Loconto, INRAE

Pour en savoir plus, visitez: http://www.dudutech.com 


\subsubsection{Services de gestion du cycle de l'eau}

Due aux changements climatiques, la gestion des cycles de l'eau devient de plus en plus difficile. Bien que l'irrigation à grande échelle ne soit souvent pas possible dans plusieurs régions du monde, les agriculteurs ont trouvé des solutions alternatives et innovantes pour récupérer l'eau et de réorienter son cycle naturel pour l'usage agricole.

\section{Deux exemples de gestion d'eau (Zimbabwe, Inde, Tanzanie)}

1. Fosses de Phiri (Zimbabwe): Dans les zones prédisposées à la sécheresse, une pratique traditionnelle consiste à construire des fosses souterraines avec des pierres qui peuvent à la fois filtrer l'eau de pluie et augmenter la capacité de rétention du sol. Dans les années 1960, M. Phiri a commencé à le faire sur sa ferme avec une série de fosses situées à différents endroits de la ferme, qui a résulté en une résilience à la sécheresse sur le long terme.

Pour en savoir plus, visitez: https://youtu.be/cXLDOakTmrl https://youtu.be/ieqYZaTOJwA

2. Pompes à gravité (Inde/Tanzanie): Les régions montagneuses et de hauts-plateaux peuvent également souffrir de pénuries d'eau parce que l'écoulement naturel de l'eau est tiré vers le bas par la gravité. En Inde, des ingénieurs de technologie à petite échelle ont commencé à utiliser la force de gravité en plaçant une pompe dans un ruisseau au pied de la colline. L'impulsion de la chute de l'eau entre en collision avec la pompe qui, étant assez robuste, repousse l'eau vers le haut de la colline à travers un tube qui approvisionne la ferme. Cette technologie est activement utilisée dans les hauts plateaux tanzaniens.

Source: Allison Loconto, INRAE.

\section{Avantages}

* Ces technologies et d'autres technologies de captage de l'eau de pluie aident les agriculteurs à créer une irrigation à petite échelle et à faible coût;

+ les modèles de pompes hydrauliques à piston (la pompe à gravité étant la plus connue) se trouvent facilement sur internet.

\section{Inconvénients}

- Les pompes à gravité nécessitent un débit et une chute d'eau, d'où elles ne fonctionnent pas efficacement dans les zones planes ou pendant les longues saisons sèches;

- les fosses de phiri nécessitent un entretien, on ne peut pas les construire une fois pour toutes et les oublier le nouveau sol qui y tombe doit être pelleté.

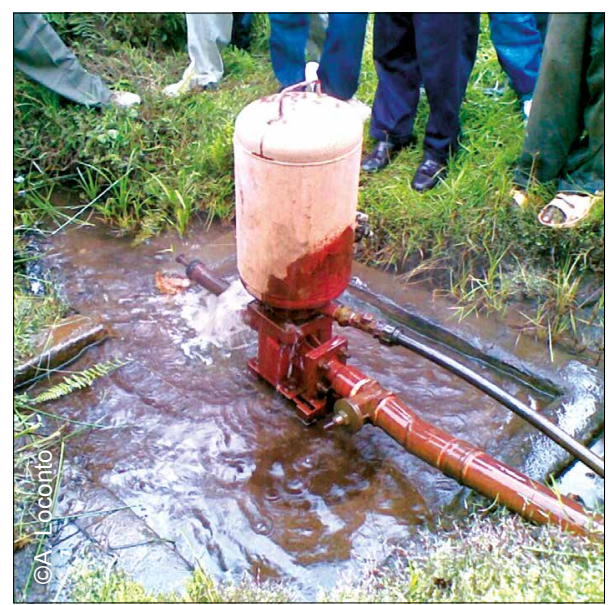




\subsubsection{Les intrants qui favorisent la pollinisation}

Pour la production agricole mondiale, les pollinisateurs fournissent un service essentiel à l'écosystème. Les interactions complexes entre les espèces végétales et les espèces pollinisatrices comme les abeilles, les oiseaux, les papillons et les chauves-souris, constituent un cycle précieux à maintenir pour une ferme durable. Les agriculteurs doivent de se tenir au courant des systèmes de pollinisation et des pollinisateurs appropriés pour leurs cultures et agroécosystèmes spécifiques. Par exemple, les abeilles sont les principales espèces pollinisatrices pour les grains de café.

\section{疍 Contribuer au cycle naturel des pollinisateurs (Malaisie)}

48

S'impliquer dans le commerce des pollinisateurs peut encourager et maintenir des cycles de pollinisation naturels dans votre ferme. Il s'agit de transporter du matériel vivant dans des écosystèmes où il est habituellement absent. En revanche, cela aidera à établir un système de pollinisation qui peut «gratuitement» augmenter les rendements de cultures dans le champ. Par exemple, le charançon, un coléoptère responsable de la pollinisation du palmier à huile, a été introduit en Afrique et en Malaisie où le palmier à huile est une culture principale. La pollinisation se faisait manuellement avant son introduction et les rendements des cultures ont considérablement augmenté depuis !

\section{Avantages}

+ Les services de pollinisation sont es «intrants agricoles» essentiels qui assurent la production des cultures;

+ l'amélioration de la densité et de la diversité des pollinisateurs a un impact positif direct sur le rendement des cultures.

\section{Inconvénients}

- L'introduction de nouvelles espèces inclue toujours le risque que les nouvelles espèces deviennent envahissantes et déséquilibrent l'écosystème. L'introduction de nouveaux insectes (même s'ils sont bénéfiques) nécessite des soins et de l'attention particuliers. Commencez par rechercher des espèces locales qui peuvent jouer le même rôle.

Pour en savoir plus, visitez: http://www.fao.org/pollination/en

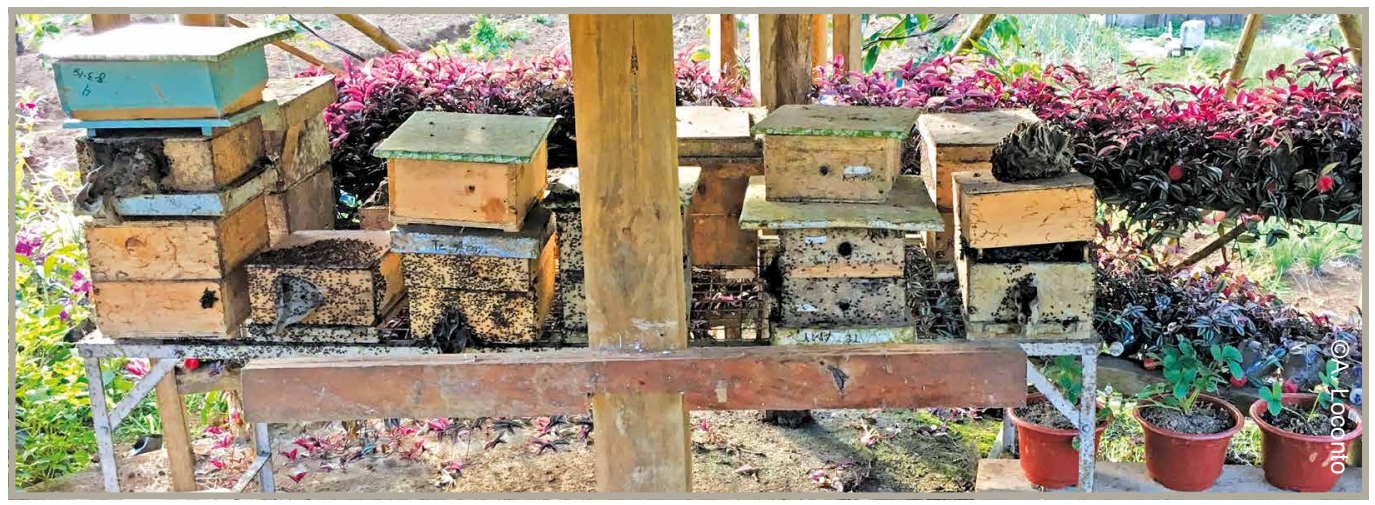




\section{COMMENT GÉREZ-VOUS VOS INTRANTS?}

Une fois que vous avez compris vos besoins en intrants et la portée des techniques ou technologies émergentes, commencez à réfléchir à l'accessibilité. Comment accédez-vous actuellement à vos intrants ? Comment gérez-vous leur utilisation? Quelles sont les options alternatives?

\section{LISTE DE VÉRIFICATION}

Répondre aux questions suivantes vous aidera à identifier les pratiques qui peuvent être durables et celles qui doivent être modifiées.

1. Comment gérez-vous vos terres?

a. Êtes-vous à jour dans vos paiements, titres ou contacts?

b. Avez-vous besoin de collaborer ou de négocier avec quelqu'un d'autre pour vous assurer que votre utilisation des terres est durable?

2. Comment maitrisez-vous vos ressources en eau ?

a. Avez-vous des ressources naturelles ou faut-il payer pour l'accès?

b. Avez-vous besoin de collaborer ou de négocier avec quelqu'un d'autre pour vous assurer que votre plan de gestion des ressources en eau est durable?

3. Comment gérez-vous vos cycles nutritifs ?

a. Utilisez-vous des engrais synthétiques ou biologiques ?

b. Les achetez-vous, les fabriquez-vous vous-mêmes ou les échangez-vous avec d'autres agriculteurs?

4. Comment gérez-vous les parasites et les maladies ?

a. Utilisez-vous des produits phytosanitaires synthétiques ou biologiques ?

b. Les achetez-vous, les fabriquez-vous vous-mêmes ou les échangez-vous avec d'autres agriculteurs?

5. Comment gérez-vous la main-d'œuvre agricole?

a. Utilisez-vous votre propre main-d'œuvre (ou celle de votre famille) ou embauchezvous d'autres personnes?

b. Est-ce que vous compensez le travail familial ?

c. Si vous embauchez des travailleurs, faites-vous votre propre recrutement ou comptezvous sur les autres?

d. Offrez-vous une rémunération équitable à vos travailleurs?

6. Comment gérez-vous votre technologie et vos machines?

a. Construisez-vous vos propres technologies/machines ou en faites-vous l'acquisition à l'extérieur de la ferme?

b. Achetez-vous, louez-vous ou partagez-vous votre technologie?

c. Comment entretenez-vous vos machines? 
Une fois que vous aurez réfléchi à ces questions, essayez de remplir la matrice ci-dessous. Elle vous aidera à déterminer pourquoi vous vous approvisionnez auprès de personnes ou de lieux précis.

Tableau 6: Matrice pour identifier vos sources d'approvisionnement d'intrants

\begin{tabular}{l|l|l|l|l|l}
\hline $\begin{array}{l}\text { Nom de } \\
\text { l'intrant }\end{array}$ & $\begin{array}{l}\text { D'où } \\
\text { provient } \\
\text { l'intrant ? }\end{array}$ & $\begin{array}{l}\text { Quel } \\
\text { pourcentage } \\
\text { est local ? }\end{array}$ & $\begin{array}{l}\text { Avantages } \\
\text { offerts }\end{array}$ & $\begin{array}{l}\text { Défis à } \\
\text { relever }\end{array}$ & Solutions alternatives durables \\
\hline L'urée & $\begin{array}{l}\text { Fournisseur } \\
\text { d'intrants }\end{array}$ & $100 \%$ & $\begin{array}{l}\text { Bon marché, } \\
\text { facile } \\
\text { d'accès }\end{array}$ & $\begin{array}{l}\text { Les nitrates } \\
\text { s'infiltrent } \\
\text { dans les } \\
\text { sources } \\
\text { d'eau, ce } \\
\text { qui nuit à la } \\
\text { santé du sol. }\end{array}$ & $\begin{array}{l}\text { Commencez à réduire } \\
\text { l'utilisation d'intrants externes } \\
\text { en appliquant d'abord du } \\
\text { fumier de bétail, puis en } \\
\text { vérifiant si vous avez besoin de } \\
\text { nutriments supplémentaires. } \\
\text { Si vous n'en avez pas à la } \\
\text { ferme, vérifiez s'il y a une ferme } \\
\text { d'élevage dans votre région. }\end{array}$ \\
\hline $\begin{array}{l}\text { Maintenant } \\
\text { vous essayez... }\end{array}$ & & & & & \\
\hline
\end{tabular}

\section{ASTUCE 25}

\section{Gérer et accéder aux intrants durables}

- Les intrants dans l'agriculture durable ne sont pas seulement des ajouts matériels au système, ils incluent aussi les terres, les écosystèmes et les services humains qui assurent la production ;

- L'option la plus durable est de maintenir tous les cycles naturels dans la ferme, si ceci n'est pas viable, essayez d'établir des échanges pour réduire les coûts des intrants ;

- Il existe de nombreuses technologies appropriées pour la production durable,les logiciels open source encouragent l'expérimentation et l'innovation ;

- Des modèles d'entreprise innovants peuvent garantir que le temps et le travail, intrants fondamentaux dans les systèmes durables, sont équitables et durables.

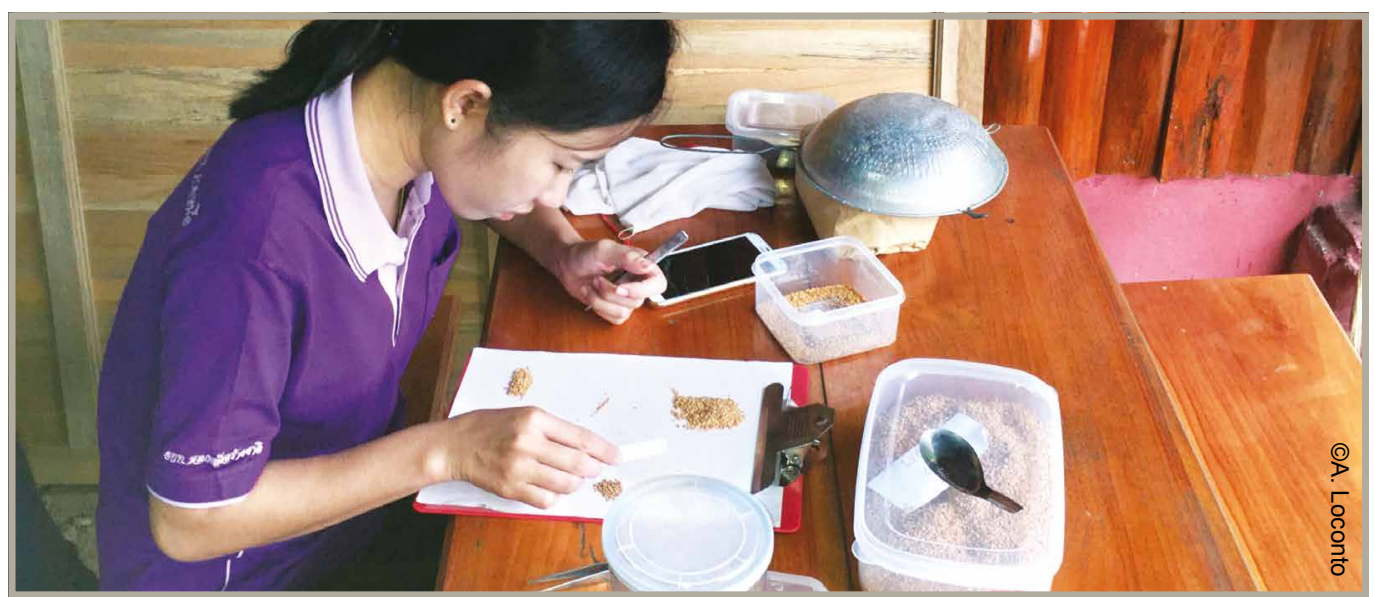




\section{LE CHEMIN D’APPRENTISSAGE, OÙ ALLER ENSUITE?}

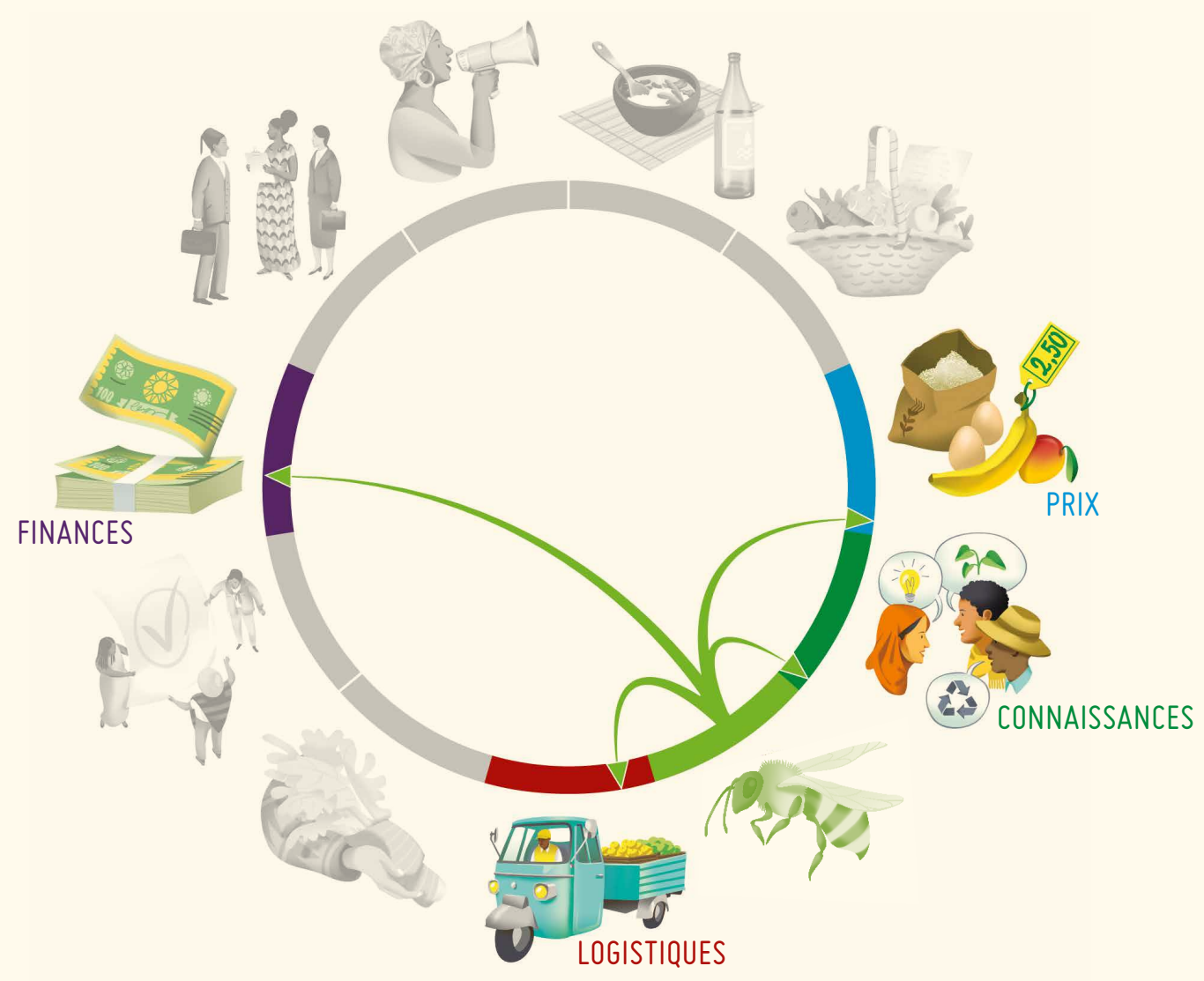

- Vous pensez que le savoir-faire et les connaissances, en tant qu'intrants n'a pas été discuté ? N'ayez crainte, ce sujet a été abordé dans le Chapitre 4: Partager et co-créer des connaissances pour une production durable.

- Si vous ne pouvez pas produire tous les intrants sur votre ferme, vous devrez peut-être les acheter. Vous avez besoin d'idées sur des mécanismes de financement innovants ? Consultez le Chapitre 9: Les financements innovants.

- Vous voulez essayer de recycler les déchets organiques non-agricoles en biogaz ou en compost ? Lisez le Chapitre 6: Rester connectés grâce à la logistique.

- Vous fabriquez plus d'intrants que vous ne pouvez utiliser et vous voulez essayer de les vendre à d'autres agriculteurs ? Le Chapitre3: Trouver le «bon» prix peut être utile.

Ou consultez un autre chapitre... à vous de choisir ! 


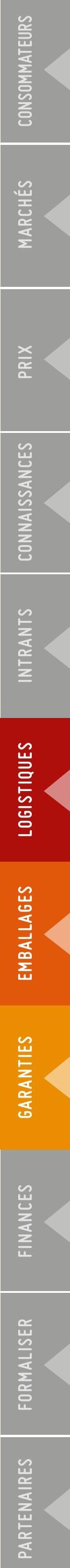



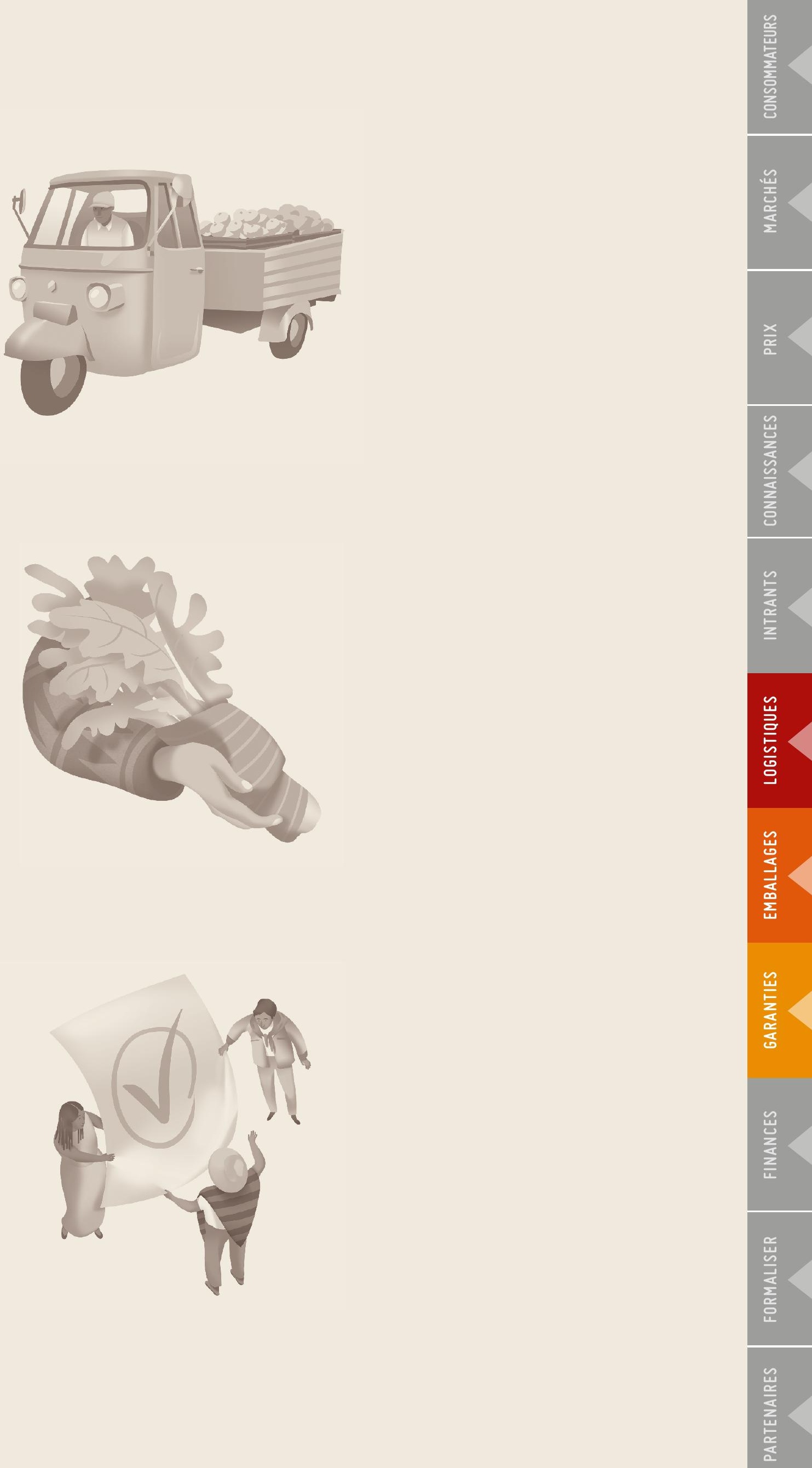



\section{POURQUOI EST-CE IMPORTANT?}

Bien que vous puissiez avoir un excellent produit, le livrer à vos clients (consommateurs ou transformateurs) en bon état, avec la qualité qu'ils ont demandée et au moment convenu, demeure un élément clé pour bâtir des relations de marché fidèles et durables.

Ces exigences sont les mêmes pour la gestion des sous-produits issus de l'agriculture durable, qui doivent également être transformés en produits secondaires. Ces derniers ajoutent de la valeur à votre système alimentaire.

Enfin, la logistique des déchets doit être gérée. Les déchets organiques peuvent être compostés en intrants agricoles ou utilisés comme source d'énergie (biogaz). Les déchets non-organiques devraient être réutilisés dans la mesure du possible (comme les bouteilles en verre) ou recyclés si l'infrastructure locale est disponible.

Une logistique bien organisée est d'une grande valeur pour les acheteurs et les distributeurs qui veulent assurer un approvisionnement constant aux consommateurs. En outre, elle est également avantageuse pour les producteurs, car la standardisation des étapes logistiques au sein d'un système de production durable génère une valeur ajoutée plus élevée, réduit les coûts et favorise la durabilité globale.

Cependant, la gestion de la logistique est difficile, vu que le moment choisi est essentiel ! Parmi les principaux défis on trouve la définition des besoins logistiques et la réduction maximale des coûts pour y répondre. Qu'est-ce que cela signifie?

Le calcul suivant vous aidera à déterminer la logistique de votre initiative:

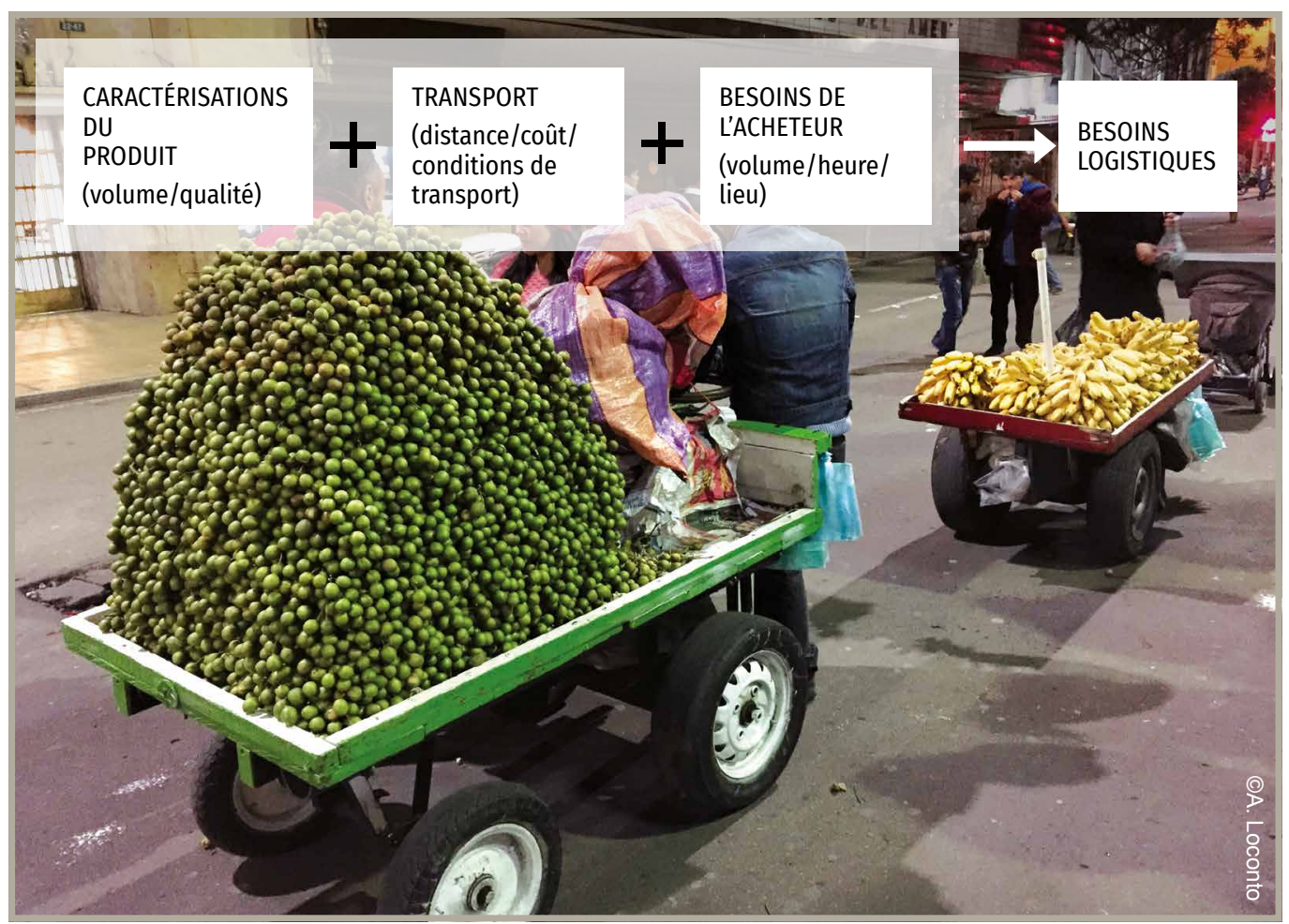


En vous basant sur vos produits, demandez-vous:

\begin{tabular}{|c|c|c|}
\hline S'ils sont: & Avec qui faites-vous des affaires? & Que recherchent vos acheteurs? \\
\hline $\begin{array}{l}\text { 1. Périssables? } \\
\text { 2. Pour les } \\
\text { particuliers ou } \\
\text { les grossistes? }\end{array}$ & $\begin{array}{l}\text { 1. Se trouvent-ils loin de vous? } \\
\text { 2. Est-ce que le transport est } \\
\text { inclus, ou avez-vous besoin } \\
\text { de l'organiser? } \\
\text { 3. Qu'en est-il des conditions } \\
\text { de transport? Par exemple, } \\
\text { avez-vous besoin d'un } \\
\text { entrepôt frigorifique ou de } \\
\text { types de caisses spéciales? }\end{array}$ & $\begin{array}{l}\text { 1. Avez-vous la bonne } \\
\text { quantité et la qualité } \\
\text { qu'ils demandent? } \\
\text { 2. Quand ont-ils besoin } \\
\text { des produits ? Ont-ils un } \\
\text { horaire flexible? } \\
\text { 3. Pouvez-vous facilement } \\
\text { vous rendre à leurs locaux } \\
\text { avec votre moyen de } \\
\text { transport habituel? }\end{array}$ \\
\hline
\end{tabular}

Si vous avez répondu à ces questions, vous pouvez construire un plan logistique simple.

\section{COMMENT COMMENCER PAR UN PLAN LOGISTIQUE?}

En général, la logistique commerciale inclut le flux complet de la production (ou de la transformation) à la vente. Ainsi, elle peut aussi impliquer des activités liées à l'achat, au stockage, à l'inventaire et/ou au transport. Pour être durable, le plan logistique doit également tenir compte des flux circulaires de sous-produits et le recyclage des déchets.

L'opérationnalisation est souvent déterminée par le contexte local, par les ressources humaines (par exemple la culture, les compétences et les

N'oubliez pas: un plan difficile à mettre en œuvre est un mauvais plan! coûts) et par l'environnement commercial et juridique. Par conséquent, il n'existe pas un plan unique pour toutes les situations, et tous les plans doivent être régulièrement révisés et mis à jour.

D’abord, commencez par remplir le tableau ci-dessous, étape par étape, avec vos produits.

\begin{tabular}{|c|c|c|c|}
\hline Les étapes & Riz & Légumes & $\begin{array}{l}\text { Mes produits } \\
\text { (à remplir } \\
\text { vous-même) }\end{array}$ \\
\hline $\begin{array}{l}\text { a. } \\
\text { Commencez par le diagramme } \\
\text { de production/d'opération } \\
\text { qui indique toutes les étapes } \\
\text { de travail, les personnes } \\
\text { impliquées et les tâches } \\
\text { principales. }\end{array}$ & $\begin{array}{l}\text { Culture et récolte du riz } \\
\text { collecte du riz }>\text { stockage } \\
\text { du riz }>\text { mouture } \\
\text { emballage }>\text { livraison aux } \\
\text { consommateurs }\end{array}$ & $\begin{array}{l}\text { Culture et récolte de légumes } \\
\text { centre de collecte } \\
\text { stockage }>\text { lavage et rognage } \\
\text { emballage livraison aux } \\
\text { consommateurs }\end{array}$ & \\
\hline
\end{tabular}




\begin{tabular}{|c|c|c|c|}
\hline Les étapes & Riz & Légumes & $\begin{array}{l}\text { Mes produits } \\
\text { (à remplir } \\
\text { vous-même) }\end{array}$ \\
\hline $\begin{array}{l}\text { b. } \\
\text { Indiquez le nombre de } \\
\text { personnes, l'espace, le temps, } \\
\text { l'équipement et les coûts } \\
\text { nécessaires pour chaque } \\
\text { étape. }\end{array}$ & $\begin{array}{l}\text { - Le broyage est effectué } \\
\text { par un entrepreneur } \\
\text { privé, nécessitant un } \\
\text { minimum de } 5 \text { tonnes de } \\
\text { riz, produisant environ 3,5 } \\
\text { tonnes de riz blanchi. } \\
\text { - Le camion peut } \\
\text { transporter un maximum } \\
\text { de } 1 \text { tonne (fixé par la } \\
\text { réglementation locale). } \\
\text { - Les frais pour le camion } \\
\text { sont de } 200 \text { USD par } \\
\text { voyage. } \\
\text { - Emballage du riz au } \\
\text { bureau, l'espace peut } \\
\text { stocker environ } 2 \text { tonnes. }\end{array}$ & $\begin{array}{l}\text { - Réception de } 100 \text { kg de } \\
\text { légumes le jeudi après-midi. } \\
\text { - Besoin de } 10 \text { personnes pour } \\
\text { nettoyer et emballer les } \\
\text { légumes avec le taux horaire } \\
\text { de } 2 \text { USD. } \\
\text { - Besoin de } 4 \text { heures pour le } \\
\text { nettoyage et l'emballage. } \\
\text { - Besoin de } 2 \text { heures pour la } \\
\text { livraison aux consommateurs. } \\
\text { - Les acheteurs } \\
\text { (consommateurs) veulent } \\
\text { ramasser des légumes à } \\
\text { 17h00 en ville. }\end{array}$ & \\
\hline $\begin{array}{l}\text { c. } \\
\text { Vérifiez si la logistique a un } \\
\text { sens, demandez-vous «Tout } \\
\text { s'accorde-t-il ?» }\end{array}$ & $\begin{array}{l}\text { - Un des camions est à } \\
\text { moitié-plein, donc les } \\
\text { coûts sont plus élevés. } \\
\text { - Pas assez d'espace pour } \\
\text { entreposer le riz blanchi. }\end{array}$ & $\begin{array}{l}\text { - La livraison n'a pas pu être } \\
\text { effectuée à temps le jeudi. }\end{array}$ & \\
\hline $\begin{array}{l}\text { d. } \\
\text { Examinez d'autres options } \\
\text { et leurs implications, vérifiez } \\
\text { la faisabilité d'une nouvelle } \\
\text { configuration logistique. }\end{array}$ & $\begin{array}{l}\text { - Option 1: négociez avec } \\
\text { la rizerie pour réduire le } \\
\text { minimum à } 2,8 \text { tonnes de } \\
\text { paddy (1,9 tonne de riz } \\
\text { blanchi), mais les coûts } \\
\text { de mouture seraient plus } \\
\text { élevés. } \\
\text { - Option 2: trouvez de } \\
\text { l'espace de stockage } \\
\text { supplémentaire près du } \\
\text { bureau pour } 1,9 \text { tonnes } \\
\text { mais prenez garde à } \\
\text { payer le loyer et les frais } \\
\text { de main d'œuvre pour } \\
\text { charger deux fois du riz, } \\
\text { puis augmenter le volume } \\
\text { de mouture à 5,6 tonnes } \\
\text { (ce qui équivaut à 3,9 } \\
\text { tonnes de riz blanchi). }\end{array}$ & $\begin{array}{l}\text { - Option 1: demandez aux } \\
\text { agriculteurs d'effectuer la } \\
\text { livraison plus tôt dans la } \\
\text { journée, avant } 10 \text { h00 le jeudi } \\
\text { matin. Alors, les agriculteurs } \\
\text { doivent commencer la récolte } \\
\text { à 5h00. } \\
\text { - Option 2: augmentez le } \\
\text { nombre de travailleurs à 20, } \\
\text { réduisant ainsi le temps de } \\
\text { coupe et d'emballage de } \\
\text { moitié, mais augmentant les } \\
\text { coûts de main-d'œuvre; } \\
\text { - Option 3: demandez aux } \\
\text { agriculteurs de récolter le } \\
\text { mercredi soir et de livrer le } \\
\text { même jour, mais ils auraient } \\
\text { besoin d'un entrepôt } \\
\text { frigorifique pendant la nuit. }\end{array}$ & \\
\hline $\begin{array}{l}\text { e. } \\
\text { «Tout ce qui peut mal } \\
\text { tourner, tournera mal». Par } \\
\text { conséquent, votre calcul } \\
\text { devrait inclure une marge } \\
\text { d'erreur, un facteur de } \\
\text { sécurité, ou un plan d'urgence. }\end{array}$ & $\begin{array}{l}\text { - L'espace d'entreposage } \\
\text { doit être } 10 \% \text { plus } \\
\text { grand que prévu afin } \\
\text { de permettre une plus } \\
\text { grande flexibilité. }\end{array}$ & $\begin{array}{l}\text { - Lorsque vous commandez } \\
\text { des légumes auprès des } \\
\text { agriculteurs, ajoutez } 5 \text { à } 10 \% \\
\text { de plus que ce dont vous } \\
\text { avez besoin. }\end{array}$ & \\
\hline
\end{tabular}

Il n'est pas toujours vrai que le produit doit déterminer la logistique que nous utilisons. Dans certains cas, la logistique (surtout les coûts et la distance au marché) détermine ce que nous pouvons produire ou vendre. Considérez ceci en élaborant votre plan. 


\section{QUI GÈRE LA LOGISTIQUE?}

Certains agriculteurs vendent leurs produits directement à leur ferme en installant un stand pour accueillir les consommateurs. Pour certains fruits, les fermes d'auto-cueillette permettent aux consommateurs de les cueillir eux-mêmes à un prix inférieur. La plupart du temps, l'agriculteur déplace le produit ailleurs, par exemple à un marché fermier, un hôtel, un restaurant, une école, un centre de distribution de supermarché, un point de collecte d'une AMAP, une maison d'acheteur ou un entrepôt pour un transport supplémentaire dans le pays ou à l'étranger.

Tout cela nécessite une logistique et un intermédiaire transparent. Bien qu'il y ait des intermédiaires malveillants, ce qui est souvent le cas dans une chaîne d'approvisionnement non organisée, les intermédiaires transparents jouent un rôle clé dans l'assemblement et l'acheminent de produits vers leurs marchés. Cela implique des frais généraux pour le service (transport/stockage/remballage/remballage occasionnel) et les risques (pertes/dommages/ inaptitude à la vente). Les agriculteurs peuvent recevoir un prix inférieur s'ils impliquent un intermédiaire. Par contre, ce prix ne tient pas compte des frais de transport, de stockage ou des risques liés à la logistique.

Une voie possible pour votre initiative est de développer vos compétences et trouver l'équipement nécessaire pour autogérer votre logistique, sans avoir besoin d'un intermédiaire transparent. Cependant, cette option n'est souvent pas la plus facile ou la meilleure. Pour de petits volumes, avoir son propre camion peut coûter plus cher que de payer une entreprise de transport. Les acheteurs de gros volumes ainsi que les groupes de consommateurs concernés peuvent déjà avoir l'équipement et la capacité de récupérer les produits à la ferme, vous permettant d'éviter cet investissement et ce défi. Tant que les agriculteurs pourront produire des produits de qualité et des volumes constants qui répondent aux besoins des acheteurs, et encore plus s'ils ont des produits écologiques exceptionnels, ils pourront négocier plus pour garantir des rendements équitables.

«Partager la poire en deux», transformer le désavantage en avantage (RDP Lao)

Basée à Vientiane, RDP Lao, Xaobaan est une petite entreprise socialement engagée qui produit des yaourts naturels et des produits laitiers de qualité. En essayant d'intégrer davantage des groupes marginalisés, la directrice, Mme Nongnut Foppes Ayamuang, a embauché des personnes de la communauté sourde qui gèrent désormais toute leur logistique quotidienne, livrant le yaourt et les autres produits vers différents points de distribution en ville, souvent en motocycle. En milieu bruyant, particulièrement dans les rues, leur audition limitée et leur capacité à communiquer par le langage des signes sont des atouts. La messagerie texte par téléphone mobile leur permet de communiquer facilement avec d'autres personnes qui ne parlent pas le langage des signes. Au final, ce qui aurait pu être considéré comme un obstacle au travail s'est avéré un atout stratégique pour leurs opérations.

Source: Michael Commons, Earthnet Foundation, Thaïlande 


\section{PRÉOCCUPATIONS LOGISTIQUES}

\subsection{Stockage}

Une règle de base pour la logistique est «moins de travail, moins de problèmes». Il est donc préférable de choisir un plan logistique sans stockage.

Pourtant, le stockage est essentiel pour les intrants (tels que les semences, semis, engrais organiques), les matières premières (tel que le riz paddy), les produits semi-transformés (tel que le riz blanchi non-conditionné) et les produits finis (tel que le riz conditionné prêt à la vente aux consommateurs). La plupart du temps, le stockage est géré par un intermédiaire ou un groupement de producteurs et parfois, par une communauté avec un entrepôt de stockage public. Assurez-vous de connaître les options disponibles dans votre région!

Vous trouverez ci-dessous les éléments à prendre en compte pour gérer le stockage:

- Quantité: En plus de disposer d'espace suffisant pour stocker vos produits, certains d'entre eux, particulièrement les matières premières et les produits semi-transformés, peuvent perdre du poids en période de stockage. Ceci est dû aux parasites, à la perte d'humidité et à l'usure avec le temps. Tenez compte de ces éléments lorsque vous installez l'espace afin de pouvoir réduire ces pertes.

- Qualité: Différents produits nécessitent des conditions de stockage différentes. Par exemple, différents types de légumes nécessitent divers températures et niveaux d'humidité. Certains produits (p. ex. le café, le thé) absorbent les odeurs et les parfums du milieu environnant. Une gestion efficace du stockage considère les caractéristiques du produit et définit l'espace en conséquence.

- Inventaire: Il y a quelques aspects à prendre en compte à cet égard, tels que:

- la règle du «premier entré, premier sorti»;

- la personne autorisée à sortir des produits de l'entrepôt;

- le contrôle de vérification de l'inventaire. Souvent, il est soit complètement ignoré, soit sur-réglementé. Trouvez un niveau d'activité approprié.

Coûts: Pour être durable, l'entreprise doit maintenir ses coûts au plus bas à l'aide de l'efficience et de l'efficacité. Les frais d'entreposage peuvent comprendre de nombreux éléments, tels que ceux-ci-dessous:

- coûts d'investissement initial, comme l'achat et la construction des installations de stockage;

- coûts d'amortissement (surtout à des fins comptables);

- coûts d'entretien (c.-à.-d. pour la réparation de murs, de toits et/ou de machines);

- électricité et commodités (par exemple,, l'entreposage frigorifique a besoin d'un climatiseur fonctionnant 24 heures sur 24,7 jours sur 7,365 jours par année);

- nettoyage régulier;

- services de lutte contre les parasites;

- rayons ou étagères;

- main d'œuvre pour le déchargement;

- personnel de contrôle des stocks. 


\subsection{Transformation artisanale ou à petite échelle}

Voici la règle de base où la logistique ne s'applique pas: «plus de transformation, moins de problèmes». Il y a une différence entre le transport de produits frais et de produits semitransformés ou entièrement transformés. En transformant certains produits à la ferme ou dans des points de collecte locaux, ils peuvent être conservés sur une durée prolongée et transportés sur de plus longues distances. Ces types d'activités à valeur ajoutée peuvent également générer des prix plus élevés pour les producteurs. La transformation peut se faire à l'aide d'équipement industriel ou moderne, mais cela exige beaucoup d'investissement initial. La transformation traditionnelle ou artisanale peut être tout aussi efficace si vous respectez les normes d'hygiène et de sécurité alimentaire. Elle peut également offrir des possibilités de commercialisation car vous pouvez revendiquer la caractéristique «fait à la main», «traditionnel» ou «artisanal», que certains consommateurs préfèrent.

\section{Le traitement du riz étuvé au Centre Songhaï (Bénin)}

Le Centre Songhaï au Bénin, un centre de formation et de production pour les jeunes, est considéré comme un modèle d'innovation exemplaire qui permet la transformation des produits à travers son réseau. Dans le pôle central situé à Porto Novo, des équipements modernes de transformation sont utilisés pour embouteiller l'eau de source et les jus de fruits et pour produire et/ou emballer le riz, le pop-corn, les biscuits et les snacks salés. Une boulangerie située sur place prépare du pain et des pâtisseries, un fumoir fait sécher et vieillir la viande, et des moulins transforment le soja en huile.

Ce pôle central de transformation s'approvisionne en matières premières à partir de sa propre ferme et de ses étangs piscicoles, ainsi qu'auprès des cinq sites satellites régionaux situés dans différentes zones agroécologiques. Chaque site satellite, également centre de formation, soit s'autoapprovisionnent, soit s'approvisionnent auprès de ses anciens élèves qui ont leur propre ferme dans la région. Les agriculteurs transportent leurs stocks vers les centres régionaux où les produits tels que les mangues séchées et la pulpe de mangue sont transformés. Les résidus de farine des moulins sont mélangés à d'autres matériaux pour préparer des aliments pour le bétail. Du jus de fruits et des collations sont produits dans ces pôles en utilisant des méthodes artisanales, dont une partie est vendue sur place au stand de la ferme.

Songhaï a un programme de formation de techniciens dans leur département de machines. Les techniciens développent et fabriquent toutes sortes de machines adaptées aux besoins locaux et aux divers produits (riz, huile de palme, jus de fruits, gari [à partir de tubercules de manioc], alimentation animale, séchage, etc.). Ces machines sont également vendues à un prix réduit et abordable. Elles peuvent donc être achetées par d'autres qui souhaitent dynamiser leurs systèmes de production et de transformation primaire. Pour la transformation du riz, Songhaï possède ses propres camions qui se déplacent entre les sites satellites et le pôle pour ramasser les produits bruts et déposer les produits transformés. Le riz, par exemple, est transporté des satellites jusqu'à Porto Novo pour être étuvé, séché au soleil et emballé dans des sacs standardisés et étiquetés pour être vendus sur le marché intérieur ou à l'exportation. Le riz étuvé est facile à transformer sans avoir besoin de machines modernes, et le prix du marché est beaucoup plus élevé grâce à son temps de cuisson court.

Source: Allison Loconto, INRAE et Belvue Akpatcho, Centre Songhaï, Bénin http://www.songhai.org/index.php/en/homeen/16-songhai/191-biotransformation-en 


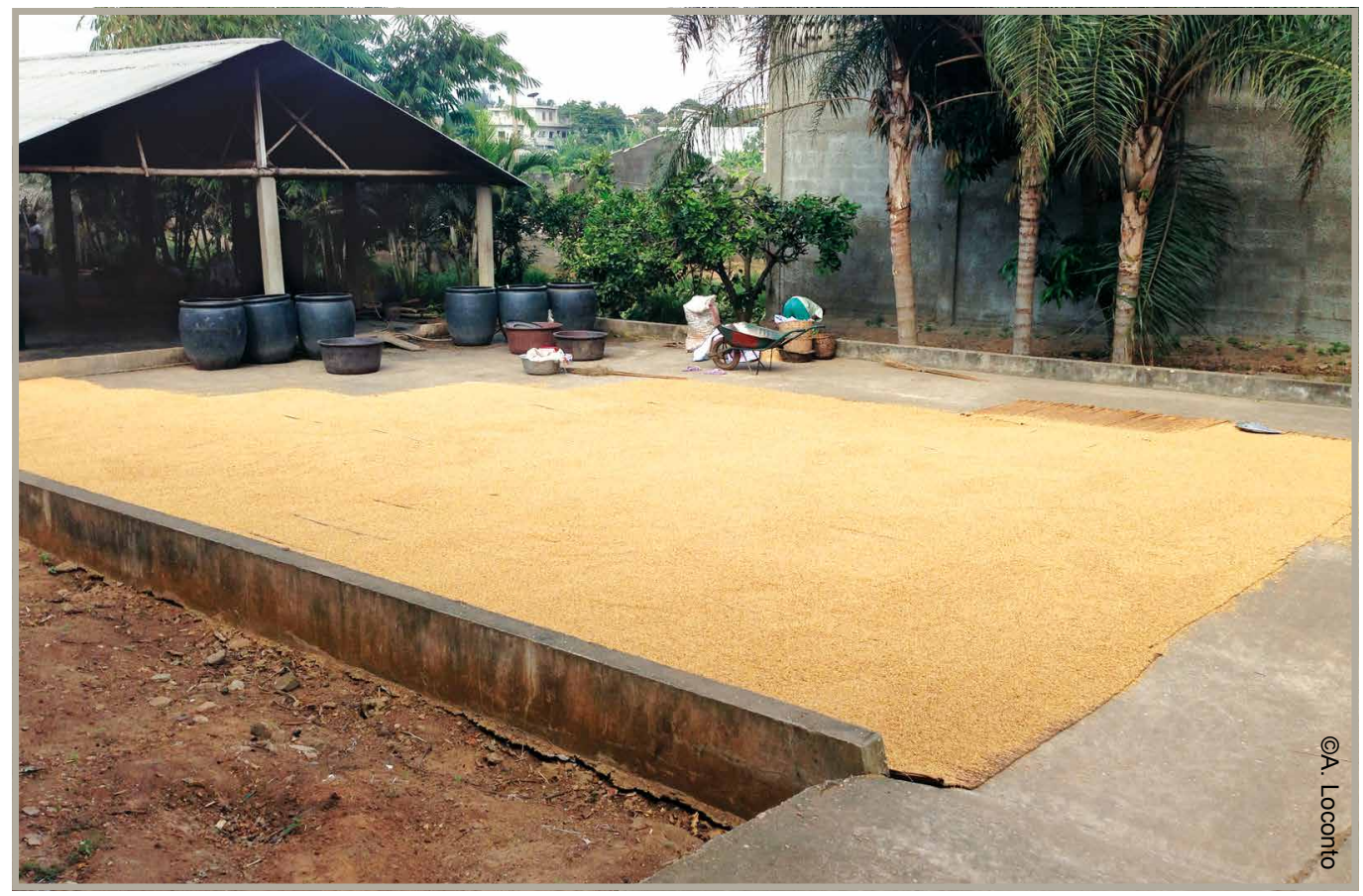

\subsection{Transport et livraison}

Là encore, la règle de base en logistique s'applique: «moins de transport, moins de problèmes».

En effet, les facteurs qui influencent la gestion du transport sont similaires à d'autres problèmes logistiques, car il s'agit aussi d'une forme de stockage temporaire. Les éléments supplémentaires à considérer sont le camion, le conducteur et le trafic.

Pour une start-up, il est souvent préférable d'utiliser, autant que possible, des services de livraison et de transport spécialisés puisqu'ils ne nécessitent pas d'investissement initial et les coûts pour des petites quantités sont minimes. Une fois que les quantités augmentent, des options plus durables sont nécessaires.

En considérant la durabilité absolue, essayez de choisir des services de livraison qui utilisent moins d'énergie fossile, par exemple dans l'ordre de préférence: vélo, train, bateau, véhicule électrique ou hybride, ou camion. Essayez de partager le transport autant que possible pour maximiser l'espace et réduire le nombre de déplacements nécessaires (par exemple, le covoiturage ou le partage d'un fret de camions). Assurez-vous que ces décisions sont également basées sur la destination de votre marché final. Lorsque cette option n'est pas réalisable, il est nécessaire d'avoir son propre moyen de transport. Dans cette situation, un calcul adéquat de vos coûts de transport et de leur empreinte carbone est essentiel au succès de votre entreprise. 


\section{EcoVida, un système de transport partagé (Brésil)}

EcoVida est un réseau de 4500 familles qui vendent des produits agroécologiques dans quatre états du sud du Brésil. Leur réseau de distribution s'étend sur plus de $1600 \mathrm{~km}$. Chaque semaine, les agriculteurs apportent leurs produits à un pôle central (nucléo) géré par un agriculteur). Chaque responsable de nucléo envoie un message via WhatsApp aux autres responsables pour leur informer de la quantité courante de chaque produit ainsi que des commandes. Par exemple, un nucléo a $10 \mathrm{~kg}$ d'oranges et $20 \mathrm{~kg}$ de bergamotes et recherche $5 \mathrm{~kg}$ de bananes, $1 \mathrm{~kg}$ de noix de cajou et $10 \mathrm{~kg}$ de riz. Un autre gérant de centre peut avoir $15 \mathrm{~kg}$ de riz et $10 \mathrm{~kg}$ de noix de cajou et a besoin de $5 \mathrm{~kg}$ d'oranges, de bergamotes et de bananes chacun, et ainsi de suite.

Les commandes sont placées et l'itinéraire du camion est déterminé en conséquent, s'arrêtant d'abord au nucléo 1 pour ramasser les oranges, au nucléo 2 pour aller chercher le riz et les bananes et déposer les oranges, puis au nucléo 3 pour aller chercher/déposer les autres

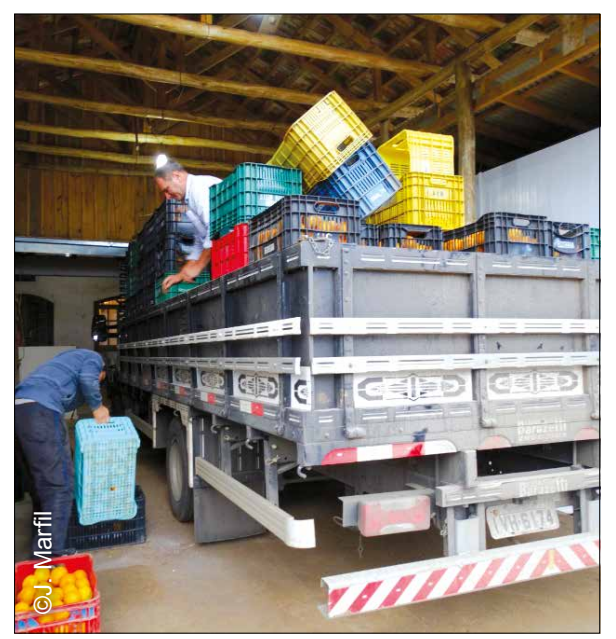

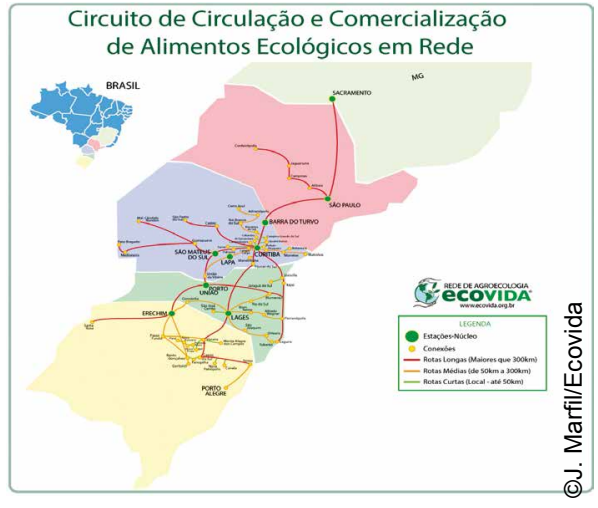

produits. Au retour, le nucléo 1 recevra les bananes, les noix et le riz dont ils ont besoin.

Tous les camions doivent appartenir à un agriculteur membre d'Ecovida; il n'y a pas distributeurs externes pour gérer la livraison.

Pour tous les produits échangés chaque semaine, les prix sont négociés. Des frais de transport fixes sont également inclus: 0,30 BRL par kg pour les trajets courts et 0,60 BRL par kg pour les trajets longs (comme, par exemple, de Curitiba à São Paulo).

Source: José Antonio da Silva Marfil, Ecovida, Brésil

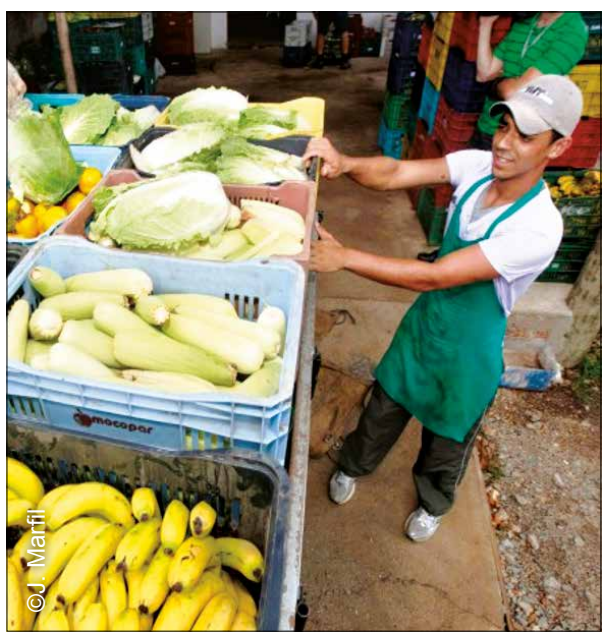


Tableau 7: Typologie des différents systèmes de distribution

\begin{tabular}{|c|c|c|c|}
\hline Système de livraison & Exemples & Avantages & Défis à relever \\
\hline PRODUCTEURS & $\begin{array}{l}\text { Marchés de } \\
\text { producteurs }\end{array}$ & $\begin{array}{l}\text { Permet aux } \\
\text { consommateurs } \\
\text { de connaître leurs } \\
\text { producteurs. } \\
\text { Aucun intermédiaire } \\
\text { dans la chaîne } \\
\text { d'approvisionnement. } \\
\text { Plus de quantité et de } \\
\text { variété à la disposition } \\
\text { des consommateurs. } \\
\text { Le stockage peut être } \\
\text { inclus ou non. }\end{array}$ & $\begin{array}{l}\text { Aucune garantie que } \\
\text { ce que le producteur } \\
\text { apporte sur le marché } \\
\text { sera vendu. } \\
\text { Tant les producteurs } \\
\text { que les consommateurs } \\
\text { doivent utiliser leur } \\
\text { propre moyen de } \\
\text { transport, ce qui signifie } \\
\text { des coûts plus élevés. }\end{array}$ \\
\hline PRODUCTEURS & $\begin{array}{l}\text { Magasins } \\
\text { coopératifs; } \\
\text { magasins } \\
\text { d'agriculteurs; } \\
\text { dépôt AMAP; } \\
\text { assemblées } \\
\text { alimentaires; } \\
\text { groupes d'achats } \\
\text { collectifs, } \\
\text { systèmes de } \\
\text { boîtes. }\end{array}$ & $\begin{array}{l}\text { Peut permettre des } \\
\text { prix dirigés par les } \\
\text { agriculteurs. } \\
\text { Peut être un débouché } \\
\text { régulier et utile pour les } \\
\text { producteurs locaux. } \\
\text { - Plus de quantités et de } \\
\text { variétés à la disposition } \\
\text { des consommateurs. } \\
\text { Les agriculteurs peuvent } \\
\text { s'organiser pour minimiser } \\
\text { les coûts de transport } \\
\text { jusqu'au point de } \\
\text { distribution. } \\
\text { La livraison des boîtes aux } \\
\text { consommateurs peut être } \\
\text { organisée de manière à } \\
\text { minimiser les coûts. }\end{array}$ & $\begin{array}{l}\text { Un stockage minimum } \\
\text { doit être inclus dans ce } \\
\text { modèle. } \\
\text { Il peut être difficile de } \\
\text { gérer le calendrier, car } \\
\text { il dépend de l'afflux de } \\
\text { consommateurs. } \\
\text { L'offre est saisonnière } \\
\text { et risque de ne } \\
\text { pas satisfaire les } \\
\text { consommateurs réguliers. } \\
\text { La demande peut être } \\
\text { très faible à certaines } \\
\text { périodes de l'année (par } \\
\text { exemple, pendant les } \\
\text { vacances d'été). }\end{array}$ \\
\hline PRODUCTEURS & $\begin{array}{l}\text { Ventes à la } \\
\text { ferme, Parts de } \\
\text { la ferme dans } \\
\text { une AMAP }\end{array}$ & $\begin{array}{l}\text { Dirigé par des paysans. } \\
\text { Consommateurs locaux. } \\
\text { Pas de frais de transport } \\
\text { pour les agriculteurs. } \\
\text { Pas de stockage } \\
\text { nécessaire. }\end{array}$ & $\begin{array}{l}\text { L'approvisionnement } \\
\text { irrégulier et saisonnier } \\
\text { peut être une difficulté } \\
\text { pour fidéliser les } \\
\text { consommateurs réguliers. }\end{array}$ \\
\hline
\end{tabular}

Source: M. Vicovaro, FAO et les auteurs 


\subsection{Transformation des pertes et des déchets en sous-produits et intrants pour la production ou la transformation}

Une gestion bien organisée et créative des sous-produits peut les transformer en produits de grande valeur, évitant ainsi le gaspillage. Le conseil clé pour la gestion des sous-produits est de «ne jamais cesser d'innover».

Tout comme les produits principaux, les sous-produits doivent aussi être considérés de façon créative afin d'ajouter plus de valeur et d'obtenir de meilleurs rendements. Ils peuvent être utilisés pour développer des emballages innovants, être transformés en nouveaux produits ou simplement être vendus sur différents marchés. N'oubliez pas: Comme pour vos produits principaux, comprenez où se trouvent les marchés des sous-produits, vu qu'ils ils peuvent être différents. Ils peuvent aussi nécessiter un travail supplémentaire pour organiser des réunions entre agriculteurs, transformateurs, intermédiaires et consommateurs.

Si votre objectif est d'atteindre une durabilité environnementale exhaustive, réduisez vos déchets et à la fois, recyclez les déchets qui ne peuvent être réduits. Il est possible de composter les déchets organiques en intrants agricoles ou les utiliser comme source d'énergie pour le biogaz. D'autre part, les déchets non-organiques, comme les bouteilles en verre, peuvent être réutilisés ou recyclés pour être transformés en produits nouveaux.

\section{ASTUCE 26}

\section{Gérer les déchets efficacement}

1. Les déchets peuvent être réduits grâce à une bonne conception et à un bon contrôle de la production ou des procédés. Déterminez quelles étapes de votre production génère des déchets et cherchez des solutions alternatives pour les réduire. Parfois, cela implique un changement du système et de machines, tandis que d'autres fois, il s'agit simplement d'un changement de comportement. D'abord, mettez en œuvre l'alternative facile ou peu coûteuse et vérifiez les résultats avant de décider d'investir dans des solutions plus coûteuses.

2. Recyclez des matières organiques à l'aide des animaux. Plusieurs déchets organiques servent de nourriture pour les poulets, les chèvres, les porcs, les poissons ou les vers de terre (lombricompostage). Une grande partie des déchets organiques non-mangeables par les animaux peuvent être «mangés» (utilisés comme substrat) par les champignons.

3. Recyclez des matières organiques par le compostage. Il peut s'agir d'un simple compostage (compost à fosse ouverte) ou d'un digesteur de biogaz. Les matières compostées peuvent être utilisées dans la production agricole pour clôturer les cycles des éléments nutritifs ou vendues aux jardiniers urbains (cela pourrait être une bonne activité promotionnelle pour votre entreprise).

4. Recyclez les matières non-compostables (papier, bouteilles de verre, sacs de plastique, etc.) avec les services publics d'ordures, dans la mesure du possible. Une autre opportunité commerciale pourrait être d'investir dans des machines de recyclage de plastique ou de verre et d'offrir ces services aux entreprises locales et/ou au gouvernement ! 


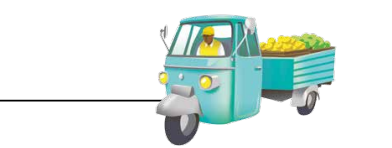

\section{䍗 \\ Les déchets de la foire deviennent de l'engrais en ville (Brésil)}

52

La ville de São Mateus, capitale de l'État d'Espírito Santo dans le sud-est du Brésil, a conçu un plan de collecte des déchets organiques de ses 900 marchés hebdomadaires pour les composter localement. Cela permet d'atténuer le flux annuel des décharges de 62000 tonnes de déchets. La ville a établi quatre usines de compostage, chacune coûtant environ 140 USD par mois pour fonctionner. Il s'agit d'un montant équivalent aux dépenses que la mairie paie pour enterrer les ordures des marchés dans les décharges. Ces usines disposent d'une multitude de solutions de collecte et de traitement des déchets. Ils vendent leurs matières de compostage aux petits agriculteurs et, de ce fait, ils réduisent la production de déchets, augmentent la durée de vie des sites de décharge et économisent environ 30 pour cent des coûts de nettoyage des marchés. Alors que ces économies au marché plein-vent d'Ursa Minor sont dues à la réduction de main d'œuvre, deux parmi leurs huit nettoyeurs de rue se sont reformés pour devenir des agents environnementaux qui accompagnent la collecte des déchets, le transport des déchets à l'usine de compostage et l'assemblage des déchets en bûches de compost (c.-à.-d. les déchets organiques sont mélangés avec de la sciure pour se transformer en compost).

Source: José Antonio da Silva Marfil, Ecovida, Brésil

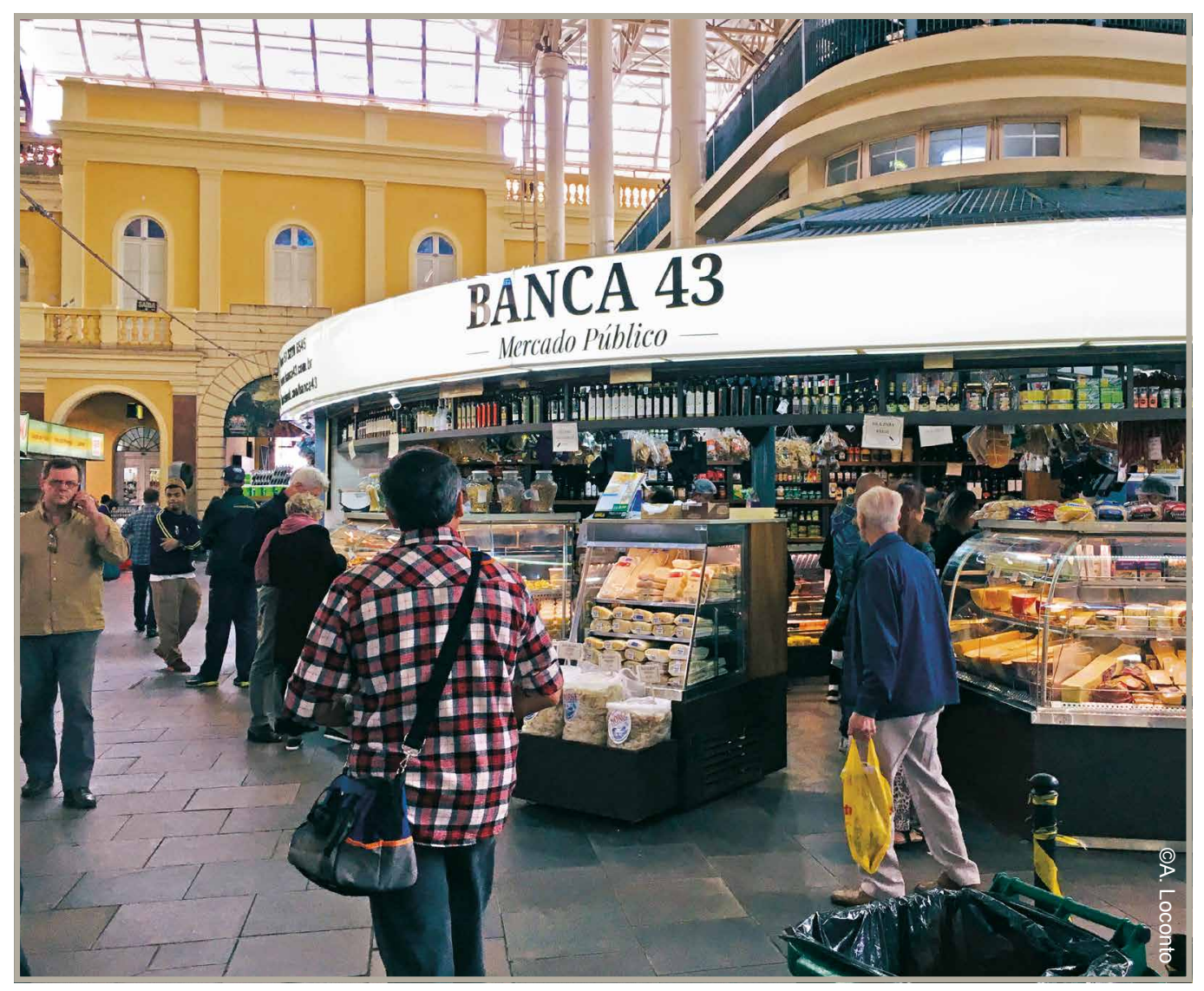




\section{UTILISER LE PLAN LOGISTIQUE POUR RÉPONDRE AUX NORMES}

\subsection{Traçabilité}

La traçabilité est un processus qui utilise la documentation et l'étiquetage pour permettre aux utilisateurs finaux de vérifier le parcours des produits partants de la ferme. Généralement, il s'agit d'une exigence pour certifier la securité sanitaire et la durabilité des aliments. La mise en place d'un système de traçabilité permet d'identifier l'origine des crises de sécurité sanitaire des aliments et de rappeler les produits.
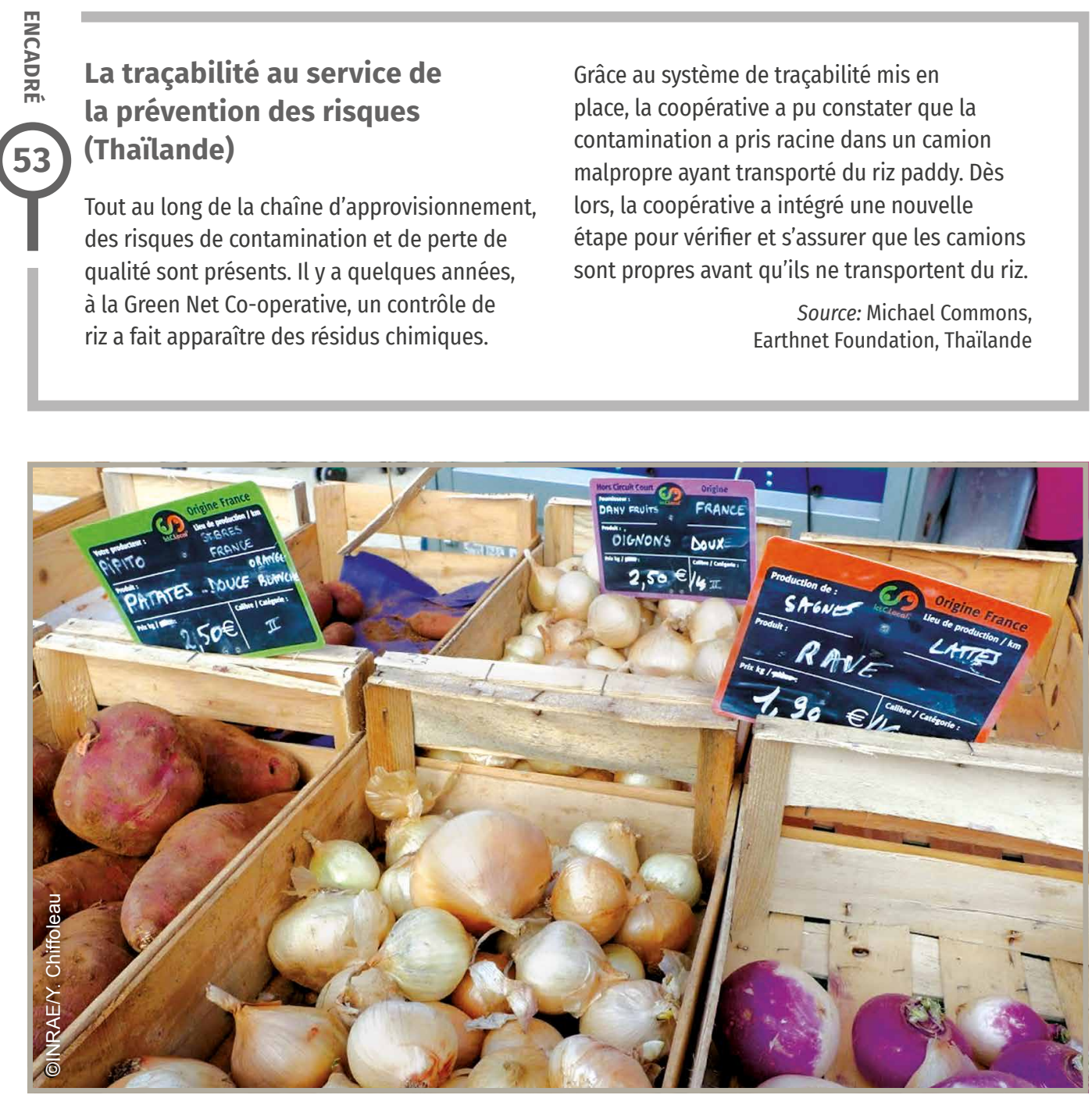


\section{Un code couleur servant de système de traçabilité (Tanzanie)}

La Wakulima Tea Company (WATCO) a mis en place un système de pesage numérique avec un programme conçu pour séparer les agriculteurs certifiés et non-certifiés Rainforest Alliance aux points de collecte du village. Chaque agriculteur se voit attribuer un numéro avec les préfixes $00=$ non-certifié et $01=$ certifié. Après la pesée, le thé certifié est chargé dans des sacs verts ou d'autres couleurs tandis que le thé non-certifié est chargé dans des sacs jaunes. Pendant le transport, les noncertifiés sont chargés dans le casier inférieur du véhicule et les certifiés sont chargés dans le casier supérieur, tandis que le déchargement en usine commence avec le casier supérieur se termine avec le casier inférieur. Par conséquent, les non-certifiés sont les derniers à être placés dans des cuvettes spéciales pour le flétrissement respectivement marquées (et numérotées). Pendant la transformation, ils commencent avec des thés certifiés. Ensuite, ils nettoient complétement la zone de traitement et attendent 45 minutes avant de commencer à traiter les thés non-certifiés. Cette séparation entre le thé certifié et le thé non-certifié est fondamentale pour encourager l'adoption de pratiques durables. Les agriculteurs avec des sacs jaunes questionnent leur distinction, vu

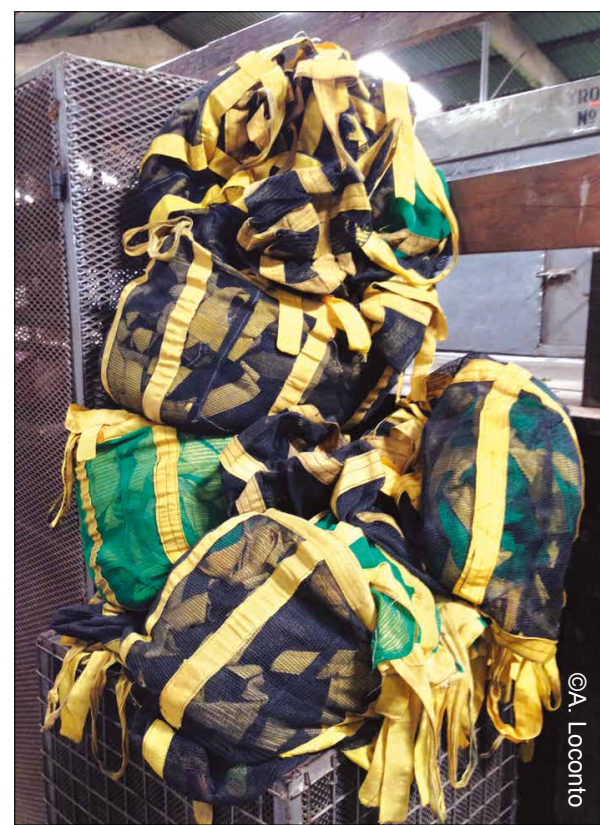

que la couleur est évidemment remarquée par les autres agriculteurs présents au point de collecte. De plus, des prix différents sont attribués à chaque couleur, encourageant les agriculteurs non-certifiés à se demander comment obtenir la certification, afin d'atténuer le sentiment d'exclusion.

Source: Allison Loconto, INRAE et Emmanuel Simbua, TRIT, Tanzanie

\subsection{Réduction du risque de contamination}

Tout au long de la chaîne de valeur, les aliments risquent d'être contaminés. Le risque est généralement faible dans les fermes durables, étant donné l'utilisation de bonnes pratiques. Cependant, le risque est proportionnellement plus élevé à l'extérieur de la ferme, puisque les produits sont transportés sur de longues distances avant d'atteindre les consommateurs. Tous les acteurs impliqués dans la chaine alimentaire doivent éviter qu'une telle contamination se produise. Sur les marchés éloignés, ces bonnes pratiques sont appliquées par le biais d'un certificat de transaction, qui retrace les produits au fur et à mesure qu'ils passent d'un acteur à l'autre. Cette trace écrite est ensuite vérifiée par une tierce partie qui teste le produit afin de repérer des toxines ou des bactéries. Toutefois, d'un point de vue pratique, chaque lot de 
marchandises ne peut pas être inspecté en raison du volume trop élevé des transactions qui peuvent être couteuses et augmenter le prix final pour les consommateurs.

D'autres méthodes méritent aussi d'être explorées. Une fois qu'un système de garantie considère qu'une source de produit est durable, le produit final sur le marché peut être testé pour des résidus (un procédé appelé «surveillance du marché»). Cependant, les tests effectués sur les produits finis du marché sont également coûteux. S'il existe un niveau de confiance élevée entre les acteurs impliqués dans l'initiative (principalement entre les producteurs et les consommateurs), le coût des tests pourrait être réduit. Cela peut être accompli en rendant aléatoire le prélèvement d'échantillons de manière participative et en partageant les coûts entre les membres de l'initiative.

\section{Des tests participatifs de contamination (Inde)}

En Inde, dans le cadre de la campagne BhoomiKa et du marché des producteurs biologiques de Delhi, les consommateurs sont invités à sélectionner périodiquement des échantillons aléatoires de fruits, légumes et autres céréales, tandis qu'un représentant du laboratoire prélève un échantillon. Tous les échantillons sont mis dans des sacs scellés et le rapport du test est volontairement partagé avec le public. L'inclusion d'un tel mécanisme de transparence invite les commentaires et les suggestions de tous les acteurs sur la manière d'améliorer la transparence tout en évitant des coûts de conformité élevés. Les consommateurs finaux sont également invités à faire partie du SPG utilisé pour certifier les fermes et certains d'entre eux visitent aussi les fermes pour renforcer le sentiment de confiance. À ce jour, aucun résidu n'a été trouvé dans le protocole d'essai. Dans le cas échéant, les acteurs du système alimentaire durable sont responsables pour rendre ces rapports publics, prendre des mesures correctives, et rembourser les consommateurs. Si un producteur subit une perte, une simple excuse peut largement suffire pour maintenir la transparence et bâtir la confiance. De tels modèles de transparence pourraient également être testés pour des longues chaînes d'approvisionnement, pour réduire les risques de contamination. Cependant, en règle générale, les gens font plus confiance aux personnes qu'ils connaissent qu'aux entreprises inconnues.

Source: Ashish Gupta, Jaivik Haat
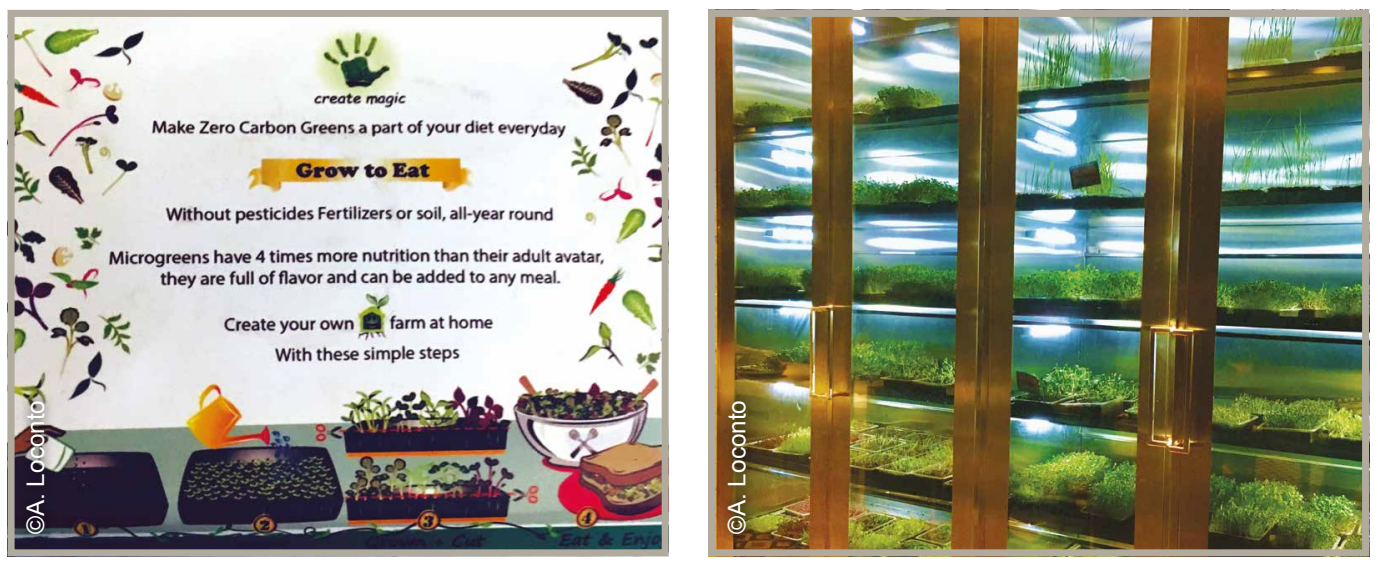


\subsection{Préoccupations et réglementation en matière de securité sanitaire des aliments}

Bien que les réglementations sur la sécurité alimentaire régissent tous les systèmes, dans un système alimentaire durable, des mesures prudentes sont nécessaires pour éviter toute contamination par les aliments conventionnels. De la production à la consommation, les directives réglementaires précisent les règles de ségrégation des aliments. Cependant, il est important de rester à jour avec les règles de sécurité puisqu'elles évoluent avec le temps.

罗

\section{La réglementation participative} de la sécurité sanitaire des

\section{6} aliments (Inde)

Un moyen efficace de tenir les producteurs et les consommateurs informés est d'assurer une représentation adéquate dans les processus participatifs de révision réglementaire. En Inde, l'organisme de réglementation de la securité sanitaire des aliments (Food Safety and Standards Authority of India) a invité des représentants de diverses organisations de société civile (OSC) à participer à la révision de la réglementation biologique. Des projets de réglementation ont été envoyés et les OSC ont eu de multiples discussions avec l'organisme de réglementation. Suite à cette réunion, de nombreux changements importants ont été acceptés par le régulateur, tels que la reconnaissance des Systèmes Participatifs de Garantie (SPG) comme un mécanisme de certification valide, l'harmonisation des différents mécanismes de conformité sous un code réglementaire biologique unifié et la création d'un logo officiel «Jaivik Bharat». L'organisme de réglementation a également lancé un portail appelé «India Organic Database Integrity Portal» pour que les consommateurs puissent retracer les aliments des certificateurs (tiers et SPG). Le règlement comprend aussi certains éléments clés qui visent à augmenter la compétitivité des producteurs locaux, tels que:

1. Les petits agriculteurs qui vendent directement aux consommateurs ont été exemptés du règlement et peuvent toujours vendre leurs produits comme étant biologiques dans leurs ventes directes.

2. La réglementation relative à l'importation des aliments a été renforcée afin de garantir que les aliments transformés importés ne soient pas vendus sur les marchés indiens s'ils sont disponibles auprès des producteurs locaux.

Source: Ashish Gupta, Jaivik Haat

\section{ASTUCE 27}

\section{Gérer la logistique}

- L'échéance est fondamentale - être présent au bon endroit au bon moment peut vous économiser de l'argent, réduire le gaspillage et assurer la qualité !

- La transformation des produits sur-place à la ferme ou dans des installations locales de collecte et de stockage peut augmenter les revenus des producteurs et réduire les coûts de transport.

- La traçabilité est essentielle pour garantir la sécurité sanitaire de vos aliments, mais elle n'a pas besoin d'être complexe; il s'agit surtout de savoir qui a livré quoi et quand.

- Le partage des responsabilités, même en matière de logistique, peut contribuer à renforcer la confiance entre producteurs et consommateurs, ce qui rendra les systèmes durables à long terme. 


\section{LE CHEMIN D’APPRENTISSAGE, OÙ ALLER ENSUITE?}

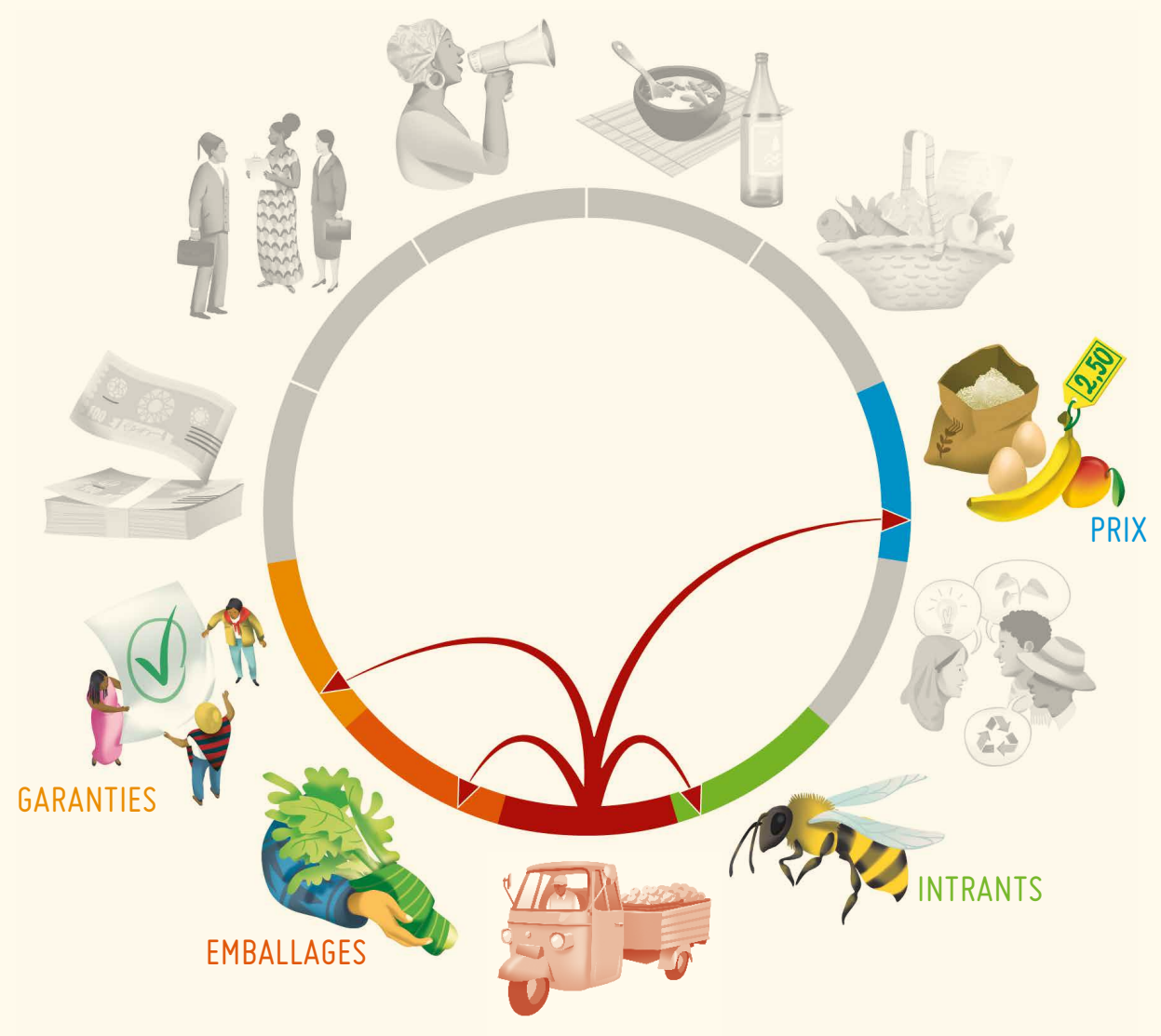

- Vous avez besoin d'assurer la traçabilité pour une exigence de certification, mais vous n'êtes pas satisfait de la façon dont elle fonctionne ? Visitez le Chapitre 8: Garantir la durabilité pour découvrir d'autres formes de garantie.

- Vous souhaitez en savoir plus sur les intermédiaires transparents et sur la manière dont ils pourraient soutenir votre initiative ? Retournez au Chapitre 3: Trouver le «bon» prix.

- Et quand est-il des déchets organiques ? Essayez de les transformer en compost. Pour en savoir plus sur ce sujet, lisez le Chapitre 5: Gérer et accéder aux intrants durables.

- Est-il sécuritaire de réutiliser des déchets non-organiques pour l'emballage ? Continuez au Chapitre 7: Emballage de qualité et durable pour en savoir plus.

Ou allez à un autre chapitre...c'est à vous de décider ! 


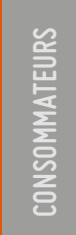

\section{EMBALLAGE
DE QUALITÉ \\ ET DURABLE}

r

$\frac{x}{a}$

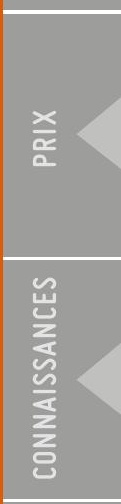

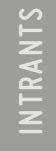

쁠
음
$\stackrel{5}{6}$
의

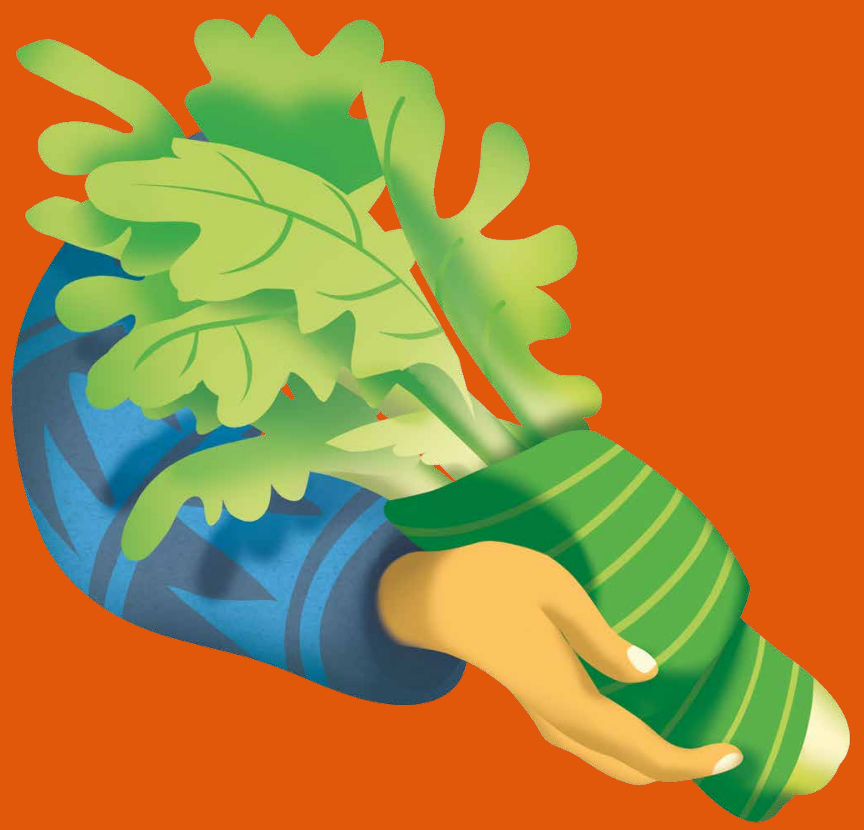

岕

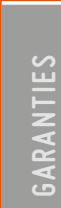

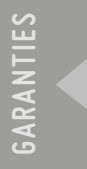

岕

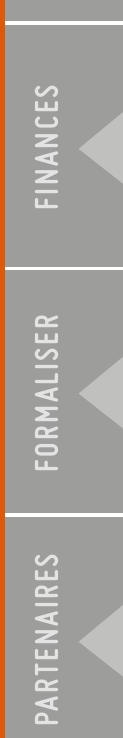



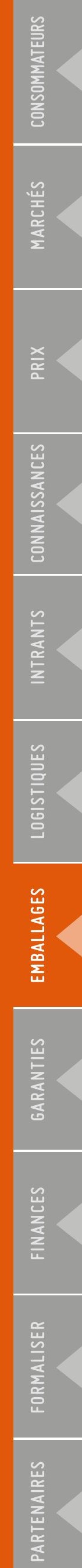


\section{POURQUOI EST-CE IMPORTANT?}

Dans un système alimentaire durable, l'emballage a deux fonctions principales:

1. conserver votre produit;

2. communiquer sa valeur durable aux consommateurs.

Ces deux fonctions génèrent plus de valeur tant pour les producteurs que pour les consommateurs, car l'emballage offre une image reconnaissable de votre initiative. Un emballage uniforme assure une stabilité dans le temps où les consommateurs reconnaîtront facilement vos produits sans perdre trop de temps à lire les étiquettes ou à chercher parmi des produits similaires.

罗

Réduire, réutiliser, recycler (Chine)

«Réduire, réutiliser, recycler» sont les trois

messages de la stratégie d'emballage de produits (y compris les fruits et légumes) de Shared Harvest Farm. Il s'agit d'une ferme en AMAP à Beijing constituée d'environ 800 familles-membres. Elle livre des aliments en porte à porte chaque semaine. Tout d'abord, la ferme n'utilise que de la paille pour emballer la plupart de ses produits comme les légumes et très peu de sacs en plastique sont utilisés pour le reste. Deuxièmement, elle travaille avec une entreprise de logistique locale qui collecte les boîtes et les autres matériaux d'emballage pour les retourner à la ferme et les réutiliser. Elle utilise les réseaux sociaux pour promouvoir les avantages de l'utilisation réduite des emballages auprès de ses consommateurs. Enfin, elle essaie d'utiliser des matériaux recyclables autant que possible.

Source: Shi Yan, Récolte partagée
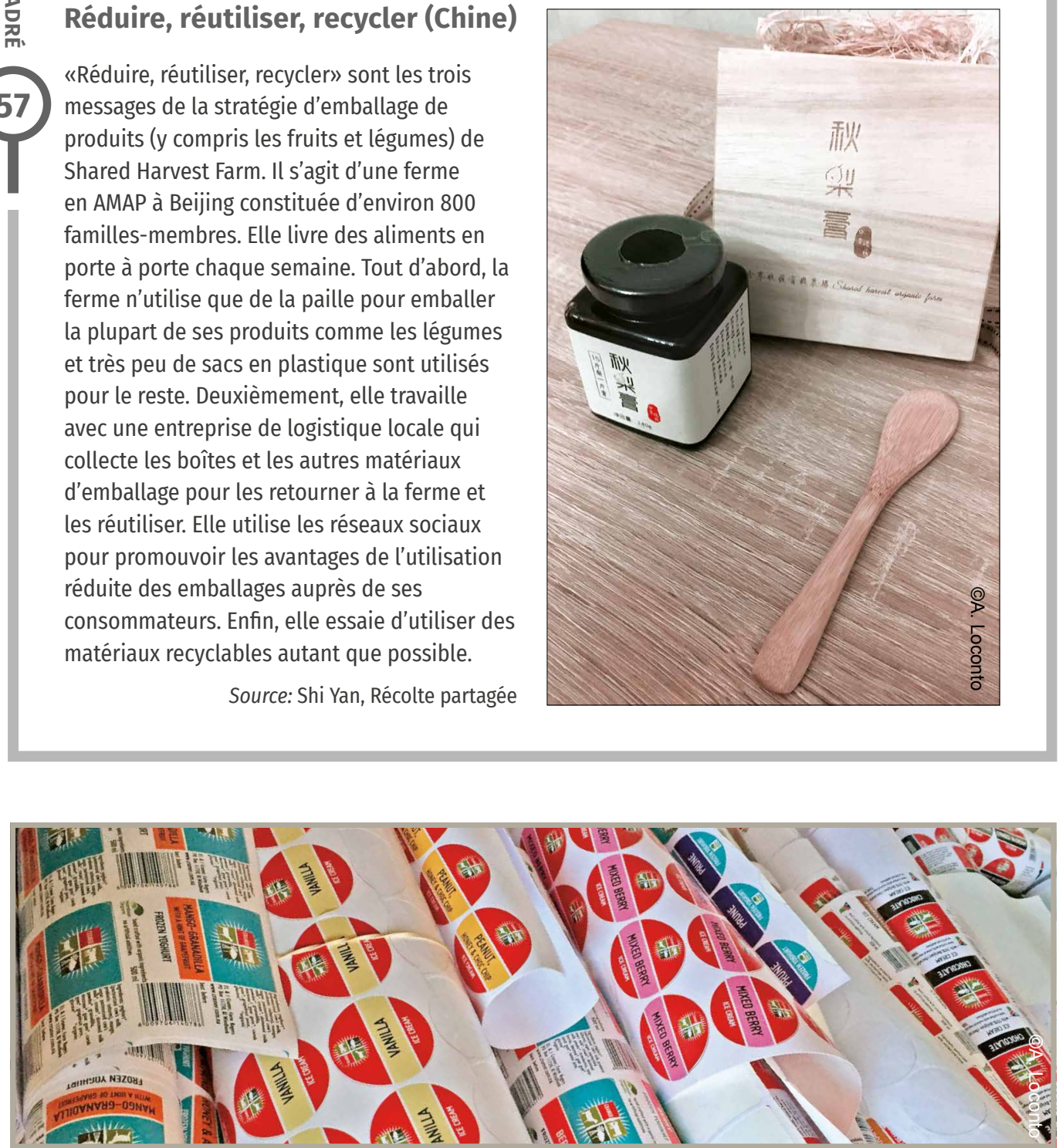


\section{COMMENT DÉTERMINER L'EMBALLAGE DE VOS PRODUITS?}

Lorsque vous décidez de la façon d'emballer votre produit de façon durable, tenez compte de deux éléments principaux:

1. La périssabilité du produit à vendre et le but de son emballage, c'est-à-dire vendre le produit au consommateur final ou le transporter à un autre point de transformation ou de vente.

2. Lorsqu'il s'agit de matériaux d'emballage durables, il est également important de considérer la durabilité environnementale. Pour ce faire, adoptez une parmi deux stratégies: recherchez soit des matériaux d'emballage biodégradables, comme les emballages en fibres naturelles, soit des emballages réutilisables ou entièrement recyclés (par exemple, le verre). Dans la mesure du possible, évitez les emballages en plastique.

Tableau 8: Exemples d'emballages différents selon les caractéristiques du produit

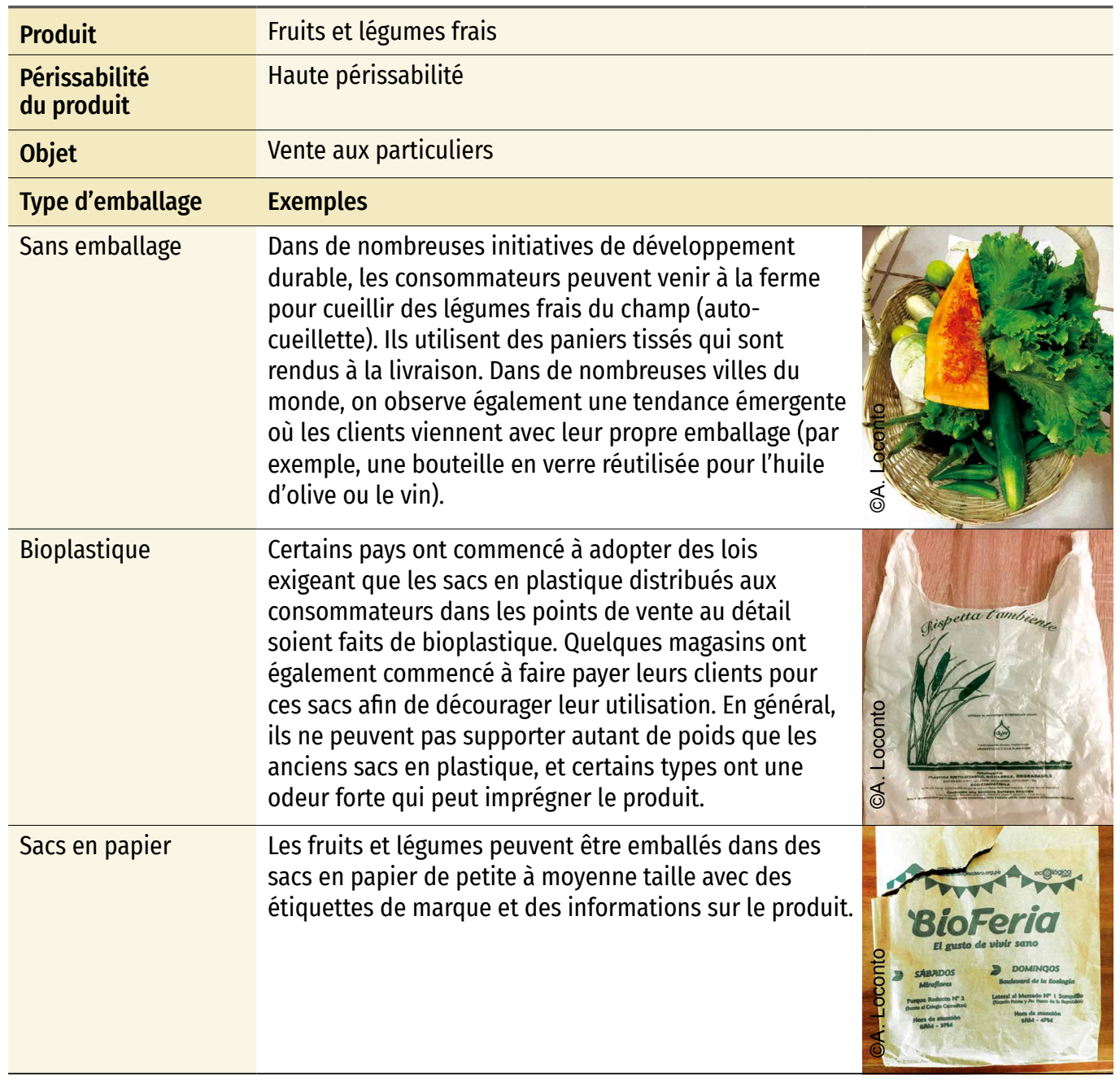




\begin{tabular}{|c|c|}
\hline Produit & Fruits et légumes frais \\
\hline $\begin{array}{l}\text { Périssabilité } \\
\text { du produit }\end{array}$ & Haute périssabilité \\
\hline Objet & Transport vers les points de vente ou de transformation \\
\hline Type d'emballage & Exemples \\
\hline Carton pour fraises & $\begin{array}{l}\text { Pour les produits très périssables comme les fraises, } \\
\text { des boîtes en carton de petite taille peuvent être } \\
\text { utilisées pour réduire l'usage du plastique. Vu } \\
\text { que les boîtes en carton ont tendance à absorber } \\
\text { l'humidité, c'est mieux de les garnir de feuilles. } \\
\text { Dans le cas des fraises, cela permet de conserver le } \\
\text { produit plus longtemps et d'éviter qu'il périme. Pour } \\
\text { les fraises biologiques, les feuilles de fraisier sont } \\
\text { utilisées. Dans la production conventionnelle, cette } \\
\text { méthode n'est pas autorisée. }\end{array}$ \\
\hline $\begin{array}{l}\text { Paniers tissés de } \\
\text { feuilles de palmier }\end{array}$ & $\begin{array}{l}\text { Les paniers sont biodégradables, facilement } \\
\text { recyclables et très abordables. De plus, l'esthétique } \\
\text { des paniers a tendance à attirer les consommateurs. }\end{array}$ \\
\hline Caisses empilables & $\begin{array}{l}\text { Ce type de caisse peut être réutilisé plusieurs fois } \\
\text { et peut maximiser l'espace disponible en prévenant } \\
\text { que les produits ne s'endommagent pendant } \\
\text { le transport. Certaines marques de caisse sont } \\
\text { fabriquées à partir de bioplastiques solides (par } \\
\text { exemple, l'amidon de maïs). }\end{array}$ \\
\hline
\end{tabular}

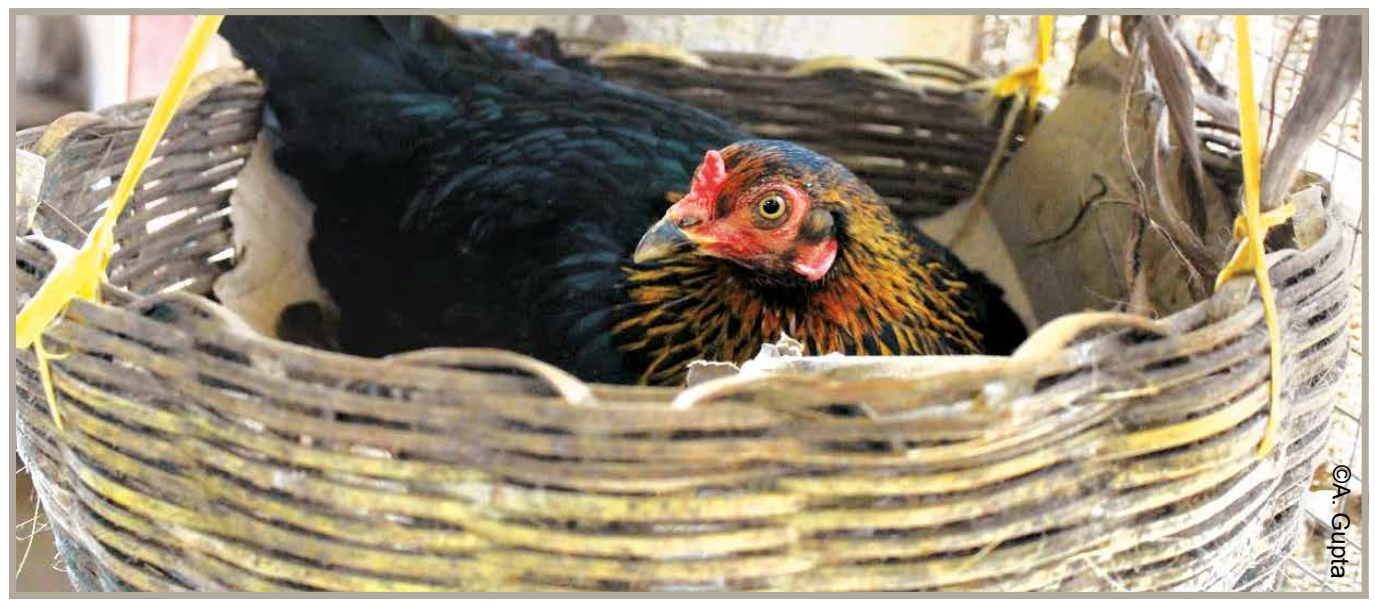




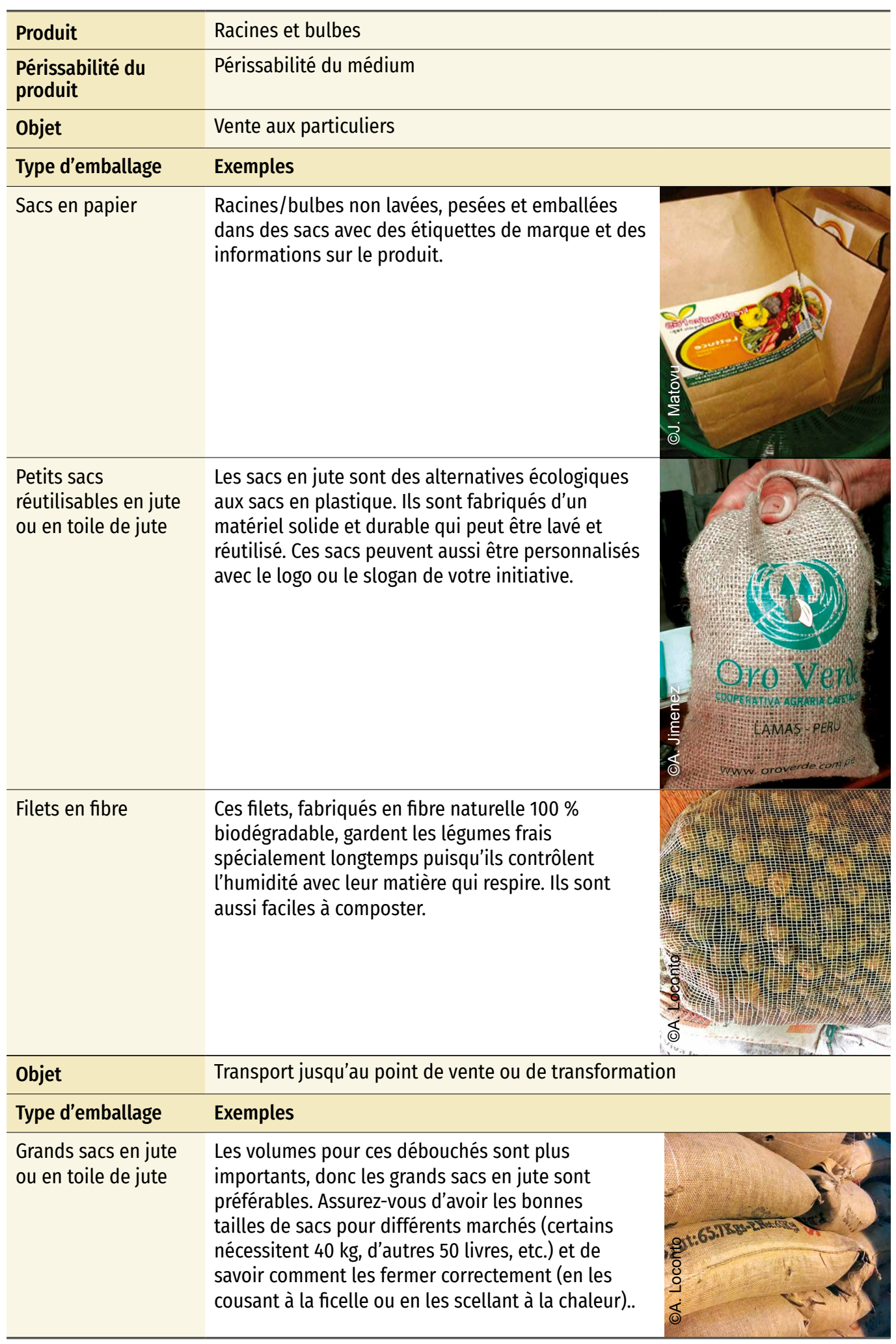




\begin{tabular}{ll}
\hline Produit & Liquides et confitures \\
\hline Product perishability & Périssabilité moyenne à faible \\
\hline $\begin{array}{l}\text { Périssabilité du } \\
\text { produit }\end{array}$ & Vente aux particuliers \\
\hline Type d'emballage & Exemples \\
\hline $\begin{array}{l}\text { Bouteilles et pots en } \\
\text { verre }\end{array}$ & $\begin{array}{l}\text { Les emballages en verre pour le sirop Yacon des } \\
\text { producteurs de Sumapaz en Colombie conservent les } \\
\text { propriétés nutritionnelles de ce produit. Le verre est } \\
\text { un emballage approprié car il respecte les normes } \\
\text { d'hygiène et est esthétiquement propre. }\end{array}$ \\
\hline
\end{tabular}

塄

L'emballage dans un projet de pêche artisanale biologique

58 (Thaïlande)

Le projet de pêche artisanale biologique de Earth Net Foundation vise à connecter les pêcheurs traditionnels à petite échelle respectant les pratiques durables dans différentes provinces du sud de la Thaïlande aux consommateurs de Bangkok. Avec des quantités beaucoup trop faibles pour louer un camion frigorifique, les poissons sont emballés dans des caisses en mousses isolantes réutilisables pour préserver leur qualité et leur fraîcheur pendant le transport. Bien qu'il existe de nombreux services logistiques, ceux-ci n'étaient pas disposés à transporter des poissons et fruits de mer avec d'autres marchandises. Heureusement, certains bus publics circulent régulièrement depuis et vers Bangkok et d'autres destinations et acceptent de transporter les caisses en mousse dans leurs conteneurs de fret. Ensuite, le personnel du projet récupère les caisses et les transporte au marché.

Source: Michael Commons, Earthnet Foundation
Les emballages en fibres naturelles pour la livraison à domicile de FreshVeggie (Ouganda)

Dans le programme de livraison FreshVeggie, les paniers sont fabriqués à la main à partir de feuilles de palmier non-teintes et de fibres de bananes séchées. Tissés en plusieurs couches, ils assurent la solidité et la durabilité. Provenant d'un collectif local de femmes, les paniers soutiennent les revenus des ménages, ainsi que l'utilisation durable des zones humides (les palmiers poussent dans les zones marécageuses et humides). Ces paniers naturels sont attrayants, réutilisables, sûrs pour transporter des légumes frais et d'autres produits alimentaires, abordables et respectueux de l'environnement. De plus, ils préservent la fraîcheur en permettant l'évapotranspiration naturelle des légumes. En revanche, les sacs en plastique étouffent les produits et réduisent leur durée de conservation et ont un impact négatif sur l'environnement tout au long de leur cycle de vie.

Source: Julie Matovu, SPG FreshVeggie, Ouganda 


\section{CONCEVOIR LES BONS EMBALLAGES}

\subsection{Le design}

La conception de l'emballage sert de "façade» du produit et doit se distinguer par rapport aux autres produits de la même catégorie. De plus, elle doit afficher les informations et les caractéristiques du produit qui le rendent plus attrayant que ses concurrents.

L'emballage est aussi un moyen de communiquer les valeurs de votre initiative et de vos produits (écologique, innovant, économie solidaire, artisanal, local, etc.) aux consommateurs.

Rappelez-vous que la conception est essentielle au développement de produits et à la création de valeur.

\section{ASTUCE 28}

Développer un design attrayant

1. Pensez à l'ergonomie du produit (facile/difficile à ouvrir, facile à manipuler, facile à transporter, poids adapté à sa taille, etc.).

2. Parlez directement à vos consommateurs au cours de la conception de votre emballage. Pensez à la façon dont il incarne le message visuel clé que vous souhaitez communiquer (santé, produit local, écologique, etc.) et aux façons de le faire (couleur/images/texte/relation entre les différents éléments de l'emballage, etc.).
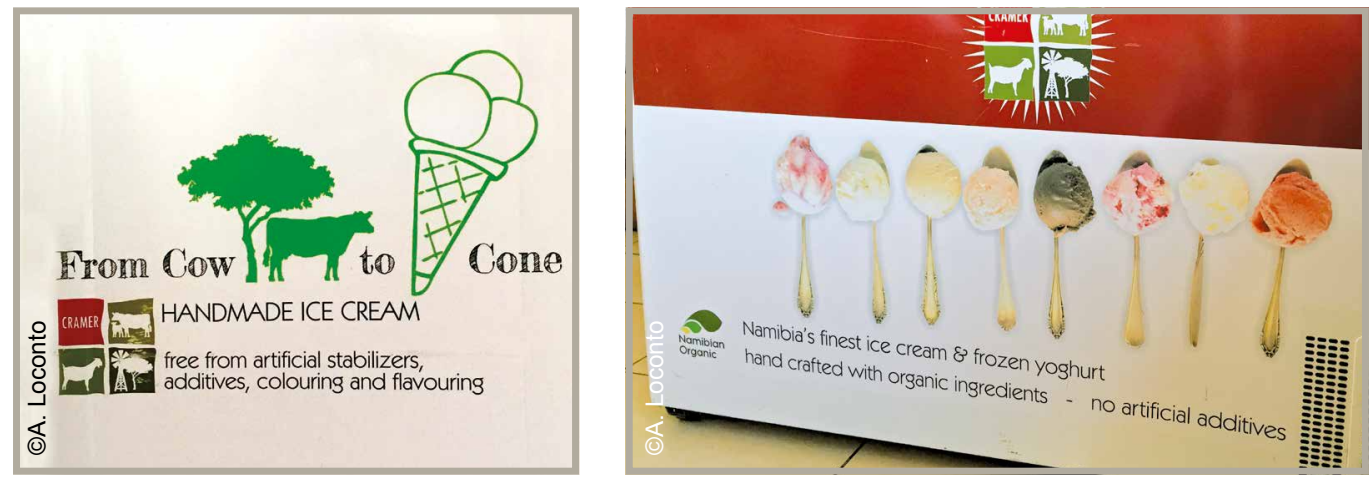


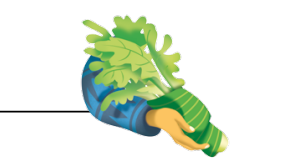

\section{Comment créer un design original et attrayant? (Colombie)}

Dans le cas des chips Ancestrales, fabriqués à partir de variétés de pommes de terre autochtones, Familia de la Tierra a commencé à développer son produit en identifiant le concept principal qu'elle souhaitait partager avec ses clients: promouvoir la préservation de l'écosystème de Páramo et de la culture paysanne traditionnelle. Ancestrales est apparu comme un nom de qui encapsule ces valeurs.
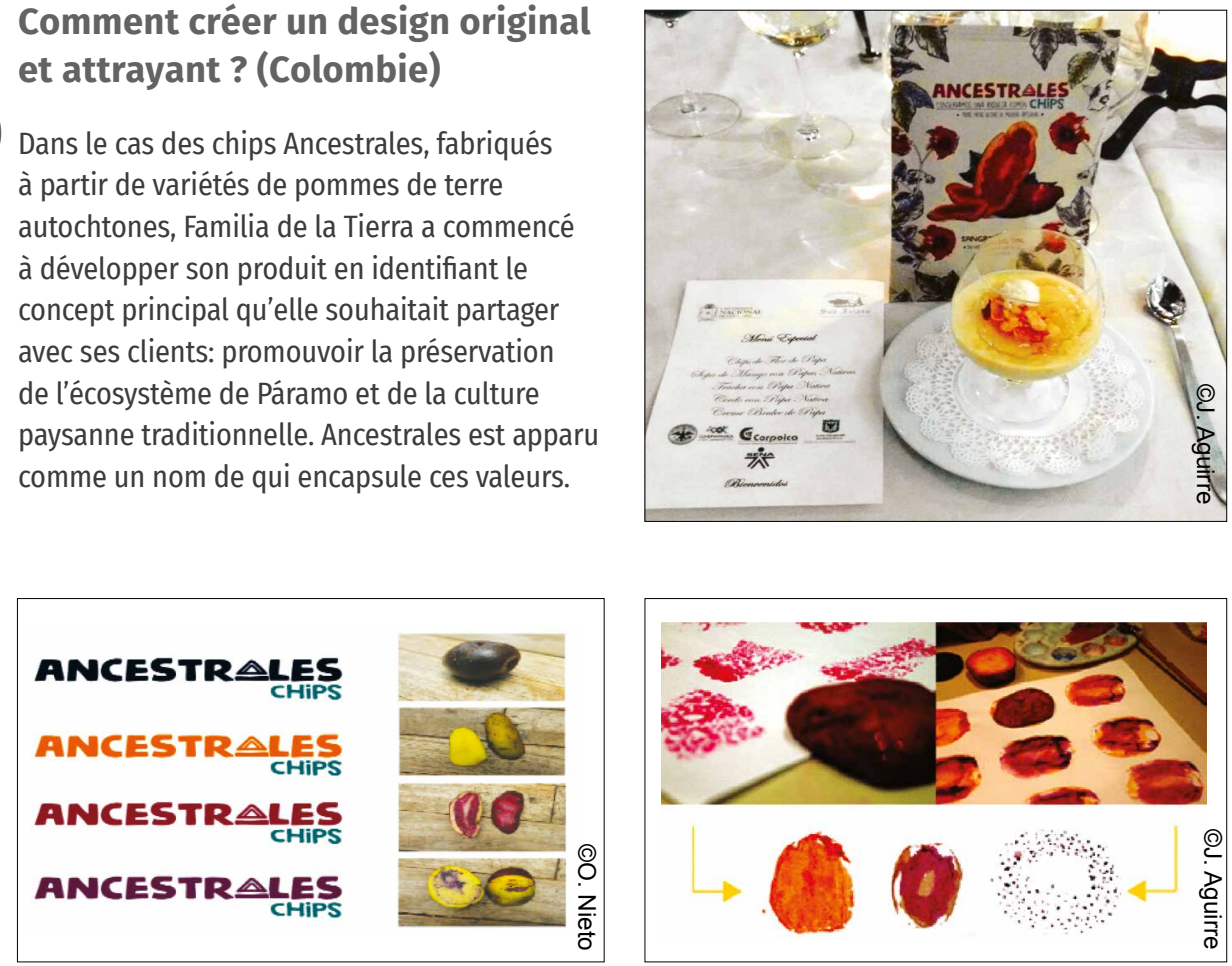

Ensuite, une équipe de designers a apporté son aide pour réaliser l'emballage. Les textures de pommes de terre ont été reproduites à l'aquarelle, puis numérisées et incorporées dans la conception finale.

La dernière étape a consisté à finaliser le logo de la marque et à rassembler tous les éléments pour compléter l'emballage.

Source: Oscar Nieto, FdT
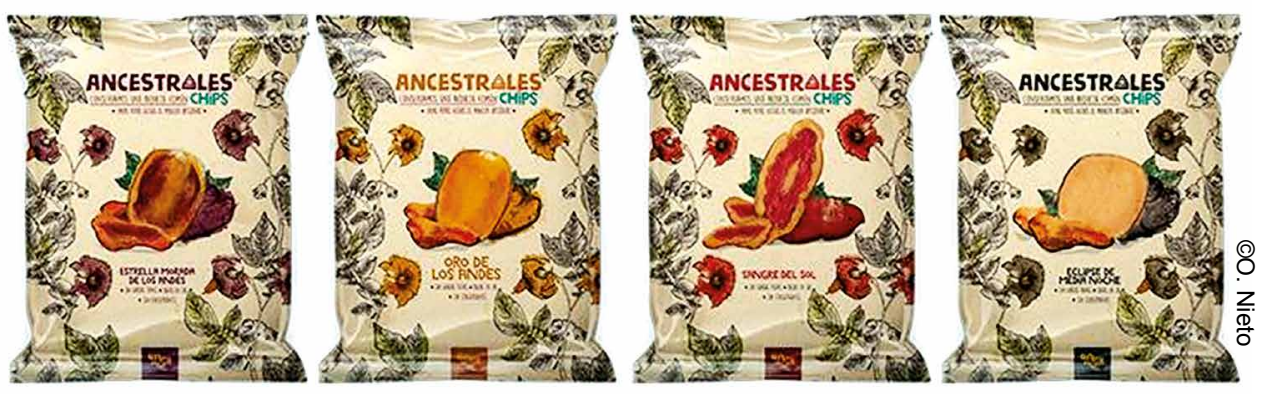


\subsection{La taille}

La taille de l'emballage est également d'une importance fondamentale pour assurer l'attractivité, l'ergonomie et la consommation durable (c'est-à-dire la réduction des déchets alimentaires, une portion nutritionnelle appropriée) de vos produits. Pensez au consommateur final pour déterminer la taille appropriée. Par exemple, si le produit est destiné aux enfants (ou à un consommateur individuel plutôt qu'à une famille), réduisez la taille afin d'éviter le gaspillage.

Tableau 9: Exemples de différents formats d'emballage pour différents consommateurs

\begin{tabular}{l|l|l}
\hline Produit & $\begin{array}{l}\text { Consommateurs ciblés } \\
\text { (et débouchés) }\end{array}$ & Taille \\
\hline \multirow{2}{*}{$\begin{array}{l}\text { Canne } \\
\text { à sucre } \\
\text { fraîche }\end{array}$} & $\begin{array}{l}\text { Consommateurs individuels } \\
\text { (épiceries de détail, marchés } \\
\text { d'alimentation ouverts) }\end{array}$ & $\begin{array}{l}\text { Fagots de } 10 \text { à } 12 \text { morceaux entiers de canne à sucre } \\
\text { de la taille d'une plante attachés avec des ficelles } \\
\text { de sisal/fibre. }\end{array}$ \\
\cline { 2 - 3 } & $\begin{array}{l}\text { Enfants (marchés de producteurs } \\
\text { et ventes à la ferme) }\end{array}$ & Pièces uniques de tiges coupées. \\
\cline { 2 - 3 } & $\begin{array}{l}\text { Les familles } \\
\text { (paniers) }\end{array}$ & $\begin{array}{l}\text { Décortiquée, pesée en kilogrammes }(0,5 \mathrm{~kg}, 1 \text { à } 4 \mathrm{~kg}) \\
\text { et emballée dans un sac en papier recouvert d'un } \\
\text { sac en polyéthylène de qualité alimentaire. }\end{array}$ \\
\hline
\end{tabular}

\subsection{La transformation des matériaux d'emballage}

Des investissements importants sont souvent nécessaires pour l'équipement d'emballage ainsi que pour le développement des matériaux d'emballage. Des machines spécifiques sont requises pour chaque processus, ainsi qu'un personnel qualifié pour s'en servir. Généralement, le coût de l'emballage est l'une des principales raisons pour lesquelles les petits agriculteurs préfèrent externaliser la tâche. Cependant, il existe de nombreuses techniques artisanales de transformation, de stockage et d'emballage des produits (par exemple, la mise en conserve des confitures à la maison) et il y a une tendance croissante à utiliser une approche minimaliste qui offre de nouvelles possibilités. Ainsi, vous pouvez soit développer un emballage simple et attrayant, soit le confier à une entreprise spécialisée.

\section{ASTUCE 29}

Concevoir un emballage simple et attrayant

Un emballage attrayant ne nécessite pas forcément un investissement important. Avec une imprimante (couleur) et un peu d'attention au design, vous pouvez créer une marque unique et fournir toutes les informations nécessaires. Pour les produits partageant une taille et un emballage communs, comme les paquets de semences de légumes, il peut être utile de concevoir un emballage imprimé standard, puis d'apposer de petites étiquettes autocollantes pour indiquer la variété, le poids et d'autres informations spécifiques. Les feuilles d'étiquettes autocollantes blanches (et colorées) au format imprimante A4 sont faciles à trouver. Autre alternative, du papier recyclé peut être utilisé et collé avec un autocollant ou une colle transparente. C'est un choix beaucoup plus approprié jusqu'à ce que des volumes plus importants rendent l'impression professionnelle intéressante. Une écriture attrayante ou l'apposition d'un tampon en caoutchouc peut ajouter du caractère à un produit et à son côté artisanal. 

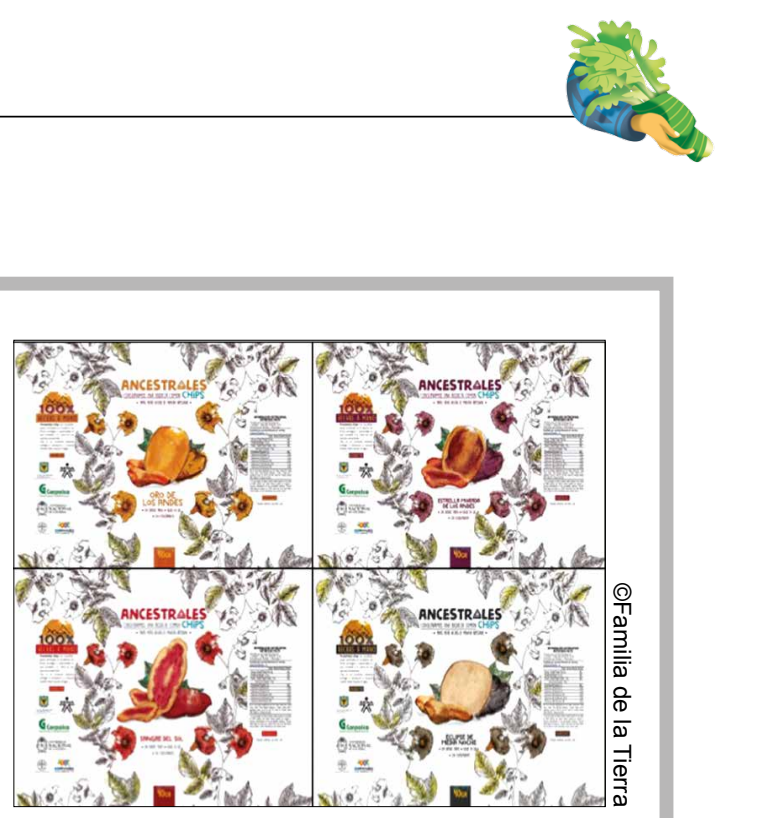

insérés sur une seule feuille d'impression, obtenant ainsi 12000 emballages pour chaque type de chips. Les coûts d'emballage ont réduit de 200 COP à 105 COP par sac (environ 0,03 USD), économisant ainsi une somme d'argent importante. Pour Ancestrales, une somme initiale de 2400 USD a été investie

pour la conception des modèles exemplaires, le découpage de moules et l'impression. Le deuxième cycle d'impression a couté environ 1700 USD.

Source: Oscar Nieto, FdT

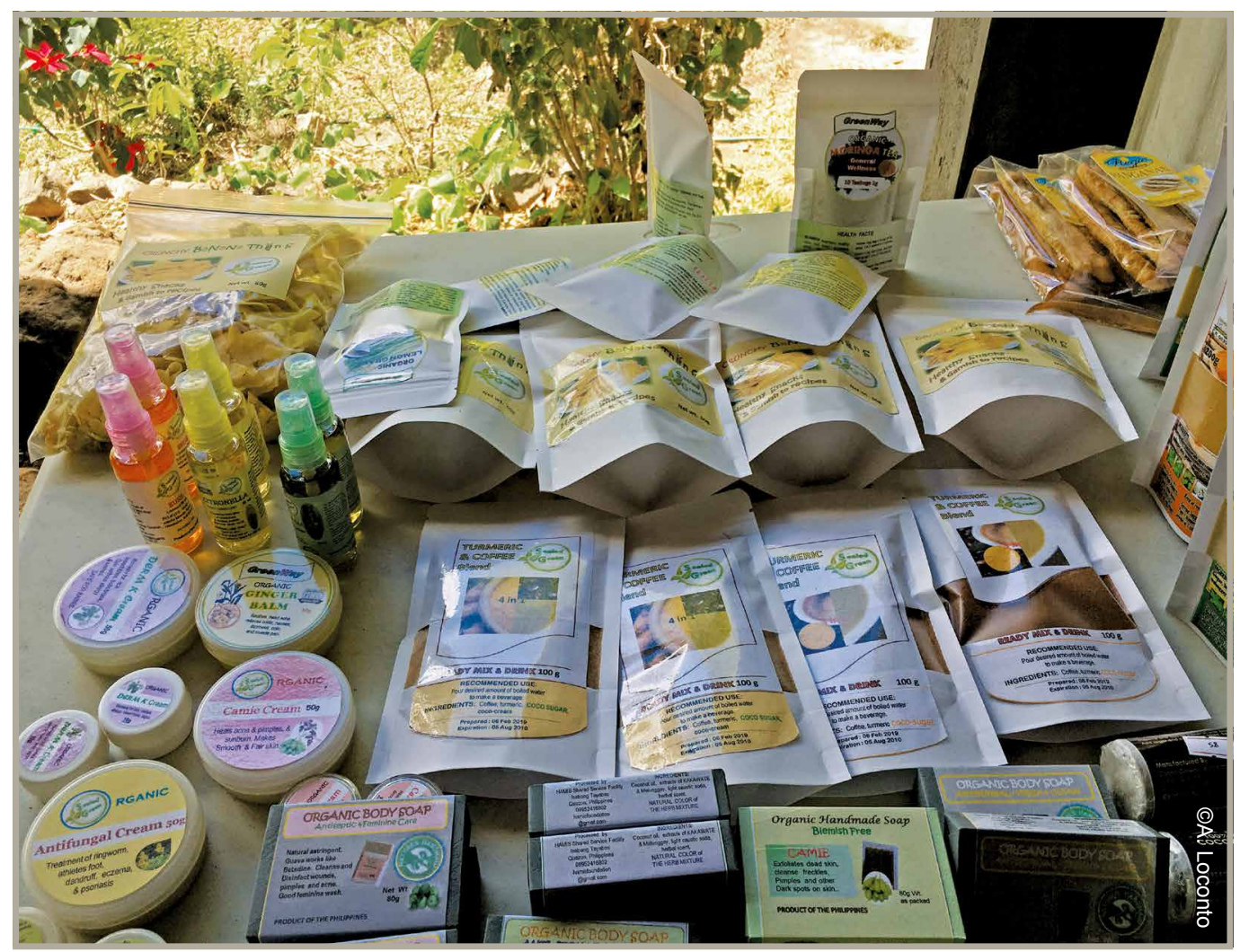




\section{COMMENT POUVEZ-VOUS ÉTIQUETER VOS EMBALLAGES?}

Les messages écrits et les images que vous incluez sur vos emballages ont deux objectifs: de communiquer les informations légalement requises par la loi sur le produit, mais aussi vos valeurs et les qualités de vos produits.

\subsection{Les aspects juridiques de l'emballage}

Même si les réglementations varient d'un pays à l'autre, les informations suivantes doivent normalement figurer sur l'emballage: le nom du produit, la liste des ingrédients, le poids, les valeurs nutritionnelles, les coordonnées du producteur ou de la société, le lieu d'origine, le numéro de lot, la date de production et la date d'expiration, les instructions de conservation et le mode d'emploi.

\section{Les informations obligatoires sur l'étiquette (Colombie)}

Les informations à inclure sur l'étiquette d'un produit sont spécifiées dans les lois nationales sur l'étiquetage. En Colombie, les informations suivantes sont obligatoires: 1) les propriétés nutritionnelles; et 2) les ingrédients énumérés dans l'ordre, de la plus grande quantité à la plus petite. L'accès à ces informations, notamment sur les valeurs nutritionnelles, n'est pas toujours une tâche facile pour les petits agriculteurs. La façon la plus précise d'obtenir cette information est de faire tester vos produits dans un laboratoire accrédité. Si cette option n'est pas faisable, des copies des tableaux de composition des aliments peuvent être obtenues auprès des autorités nationales de sécurité alimentaire et de nutrition. De plus, certains outils web permettent aussi de créer des étiquettes de valeur nutritive pour des produits basés sur des recettes standard. Avant de les utiliser, assurez-vous que vos chiffres soient valides. Voici quelques outils utiles pour ce faire:

- Le répertoire international des tables/ bases de données internationales sur la composition des aliments (INFOOD) contient tous les tableaux officiels de composition nutritionnelle des produits alimentaires mesurés et a été constamment mis à jour depuis 1988. Il existe des liens vers des bases de données régionales qui contiennent de l'information sur les aliments propres aux régions.

http://www.fao.org/infoods/infoods/tablesand-databases/en

Wolphram alpha est un exemple d'outil gratuit où, en saisissant votre produit, vous pouvez obtenir les valeurs nutritionnelles, même si les valeurs fournies sont des "produits standards» et qu'il peut exister des différences avec ces valeurs, selon les caractéristiques spécifiques du produit. https://www.wolframalpha.com/examples/ society-and-culture/food-and-nutrition

Recipal est un outil qui vous permet d'obtenir, moyennant des frais minimes, non seulement la valeur nutritive de vos produits/recettes, mais aussi de vous aider à concevoir votre propre étiquette. https://www.recipal.com

Source: Orcar Nieto, Familia de la Tierra, Colombie 


\subsection{Les aspects commerciaux de l'emballage}

Vous pouvez inclure des renseignements supplémentaires non-obligatoire sur l'emballage pour le rendre plus attrayant. Ces informations comprennent:

- des labels environnementaux et/ou sociaux attribués aux agriculteurs qui respectent des normes spécifiques de production ou de commerce (par exemple, biologique, équitable, sans OGM, vérifié) - ceux-ci sont facilement reconnus par de nombreux consommateurs qui les associent à la durabilité et au commerce éthique;

- votre marque, pour aider vos consommateurs à différencier vos produits de ceux de vos concurrents;

- des déclarations sur les valeurs nutritionnelles spécifiques de votre produit (par exemple: faible ou inexistante en gras, riche en fibres, faible teneur en sucre, sans gras trans, etc.)

- des déclarations des propriétés bénéfiques pour la santé de votre produit (par exemple: Yacón aide à réguler la glycémie, ce qui a un effet positif pour le traitement du diabète);

- une histoire personnelle sur qui vous êtes, comment vous vivez, comment vous cultivez ou produisez le produit - ce texte est normalement accompagné d'une photo de l'agriculteur ou du producteur (en partageant l'histoire du produit, le consommateur se rapproche de vous). N'oubliez pas de faire très attention aux allégations commerciales que vous faites sur vos produits - certaines d'entre elles sont réglementées par les lois alimentaires. Les allégations concernant les bienfaits pour la santé sont généralement très strictement réglementées et vous pouvez avoir des problèmes si vos allégations ne sont pas appuyées par des preuves scientifiques.

Attention: Certains pays réglementent strictement les déclarations des propriétés bénéfiques pour la santé faites sur les produits, vérifiez donc les règles de votre pays et celles du pays d'export (par exemple, l'UE a des lois très strictes sur les allégations santé).

\section{ASTUCE 30}

Emballer des produits durables qui se vendent

- Bien que l'emballage joue un rôle important dans la conservation des produits, il est également très important pour la communication de la valeur.

- Pour répondre au mieux aux besoins des intermédiaires et des consommateurs, différentes formes d'emballage à différents stades de la chaîne d'approvisionnement peuvent être utilisées. Certains matériaux d'emballage sont même réutilisables.

- Utiliser des emballages respectueux de l'environnement peut réduire les coûts (en particulier si vous utilisez des matériaux recyclés), réduire l'impact environnemental et les déchets et être à la fois attrayant.

- L'espace pour les étiquettes sur un produit emballé est limité - choisissez ce que vous communiquez judicieusement! 


\section{LE CHEMIN D’APPRENTISSAGE, OÙ ALLER ENSUITE?}
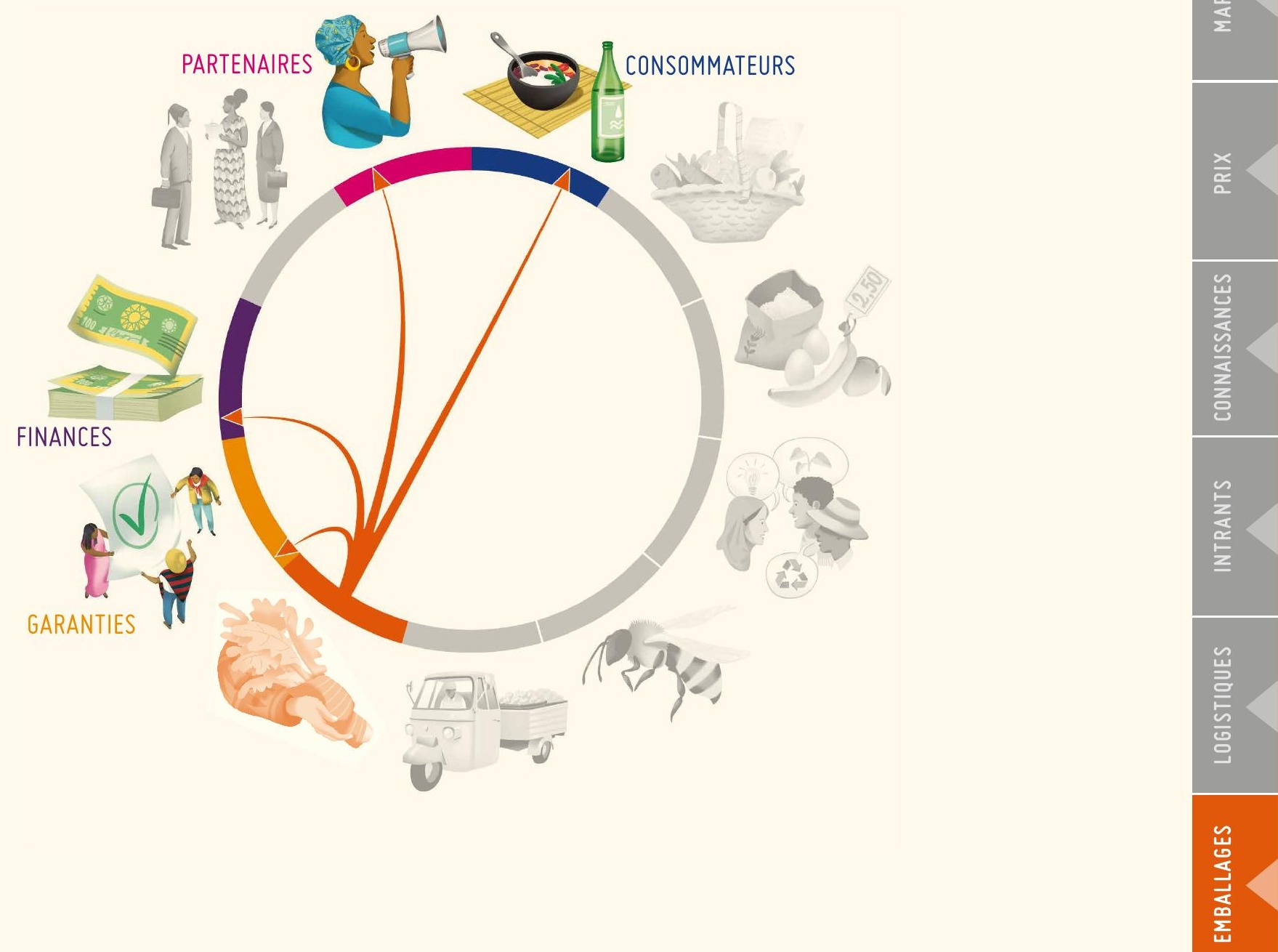

- Vous voulez savoir quelles valeurs inclure sur votre emballage ? Retournez au Chapitre 1: Attirer les clients et les fidéliser

- Vous pensez vouloir collaborer avec un imprimeur professionnel pour concevoir vos emballages ? Consultez le Chapitre 11: La place des partenaires et des plaidoyers pour apprendre comment faciliter un partenariat.

- Vous avez l'idée d'investir dans les procédés et la technologie d'emballage, mais vous n'avez pas les fonds nécessaires ? Sautez au Chapitre 9: Les financements innovants pour vous faire une idée.

- Vous souhaitez inclure des étiquettes liées au développement durable, comme les labels biologiques, sur votre emballage ? D’abord, assurez-vous de pouvoir garantir cette qualité... le Chapitre 8: Garantir la durabilité vous en dira plus.

Ou dirigez-vous vers un autre chapitre... vous êtes le mieux placé pour le savoir ! 


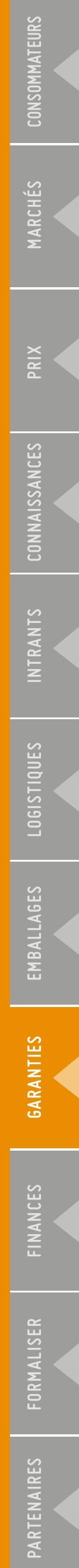




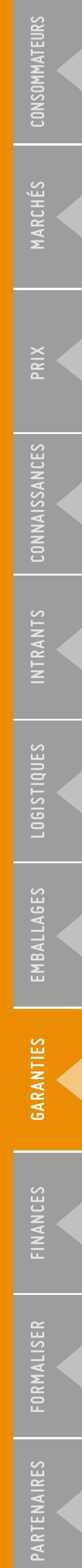




\section{POURQUOI EST-CE IMPORTANT?}

Vu que l'agriculture durable n'est pas pratiquée par tous les producteurs, il est fondamental de se différencier des autres.

Les producteurs, les intermédiaires et les consommateurs utilisent un système de garantie pour confirmer que les aliments échangés ont été produits de manière durable.

\section{QU'EST-CE QU'UNE GARANTIE?}

Une garantie est une promesse que quelque chose a été fait ou que quelque chose se produira. Cela peut être aussi simple que de dire qu'on promet de produire de façon durable (une garantie informelle) ou aussi complexe qu'une allégation formelle, certifiée par un tiers, qu'un produit, une personne ou un procédé répond à certaines normes préétablies de durabilité ou de qualité.

Une garantie est nécessaire pour instaurer la confiance des consommateurs. Elle assure a cohérence de la qualité des produits, protège les consommateurs contre la fraude et confirme que le produit respecte les pratiques de production durable.

Un système de garantie est la fourniture systématique d'une garantie. Il suit généralement une norme de production et des règles opérationnelles écrites et les producteurs ou transformateurs obtiennent un certificat démontrant leurs pratiques durables. Il existe différentes façons d'attester le respect de ces pratiques.

\subsection{Auto-déclaration}

Dans ce type de garantie, les producteurs s'auto-certifient. L'auto-déclaration peut avoir lieu sous différents degrés de formalité. Il peut s'agir d'une simple garantie informelle, communiquée verbalement aux consommateurs comme, par exemple, la «garantie de remboursement» (si le consommateur est insatisfait du produit, le vendeur promet de le rembourser). Une autodéclaration plus formelle consiste à remplir un formulaire d'auto-évaluation écrit ou un engagement soumis à l'intermédiaire ou à l'acheteur transparent. Ce type de garantie est avantageux puisqu'il renforce la confiance entre les producteurs et les consommateurs qui se rencontrent et communiquent régulièrement. Par contre, les fausses déclarations sont fréquentes et difficiles à détecter lorsque les producteurs et les acheteurs se côtoient rarement.

Avantages

+ Les coûts sont bas;

+ La plupart des formulaires sont simples à remplir;

+ il n'y a pas d'audits externes.

\section{Inconvénients}

- Les fausses déclarations sont fréquentes;

- certains formulaires sont difficiles à remplir;

- habituellement, il n'y a pas d'étiquettes sur l'emballage. 


\subsection{Audit d'acteurs}

Dans ce type de garantie, également appelée «certification par une deuxième partie», l'évaluation est effectuée par un intermédiaire transparent ou un transformateur qui est intéressé à auditer les producteurs. Il peut s'agir, par exemple, d'un transformateur qui souhaite auditer les agriculteurs qui le fournissent, ou d'une organisation certifiant ses membres et, dans ce dernier cas, le processus est aussi appelé «système de contrôle interne» (SCl).

\section{Avantages}

+ Les coûts sont bas.

+ Les agriculteurs n'ont pas besoin de remplir des formulaires, ceux-ci sont généralement remplis par la deuxième partie.

+ Il n'y a pas d'audits externes.

+ Parfois, les agriculteurs peuvent disposer de leurs propres certificats.

+ Cela permet parfois aux producteurs d'apposer une étiquette sur l'emballage.

\section{Inconvénients}

- Toutes les fermes ne sont pas visitées.

- Il arrive parfois que de fausses déclarations soient faites.

- L'inspection aléatoire (en vertu du SCI) est ouverte aux abus et à la non-conformité.

- Les certificats sont souvent utilisés en interne par l'acheteur ou la coopérative/association et ne peuvent être utilisés sur d'autres marchés.

\subsection{Système d'inspection, de certification et d'accréditation}

Ce système de garantie, également appelé «certification par un tiers», est le plus formel, puisqu'il comprend plusieurs niveaux de contrôle. L'évaluation est effectuée par un organisme de certification externe qui doit être accrédité par le gouvernement et qui n'a aucun intérêt financier ou commercial dans la ferme ou l'organisme en question.

\section{Avantages}

+ C'est la seule garantie reconnue par la réglementation publique (sauf pour quelques pays).

+ Il y a des exigences en matière d'exportation (pour accéder aux marchés des États-Unis, de l'UE, du Japon, de la Chine, du Canada, etc.).

+ Certaines certifications sont acceptées sur plusieurs marchés.

+ Les agriculteurs organisés en coopératives peuvent utiliser un $\mathrm{SCl}$ secondaire pour le contrôle interne et faire auditer la certification de leur SCl par une tierce partie.

+ Les producteurs peuvent souvent apposer une étiquette sur l'emballage.

\section{Inconvénients}

- Les coûts sont élevés.

- Beaucoup de documentation est requis.

- La participation n'est pas promue.

- Le contrôle social est absent.

- Le certificat est remis au groupe (ICS), à l'ONG ou à l'entreprise exportatrice et non aux agriculteurs individuels.

- Les normes sont systématiquement revues et parfois assouplies pour faciliter l'obtention de la certification.

- L'inspection aléatoire (en vertu du SCI) est ouverte aux abus et à la non-conformité.

- Il y a un conflit d'intérêts inhérent puisque les agriculteurs paient directement les certificateurs pour l'audit (des certificats sont parfois achetés). 


\subsection{Systèmes hybrides}

Il s'agit de systèmes qui utilisent plusieurs types de garanties. Par exemple, l'auto-déclaration et la certification par un tiers peuvent être combinées - c'est le cas dans certaines tables rondes sur les produits de matière première dans le secteur privé. Dans ce système, les agriculteurs remplissent des formulaires sur leur rendement, puis un échantillon représentatif de fermes est choisi au hasard pour être vérifié par un tiers. Des garanties secondaires et des garanties de tiers peuvent aussi être combinées, comme le cas de la certification Rainforest Alliance. Dans tous les systèmes, les garanties informelles jouent souvent un rôle.

\section{Avantages}

+ Ces systèmes mixtes sont souvent adoptés par des marques privées - il existe donc de grands marchés pour des produits tropicaux uniques (par exemple, le thé, le café, l'huile de palme, le soja et le cacao).

+ La combinaison de différentes formes de garantie peut contribuer à éviter la fraude qui est fréquente dans les systèmes reposants sur un seul type de garantie.

+ Il y a une collaboration accrue entre les systèmes mixtes, de sorte que la possibilité d'utiliser des rapports pour plus d'un système de garantie peut réduire la tâche administrative des producteurs.

\section{Inconvénients}

- Certaines difficultés liées à la certification par une seconde ou une tierce partie persistent, particulièrement en termes de coûts et de documentation.

- Les multiples formes de garanties créent beaucoup de bureaucratie et ralentissent le flux des retours/ recommandations.

- Certains consommateurs considèrent le mélange des garanties comme une stratégie d'entreprise visant à réduire la rigueur des vérifications par des tiers, qu'ils estiment comme étant les plus rigoureuses. Cela peut diminuer la confiance des consommateurs.
塄

63

Rainforest Alliance combine la certification par

une deuxième partie et une tierce partie pour

attribuer l'utilisation de son label «grenouille verte». Chaque année, les fermes sont évaluées par l'équipe de vérification de Rainforest Alliance. Les audits secondaires de Rainforest Alliance consistent principalement en des audits documentaires (pour s'assurer que des rapports sur les plans d'amélioration sont effectués) ainsi que des visites occasionnelles à la ferme pour discuter des principales non-conformités. Tous les trois ans, un tiers certificateur audite la ferme, choisit de renouveler ou de révoquer le certificat et envoie le rapport final à Rainforest Alliance. Sur la base de ce rapport et de ses propres audits, Rainforest Alliance décide si la ferme sera certifiée pour la période suivante de trois ans.

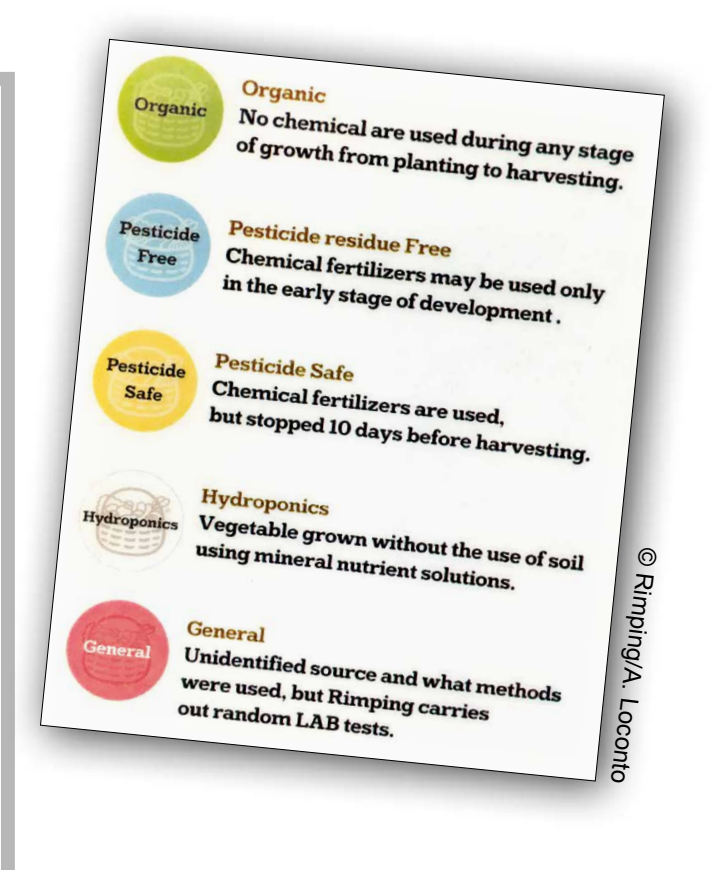




\subsubsection{Système participatif de garantie (SPG)}

Les SPG utilisent des éléments d'auto-déclaration et de certification par une tierce partie. Ce sont des systèmes d'assurance de la qualité axés sur les conditions locales. Ils certifient les producteurs sur la base de la participation active des agriculteurs, des consommateurs et d'autres acteurs locaux. Les agriculteurs s'engagent à respecter les normes biologiques et un groupe d'acteurs (généralement composé d'agriculteurs ou un mélange d'agriculteurs, de consommateurs et d'agronomes) effectuent des visites sur le terrain à intervalles réguliers, soit mensuelles, semestrielles ou annuelles. Un comité de SPG est établi avec des représentants de tous les groupes d'intervenants qui examinent les rapports et déterminent si la certification mérite d'être accordée ou non. Ces groupes travaillent de manière autonome pour garantir le respect des marques privées de distributeurs, ou ils peuvent être associés à des organismes publics pour garantir le respect des normes biologiques nationales.

\section{Avantages}

+ Les coûts sont faibles.

+ L'accès et le partage de l'information et des connaissances sont fournis.

+ Tous les acteurs participent et les consommateurs s'engagent à fournir des garanties de produits, ce qui renforce le soutien à la production.

+ L'accès au marché est facilité grâce à des contacts plus directs dans des chaînes d'approvisionnement plus courtes.

+ Lorsque les grands intermédiaires, les hôtels et les restaurants comprennent les processus que les producteurs subissent, ils deviennent alors plus favorables à la production. Leur engagement est renforcé au sein d'un SPG.

+ Le marché et les économies locales sont soutenus.

+ La transparence, l'autonomisation des agriculteurs et le contrôle social sont favorisés.

+ Il y a des règles de non-conformité prédéterminées.

+ Il est développé en fonction des conditions et des besoins des petits agriculteurs.

+ La méthode de certification n'est pas hiérarchique.

\section{Inconvénients}

- Il est non-reconnu par certains organismes de réglementation publics.

- Les producteurs ne connaissent pas les SPG à moins qu'une ONG ou un chercheur ne leur en parle.

- Il y a un manque d'information nationale et internationale sur les SPG.

- Il faut beaucoup de temps et d'efforts (de la part des producteurs, des consommateurs et d'autres acteurs) pour élaborer un système véritablement participatif.

- Des ressources importantes sont nécessaires pour financer d'autres activités d'appui aux agriculteurs, comme la formation continue, les mises à jour technologiques et les efforts orientés vers la reconnaissance globale des SPG (lorsque le soutien des ONG est limité).

- Il y a souvent un manque de participation active des consommateurs, à moins qu'ils ne soient soutenus par des ONG ou par le gouvernement. 


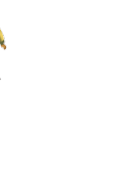

罗

\section{Le SPG de Quezon (Philippines)}

Le SPG de Quezon est un organisme de

certification multi-acteurs qui a été établi pour garantir aux consommateurs que tous les produits vendus au marché fermier hebdomadaire sont biologiques. Son processus de certification comprend les étapes suivantes:

1. L'auto-examen: l'agriculteur est formé sur les normes biologiques, les règles et les processus relatifs au SPG avant de soumettre une demande à un pair examinateur qui détermine si l'exploitation est qualifiée pour l'inspection.

2. Le pair examinateur procède à l'inspection de la ferme et soumet un rapport au comité d'examen.

3. L'examinateur du comité évalue le rapport et décide: a. s'il est approuvé: le comité transmet la décision au Comité de gestion du SPG de Quezon pour délivrer le certificat;

b. s'il est rejeté: le rapport est renvoyé à l'examinateur qui avise l'agriculteur et le Comité de gestion du SPG de Quezon. Dans ce cas, les agriculteurs du groupe peuvent faire des corrections et soumettre une demande pour une autre visite d'examen par les pairs dans six mois.

4. Le Comité de gestion du SPG de Quezon délivre la certification.

5. Le pair examinateur remet la certification à l'agriculteur.

Source: Carmen Cabling, SPG de Quezon, Philippines

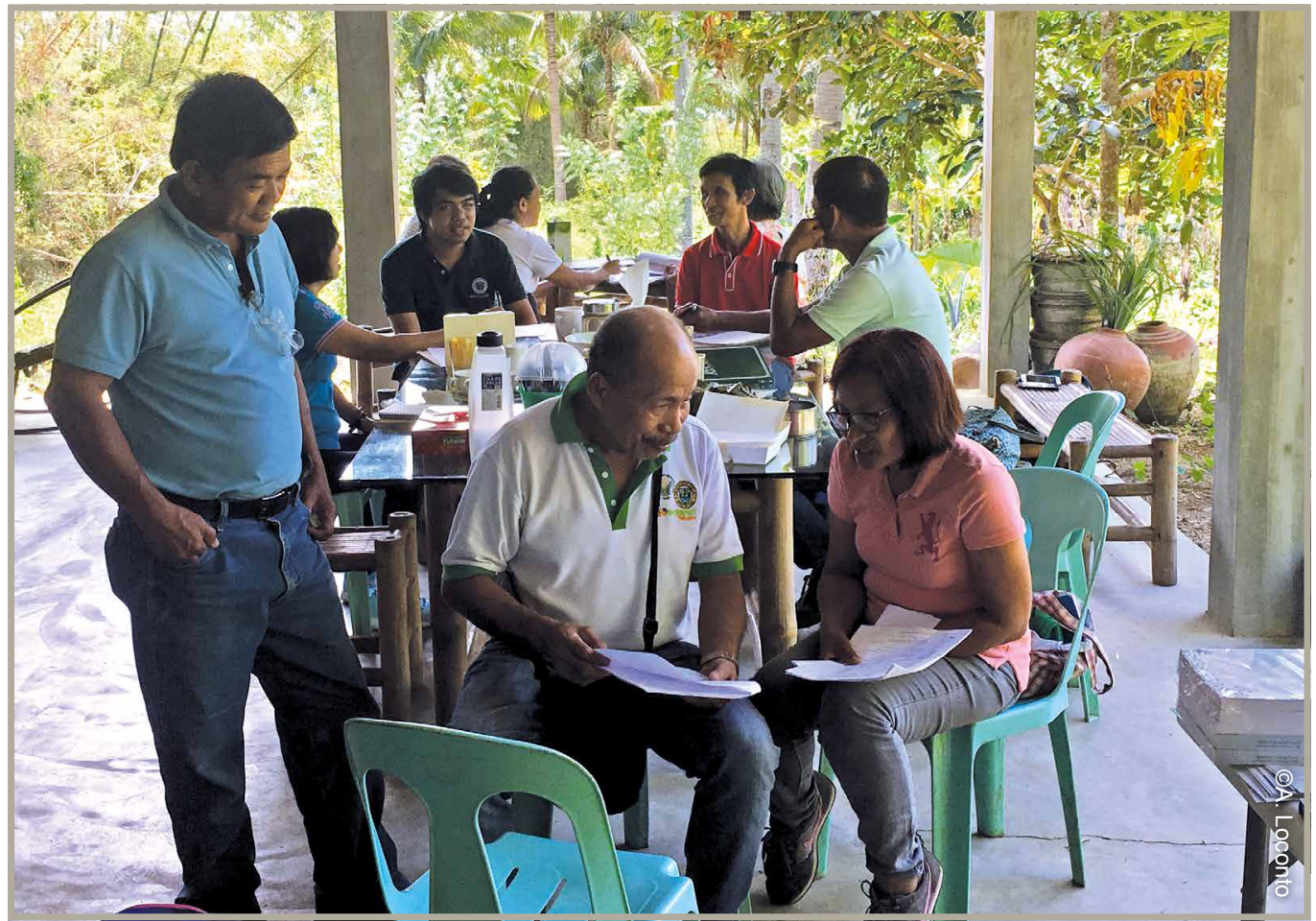




\section{CHOISIR VOTRE SYSTÈME DE GARANTIE}

Avant de choisir le système de garantie qui vous convient le mieux, informez-vous sur les systèmes de certification déjà mis en place dans votre région, afin de prendre la meilleure décision.

\section{ASTUCE 31}

Recueillir des informations sur les systèmes de garantie opérant dans votre région

a. Quelles sont les initiatives qui adoptent des systèmes de certification pour les produits fabriqués de manière durable?

b. Quel est le profil organisationnel de l'initiative?

c. L'initiative est-elle enregistrée légalement ?

d. Combien de systèmes ou de produits agricoles ont été certifiés?

e. Quels sont les frais de participation?

f. Y a-t-il d'autres services offerts aux membres à travers le système de certification mis en place ?

g. Sur quels marchés vendent-ils leurs produits?

h. Pour voir où fonctionnent les différents systèmes de garantie, consultez la page:

- La carte des normes de l'ITC_http://www.standardsmap.org)

- L'index du label écologique (http://www.ecolabelindex.com)

- Carte des SPG IFOAM_https://pgs.ifoam.bio)

Une fois que vous avez suffisamment d'informations, réfléchissez au type de garantie que vous pouvez obtenir pour vos marchés cibles et au coût de chaque type.

\subsection{Comment la confiance se construit-elle dans chaque système de garantie et dans quel marché est-elle la plus effective?}

Les marchés définissent souvent le type de système de garantie à utiliser pour une certification. Les différents marchés et les consommateurs qui les fréquentent exigent différents types de garanties; cela est généralement lié à la manière dont la confiance se construit au sein du système de garantie. Par exemple, dans les marchés de quartier, les auto-déclarations pourraient suffire, étant donné que les producteurs et les consommateurs se connaissent. Par contre, pour les marchés éloignés tels que les marchés d'exportation, où il est impossible pour les consommateurs et les producteurs de se rencontrer, un SPG ou une certification par un tiers peut être acceptées. 
Tableau 10: Comment la confiance se construit-elle dans les différents systèmes de garantie ?

\begin{tabular}{|c|c|c|}
\hline Quel système de garantie? & Comment la confiance se construit-elle? & Quel marché ? \\
\hline Auto-déclaration & $\begin{array}{l}\text { La confiance repose sur des interactions } \\
\text { directes et fréquentes entre producteurs } \\
\text { et consommateurs. }\end{array}$ & $\begin{array}{l}\text { Les marchés locaux et de } \\
\text { quartier où des relations } \\
\text { directes entre producteurs et } \\
\text { consommateurs sont possibles. }\end{array}$ \\
\hline Audit des acteurs & $\begin{array}{l}\text { La confiance repose sur des interactions } \\
\text { directes et récurrentes entre } \\
\text { l'intermédiaire ou le transformateur } \\
\text { transparent et les consommateurs. }\end{array}$ & $\begin{array}{l}\text { Les marchés locaux ou } \\
\text { nationaux où des relations } \\
\text { directes entre intermédiaires ou } \\
\text { transformateurs transparents et } \\
\text { consommateurs sont possibles. }\end{array}$ \\
\hline $\begin{array}{l}\text { Vérification par une tierce } \\
\text { partie }\end{array}$ & $\begin{array}{l}\text { La confiance repose sur l'indépendance } \\
\text { de l'organisme de certification et } \\
\text { sur l'accréditation par les autorités } \\
\text { publiques. }\end{array}$ & $\begin{array}{l}\text { Des marchés nationaux } \\
\text { et internationaux où les } \\
\text { consommateurs ont peu de } \\
\text { possibilités de rencontrer } \\
\text { directement les opérateurs de } \\
\text { systèmes alimentaires durables } \\
\text { et doivent faire confiance à des } \\
\text { certificateurs professionnels. }\end{array}$ \\
\hline $\begin{array}{l}\text { Systèmes de garantie } \\
\text { participative }\end{array}$ & $\begin{array}{l}\text { La confiance repose sur la participation } \\
\text { directe de tous les acteurs, ce qui } \\
\text { signifie que les consommateurs font } \\
\text { confiance à ce système soit parce qu'ils } \\
\text { participent activement au processus } \\
\text { de certification, soit parce qu'ils ont } \\
\text { des relations directes avec les autres } \\
\text { acteurs participant au système de } \\
\text { garantie (producteurs, intermédiaires } \\
\text { transparents ou autres consommateurs). }\end{array}$ & $\begin{array}{l}\text { Des marchés locaux et nationaux } \\
\text { où des relations directes entre } \\
\text { les acteurs du système sont } \\
\text { possibles. }\end{array}$ \\
\hline D'autres? & $\ldots$ & $\ldots$ \\
\hline
\end{tabular}

\section{Les supermarchés SPAR/ SUPERSPAR (Namibie)}

SUPERSPAR est une chaîne d'épiceries en Namibie et une filiale de la marque néerlandaise SPAR. Dans le magasin situé à Maerua, SUPERSPAR a ouvert «The Health Nut». Il s'agit d'une section spéciale du supermarché qui offre une gamme de produits biologiques tels que des produits frais, des produits transformés et des cosmétiques.

Sur son site web, SUPERSPAR atteste que «nos herbes et légumes biologiques produits localement sont cultivés selon des règlements stricts de l'association Namibia Organic
Association (NOA) et sont donc certifiés biologiques. Gâtez-vous avec une variété de légumes frais, de salades croquantes ou d'herbes intenses.»

Les normes privées NOA sont les seules normes biologiques namibiennes puisqu'il n'y a pas de réglementation biologique national. La NOA accorde une certification issue d'un SPG pour garantir la qualité biologique de ses produits. Cet exemple démontre qu'il est possible pour les supermarchés d'utiliser des types de garanties alternatifs.

Source: Manjo Krige, Namibian Organic Agriculture Movement, http://www.weckevoigtsspar.com/ in-store/health-nut 


\subsection{Combien cela coûte-t-il ?}

Les frais et les structures de frais varient selon le type de système de garantie et le domaine d'activité. Alors que l'auto-déclaration et l'audit des acteurs n'entraînent généralement pas ou très peu de frais, la certification par une tierce partie exige des frais. Malheureusement, les frais ne sont pas réglementés et les certificateurs ne sont pas obligés de les publier ou de les partager. S'informer sur les coûts de certification nécessite un peu de recherche:

1. Tout d'abord, consultez les sites web des organismes de standardisation pour savoir lesquels se trouvent dans votre région; puis, consultez les sites web des organismes de certification pour savoir s'ils ont publié leurs frais.

2. Ensuite, communiquez avec les antennes locales afin d'obtenir une estimation du coût de la certification. S'il y a plus d'un certificateur dans votre région, magasinez et comparez les frais divers.

Tableau 11: Comment les coûts sont-ils comparés d'un système de garantie à un autre ?

\begin{tabular}{|c|c|c|}
\hline Quel système de garantie? & Quels sont les frais ? & Quel est la structure des frais? \\
\hline Auto-déclaration & $\begin{array}{l}\text { Pas de frais requis pour ce système de } \\
\text { certification. }\end{array}$ & Pas de frais. \\
\hline Audit des acteurs & $\begin{array}{l}\text { Pas de frais ou des frais très limités sont } \\
\text { requis pour ce système de certification. }\end{array}$ & $\begin{array}{l}\text { La structure des frais peut } \\
\text { varier considérablement selon } \\
\text { le vérificateur. Elle peut être } \\
\text { calculée à la fois sur la base de } \\
\text { l'ensemble du système agricole } \\
\text { ou seulement pour des produits } \\
\text { individuels. }\end{array}$ \\
\hline $\begin{array}{l}\text { Vérification par } \\
\text { une tierce partie }\end{array}$ & $\begin{array}{l}\text { Des frais sont exigés et peuvent être } \\
\text { assez élevés. Il y a parfois des tarifs } \\
\text { spéciaux pour les groupes de petits } \\
\text { exploitants agricoles. }\end{array}$ & $\begin{array}{l}\text { La plupart des tiers certificateurs } \\
\text { calculent leurs honoraires en } \\
\text { fonction du nombre de produits } \\
\text { à certifier sur la ferme, du } \\
\text { nombre de normes nécessaires } \\
\text { (p. ex., les normes biologiques } \\
\text { de l'UE, la NOP et la Soil } \\
\text { Association peuvent toutes être } \\
\text { évaluées au cours du même } \\
\text { audit), de la taille de la ferme et } \\
\text { du nombre de jours de travail } \\
\text { qui seront nécessaires pour } \\
\text { effectuer la certification. }\end{array}$ \\
\hline $\begin{array}{l}\text { Systèmes de garantie } \\
\text { participative }\end{array}$ & $\begin{array}{l}\text { Les frais sont convenus par les groupes } \\
\text { SPG, mais tous les SPG n'exigent pas de } \\
\text { frais. }\end{array}$ & $\begin{array}{l}\text { Dans les SPG, les frais couvrent } \\
\text { la certification de l'ensemble du } \\
\text { système agricole, peu importe } \\
\text { le nombre de produits qu'il } \\
\text { inclut. S'il y a des frais, ils sont } \\
\text { généralement minimes afin de } \\
\text { couvrir les frais de transport } \\
\text { pour les visites à la ferme. }\end{array}$ \\
\hline Autre & .... & ...... \\
\hline
\end{tabular}




\section{La certification Fairtrade (FLOCERT)}

FLOCERT, l'organisme de certification Fairtrade, met à disposition un calculateur de frais de certification facile à utiliser sur son site web. À l'aide d'un questionnaire à choix multiples, la plateforme FloCert vous pose les questions suivantes:

1. Quel est votre type d'organisation (c.-à-d. petit producteur)?

2. Quel est le type de frais que vous voulez calculer (initial ou annuel)?

3. À quel «catégorie» appartiennent vos membres?

a. Première année: une organisation de petits producteurs dont les membres sont exclusivement des petits agriculteurs individuels;

b. Deuxième année: une organisation de petits producteurs dont les membres sont des organisations de première année; c. Troisième année: une organisation de petits producteurs dont les membres sont des organisations de deuxième année.

4. Combien y a-t-il de membres dans votre organisation?

5. Combien de produits produisez-vous ?

6. Combien d'installations de transformation avez-vous?

a. Combien d'ouvriers avez-vous dans chaque installation?

7. Combien d'entités sous-traitées utilisez-vous? Après la dernière question, FloCert vous fournit instantanément une estimation des frais totaux (ainsi qu'une copie PDF à télécharger) divisée en coûts pour la demande, la certification et les frais supplémentaires.

Source: http://www.flocert.net/fairtrade-services/ fairtrade-certification/fees

\subsection{Quoi d'autre est garanti ?}

\subsubsection{Prestations de services}

Parfois, dans le cadre du système de garantie, des services tels que des formations sont offerts aux producteurs afin de renforcer leur capacité d'adopter des pratiques agricoles durables.

\section{LISTE DE VÉRIFICATION}

Questions sur les services offerts

1. Les orientations et/ou formations sont-elles incluses dans le système de garantie ?

2. Le suivi et le mentorat sont-ils inclus dans le système ? Si oui, à quelle fréquence (trimestriellement, à chaque cycle de culture, semestriellement) ?

3. Suite à la remise des certificats, les responsables du système de garantie fournissentils régulièrement des mises à jour sur les nouveaux développements des systèmes alimentaires durables?

4. Est qu'il ya d'autres services supplémentaires offerts dans le cadre du système de garantie? 


\subsubsection{Portée et équivalence}

Certains systèmes de normes et de garanties ont établi des équivalences entre les différentes normes nationales, tandis que d'autres fonctionnent dans plusieurs pays. Le système de garantie que vous choisissez d'adopter dictera les limites de votre marché en fonction de sa zone de couverture. Lorsque vous cherchez le système le plus approprié, demandez aux certificateurs la portée et l'équivalence de la norme.

\subsubsection{Conformité légale}

Assurez-vous que le système de garantie que vous souhaitez adopter respecte les lois locales et nationales régissant la durabilité dans votre pays.

Si vous souhaitez vérifier l'équivalence des différents systèmes de garantie, rendez-vous sur: http://www.ifoam.bio/sites/default/files/irocb_equitool_2012_0.pdf

\section{LISTE DE VÉRIFICATION}

Conformité légale

1. Est-ce qu'il existe des lois dans votre pays qui ont des exigences spécifiques pour les systèmes de garantie?

2. Est-ce que le système choisi respecte les codes et principes du CODEX, de l'IFOAM, de l'ISEAL, de l'ISO ou des programmes et accords bilatéraux entre pays ?

3. Les termes (biologique, naturel, certifié, etc.) sont-ils réglementés par le gouvernement et, le cas échéant, quel type de système de garantie est requis pour les utiliser?

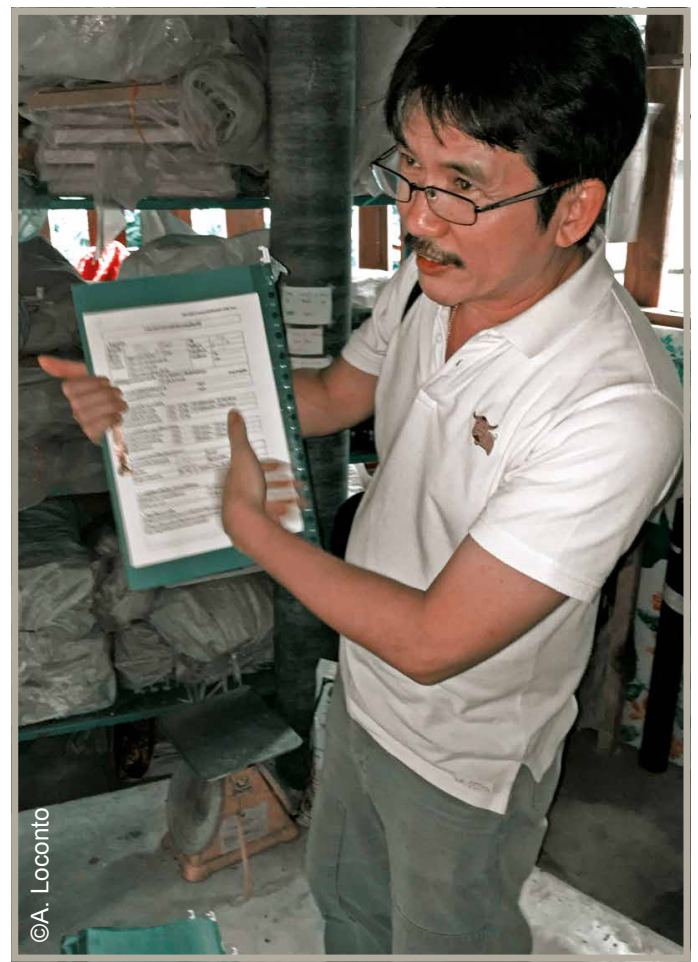




\section{COMMENT COMMUNIQUER VOTRE GARANTIE?}

Étant donné que des systèmes de garantie sont mis en place pour différencier les producteurs durables non-durables, la communication avec les consommateurs est fondamentale. Selon le système de garantie que vous décidez de mettre en place, la manière d'informer les consommateurs peut être verbale, visuelle ou un mélange des deux.

1. Si vous ne communiquez que verbalement (par exemple, avec une auto-certification informelle), utilisez quelques techniques pour être plus efficace: communiquez avec des messages simples et clairs pour expliquer vos pratiques durables.

2. La plupart des systèmes de garantie permettent l'utilisation d'étiquettes que les consommateurs peuvent immédiatement associer à un système de garantie particulier. Si vous utilisez une étiquette, assurez-vous de l'utiliser sur vos emballages et dans vos communications écrites.

\section{COMMENT VOTRE SYSTÈME DE GARANTIE PEUT-IL AIDER LES AGRICULTEURS À RESPECTER D'AUTRES NORMES?}

Dans la mesure du possible, les systèmes de garantie simplifient les questions administratives pour les producteurs et les intermédiaires transparents et des échanges entre les acteurs publics et privés devraient être orientés vers une reconnaissance mutuelle des garanties. Outre les normes de durabilité, les agriculteurs doivent également respecter les normes de sécurité alimentaire, de marchés publics et de protection de l'environnement.

\section{ASTUCE 32}

Simplifier et standardiser le processus administratif

1. Vos groupes d'agriculteurs peuvent-ils être considérés comme des coopératives légales éligibles pour des subventions publiques ou des services de vulgarisation?

2. Vos garanties peuvent-elles être utilisées pour collecter des informations sur la sécurité alimentaire et enregistrer les agriculteurs auprès de ces autorités?

3. Pouvez-vous utiliser un certificat SPG pour vous inscrire auprès des autorités responsables des marchés publics?

4. Pouvez-vous utiliser votre système de garantie pour tenir un registre des services écosystémiques ou de la séquestration du carbone et enregistrer vos agriculteurs pour ces subventions ou marchés? 


\section{Le système participatif de} garantie, l'enregistrement de la

67 sécurité sanitaire des aliments et les marchés publics (Bolivie)

En Bolivie, la loi nationale sur l'agriculture «écologique» (loi numéro 3525) a autorisé un régime réglementaire pour les systèmes de garantie participatifs. Chaque SPG est enregistré auprès des bureaux municipaux et des autorités nationales afin d'accéder aux services de vulgarisation locaux (même pour la production écologique) et afin d'utiliser le label biologique national. En outre, tous les SPG sont enregistrés auprès de l'autorité de la sécurité alimentaire, ce qui garantit le processus de certification des agriculteurs SPG, en leur autorisant à vendre à des programmes de marchés publics et à accéder aux marchés officiels dans tout le pays. Bien que les agriculteurs mettent en œuvre leur SPG au sein de leurs communautés, ils ont accès aux marchés nationaux.

Source: Pilar Santacoloma, FAO, Panama

\section{QUE FAIRE SI PERSONNE NE DEMANDE DE GARANTIE?}

Certains consommateurs ne sont tout simplement pas intéressés à la durabilité de leurs aliments. Mais si vous produisez déjà de façon durable, préparez-vous pour le moment où vos consommateurs commenceront à vous poser la question. Commencez par intégrer la vente directe dans votre stratégie de marketing, afin que vous puissiez construire un système de garanties informelles avec un groupe de consommateurs.

\section{ASTUCE 33}

\section{Mettre en place un système de garantie lorsque personne ne le demande}

Le groupe de producteurs en question peut:

- publier un bulletin d'information pour informer et communiquer avec les consommateurs sur les pratiques agricoles et leur permettre de demander des questions liées à la sécurité sanitaire, à la santé et à la garantie d'approvisionnement;

- partager des recettes, annoncer des points d'intérêt commun, ainsi que des événements sociaux pour attirer des consommateurs et bâtir la confiance;

- introduire une politique de remboursement raisonnable au cas où les consommateurs ne sont pas entièrement satisfaits de la qualité des produits. Ce système doit être soigneusement planifié et correctement exécuté avec des conditions qui décourageront toute forme d'abus flagrant de la part de consommateurs non-honnêtes;

- inviter les consommateurs à jouer un plus grand rôle dans l'exploitation agricole par le biais de visites, de projets de développement collectif, ou même en vendant des parts de membre dans la récolte (par exemple, en créant un modèle d'AMAP); 
- agir de façon proactive en déclarant leurs pratiques durables, en les affichant aux points de collecte, sur les camionnettes de livraison ou sur les emballages afin de sensibiliser les consommateurs et renforcer leur confiance et leur fidélité;

- être plus transparent: la divulgation volontaire d'informations sur les pratiques agricoles (les bonnes et les mauvaises) aide à instaurer la confiance, ce qui est la première étape de la mise en place d'un système de garantie;

- s'allier avec d'autres fermes qui utilisent des pratiques semblables :cela peut aider à établir de la confiance entre les agriculteurs et à diversifier les produits offerts aux consommateurs. Au début, il est important d'organiser des visites d'exploitations agricoles pour en apprendre davantage sur les pratiques de production et de commercialisation des uns et des autres.

\section{ASTUCE 34}

\section{Fournir une garantie}

- Tous les marchés n'exigent pas le même type de garantie.

- Lorsque vous mettez en place un système de garantie, choisissez celui auquel vos consommateurs principaux font le plus confiance.

- Assurez-vous de comparer les frais et les structures tarifaires des différents certificateurs et comparez-les à ce que vos consommateurs sont prêts à payer pour vos produits.

- Soyez prêts! Si vos consommateurs ne vous demandent pas de garantir vos pratiques durables, commencez à mettre en place votre système de garantie de toute façon, car quelqu'un finira par vous le demander.

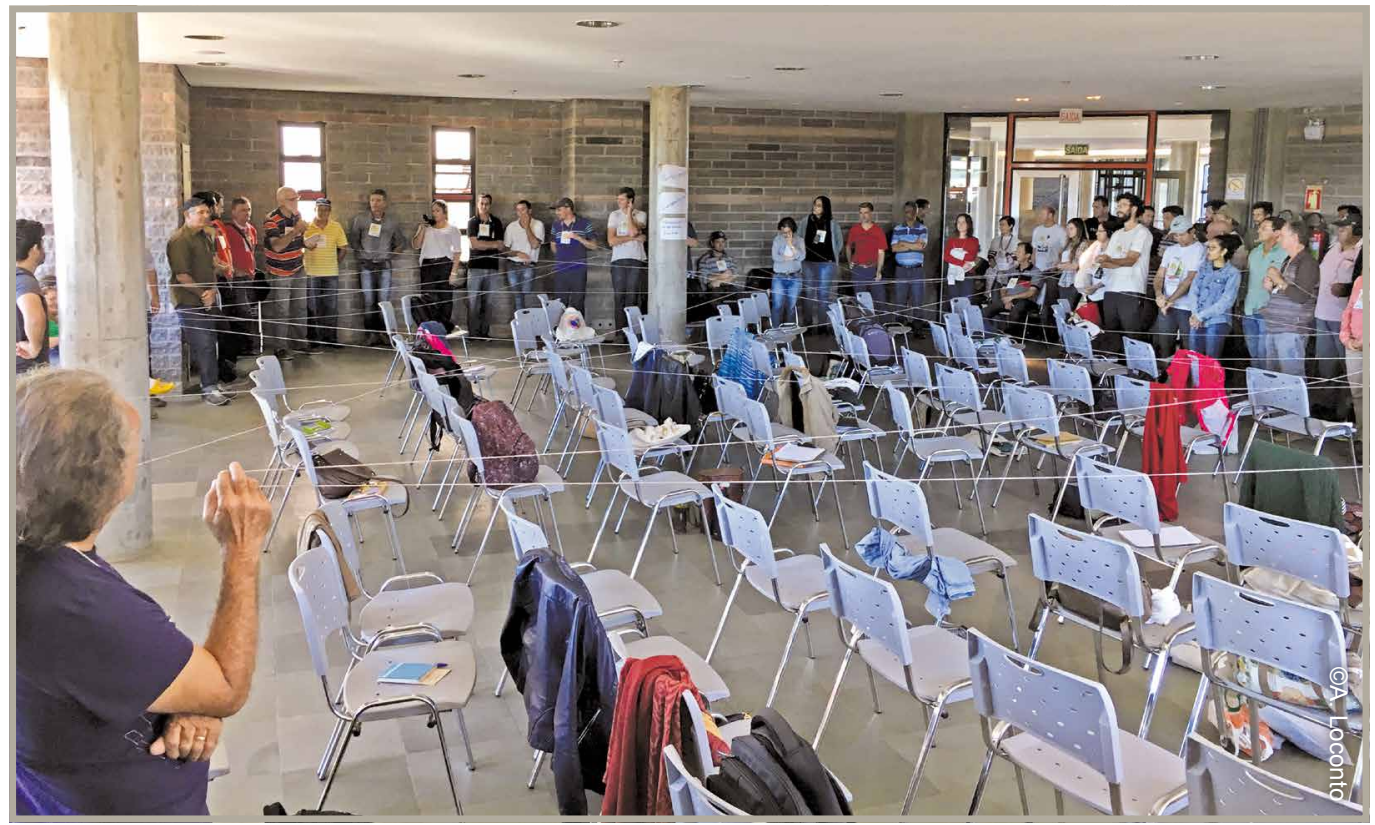




\section{LE CHEMIN D’APPRENTISSAGE, OÙ ALLER ENSUITE?}

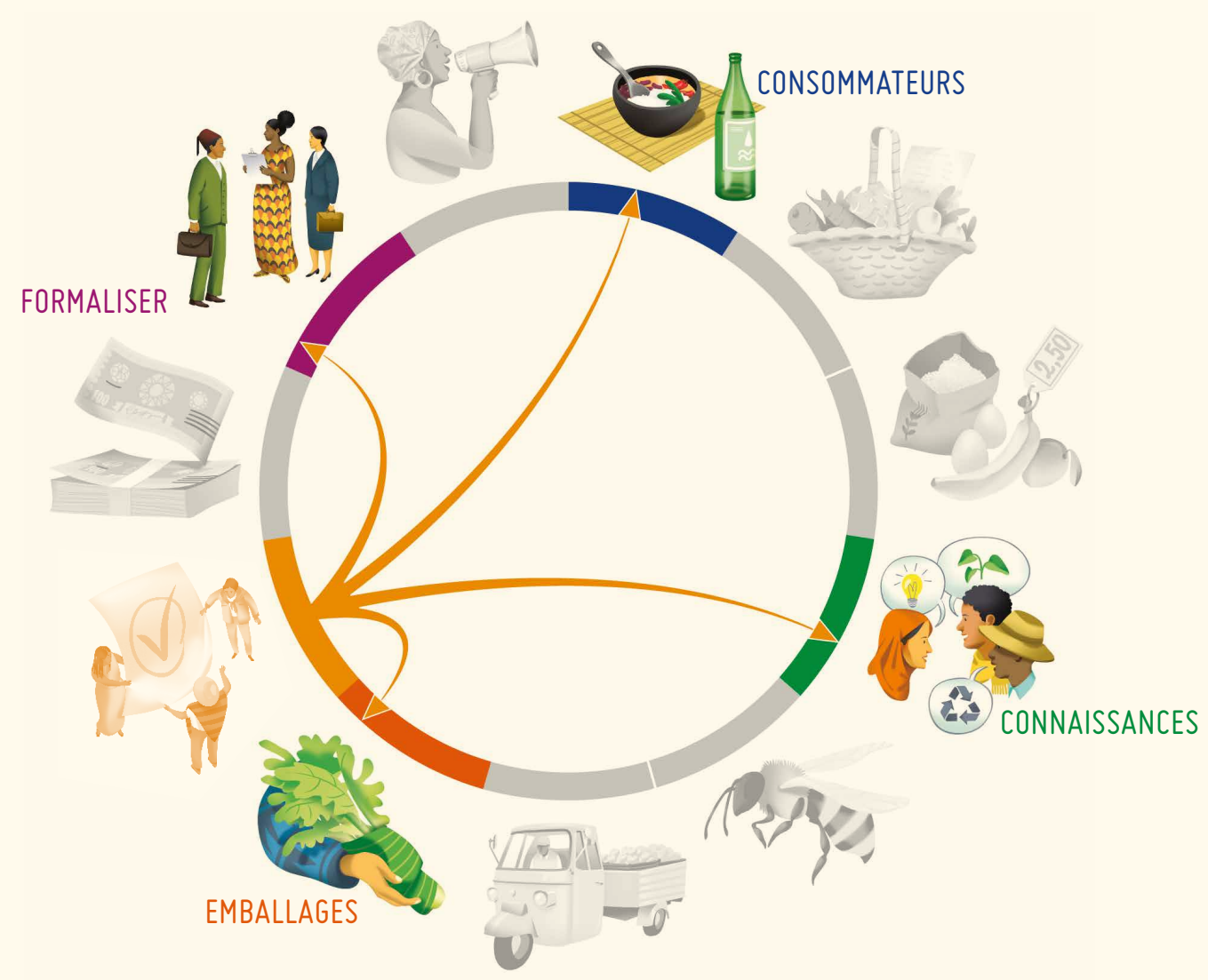

- Vous voulez en savoir plus sur la façon dont les étiquettes peuvent mieux communiquer les valeurs de vos produits ? Lisez leChapitre 7: Emballage de qualité pour la durabilité.

- Vous avez besoin d'aide pour communiquer avec vos consommateurs ? Essayez certaines astuces qui se trouvent dans le Chapitre 1: Attirer les clients et les fidéliser.

- Vous souhaitez fournir des services de vulgarisation dans le cadre de votre système de garantie ? Allez au Chapitre 4: Partager et co-créer des connaissances pour une production durable pour découvrir comment en développer une.

- Vous voulez transformer votre système de garantie en un système plus formalisé, mais vous n'êtes pas sûr du modèle organisationnel approprié pour le faire ? Aventurez au Chapitre 10: Formaliser votre travail collectif.

.... ou consultez un autre chapitre de votre choix... 


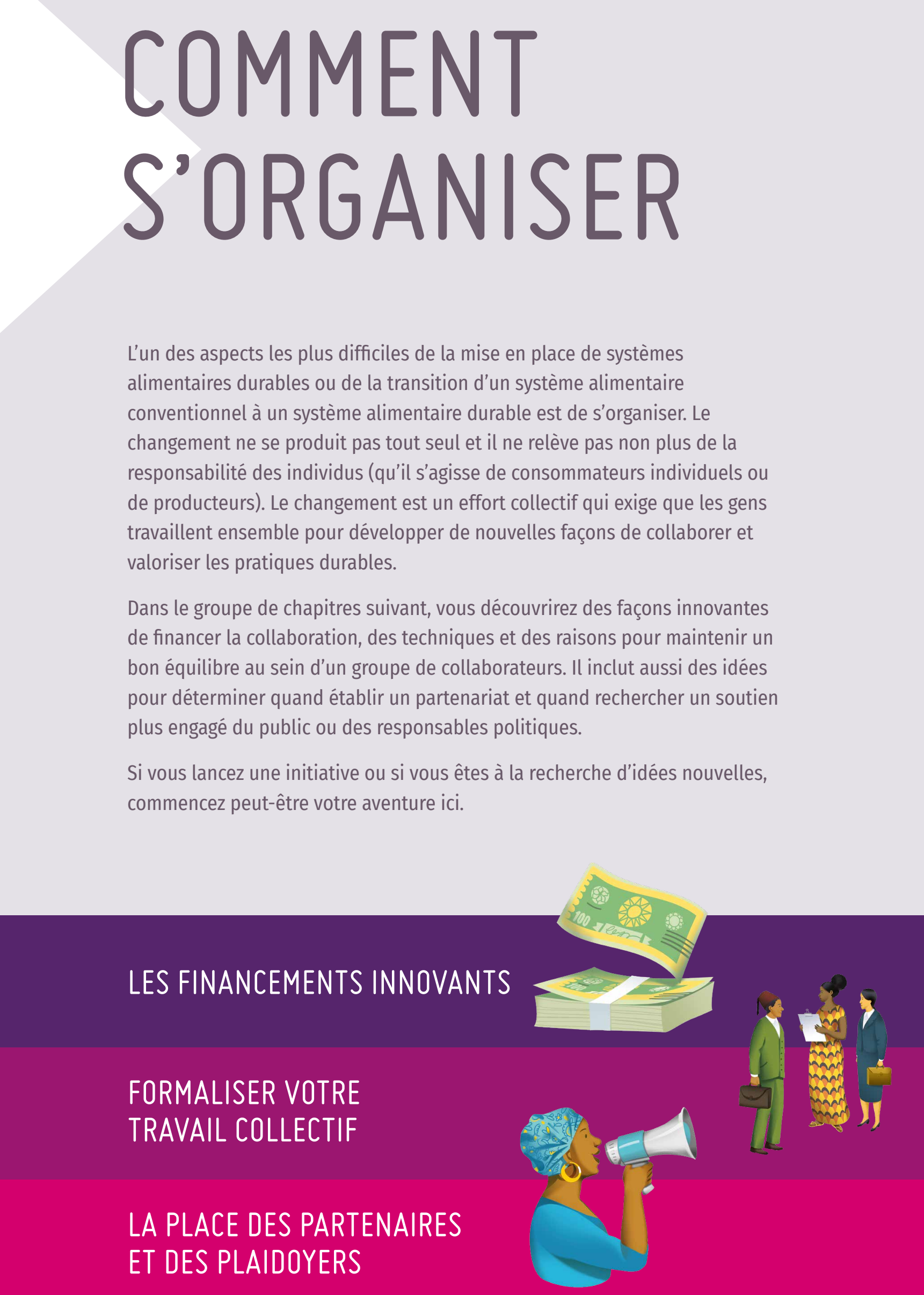



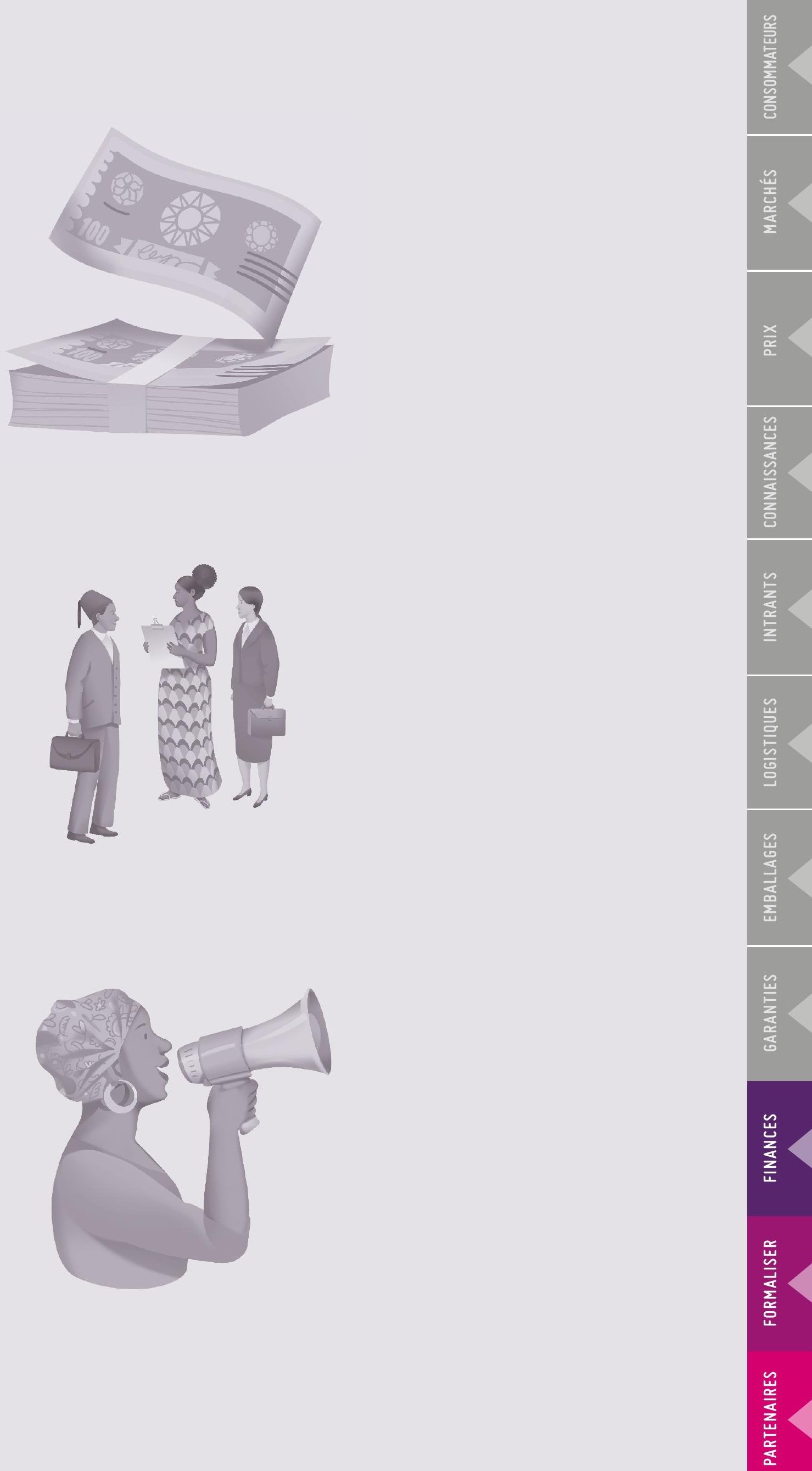


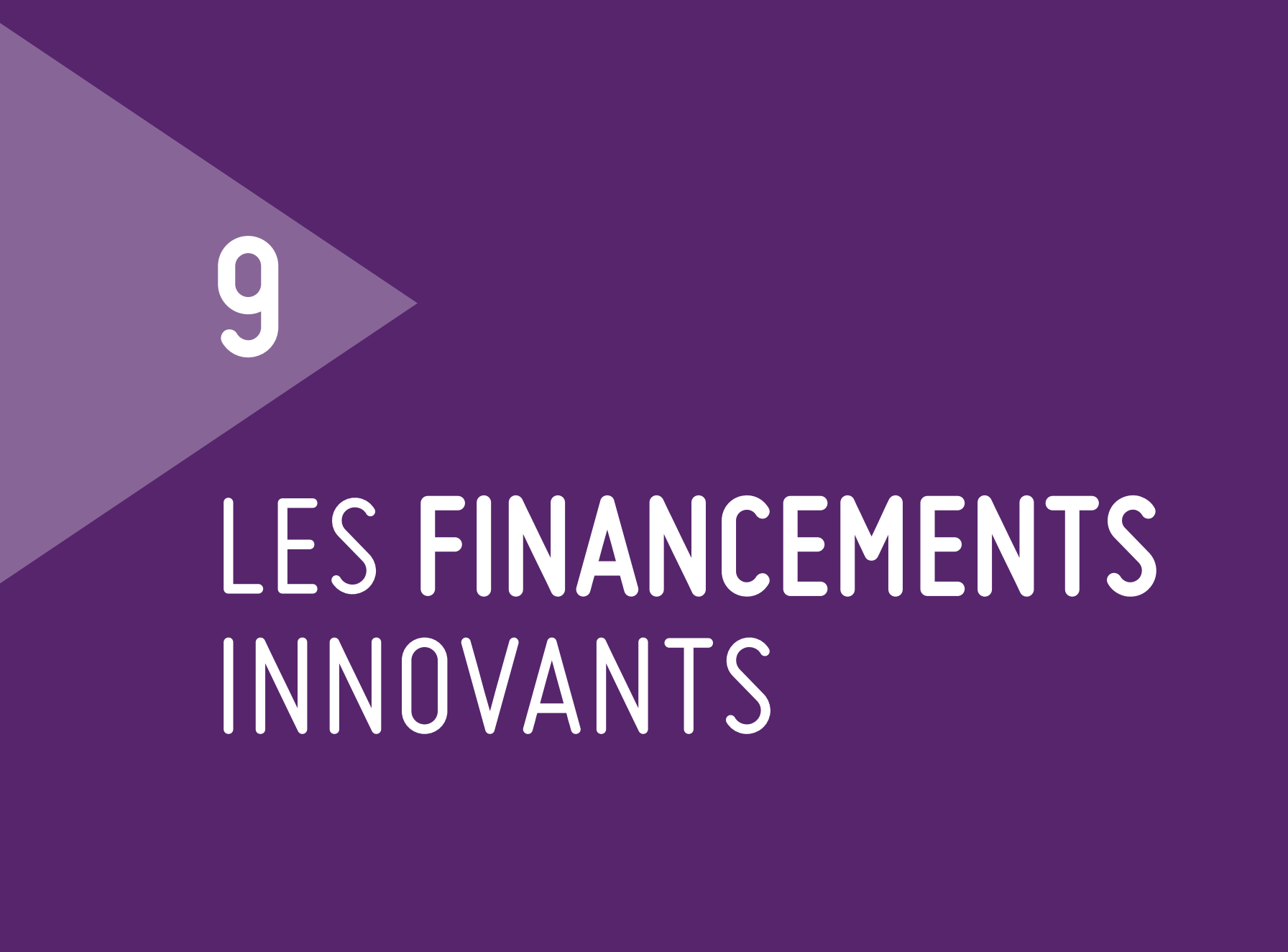




\section{POURQUOI EST-CE IMPORTANT?}

Assurer la stabilité financière de collaborations innovantes pose un défi récurrent pour de nombreux acteurs du système alimentaire durable. Le modèle d'affaires et l'échelle de ce type d'initiative sont souvent considérés comme étant trop risqués ou trop petits pour être éligibles aux prêts des banques commerciales et à la fois trop grands pour bénéficier de la microfinance.

Pour certaines initiatives, la capacité d'instaurer une indépendance financière vis-à-vis des banques commerciales est incluse dans leur vision d'un système alimentaire durable.

\section{QUELLES SONT LES OPTIONS?}

Une initiative a besoin de financement pour trois raisons principales:

1. Garder les portes ouvertes: Il doit y avoir suffisamment d'argent pour payer les frais de fonctionnement (c.-à-d. le personnel, l'électricité, le transport, les locaux de bureaux) qui permettent à l'initiative de relier les consommateurs et les producteurs durables.

2. Faire des investissements: les innovations, les améliorations, les rénovations, les remplacements, les agrandissements et les autres activités vont au-delà des coûts opérationnels de base.

3. Recevoir des services: Les producteurs, les consommateurs, les fonctionnaires et les autres acteurs du système alimentaire ont besoin d'un certain nombre de services facilitant la transition vers la durabilité (éducation, formation, événements, assistance technique, systèmes de garantie, espaces communs, etc.). Certains services peuvent exiger des frais, mais la majorité aura besoin d'un financement externe.

Généralement, ce financement peut être acquis par deux sources de revenus: Interne et externe.

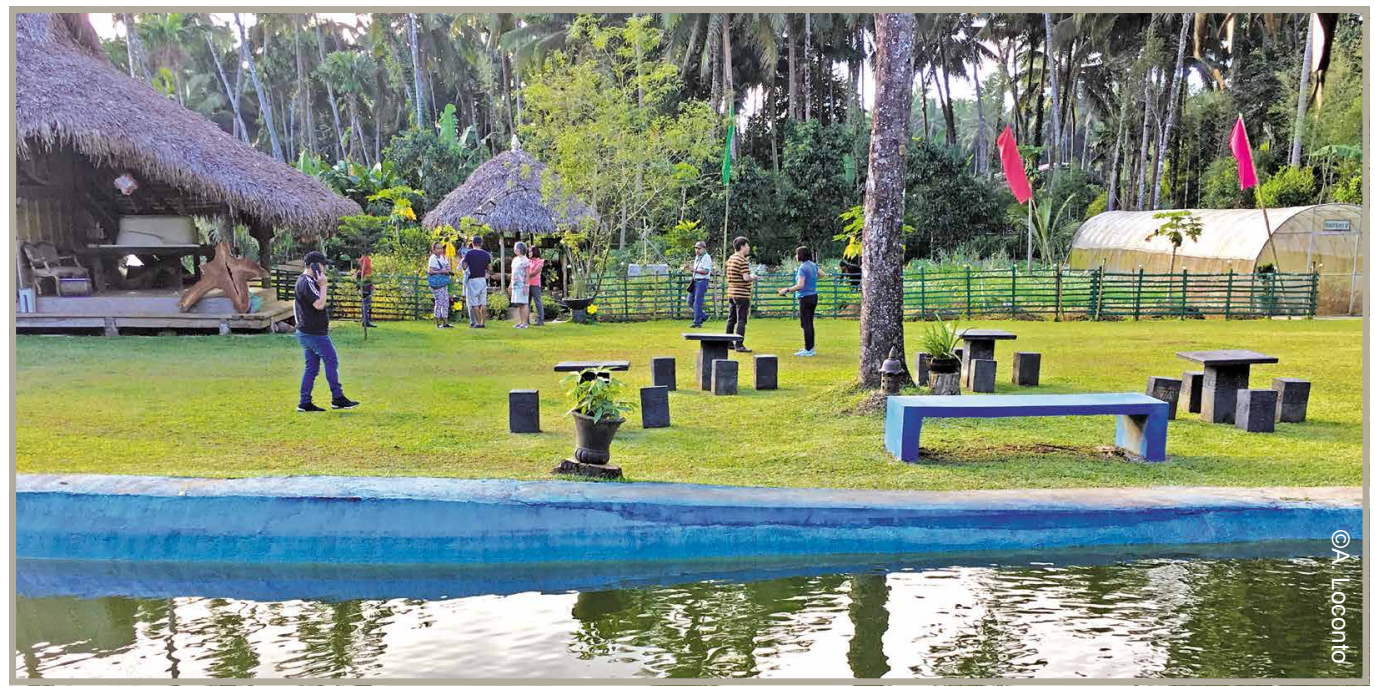




\subsection{Fonds internes}

Les fonds internes proviennent de ceux qui investissent dans votre initiative et qui partagent les risques de votre initiative (par exemple, les acteurs, les agriculteurs, les consommateurs dans une AMAP, les actionnaires). Cet argent n'est généralement pas remboursé. Bien que le profit issu de vente de produits et services soit la principale source de financement interne, les membres et les actionnaires peuvent aussi bénéficier de tout profit non-réinvesti dans l'initiative.

\subsubsection{Ventes de produits}

L'autofinancement est une approche courante pour les initiatives de financer leurs opérations en réinvestissant les revenus de l'entreprise (vente de produits et services) pour payer ses frais d'exploitation. L'utilisation des revenus en espèces ou en nature (par exemple, un sac de riz ou de café provenant des producteurs-membres) est une source de financement stable qui devrait être la base de toute initiative.

\section{ASTUCE 35}

Réinvestir le revenu issu de la vente de produits

- Conservez un bilan avec une colonne dédiée aux revenus et aux dépenses. Commencez par payer vos frais d'exploitation avec vos revenus issus de la vente de produits.

- Si vous avez plus de revenus que de dépenses, pensez aux investissements possibles pour augmenter les ventes de produits et faire croître l'initiative:

- Vous voulez acheter de l'équipement de transformation pour ajouter de la valeur à vos produits?

- Vous avez besoin d'un camion pour transporter des marchandises?

- Vous avez besoin d'installer des points de collecte et de stockage pour élargir la portée de votre réseau de production?

- Est-ce que vous pouvez offrir un renforcement des capacités ou une formation aux producteurs, aux consommateurs ou au personnel de l'initiative?

- Vous voulez participer à des conférences ou à des foires commerciales pour élargir vos marchés, vos réseaux et vos connaissances ? Vous aurez besoin d'un budget de voyage pour cela.

- Est-ce que vous pouvez embaucher des personnes qui se spécialisent dans les services que vous offrez aux producteurs et aux consommateurs?

- Vous avez besoin de technologies de l'information, de fournitures et d'espace de bureau ?

- Est-ce que vous pouvez commencer à conserver une partie des fonds dans un compte à intérêt pour de plus longues périodes afin de constituer une réserve d'urgence?

- Vous avez besoin d'un espace de vente à temps plein ? Être installé dans un magasin permanent aide les consommateurs à vous trouver. 


\subsubsection{Groupes d’épargne des membres producteurs}

De nombreuses initiatives sont basés sur une collaboration entre des producteurs qui se sont récemment associés pour regrouper de plus grandes quantités de leurs produits. Une pratique courante des coopératives consiste à verser de petites contributions «en capital» dans un fonds commun géré par ses membres. Lors des assemblées générales, les membres votent sur la façon dont l'épargne va être dépensée. Souvent, elle est utilisée pour les besoins individuels des membres. Elle peut aussi être consacrée à des investissements plus importants dans l'intention d'accroître les capacités individuelles ou collectives. Les économies servent aussi pour garantir un fonds d'urgence pendant des périodes difficiles.

\section{Des groupes d'épargne et de crédit communautaires (Kenya)}

68

Pour les petits exploitants agricoles, l'accès au financement est l'un des principaux moteurs du développement entrepreneurial. Au fil des ans, au Kenya, des programmes communautaires d'épargnes et de prêts ont été adoptés pour renforcer la capacité du groupe à accéder à ces services. Il en résulte une capacité collective à accroître les investissements agricoles et les revenus de petites entreprises. PELUM
Kenya s'est engagé à promouvoir la mise à l'échelle de ce modèle pour le développement des entreprises. Certains exemples incluent des groupes d'agriculteurs tels que Kamicha Kabondo Farmers Group et Ti-Kwenda Kwitu Self-Help Group. Ces collectifs utilisent l'épargne comme un investissement collectif pour stimuler les capacités commerciales, plutôt que comme un fonds rotatif individuel.

Source: Rosinah Mbenya, PELUM Kenya

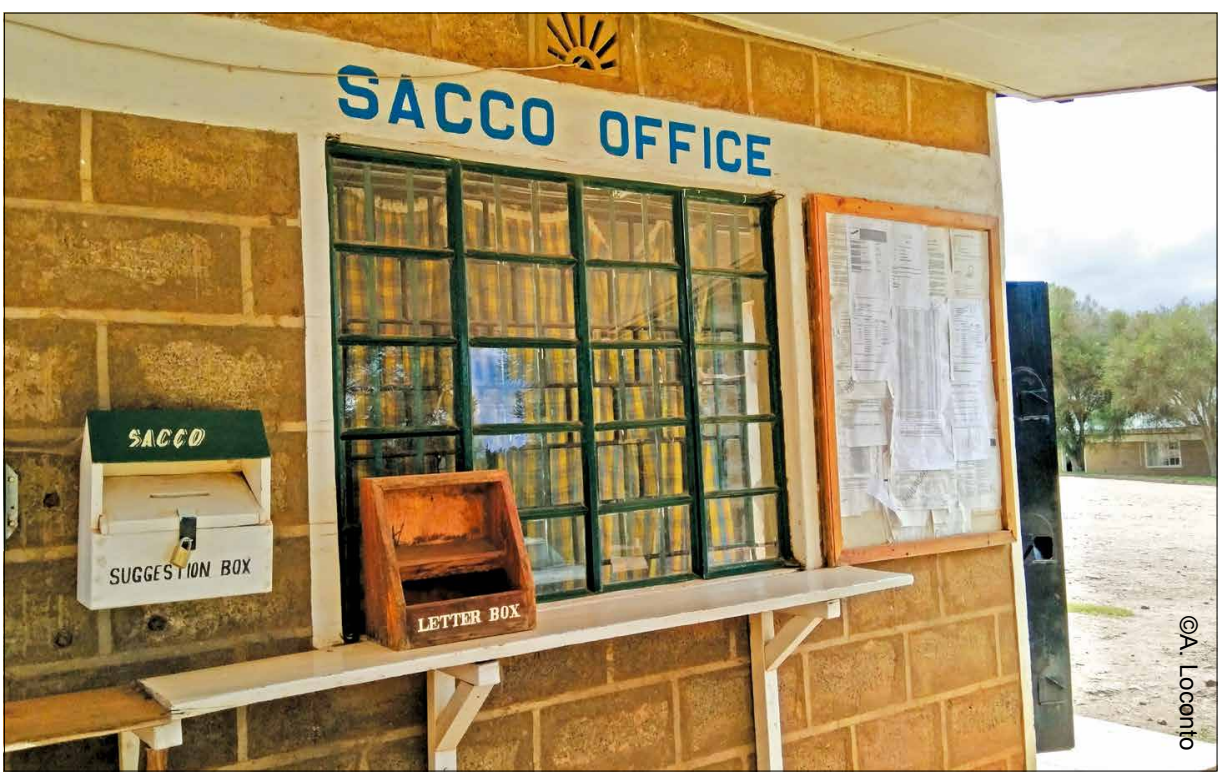




\subsubsection{Investissement des consommateurs}

Convaincre les consommateurs d'investir dans la production de biens et de services est une approche récente pour créer des actionnaires d'initiatives locales. Couramment pratiquée dans les modèles coopératifs pour renforcer l'engagement producteur-consommateur, les membres paient une cotisation annuelle que l'initiative utilise ensuite pour payer ses frais opérationnels. Dans les initiatives d'AMAP, les consommateurs achètent une action de la ferme au début de la saison de croissance afin d'amasser l'argent nécessaire pour la production.

\section{Il y a plusieurs options pour les contributions des consommateurs - soyez créatifs !}

\section{Crowdfunding ou financement participatif (Chine)}

69

Shared Harvest Farm a utilisé un financement participatif basé sur des relations personnelles. Au début de l'initiative, l'équipe a calculé leurs besoins financiers et déterminé qu'un minimum de 150 membres était nécessaire au cours de la première année, en plus d'environ deux mois de liquidités (l'équivalent de 300000 CNY). Shi Yan a réuni dix personnes qu'elle connaissait personnellement et qui étaient prêtes à investir dans les cinq premières années d'opération. Le prix estimé des légumes pour un an étant d'environ 6000 CNY par famille (940 USD), une avance de paiement de cinq ans équivalait à 30000 CNY ( 4700 USD). Cette source de financement était fondamentale pour Shared Harvest Farm parce que tous ces «investisseurs» s'attendaient à manger des légumes biologiques pendant cinq ans et se consacraient à soutenir une nouvelle initiative durable.

Source: Shi Yan, Shared Harvest, Chine

\section{Des contrôles verts menés par les consommateurs (Brésil)}

70

ACOPA (Association of consumers of agroecological products of Paraná), qui fait partie du Réseau de certification participative Ecovida, relie les consommateurs et les agriculteurs agroécologiques. En organisant pour les consommateurs des visites de terrain sur les exploitations des agriculteurs, l'ACOPA s'efforce d'organiser les consommateurs et d'encourager le partage de connaissances et d'expériences entre les consommateurs et les producteurs. Elle a également créé un mécanisme de contrôle vert qui aide les agriculteurs à réaliser des investissements axés sur les consommateurs. Grâce à ce mécanisme, un groupe de consommateurs paie un quota pour financer les activités des agriculteurs. Ayant eu le contrôle vert, les agriculteurs doivent rembourser un montant équivalent avec leurs produits. Ce système favorise des relations de solidarité et de confiance.

Source: José Antonio da Silva Marfil, Ecovida 


\subsubsection{Participation des entreprises communautaires/Prêts aux membres}

Les entreprises communautaires, sous forme de coopératives ou de groupes d'agriculteurs légalement enregistrés, lèvent un capital auprès de leurs membres-actionnaires. Au fil du temps, les membres augmentent leurs actions et les profits sont annuellement distribués sous forme de dividendes. Cette propriété collective offre à la fois un capital de base important et équivaut souvent à un engagement et une participation accrus de la part des membres pour franchir les périodes difficiles.

Les achats de récolte saisonnière (comme le riz ou le café) nécessitent de grandes quantités de capitaux pour stocker la matière première. Au Nature Care Club, à Kut Chum, dans la province de Yasothorn, en Thaïlande, les membres économisent de l'argent à un taux d'intérêt plus favorable que celui offert par les banques, tandis que l'entreprise paysanne paie beaucoup moins d'intérêts que pour un prêt bancaire. Cela permet à l'entreprise d'économiser beaucoup tout en soutenant la richesse de la communauté.

\section{ASTUCE 36}

Utiliser la budgétisation participative

La budgétisation participative provient d'efforts du secteur public ayant pour but d'inclure les citoyens dans les décisions liées aux dépenses de fonds publics. Cependant, lorsqu'il n'y a pas de structure organisationnelle dominant, l'approche participative s'applique facilement aux initiatives durables privées. Voici quelques conseils pour réaliser un exercice de budgétisation participative:

1. Ne préparez pas un budget trop détaillé: Même si vous disposez de chiffres précis, essayez de simplifier votre processus participatif. Le but principal est de comprendre les priorités. En partant de votre budget réel, commencez par des chiffres pairs entiers et arrondis, et utilisez-les pour discuter les possibilités.

2. N'ouvrez le débat que sur les éléments budgétaires que vous êtes prêt à modifier: certaines parties de votre budget sont tout simplement inflexibles, généralement celles liées aux coûts d'exploitation (factures d'électricité, loyer, etc.). Concentrez la discussion sur les investissements possibles et sur toute augmentation/diminution des frais de personnel. Rendez le budget complet accessible pour assurer la transparence et la confiance au sein de votre initiative.

3. Soyez prêt à expliquer les conséquences réelles de certaines décisions budgétaires: avant de commencer, réfléchissez aux conséquences potentielles des réductions budgétaires (c.-à-d. réduire le budget de transport de 20 pour cent signifie que le nombre de livraisons devra être réduit), des augmentations budgétaires ou des investissements. Précisez les types d'avantages qu'apportera un nouvel espace de bureaux à court et à long terme. Si vous demandez aux gens de participer au budget de manière significative, les conséquences de leurs choix doivent être clairement comprises.

4. Essayez de faire un lien entre les discussions en ligne et celles en personne: Avant de discuter du budget lors de vos assemblées générales ou d'autres réunions, des outils de budgétisation participative en ligne peuvent aider à initier ce processus. Ces outils peuvent éviter un sentiment d'exclusion parmi les membres qui ne peuvent pas y assister en personne. Utilisez toujours des outils TIC adaptés à vos communautés. Si une grande partie de vos producteurs et consommateurs n'ont pas accès à internet, un outil en ligne est inutile. 


\subsection{Fonds externes}

Les fonds externes proviennent d'investisseurs n'ayant pas d'intérêt dans l'initiative, considérés essentiellement comme des «créanciers» - des personnes et des organisations qui donnent ou prêtent de lıargent. Selon le type de source externe, vous devrez peut-être rembourser lıargent qui vous a été prêté (les subventions nıont pas besoin d'être remboursées).

\subsubsection{Fonds publics}

À l'échelle mondiale, les gouvernements fournissent des fonds aux citoyens pour subventionner certains types de production agricole ou pour stimuler les économies locales. Les subventions importantes sont généralement gérées au niveau national par de grandes organisations publiques ou privées. Ces fonds ne sont généralement pas faciles d'accès pour les petits producteurs ou les innovateurs qui font la transition vers des systèmes alimentaires durables. Toutefois, il existe des sources de financement souvent méconnues qui peuvent subventionner certaines activités au niveau municipal, national, ou supranational. Pour les trouver, il faut dédier du temps et des efforts pour soumettre des candidatures, et pour trouver des partenaires fiables dans les secteurs public et privé !

\section{疍 Le projet Paramparagat Krishi Vikas Yojana (PKVY) du Ministère de l'agriculture (Inde)}

Au cours de la dernière décennie, le gouvernement indien a développé des programmes novateurs pour encourager, promouvoir et financer l'agriculture durable.

L'un de ces projets s'inscrit dans le cadre plus large de l'initiative de la Mission nationale pour une agriculture durable (NMSA); il s'appelle Paramparagat krishi vikas yojana (PKVY) qui signifie «régime progressif de l'agriculture traditionnel». Dans ce cadre, le gouvernement subventionne des groupes d'agriculteurs pour qu'ils collaborent et adhèrent au système participatif de garantie (SPG). Ils sont soutenus pendant trois ans avec des fonds alloués à des activités telles que la formation, le renforcement des capacités, l'achat d'intrants biologiques, l'analyse d'échantillons de sol, la conversion de terres en production biologique et l'achat d'outils. Le régime prévoit également des fonds pour les activités servant à construire des marchés telles que le matériel d'emballage, le marquage et l'étiquetage des produits, le soutien à l'achat de véhicules et l'organisation de foires biologiques pour la vente et la promotion de produits. En conséquence, plus de 200000 agriculteurs indiens et plus de 100000 hectares de terres agricoles ont été inscris dans le SPG. Le marché est également approvisionné en produits transformés destinés au consommateur final. Au cours de la prochaine décennie, l'objectif est d'atteindre plus d'un million d'agriculteurs et un million d'hectares de terres qui bénéficieront directement de ce programme. D'autres programmes gouvernementaux sont conçus pour le développement de villages biologiques, créant un effet multiplicateur et permettant aux acteurs locaux de mobiliser ces fonds pour promouvoir l'agriculture durable. Enfin, le Ministère du développement rural a décidé de construire des SPG pour les petites agricultrices dans le cadre de son Programme Mahila kisan sashaktikaran (MKSP).

Source: Ashish Gupta, Jaivik Haat 


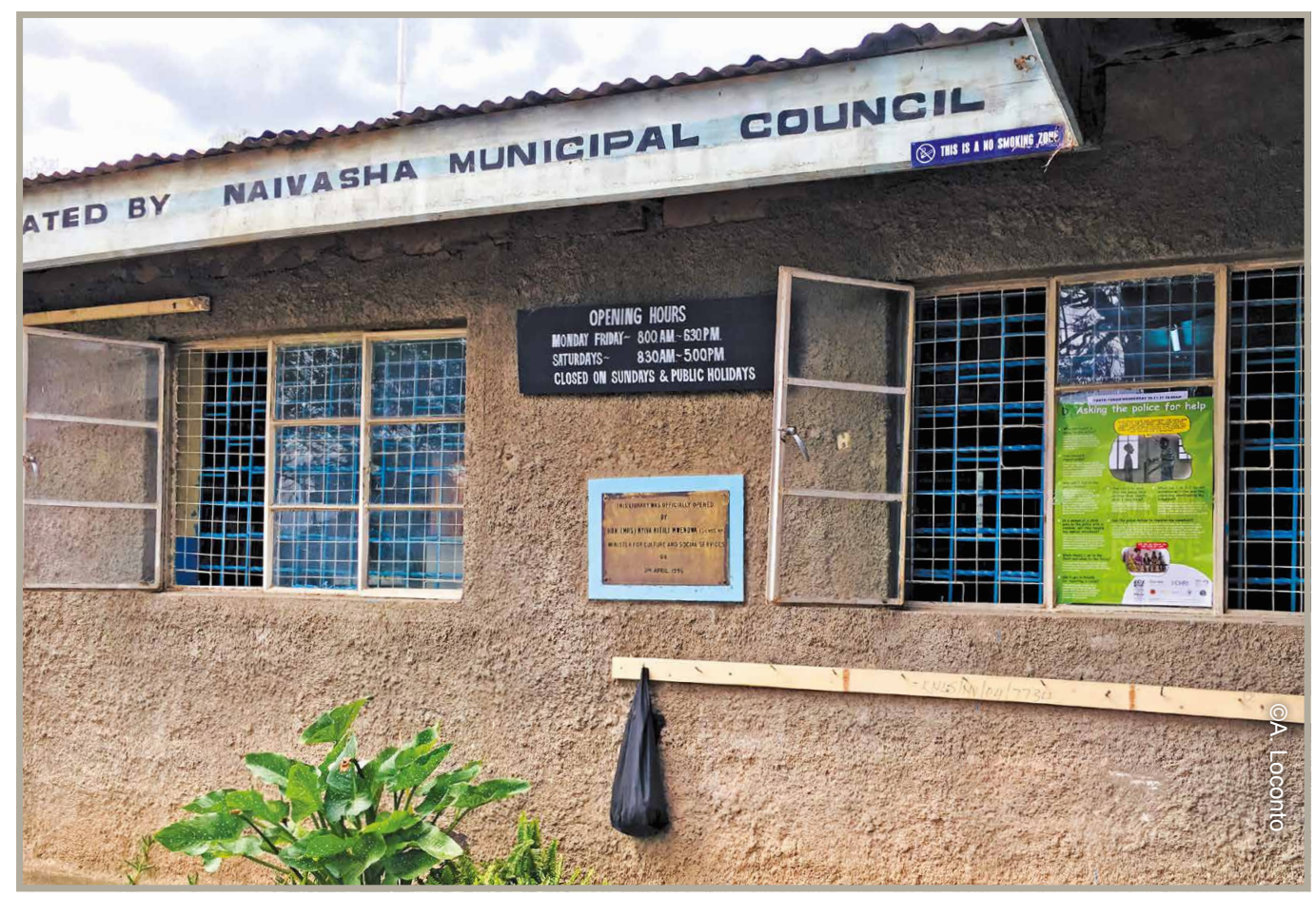

\section{Utiliser des célébrités pour soutenir votre initiative (Pérou)}

Frutos de la Tierra est une marque collective de l'Association nationale des producteurs biologiques du Pérou (ANPE PERU). Elle a été créée pour soutenir l'agriculture familiale biologique et la biodiversité associée à leurs systèmes agricoles. La marque a été lancée en septembre 2013 par Gastón Acurio, chef péruvien de renommée mondiale et leader dans le secteur gastronomique, pour valoriser et faire reconnaître la cuisine péruvienne, les petits producteurs biologiques et les traditions culturelles. Il est le principal ambassadeur de la marque collective au sein de l'alliance cuisinechef promue par l'ANPE Pérou et l'APEGA (Association péruvienne de gastronomie). Les agriculteurs dotés de cette marque collective fournissent les restaurants de

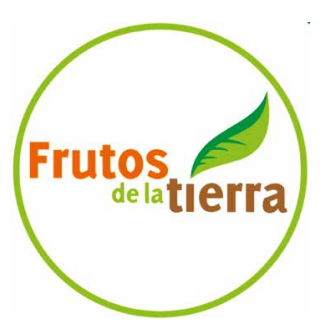

Gastón Acurio, participent à la foire alimentaire Mistura et représentent quasiment toutes les régions du Pérou avec plus de 200 produits biologiques différents. Cette stratégie a permis à l'ANPE Pérou d'accéder à des fonds de coopération internationale et a attiré l'attention du Ministère de l'agriculture. Ils ont utilisé ces fonds pour améliorer la commercialisation de leurs produits, y compris la création d'une plateforme de vente en ligne.

Source: Patricia Flores, IFOAM Organics International 


\subsubsection{Accès aux sources de financement publique non-traditionnelles}

La majorité des fonds publiques non-traditionnels consiste en des «appels d'offres» annoncés régulièrement par différents organismes gouvernementaux. Bien qu'il y ait des directives précises pour les types d'initiatives éligibles, il est possible de créer des consortiums, de soutenir le renforcement des compétences et de construire du matériel (par exemple, un stand de marché). Selon le pays, la disponibilité de ces fonds est annoncée différemment et, dans la plupart des cas, il est important d'avoir des relations positives avec les acteurs publics.

\section{Le financement de projets d'innovation nationaux (Chili)}

73 En 2010, au Chili, le Ministère de l'agriculture a lancé un appel à projets dans le cadre de son «fonds d'innovation». La coopérative Kom Kelluhayin a proposé une initiative ayant pour but de lier la communauté agricole autochtone aux restaurants locaux et aux groupes de consommateurs. Son objectif était d'augmenter la disponibilité du quinoa biologique pour le développement du tourisme local et la promotion de la cuisine traditionnelle mapuche. Le financement a permis à Kom
Kelluhayin de construire une usine de transformation du quinoa qui sert de bureau pour la coopérative, d'usine de transformation pour les confitures et d'espace de stockage pour les autres produits des agriculteurs. Le projet a également permis à Kom Kelluhayin d'avoir les fonds de base nécessaires pour stimuler la demande des consommateurs locaux. Plus précisément, elle a pu louer un stand sur le marché touristique local et développer le label Wemapu pour les produits de ses membres.

Source: Gabriel Curilef, Wemapu
Mobiliser des fonds publics de développement régional (Croatie)

74 En 2013, l'institution de développement et de coordination de la Région SplitDalmatie (RERA SD) a créé un programme d'accompagnement de projet pour les petits et jeunes agriculteurs afin de leur faciliter l'accès aux subventions publiques. Ce projet combinait le développement d'infrastructures soutenant les agriculteurs et les agro-entrepreneurs, un programme de renforcement de compétences et de l'appui technique (aide à l'identification et au remplissage des formulaires pour la demande). Comme la Croatie est rentrée dans l'Union européenne (État Membre de la FAO) en juillet 2013, le RERA SD a principalement ciblé le financement européen, en particulier le Fonds social européen pour le renforcement des capacités, le Fonds européen de développement régional pour les infrastructures de soutien et le Fonds européen agricole pour le développement rural pour les subventions agricoles.

En tant qu'organisation publique régionale, RERA SD a utilisé son réseau d'institutions publiques pour fournir des services aux agriculteurs locaux. Avec cet ensemble complet d'appuis, l'argent public mobilisé à partir de 
trois sources différentes a permis à RERA SD de fournir deux services clés aux producteurs. Premièrement, le programme de renforcement des compétences mis en place par le projet EkoBiz en 2015, a utilisé la formation, le soutien aux entreprises et le développement des compétences entrepreneuriales pour mieux transférer le savoir-faire dans le secteur biologique. Jusqu'à présent, plus de 100 jeunes ont été formés et ont bénéficié d'un soutien aux entreprises, (par exemple, la conception de plans d'affaires et des conseils sur la soumission de leurs demandes de financement de l'UE). EkoBiz a directement contribué à la création de 15 nouvelles entreprises. Deuxièmement, le centre d'agro-entreprenariat lancé en 2013, Čaporice (ville de Trilj), a élargi la «zone d'activités Čaporice» pour inclure 23 nouveaux hectares et la construction de trois bâtiments. Ces derniers abritent des start-ups de l'industrie agroalimentaire (incubateurs), un laboratoire moderne pour les tests alimentaires, des entrepôts, un centre de formation et un centre d'exposition. La phase finale du projet, intitulée «Centre de Compétences $3 \mathrm{LJ} »$, consistera en un centre de recherche agricole appliquée.

Source: Jelena Petrov, rera sd, Croatie 


\subsubsection{Banques de développement}

Les banques régionales de développement sont de grands fonds d'investissement, souvent consacrés au développement rural. Quoiqu'ils soient généralement axés sur les infrastructures de grande échelle, il existe des opportunités dédiées aux marchés et au soutien des initiatives innovantes de petite et moyenne échelle. Il est important de se renseigner auprès des banques de développement rural et de contacter les autorités publiques impliquées dans la mise en œuvre de ces projets.

\section{Le Fonds national de
développement agro-sylvo-
pastoral (Sénégal)}

En 2011, le Sénégal a créé le Fonds national de développement agro-sylvo-pastoral (FNDASP) (arrêté nº 10203 du 2 novembre 2011), devenu opérationnel en 2013 pour une durée illimitée et supervisé par le Ministère de l'agriculture et la tutelle financière du Ministère de l'économie et des finances. Les fonds gérés et distribués par le FNDASP proviennent de sources diverses: une dotation annuelle du budget de l'État; des contributions d'associations interprofessionnelles sous forme de prélèvements des secteurs d'agro-sylvopastoralisme et de la pêche; du cofinancement de projets avec les communautés locales; du cofinancement de projets avec le secteur privé; de l'appui des partenaires techniques et financiers bilatéraux (CEDEAO, FAO, FIDA,
Banque mondiale, UEMOA); des dons, des héritages et des subventions autorisés.

L'objectif du FNDASP est d'offrir un financement compétitif et contractuel en: 1) services de vulgarisation sylvo-pastorale et halieutique par moyen «d'associations interprofessionnelles» dans plusieurs secteurs; 2 ) formation des producteurs; 3 ) soutien institutionnel aux organisations de producteurs; et 4) financement des programmes de recherche agro-sylvo-pastorale et halieutique par le Fonds national de recherche agricole et agroalimentaire (FNRAA). À ce jour, le fonds a financé l'organisation de filières d'élevage (lait et viande), de céréales (maïs, riz) et de légumes (tomate, oignon), en promouvant spécialement les pratiques durables (tels que les variétés et les races traditionnelles et l'utilisation de l'énergie solaire) et la création de marchés locaux pour ces produits.

Source: FNDASP, http://fndasp.sn

\subsubsection{Projets de recherche}

La recherche participative est de plus en plus pratiquée et financée. Elle peut être axée sur différents aspects des systèmes alimentaires durables ( $p$. ex. la production, la transformation, la commercialisation, ou la santé) où les chercheurs et les praticiens coproduisent et partagent leurs connaissances. La subvention accordée à ces projets finance souvent le personnel, les services et l'investissement dans l'infrastructure. L'accès à ces fonds est un processus compétitif marqué d'une période d'attente d'environ six mois pour le résultat. Répondre à un appel à projets nécessite beaucoup d'effort ainsi qu'une collaboration avec des chercheurs locaux (et souvent internationaux). 
Une première étape consiste à contacter des chercheurs dans votre réseau et à rechercher ensemble les appels annoncés par des fondations scientifiques nationales et internationales.

\subsubsection{Paiements pour les services écosystémiques}

Face aux défis du changement climatique, plusieurs gouvernements nationaux instaurent des programmes de récompenses pour les services écosystémiques. Cette approche s'est ancrée sur l'idée que les gouvernements peuvent contribuer à la préservation de la biodiversité et des ressources naturelles en récompensant les agriculteurs qui respectent des pratiques durables. La structure de ces programmes varie selon le pays - elle peut se manifester par le biais d'organisations internationales ou supranationales (comme les programmes de réduction des émissions liées à la déforestation et à la dégradation des forêts [REDD+]) ou au niveau municipal. La plupart sont gérés par les Ministères de l'environnement ou du tourisme.

\section{总 Le programme du Fonds communautaire pour \\ 77 l'écotourisme (Trinité-et-Tobago)}

En 1997, la Tourism and Industrial Development Company de Trinité-et-Tobago (TIDCO), dirigée par le gouvernement national, a financé 12 collectivités rurales au moyen d'un programme d'action communautaire pour la gestion de ressources touristiques. Dans chaque communauté, des comités d'action touristique (CAT) ont été formés. Le CAT de Brasso Seco fut parmi les rares à réussir à devenir durable en développant une approche de préservation de leur environnement naturel et des traditions culturelles locales. En 2000, TIDCO a organisé un concours culinaire intercommunautaire à Brasso Seco. L'année suivante, l'événement est devenu un festival culinaire communautaire ayant lieu en octobre pendant la saison traditionnelle de gibier. Grâce aux recettes de cet événement et de l'aide de TIDCO, Brasso Seco a construit un espace-visiteurs afin d'améliorer l'expérience écotouristique des randonneurs et des ornithologues. Le comité a aussi pu élargir sa gamme de produits et de services de manière à inclure des aliments et de l'artisanat fabriqués par les membres de la communauté. L'établissement emploie huit résidents. En 2005, la communauté a remporté un appel d'offres pour devenir entrepreneur dans le cadre du Programme national de reboisement et de restauration des bassins hydrographiques afin d'aider à conserver la forêt tropicale voisine. Ce contrat continu emploie 35 membres de la communauté responsables de la création et de l'entretien de 12 sentiers de randonnée pédestre, de bancs et d'abris faits de matériaux forestiers et de la signalisation placée le long des sentiers. En 2006, le CAT de Brasso Seco a obtenu une aide supplémentaire du Ministère du tourisme pour accéder à un terrain de 15 acres, ancienne plantation de cacao et de café. En collaboration avec l'Institut interaméricain de coopération pour l'agriculture (IICA) et l'Institut du cacao de l'Université des Antilles, la plantation a été réhabilitée pour produire du cacao et du café durables. Une infrastructure traditionnelle de séchage au soleil a été construite dans la forêt, lieu où le collectif organise aussi un festival annuel de récolte, pratiquant la «danse du cacao» traditionnelle pour écraser collectivement les fèves de cacao. Ils emballent le cacao dans l'unité de transformation adjacente et l'exportent en utilisant leur propre marque ainsi que les étiquettes de certification biologique américains.

Source: Roxanne Waithe, IICA et Elaine Philipps, Brasso Seco Paria TAC, Trinité-et-Tobago 


\section{Un cas d'éco-services fournis par} le gouvernement (Chine)

La ville de Hulun Buir, en Chine, est située au sein des trois plus grandes superficies de sol noir au monde, couvrant 11 millions d'hectares. Elle est considérée comme l'une des plus grandes zones de production céréalière du pays et dépend considérablement des engrais chimiques depuis longtemps. Au fur et à mesure, ces produits ont dégradé la matière organique du sol à un taux annuel de 0,5 point. Afin d'utiliser la terre durablement et de produire une quantité stable de céréales biologiques pour assurer la sécurité alimentaire nationale, le gouvernement de Hulun Buir a coopéré avec l'entreprise Beijing Jia Bowen Biological Technology Corporation pour élaborer un plan de restauration. La préférence des consommateurs pour un type de céréale spécifique a servi de point de départ. En s'appuyant sur l'industrie du recyclage et en se concentrant sur l'innovation pour maintenir la haute qualité des terres, ils ont protégé et revitalisé le sol noir. Ils ont aussi augmenté l'utilisation du fumier de bétail et de volaille via une stratégie de «réduction du fumier trois en un» et mis en place un service de surveillance des données pédologiques.

Après trois années de production de blé noir biologique, des progrès remarquables ont été observés. Le niveau des terres en péril est passé de quatre à trois, la teneur en matière organique du sol a augmenté de 0,2 point, la couche arable est passée de $20 \mathrm{~cm}$ à $30 \mathrm{~cm}$, la quantité de nutriments dans le sol a doublé et la résistance à la sécheresse a augmenté. En 2017, la ville a eu recours à un appel d'offres du gouvernement pour mettre en œuvre cette même stratégie sur 13300 hectares de terrain. Grace au projet, 48000 tonnes de fumier de bétail, de volaille et de paille ont été recyclés et redistribués dans le système de production et dans les cycles nutritifs naturels. Cette réutilisation a résolu le problème d'avoir une quantité de fumier trop élevée (équivalente à celle produite dans une exploitation intensive de 200000 porcs), ce qui impacte négativement l'environnement.

Source: Shi Yan, Shared Harvest, Chine

\subsubsection{Fonds privés}

Bien que les banques commerciales soient la source la plus courante de fonds privés, les systèmes alimentaires durables peuvent rechercher d'autres sources de financement privé, en dehors du secteur financier traditionnel. Puisque la durabilité est valorisée dans les futures systèmes alimentaires, les initiatives peuvent trouver des partenaires du secteur privé enthousiastes de soutenir des pratiques de consommation et de production durables.

\subsubsection{Banques éthiques}

Des banques éthiques existent dans certains pays. Elles se distinguent des banques commerciales parce qu'elles tiennent compte des préoccupations sociales dans leur développement financier. Leurs conditions de prêt sont généralement plus souples et elles sont prêtes à accepter d'autres formes de garantie. Identifiez les banques éthiques opérant dans votre région et déterminez les types de soutien financier qu'elles peuvent offrir. 


\section{(1) \\ La certification du commerce équitable comme garantie de crédit (Amérique latine et Afrique)}

Dès l'Année internationale de la microfinance en 2005, les certificats de durabilité ont commencé à être utilisés comme garanties pour les prêts bancaires (parce qu'ils démontrent la fiabilité des producteurs et la promesse de contrats de vente éthiques). Cependant, ce n'est qu'en 2012 que le Fairtrade Access Fund a été créé. Incofin Investment Management, Fairtrade International et Grameen Foundation USA se sont associés dans un fonds d'investissement international avec une avance de 1,3 millions d'USD de Starbucks. Incofin gère le fonds; Grameen fournit une gamme unique de prêts (à court et à long terme) et de l'assistance technique conçue pour répondre aux besoins des coopératives de petits producteurs; Fairtrade facilite l'accès aux marchés internationaux et fournit un filet de sécurité à prix minimum ainsi que d'autres services de soutien pour le développement démocratique des entreprises; et Starbucks, dans le cadre de son Programme de responsabilité sociale des entreprises (RSE), accorde l'argent pour soutenir ses fournisseurs (Starbucks achète de grandes quantités de café certifié Fairtrade). En 2018, le Fonds a prêté 128 millions d'USD à des agriculteurs cultivant 11 plantes dans 19 pays.

Source: http://incofinfaf.com/\#mission

\subsubsection{Concours d'innovation}

Au cours des cinq à dix dernières années, plusieurs concours et prix ont été créés pour stimuler l'innovation. Ceux-ci sont souvent axés sur les jeunes entreprises et sur des technologies spécifiques. Dans l'alimentation durable, les structures organisationnelles sont également

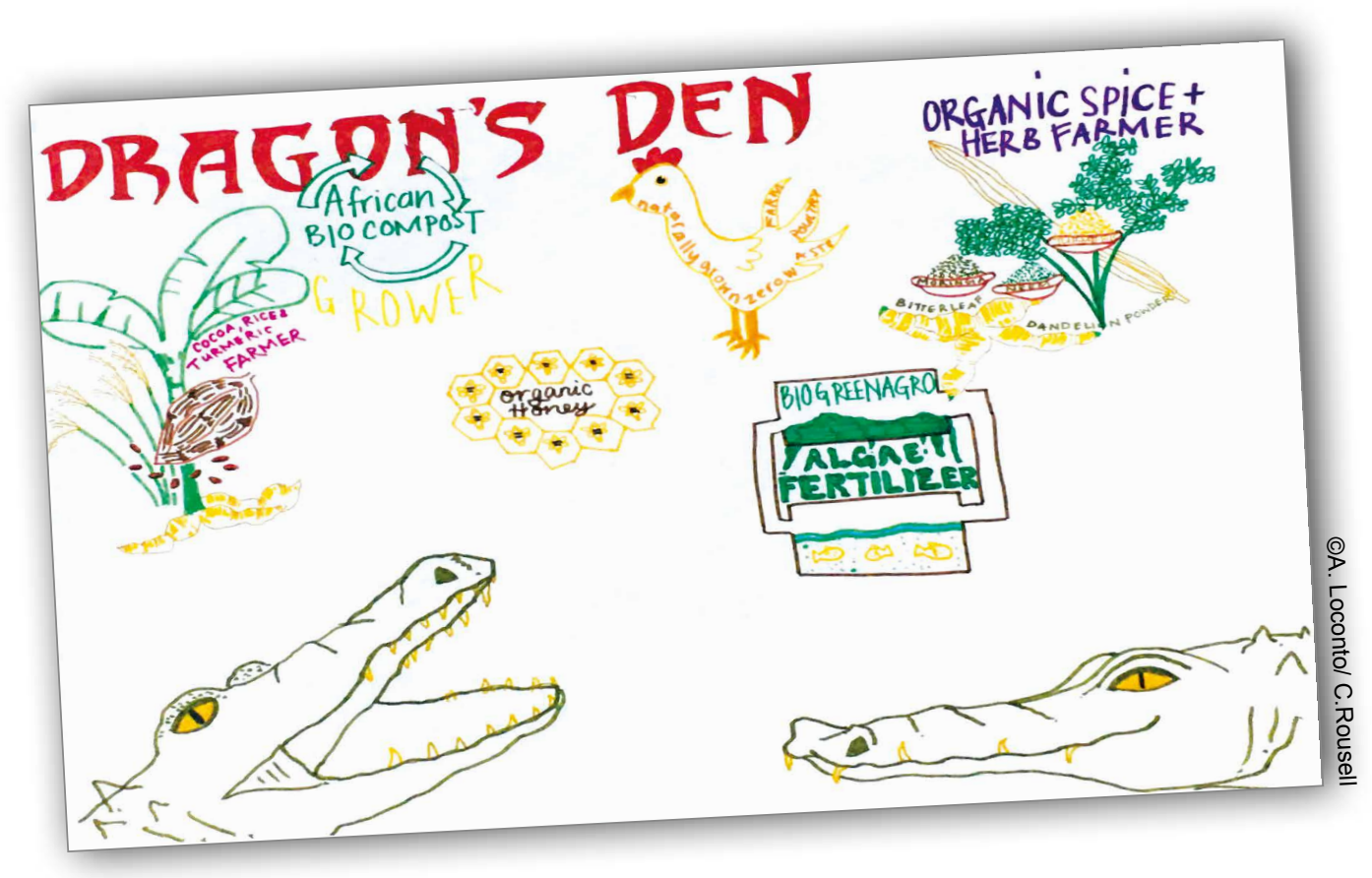


reconnues comme des innovations. Chaque concours est différent et conçu pour offrir une bourse pour récompenser soit un travail déjà accompli, soit de nouvelles idées. Lorsqu'il n'y a pas de bourse, un événement est organisé pour réunir les innovateurs afin de générer de nouvelles idées et de les faire rencontrer des investisseurs. Les informations sur ces concours se trouvent souvent dans les journaux, sur internet et dans les réseaux sociaux.

\subsubsection{Programmes de responsabilité sociale des entreprises (RSE) et fondations privées}

Les fondations privées et les programmes de RSE s'intéressent de plus en plus à financer des innovations axées sur le développement de systèmes alimentaires durables. Leurs investissements s'orientent particulièrement dans les communautés où leurs chaînes d'approvisionnement opèrent déjà. Si vous vendez certains de vos produits dans des chaînes d'approvisionnement mondiales, voyez si vos clients sont prêts à financer certaines de vos activités.

\section{Les programmes RSE de l'industrie chocolatière (Côte d'Ivoire)}

Certains produits tropicaux, tels que le cacao, le café, le thé, le sucre et l'huile de palme, servent d'ingrédients clés pour les produits des grands transformateurs alimentaires. Suite à la pression exercée par les ONG concernant les pratiques non-durables de la majorité des fermes en monoculture, les transformateurs ont établi des partenariats avec des organismes de certification tiers tels que Fairtrade International et Rainforest Alliance pour rendre ces systèmes plus durables. Ces programmes ont de plus en plus reconnu l'impact négatif des monocultures, en particulier dans les environnements forestiers, ainsi que la capacité et le devoir des producteurs de diversifier leurs exploitations.

ECOOKIM, un syndicat de 23 coopératives de cacao et de noix de cajou en Côte d'Ivoire, a développé un programme de production de manioc financé par les programmes RSE des chocolatiers Ferrero, Mars et Tony's
Chocolonely. Ce programme, qui s'intitule «autonomisation des femmes», fournit du matériel de plantation et propose une formation aux agricultrices. Celles-ci se sont organisées en coopératives dans leurs villages pour gérer la production, la transformation et la vente du manioc sur le marché local. Initialement, le vecteur moteur de la production du manioc était de stabiliser la sécurité alimentaire des ménages. Cependant, les femmes ont si bien réussi qu'elles ont produit une quantité de manioc plus élevée que leur consommation. En conséquence, les communautés ont utilisé l'argent reçu du Fonds social du commerce équitable pour investir dans des machines de broyage et de transformation de manioc, afin de diversifier leurs produits et de réduire le travail effectué par les femmes. Ils cherchent aussi à diversifier leurs systèmes de production pour inclure des cultures vivrières pouvant être vendues sur les marchés locaux.

Source: Allison Loconto, INRAE et Aminata Bamba, ECOOKIM, Côte d'Ivoire 


\subsubsection{Fonds citoyens}

La majorité des initiatives locales qui ont fait la transition vers des systèmes alimentaires durables se sont appuyées sur des collectes de fonds communautaires et sur d'autres efforts menés par les citoyens. Bien que de tels mécanismes de financement soient efficaces, quelques nouvelles approches permettent aux initiatives de dépasser le cadre de leur communauté immédiate. En même temps, certaines communautés expérimentent avec la mise en place de systèmes locaux autofinancés et autonomes.

\subsubsection{Crowdfunding}

Le financement participatif (ou crowdfunding) devient un moyen courant de recevoir de petites contributions provenant de nombreuses personnes via des plateformes en ligne. En général, ces sites incluent un bref résumé du profil de l'organisation et de sa demande de financement. Il existe des sites internationaux tels que Kickstarter, Gofundme, Indiegogo, Crowdrise ainsi que des sites nationaux et régionaux émergents tels que M-changa, Afrikwity, SliceBiz, et Projet Iroko. Effectuez une recherche internet sur le financement participatif pour voir s'il en existe déjà dans votre région.

\section{Cargonomia, un réseau de trois entreprises sociales et \\ 81}

Située à Budapest, en Hongrie, Zsamboki Biokert est une ferme maraîchère biologique et un centre d'éducation communautaire d'agriculture durable qui distribue des paniers de légumes hebdomadaires aux communautés locales. D'autre part, Kantaa est une entreprise autogérée de livraison à vélo. Ensemble, ils ont créé plus de 20 emplois locaux durables dans la région de Budapest et se sont engagés à distribuer les aliments en utilisant des vélos cargo fabriqués localement. De plus, ils ont aussi construit un centre logistique offrant des solutions de transport urbain durable pour les membres de la communauté.

Afin d'élargir leur portée, Cargonomia a lancé une campagne de financement participatif pour amasser des fonds d'investissements spécifiques tels que:

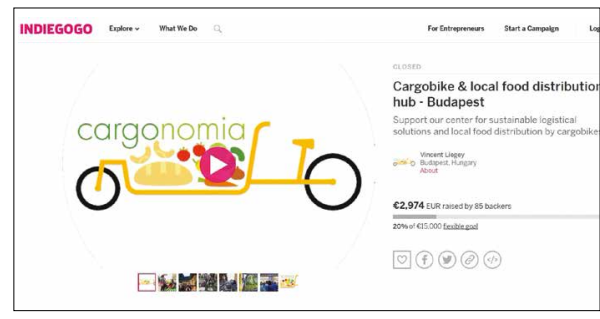

quatre nouveaux vélos de cargaison type «Long John» fabriqués à la main à Cyclonomia;

> deux moteurs électriques pour faciliter la livraison des vélos dans des quartiers urbains vallonnés;

$\checkmark$ un prototype d'essoreuse à salade faite à la main et à propulsion humaine pour le partenaire Zsambok's Organic Garden;

> du matériel supplémentaire pour développer des unités de transport et de stockage des légumes et de pain.

Source: Orsolya Lazanyi, Cargonomia 


\subsubsection{Monnaie locale}

Certaines communautés expérimentent la création de monnaies locales pour échanger des produits et des services. Cette approche a pour but de conserver une certaine valeur au sein de la communauté, tout en aidant les membres marginalisés à s'impliquer davantage dans le système alimentaire local. Il peut s'agir, par exemple, de simples systèmes de troc utilisés depuis des millénaires pour échanger des aliments. D'autres systèmes sont plus complexes, reposant plutôt sur des billets imprimés. Il est important de noter que ces initiatives sont très difficiles à maintenir et doivent souvent inclure des biens et services autres que la nourriture.

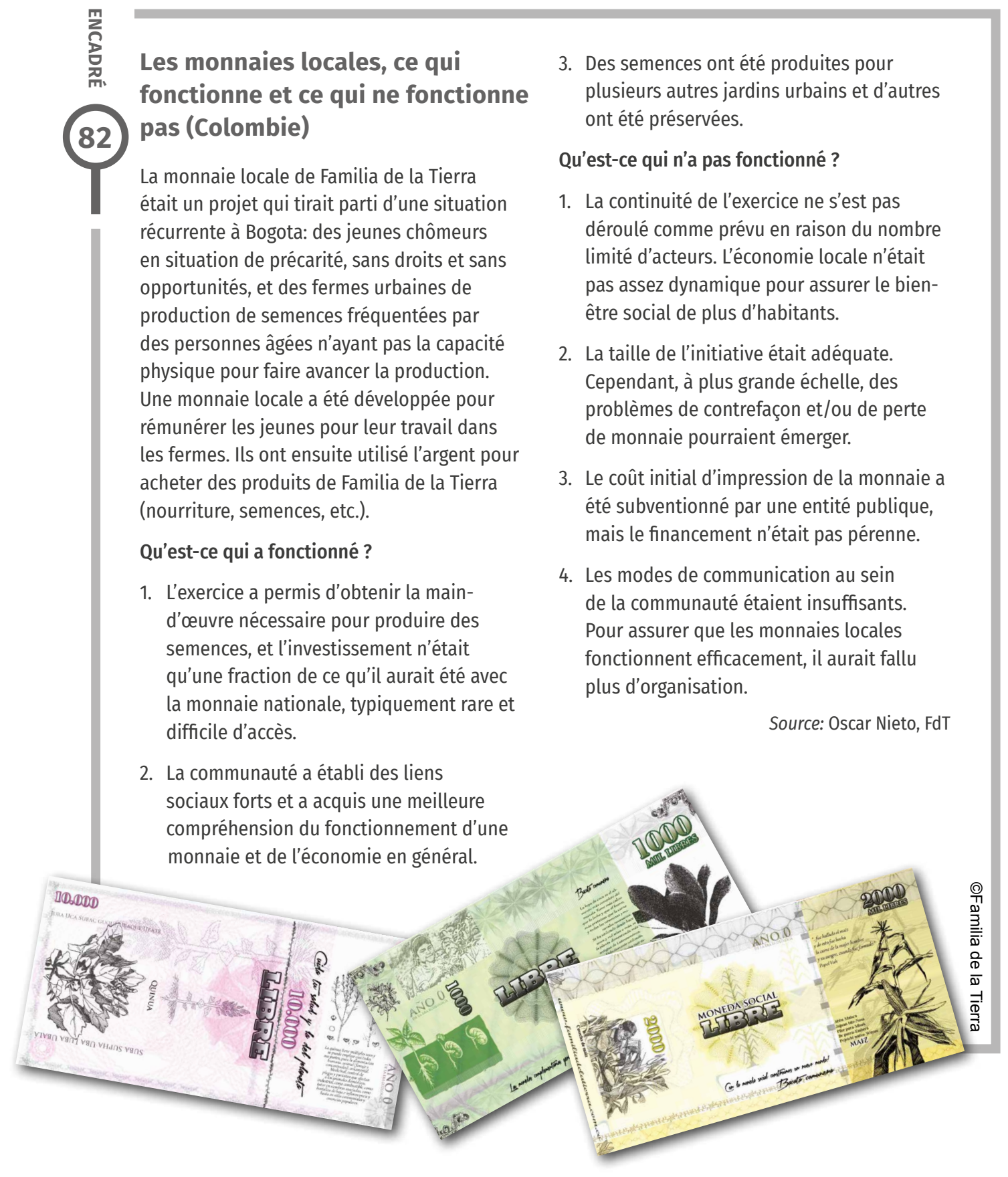




\section{COMMENT TROUVER LES FONDS NÉCESSAIRES?}

N'oubliez pas qu'un financement peut être accordé en espèces ou en nature (p. ex. services, maind'œuvre, équipement, produits alimentaires). Les points suivants peuvent vous aider à reconnaître une opportunité de financement quand elle se présente:

1. Analysez les sources de financement internes et externes, en particulier leurs forces et faiblesses, et essayez de combiner plusieurs sources pour trouver la meilleure solution (avant l'analyse, concevez un plan d'affaires). Cela peut changer avec le temps.

2. Élaborez des critères pour évaluer les sources de financement, tels que la durabilité des normes des créanciers (par ex. les types de banques, les types de programmes de RSE) et soyez conscient que les start-ups ont souvent des options limitées. Deuxièmement, le financement devrait s'orienter vers la pratique sans être trop idéaliste. Il est plus probable que cela commence par l'autofinancement (interne), étant donné que les nouvelles initiatives ne disposent généralement pas de profil financier officiel.

3. Élaborez quelques plans de secours (p. ex. des plans A et B), car il est peu probable de remporter tous les concours ou appels à projets.

4. Gérez l'adhésion de vos membres de manière à renforcer leur engagement envers l'initiative et leur volonté d'y investir.

5. Développez, avec les acteurs publics et privés, des partenariats basés sur la confiance et les garanties mutuelles.

\section{ASTUCE 37}

\section{Financer votre initiative}

- Bien qu'il existe de nombreuses options de financement, y accéder n'est pas toujours facile. Sortez des sentiers battus et soyez créatifs avec la façon de combiner vos idées.

- Le cycle de vie d'une initiative n'est pas stagnant, vous avez donc besoin de différentes sommes d'argent à différents moments. Essayez de maintenir une liste de sources de financement possibles pour la consulter au besoin.

- Vos membres sont une excellente ressource pour mobiliser des fonds internes et pour trouver des fonds externes. Maintenez des relations transparentes avec vos membres pour renforcer leur fidélité à long terme.

- Si l'autonomie financière est un objectif, le financement devrait provenir principalement de sources internes plutôt que de sources externes, car cela est plus durable dans le long terme. Cependant, si vous envisagez de faire croitre votre activité, cherchez plutôt des fonds externes. 


\section{LE CHEMIN D’APPRENTISSAGE, OÙ ALLER ENSUITE?}

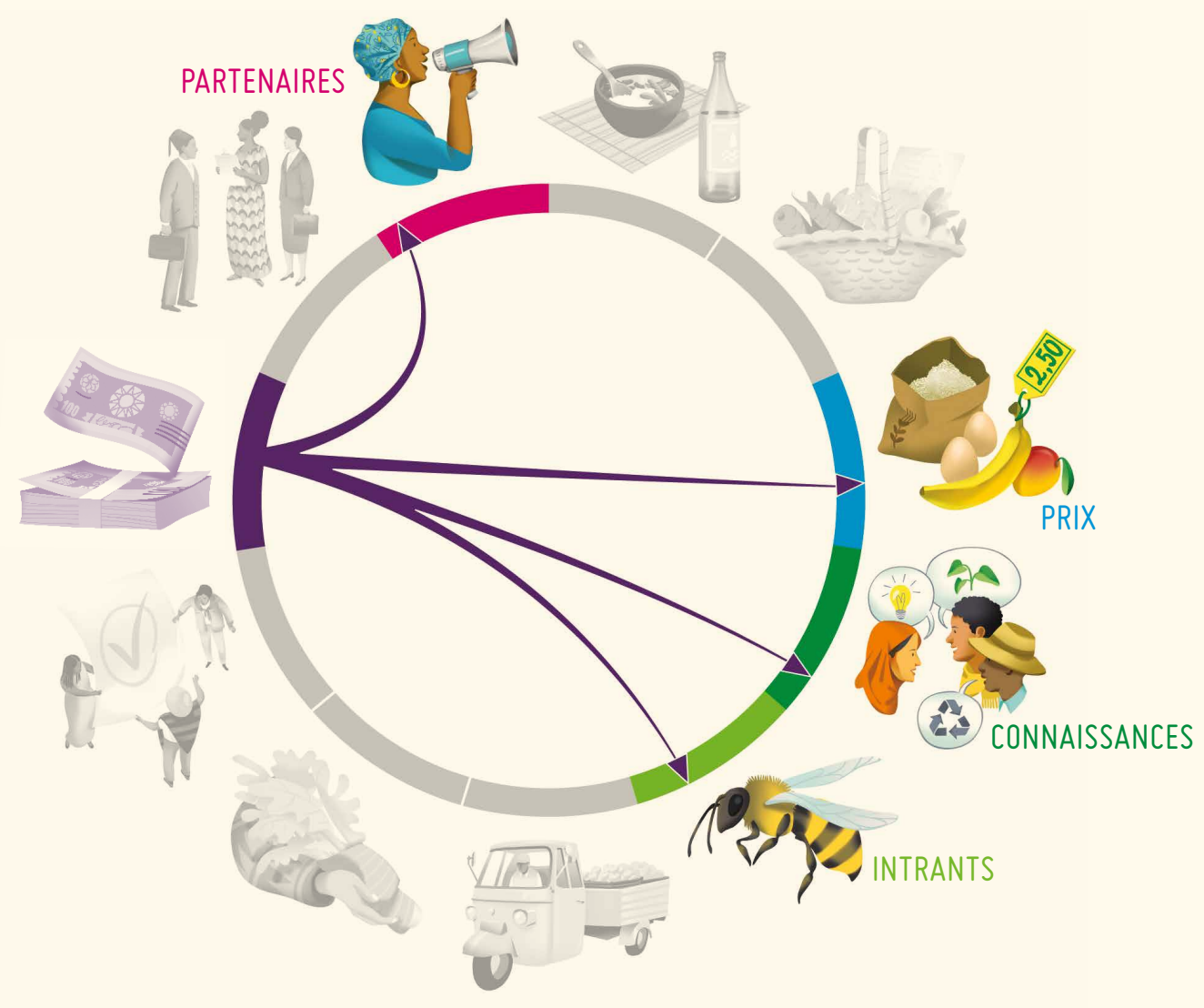

- Vous cherchez à mobiliser les réseaux de vos membres pour financer un nouvel investissement? Trouvez des conseils sur comment y arriver au Chapitre 11: La place des partenaires et des plaidoyers.

- Vous préparez une demande de financement de recherche et vous avez besoin de savoir comment collaborer avec des chercheurs ? Explorex quelques options dans le Chapitre 4: Partager et co-créer des connaissances pour une production durable.

- Vous voulez utiliser la vente de produits comme stratégie de financement de base et vous n'êtes pas certain du montant que vous pouvez demander ? Allez au Chapitre 3: Trouver le «bon» prix pour en savoir plus.

- Vous financez des intrants pour vos membres et vous cherchez des solutions plus durables? Consultez le Chapitre 5: Gérer et accéder aux intrants durables.

Ou, lisez un autre chapitre qui attire votre attention ! 


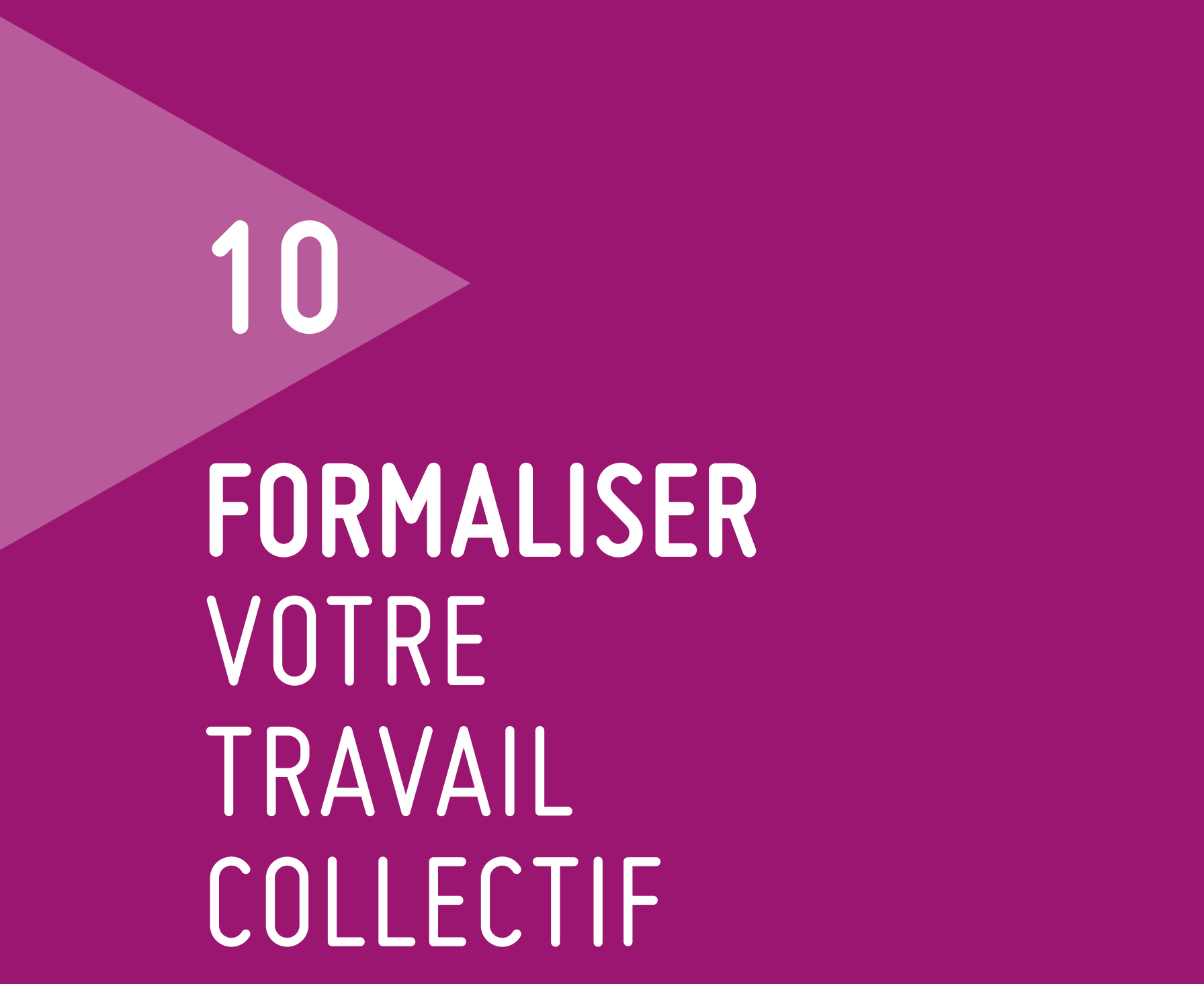




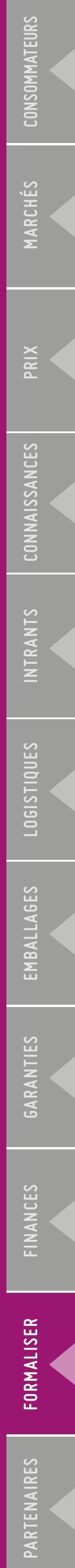




\section{POURQUOI EST-CE IMPORTANT?}

Vous envisagez de lancer une nouvelle initiative ou de formaliser votre travail collectif ?

L'élaboration d'un nouveau projet est un défi qui exige des stratégies très diverses. La manière de s'organiser est un aspect clé pour le succès d'une initiative. La répartition des responsabilités entre les différentes personnes au sein d'une organisation ou entre un réseau d'organisations, influencera considérablement vos capacités (par exemple, déterminer qui participe à la prise de décision, a la responsabilité financière ou juridique et comment enregistrer l'organisation auprès des autorités publiques).

Chaque modèle offre des possibilités et des défis différents et, selon ce que vous essayez de faire, vous choisirez un modèle différent.

\section{FIXER DES OBJECTIFS COMMUNS}

Faciliter un processus de groupe nécessite toujours une première étape d'autodéfinition. Voici quelques questions clés pour réfléchir collectivement sur les objectifs communs de votre initiative:

1. Qui êtes-vous?

2. Qui voulez-vous devenir?

3. Que voulez-vous réaliser ? Créer ? Produire ?

4. Combien de temps voulez-vous que ce processus dure ?

5. Comment allez-vous y arriver?

6. Comment saurez-vous quand vous aurez atteint ces objectifs?

\subsection{Vision, mission, objectifs et valeurs}

La vision, la mission, les objectifs et les valeurs (VMOV) d'une organisation sont des outils de base pour bâtir une collaboration durable. Certains pays ont des exigences légales pour l'enregistrement d'une organisation. Articuler la façon dont VMOV relient les différents aspects de votre travail aidera à maintenir le groupe sur la bonne voie.

- La vision est une image conceptuelle de l'avenir. Elle projette ce que l'organisation veut atteindre à long terme. C'est le rêve guidant l'organisation dans l'avenir.

- La mission est la raison d'être d'une organisation. C'est le but pour lequel les stratégies de l'organisation sont construites. Elle répond à la question: «pourquoi l'organisation a-t-elle été créée ?» 
- Les buts (objectifs) indiquent ce que l'organisation prévoit de réaliser. Toutes les activités visent à atteindre ces objectifs. Les objectifs reflètent des plans spécifiques à court ou à long terme. Ils sont SMRRO (spécifiques, mesurables, réalisables, réalistes, opportuns) et périodiquement révisés par le groupe. Un processus annuel de planification stratégique peut être un outil utile et efficace.

Gardez à l'esprit que dans les groupes de production communautaires, tous les producteurs devraient partager des objectifs et des buts similaires. Être d'accord sur un objectif de production est essentiel pour maintenir l'engagement des agriculteurs et le niveau des rendements et aide aussi à répondre aux attentes des clients.

- Les valeurs guident la prise de décision des systèmes alimentaires durables et aident les membres à former une identité collective de base. Ils orientent également les non-membres sur leurs attentes d'engagement. Ces valeurs constituent le fondement de toutes les règles et normes adoptées par l'organisation.
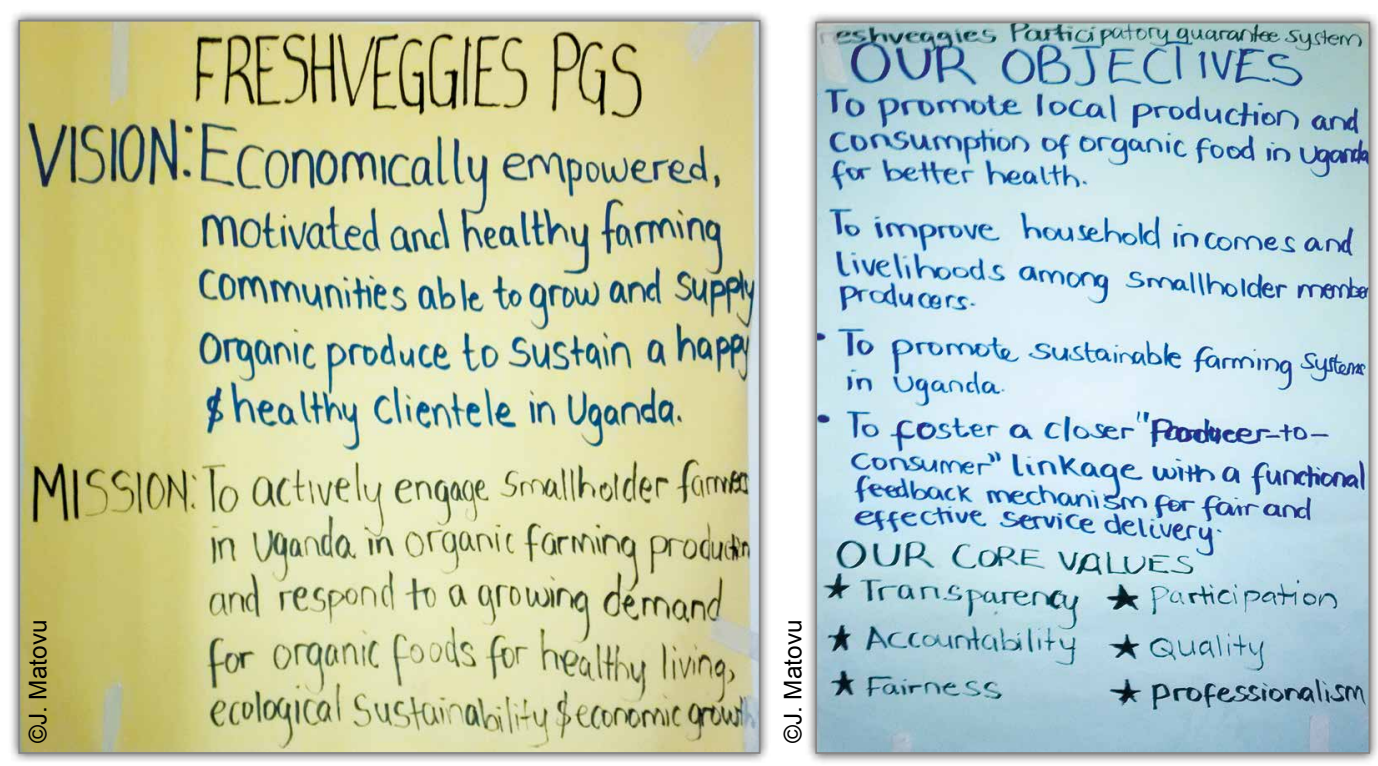

Source: SPG FreshVeggie Group Vision/Mission/Mission/Objectifs/Valeurs travail d'établissement des valeurs

\section{LISTE DE VÉRIFICATION}

Questions clés pour définir votre VMOV

1. Vous voulez vendre seulement une petite quantité pendant la fin de semaine ?

2. Vous travaillez pour produire à temps plein?

3. Quel type de croissance voulez-vous atteindre ?

4. Combien de marchés/restaurants/magasins voulez-vous desservir? 


\subsection{Définir l'identité du groupe}

Définir le groupe façonne sa composition et par conséquent, définit ses objectifs. Cette identité s'appuie sur les valeurs fondamentales de l'organisation. Que se passe-t-il s'il y a d'autres acteurs (c.-à-d. des partisans, des membres de l'académie, des personnalités religieuses ou des agents gouvernementaux) qui veulent devenir membres de l'organisation et qui ne partagent pas les mêmes valeurs? Dans une organisation multi-acteurs, le groupe doit se mettre d'accord sur les critères d'adhésion et de partenariat et sur la manière de distinguer les deux catégories.

Pour le faire, invitez tous les collaborateurs potentiels à discuter ensemble des VMOV afin de construire une stratégie et une identité. Sinon, vous risquez de ne pas savoir si les autres partagent les mêmes intérêts que vous. Dans ce cas, lancez un appel à projet pour un sujet (organisez peutêtre une discussion publique ou un séminaire) et voyez si les personnes intéressées sont prêtes à formaliser collectivement le projet.

\section{ASTUCE 38}

Développer une initiative de système alimentaire durable

1. Le projet est-il guidé par la perspective du producteur ou du consommateur (mangeur)? Savoir qui motive votre projet permettra d'orienter la structure globale et d'aboutir à un consensus quant au rôle de décideur principal.

2. Y a-t-il un accès adéquat à la terre, à l'eau, à la main-d'œuvre, aux finances ou aux intrants ? Ces ressources sont-elles adaptées au type de production prévu?

Bien que cela puisse sembler évident, c'est l'un des principaux obstacles à la mise en route d'un projet agricole.

3. Y-a-t-il un plan d'affaires bien élaboré (basé sur un plan d'environ trois ans ou saisonnier) ? Si l'agriculteur est employé à l'extérieur ou s'il entreprend le projet, estimez la taille du marché nécessaire à la viabilité de l'entreprise et comment satisfaire les besoins de consommation avec une exploitation diversifiée. Ce plan d'affaires, fondé sur une stratégie financière, impacte aussi la durabilité globale. La thématique de la diversification des sources de revenus devrait aussi être abordée.

4. Vous voulez élargir votre mission pour inclure les jeunes et l'éducation, et si oui, comment ? Les projets éducatifs destinés aux consommateurs adultes et enfants peuvent ajouter de la valeur et de la profondeur aux systèmes alimentaires durables locaux. Ils exigent une planification pédagogique détaillée et une coordination avec les autorités éducatives locales. 


\subsection{Un bon leadership}

Dans un système alimentaire durable, tous les membres du groupe jouent un rôle, mais il faut aussi guider les autres dans la bonne direction. Le style de leadership est très important, car il peut encourager ou contraindre le travail d'équipe et la réalisation des objectifs collectifs.

\section{Le leadership organique: un concept de style de vie}

83

Le leadership organique est un hybride de styles de leadership serviteur, relationnel et transformationnel. Un leader organique influence les autres à travers la compassion. Il s'agit autant d'atteindre et de réaliser les objectifs définis que de la façon qu'ils sont atteints. Le leadership organique est généralement composé de six principes:
1. des relations fondées sur la collaboration;

2. une communauté formée par le récit;

3. l'influence façonnée par la compassion;

4. La directive façonnée par la découverte;

5. l'authenticité façonnée par la responsabilité;

6. les réseaux façonnés par les relations personnelles.

Source: Patricia Flores, IFOAM Organics International (extrait de Organic Leadership: Un nouveau style de leadership, K. Heitzman)

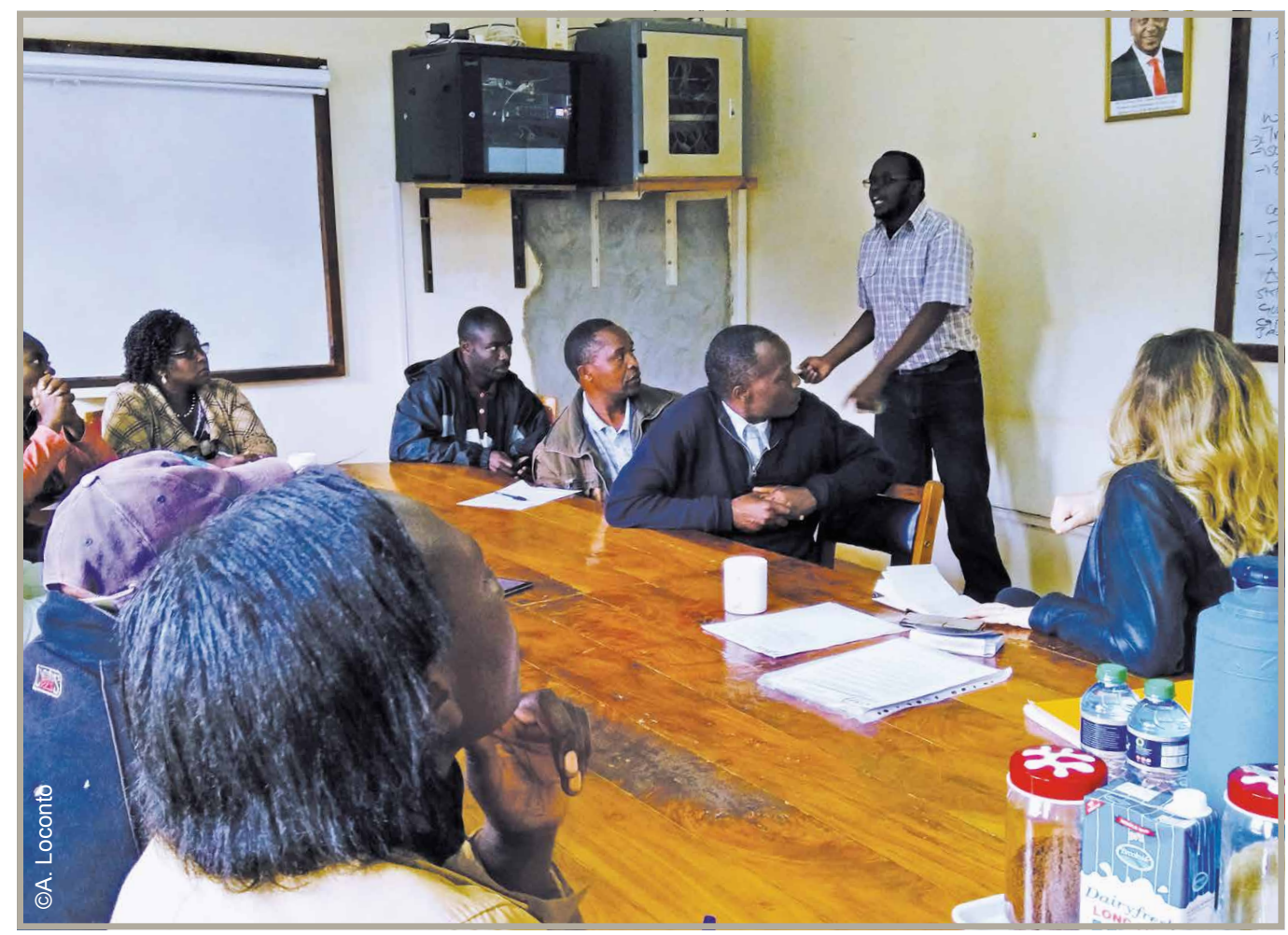




\section{2. ÉTABLIR UN MODÈLE ORGANISATIONNEL POUR VOTRE GROUPE}

La façon dont les acteurs se réunissent et définissent une structure commune pour leurs opérations dépend largement du contexte local. Quel que soit le degré de créativité des acteurs, les organisations formelles sont façonnées par le cadre juridique national.

\section{LISTE DE VÉRIFICATION}

Déterminer la flexibilité nécessaire dans votre organisation

1. Quel est le degré de formalité de votre engagement ?

2. Vous essayez de garantir un revenu décent au producteur dans un contexte global durable?

3. Vous essayez de minimiser les tensions et les conflits en fixant des règles opérationnelles précises?

4. Vous essayez de rendre l'initiative durable à long terme ?

5. Vous essayez de structurer les opérations et de repartir les responsabilités pour encourager plus d'efficacité et de clarté au sein de l'organisation?

6. Vous souhaitez de changer votre échelle d'opération en élargissant votre organisation ou vos activités (en prenant de l'expansion ou en incitant d'autres personnes à essayer des approches similaires) ?

Lorsque les agriculteurs, les intermédiaires transparents et les consommateurs coopèrent pour faire évoluer le système alimentaire, leur engagement doit être officiellement enregistré conformément aux lois et réglementations nationales. Dans certains pays, par exemple, un groupe d'agriculteurs n'est pas autorisé à vendre ses produits à moins qu'il ne soit enregistré en tant que coopérative agricole.

Renseignez-vous sur les options possibles auprès de vos organismes publics locaux. Ces options dépendent généralement de la taille prévue de l'entreprise, du montant du capital, du type d'entreprise (nationale ou internationale) et du nombre et du type de propriétaires. Vous pouvez trouver ces informations en ligne ou en visitant des bureaux spécifiques dans votre région, tels que:

- chambres de Commerce;

- bureaux d'affaires locaux.

ASTUCE 39

\section{Créer une entreprise}

Est-il facile d'immatriculer une entreprise dans votre pays ? Consultez le site web suivant pour savoir combien de jours, en moyenne, vous devez attendre afin d'obtenir différents types d'autorisations: https://www.doingbusiness.org/en/reports/global-reports/doing-business-2020 
Chaque pays utilise des modalités et des conditions différents pour régir les types d'organisation possibles. Par exemple, une coopérative n'a pas la même signification dans tous les pays: elle peut être à but non lucratif, à but lucratif ou même interdite. Cependant, il existe quelques «grandes familles de modèles organisationnels» qui vous aident à comprendre les diverses options. Des exemples de modèles déjà établis sont fournis ci-dessous.

\subsection{Entreprises sociales et autres types d'entreprises}

\subsubsection{Entreprise sociale}

Les entreprises sociales génèrent des revenus en ayant deux objectifs principaux: 1) avoir un impact social, culturel, économique communautaire et/ou environnemental positif; et 2) apporter des revenus. Elles peuvent avoir un but lucratif ou non-lucratif. Bien qu'en apparence, bon nombre de ces entreprises ressemblent à des entreprises traditionnelles, elles sont exceptionnellement axées sur les missions de l'entreprise tandis que la production de revenus est souvent une priorité secondaire.

\section{ASTUCE 40}

Vérifier le procédé d'immatriculation des entreprises sociales de votre pays.

\subsubsection{Entreprise détenue par ses salariés}

Il y a plusieurs types d'entreprises détenues par les salariés, notamment: l'actionnariat direct des employés (les employés sont les principaux actionnaires de l'organisation), l'actionnariat indirect des employés (les employés détiennent des actions collectives de l'organisation par le biais d'une fiducie) ou une combinaison de ces deux modèles. Dans ce type d'entreprise, les employés sont aussi les propriétaires, se transformant de travailleurs en acteurs engagés ayant un intérêt dans le succès de l'entreprise.

\section{兑 ChocoTogo - une entreprise de transformation détenue par ses \\ 84. employés (Togo)}

Créée par un groupe de jeunes entrepreneurs en 2012, ChocoTogo est la première entreprise de transformation du cacao détenue collectivement par ses salariés. Elle est aussi la première marque de chocolat en barre au Togo. Comblant une lacune sur le marché national de la production et de la distribution du chocolat, ces chocolatiers ont installé une petite unité de production et un centre de formation à petite échelle. L'emploi des femmes est l'un des leurs principaux piliers. Depuis sa fondation, ChocoTogo a ouvert le marché aux chocolats fins contrôlés et produits au Togo.

Source: Nathalie Kpante, CHOCOTOGO, Togo http://www.chocofair.org/en/chocotogo 


\subsubsection{Entreprise familiale}

Les entreprises familiales constituent la forme d'entreprise la plus courante dans le monde (http://www.ffi.org/). Étant donné qu'elles varient considérablement à l'échelle mondiale et d'une entreprise à l'autre, il est difficile d'établir une définition unique. Cependant, elle consiste en un modèle dans lequel plusieurs membres d'une même famille sont les propriétaires ou gestionnaires principaux, et/ou dans lequel la majorité des décisions sont prises par des membres de la famille du fondateur de l'entreprise. Certains pays exigent que ce genre d'entreprise s'immatricule en tant que société de capital-investissement (private equity).

\subsubsection{Société commerciale}

Une société commerciale est une entreprise qui travaille avec une variété de produits vendus aux consommateurs, à d'autres entreprises ou directement aux gouvernements. Dans ce modèle, l'entreprise négocie l'achat de produits et coordonne la livraison des produits aux consommateurs. Pourtant, elles ne possèdent pas ou ne stockent pas de produits. Une société de transport, en revanche, peut posséder des camions et des entrepôts et peut également s'immatriculer en tant que société commerciale. Les sociétés commerciales peuvent fonctionner dans divers cadres juridiques, y compris des structures de société à responsabilité limitée, des entités non constituées en société, ou des structures avec des droits territoriaux.

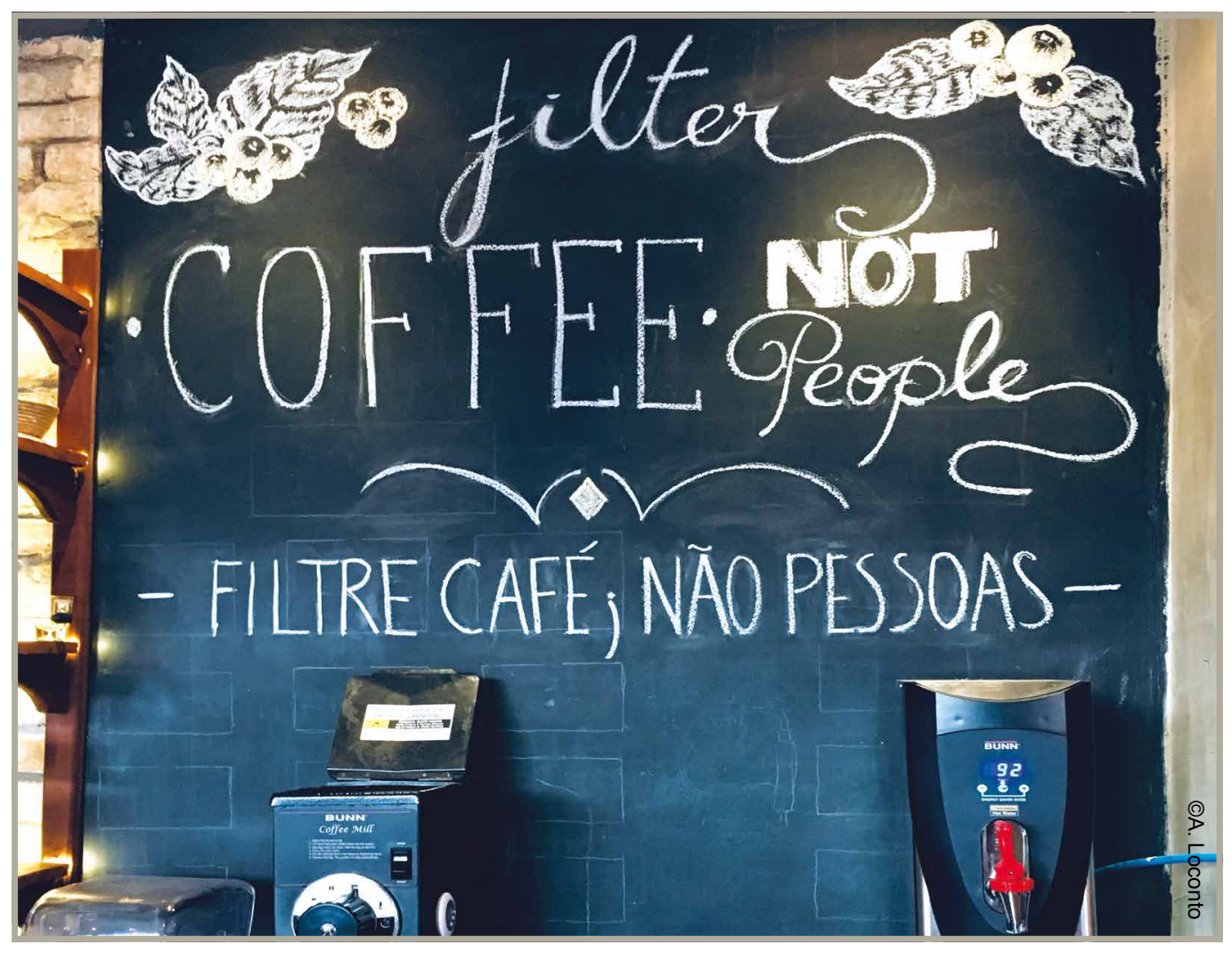




\subsection{Coopératives}

Selon la définition officielle de l'Alliance Coopérative Internationale ( $\mathrm{ACl})$, «une coopérative est une association autonome de personnes volontairement unies pour répondre à leurs besoins et aspirations économiques, sociaux et culturels communs par le biais d'une entreprise commune et sous contrôle démocratique». Les coopératives peuvent s'établir pour des producteurs, des consommateurs et/ou des commerçants. Elles fonctionnent selon les principes suivants:

1. adhésion volontaire et ouverte;

2. contrôle démocratique des membres;

3. participation économique des membres;

4. autonomie et indépendance;

5. éducation, formation et information;

6. coopération entre coopératives;

7. préoccupation pour la communauté.

\section{Hansalim, une structure coopérative agriculteurs-}

\section{5} consommateurs (Corée du Sud)

Hansalim est l'une des plus grandes coopératives agricoles durables au monde. Basé en Corée du Sud, elle a été créée en 1986. En 2016, ses consommateurs constituaient plus d'un demi-million de ménages. Avec 22 coopératives de consommateurs, 204 vitrines et un chiffre d'affaires total de 362 millions d'USD, Hansalim a réussi à connecter 112 communautés agricoles avec plus de 200 familles sur le marché. Ces échelles sont gérées en cooptant les structures existantes telles que les coopératives régionales de consommateurs et les associations d'agriculteurs. Chaque organisme a sa propre structure de gouvernance et nomme des personnes au conseil d'administration de la coopérative. Chaque année, les membres des deux structures se rencontrent et prennent des décisions sur diverses thématiques telles

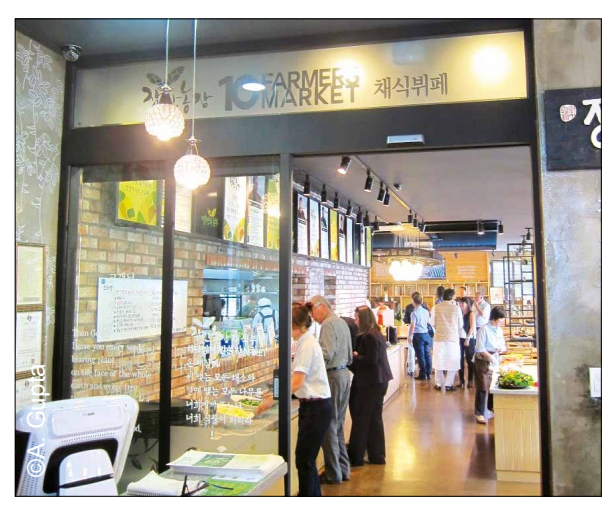

que le prix des produits, la disponibilité, les fonds pour la stabilisation des prix et de la production, et organisent d'autres réunions sociales comme des festivals. Chacune des sous-structures est gérée selon des modalités de gouvernance démocratiques. Hansalim défend les intérêts des consommateurs et des producteurs.

Source: Hansalim, Corée du Sud, http://eng.hansalim.or.kr 


\subsection{Organismes sans but lucratif (OSBL) et fondations}

Un organisme sans but lucratif ayant une structure à but lucratif ou de coopérative, permet d'accéder aux ONG et aux fonds publics/donateurs, en plus des revenus privés.

\subsubsection{ONG qui fournit un soutien/espace de marketing}

Les ONG sont des groupes du secteur privé (généralement sans but lucratif et non-sectaires) qui mènent et participent à des projets d'éducation, de formation, d'aide humanitaire et/ou de coopération. Il peut s'agir de grandes organisations mondiales ou de petits groupes locaux. Certaines ONG encouragent l'activité autour d'une question spécifique (comme les droits de l'homme ou les préoccupations environnementales), tandis que d'autres sont plutôt axés sur des activités d'analyse ou de recherche.

\section{Maputo Earth Market
(Mozambique)}

86

Créé en 2013, le marché Maputo Earth Market est né d'une collaboration entre l'ONG italienne Gruppo di Volontariato Civile (GVC) et Slow Food International. Avec 13 producteurs et agriculteurs de la province de Maputo au Mozambique, il est un point de distribution central pour les fruits et légumes, les œufs frais et les confitures, tout en respectant le mantra «local, saisonnier, propre et artisanal». Ce marché a été créé dans le cadre d'un consortium d'ONG (dont le GVC et Essor), l'Union nationale des agriculteurs du Mozambique et le convivium local de Slow Food. Ce partenariat souligne l'interaction entre les secteurs public et privé, ainsi que le rôle que les ONG peuvent jouer dans le soutien relationnel, analytique, financier et de recherche.

Source: Stelio Miguel Joaquim, Maputo Earth Market, Mozambique http://www.earthmarkets.net/network/maputo

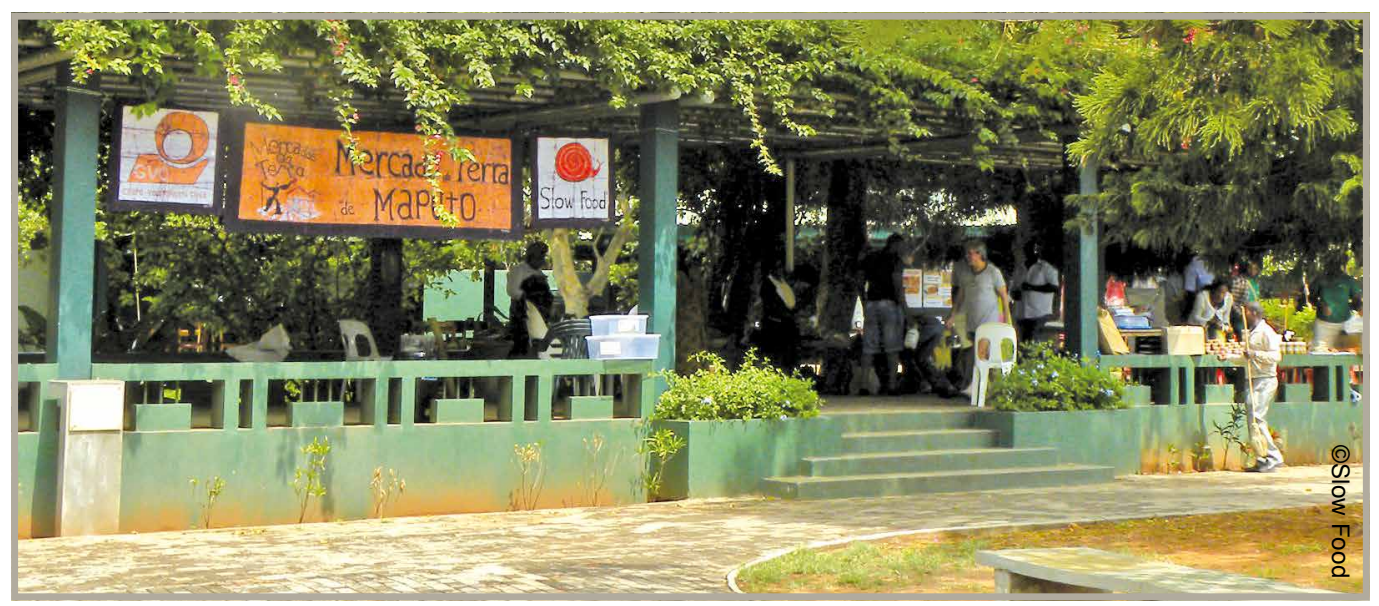




\subsubsection{Fondations}

Les fondations appartiennent à deux catégories: les fondations caritatives et les fondations privées. Les fondations caritatives sont une catégorie légale d'OSBL spécialisées dans les dons ou dans le soutien financier d'organismes publics ou privés. Les fondations privées remplissent une fonction similaire, bien qu'elles soient traditionnellement dotées par un individu ou une famille, ainsi que par un statut et des exigences juridiques différents.

\section{Green Net Cooperative/ Earth Net Foundation (Thaïlande)}

87

Comme le cas de Green Net, en Thaïlande, différentes structures organisationnelles répondent à différents besoins. Green Net s'est d'abord immatriculé sous le nom Green Net Cooperative, étant donné que cette structure correspondait à ses valeurs de commerce équitable et la permettait d'être légalement reconnue en tant qu'organisation commerciale et exportatrice. Plus tard, Green Net s'est enregistré comme Earth Net Foundation, un OSBL. Ce type de structure était le plus éligible à bénéficier de fonds externes de développement en vue de former et de soutenir les nouveaux agriculteurs à la conversion en agriculture biologique, de développer des entreprises de groupes d'agriculteurs et de sensibiliser les consommateurs aux produits biologiques et au commerce équitable. Une troisième organisation, Green Net SE Co. Ltd, une entreprise sociale, a été créée pour développer le Projet de café forestier biologique, puisque de larges investissements étaient nécessaires pour le savoir-faire, l'infrastructure et l'achat équitable des graines de café. En tant que société en commandite, les investisseurs sociaux peuvent accorder des montants de capital plus importants, permettant au projet de décoller.

Source: Michael Commons, Green Net, Thaïlande http://www.greennet.or.th/en/about/earthnet

\subsection{Autres modèles organisationnels}

\subsubsection{Fermes communautaires}

Au cours des 50 dernières années, de nouvelles structures collectives ont émergé. Par exemple, les fermes collectives contemporaines ont évolué à partir des fermes communales des années 1960. La ferme collective repose sur la récupération des terres communes et de la protection de la propriété foncière commune, ou de la location de terres par le biais de fiducies foncières communautaires ou d'autres accords similaires garantissant des terres pour la production alimentaire collective. 


\subsubsection{Association pour le maintien d'une agriculture paysanne (AMAP)}

Une AMAP est un partenariat entre une ferme et des consommateurs, où les risques et les avantages de l'agriculture sont partagés. S'inscrivant dans une démarche active de la relocalisation de l'économie, l'AMAP repose sur un partenariat typiquement formalisé sous forme de contrat entre chaque consommateur et le producteur. Cet accord est caractérisé par un engagement d'approvisionnement mutuel (en argent et en nourriture) sur une longue période de temps. Les AMAP sont motivés par les principes fondamentaux suivants:

- restaurer la souveraineté alimentaire locale;

- créer une solidarité entre producteur et consommateur pour construire des communautés plus socialement justes et durables;

- partager les risques et les avantages de la production;

- défendre la santé et la nutrition par l'alimentation;

- développer la responsabilité civique.

\subsubsection{Réseau (structure informelle)}

Dans un modèle de réseau, il n'y a pas de secrétariat ni de système de coordination central pour la prestation de services. Traditionnellement, les réseaux reposent sur des relations informelles d'organisations ou d'individus travaillant ensemble autour d'une idée ou d'un objectif commun. Certains réseaux sont plus légalement reconnus pour assurer la sécurité des parties impliquées.

\section{尊 Des contrats de réseaux (Italie)}

Le «Contratto di Rete» italien est un outil

88

innovant qui permet la collaboration entre les entreprises, tout en préservant leur indépendance commerciale individuelle. L'objectif est d'accroître l'innovation et la compétitivité globale du marché. Toutes les parties impliquées signent un contrat au moyen d'un acte public, d'un notaire privé, ou d'une signature électronique approuvée. Avec près de 20000 de ces contrats actuellement utilisés en Italie, ils constituent un exemple innovant de cadre juridique pour la collaboration entre les entreprises.

Source: Contratto di Rete Registro http://contrattidirete.registroimprese.it/ reti/index.action
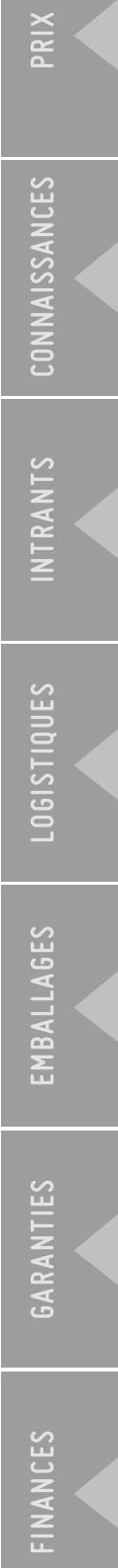


\section{GÉRER LA CROISSANCE ET TROUVER LA BONNE ÉCHELLE ORGANISATIONNELLE}

Il est essentiel de décider comment augmenter ou réduire l'échelle de votre opération, et si cela en vaut la peine. Selon le modèle organisationnel choisi, il est souvent plus avantageux d'élargir votre réseau d'une façon créative, en collaborant avec plus de producteurs complémentaires, que d'augmenter la taille de l'initiative. Différents marchés locaux peuvent se relier de manière cohérente en travaillant avec les autorités locales, y compris des initiatives de vente directe aux consommateurs et de marchés publics locaux pour les écoles, les hôpitaux et les maisons de retraite. Un changement d'échelle peut également se faire à l'aide de projets de transformation alimentaire coopératifs à petite échelle.

L'évaluation systématique de vos objectifs doit tenir compte de l'échelle d'opération souhaitée, qui peut être divisée en différentes sous-échelles conçues pour différentes phases.

Rappelez-vous que des échelles différentes exigent des structures physiques et organisationnelles différentes.

Tierra Viva, Agricultores Orgánicos (Chili)

Tierra Viva est une association d'agriculteurs biologiques basée à Santiago, au Chili. Fondée il y a 23 ans, elle est pionnière du mouvement biologique chilien, dont le but est d'organiser les agriculteurs biologiques pour les marchés domestiques locaux. Avec seulement dix clients au départ, elle fournit à présent de la nourriture à plus de 500 clients, y compris des restaurants et des hôtels. Tierra Viva est un SGP accrédité par l'État depuis 2008. Andrea Tuczek, fondatrice et agricultrice principale, déclare:

«Avec la nouvelle réglementation biologique intégrant les systèmes d'auto-certification des organisations d'agriculteurs (soit SGP), de nouveaux agriculteurs et de nouvelles organisations ont demandé à faire partie de notre initiative. Nous avons remarqué qu'après quelques années, une fois qu'ils ont été formés et qu'ils savent comment gérer le système, ils retournent dans leurs communautés pour

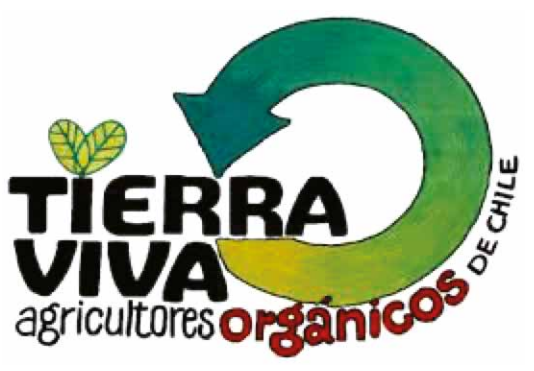

établir leurs propres systèmes. Nous avons compris que même si nous n'augmentons pas notre taux d'adhésion de membres, nous pouvons aider les nouveaux arrivants à établir un système de garantie crédible».

En d'autres termes, Tierra Viva, sans avoir l'intention de jouer ce rôle, est un incubateur d'initiatives de SPG pour les agriculteurs biologiques essayant de se conformer aux normes prescrites du Servicio Agrícola y Ganadero (SAG), l'autorité nationale pour l'agriculture biologique au Chili.

Source: Patricia Flores, IFOAM, Pérou 


\subsection{Gérer la croissance}

En général, les groupes communautaires commencent petits et se développent au fur et à mesure. Certains deviennent des grandes entreprises. Dans tous les cas, gérer la croissance est un élément essentiel du groupe. La croissance signifie l'augmentation du nombre d'agriculteurs ainsi que la capacité à atteindre les objectifs du marché.

\section{LISTE DE VÉRIFICATION}

Facteurs impactant les dimensions de votre groupe

Plusieurs facteurs affectent la taille de l'organisation et il est difficile d'en identifier seulement quelques-uns. Néanmoins, les questions suivantes peuvent vous aider à définir l'échelle qui reflète vos objectifs:

1. Quelle est la portée du mouvement alimentaire durable dans votre ville/région (c.à-d. y a-t-il des intrants facilement disponibles pour les traitements écologiques, les semences, les marchés, la recherche, etc.) ?

2. Y a-t-il une demande spécifique pour vos produits?

3. Quelle est la tendance de la consommation alimentaire (locale et mondiale) ?

4. Est-ce que vous avez les fonds nécessaires pour maintenir un réseau ?

5. Quelle est l'échelle de l'organisation que vous souhaitez développer?

6. Votre organisation doit-elle croître en nombre ou être consolidée?

7. Y a-t-il d'autres personnes qui veulent se joindre à l'organisation ?

8. Quels sont les moyens que vous pouvez utiliser pour la communication interne avec les membres (téléphone portable, applications de chat, Facebook, etc.) ?

\subsubsection{Choisir la bonne taille}

Tout le monde ne s'intègre pas dans un groupe donné. En tant que producteur, identifiez les membres du groupe qui partagent des objectifs similaires et collaborez avec eux. Cela améliorera le succès et la croissance collectifs. Par exemple, une personne retraitée élévant de la volaille pour sa propre consommation n'a pas nécessairement le même intérêt et le même désir de croissance qu'un jeune couple qui souhaite instaurer une exploitation professionnelle. Beaucoup de déceptions et de conflits peuvent être évités si les membres du groupe partagent le même objectif. 


\section{La croissance d'un modèle de réseau (Colombie)}

Le modèle de réseau de Familia de la Tierra (FdT) leur permet de répondre aux dynamiques socio-économiques locales, tels que: les nouveaux marchés, les projets de recherche impliquant plus de familles, les innovations de produits impliquant l'action de plus de familles, les nouveaux produits cultivés et/ou en demande.

Actuellement, 25 familles rurales à Bogota participent au réseau. Ils cultivent des variétés traditionnelles de pommes de terre et des semences andines, au service de 12 restaurants, 3 hôtels et 10 magasins à Bogota, Cali et Medellin. FdT joint ses efforts avec ceux de trois organisations partenaires opérant dans les zones rurales de Cundinamarca, qui comprennent environ 118 familles au total. Elle travaille également avec la Faculté des sciences agraires de l'Université nationale de Colombie, avec des professeurs du SENA (Service National d'Apprentissage), avec des cuisiniers indépendants, avec des professeurs de gastronomie ainsi qu'avec des étudiants universitaires qui étudient le système alimentaire tel que développé par FdT.

FdT a évolué au rythme du marché et de l'économie locale axée sur l'agriculture biologique, permettant d'impliquer de plus en plus d'acteurs et pas seulement des producteurs biologiques. Elle a surtout été marquée par la demande croissante des hôtels et des restaurants pour des aliments sains et locaux.

Source: Oscar Nieto, Familia de la Tierra, Colombie

\subsubsection{Votre groupe est-il prêt à accueillir de nouveaux membres?}

Le groupe doit reconnaitre que des nouveaux membres peuvent créer de l'animosité, quitter ou faire partir les anciens. Le groupe doit être prêt à affronter un niveau d'inconfort minime qui peut accompagner l'introduction de nouveaux membres, ou la perte d'anciens.

\subsubsection{Comment gérer les nouveaux membres}

Le suivi avec les nouveaux membres est utile pour mieux comprendre les raisons de désistement et la dynamique du groupe. Si les nouveaux membres aiment le groupe, informez-les de la prochaine réunion. Si les membres partent et ne sont pas remplacés par de nouveaux, il sera difficile de maintenir la croissance de votre organisation.

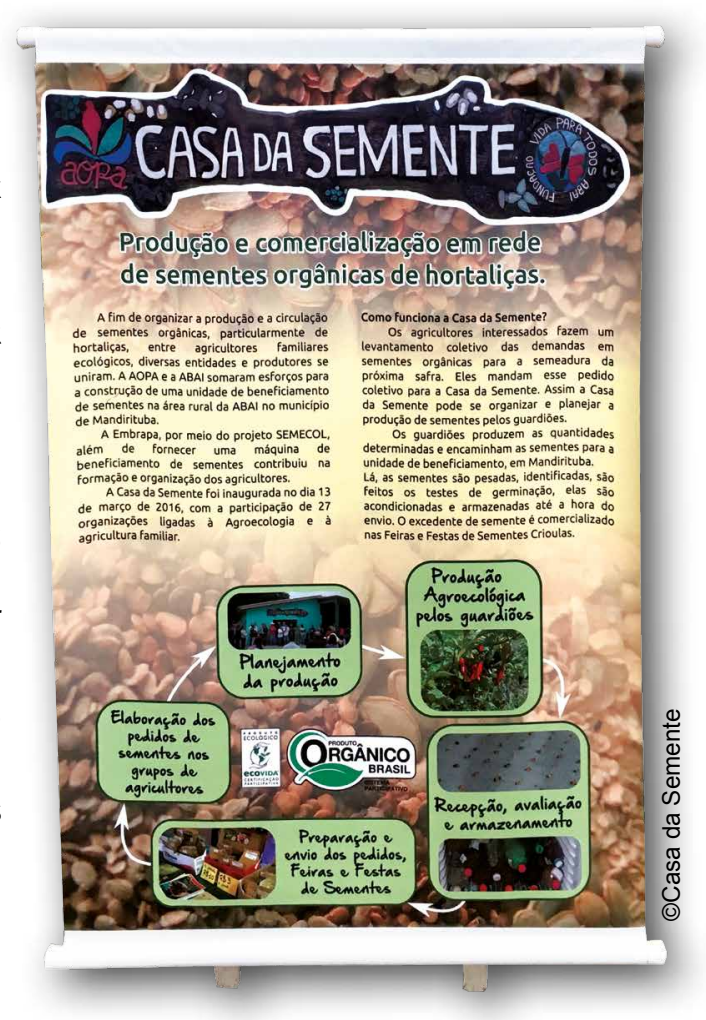




\subsection{Comment équilibrer objectifs et capacités?}

Affirmez vos objectifs et reconnaissez vos capacités. Utilisez les quelques astuces suivantes pour trouver cet équilibre:

- La transparence est un facteur clé de succès pour toute entreprise collective.

- L'équité est cruciale.

- Évitez un sentiment d'inégalité et de frustration parmi les membres plus diligents en compensant leur temps.

\subsubsection{Prise de décision collective sur des questions clés}

Mise à part le statut qui peut en résulter, un meilleur équilibre de pouvoir signifie des processus de prise de décision collectifs. Ces derniers abordent des sujets importants comme la fixation des prix. Cela peut être un facteur majeur d'autonomisation et de sentiment d'appropriation pour tous ceux qui participent à l'opération.

N'oubliez pas que les groupes évoluent constamment et par conséquent, ils essaient d'établir des structures souples afin de pouvoir réviser les règles et les priorités si nécessaire. Confier des différentes tâches à différentes personnes aide à répartir le pouvoir et la voix et développe un système de gouvernance plus "naturellement" structuré.

\subsubsection{Dynamique}

Les objectifs communs ne sont pas fixes. À mesure que l'organisation évolue et grandit, ils doivent être régulièrement et collectivement réévalués. L'outil d'analyse FFPM peut être utilisé pour évaluer la performance d'une organisation par rapport à ses objectifs. FFPM est un acronyme pour les forces et les faiblesses internes de l'organisation et la façon dont elle fonctionne. Les opportunités et les menaces sont des pressions externes qui conditionnent le fonctionnement de l'organisation.

- Créez un tableau de format 2x2 et remplissez les FFPM auxquels votre organisation est confrontée.

- Vous pouvez ensuite déterminer si vous avez des forces et des opportunités qui peuvent compenser les faiblesses et les menaces.

- Enfin, priorisez les problèmes urgents en mobilisant vos forces et vos opportunités. Mettez en place des plans de renforcement de compétences pour réduire les faiblesses et éviter les menaces. 


\section{COMMENT ASSURER L'AVENIR DE L'ORGANISATION ? IMPLIQUEZ LES JEUNES!}

\subsection{Créer des emplois attrayants pour les jeunes}

Les jeunes ne sont pas toujours attirés par l'agriculture. Pour autant, cela ne signifie pas que tous les emplois dans un système alimentaire durable ne sont pas attrayants.

La transformation des aliments durables est un exemple d'emploi des jeunes dans l'agriculture. Le recours croissant aux technologies de l'information, à la communication par smartphone, aux panneaux solaires, aux transports et aux technologies de surveillance électronique, incite de plus en plus de jeunes diplômés à devenir des entrepreneurs agricoles.

\section{罗 \\ Impliquer les jeunes et les femmes dans les initiatives agroécologiques durables (Brésil)}

Afin de saisir plus de valeur ajoutée et de créer des emplois, le Réseau Ecovida a réussi à développer plusieurs petites usines de transformation agroécologique au cours des dernières années. Alors qu'il y avait environ dix de ces usines en 2000, il y en a à présent plus de 150 , dont 90 pour cent qui sont gérées par des femmes et des jeunes. Ces derniers bénéficient de nombreux avantages issus de ces nouveaux emplois:

- une confiance en soi et une autonomie plus prononcées;

- la création de nouvelles lignes de crédit dans les banques locales conçues spécifiquement pour les jeunes et les femmes de milieux rurales;

- des nouvelle sources de revenus et possibilités d'accès aux marchés agroécologiques et aux programmes de marchés publics (aujourd'hui, un jeune homme ou femme qui travaille dans une usine de transformation agroécologique et qui vend aux programmes institutionnels peut gagner jusqu'à 7700 USD par an);

- la permanence des jeunes dans l'agriculture et dans les zones rurales;

- la création de nouvelles connaissances techniques liées à la transformation des aliments.

Source: José Antonio da Silva Marfil, Ecovida, Brési

\subsubsection{L’éducation communautaire}

L'éducation communautaire est un outil clé pour impliquer les générations futures dans votre organisation. Réfléchissez aux possibilités d'inclure les enfants et les adolescents dans votre programmation, en sachant que cela s'accompagne souvent d'exigences légales spécifiques.

N'oubliez pas de vérifier les définitions légales du travail, de l'apprentissage et du bénévolat dans votre pays et de les respecter lorsque vous élaborez votre programme. 


\section{LISTE DE VÉRIFICATION}

1. Avez-vous vérifié les exigences légales d'immatriculation dans votre pays ?

2. Avez-vous utilisé un processus participatif pour définir la mission de votre groupe ?

3. Avez-vous trouvé le modèle qui correspond le mieux à votre mission et à vos valeurs ?

4. Avez-vous adapté le modèle à votre contexte local ?

5. Avez-vous réparti les responsabilités entre les différents acteurs de votre groupe?

6. Avez-vous mis en place un système pour traiter les problèmes au fur et à mesure qu'ils se présentent?

7. Avez-vous enregistré votre groupe auprès des autorités ?

\section{ASTUCE 41}

Trouver et maintenir le bon équilibre

- Trouver le bon équilibre au sein de groupe et le maintenir n'est pas une tâche simple. Le partage des valeurs et des responsabilités fondamentales facilite ce processus.

- Bien que l'informalité présente des avantages, l'enregistrement formelle de votre initiative vous permettra d'accéder aux sources de financement public et privé.

- Différentes formes d'organisation sont requises à différents stades du cycle de vie de votre initiative. Maintenez vos structures aussi flexibles que possible afin de gérer efficacement les opportunités et les menaces émergentes.

- Changer l'échelle des opérations fait naturellement partie du développement organisationnel. Assurez-vous que votre groupe est d'accord sur la meilleure façon de croître ou d'élargir ses activités afin de pouvoir maintenir la cohésion collective à long terme.

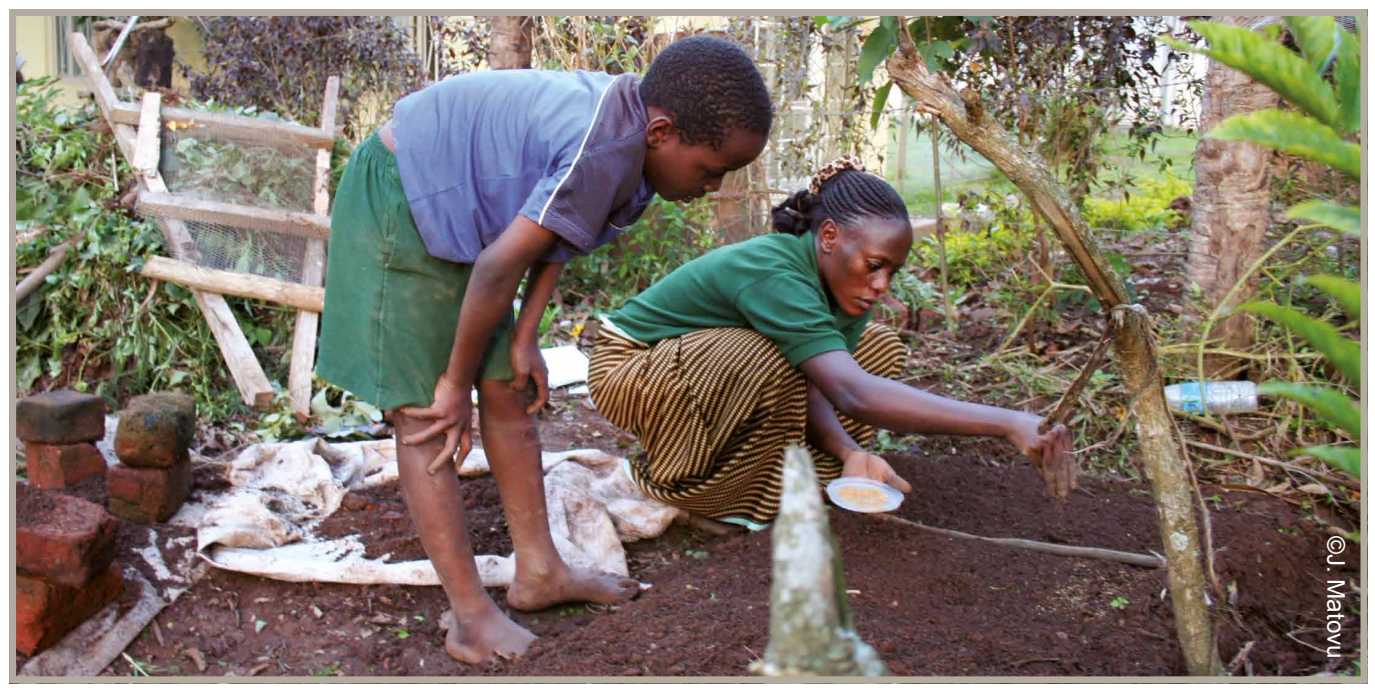




\section{LE CHEMIN D’APPRENTISSAGE, OÙ ALLER ENSUITE?}
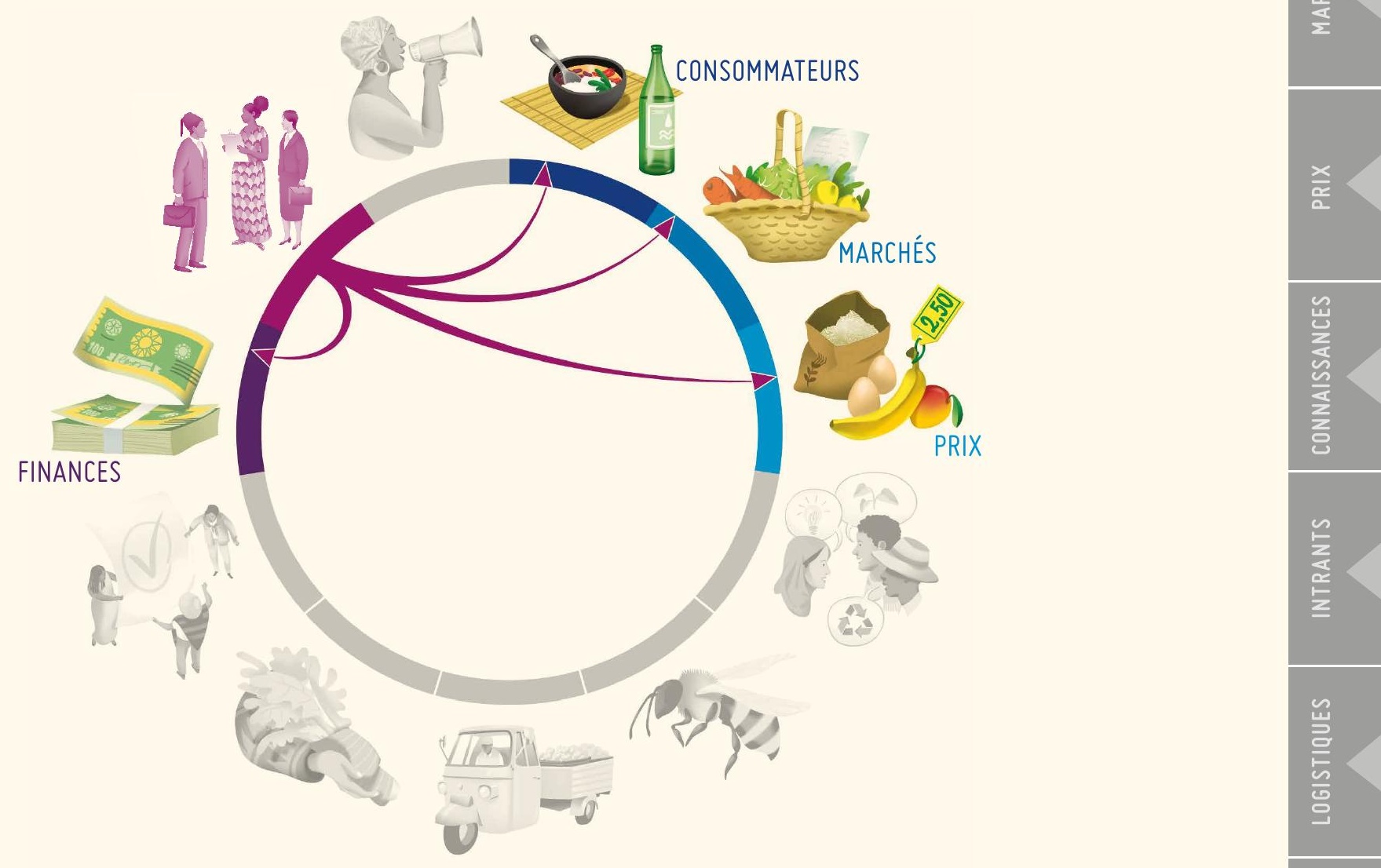

- Pour en savoir plus sur l'élaboration d'une AMAP, consultez le Chapitre 2: Connaître vos marchés.

- Est-ce que votre travail comporte un volet sur l'éducation des jeunes en milieu rural ? Retournez au Chapitre 1: Attirer les clients et les fidéliser pour voir d'autres exemples.

- Vous souhaitez mettre en place un comité d'établissement des prix comme moyen d'intégrer des processus démocratiques dans votre organisation ? Passez au Chapitre 3: Trouver le «bon» prix.

- Maintenant que votre initiative collective est enregistrée, avez-vous les fonds d'exploitation ou d'investissement nécessaires ? Retournez au Chapitre 9: Les financements innovants pour vous faire une idée.

Ou peut-être un autre chapitre vous vient à l'esprit ? Allez-y ! 



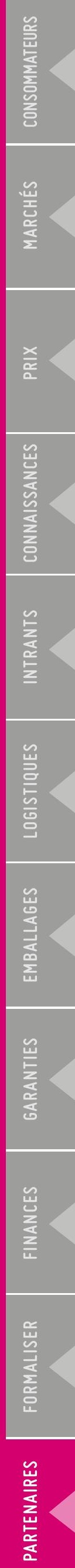




\section{POURQUOI EST-CE IMPORTANT?}

L'innovation est le processus d'apporter de nouvelles idées aux utilisateurs, à travers de nouvelles structures organisationnelles et de nouvelles relations collaboratives. Les partenaires et les plaidoyers peuvent aider à cultiver ces nouveaux liens et à susciter l'intérêt des consommateurs et des producteurs.

Un partenariat signifie construire des réseaux, des accords, des alliances, des collaborations et des coalitions pour échanger de l'information et faire parler les gens de votre innovation. Il doit être avantageux pour tous les partenaires.

Un plaidoyer s'agit de faire appel à de l'aide pour défendre une cause ou un groupe de personnes. Il est nécessaire pour empêcher qu'une innovation ne soit appropriée par une autre organisation ou entreprise privée, évinçant ses praticiens réels et profitant d'une initiative qui a du succès. Cela pourrait être fait par n'importe qui, surtout les grandes entreprises ayant une grande expérience avec des lobbyistes spécialisés, qui peuvent prétendre vous représenter.

\section{ASTUCE 42}

\section{Convaincre les parties prenantes}

1. Les décideurs politiques (le gouvernement) ont tendance à écouter davantage les groupes organisés. Institutionnaliser un système alimentaire innovant et durable est un défi majeur. Une fois institutionnalisée, une innovation perd sa nouveauté et les innovateurs risquent de perdre le contrôle de leur système. Afin de diminuer ce risque, il est nécessaire de s'engager sur le long terme avec le gouvernement et les acteurs du marché. Les coalitions et les alliances entre les acteurs d'un secteur (associations interprofessionnelles) peuvent fournir un mécanisme de soutien à long terme pour un plaidoyer. D'après notre expérience, les décideurs politiques écoutent ces groupes, répondent à leurs recommandations ou à leurs demandes, ou négocient un accord pour apaiser les tensions sociales potentielles. Dans certains pays, les groupes organisés sont obligés d'aider les décideurs politiques à rédiger des directives précises pour la mise en œuvre de leurs innovations.

2. Les données/informations provenant de sources fiables (c.-à.-d. des leaders d'opinion, des chercheurs, scientifiques, des personnalités religieuses), crédibilisent votre initiative. Étant donné que les innovations remettent en question l'ordre des systèmes alimentaires existants, le soutien de ces sources est crucial pour démontrer l'efficacité d'une innovation (p. ex., en termes de rentabilité, d'avantages environnementaux et sociaux, de sécurité alimentaire).

3. Accéder à d'autres marchés et/ou à des marchés plus importants. Lorsqu'il y a une forte demande, la production augmente. Cette demande provient des points de vente établis et/ou traditionnels tels que les grandes chaînes de supermarchés, les restaurants et les magasins spécialisés. Toutefois, entrer sur ces marchés, signifie qu'il faut adhérer à certaines exigences qualitatives et quantitatives de l'offre, celles-ci étant généralement plus strictes que pour la vente directe. C'est pourquoi le soutien d'un réseau et le partenariat avec d'autres groupes d'agriculteurs ou transformateurs peut être utile. Parfois, une approbation ou une lettre d'approbation d'un réseau ou d'un partenaire respecté suffit pour convaincre, par exemple, le responsable de la logistique ou du produit de s'approvisionner auprès d'un nouveau fournisseur. 
4. Les partenariats et les réseaux permettent aux innovateurs de mobiliser des fonds. Il est essentiel d'avoir suffisamment d'argent pour opérer. Dans la plupart des cas, les innovations sont autofinancées, bien que cela risque de limiter leur capacité d'expansion. Les partenaires de financement locaux et internationaux exigent généralement la preuve d'un profil professionnel satisfaisant (c.-à-d. trois années d'activités, le nombre de membres, la portée des activités et les répercussions sociales) pour pouvoir bénéficier d'une aide financière. Le soutien d'un réseau peut améliorer l'accès à des sources de financement externes.

5. La confiance est cruciale pour le partenariat. Les partenariats reposent sur des objectifs communs et une vision commune qui sont créés par un engagement réel et soutenu par tous les partenaires, c'est-à-dire uniquement au moyen de relations de confiance.

\section{SPG: Réseautage, partenariat et plaidoyer (Philippines)}

Le modèle du système participatif de garantie s'est introduit aux Philippines en 2004 avec le SPG MASIPAG Farmers Guarantee System (MFGS), établi par le réseau de producteurschercheurs Magsasaka at Siyentista Para sa Pag-unlad ng Pagsasaka (MASIPAG). Le MASIPAG a aidé à organiser et à soutenir les groupes d'agriculteurs responsables de l'implémenter. En 2012, la province de Quezon était à la recherche d'un système de certification adapté aux petits producteurs biologiques locaux vendant sur le marché hebdomadaire de la capitale provinciale. Elle a donc adapté le SPG au contexte local en établissant un partenariat avec MASIPAG pour former les producteurs biologiques de la province. L'expérience du SPG de Quezon a inspiré les décideurs politiques d'autres provinces à utiliser le modèle du SPG au lieu de la certification par une tierce partie pour leurs propres marchés locaux.

En 2013, le SPG Pilipinas a été officiellement créé comme un réseau d'initiatives de SPG à travers le pays. Depuis sa création, il a établi un partenariat avec l'IFOAM pour que le gouvernement national reconnaisse officiellement le SPG. Cette activité de plaidoyer a abouti à un moratoire de trois ans sur la mise en œuvre de la Loi de la république (RA) 10068, celle-ci étant la réglementation nationale sur les produits biologiques et ne reconnait que la certification par un tiers pour la certification biologique. Ce moratoire a permis aux SPG dans tout le pays de se renforcer et de se développer.

En 2017, à la fin du moratoire, le Département de l'agriculture a, par l'intermédiaire du Bureau des normes pour l'agriculture et la pêche (BAFS), invité le SPG Pilipinas et d'autres membres du réseau au Groupe de travail technique (GTT) à élaborer des directives visant à établir un SPG national, préparant ainsi une révision du RA10068 qui reconnaisse officiellement le SPG. En juin 2017, la version finale des directives a été approuvée par les membres du GTT et servira de référence pour les révisions juridiques.

Source: Carmen Cabling, SPG de Quezon 


\section{COMMENT CONSTRUIRE DES PARTENARIATS?}

Les partenariats avec d'autres organisations peuvent prendre la forme de relations informelles par le biais desquelles les membres collaborent temporairement pour atteindre un but précis. Dans d'autres cas, les partenariats peuvent durer longtemps et peuvent éventuellement devenir de nouvelles organisations dotées de leur propre structure de gestion et de gouvernance. Aucune règle ne stipule qu'un partenariat à court terme ne peut pas devenir un partenariat à long terme, ou vice versa.

Avant toute chose, il est important de se poser deux questions:

- «Quels intérêts communs les partenaires potentiels ont-ils avec mon initiative ?» Deux (ou plusieurs) entités ou individus décideront de devenir partenaires une fois qu'ils auront reconnu leurs intérêts communs.

- «Quel type de subsidiarité y a-t-il avec les partenaires potentiels ?» Ici, la subsidiarité est sous-entendue comme le principe que les partenariats aideront à résoudre les problèmes que les organisations ne peuvent pas résoudre localement.

Une fois que vous avez répondu à ces deux questions, identifiez vos principales activités de partenariat. Celles-ci influencent la sélection de partenaires potentiels et elles incluent en général:

1. Le partage d'expériences: Les besoin de soutien mutuel et de partage d'expériences sont la raison la plus évidente pour laquelle différentes initiatives innovantes s'associent. Il est difficile d'imaginer un système alimentaire durable qui se développerait de manière isolée, séparément des autres initiatives alimentaires locales. Les nouveaux agriculteurs ont besoin que les agriculteurs expérimentés partagent leur expertise; les logisticiens des chaînes d'approvisionnement courtes doivent apprendre des systèmes de distribution préexistants; les groupes de consommateurs ont besoin de cadres organisationnels et d'outils pratiques pour commencer à travailler ensemble. Pour toutes ces raisons, la réponse à la question «avec qui établir un partenariat ?» est: un collègue agriculteur, un collègue consommateur, un collègue logisticien, un collègue détaillant et un collègue transformateur.

2. La communication externe: elle s'attache à toucher un public plus large». L'un des défis initiaux est le recrutement de membres ou de consommateurs. Par exemple, un festival de produits locaux basé sur une cuisine traditionnelle serait beaucoup plus efficace que toute action mise en place par un groupe solitaire. L'organisation d'un tel festival serait le résultat d'un partenariat ou d'un organisme-cadre, déjà bien informé sur les groupes cibles et les moyens de les atteindre. S'il n'y a pas de réseau aussi actif dans la région, les ONG ou les autorités locales pourraient organiser ce type d'activité annuellement.

3. Le plaidoyer: certains sujets ne peuvent pas être traitées séparément par un seul partenariat. C'est le cas pour des questions juridiques complexes telles que l'accès à la terre, qui est un facteur clé des systèmes alimentaires durables. Des alliances solides, généralement sur de plus longues périodes, sont nécessaires pour que le plaidoyer soit efficace. Afin d'influencer les décideurs et les cadres juridiques, la coopération avec les organisations de plaidoyer spécialisées pourrait être plus efficace que des liens directs avec des représentants élus.

4. La recherche: elle accompagne le plaidoyer. Très tôt dans leurs démarches, plusieurs pionniers des systèmes alimentaires durables ont décidé d'établir un partenariat avec une équipe de 
recherche. Les principaux objectifs sont de mener des recherches afin de soutenir la crédibilité de l'innovation sous différents points de vue et de cultiver de nouvelles connaissances sur la durabilité. Les preuves scientifiques produisent des données importantes servant d'appui pour les campagnes de plaidoyer. Cependant, la recherche ne peut pas servir uniquement ces objectifs. Il est essentiel d'étudier systématiquement les problèmes auxquels sont confrontés les acteurs du système alimentaire durable afin de trouver des solutions.

5. L'accès aux marchés: Sans un accès stable aux marchés, aucune entreprise ne peut être économiquement durable. L'accès aux marchés repose sur des partenariats et chaque transaction peut être considérée comme un partenariat. Afin de faire durer ces partenariats, il est nécessaire de mettre en place des chaînes d'approvisionnement fiables avec des objectifs à long terme. Les modèles basés sur la vente directe peuvent garantir des partenariats durables entre producteurs et consommateurs. Toutefois, il existe d'autres moyens de garantir l'accès aux marchés, y compris ceux qui reposent sur des intermédiaires transparents. Il est important de se rappeler que les échanges dépendent de la confiance.

6. La finance: certains partenariats sont créés uniquement pour mobiliser des fonds, tels que la collecte de fonds pour le plaidoyer, pour des opérations ou pour des investissements. Selon l'usage prévu des fonds, il est utile d'établir des partenariats avec différentes organisations.

\section{La campagne de consommateurs ¡Que Rico Es ! (Équateur)}

En 2009, le Colectivo Agroecológico d'Équateur et le Mouvement pour une économie solidaire et sociale ont lancé une campagne ouverte et citoyenne intitulée Qué rico es comer sano y de nuestra tierra (www.QueRicoEs.org). L'initiative relie les consommateurs et a pour objectif principal de lutter contre l'omniprésence des aliments industrialisés. Pour ce faire, elle crée des marchés pour les produits agroécologiques des agriculteurs familiaux, tout en améliorant la santé et la qualité de vie des populations rurales et urbaines. La responsabilité de la transition vers des systèmes alimentaires durables est donc confiée aux «mangeurs».

Inspiré par le potentiel de mobiliser le «consommateur-citoyen» en une force politique, le Colectivo Agroecológico a lancé, en octobre 2014, une nouvelle stratégie nationale appelée les «250 000 familles». La campagne, toujours en cours, s'appuie sur la notion de «consommation responsable» et vise à mobiliser une masse critique de «consommateurs-citoyens» pour soutenir les efforts de politiques publiques en faveur d'une «alimentation agroécologique saine pour une société saine». Le collectif demande aux consommateurs d'investir au moins la moitié du budget mensuel alimentaire/de boissons de leur famille dans les achats directs aux producteurs ou dans la vente de produits biologiques/ agroécologiques andins. L'objectif principal de la campagne est d'atteindre et d'assembler 250000 familles qui mangent de manière «savoureuse, saine et locale». Pour l'atteindre, plusieurs stratégies sont appliquées en mettant l'accent sur la visibilité, la connectivité et l'identité. Le Colectivo Agroecológio a organisé divers ateliers et des forums, élaboré un guide répertoriant tous les marchés de producteurs du pays, et développé un bulletin d'information bimensuel sur la consommation responsable.

Source: Ross Mary Borja, Fundación Ekorural, Équateur

Pour en savoir plus: http://www.fao.org/3/a-bs916e.pdf http://www.quericoes.org 


\section{ASTUCE 43}

\section{Établir des partenariats pour le plaidoyer}

1. Créer des partenariats volontaires: Avec de nombreuses stratégies de partenariat possibles, sélectionnez stratégiquement celles qui conviennent le mieux à votre organisation. Soyez très clair quant aux personnes avec qui vous souhaiter travailler (c.-à-d. les personnes qui partagent vos valeurs et/ou les mêmes objectifs) et ciblez spécifiquement ces personnes et ces groupes à travers différents modes de communication. Ceci vous aidera à mobiliser des ressources et à apporter des changements dans votre système alimentaire.

2. Élaborer une stratégie de plaidoyer: Faites un plan ! Une stratégie de plaidoyer aide tous ceux qui souhaitent s'impliquer à comprendre la situation, à analyser les acteurs, à déterminer comment le changement doit se produire et à fixer des objectifs précis. Une stratégie bien conçue permet d'aligner les objectifs clés à des points précis du cycle de vie du projet.

3. Impliquer les partenaires dans toutes les étapes du plaidoyer: Un engagement solidaire et actif, dès les premières phases du projet, permet d'élaborer des solutions et d'impliquer des personnes en dehors du groupe principal (par exemple la construction de relations externes avec les responsables de politiques gouvernementales et/ou les décideurs politiques).

4. Identifier les forces de tous les partenaires: La prise en compte des atouts de chaque partenaire maximise la dynamique des relations et les résultats du projet. Certains partenaires apportent leurs compétences financières, tandis que d'autres partagent leurs capacités de réseautage ou de réflexion stratégique. Tirez parti de ces forces et de ces intérêts pour assurer que tous les acteurs soient satisfaits et engagés. Certains partenaires sont naturellement plus actifs, tandis que d'autres ont besoin d'un peu plus d'encouragement... c'est normal ! Établir des partenariats stratégiques exige de la négociation, de la patience et une certaine flexibilité.

5. Établissez des liens innovants: Sortez des sentiers battus pour créer un réseau de partenariats unique. Différents outils s'appliquent pour différents contextes - identifiez les meilleurs et utilisez-les. Par exemple, organisez une conférence ou un congrès pour réunir les personnes intéressées, rédigez un article de journal pour une publication universitaire, ou participez à un atelier éducatif.

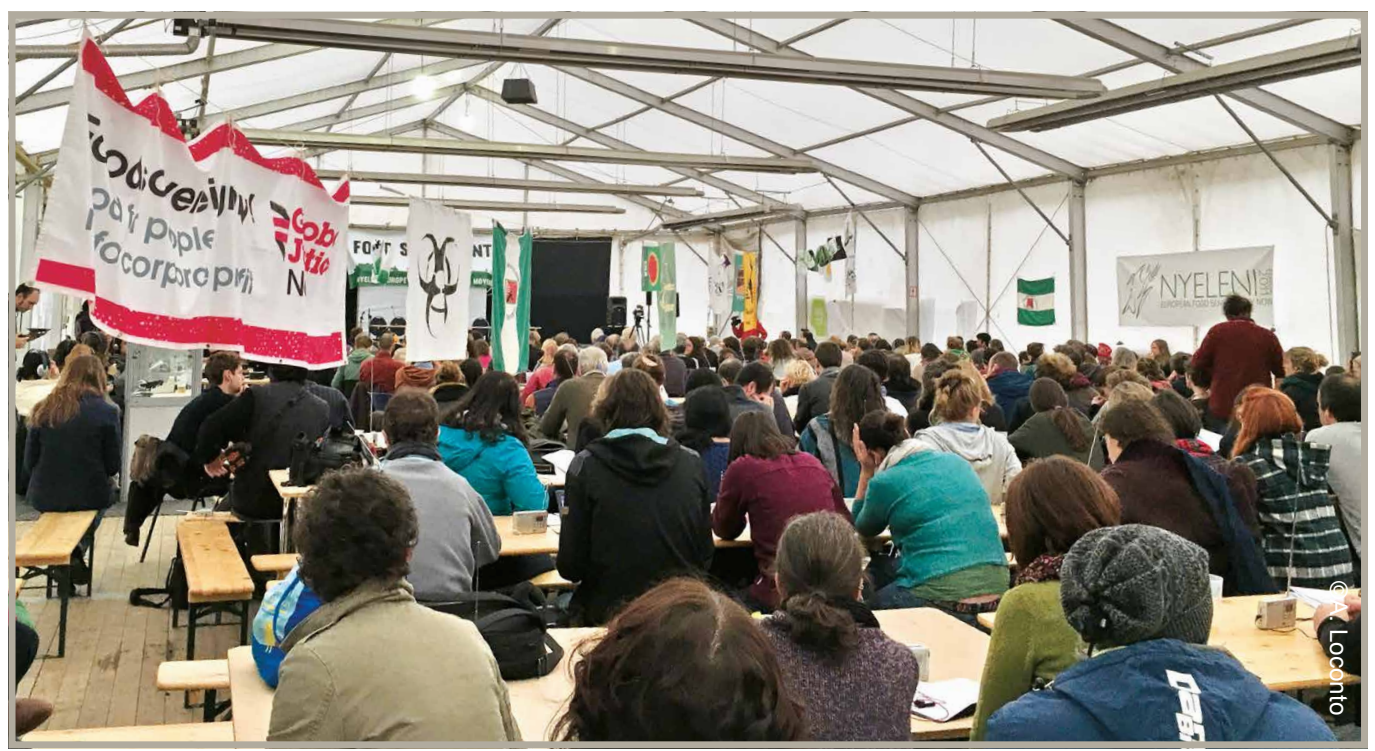




\section{QUI MOBILISER POUR LES PARTENARIATS ?}

\subsection{Autres acteurs du système alimentaire durable}

Obtenir le soutien d'autres acteurs du système alimentaire durable est un défi. S'il y a un sentiment de concurrence dans un petit marché de niche saturé, il est peu probable qu'une volonté de partager les succès, les échecs et les plans pour l'avenir soit présente. Au contraire, si un sentiment d'appartenance communautaire se construit par la création de liens sociaux, la collaboration devrait être plus facile.

Un partenariat entre les acteurs peut être établi:

a. à travers un mécanisme formel tel qu'un système participatif de garantie (nous avons constaté que le SPG est efficace pour fournir des garanties, mais qu'ils est aussi un outil pédagogique pour l'apprentissage collectif et la construction de liens sociaux).

b. à travers des réunions de partage d'expériences pour recueillir les nouvelles récentes de toutes les différentes opérations et pour discuter des doutes et des préoccupations (nous avons constaté que des réunions multilatérales régulières pour échanger sur des sujets communs sont utiles).

D'après notre expérience, les restaurants peuvent devenir d'excellents partenaires si leurs chefs recherchent des relations à long terme avec les fournisseurs. Bien entendu, il existe un risque que le producteur ou le commerçant devienne dépendant d'un seul marché pour toute sa vente. La diversification est fondamentale afin d'éviter de créer des partenariats fondés sur des relations de dépendance.

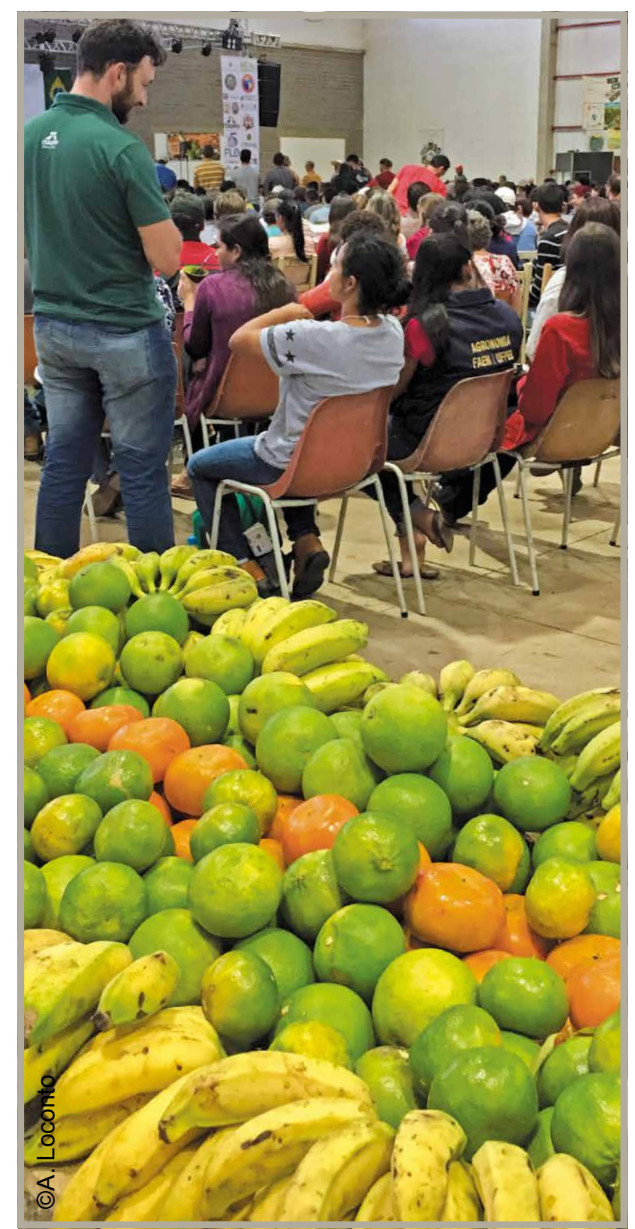




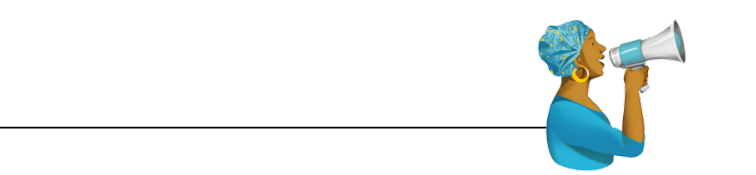

\section{Programmes de panier: créer des liens sociaux (Équateur)}

En Équateur, un partenariat a été établi entre l'association de consommateurs Canasta Comunitaria Utopia et l'organisation de producteurs Asociación de Productores Nueva Generación. Les représentants-dirigeants des deux groupes ont alors promu plusieurs stratégies pour renforcer la cohésion du groupe.

Le processus progressif était fondé sur la construction de différents types de relations de proximité. Une fois que les groupes se sont rencontrés et se sont familiarisés, un partenariat fondé sur des valeurs, des sentiments, des intérêts et des expériences partagées a été établi par le biais de visites d'échanges producteurs-consommateurs. Si ces derniers se connaissent, ils peuvent comprendre leurs processus et expériences respectifs. Après chaque livraison de panier, les producteurs ont participé aux réunions d'évaluation où les consommateurs partagent leurs impressions sur les produits livrés, la qualité, la quantité, ainsi que de tout autre sujet d'intérêt pour les deux groupes. Une autre stratégie consistait à donner un retour aux membres de l'association des producteurs lors des séances de travail. Ces membres ont également participé aux assemblées générales de Canasta Comunitaria Utopia pour fixer des prix, construire/renforcer un système de garantie et pour discuter des lois sur les semences, de la souveraineté alimentaire et de la consommation responsable.

Un espace de dialogue permet aux producteurs et aux consommateurs de se comprendre, de partager leurs expériences et, au fil du temps, d'établir et de tisser des relations de réciprocité, également appelé le capital social.

- La première étape consiste à créer une relation basée sur la confiance.

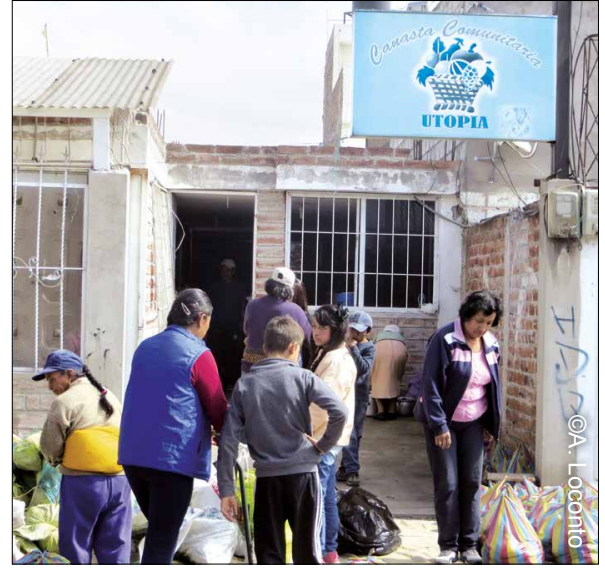

- La deuxième étape consiste à déterminer des principes ou des directives communs pour guider l'engagement des partenaires (p. ex., si des critiques ont lieu, des solutions doivent aussi être proposées).

- Troisièmement, les deux premières étapes doivent être renforcées et répandues. Les marchés et les livraisons de paniers doivent inclure d'autres activités que la vente et l'achat de produits pour d'approfondir les relations déjà établies, en créer de nouvelles, et favoriser les alliances, les interactions et les valeurs alimentaires.

Les trois étapes s'effectuent grâce à l'engagement des partenaires pour compléter les tâches requises (par exemple, prendre en charge la logistique, organiser une activité pratique) et pour représenter le groupe dans différents contextes (par exemple, gérer les relations avec les gouvernements locaux, une responsabilité exige parfois de défendre une certaine position). Les membres devraient ressentir que leur contribution, comme leurs connaissances et leurs expertises, est valorisée. Ceci aboutit souvent à des niveaux d'engagement et de soutien plus élevés.

Source: Ross Mary Borja, Fundación Ekorural 


\subsection{Organisations non gouvernementales (ONG)}

Certaines ONG ont construit une image publique positive dans leur pays d'opération. S'il est bien formé, un partenariat peut attirer l'attention publique et faire croître la visibilité de l'initiative. Les partenariats avec les ONG peuvent comprendre certains risques. Par exemple, l'ONG risque d'être perçue comme un concurrent par les acteurs du système alimentaire durable. Il est important pour une nouvelle ONG de ne pas remplacer les acteurs actuels de l'initiative de maintenir son rôle de soutien et pas plus.

\section{Les partenariats avec des ONG (Togo)}

95

Dans la capitale de Lomé, au Togo, le centre culturel Mytro Nunya a accueilli des activités d'AMAP pendant plusieurs années. Ce centre était un organisme sans but lucratif qui partageait des informations sur des sujets de solidarité internationale, qui avait une bibliothèque et qui organisait des conférences, des débats, des concerts et des représentations théâtrales. Le développement durable, la santé et les préoccupations environnementales étaient alignées sur les valeurs clés de la vision civique promues par le centre. Il semblait donc naturel pour le personnel du centre de contacter les producteurs biologiques locaux et d'établir un partenariat pour leurs consommateurs réguliers. Une collecte de paniers accompagnée d'un marché de producteurs a été mise en place régulièrement à Mytro Nunya, se déroulant simultanément avec d'autres activités: films, bibliothèque, conférences et débats.

Les consommateurs étaient attirés par ce type d'activité, puisqu'ils cherchaient à s'engager dans de nouveaux modèles de consommation responsable. Bien sûr, il n'y a pas qu'une seule solution pour rassembler les membres ou les consommateurs potentiels. Identifier les groupes d'intérêt les plus proches nécessite de la créativité.

Source: Judith Hitchman, URGENCI

\subsection{Décideurs politiques}

Les innovations attirent généralement l'attention des décideurs politiques: les représentants locaux ont intérêt à observer les activités des entrepreneurs sur leur territoire. Ils voient leur potentiel de création d'emplois locaux qui ne peuvent être délocalisés et sont enclins à soutenir des actions renforçant la cohésion communautaire et sociale. Bien entendu, certains d'entre eux seront plutôt attirés par la dimension environnementale du développement durable. Des liens étroits avec les décideurs politiques comportent également certains risques, tels que la «capture» de l'initiative durable par un parti politique, utilisée à ses propres fins. Il est donc nécessaire, dans tout partenariat de ce type, d'indiquer clairement le caractère apolitique du système innovant. La stratégie adoptée pour approcher les décideurs politiques peut être également risquée. Par exemple, si vous comptez sur une relation personnelle, tous vos efforts peuvent être perdus si un conflit personnel émerge ou si la personne démissionne de sa fonction politique. Tout comme dans vos pratiques agricoles et commerciales, la diversification des liens politiques rend l'initiative plus résiliente. 


\section{兽 Trouver et approcher les personnes pouvant influencer les décideurs politiques (Kenya)}

Le réseau PELUM Kenya a développé sa stratégie de plaidoyer pour la gestion écologique de l'utilisation du sol. Son objectif était de mettre en place une structure pour coordonner les actions de plaidoyer respectives des membres du réseau. En utilisant une approche fondée sur des données scientifiques à l'échelle des comtés et du pays, cette stratégie a abordé les défis principaux des communautés locales, tels que ceux liés à l'agriculture, à l'adaptation au changement climatique, à l'utilisation des terres, à l'allocation budgétaire pour une agriculture durable, et au renforcement des compétences.

En 2015, le réseau a fait pression pour inclure PELUM dans l'élaboration d'une politique agricole nationale. S'appuyant sur les organisations membres de PELUM et sur leurs contacts, des représentants de PELUM ont approché le Président de la Commission parlementaire de l'agriculture. Celui-ci a ensuite mobilisé quatre autres membres du
Parlement et a organisé un petit-déjeuner. Le Président du Comité de plaidoyer a également approché le Directeur des politiques du Ministère de l'agriculture, de l'élevage et de la pêche a également été contacté par qu'il avait rencontré dans des réunions auparavant. Avant ces réunions, PELUM a rédigé un compterendu/document pour exposer sa position, ses commentaires et ses contributions concernant la politique agricole nationale. Ce rapport a ensuite été partagé pendant les réunions, en plus des données soutenues par des chercheurs. Bien qu'il n'ait pas été facile de convaincre ces personnes clés rapidement, les représentants de PELUM faisaient un suivi régulier en envoyant des notes de compte-rendu et en organisant des réunions informelles, des séances d'information verbales et des appels téléphoniques. Au final, le Ministère de l'agriculture, de l'élevage et de la pêche a rajouté une clause dans la politique agricole qui reconnaît officiellement l'utilisation du savoir-faire et des connaissances traditionnelles agricoles.

Source Rosinah Mbenya, PELUM

\subsection{Chercheurs}

De plus en plus d'innovations sont identifiées à l'avance et suivies sur une longue période par une unité de recherche partenaire. Ceci est particulièrement vrai pour les nouvelles pratiques agricoles (par exemple, l'agriculture biologique, la permaculture). Les résultats scientifiques sont tout aussi cruciaux pour convaincre les agriculteurs et les décideurs d'améliorer leurs pratiques. Cependant, il y a certains risques à prendre en compte:

1. L'appropriation des connaissances peut bénéficier aux concurrents (c'est-à-dire le brevetage de la propriété intellectuelle par des acteurs malhonnêtes).

2. Les résultats de la recherche peuvent être défavorables et révéler des faiblesses dans votre initiatives, ce qui peut attirer de l'attention non-désirée.

Assurez-vous d'équilibrer l'honnêteté et la transparence avec les risques ci-dessus pour qu'un partenariat de recherche soit scientifiquement fiable, et que vous ne vous sentiez pas exploité ou trahi. Comme tout autre partenariat, établissez un rapport de confiance avec les chercheurs. 


\subsection{Secteur privé}

La plupart du temps, les partenariats avec d'autres entreprises tournent autour de besoins spécifiques (par exemple, la collaboration avec des designers pour la publicité ou l'emballage) ou de plans de développement sectoriel (par exemple, la collaboration avec d'autres agriculteurs, transformateurs, transporteurs, écoles de cuisine et détaillants). Le secteur privé est vaste et il peut y avoir des opportunités de partenariat avec des entreprises nationales ou internationales sur des campagnes de plaidoyer ou pour l'établissement de normes industrielles qui peuvent également bénéficier les acteurs du système alimentaire durable. Réfléchissez stratégiquement à la façon dont vous vous engagez avec les acteurs du secteur privé étant donné le risque de cooptation de votre message, de la propriété intellectuelle (comme les marques, les idées de produits) et du partage équitable des avantages (toutes les entreprises du secteur privé n'ont pas les mêmes modèles commerciaux; les marges et avantages seront probablement calculés différemment).

\section{ASTUCE 44}

Trouver des partenaires et des plaidoyers

- Tous les partenariats ne sont pas obligés d'être permanents. Certains peuvent s'orienter vers un objectif temporaire, tandis que d'autres peuvent s'établir à long terme.

- Choisissez judicieusement vos partenaires, quel que soit leur secteur, et assurez-vous de partager les mêmes objectifs.

- Diversifiez vos partenaires en fonction de vos sujets d'intérêt. Cependant, essayez d'éviter la surcharge de partenariats...cela peut vous faire oublier votre propre mission !

- Le plaidoyer est un partenariat. Il est donc fondamental d'institutionnaliser le changement que vous apportez à votre système alimentaire. Par contre, faites attention à vos partenaires...Assurez-vous qu'ils défendent votre cause de manière crédible !

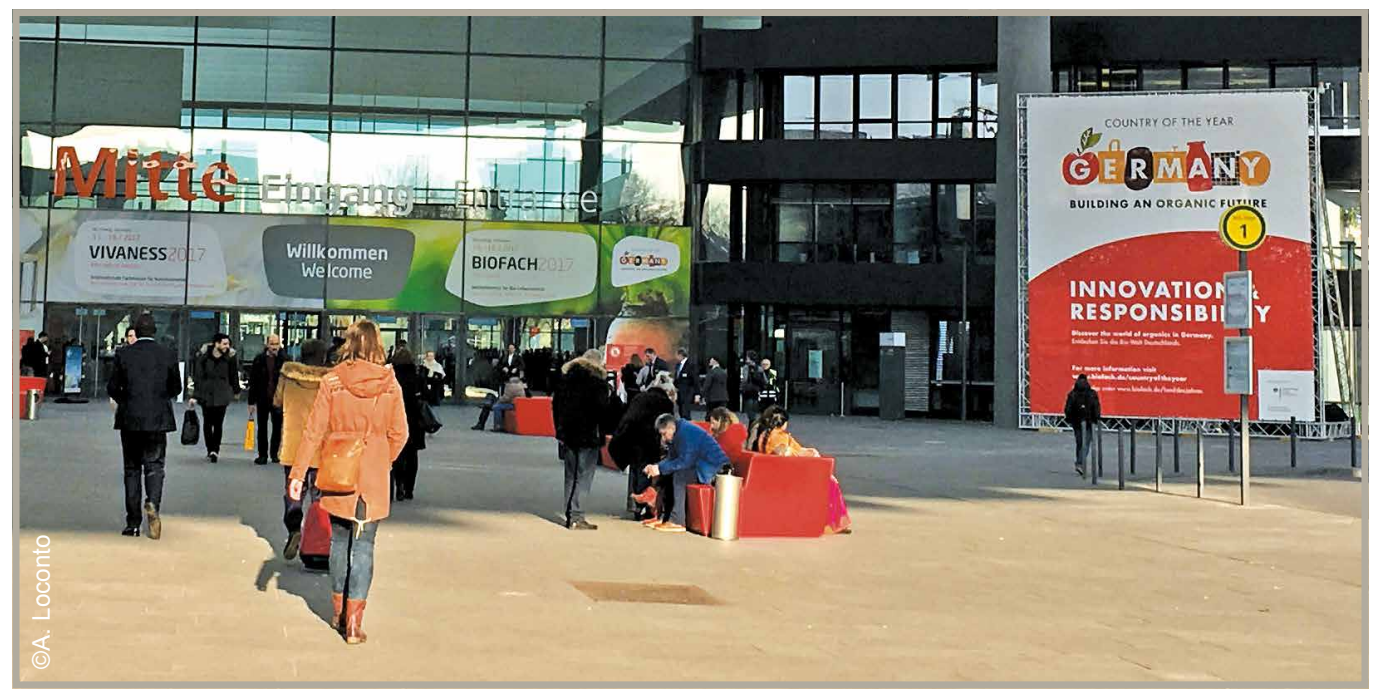




\section{LE CHEMIN D'APPRENTISSAGE, OÙ ALLER ENSUITE?}

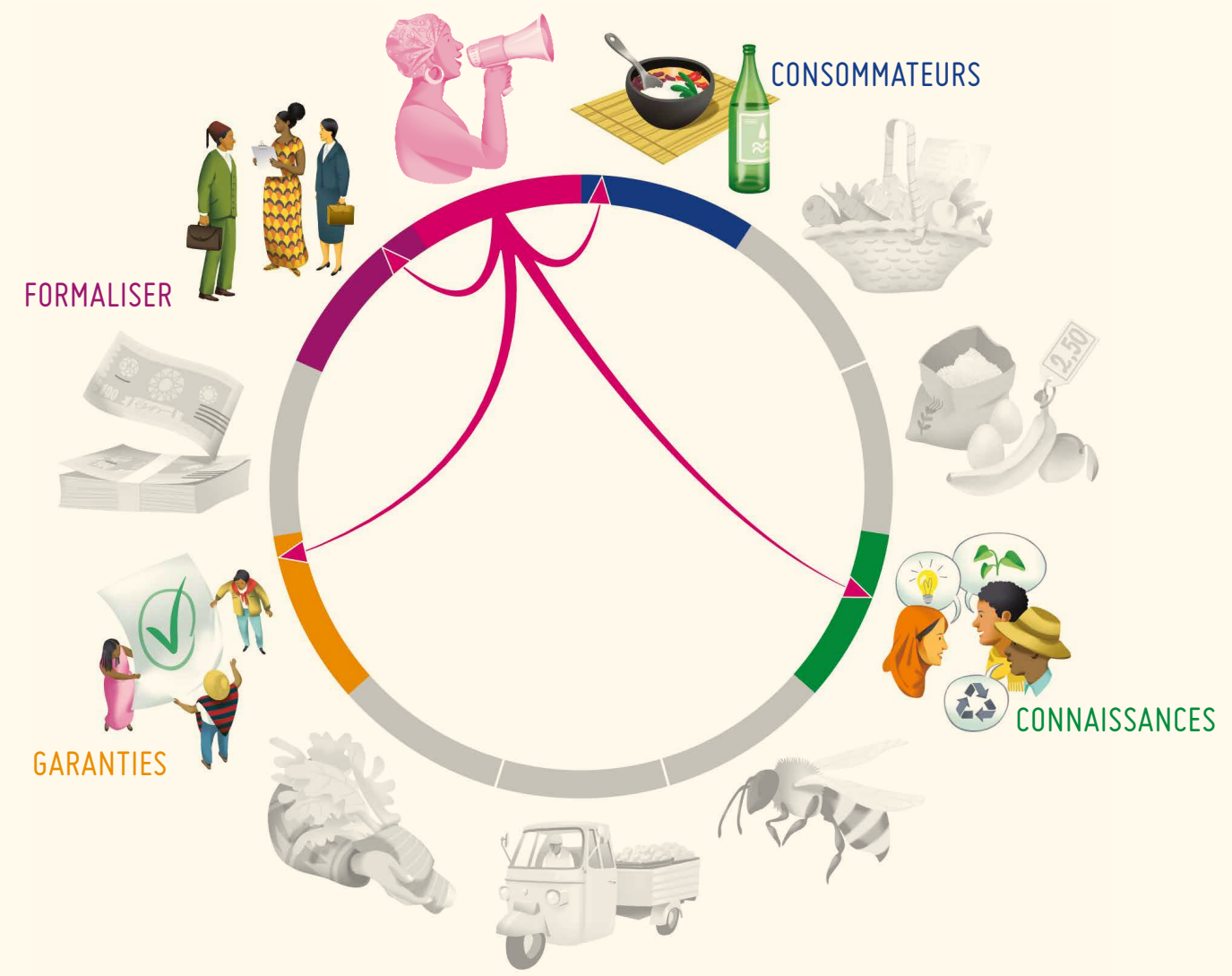

- Vous voulez essayer d'établir des partenariats avec des groupes de consommateurs? Découvrez ce qui les intéresse en consultant le Chapitre 1: Attirer les clients et les fidéliser.

- Vous cherchez des exemples de partenariats avec des chercheurs ? Retournez au Chapitre 4: Partager et co-créer des connaissances pour une production durable.

- Avez-vous besoin d'améliorer votre initiative avant d'essayer d'établir un partenariat? Retournez au Chapitre 10: Formaliser votre travail collectif.

- Vous aimerez utiliser un SPG pour établir un partenariat ? Pour en savoir plus sur ce sujet, consultez le Chapitre 8: Garantir la durabilité.

Ou peut-être vous voulez revenir à un chapitre que vous avez sauté ? Allez-y ! 



\section{RÉFÉRENCES}

Bashford, Jade, Kathleen Cross, Wolfgang Eichinger, Andreas Georgakakis, Morgane Iserte, Fabian Kern, Daniel Lešinský, et al. 2013. European Handbook on Community Supported Agriculture. Sharing experiences. Vienna. 21 pp. (également disponible à http://urgenci.net/wp-content/ uploads/2015/03/CSA4EUrope_Handbook.pdf).

Brondizio, ES, J Settele, S Díaz, \&HT Ngo. 2019. Global assessment report on biodiversity and ecosystem services of the Intergovernmental Science-Policy Platform on Biodiversity and Ecosystem Services. Bonn, DE. 1700 pp. (également disponible à https://ipbes.net/global-assessment).

Crutzen, Paul J. 2006. The "Anthropocene". In Eckart Ehlers and Thomas Krafft, eds., Earth System Science in the Anthropocene, 13-18. Berlin, Heidelberg, Springer Berlin Heidelberg.

FAO. 2003. Environmental and social standards, certification and labelling for cash crops. Rome. 120 pp. (également disponible à http://www.fao.org/3/a-y5136e.pdf).

FAO. 2007. Agro-industrial supply chain management: concepts and applications. Rome. $71 \mathrm{pp}$. (également disponible à http://www.fao.org/3/a-a1369e.pdf).

FAO. 2012. World agriculture towards 2030/2050: the 2012 revision. Rome. 154 pp. (également disponible à http://www.fao.org/3/a-ap106e.pdf).

FAO. 2014. The State of Food and Agriculture. Innovation in Family Farming. Rome. 161 pp. (également disponible à http://www.fao.org/3/a-i4040e.pdf).

FAO. 2016a. Innovative markets for sustainable agriculture: How innovations in market institutions encourage sustainable agriculture in developing countries. Rome. 390 pp. (également disponible à http:/ / www.fao.org/3/a-i5907e.pdf).

FAO. 2016b. Traceability: a management tool for business and governments. Rome. 68 pp. (également disponible à http://www.fao.org/3/a-i6134e.pdf).

FAO. 2017. Full cost accounting. [online]. Food and Agriculture Organization of the United Nations. [page web consultée le 13 mars 2020]. http://www.fao.org/nr/sustainability/full-cost-accounting/en/

FAO. 2018a. The 10 elements of agroecology guiding the transition to sustainable food and agricultural systems. Rome. 15 pp. (également disponible à http://www.fao.org/3/19037EN/ i9037en.pdf).

FAO. 2018b. Constructing markets for agroecology. An analysis of diverse options for marketing products from agroecology. Rome. 214 pp. (également disponible à http://www.fao.org/3/i8605en/ I8605EN.pdf). 
FAO. 2018c. FAO's work on agricultural innovation. Sowing the seeds of transformation to achieve the SDGs. Rome. 20 pp. (également disponible à http://www.fao.org/3/ca2460en/ca2460en.pdf).

FAO. 2018d. The International Symposium on Agricultural Innovation for Family Farmers. [en ligne]. Food and Agriculture Organization of the United Nations. [page web consultée le 13 mars 2020]. http://www.fao.org/about/meetings/agricultural-innovation-family-farmers-symposium/about/en.

FAO, IFAD, UNICEF, WFP, \&WHO. 2017. The State of Food Security and Nutrition in the World. Building resilience for peace and food security. Rome, Food and Agriculture Organization of the United Nations. 132 pp. (également disponible à http://www.fao.org/3/a-I7695e.pdf).

Fressoz, Jean-Baptiste, \&Christophe Bonneuil. 2016. L'Événement anthropocène. La Terre, l'histoire et nous: La Terre, l'histoire et nous. Paris, Points. 334 pp.

Gibson-Graham, Julie Katherine 2008. Diverse economies: performative practices for 'other worlds'. Progress in Human Geography 32 (5): 613-632.

HLPE. 2014. Food losses and waste in the context of sustainable food systems. A report by the High Level Panel of Experts on Food Security and Nutrition of the Committee on World Food Security. Rome. 8 pp. (également disponible à http://www.fao.org/3/a-av037e.pdf).

IFOAM. 2019. Definition of Participatory Guarantee Systems. [en ligne]. International Federation of Organic Agriculture Movements. [page web consultée le 13 mars 2020]. https://www.ifoam.bio/ sites/default/files/pgs_definition_in_different_languages.pdf.

ISO. 2012. Conformity assessment -- Requirements for bodies certifying products, processes and services. ISO/IEC Guide 17065. Geneva. 27 pp. (également disponible à https://www.iso.org/ standard/46568.html).

Kamaraj, R. 2015. POLICY NOTE 2015-2016. Demand No. 13. Tamil Nadu. 30 pp. (également disponible à http://cms.tn.gov.in/sites/default/files/documents/food_e_pn_2015_16.pdf).

Kanie, Norichika, \&,Frank Biermann. 2017. Governing through goals: Sustainable development goals as governance innovation. Earth System Governance. Cambridge, MA, MIT Press. 333 pp.

Ostrom, Marcia, Kathryn De Master, Egon Noe, \& Markus Schermer. 2017. Values-based Food Chains from a Transatlantic Perspective: Exploring a Middle Tier of Agri-food System Development. International Journal of Sociology of Agriculture \& Food 24 (1): 1-14.

Reinecke, Juliane, \& Ansari Shaz. 2015. What Is a "Fair" Price? Ethics as Sensemaking. Organization Science 26 (3): 867-888. https://ideas.repec.org/a/inm/ororsc/v26y2015i3p867-888.html.

Rockstrom, Johan, Will Steffen, Kevin Noone, Asa Persson, F. Stuart Chapin, Eric F. Lambin, Timothy M. Lenton, et al. 2009. A safe operating space for humanity. Nature 461 (7263): 472-475. http:// dx.doi.org/10.1038/461472a.

Swensson, Luana F. Joppert. 2015. Institutional Procurement of Food from Smallholder Farmers. The Case of Brazil. Food and Agriculture Organization of the United Nations (Rome). 
TNAU. 2015. Cost of Cultivation: Paddy (2014-15). [en ligne]. TNAU Agritech Portal. [page web consultée le 13 mars 2020]. http://agritech.tnau.ac.in/agriculture/agri_costofcultivation_rice.html.

UN Environment. 2020. One Planet Network: Sustainable Food Systems Programme. [en ligne]. [page web consultée le 13 mars 2020]. https:/ / www.oneplanetnetwork.org/sustainable-food-system.

URGENCI. 2016. Overview of Community-Supported Agriculture in Europe. Aubagne, Kernel Editions. 138 pp. (également disponible à https://urgenci.net/wp-content/uploads/2016/05/Overview-ofCommunity-Supported-Agriculture-in-Europe.pdf).

Vatin, François. 2013. Valuation as Evaluating and Valorizing. Valuation Studies Vol. 1 (1) 2013: 31.

Whitaker, Sarah, Marie Clotteau, Vlatko Andonovski, Joao Azevedo, \& Thomas Egger. 2017. Innovation and Circular Economy in the Mountain Forest Supply Chain: How to close the loop? Paris. 75 pp. (également disponible à http:/ / www.euromontana.org/wp-content/uploads/2017/03/ Innovation-and-Circular-Economy-in-the-Mountain-Forest-Supply-Chain_FINAL.pdf).

Willett, Walter, Johan Rockström, Brent Loken, Marco Springmann, Tim Lang, Sonja Vermeulen, Tara Garnett, et al. 2019. Food in the Anthropocene: the EAT-Lancet Commission on healthy diets from sustainable food systems. The Lancet 393 (10170): 447-492. 


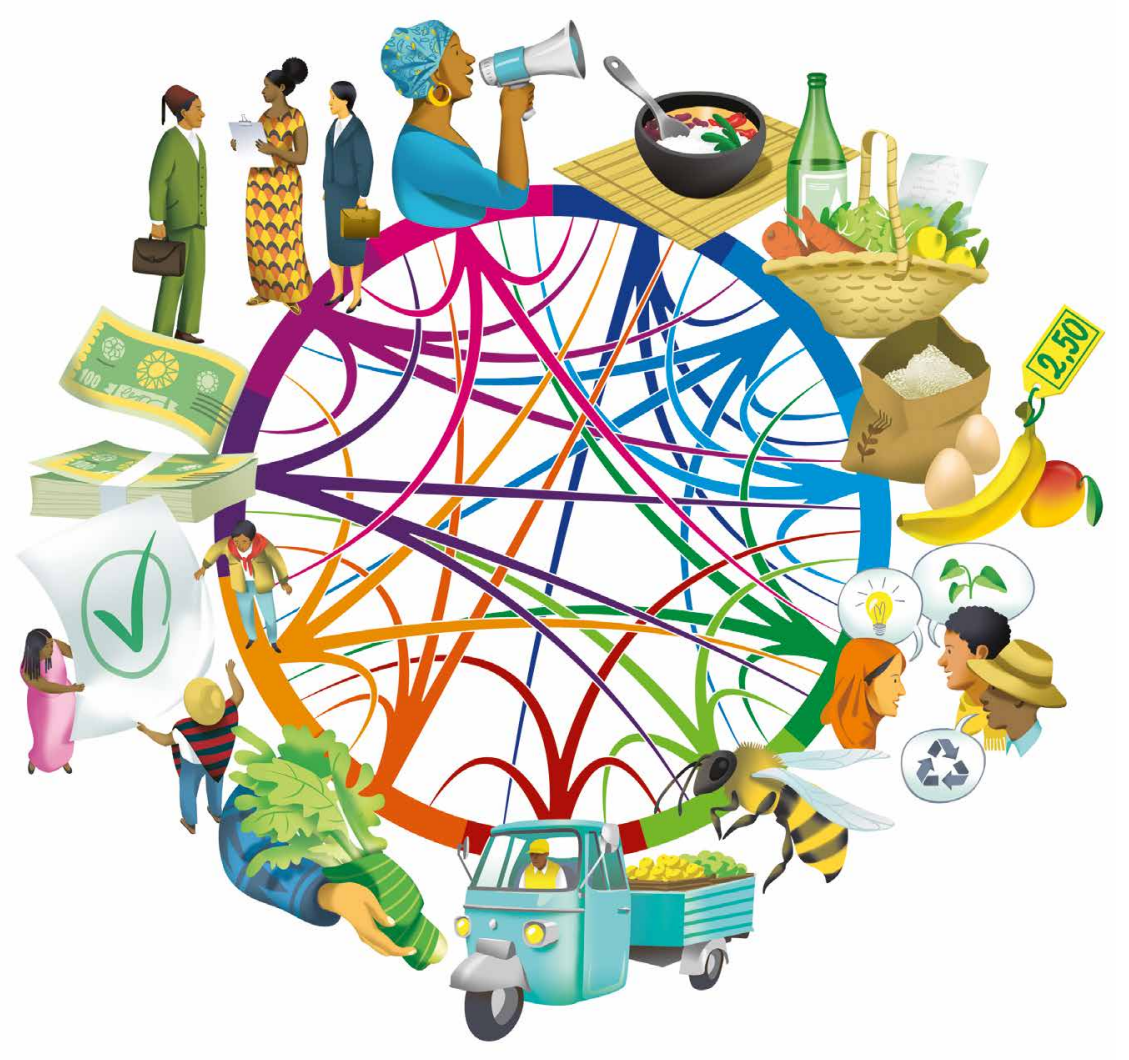


Les systèmes alimentaires durables sont

fondamentaux pour s'assurer que les

générations futures auront la sécurité

alimentaire et des régimes alimentaires sains.

Pour démarrer une transition vers des systèmes

durables, une grande partie des activités

doivent être reconçues, et une myriade

d'acteurs partout dans le monde ont déjà

commencé à mettre en oeuvre des changements

sur le plan local. Certains des changements

nécessaires sont plus faciles à mettre en oeuvre

que les autres; savoir naviguer dans ces eaux

incertaines afin de promouvoir des pratiques de consommation et production durables demande des compétences complexes.
Ce manuel est écrit pour des «innovateurs des systèmes alimentaires durables» par un groupe d'innovateurs provenant des quatre coins du monde (Asie, Afrique, Amériques et Europe) qui soutiennent des initiatives dans leurs communautés locales pour produire, partager, vendre et consommer des aliments durables.

Il inclut des expériences qui changent les structures organisationnelles de leurs systèmes alimentaires locaux afin de les rendre plus durables.

Ce manuel est organisé dans le format d'un «livre dont vous êtes le héros», chaque lecteur tout seul ou au sein d'un groupe - peut développer son propre chemin d'apprentissage et d'action selon ses intérêts. Les thèmes traités par ce manuel sont classés selon quatre types d'innovations organisationnelles, à savoir: mobiliser les consommateurs, produire durablement, mettre en marché les produits, et s'organiser équitablement.
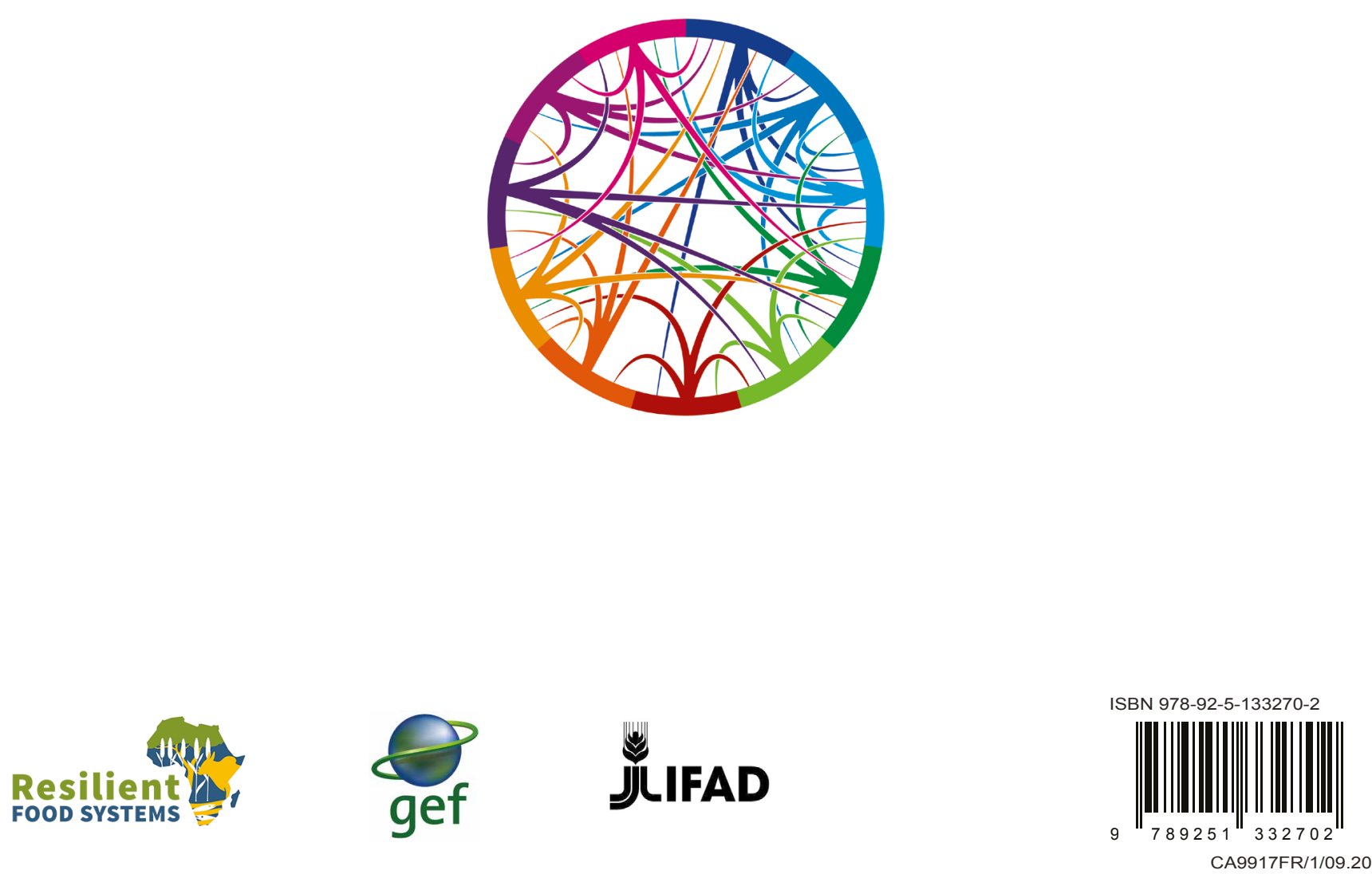